\title{
Section 7: Appendix
}

\section{Analyses}

$>$ Proposed Community Education/Outreach Plan

$>$ Solar Resource Assessment

$>$ Natural Gas Access

$>$ Preliminary Study: Laguna and Village Electric Usage

$>$ Potential Project Options

$>$ Fatal Flaw Results Summary

$>$ Solar Project Feasibility Update

$>$ Renewable Energy Development Plan

Community Information

$>$ Firming Renewable Generation with Natural Gas: A Technology Brief

$>$ Generating Electricity from the Sun: A Technology Brief

$>$ Generating Electricity from the Wind: A Technology Brief

$>$ Community Meeting

$>$ Renewable Energy Project Options

\section{Project Updates}

$>$ Study Update: Project Identification Meeting

$>$ POLUA Board of Directors: Status Report

$>$ POLUA Board and Entity Update

$>$ DOE Program Review October 2006

$>$ DOE Tribal Energy Program Review October 2007 


\section{Analyses}

$>$ Proposed Community Education/Outreach Plan

$>$ Solar Resource Assessment

$>$ Natural Gas Access

$>$ Preliminary Study: Laguna and Village Electric Usage

$>$ Potential Project Options

$>$ Fatal Flaw Results Summary

$>$ Solar Project Feasibility Update

$>$ Renewable Energy Development Plan 


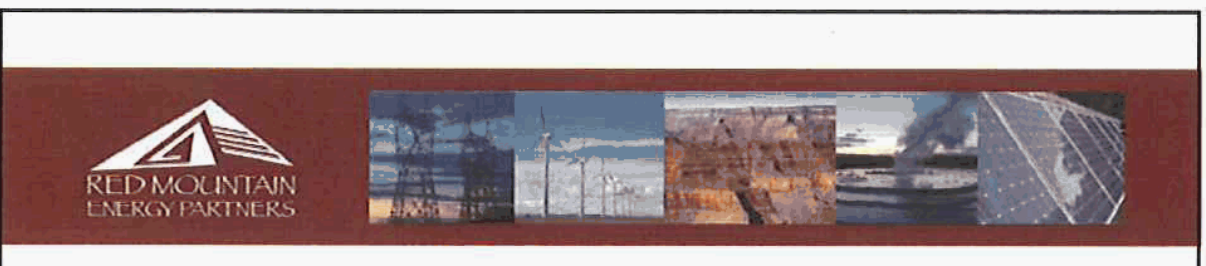

\section{Pueblo of Laguna \\ Renewable Energy Feasibility Study}

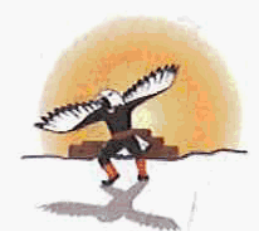

Proposed

Community Education/Outreach Plan

\section{February 2006}

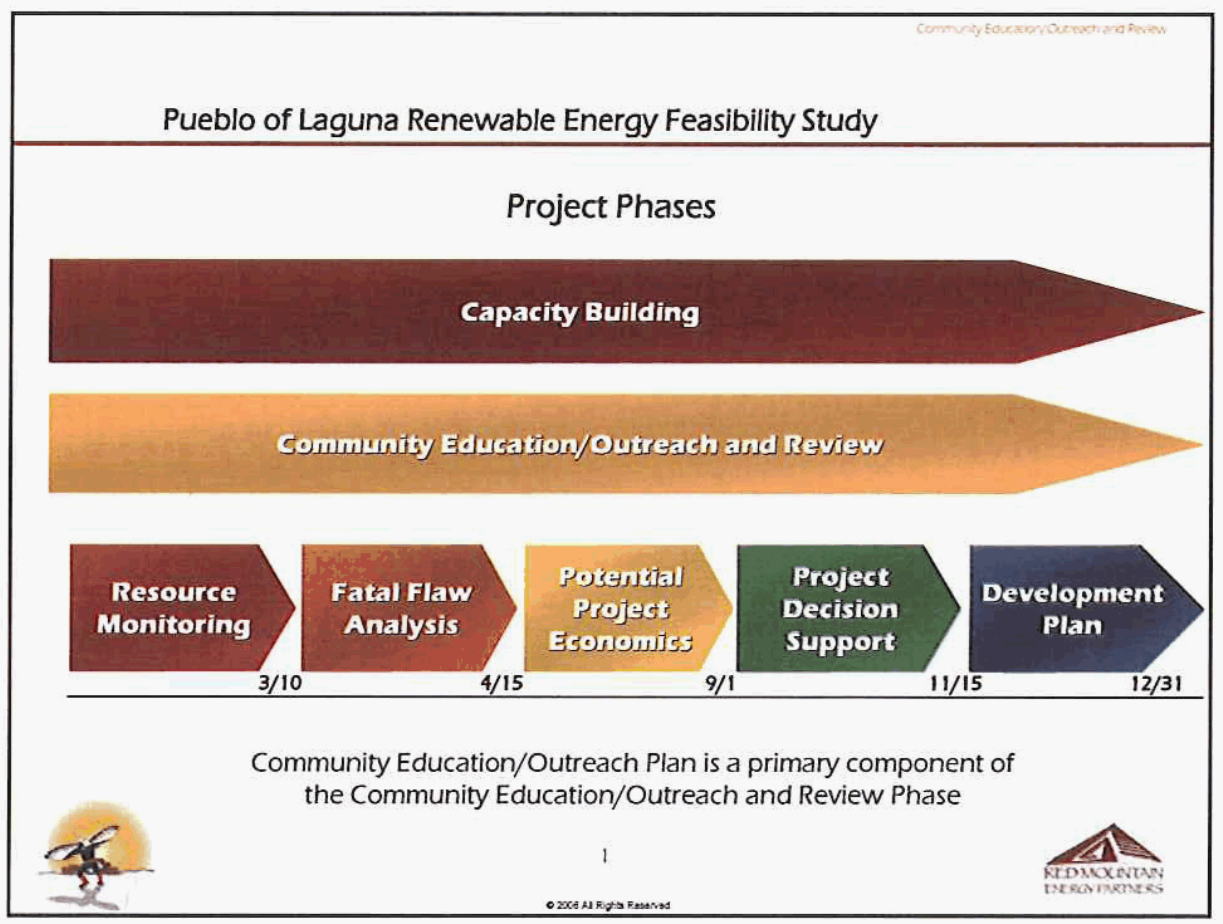


Pueblo of Laguna Renewable Energy Feasibility Study Project Objectives

- Increase energy knowledge and capacity in the Utility Authority and community

- Improve quality and reliability of electric service on the reservation

- Promote energy self-sufficiency

- Encourage economic development

- Contribute to environmentally clean energy

- Provide data needed to proceed with renewable energy development, as an owner or participant

- Provide for renewable and/or hybrid renewable generation to be a resource component in potential Utility Authority electric utility operations

- Pueblo of Laguna Background

- Community Education/Outreach Plan Purpose

- Community Education/Outreach Plan Components

$\therefore$ Utility Authority Newsletter

- Approach

- Style

- Sections

- Topics

- Preparation

- Distribution

$*$ Community Meetings

- Approach

- Content

- Agenda

- Participants

- Schedule 


\section{Pueblo of Laguna Background}

- Federally recognized Indian Tribe organized under the Indian Reorganization Act of 1934

- Project undertakes a feasibility study on Pueblo lands to develop renewable energy generation projects

- Ongoing Pueblo interest in renewable energy has focused on wind, solar and biomass resources

$\therefore$ Prior projects included solar facilities at Majors Ranch and Laguna Industries

- Feasibility Study designed to expand on previously completed studies

$*$ Utility formation

* Generation opportunities

* Energy self-sufficiency options

* Energy management integration with economic development

- Pueblo access to nearby gas pipelines could allow for a "hybrid" project, providing a "firm" power product

- Recent Utility Authority formation and operations could allow power generated to serve both Pueblo electric loads and wholesale power markets

- Renewable generation project development can have significant positive impacts

* Provide local generation to help resolve existing on-reservation power quality and reliability problems

$\therefore$ Create local short-term and long-term employment opportunities

$\therefore$ Promote energy self-sufficiency and economic development opportunities

- Provide the Laguna community (Utility Authority staff, Tribal staff and leadership and community members):

* Background information on utility service, energy, renewable and conventional generation issues

* Insights into Utility Authority infrastructure and service plans

* Insights into possible energy project types, locations, and economics

* Opportunities to provide input/ask questions about utility infrastructure/utility service plans and potential power project development plans

- Provide Pueblo of Laguna energy project development proponents:

* Insights into community utility service needs, desires and concerns

* Opportunities to generate support for project concepts and plans,

* Identification of a short-list of potential projects

* Greater assurance of successful project development 


\section{Utility Authority Newsletter Approach}

- Provide direct written communication from Utility Authority, and Pueblo of Laguna leadership to Laguna community

- Introduce Laguna community to a variety of utility and energy issues

- Provide background information, advance plans, and education on critical issues for members

- Each monthly issue will have a focus, supported by multiple articles

- Focus area will be identified as close to newsletter preparation/distribution timing as possible to ensure timeliness of topic

- Written so that members with no prior background can understand the topics and articles

- Articles will focus on customers and customer needs, where applicable

- Tone of articles to be respectful, and positive

- Look, feel and tone will be as "traditional" as possible 


\section{Utility Authority Newsletter Sections}

- Recurring sections to be included in each newsletter

$\therefore$ Lead-varies each issue

* Second lead - varies each issue

$\therefore$ Focus area - varies each issue

- Customer Corner - timely customer/senvice questions/answers

- Energy Lines - renewable energy, natural gas, propane, electric and energy management/use issues

$\therefore$ Water Reflections - water service, infrastructure updates

$\therefore$ Waste Collections - Wastewater, septic, and solid waste updates

* Employee Focus - feature on Utility Authority employees

$\therefore$ GM Message - timely topics from Utility Authority leadership

- Topics ideas identified for 2006 in monthly "units"

$\therefore$ "Unit" components can be modified, or entire "units" interchanged

- Focus Areas

* Utility Rates

* Water Services

- Energy Costs

* Solid Waste Services

-Wastewater/Septic Services

* Renewable Energy

- Telecom Services

$*$ Energy Efficiency

* Water System Improvements

- Propane/Natural Gas

* Rate and Service Changes

* Utility System Improvement Plans 
Utility Authority Newsletter Topics

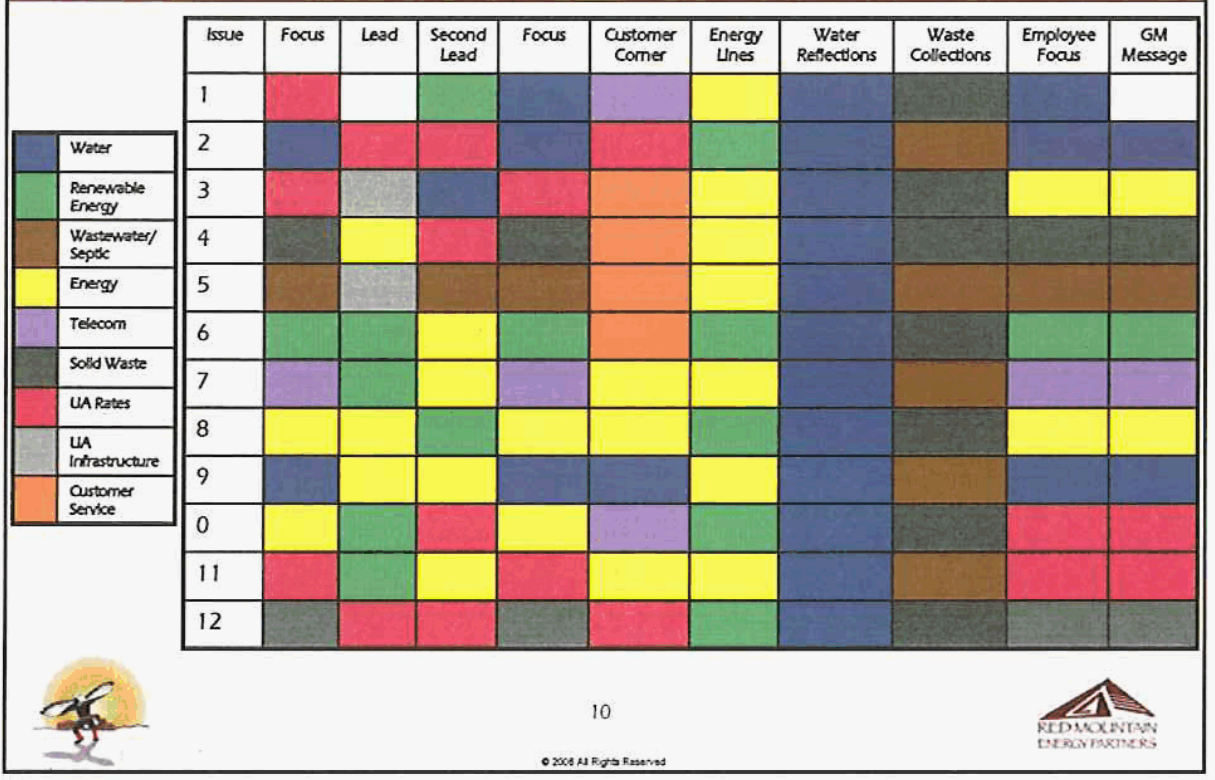

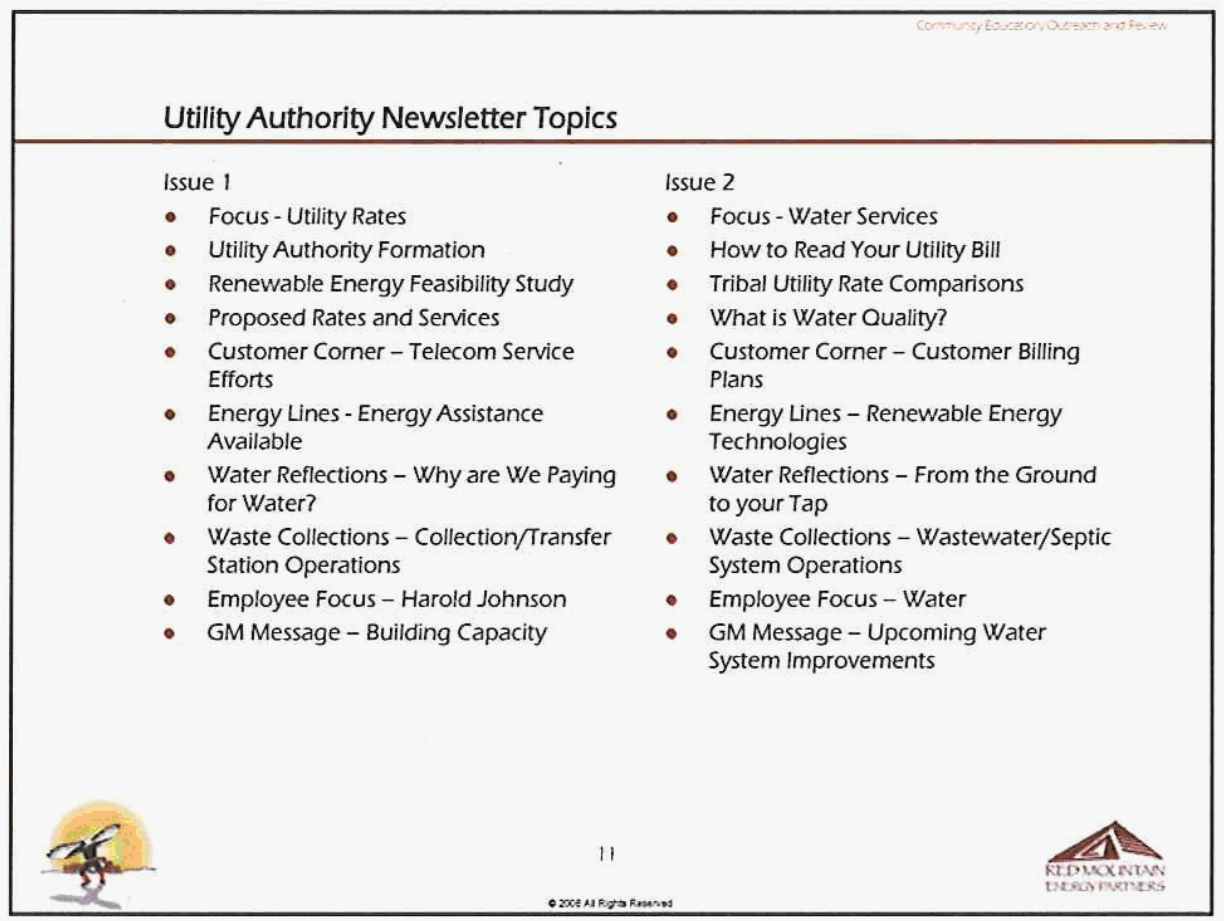




\section{Utility Authority Newsletter Topics}

Issue 3

- Focus - Energy Costs

- System Upgrades Planned

- Water System Priorities

- What You can do to Reduce Energy Costs

- Customer Corner - Billing and Customer Service Policies

- Energy Lines - How Do I Read My Electric Bill?

- Water Reflections - Planned Water System Improvements

- Waste Collections - Focus on Recycling

- Employee Focus - Energy

- GM Message - Increasing Energy Costs - What You Can Do
Issue 4

- Focus - Solid Waste Services

- Electric Service Reliability

- Water Service Rate Comparisons

- Transfer Station Operations

- Customer Corner - Customer Payment Plans

- Energy Lines - Energy Efficiency Tips

- Water Reflections - Making Water Service Available

- Waste Collections - How Recycling can Lower Solid Waste Costs

- Employee Focus - Transfer Station

- GM Message - Impact of Waste Carrier Negotiations
Issue 5

- Focus - Wastewater/Septic Services

- Laguna Infrastructure Construction Plans

- Wastewater/Septic Plans

- Wastewater/Septic System Description

- Customer Corner - Customer Support for non-UA Services

- Energy Lines - What to do when your electricity goes off

- Water Reflections - The Water Cycle

- Waste Collections - Septic System Tips

- Employee Focus - Wastewater/Septic

- GM Message - Challenges for Septic System Users issue 6

- Focus - Renewable Energy

- Community Input Sought for Renewable Energy Projects

- Nationwide Gas and Electric Cost Increases

- Renewable Energy Options at Laguna

- Customer Corner - What if I can't pay my bill?

- Energy Lines - Renewable Energy Impacts on Costs of Electricity

- Water Reflections - Water Issues in New Mexico

- Waste Collections - Special Transfer Station Programs

- Employee Focus - Renewable Energy

- GM Message - Renewable Energy Fit with Laguna Culture 
Utility Authority Newsletter Topics

Issue 7

- Focus - Telecom Services

- Community Inputs on Renewable Energy Projects

- Projected Energy Prices

- Telecom Infrastructure Project Plans

- Customer Corner - Impact on Energy Bills of Improving Energy Efficiency

- Energy Lines - Requesting Natural Gas Service

- Water Reflections - Water System Construction Update

- Waste Collections - Wastewater System Improvements Planned

- Employee Focus - Telecom

- GM Message - Telecom History at Laguna
Issue 8

- Focus - Energy Efficiency

- Energy Price Forecasts

- Renewable Energy Study Update

- Weatherization Grants Available

- Customer Corner -

Weatherization/LIHEAP for LowIncome Households

- Energy Lines - Native American Wind Energy Program

- Water Reflections - Water System Improvement Funding

- Waste Collections - Transfer Station Use Suggestions

- Employee Focus - Energy Efficiency

- GM Message - Energy Efficiency vs. Payment Assistance
Issue 9

- Focus - Water System Improvements

- What You Can do to Keep Energy Costs Down

- Propane Feasibility Study Update

- Water System Update

- Proposed Rates and Services

- Customer Corner - Water Bills as a Percentage of Income Comparison

- Energy Lines - Propane Distribution Plans

- Water Reflections - Village Water System Improvement Plans

- Waste Collections - Wastewater System Improvement Funding Sources

- Employee Focus - Water

- GM Message - Water Conservation
Issue 10

- Focus - Propane/Natural Gas

- Renewable Energy Self-Generation Options

- How Rates are Developed

- Propane Feasibility Study Results

- Customer Corner - Telecom Service Plans

- Energy Lines - Role of Renewable Energy

- Water Reflections - Water Metering Plans

- Waste Collections - Waste Collection Route Changes

- Employee Focus - Propane/Natural Gas

- GM Message - Propane Study Impacts on Customer Costs 
Utility Authority Newsletter Topics

Issue 11

- Focus - Rate and Service Changes

- Renewable Energy Study Outcomes

- Energy Price Forecast Update

- Upcoming Rate and Service Changes

- Customer Corner - Energy Bills as a Percentage of Income

- Energy Lines - Update on UA Energy Service Plans

- Water Reflections - Water and Power

- Waste Collections - Comparison of NM Wastewater/Septic Rates

- Employee Focus - Rates

- GM Message - Purpose of Rate Phase In
Issue 12

- Focus - Utility System Improvement Plans

- New Rates Effective January 1

- Utility Authority Funding Sources

- 2006 Utility Infrastructure Improvement Plans

- Customer Corner - Utility Bill Changes

- Energy Lines - Renewable Energy Project Development Process

- Water Reflections - Water Issues in the West

- Waste Collections - Planned Transfer Station Changes

- Employee Focus - Utility System Improvements

- GM Message - Importance of System Planning

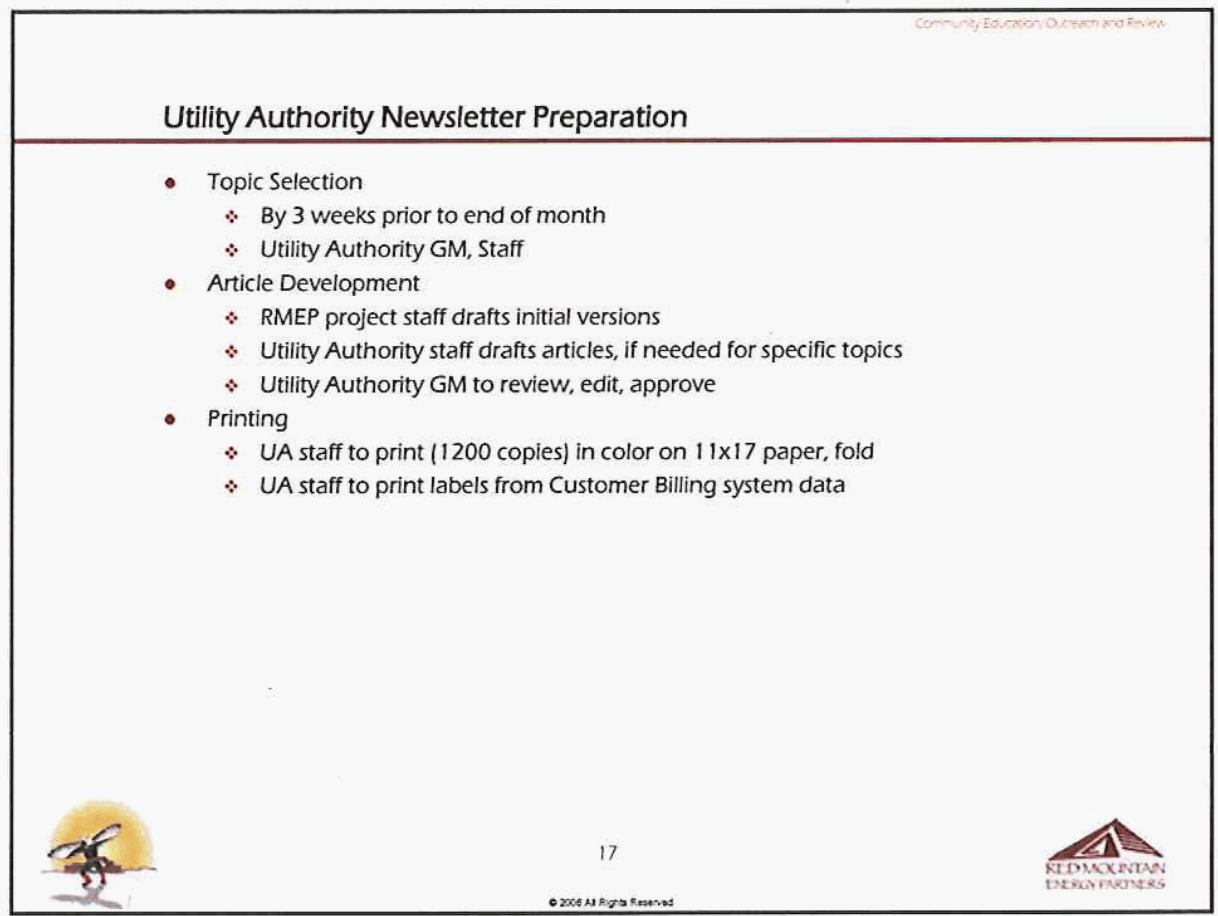




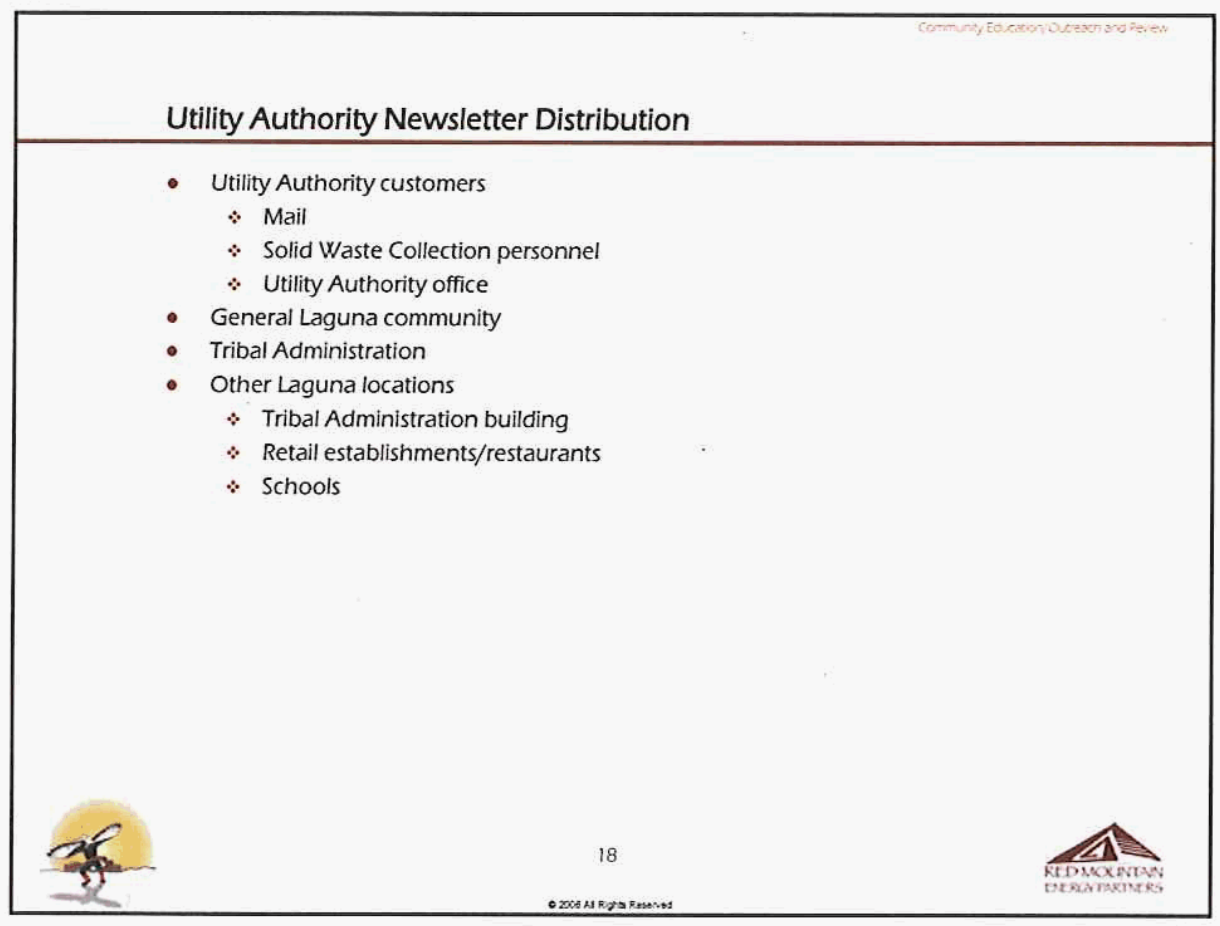

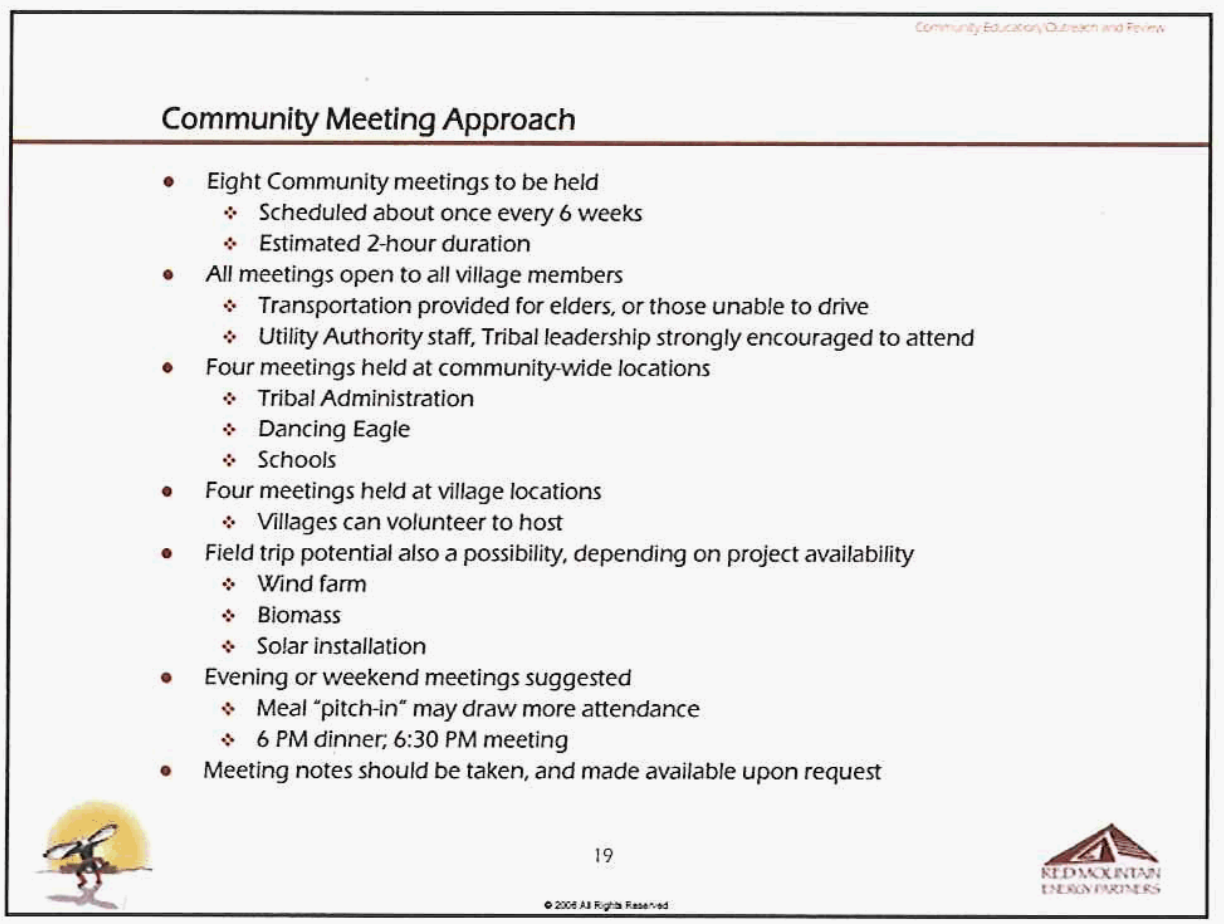



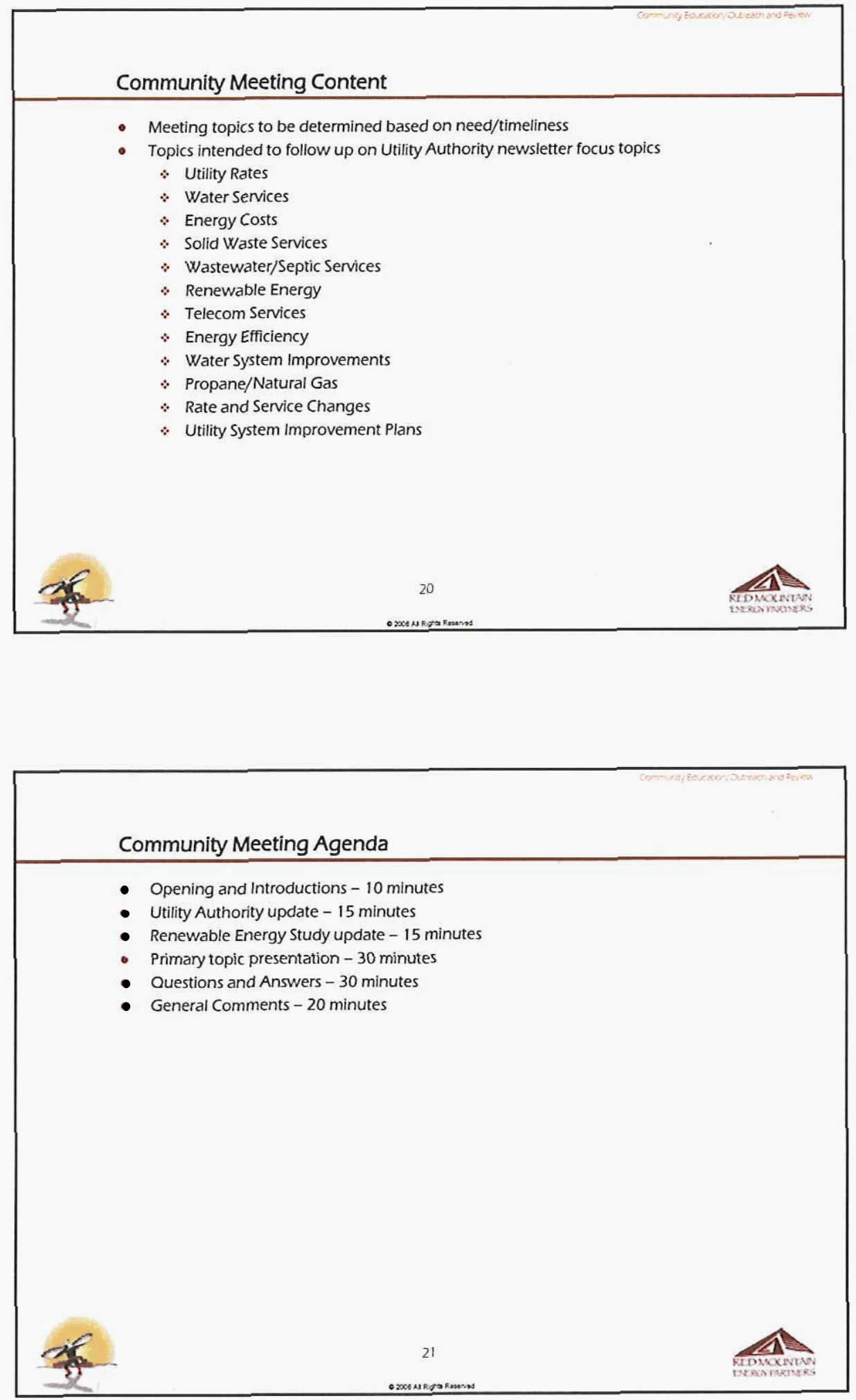


\section{Community Meeting Participants}

- Utility Authority General Manager

- Utility Authority Staff

- Utility Authority Board of Directors, Committees

- Tribal leadership

- Village officers

\section{Community Meeting Schedule}

\begin{tabular}{|c|c|c|c|c|}
\hline Newsletter Focus & $\begin{array}{l}\text { Target Meeting } \\
\text { Completion Date }\end{array}$ & Meeting Lead Toplc & $\begin{array}{l}\text { Renewable Energy } \\
\text { Content }\end{array}$ & Location \\
\hline \multicolumn{5}{|l|}{ Utilty Rates } \\
\hline Water Services & March 10 & UA Rates & RE Project Description & Community \\
\hline Energy Costs & April 15 & Water Ouality & RE Resources Identifed & Viliage \\
\hline Sold Waste Services & May 31 & Energy Pricing & RE Technologles & Community \\
\hline $\begin{array}{l}\text { Wastewater/Septic } \\
\text { Services }\end{array}$ & July 15 & $\begin{array}{l}\text { Renewable Energy Project } \\
\text { input }\end{array}$ & RE Projects identified & Community \\
\hline Renewable Energy & August 30 & infrastructure Pians & $\begin{array}{l}\text { RE Project feasibility } \\
\text { Update }\end{array}$ & Village \\
\hline Telecom Services & September 30 & UA Services Expansion & $\begin{array}{l}\text { RE Profect Feasibility } \\
\text { Update }\end{array}$ & Village \\
\hline Energy Efficiency & Novernber 15 & $\begin{array}{l}\text { Energy Efficiency/ } \\
\text { Weatherization }\end{array}$ & RE Projects Proposed & Village \\
\hline $\begin{array}{l}\text { Water System } \\
\text { Improvements }\end{array}$ & December 31 & $\begin{array}{l}\text { Renewable Energy Project } \\
\text { Pians }\end{array}$ & $\begin{array}{l}\text { RE Project Approval } \\
\text { Status }\end{array}$ & Community \\
\hline \multicolumn{5}{|l|}{ Propane/Natural Gas } \\
\hline \multicolumn{5}{|l|}{ Rate and Service Changes } \\
\hline \multicolumn{5}{|l|}{$\begin{array}{l}\text { Utility System } \\
\text { improvement Pians }\end{array}$} \\
\hline & & 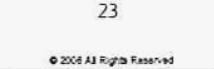 & & \\
\hline
\end{tabular}



Pueblo of Laguna

Renewable Energy Feasibility Study

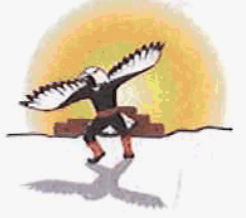

Solar Resource Assessment

February 2006

Solar Resource Assessment Outline

- Overview of Solar Energy Technology Options

$\therefore$ Residential \& Commercial (Distributed) Solar Systems

- Photovoltaic (PV) Modules

- Tracking Systems

$\therefore$ Large-Scale (Central Station) Solar Systems

- Power Tower

- Parabolic Trough

- Dish-Engine

- High Concentration Photovoltaics (HCPV)

- Solar Resource on the Pueblo

* Fixed-Tilt Flat Plate PV Measurements

* Two-Axis Tracking Concentrator Measurements

- Technology Fit with Solar Resource on the Pueblo 

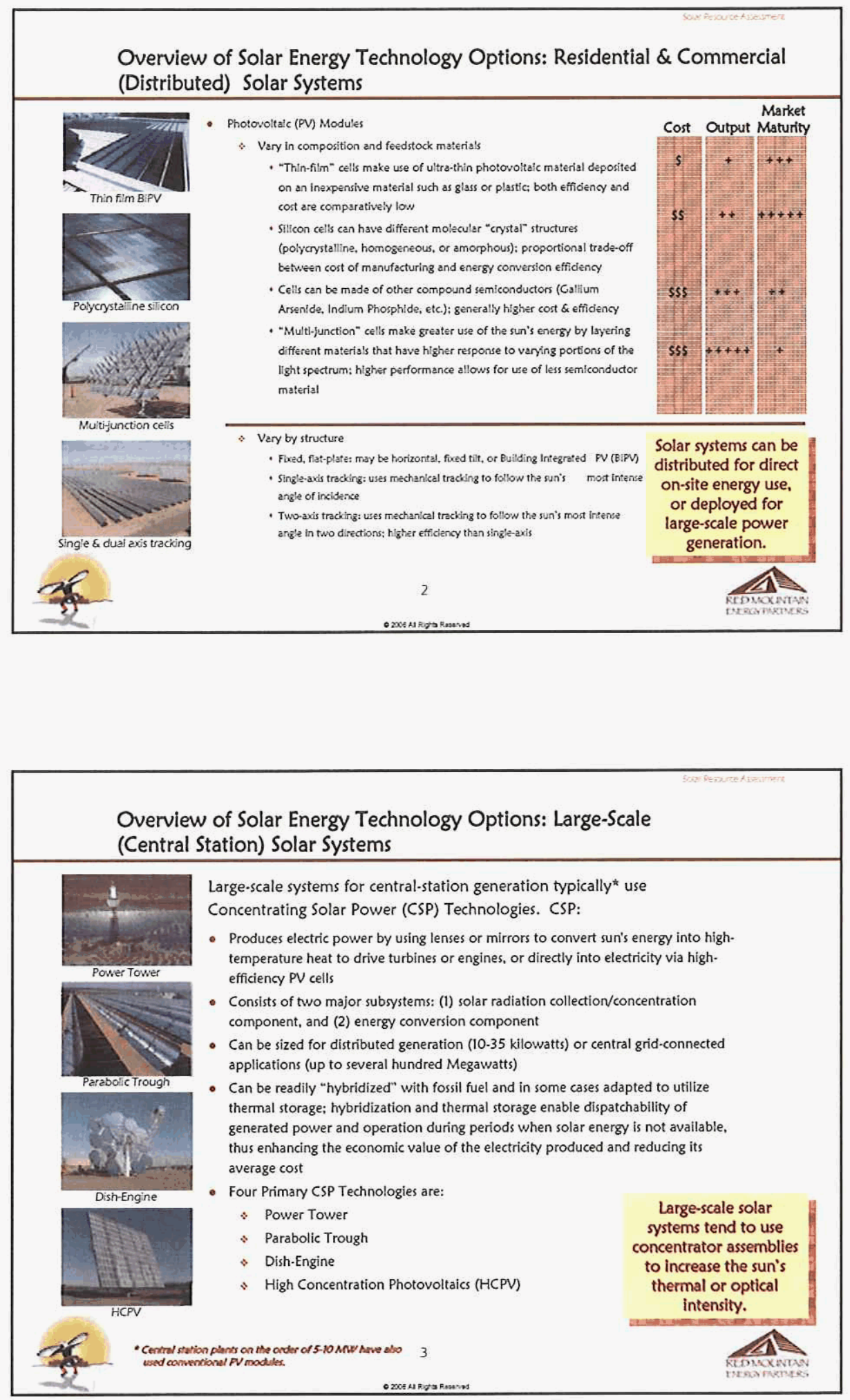


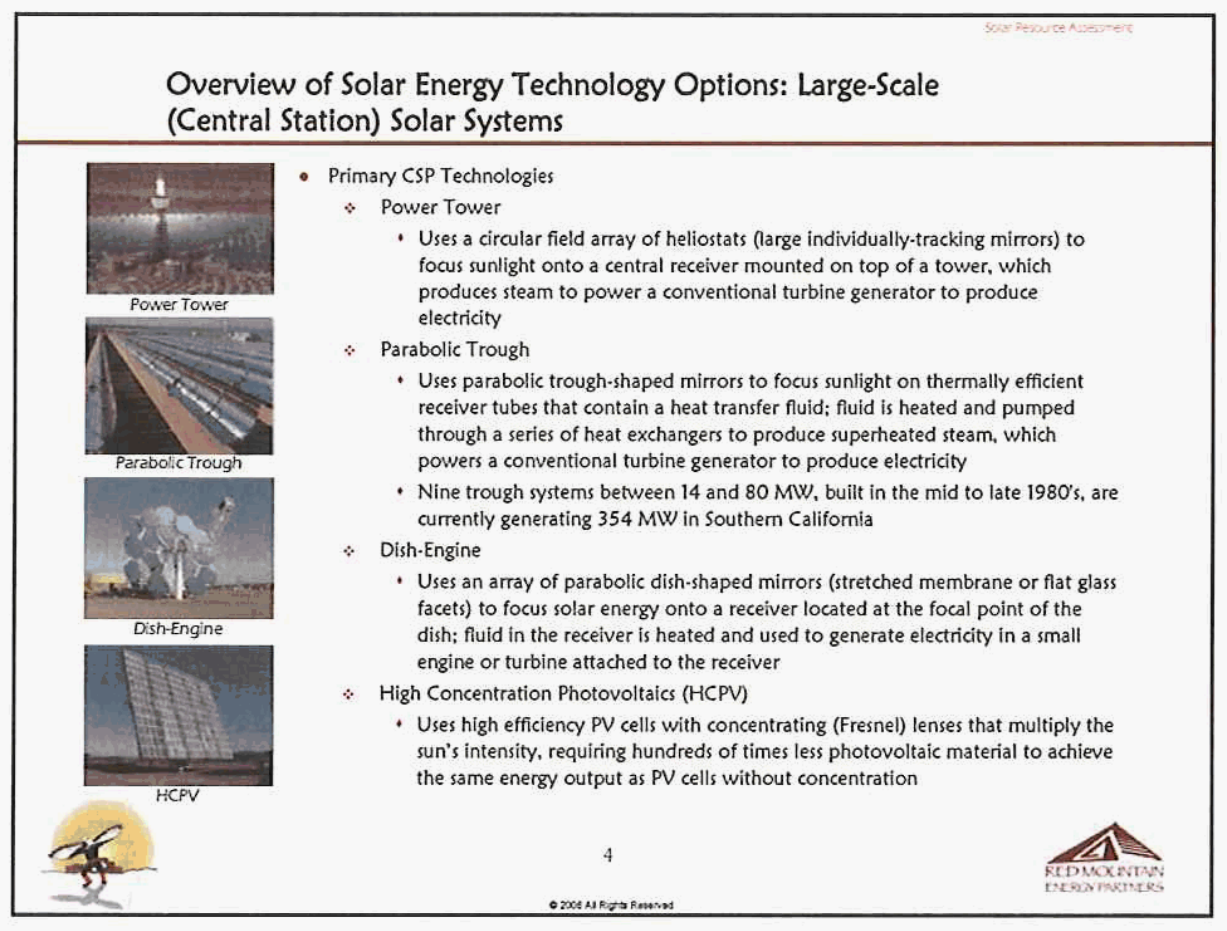

\begin{tabular}{|c|c|c|c|}
\hline \multicolumn{4}{|c|}{$\begin{array}{l}\text { Overview of Solar Energy Technology Options: Comparisons of Cost and } \\
\text { Performance }\end{array}$} \\
\hline \multicolumn{4}{|c|}{$\begin{array}{l}\text { Examination of kWh delivered per dollar expended is useful in considering technology investment } \\
\text { decisions. Some solar technologies may have lower first-costs, but also much lower performance, and } \\
\text { thus longer payback periods. }\end{array}$} \\
\hline Installation & Cost (S/Natt) & $\begin{array}{l}\text { Performance } \\
(\mathrm{kW} / \mathrm{h} / \mathrm{k} \mathrm{W} / \mathrm{yr})\end{array}$ & $\mathrm{kWh} / \mathrm{s}$ \\
\hline Fixed-Horizontal & 5.25 & 1,250 & 4.75 \\
\hline Flxed-Latitude & 5.25 & 1,630 & 6.20 \\
\hline Tracking - Horizontal & 5.50 & 2,350 & 8.55 \\
\hline Tracking - Latitude & 6.50 & 2,450 & 8.25 \\
\hline HCPV & 6.00 & 2,030 & 6.75 \\
\hline Parabolic Trough & 4.00 & 2,000 & 10.00 \\
\hline HCPV - Future & 3.00 & 2,400 & 16.00 \\
\hline Dish/Turbine - Future & 2.50 & 2,400 & 19.20 \\
\hline \multicolumn{4}{|c|}{ 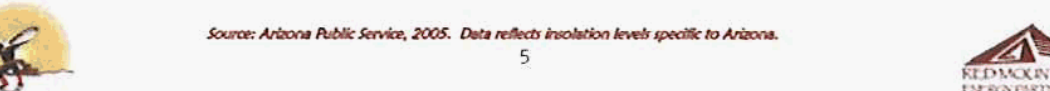 } \\
\hline
\end{tabular}




\section{Overview of Solar Energy Technology Options: Market Maturity}

Crystalline sillicon PV technology has experienced the most market penetration relative to other solar technologies. Dish engine systems may achieve significant market reach in the near term, pending the outcome of power purchase agreements currently being negotiated in California.

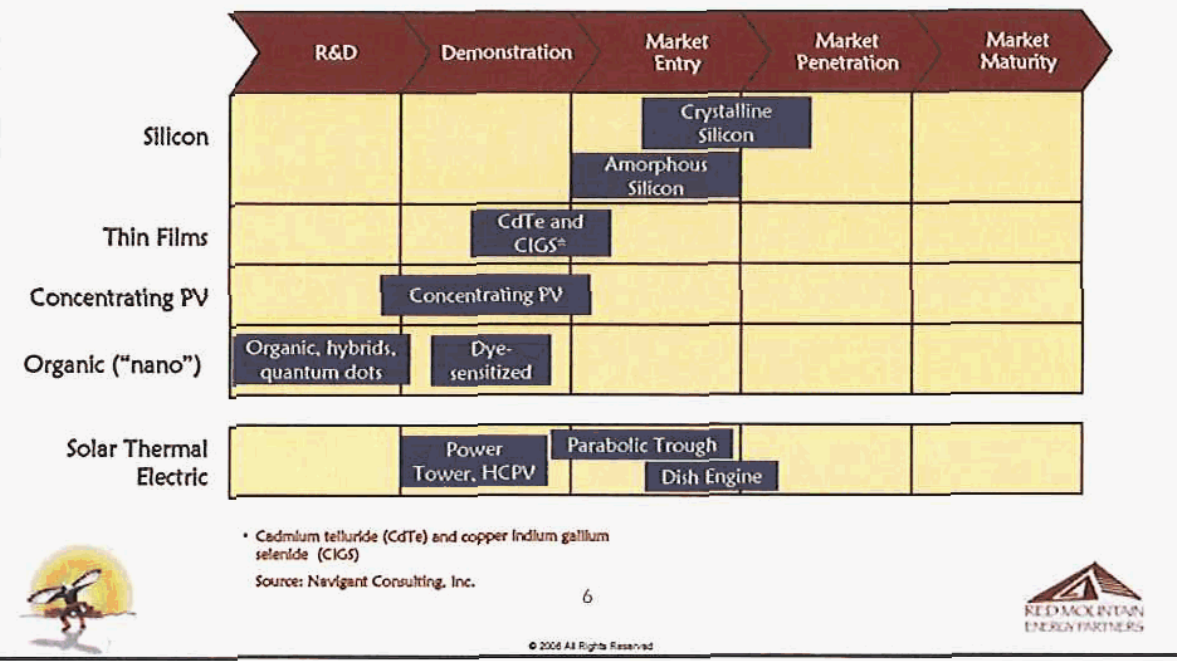

Solar Resource on the Pueblo: New Mexico's Direct Normal Solar Radiation

Solar resource, or "insolation", is measured

in different ways. Flat

plate collectors are used

to measure insolation

levels relevant for PV

systems. Average annual

insolation on the Pueblo

falls in the range of 7.25

$-7.5 \mathrm{kWh} / \mathrm{m}^{2} / \mathrm{day}$.

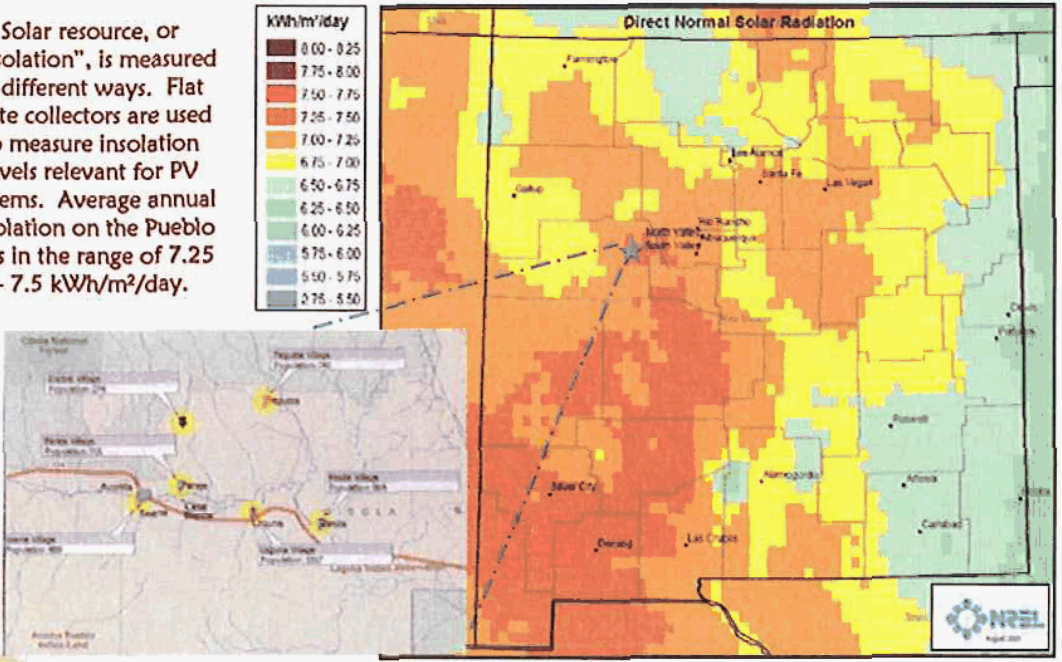

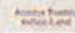



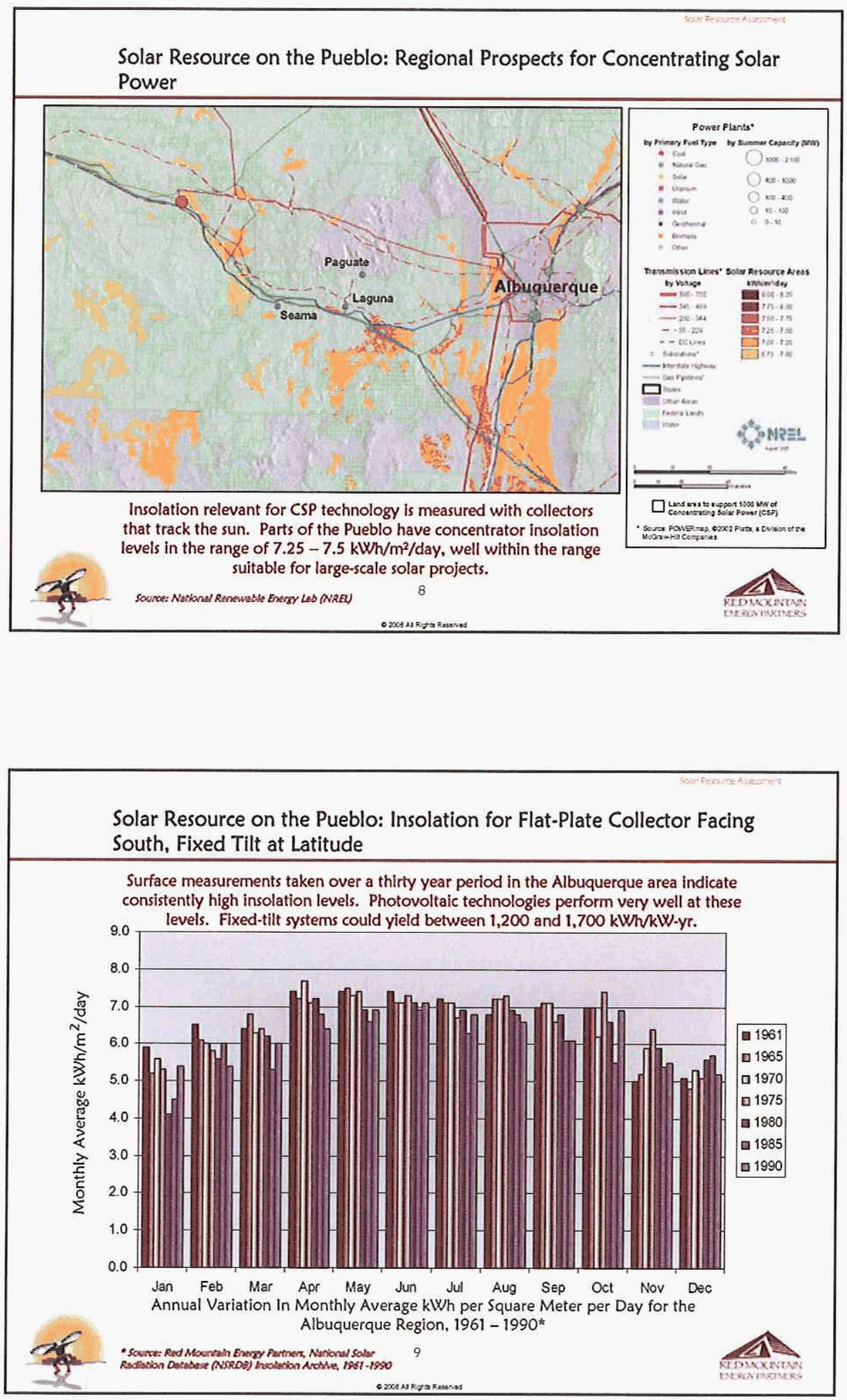

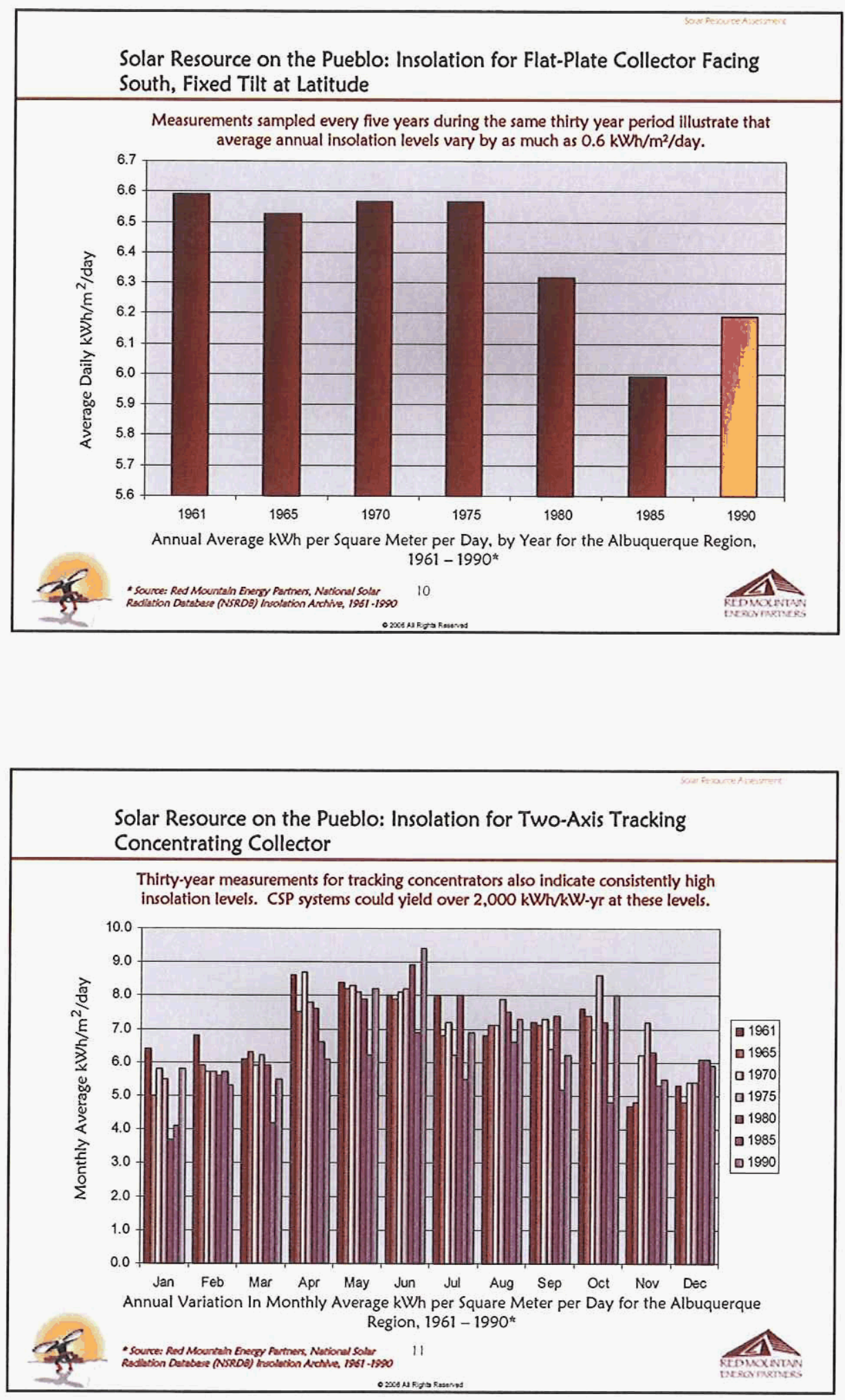


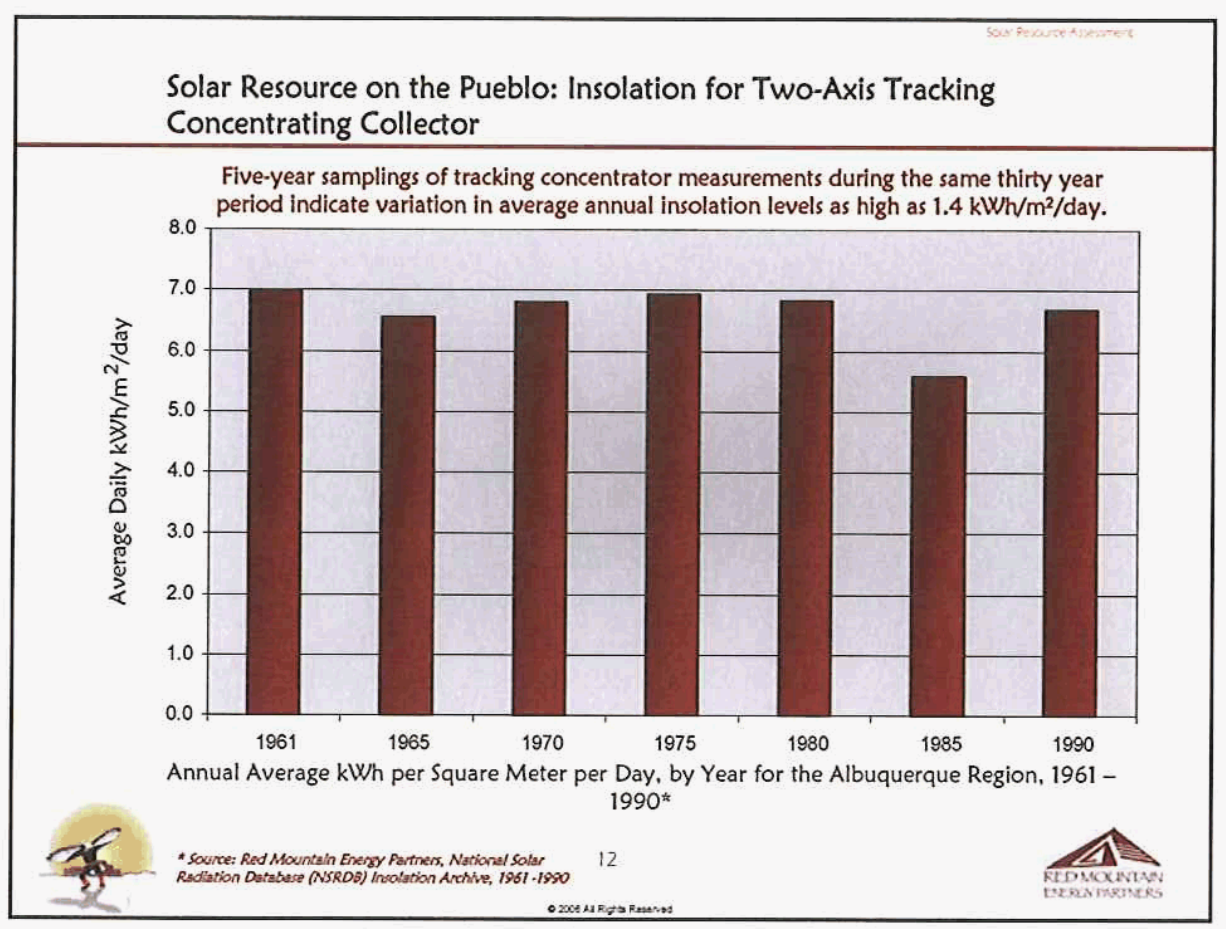

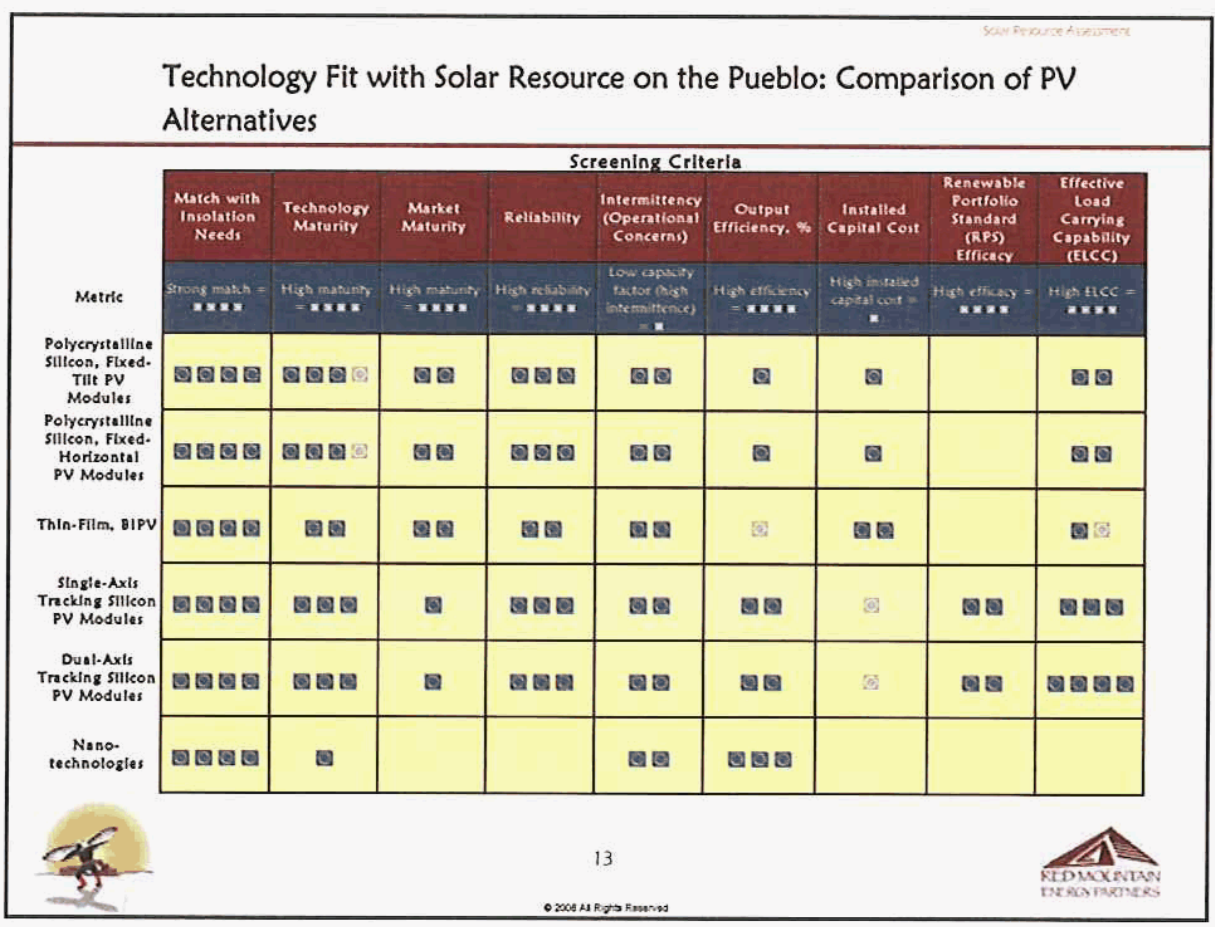




\begin{tabular}{|c|c|c|c|c|c|c|c|c|c|}
\hline \multicolumn{10}{|c|}{$\begin{array}{l}\text { Technology Fit with Solar Resource on the Pueblo: Comparison of CSP } \\
\text { Alternatives }\end{array}$} \\
\hline & \multicolumn{9}{|c|}{ Screening Criteria } \\
\hline & $\begin{array}{l}\text { March with } \\
\text { Insolution } \\
\text { Needs }\end{array}$ & $\begin{array}{l}\text { Technology } \\
\text { Maturily }\end{array}$ & $\begin{array}{l}\text { Matket } \\
\text { Maturity }\end{array}$ & Relinblity & $\begin{array}{l}\text { Intermititengy } \\
\text { (Opertionst } \\
\text { Concerms) }\end{array}$ & Eurpert: & $\begin{array}{l}\text { Intaled } \\
\text { Copital cost }\end{array}$ & 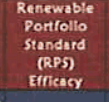 & 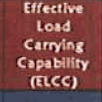 \\
\hline $\begin{array}{l}\text { Matric or } \\
\text { Welghthg } \\
\text { couffictent }\end{array}$ & 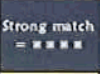 & $\begin{array}{l}\text { Hizb } \\
\text { maturify }= \\
\text { wa.n. }\end{array}$ & 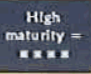 & $\begin{array}{l}\text { High } \\
\text { retiability }= \\
\text { Nen. }\end{array}$ & 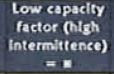 & $\begin{array}{l}\text { High } \\
\text { efriciency- } \\
\text { "rin. }\end{array}$ & $\begin{array}{l}\text { High instilied } \\
\text { clopial cost = } \\
\text {. }\end{array}$ & High efficas & $\begin{array}{l}\text { High BICC } \\
=\ldots \times \text {. }\end{array}$ \\
\hline $\begin{array}{l}\text { High } \\
\text { Concentrotion } \\
\text { PV (HCPV). } \\
\text { Sileon }\end{array}$ & 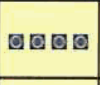 & 凹 & 目 & 回日 四 & 回回 & 回 & 回 & 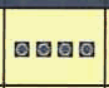 & 回回回 \\
\hline $\begin{array}{c}\text { HCPV. Multt- } \\
\text { Junction PV } \\
\text { Ceflis }\end{array}$ & 回回回 & 曰圂 & 回 & 回回 & 回 & 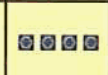 & 回 & 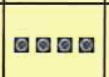 & 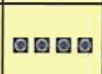 \\
\hline $\begin{array}{c}\text { Dits-Engine } \\
\text { Syrems }\end{array}$ & 日回回 & 밈ㅁㅁ & 日回? & 回回 & 밈 & 曰回回 & 回回回 & 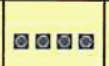 & 曰曰回曰 \\
\hline $\begin{array}{l}\text { Dish-Turbine } \\
\text { with Storase }\end{array}$ & 曰回回回 & & & & 回回 & 回回回回 & g & 回回回 & 回日回 \\
\hline $\begin{array}{l}\text { Parabolle } \\
\text { Trough }\end{array}$ & 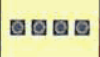 & 임임임 & 回圆 & 回回回 & 回回 & 빔임 & 回回圆 & 回圆圆圆 & 圆圆圆 \\
\hline Power Tower & 回回回 & 回回 & ह & 回圆 & 回回 & 回回圆 & 回 & 回圆圆 & 回圆圆圂 \\
\hline & & & & & 14 & & & & \\
\hline
\end{tabular}

Technology Fit with Solar Resource on the Pueblo: Comparison of CSP Alternatives

Observations

- Technology selections for any project should be weighed against considerations specific to the Tribe's needs and objectives

* Insolation levels in and around the Pueblo appear to be well-suited for most solar technologies

$\therefore$ Large scale systems are a better fit if RPS Efficacy, and sales of Renewable Energy Credits (RECs) are a goal

$\therefore$ For distributed generation applications, ELCC is an important criterion in determining a system's payback period

* Output efficiency and installed capital costs must be examined by pro forma analysis to understand the true costs and revenue potential of produced energy

* Intermittency and operational concerns are relevant to both distributed (on and off-grid) and centralized projects 
Technology Fit with Solar Resource on the Pueblo: Comparison of CSP Alternatives

Observations, continued:

- Insolation levels in and around the Pueblo villages are clearly well-suited to support distributed and centralized photovoltaic applications

- Cooler temperatures in the high-desert region yield higher operating efficiency for photovoltaics

- Insolation appears to be sufficient for larger scale CSP technologies, but actual site measurements would be required to support project investment decisions

$\therefore$ Project economics for installations in even higher insolation areas are highly dependent upon financing, project structure, enabling incentives, etc.

* As with any "free fuel" renewable project, annual kWh produced and sold will make or break project profitability, and thus accurate resource characterization is critical

* Technology advances and volumetric deployments are expected to bring capital costs down in the next several years 


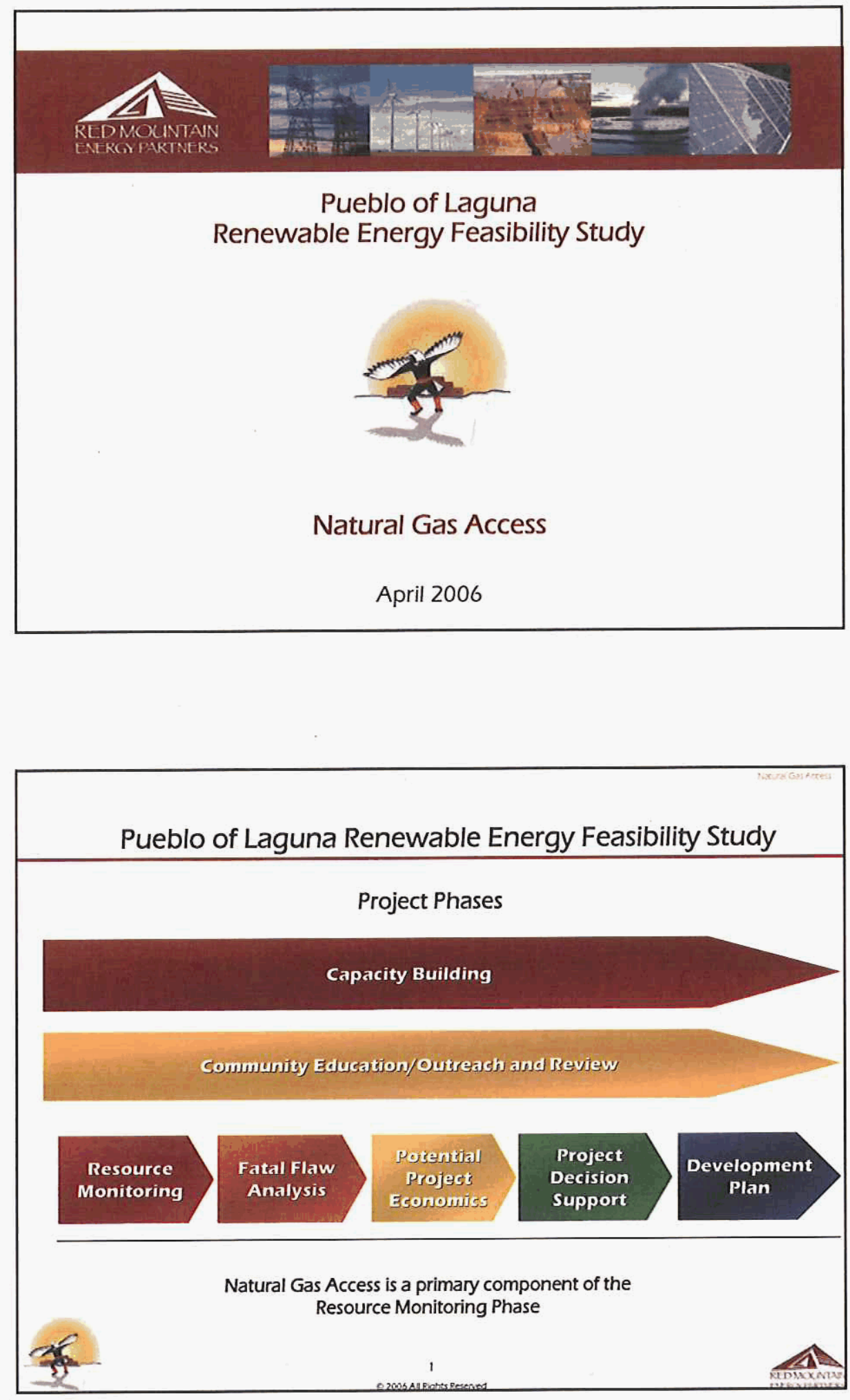


\section{Report Outline}

- Natural gas basics

- Pueblo of Laguna natural gas access

$\therefore$ Interstate pipelines

* PNM distribution system access

- Other potential natural gas access

- Natural gas supply sources

- Natural gas prices

- Natural gas applications

- Potential natural gas projects

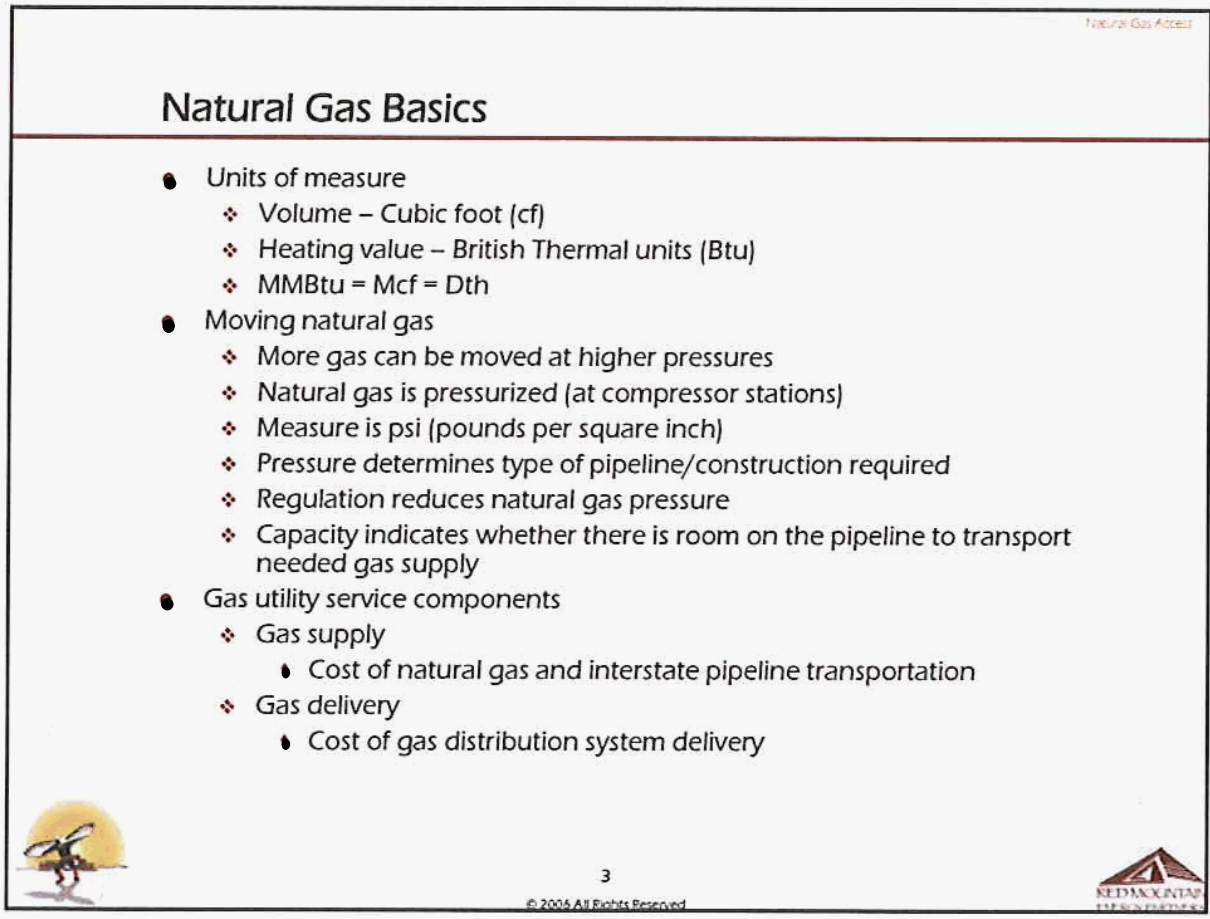




\section{Natural Gas Basics}

- Delivery system

* Gathering system (wellhead to transmission)

* Transmission system (gathering to utility distribution

- Interstate (across multiple states)

- Intrastate (within a single state)

* Distribution (transmission to service)

- Utility delivery system

- Connects

* Service

- Utility line to home/business

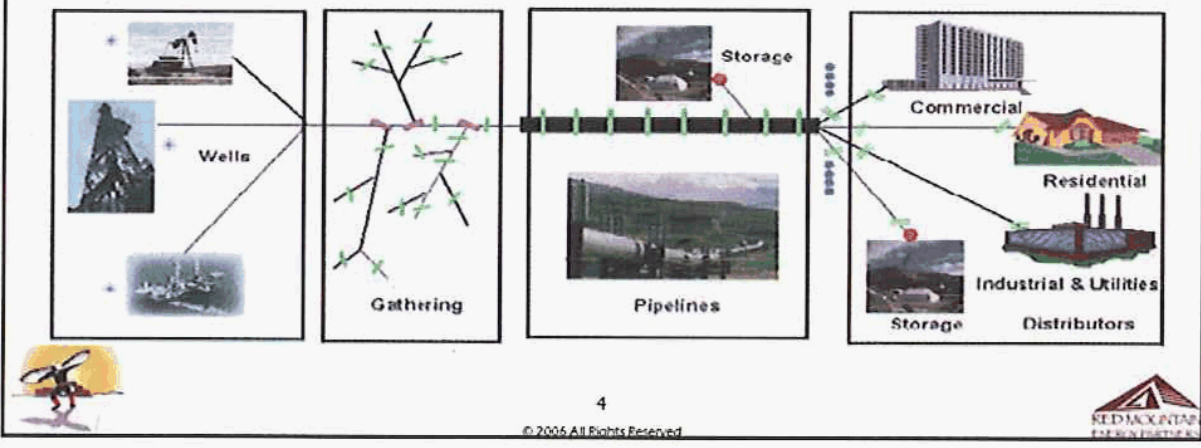

\section{Natural Gas Basics}

- Rate components

* Delivery Charge

- Reflects the cost of moving the gas from the citygate (interconnection with interstate pipeline company) to customer's meter

- Gas utility margin is included

* Gas Supply Charge

- Reflects the costs of gas supplies (gas commodity and pipeline transportation/capacity) purchased on interstate pipelines or from local production

- No gas utility margin is added

- Pipeline transportation/capacity price is set by FERC

- Commodity price is set by the marketplace; utilities use hedging instruments to moderate price volatility

- Prices typically adjusted monthly and reconciled annually
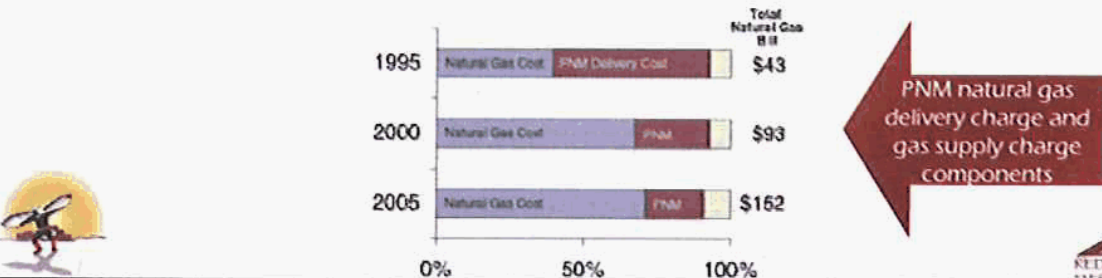

$0 \%$

$50 \%$

$100 \%$ 


\section{Pueblo of Laguna Natural Gas Line Access}

- Interstate pipelines on Laguna provide interconnection potential for very high-volume natural gas use

- TransWestern Pipeline

* El Paso Natural Gas Pipeline

- PNM transmission/distribution system service on portions of Laguna provides access for industrial. commercial, government and residential access

- Next step activities to include review of pipeline agreements and any provisions regarding rights to tap line(s), other provisions which could make interconnection cost prohibitive or cost effective

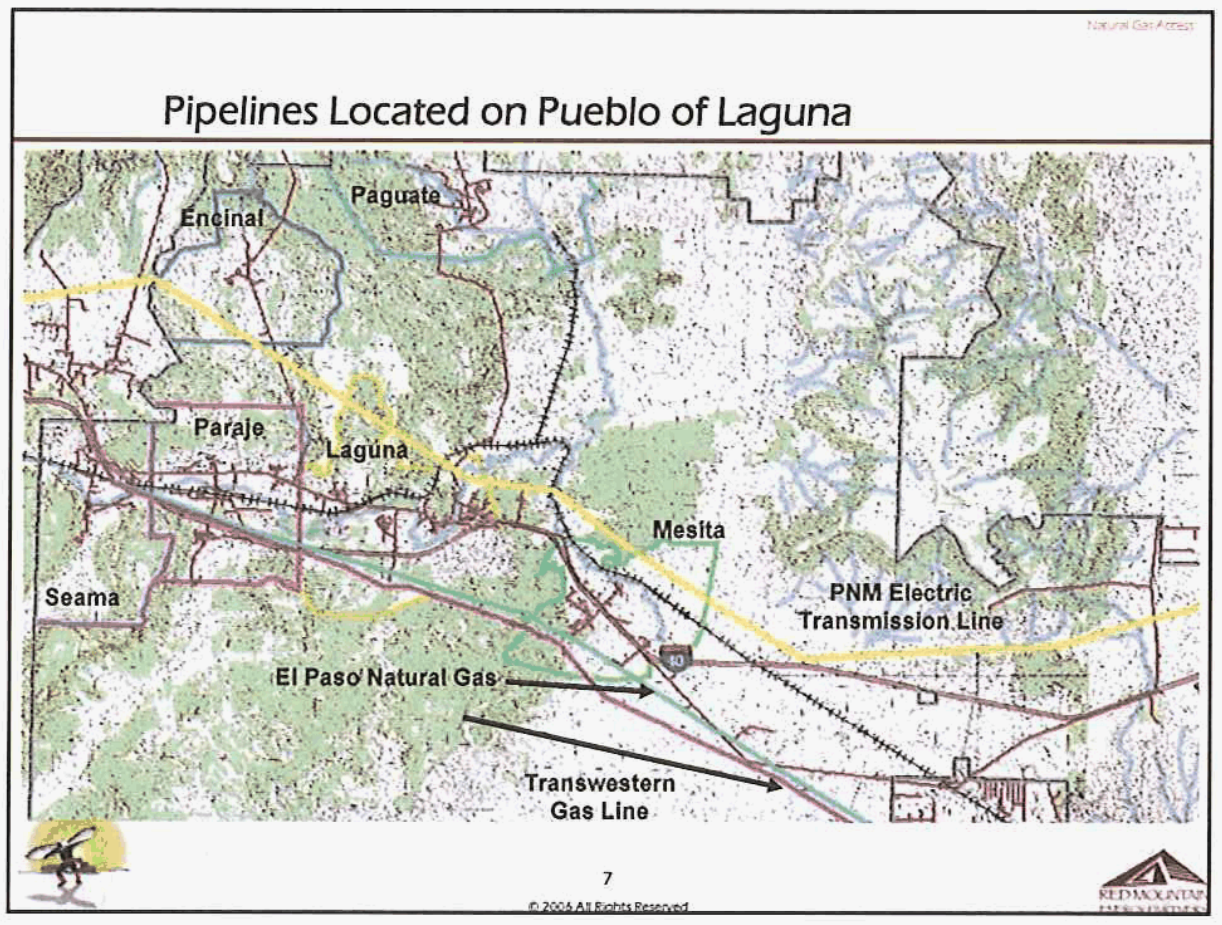




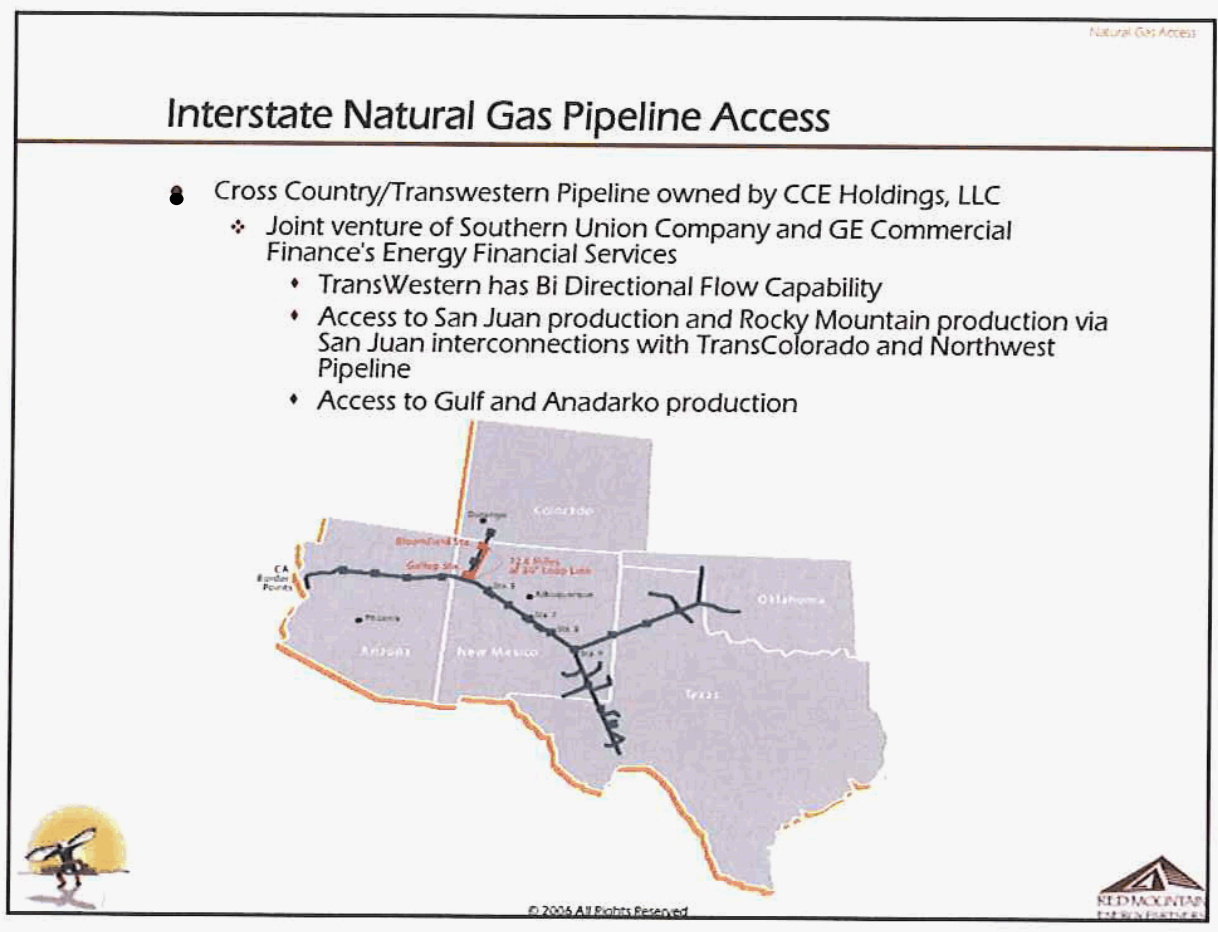

Interstate Natural Gas Pipeline Access

- El Paso Natural Gas

* Northern line crosses Central/Northern New Mexico

* Access to San Juan, Permian, and Anadarko Basin gas

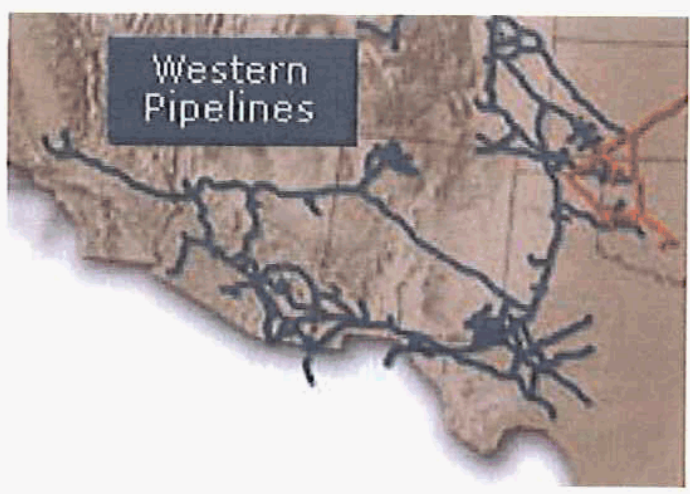




\section{Pipeline Interconnection Process and Potential Costs}

- Interconnection process

* Interstate Pipeline/Facilities -

- File Application of Public Convenience and Necessity to authorize pipeline expansions, for authority to construct any metering facilities, compressor stations, or lateral extensions to connect any facility with Federal Energy Regulatory Commission (FERC)

- For Intrastate Pipelines/Facilities -

- File application with the New Mexico Public Regulation Commission

- Interconnection costs may include

- Certificate and environmental permits

* Land rights (easements)

* Pipeline equipment and construction

* Cathodic Protection and Electrical Mitigation systems

$\therefore$ Initiation of pig launchers and receivers - equipment used to inspect the pipelines

* Installation of meter and regulator stations

* ROW cleanup, regrading, seeding, and restoration

* Completion of an "as-built" survey of the entire pipeline system

$\therefore$ Installation of pipeline markers

* Commissioning the pipeline

* Rule of thumb costs per mile: $\$ 1$ million per mile (16-inch diameter)

\section{Natural Gas Supply Sources}

- San Juan Basin

- Rocky Mountains

- Permian Basin

- Anadarko

- Gulf of Mexico

- Possible Tribal sources

* Southern Ute

* Jicarilla Apache

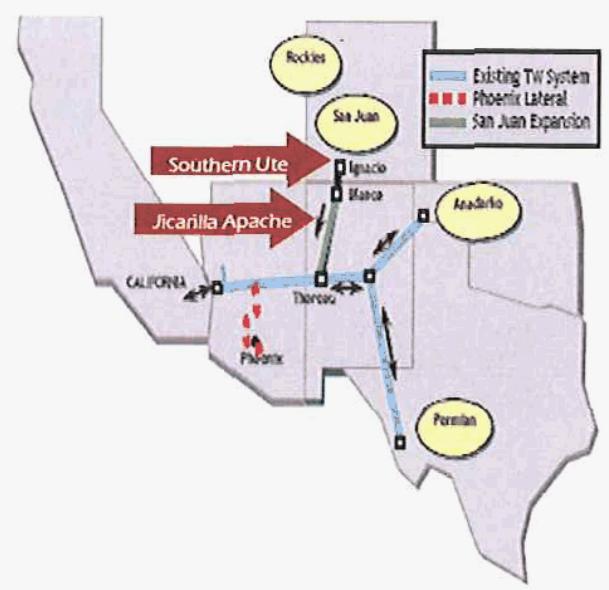

11 


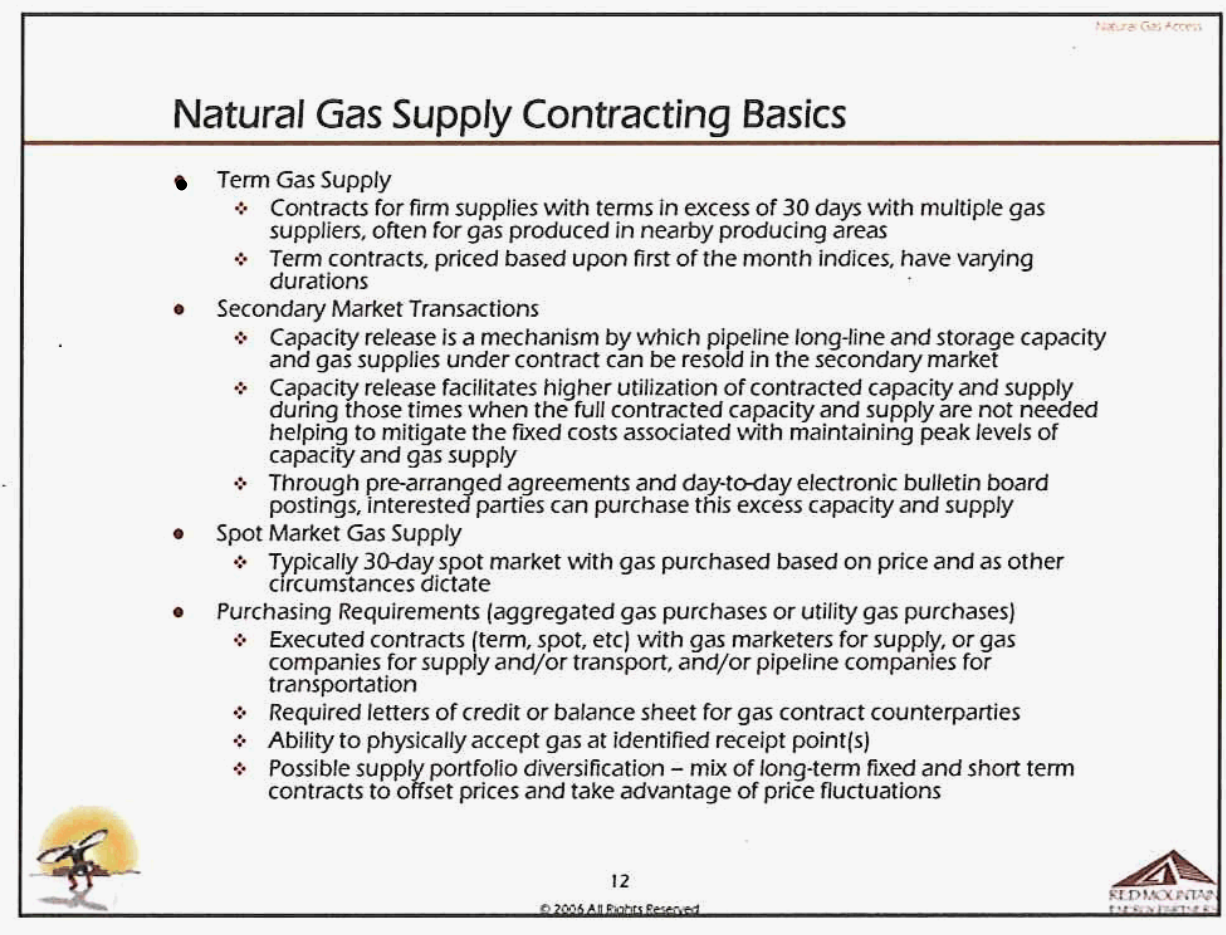

\section{Other Potential Natural Gas Access}

- Potential interest in pipeline system development (Four Nations gas project) starting at Jicarilla Apache, routing south through Pueblo of Jemez, Pueblo of Zia, and Pueblo of Sandia

- Some scenarios included continuing south through Albuquerque along existing rail rights of ways to interconnect with current pipeline infrastructure to the South; would have required additional tribal right of way partner, Pueblo of Isleta

* Potential gas producers

- Jicarilla Apache

- Southern Ute

- Project originally sought large anchor customer, e.g., gypsum plant at Zia; Intel, in order to make pipeline economically feasible

13

02005 

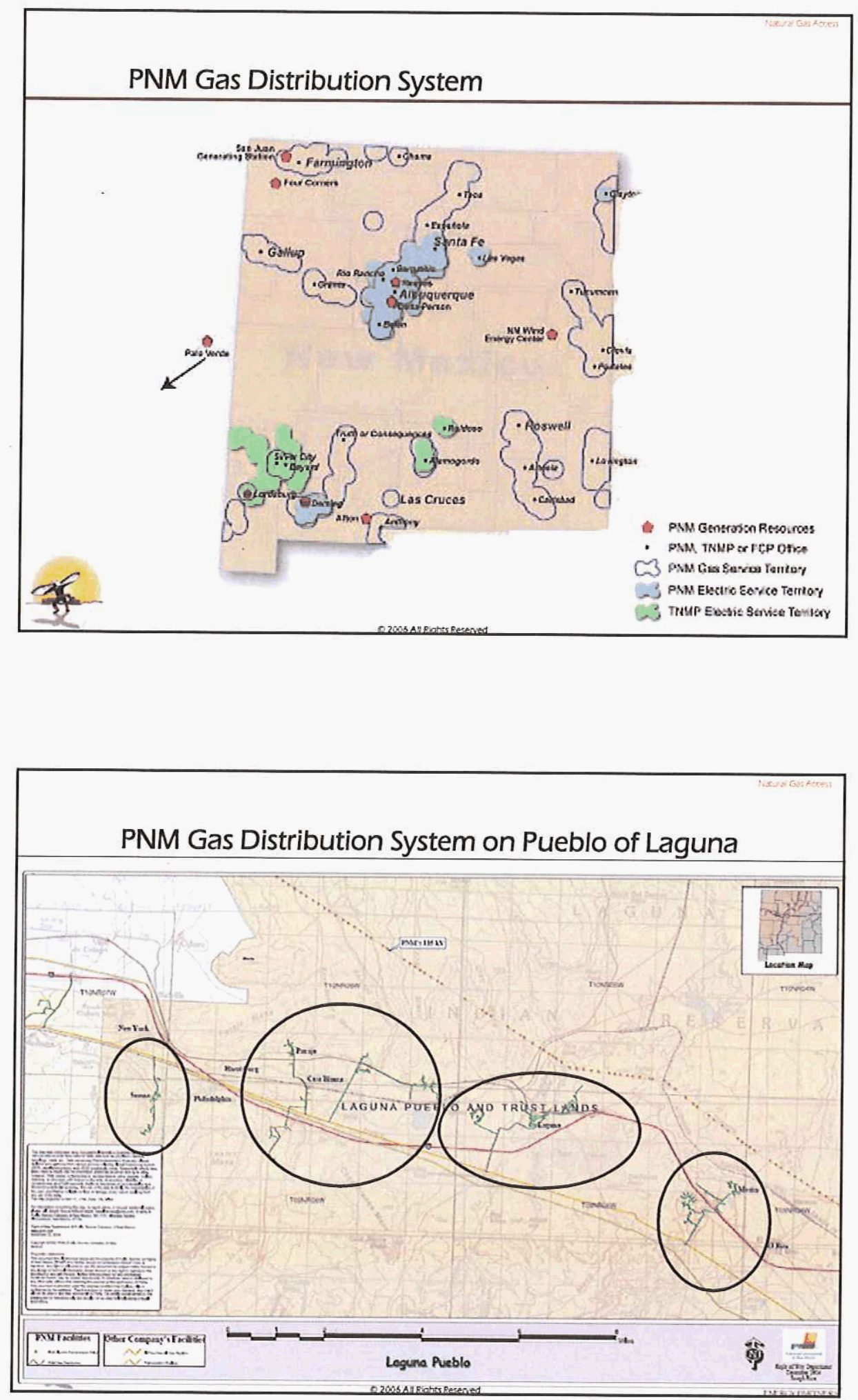


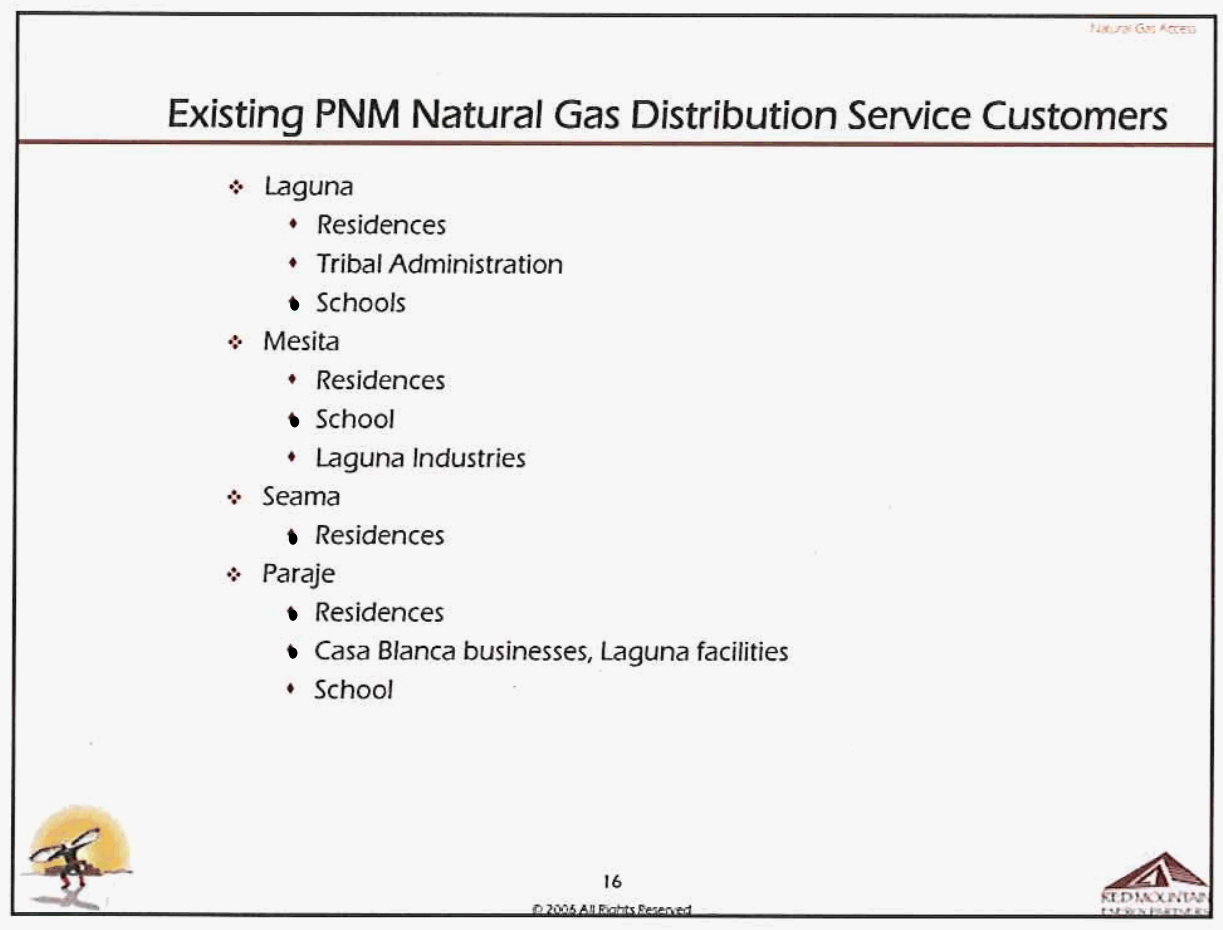

PNM Gas Distribution System Access

- PNM distribution system access

- PNM will invest in facilities up to the point of economic feasibility (potential revenues exceed system installation cost)

- Company provides cost estimate to serve customer

- If cost estimate is over $\$ 100,000$ requires a customer-paid Special Economic Study

* Customer-paid extension also available 


\section{Spot Market Natural Gas Prices}
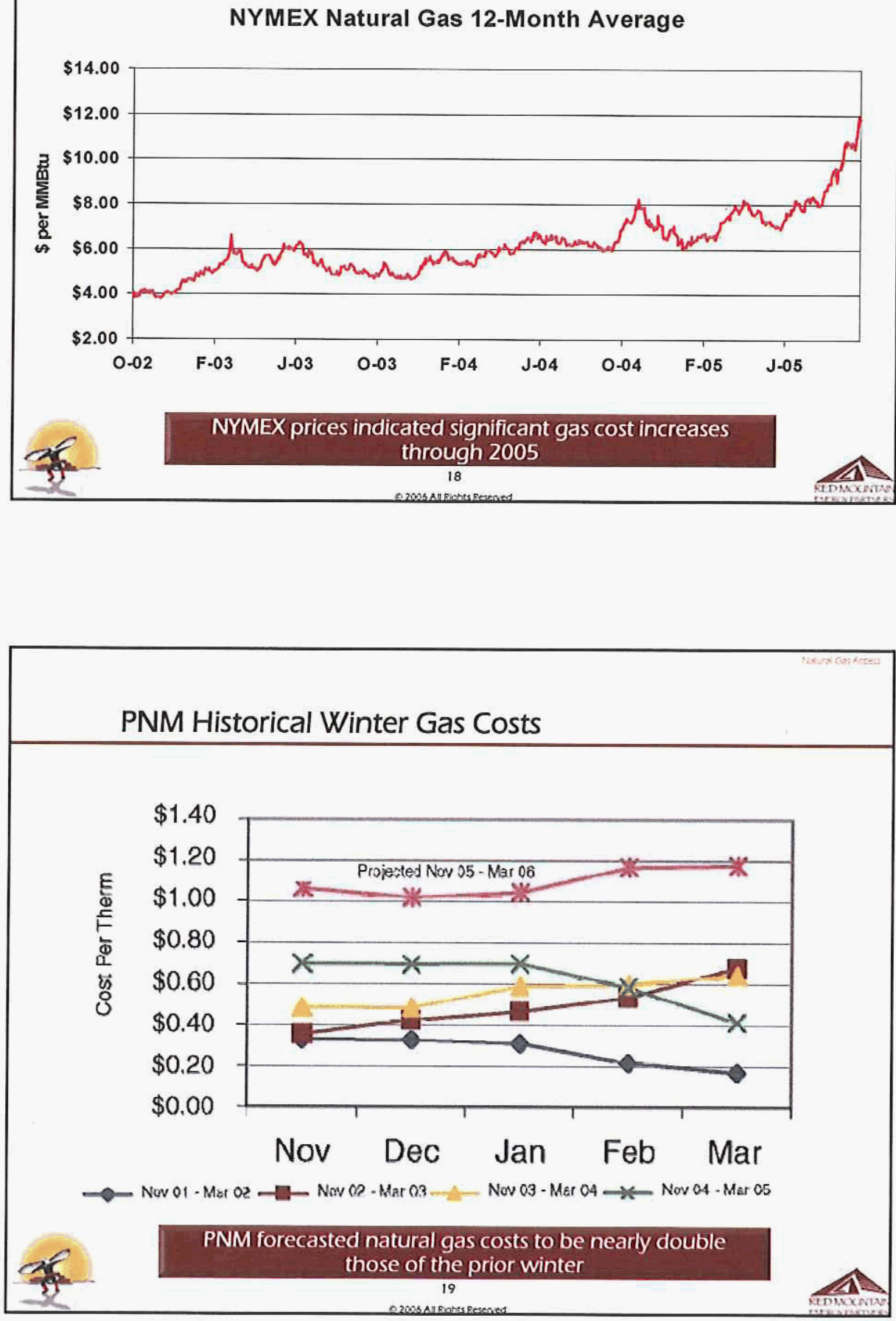


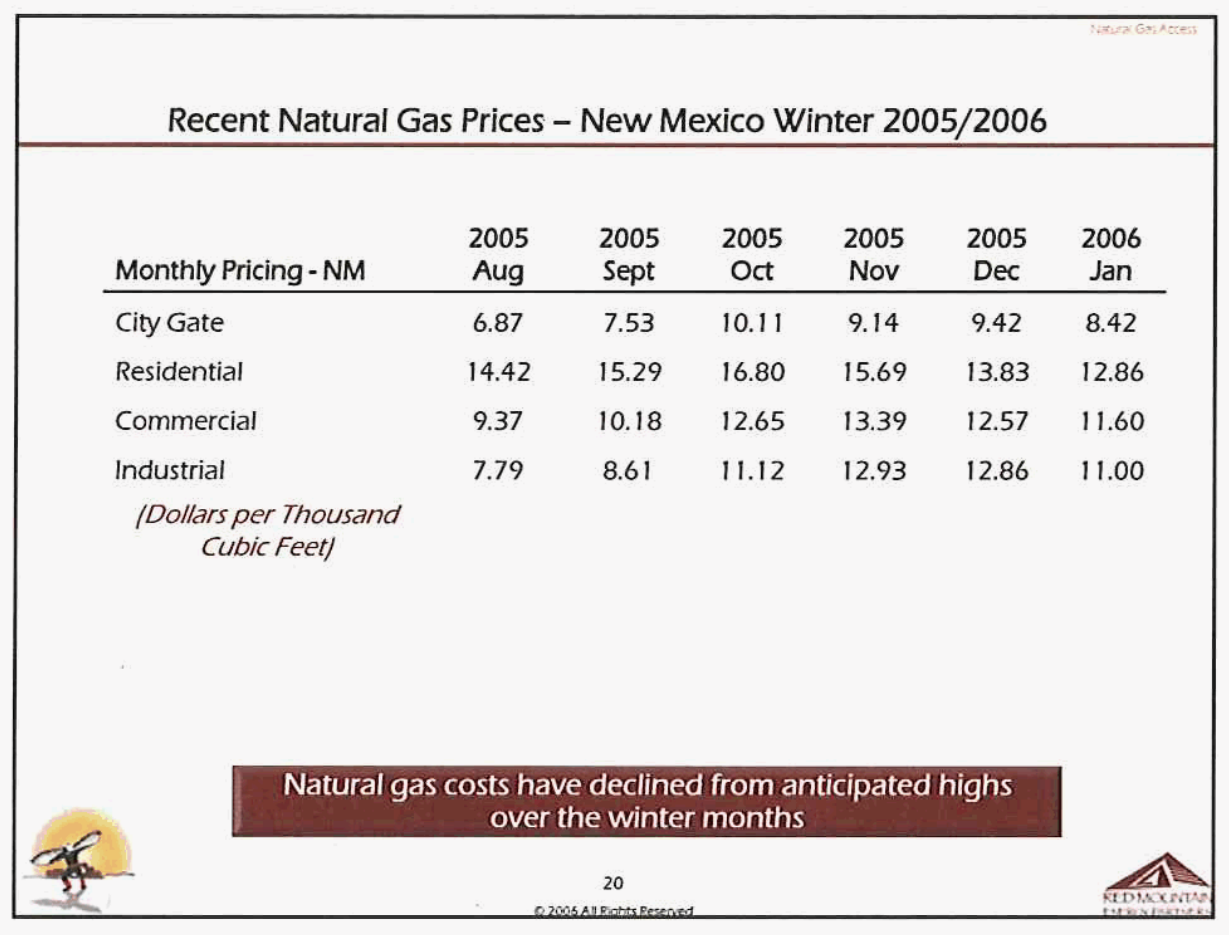

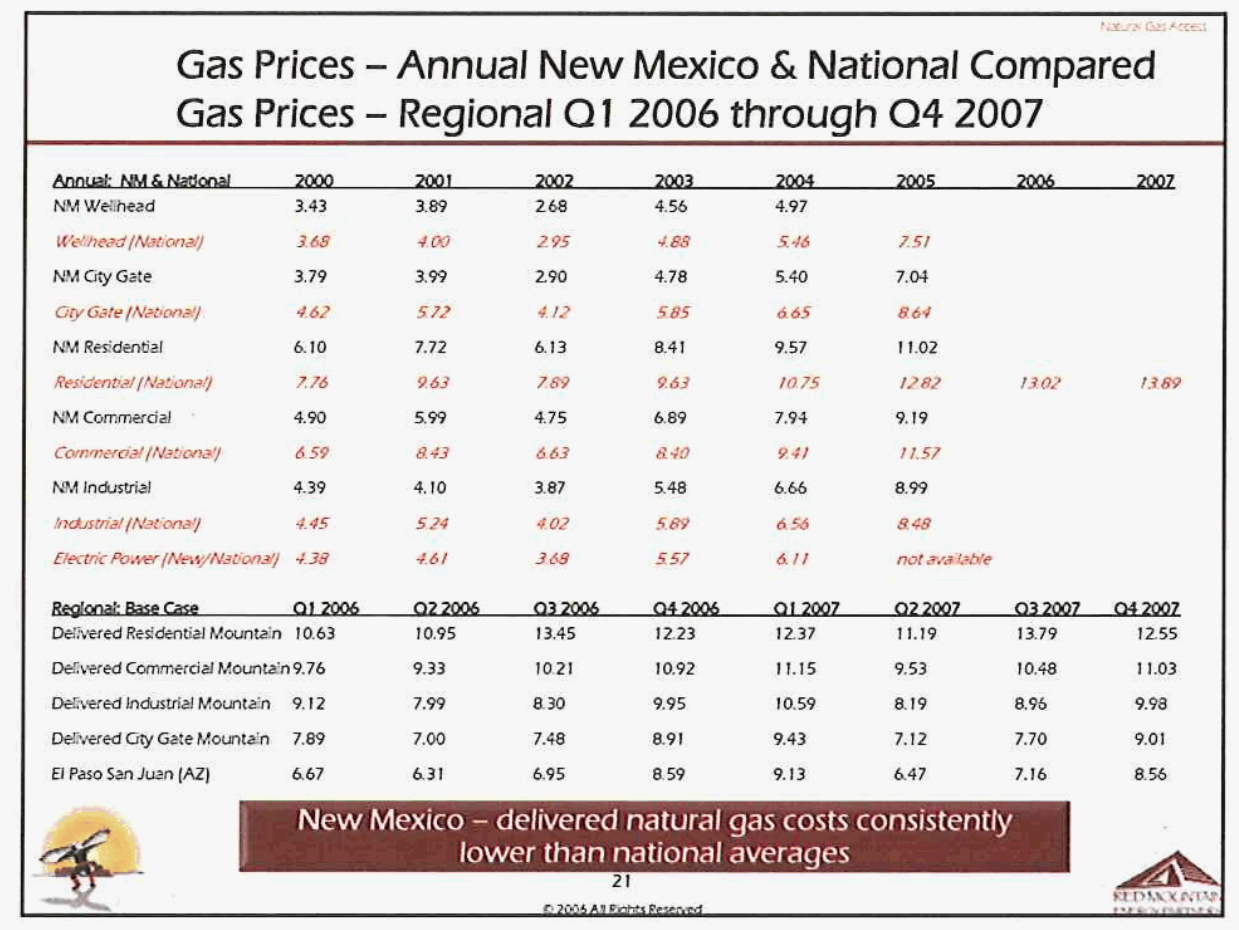




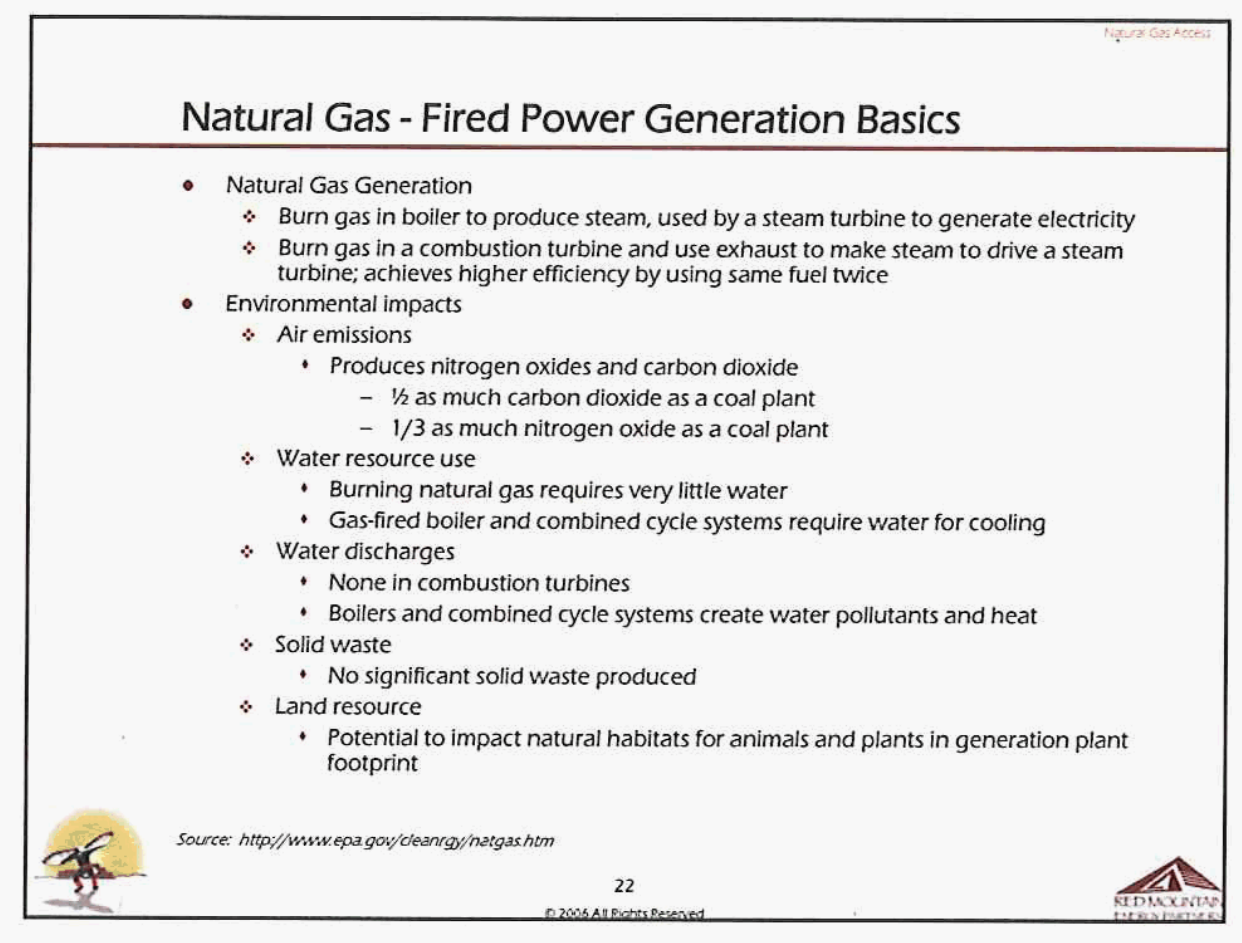

Natural Gas - Fired Generation Trends/Forecast

New Generation Capacity by Fuel 1990-2005
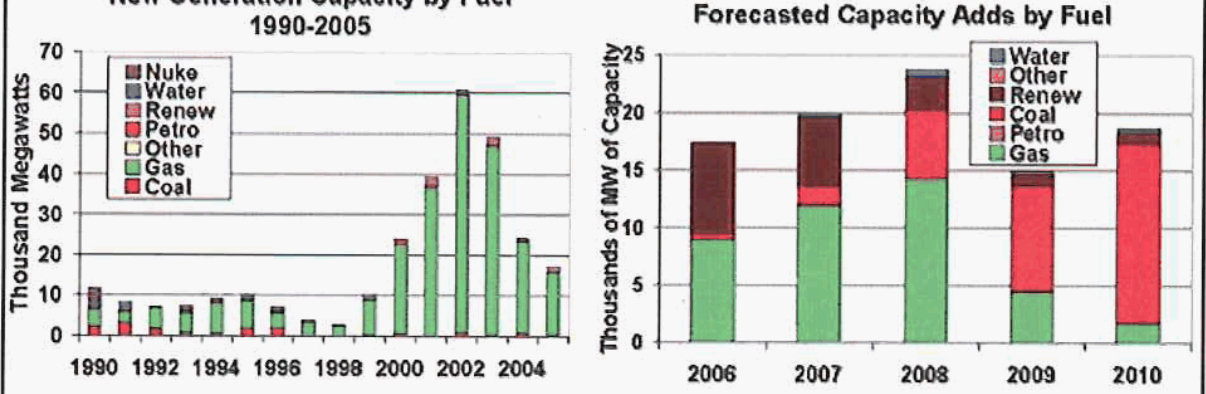

Natural gas -fired generation additions have declined in recent years; higher prices have resulted in lower forecasted natural gas capacity additions

Source: hitp//Mmw.apachecorp com/Explore/Artides/200601/Topic_Report_New_Power_Generating_Capacity/ 

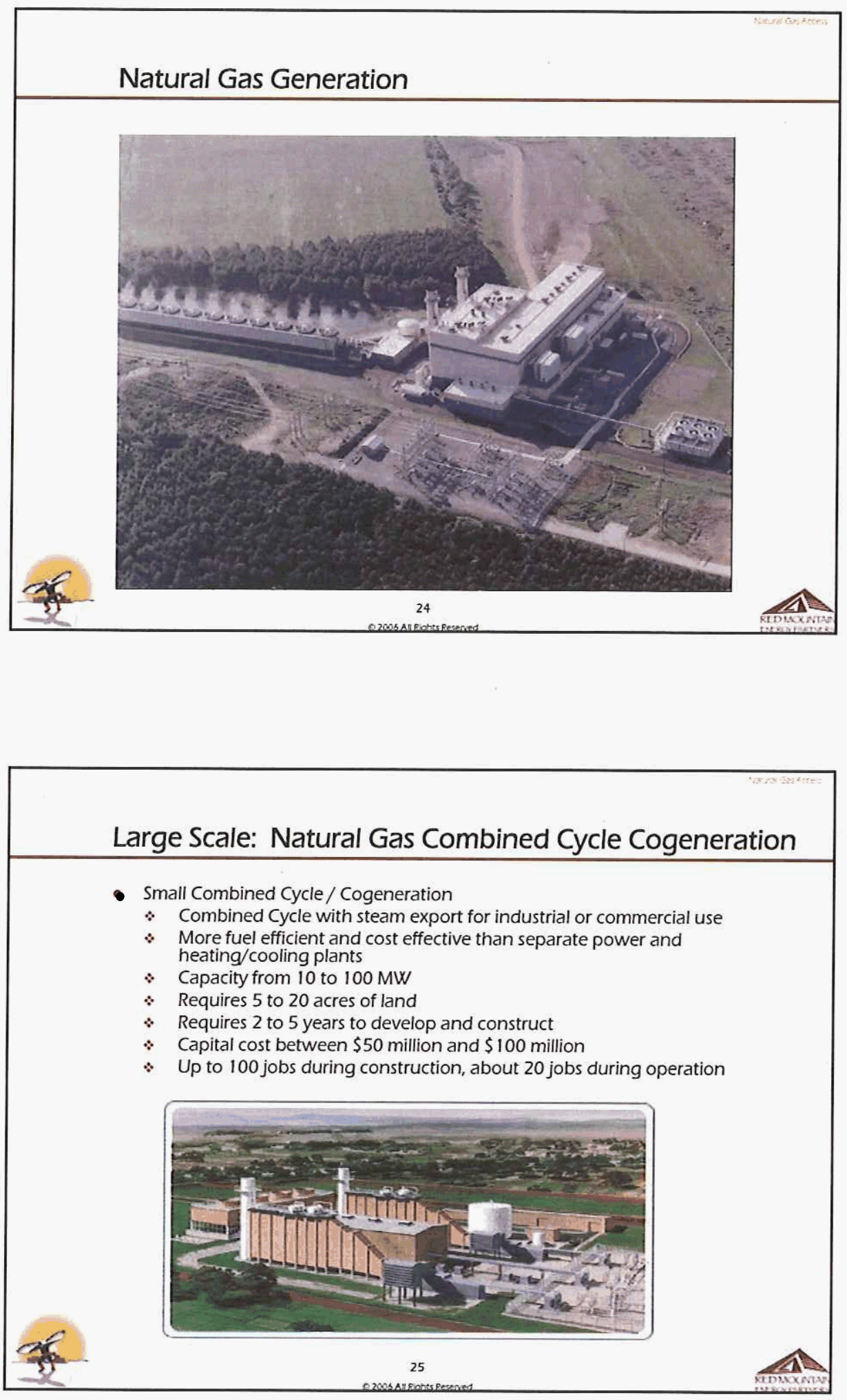


\section{Large Scale: Natural Gas Simple Cycle}

- Combustion Turbine or Reciprocating Engine only

- Lower capital cost, but lower fuel efficiency

- Appropriate for summer peak operation

- Capacity from 20 to $100 \mathrm{MW}$

- Requires 5 to 20 acres of land

- Requires 1 to 3 years to develop and construct

- Capital cost between $\$ 10$ million and $\$ 50$ million

- Up to 50 jobs during construction about 10 during operation

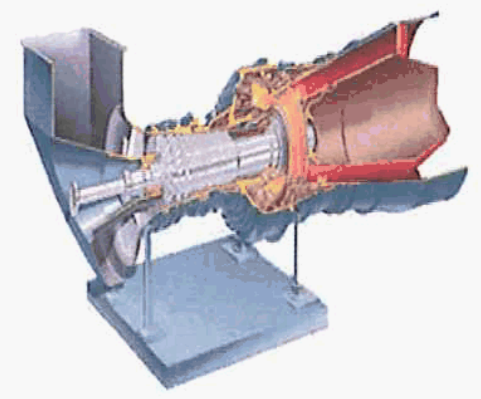

\section{Distributed Generation Options}

- Numerous natural gas-fired options available

- Several new technologies available

\begin{tabular}{|c|c|c|}
\hline Distributed Energy Technologies & $\begin{array}{l}\text { Commercialy } \\
\text { Avaliable }\end{array}$ & $\begin{array}{l}\text { Emerging } \\
\text { Technology }\end{array}$ \\
\hline Microturbines & $\checkmark$ & $\checkmark$ \\
\hline Combustion Turbines & $\checkmark$ & \\
\hline Reciprocating Engines & $\checkmark$ & \\
\hline Stirling Engines & & $\checkmark$ \\
\hline Eueicells & $\checkmark$ & $\checkmark$ \\
\hline Energy Storage / UPS Systems & $\checkmark$ & $\checkmark$ \\
\hline Photovoltaic Systems & $\checkmark$ & \\
\hline Wind Systems & $\checkmark$ & \\
\hline Hybrid Systems & & $\checkmark$ \\
\hline Combined Heat \& Power (CHP) & $\checkmark$ & $\checkmark$ \\
\hline
\end{tabular}




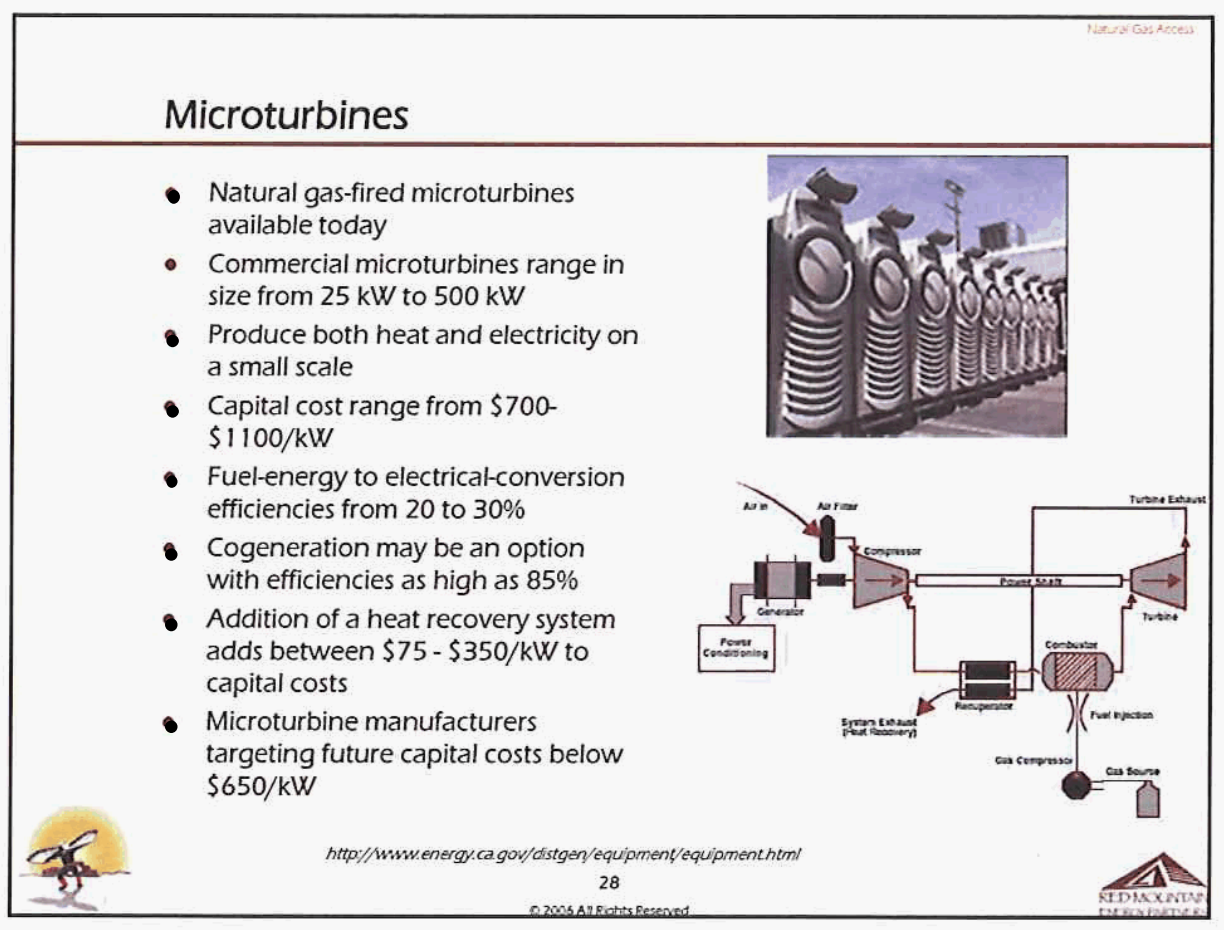

\section{Combined Heat and Power (CHP)}

- Combined heat and power (CHP) systems capture and utilize excess heat generated during the production of electric power

- Offer economic, environmental and reliability-related advantages compared to power generation facilities that produce only electricity

- Typical applications

* Hot water production

* Space heating

* Hot air/steam for industrial process heat

- Space cooling (requires an absorption chiller

* Dry air generation (with the use of a desiccant)

- CHP systems available

* Large and Medium Industrial Systems - greater than 25 MW

s Small industrial system $-50 \mathrm{~kW}$ to 25 MW

* Smaller commercial and institutional systems - $25 \mathrm{~kW}+$

s Residential - $1 \mathrm{~kW}$ to $25 \mathrm{~kW}$

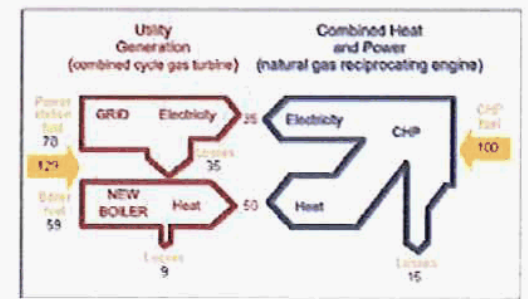




\section{Fuel Cells}

- Natural-gas supplied fuel cells available today

- Most of 600 systems producing $10+k W$ fueled by natural gas

- First cost of fuel cells high compared to other distributed technologies

* Commercially available products are about $\$ 5,500 / \mathrm{kW}$ installed

* Developers targeting costs below $\$ 1,500 / \mathrm{kW}$

* Fuel cells used in high value, "niche" markets where reliability is premium, or where electricity prices are very high and natural gas prices are low

- Fuel cells are expected to have minimum (annual) inspection and maintenance requirements
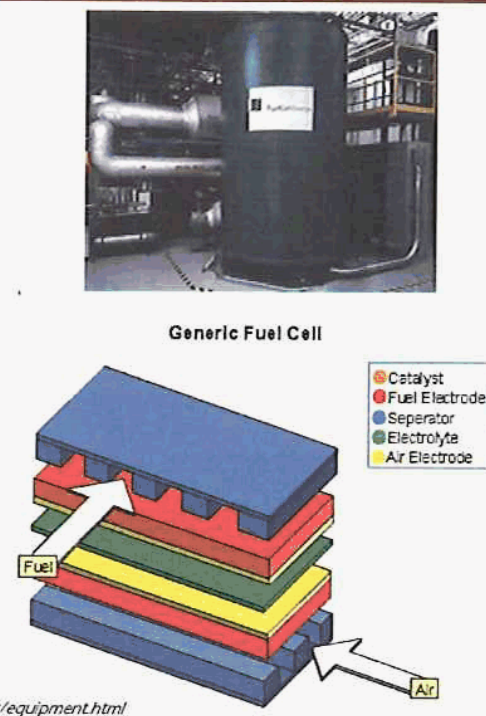

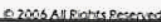

\section{Hybrid Systems}

- Hybrid systems can increase overall fuel utilization efficiency and/or reliability

- Numerous options available

* Solid oxide fuel cell/gas turbine combination

* Small Stirling engine/solar dish hybrid systems from 5 to $25 \mathrm{~kW}$

* Large solar dish/Stirling plants with outputs of 1 to $20 \mathrm{MW}$

- Wind turbines with energy storage backup generation

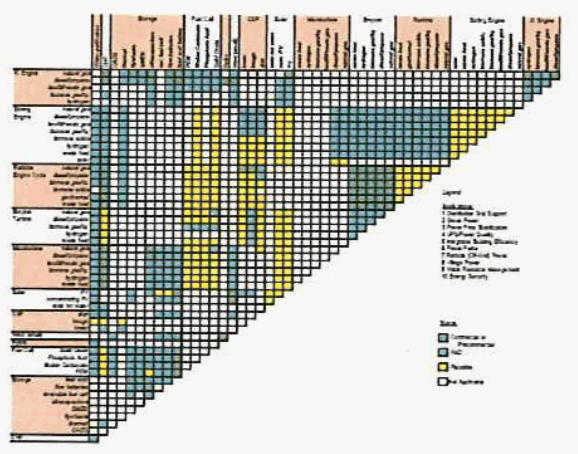
(i.e., reciprocating engine, turbine, or fuel cell) 

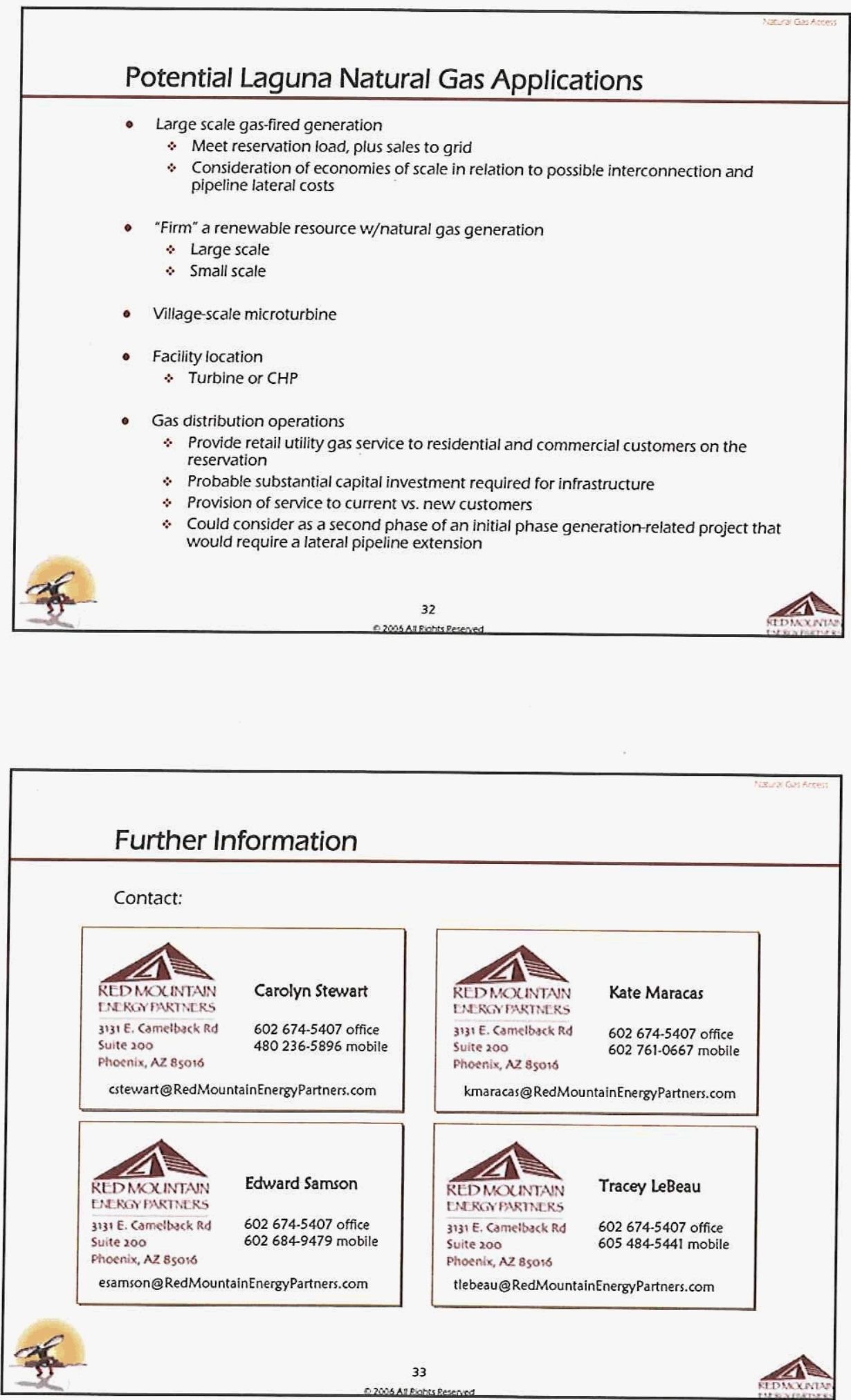


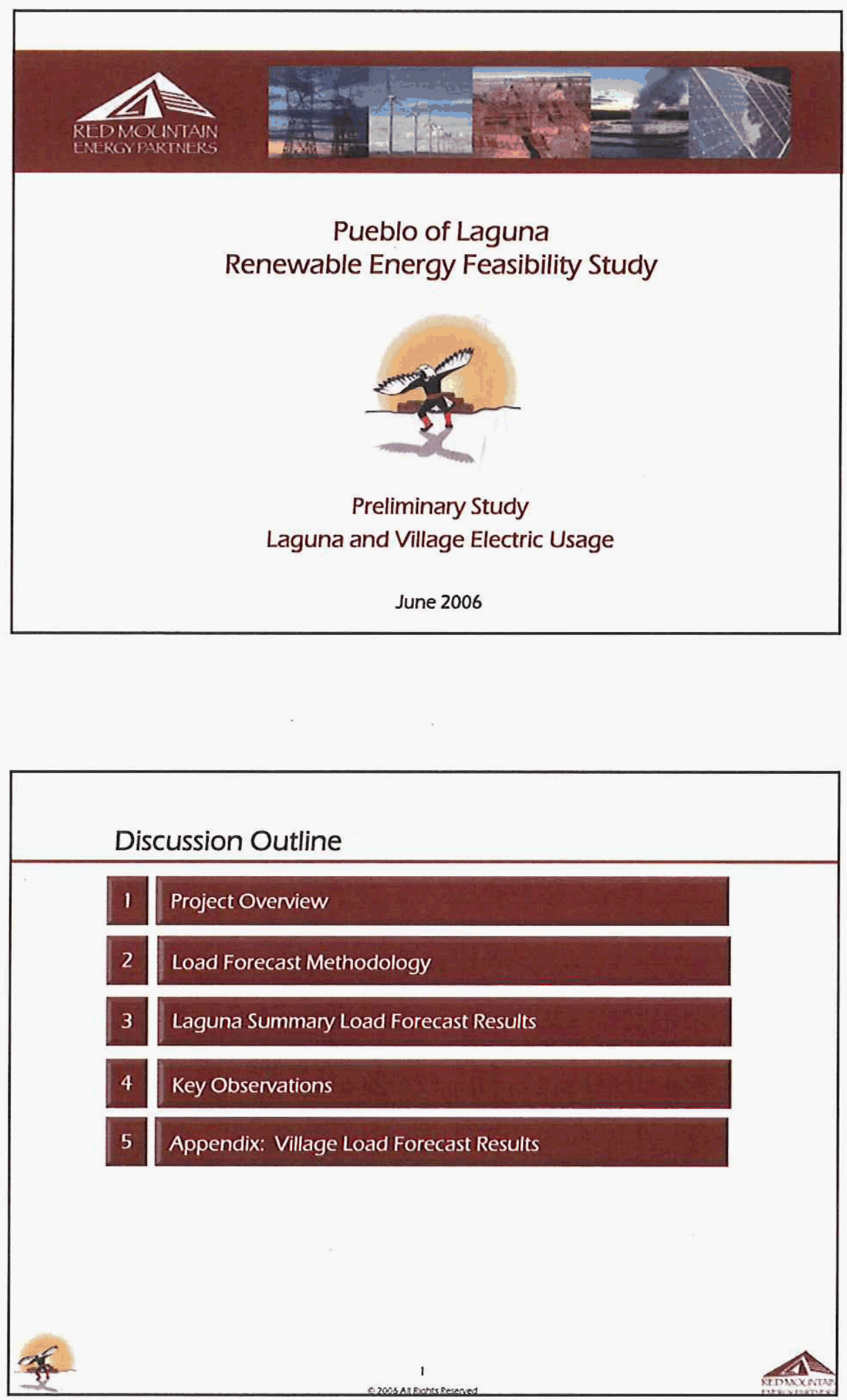


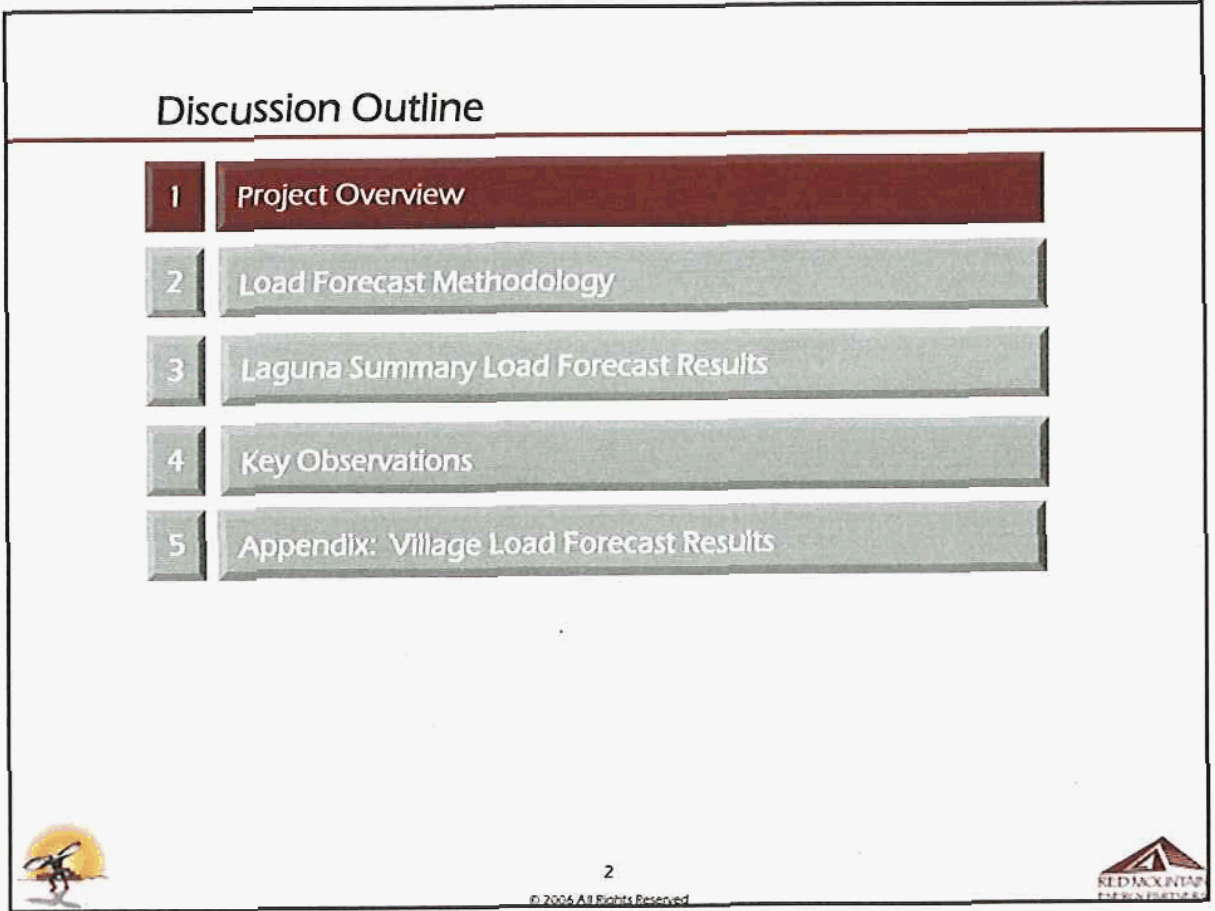

Renewable Energy Feasibility Study Project Overview

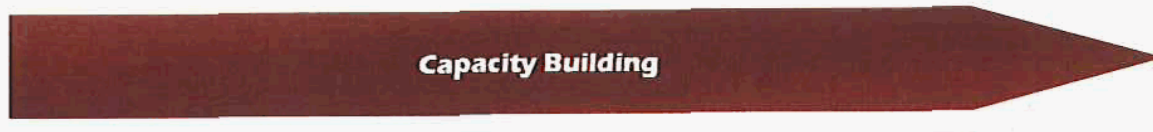

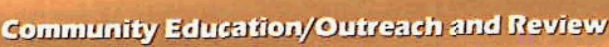

\section{Resource}

Monitoring
Fatal Flaw

Analysis
Project

Decision

Support
Development

(a)

\section{Plan}




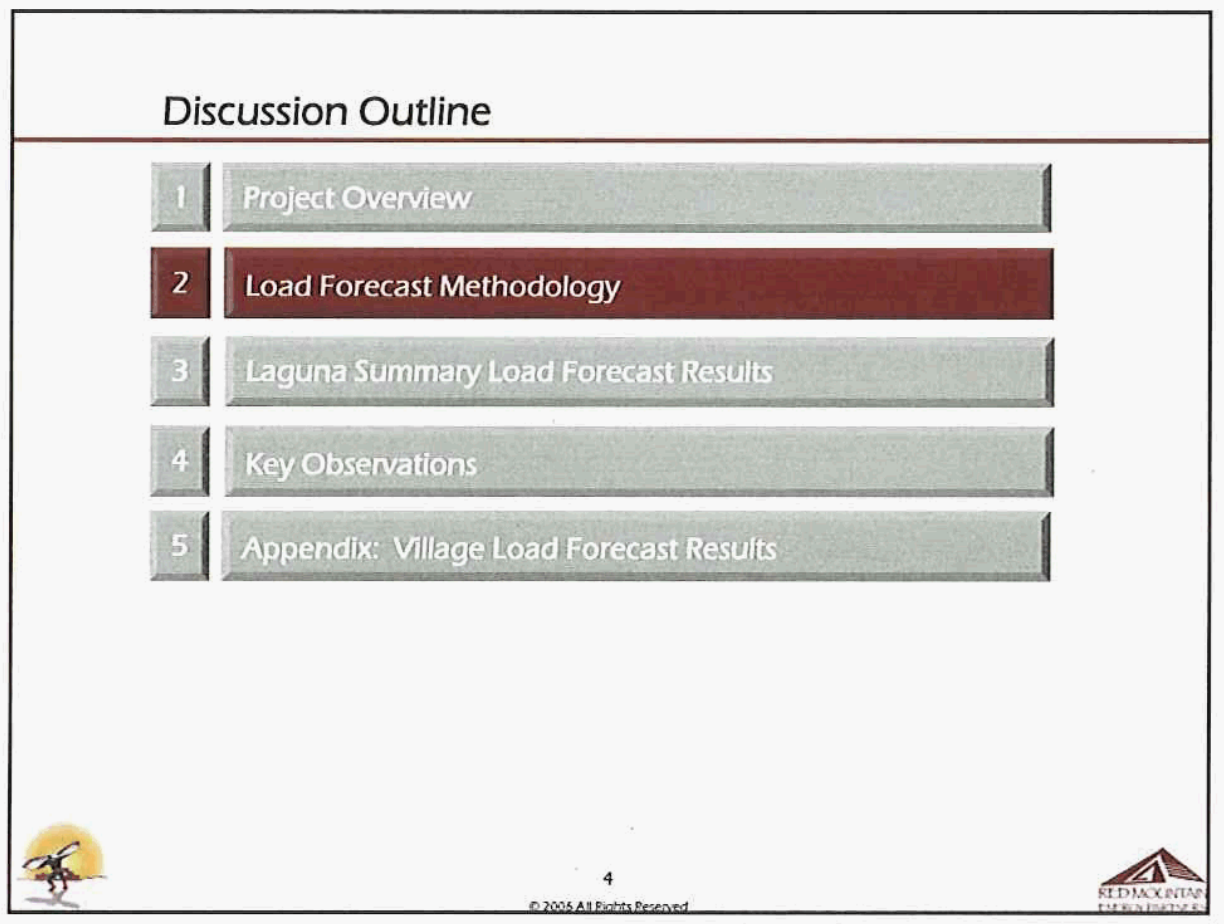

\section{Load Forecast Methodology}

- Residential: Red Mountain conservatively applied regional average household energy consumption to estimate energy use for occupied households within the Pueblo of Laguna

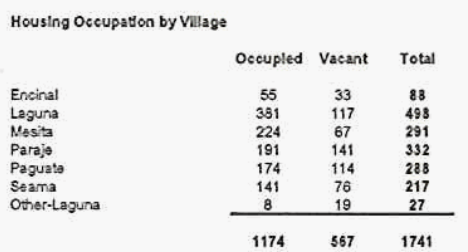

- Residential annual use patterns (load shapes) were adapted using annual energy consumption and time-of-day use patterns consistent with regional climate characteristics (cooling and heating requirements)

- Nonresidential: Energy requirements were derived for nonresidential energy consumers based upon individual electricity bills, POL annual energy consumption patterns, and prototypical building type usage patterns; Route 66 Casino electric usage was excluded from the study

- Residential and nonresidential annual hourly loads were combined to yield one composite load shape for each village and for the entire reservation 


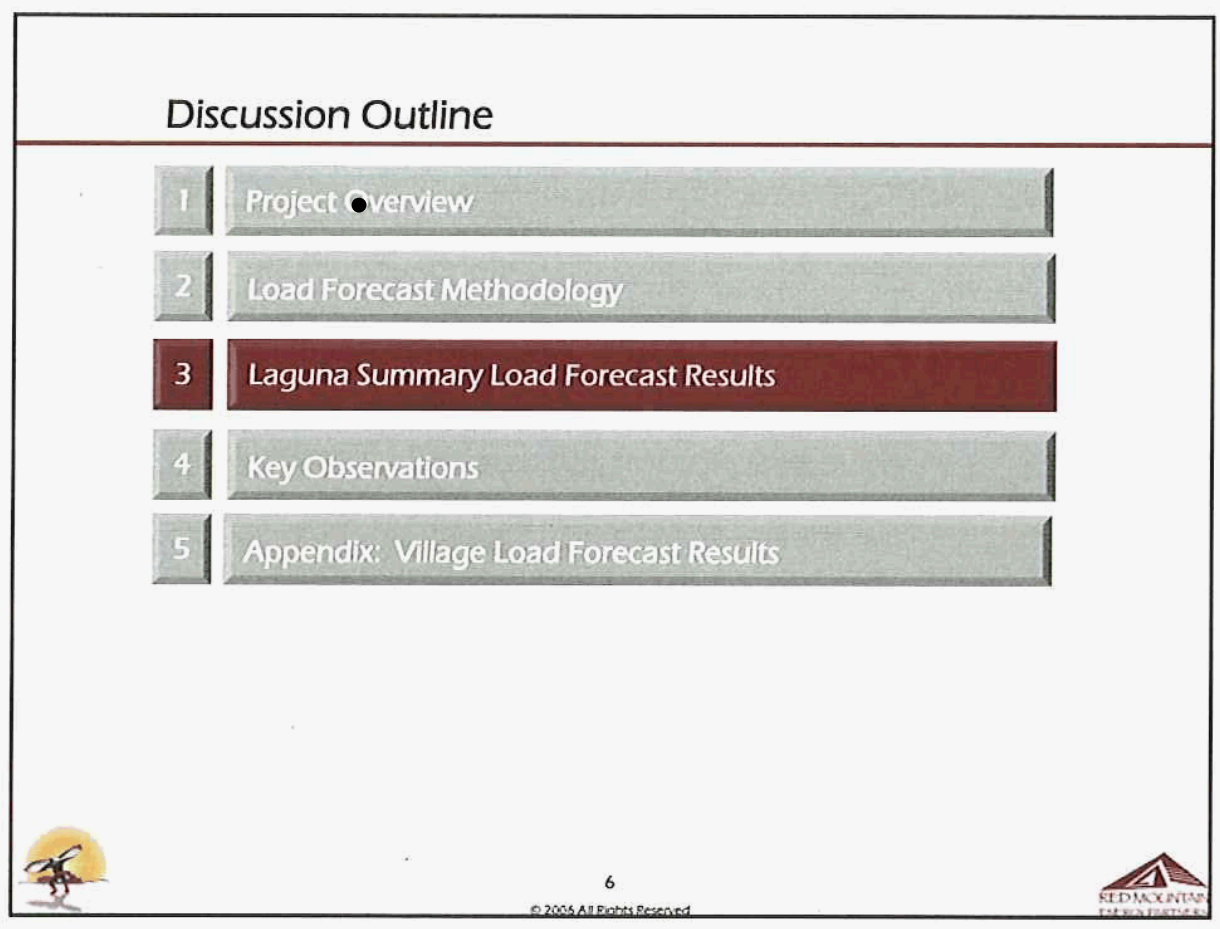

Pueblo of Laguna

Load Forecast Summary

PUEBLO OF LAGUNA

2007 Rate Class Populations, Energy Usage, and Electric Demand

Residential

Small Commercial

Medium Commercial

\begin{tabular}{|c|c|c|c|c|}
\hline Consumers & $\begin{array}{l}\text { Avg. Annual } \\
\text { Energy (kWh) }\end{array}$ & $\begin{array}{c}\text { Projected } \\
\text { Energy (kWh) }\end{array}$ & $\begin{array}{l}\text { Demand } \\
\text { kW }\end{array}$ & $\begin{array}{l}\text { Avg. } \\
\underline{\mathrm{kW}}\end{array}$ \\
\hline 1,294 & 6,230 & $8,063,459$ & 2,112 & 1.6 \\
\hline 93 & 66,676 & $6,174,853$ & 1,293 & 14.0 \\
\hline 17 & 567,186 & $9,379,839$ & 2,021 & 122.2 \\
\hline \multirow[t]{2}{*}{1,403} & & $23,618,151$ & 5,426 & \\
\hline & & Coincident Peak & 4,845 & \\
\hline
\end{tabular}




\begin{tabular}{|c|c|c|c|c|c|c|c|}
\hline \multicolumn{3}{|c|}{$\begin{array}{l}\text { Pueblo of Laguna Villages } \\
2007 \text { Electric Loads }\end{array}$} & \multirow[b]{2}{*}{ Mesita } & \multirow[b]{2}{*}{ Paquate } & \multirow[b]{2}{*}{ Parris } & \multirow[b]{2}{*}{ Seama } & \multirow[b]{2}{*}{ All Vilizaes } \\
\hline & Encinal & Laguna & & & & & \\
\hline \multicolumn{8}{|l|}{ consumers } \\
\hline $\begin{array}{l}\text { Residential } \\
\text { Smal Commercial } \\
\text { Mecium Commercial }\end{array}$ & $\begin{array}{c}61 \\
1 \\
0\end{array}$ & $\begin{array}{c}429 \\
48 \\
8\end{array}$ & $\begin{array}{c}247 \\
1 \\
2\end{array}$ & $\begin{array}{c}192 \\
10 \\
0\end{array}$ & $\begin{array}{c}211 \\
22 \\
5\end{array}$ & $\begin{array}{c}155 \\
10 \\
2\end{array}$ & $\begin{array}{l}1.294 \\
93 \\
17\end{array}$ \\
\hline $\begin{array}{l}\text { Total Consumers } \\
\text { percent of tetat }\end{array}$ & 62 & $\begin{array}{l}485 \\
345 \%\end{array}$ & $\begin{array}{l}250 \\
178 x\end{array}$ & 202 & $\begin{array}{l}238 \\
170 \%\end{array}$ & 167 & 1.403 \\
\hline \multicolumn{8}{|c|}{ Energy Requirements $(\mathrm{KW} / \mathrm{h})$} \\
\hline $\begin{array}{l}\text { Residential } \\
\text { Smal Commercial } \\
\text { Medum Commercial }\end{array}$ & $\begin{array}{c}380,347 \\
12,676\end{array}$ & $\begin{array}{l}2.634,849 \\
3.712 .765 \\
2,859,823\end{array}$ & $\begin{array}{l}1,549.074 \\
103.942 \\
2,420.635\end{array}$ & $\begin{array}{l}1.203 .288 \\
750.585\end{array}$ & $\begin{array}{l}1,320.831 \\
1,120.527 \\
3,764,809\end{array}$ & $\begin{array}{l}975.059 \\
474.358 \\
334.572\end{array}$ & $\begin{array}{l}8.063 .459 \\
6.174,853 \\
9,379,839\end{array}$ \\
\hline $\begin{array}{l}\text { Total Energy } \\
\text { percerte of toxal }\end{array}$ & $\underset{393,023}{1766}$ & $\begin{array}{l}9.207 .437 \\
32036\end{array}$ & $\begin{array}{c}4,073,652 \\
17266\end{array}$ & $\begin{array}{l}1.953,873 \\
8396\end{array}$ & $\begin{array}{c}6.206,168 \\
26396\end{array}$ & $\begin{array}{c}1.783,999 \\
7.695\end{array}$ & $23,618.151$ \\
\hline \multicolumn{8}{|l|}{ Demand $|\mathrm{kW}|$} \\
\hline $\begin{array}{l}\text { Residential } \\
\text { Smal Commercial } \\
\text { Medium Commercial }\end{array}$ & $\begin{array}{l}99.6 \\
2.7\end{array}$ & $\begin{array}{l}690.2 \\
777.2 \\
616.1\end{array}$ & $\begin{array}{l}405.8 \\
21.8 \\
521.5\end{array}$ & $\begin{array}{l}315.2 \\
157.1\end{array}$ & $\begin{array}{l}3460 \\
234.5 \\
811.1\end{array}$ & $\begin{array}{l}255.4 \\
99.3 \\
72.1\end{array}$ & $\begin{array}{l}2.1122 \\
1.2925 \\
2.020 .9\end{array}$ \\
\hline Pezk Demand & 102 & 2.083 & 949 & 472 & 1,392 & 427 & 5.426 \\
\hline $\begin{array}{l}\text { Peak Coincident Demand } \\
\text { perrentoffocal }\end{array}$ & $\begin{array}{l}101 \\
21 \%\end{array}$ & $\begin{array}{l}1,842 \\
38.0 \%\end{array}$ & $\begin{array}{l}864 \\
178 \%\end{array}$ & ${ }_{87 \%}^{421}$ & $\begin{array}{l}1.263 \\
261 \%\end{array}$ & $\begin{array}{l}382 \\
7.965\end{array}$ & 4,845 \\
\hline
\end{tabular}

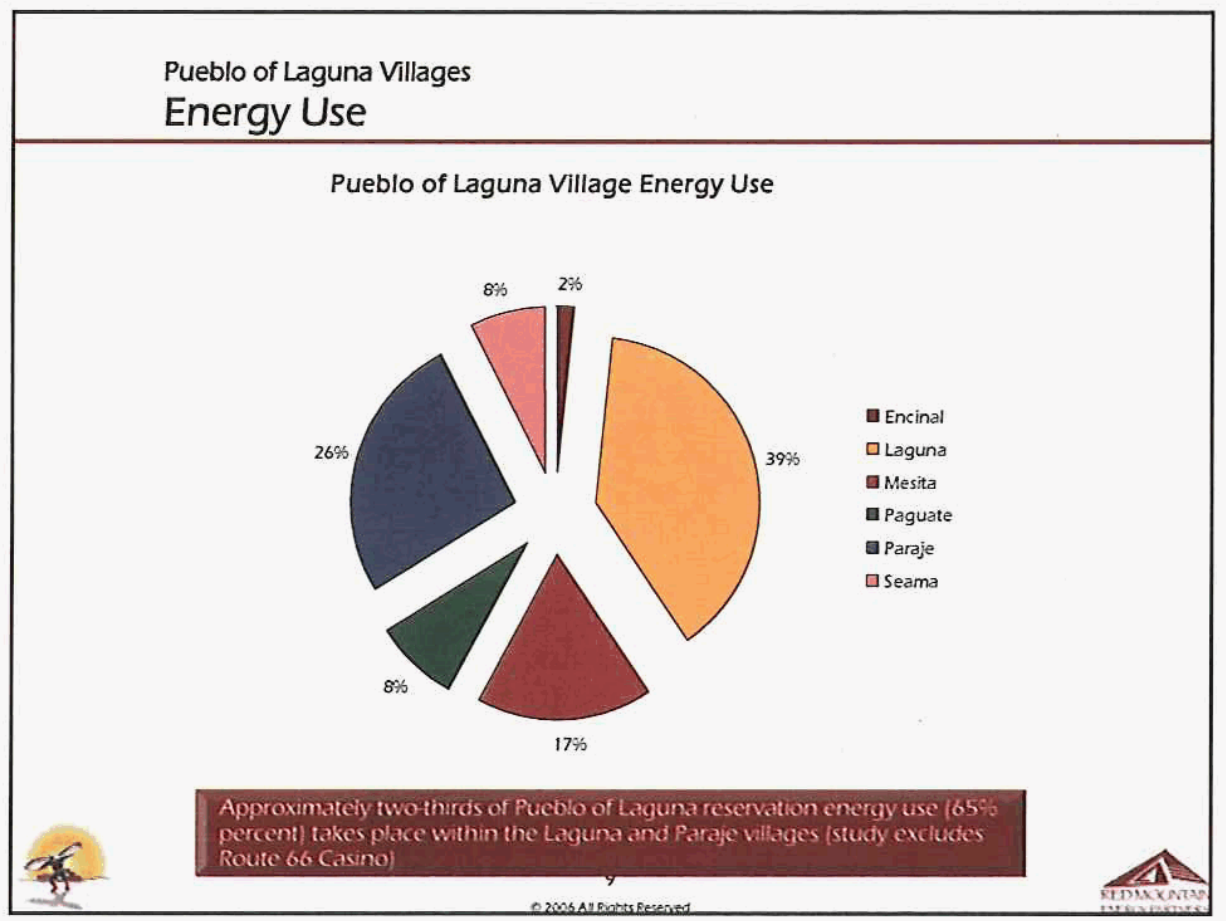



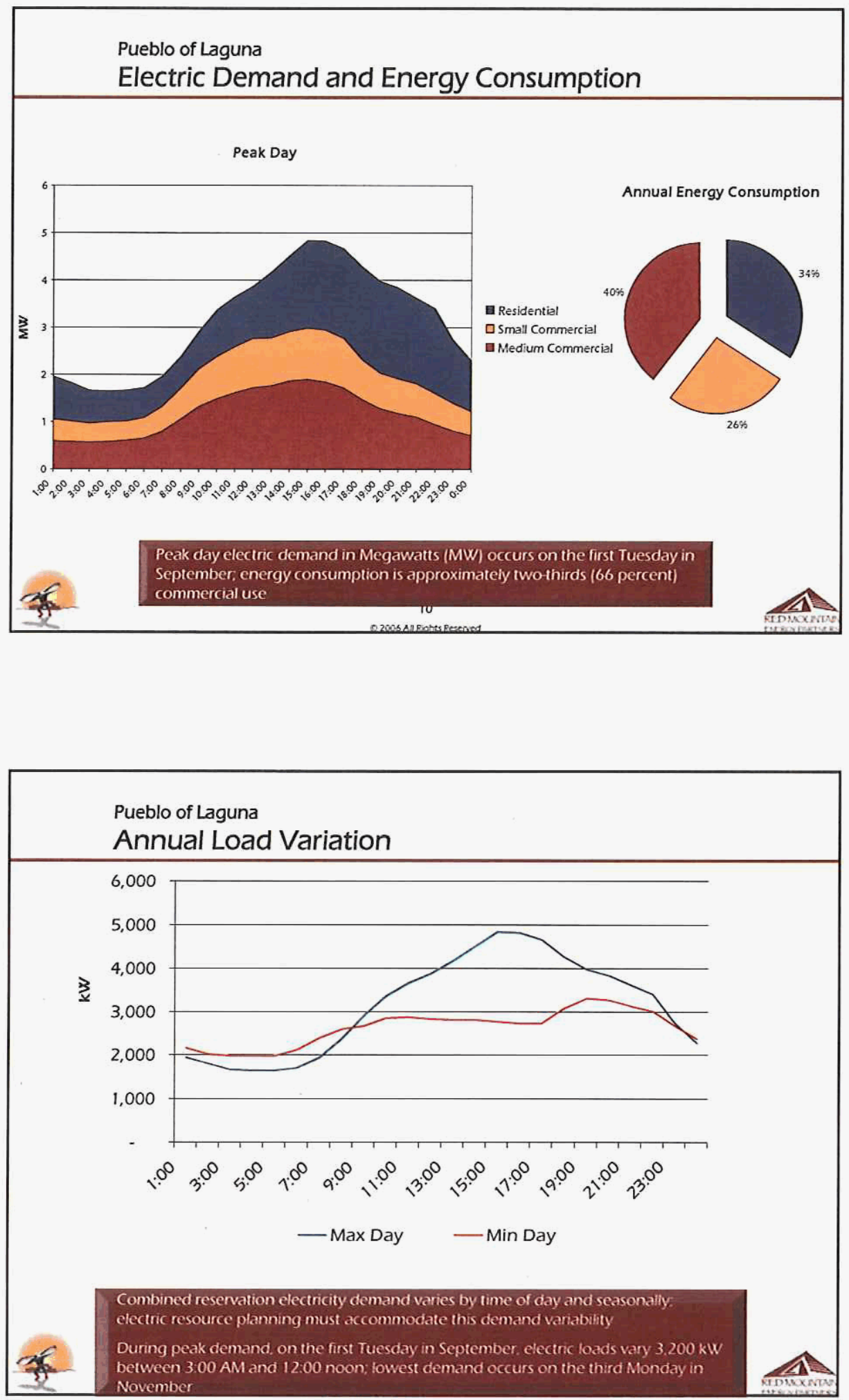

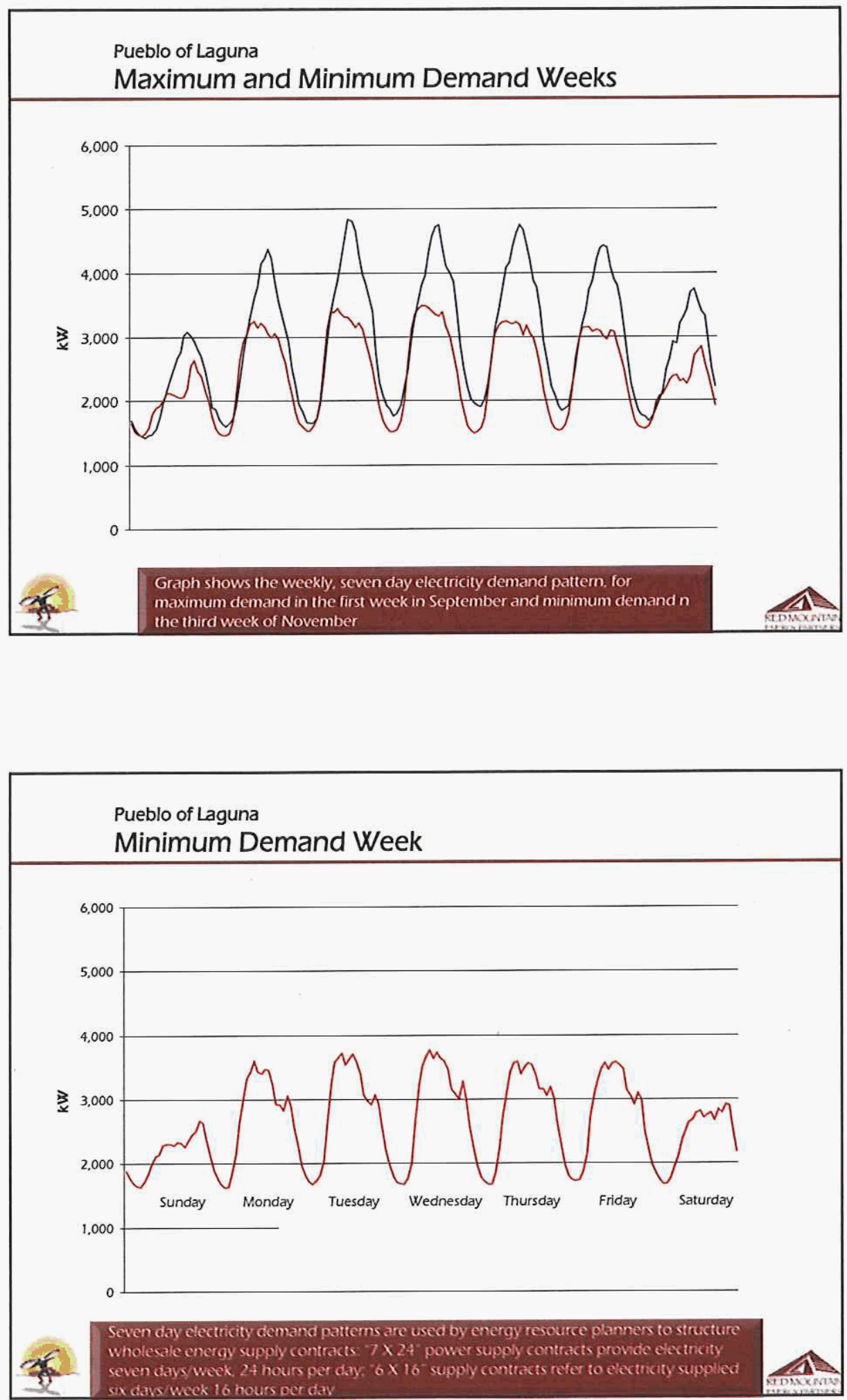

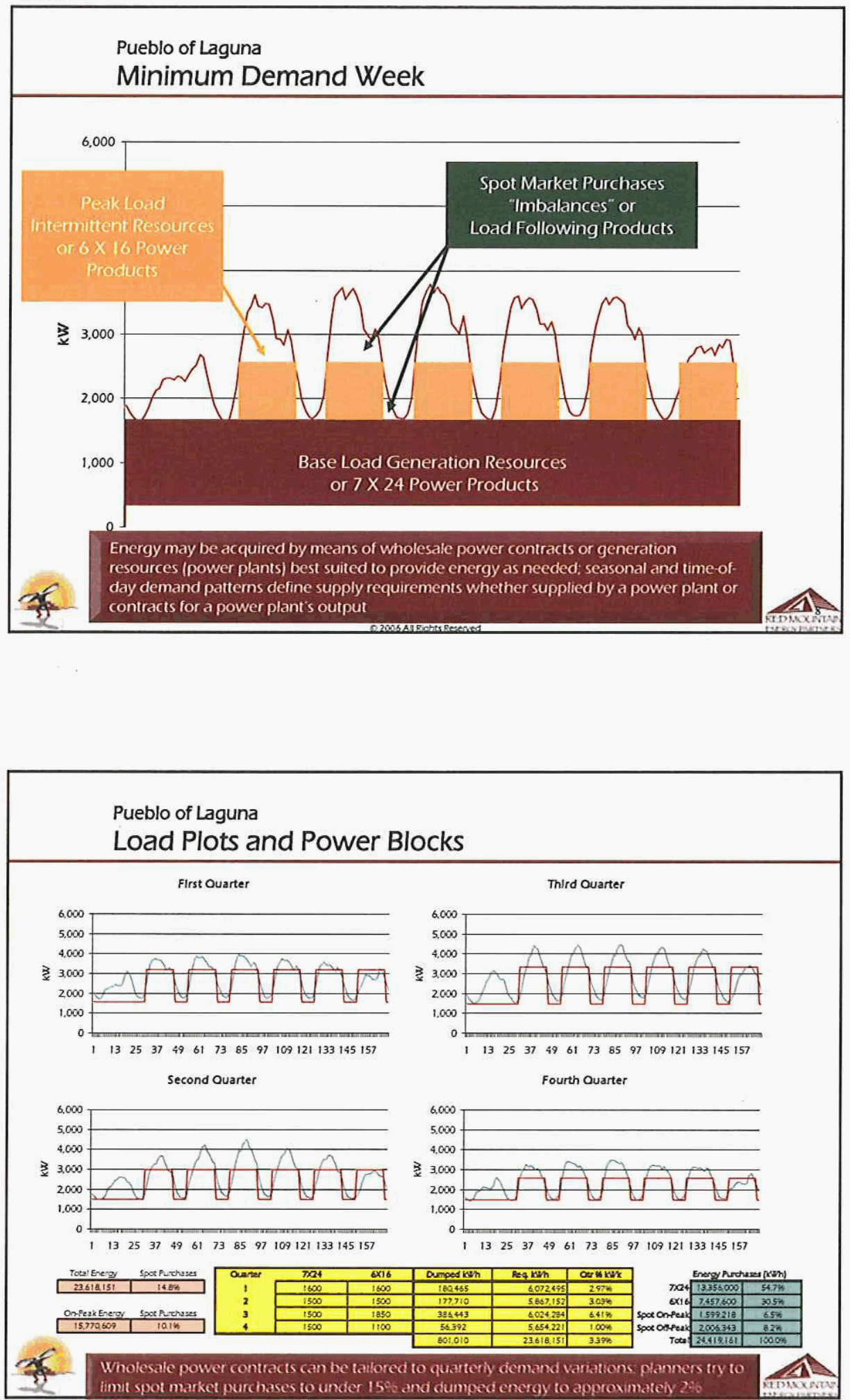

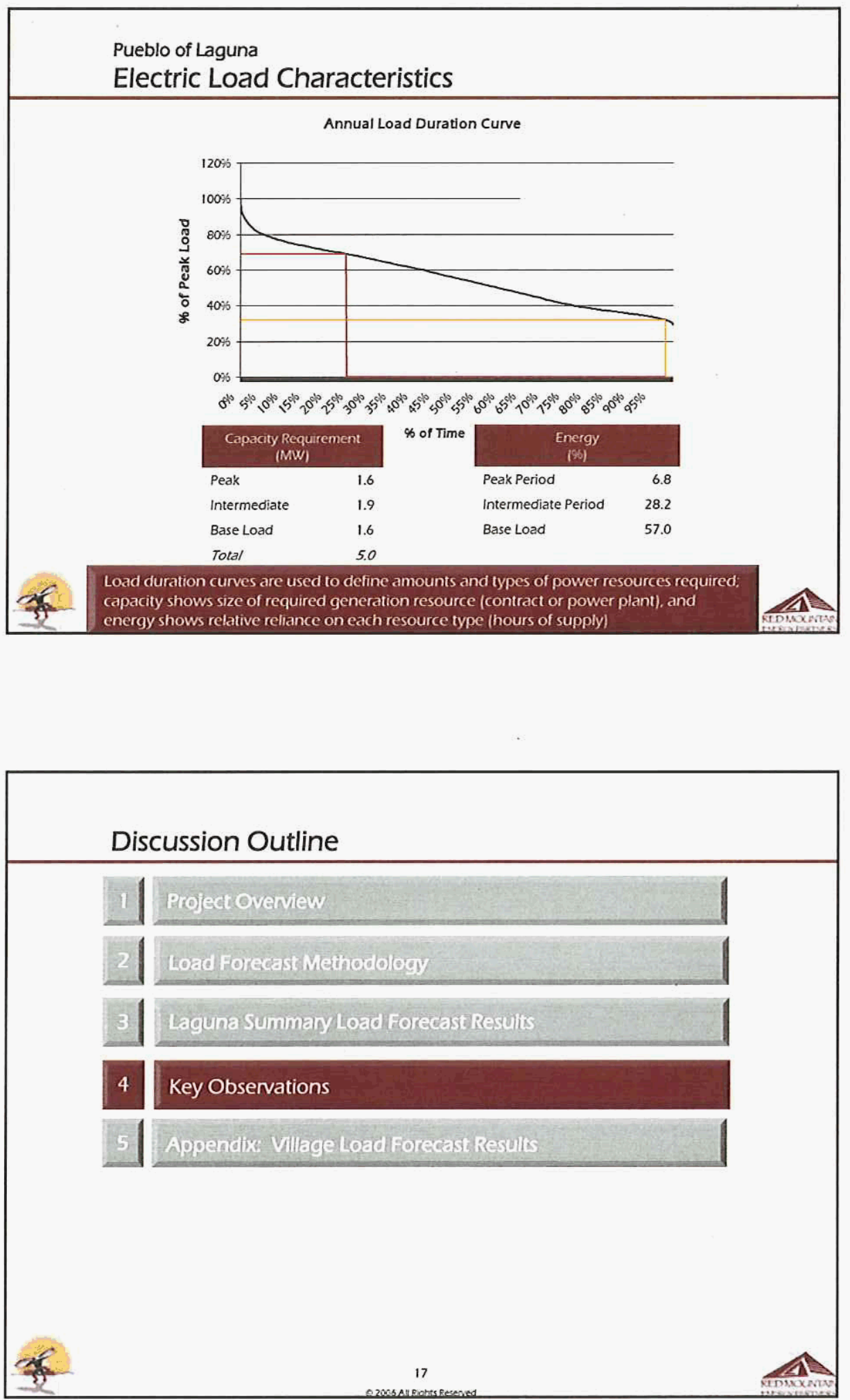


\section{Key Observations}

When addressing Pueblo of Laguna total electric energy requirements:

2 The majority of Pueblo of Laguna energy demand is associated with nonresidential activity $(66 \%)$

- Among Pueblo of Laguna villages, the villages of Laguna and Paraje comprise the majority of energy demand (65\%)

- Peak demand is approximately $5 \mathrm{MW}$, however loads can vary as much as 3.2 $M W$ in a single day

2 Resources required to serve the total reservation electric demand:

- $1.6 \mathrm{MW}$ base load supply $(7 \times 24)$

- $1.9 \mathrm{MW}$ intermediate $(6 \times 16)$

- $1.6 \mathrm{MW}$ Load following (spot market purchases)

- $57 \%$ of all energy consumed can be provided by constant duty cycle base load $(7 \times 24)$ generation resources

2 $28 \%$ of all energy consumed can be provided by intermediate resources (including solar and wind power generation)

- Remaining energy can be procured on the spot market without exceeding $15 \%$ (appropriately avoiding market uncertainties)

\section{Further Information}

Contact:
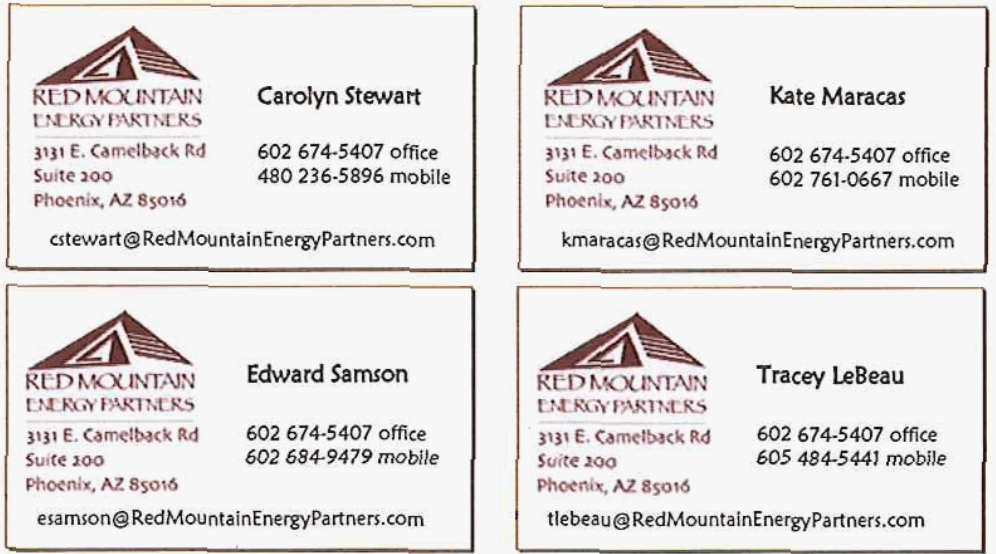


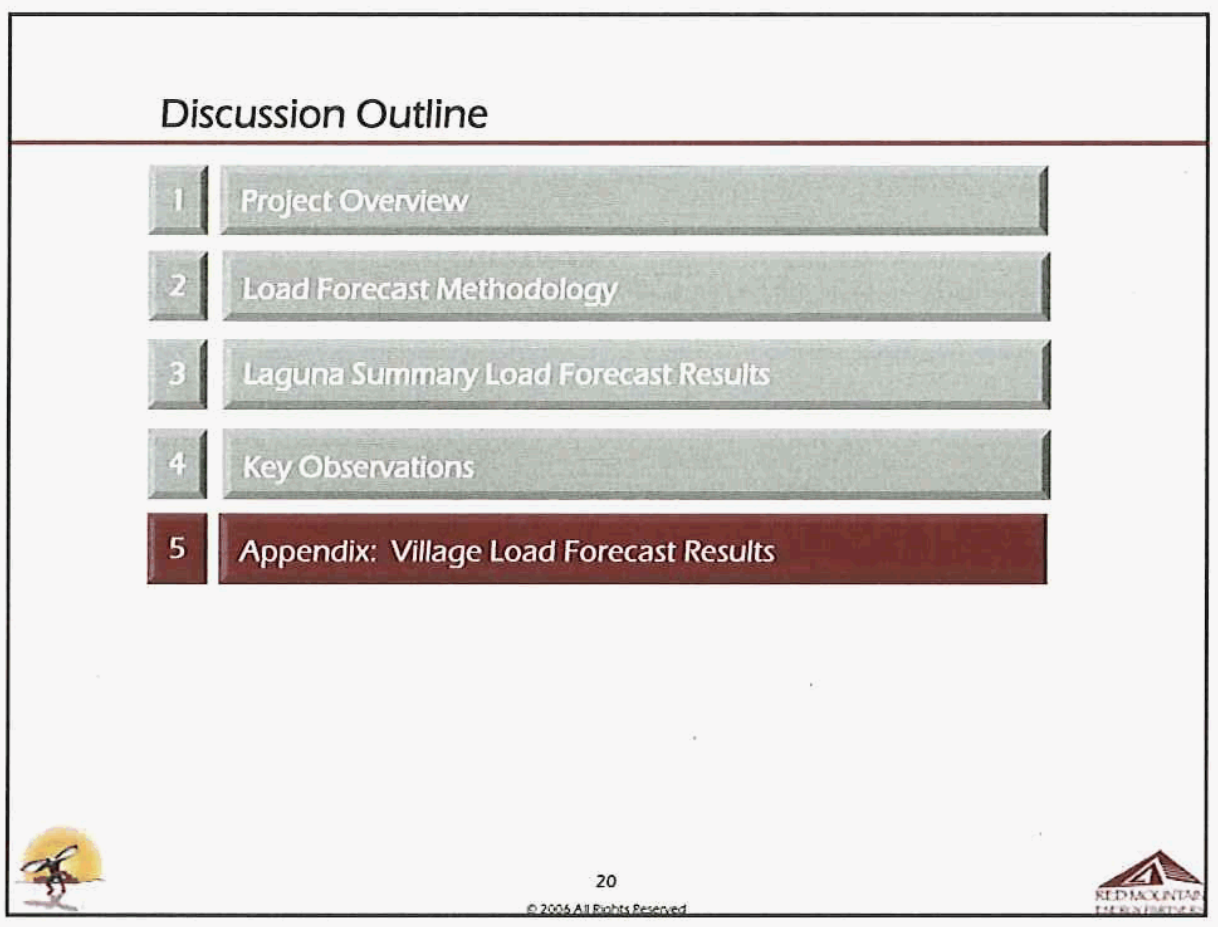

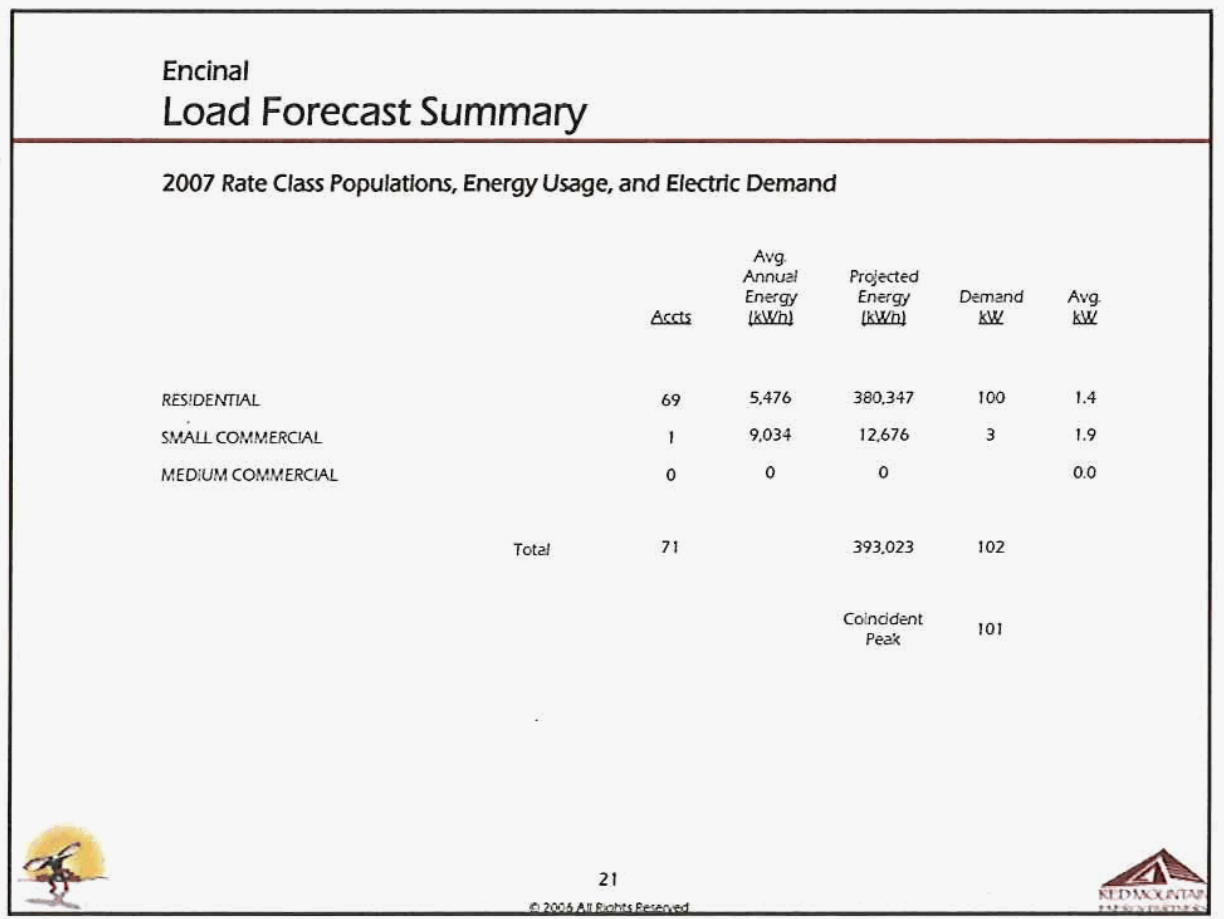




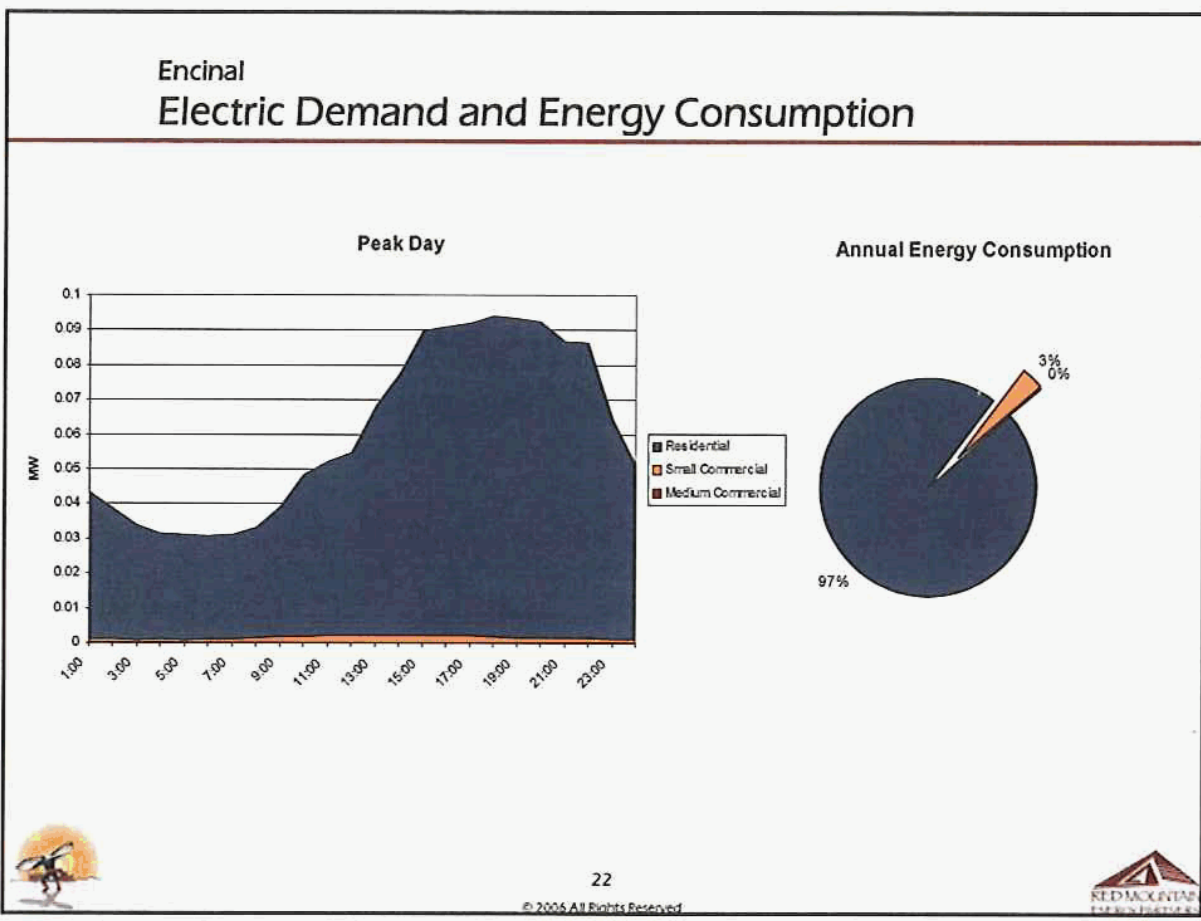

Annual Load Variation

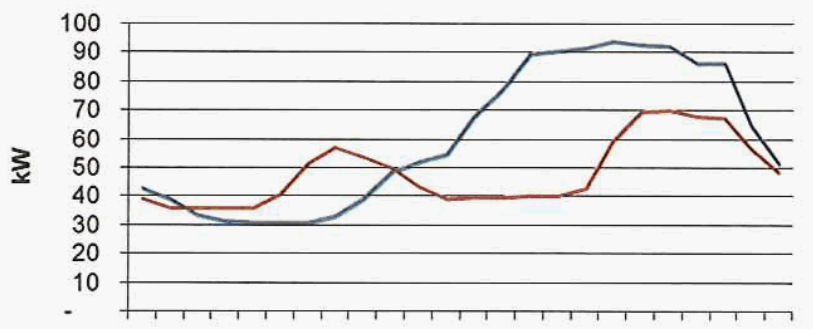

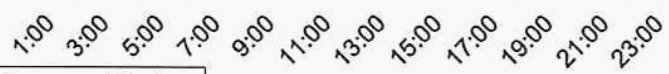

-Max Day —Min Day 


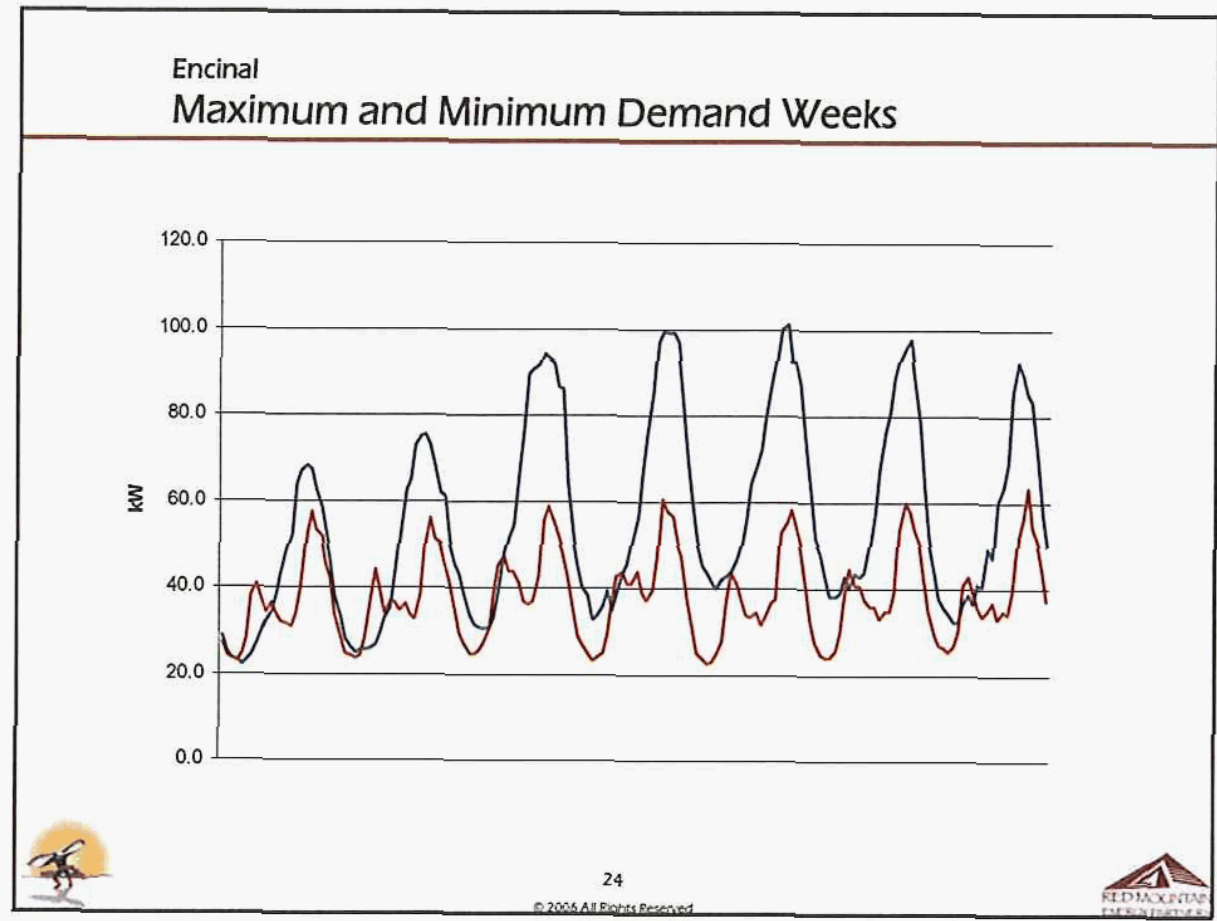

\section{Encinal}

Minimum Demand Week
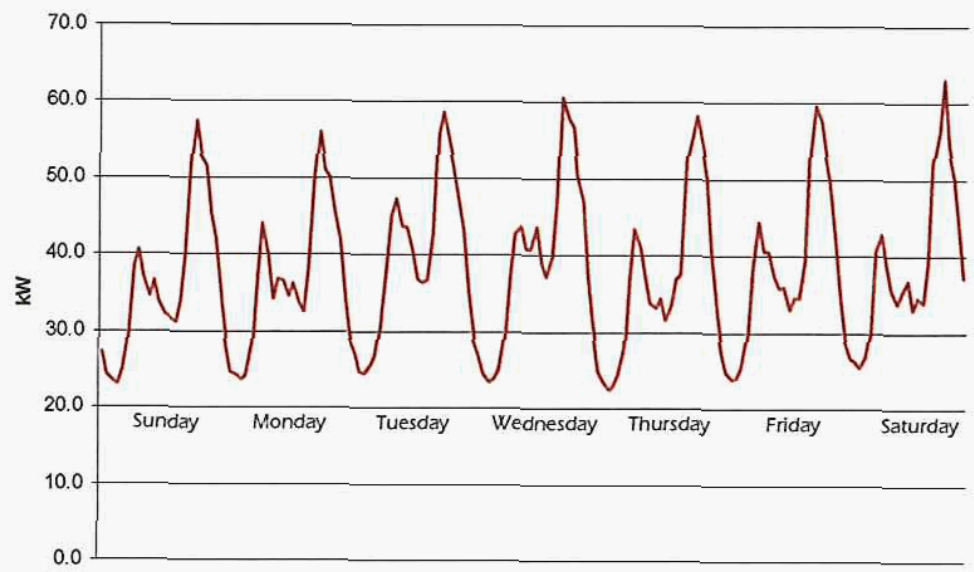

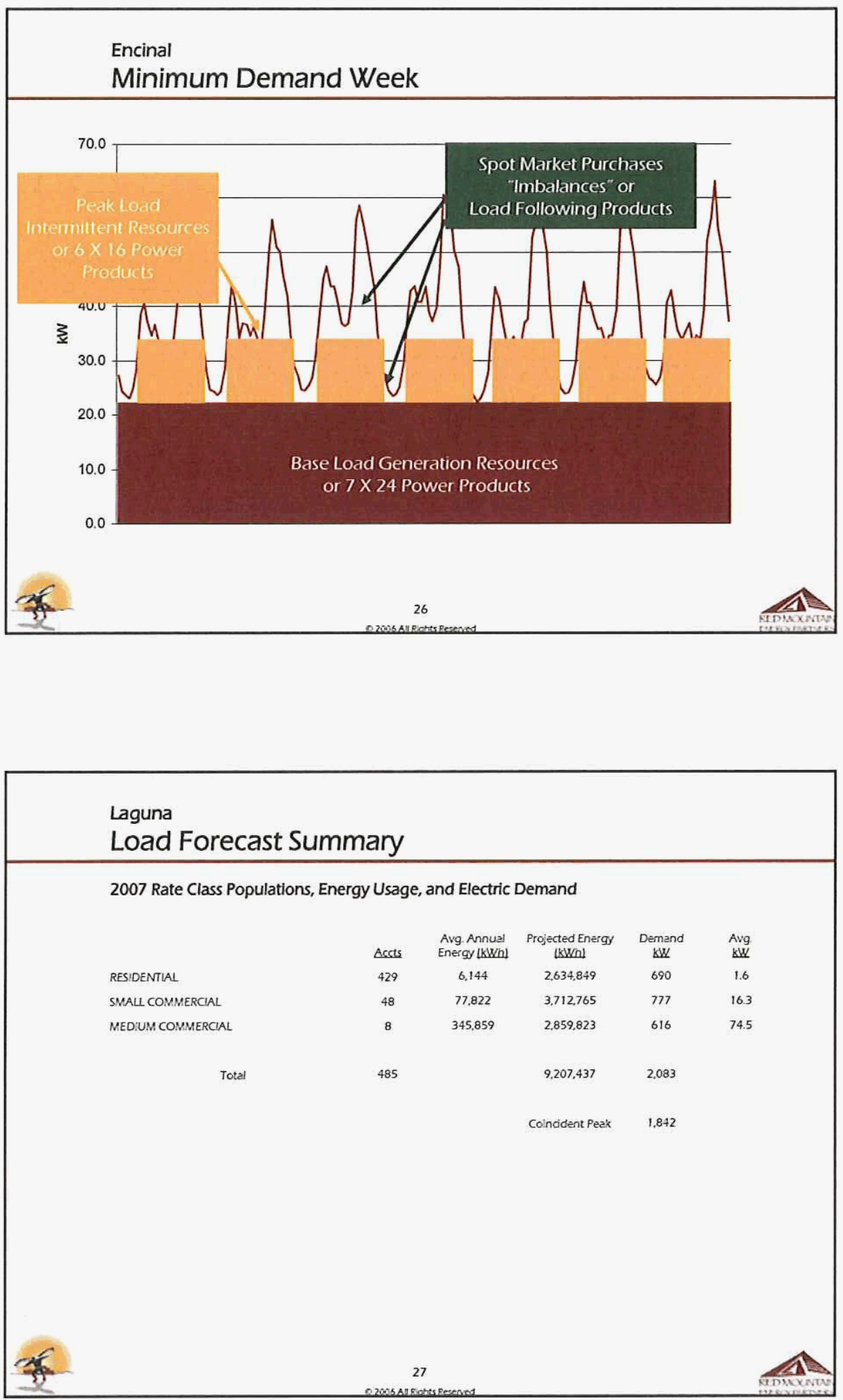

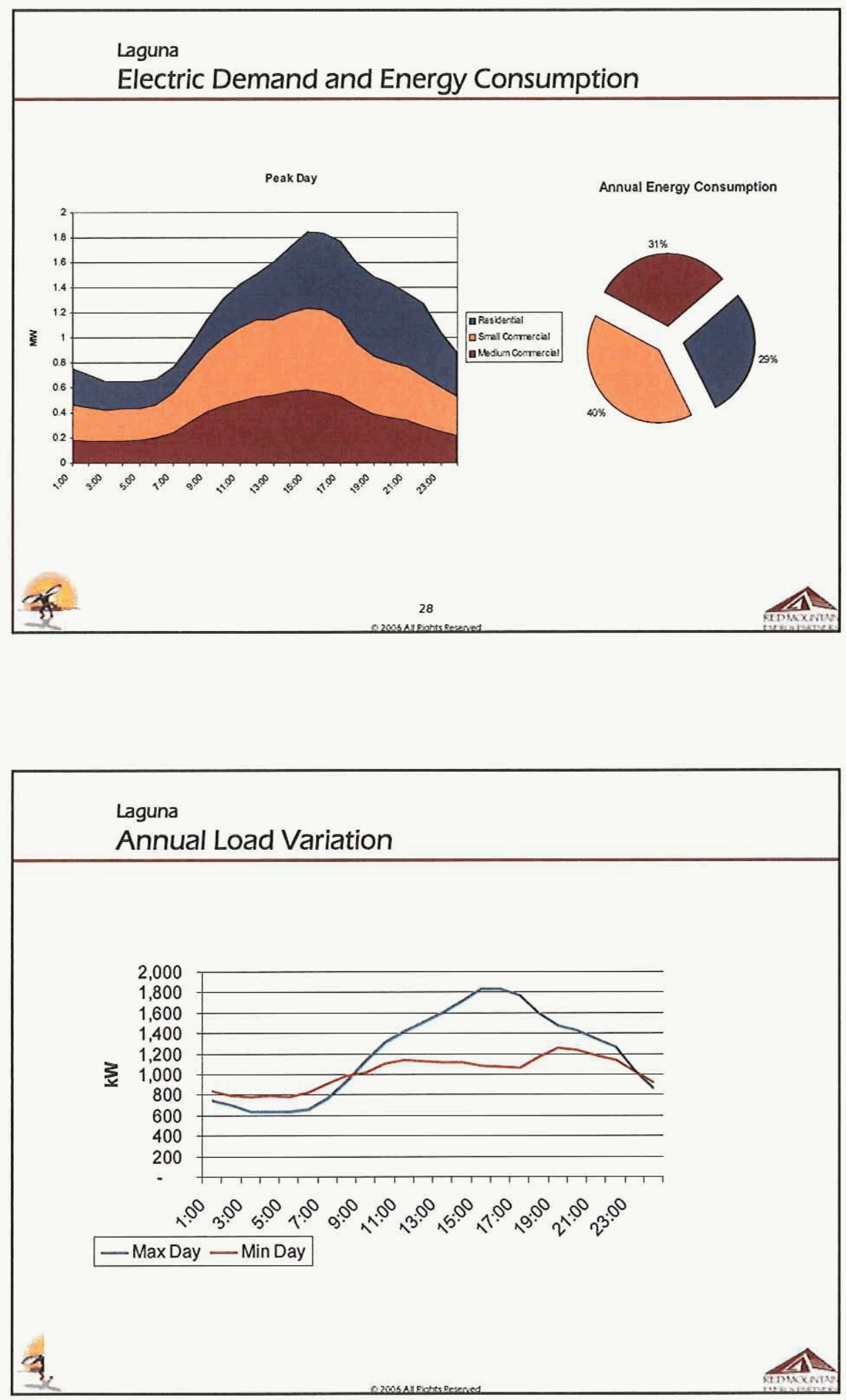

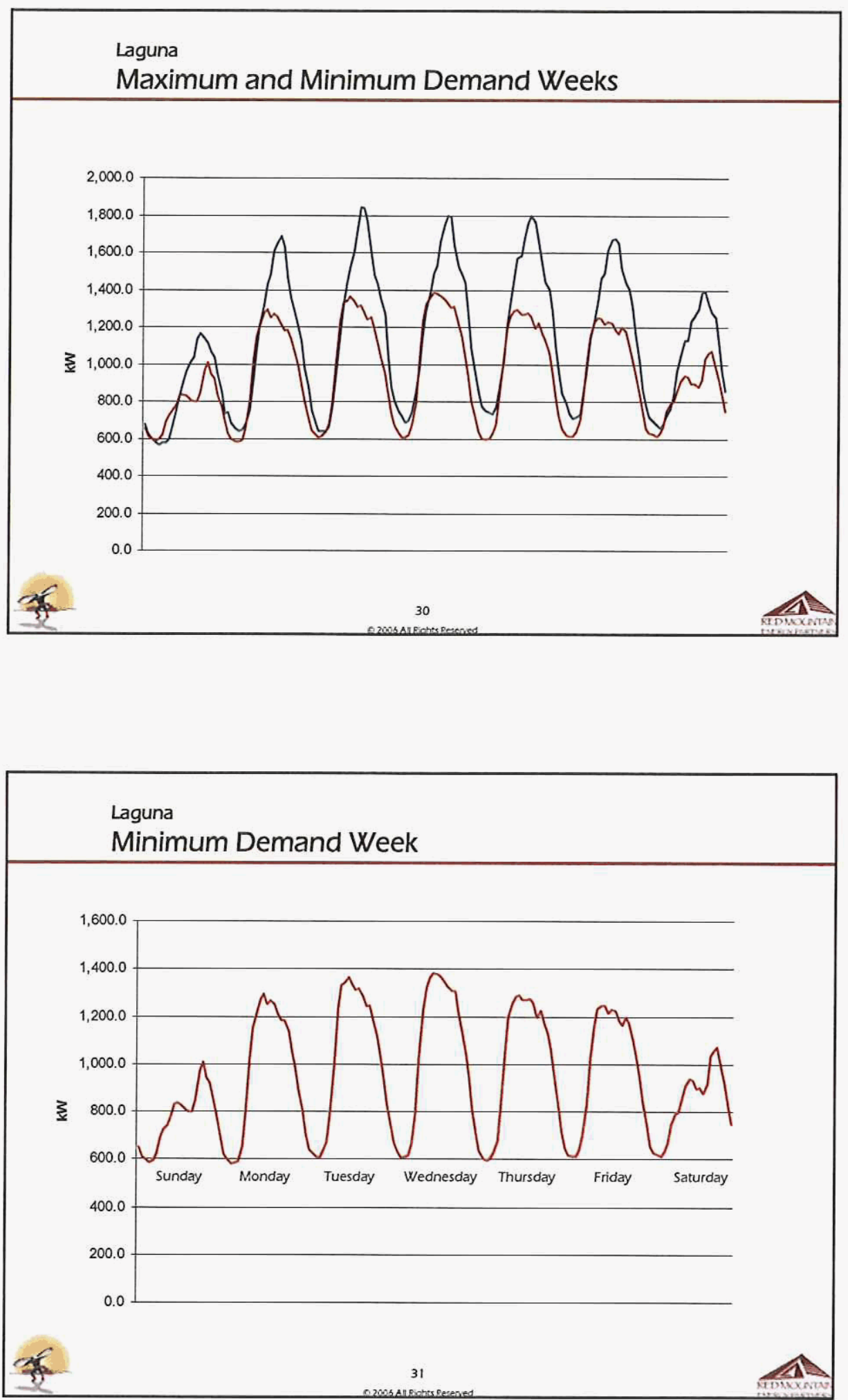

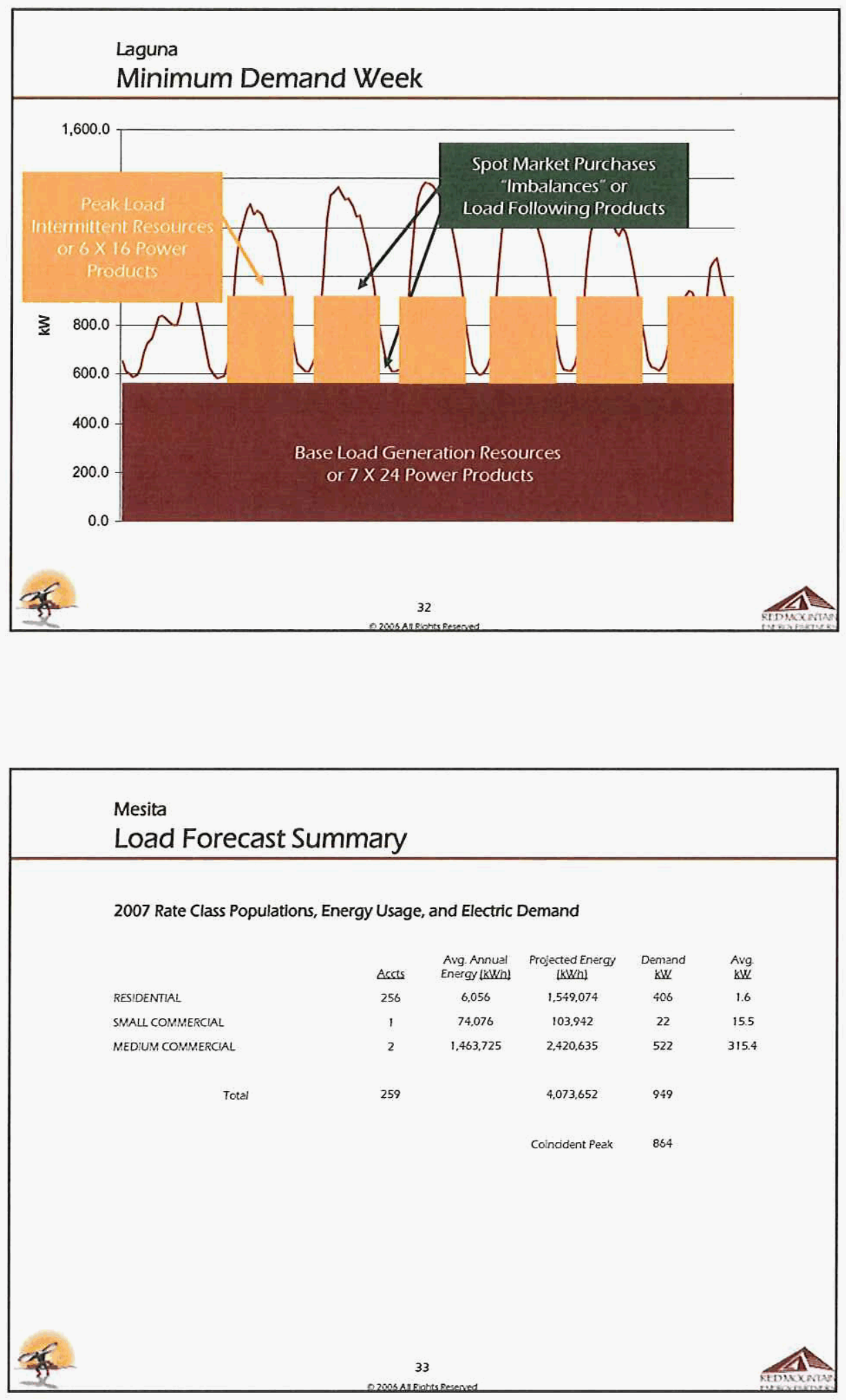


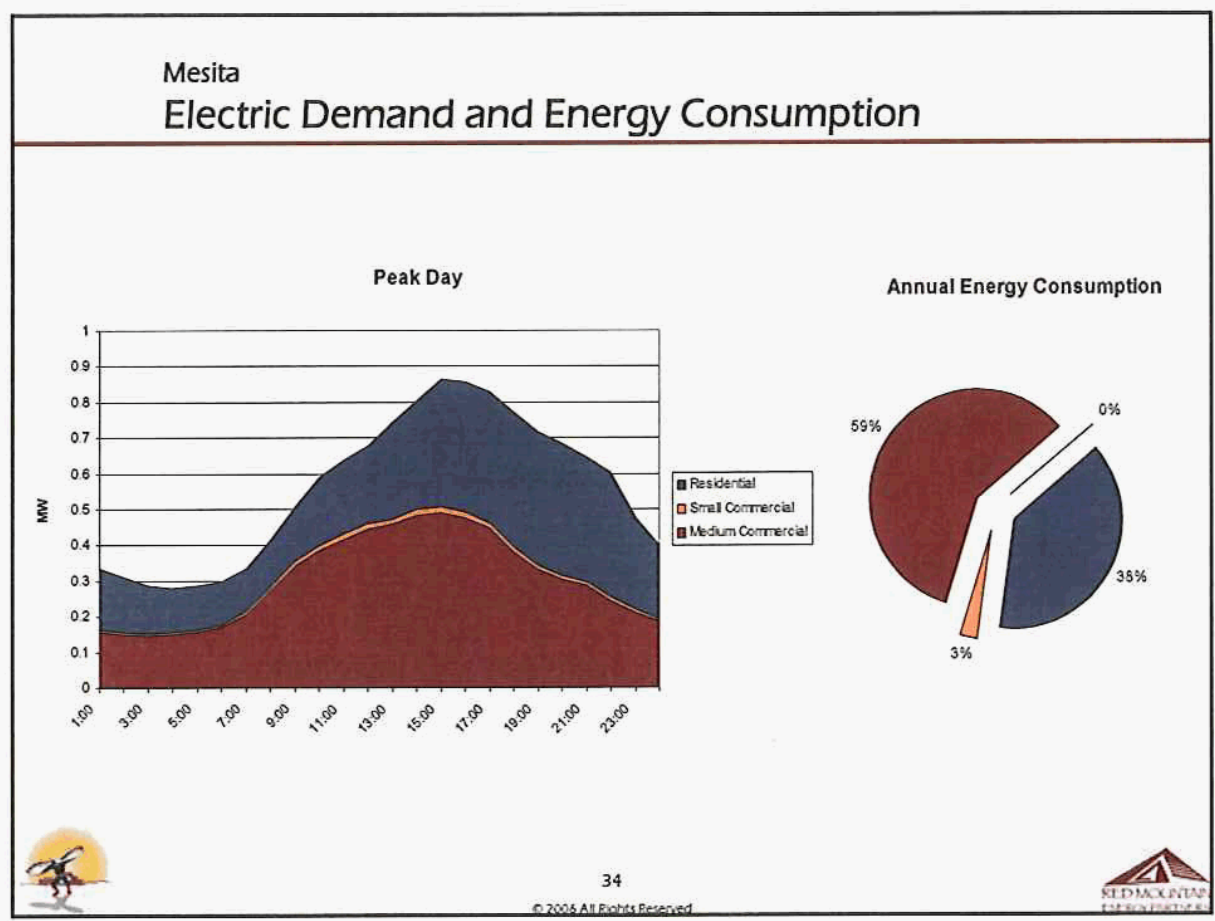

Mesita

Annual Load Variation

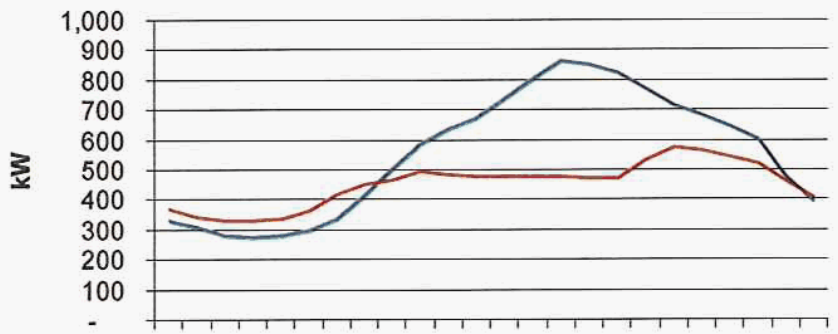

一 Max Day - Min Day 

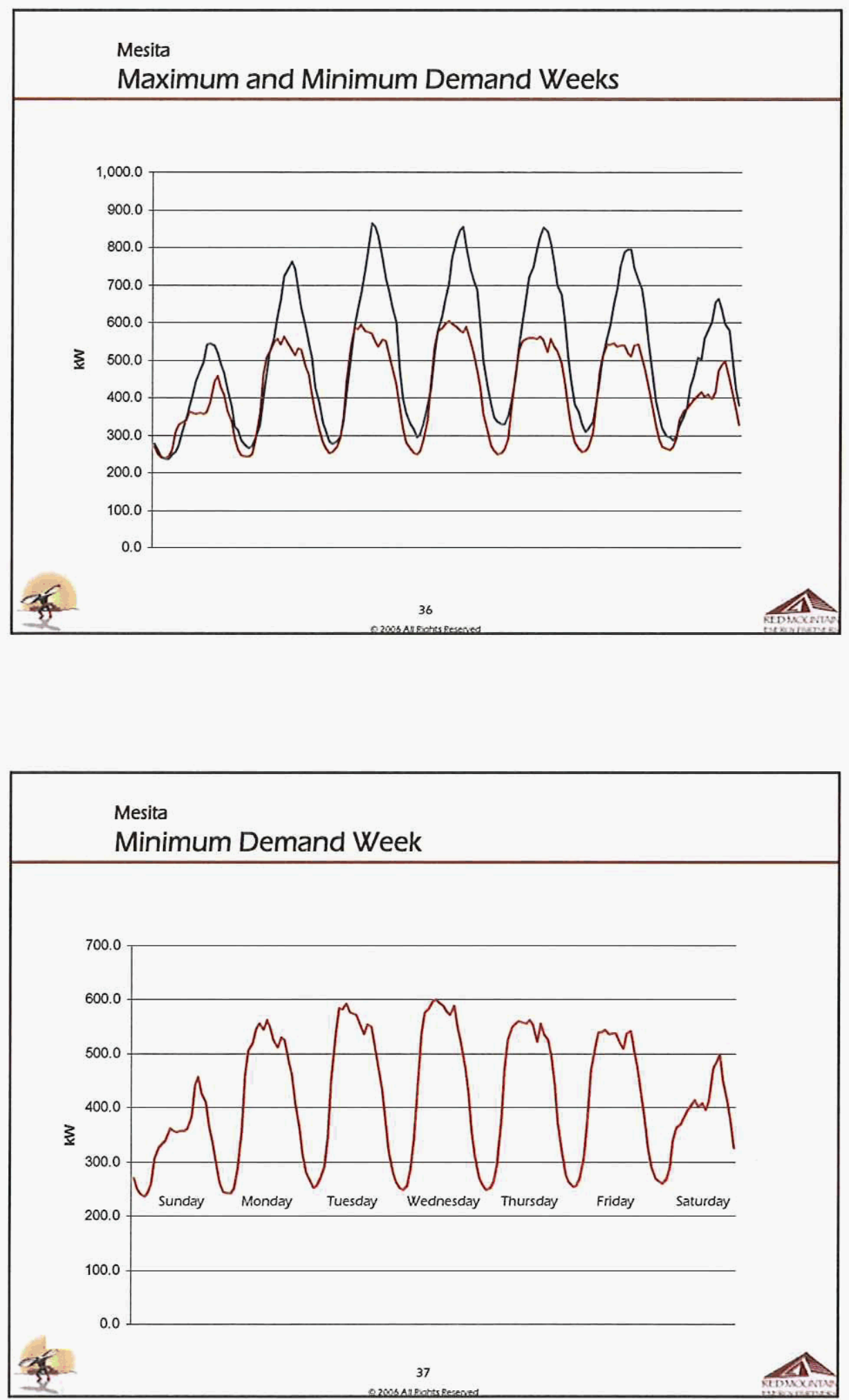

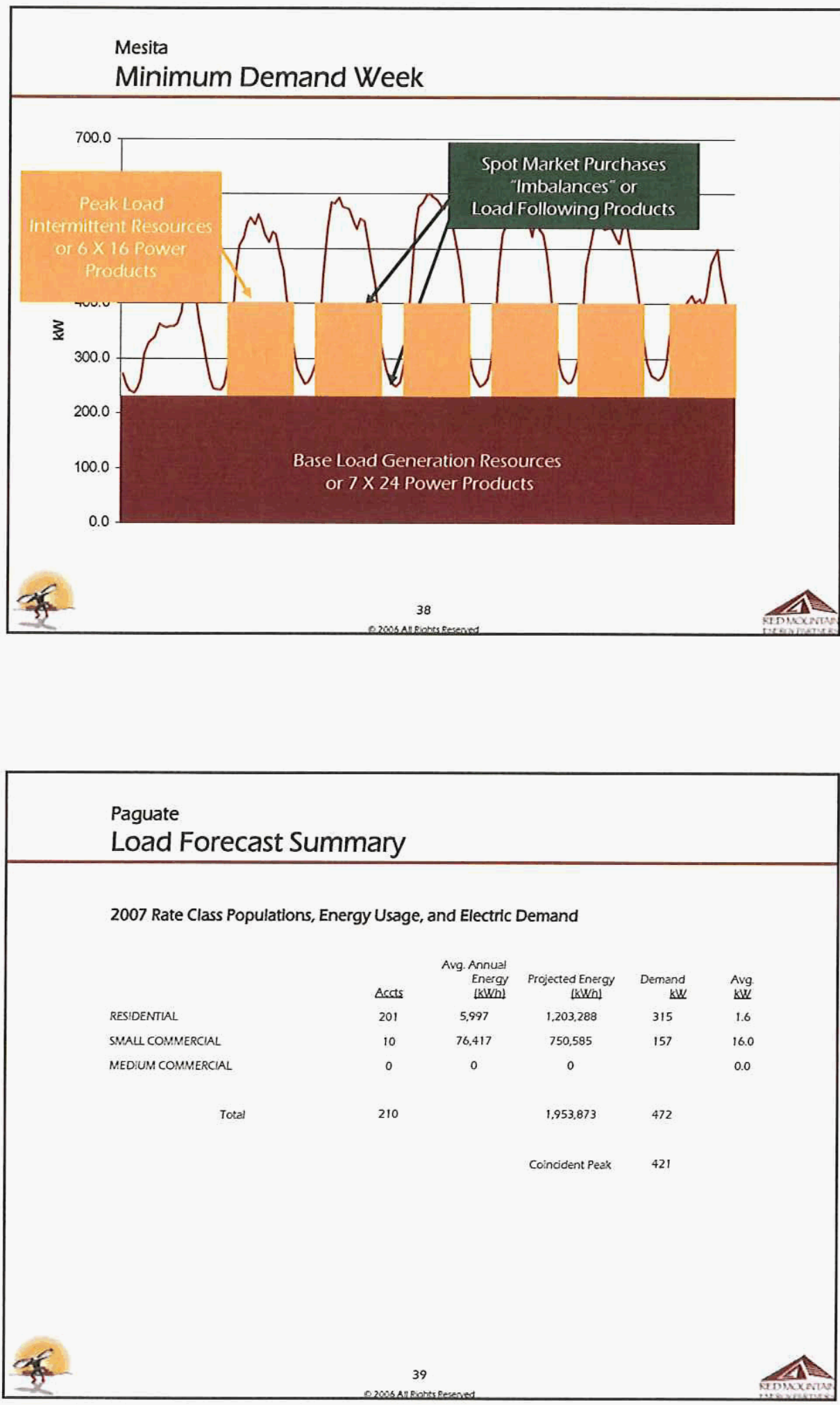


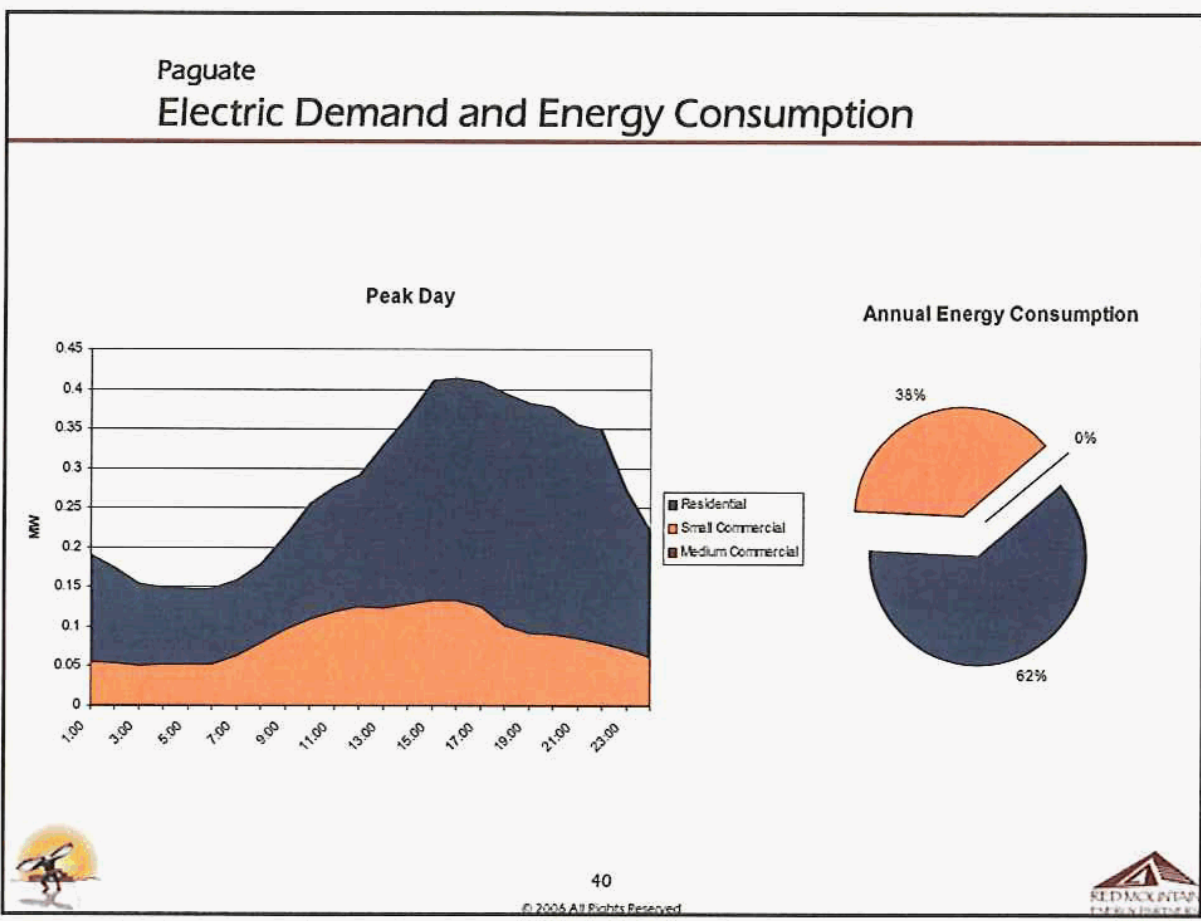

Paguate

Annual Load Variation

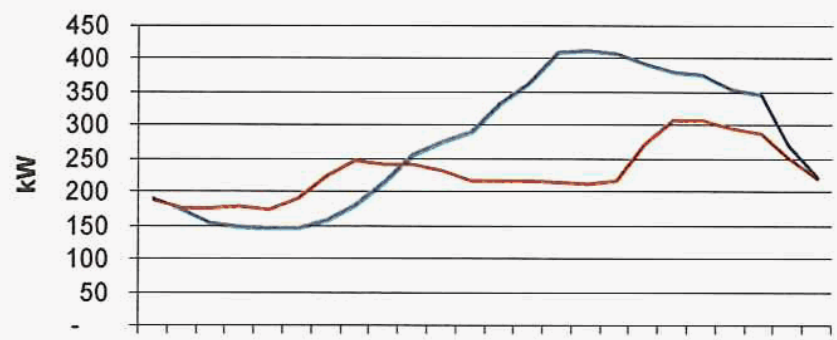

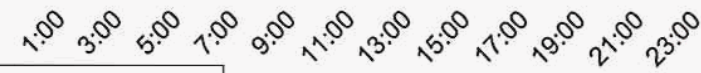

—MaxDay —Min Day

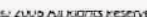



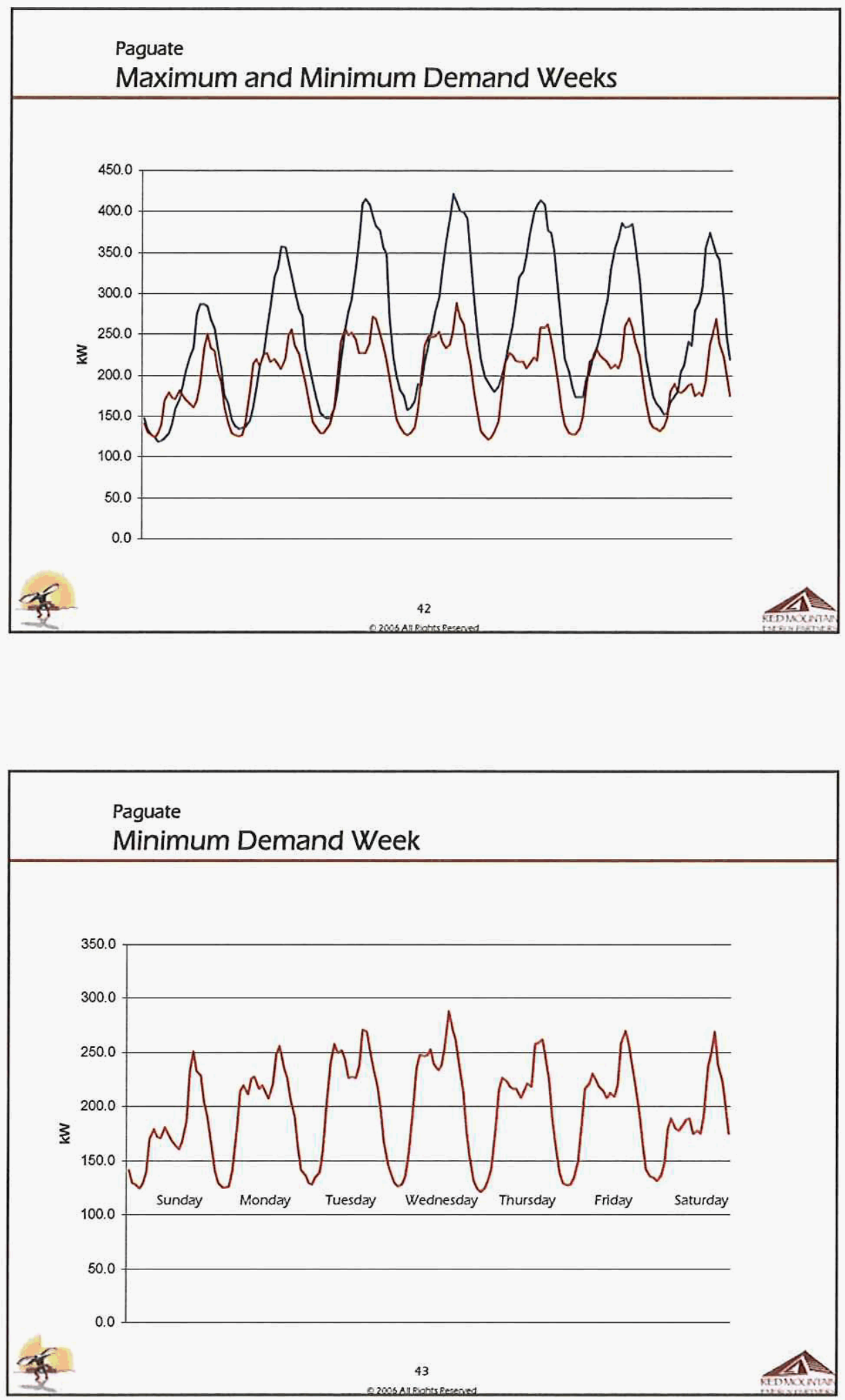

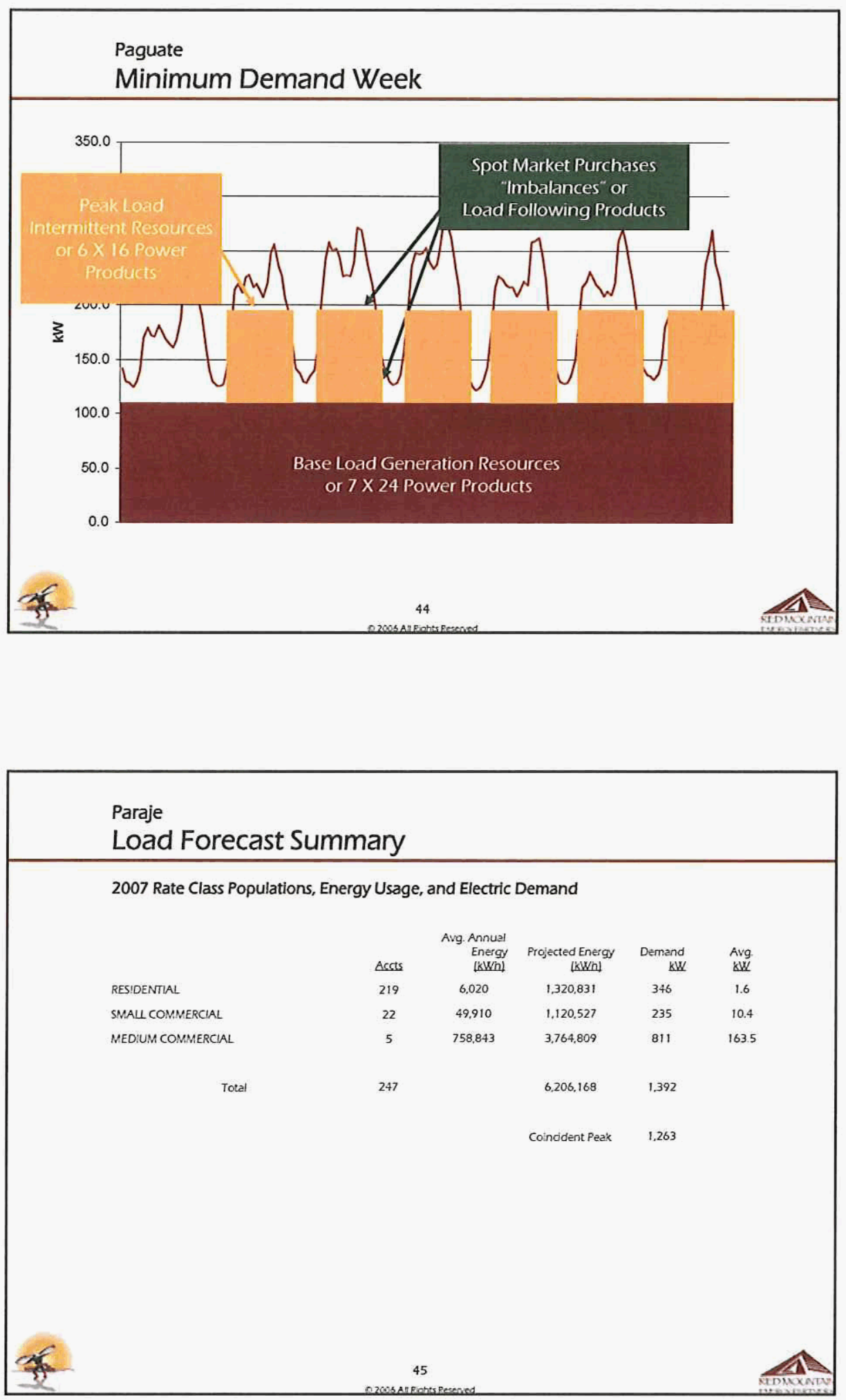


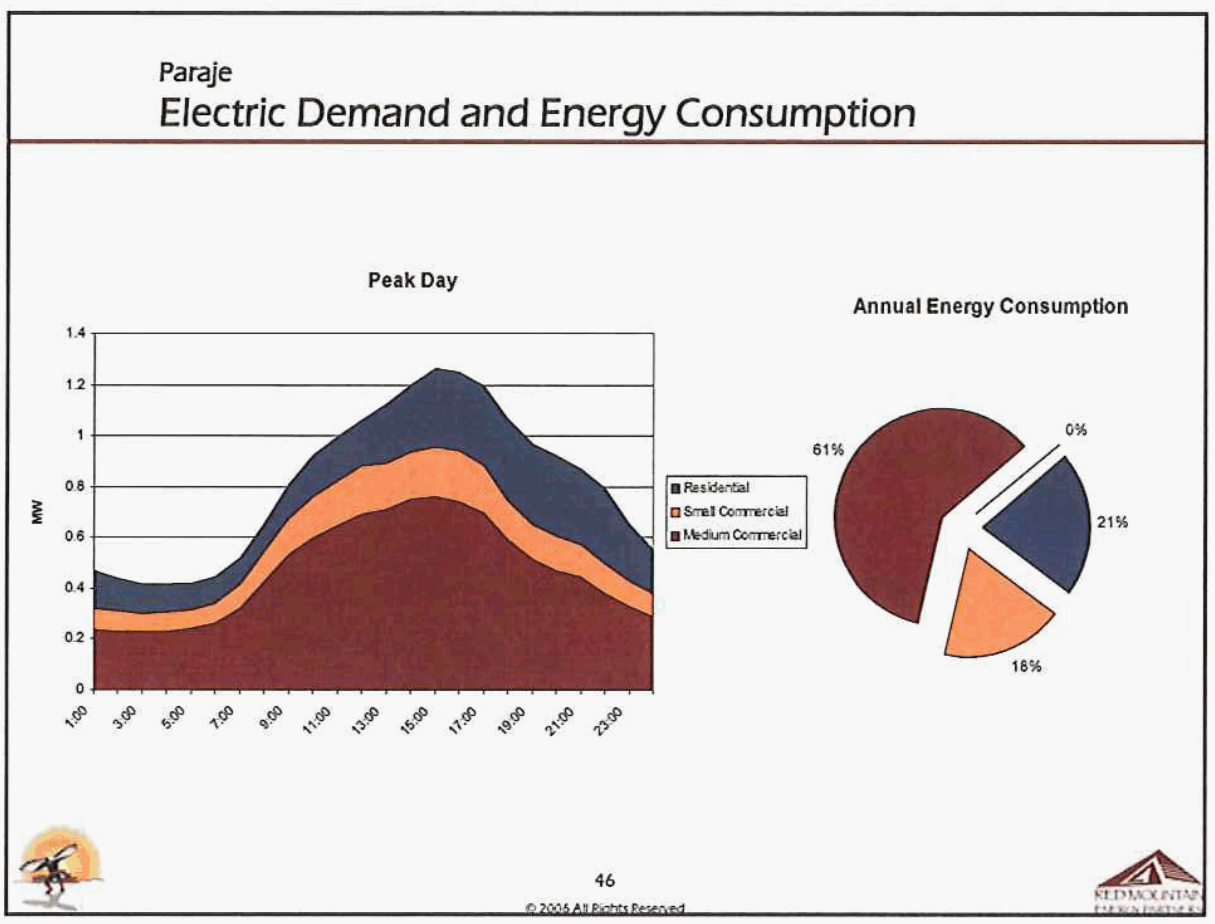

Paraje

Annual Load Variation

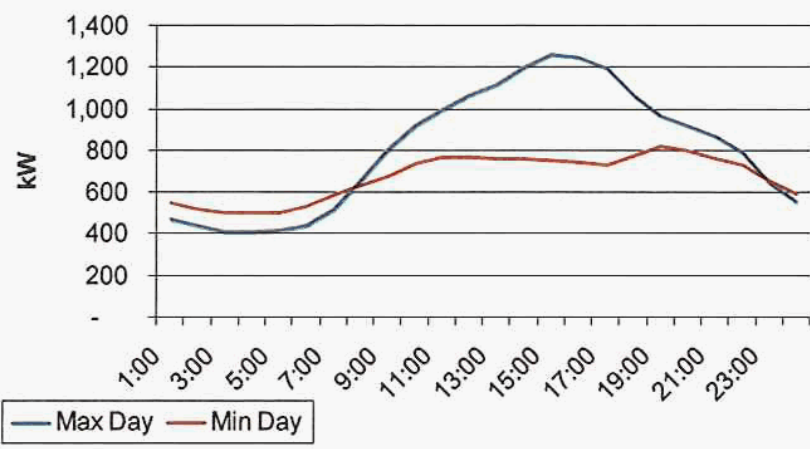

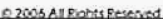



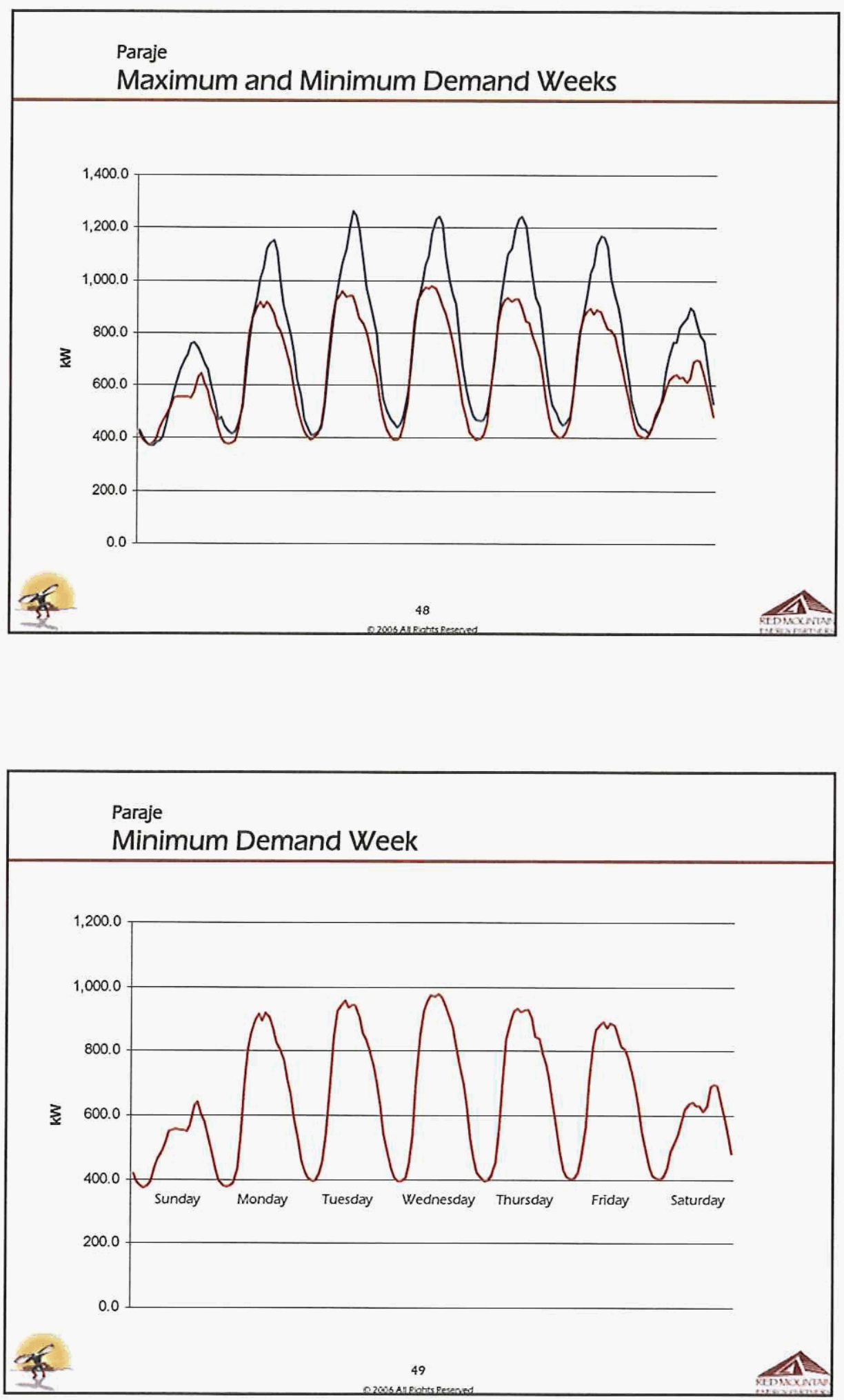


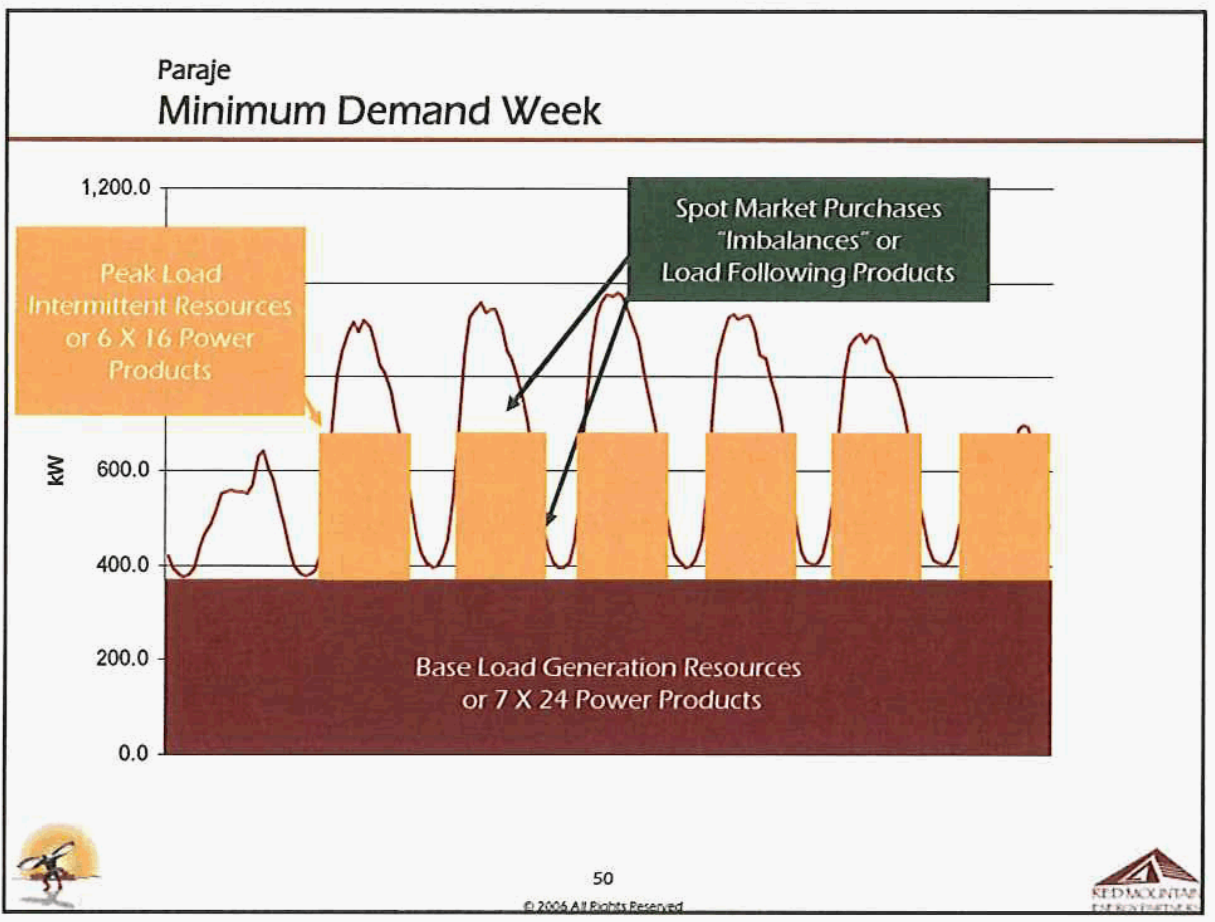

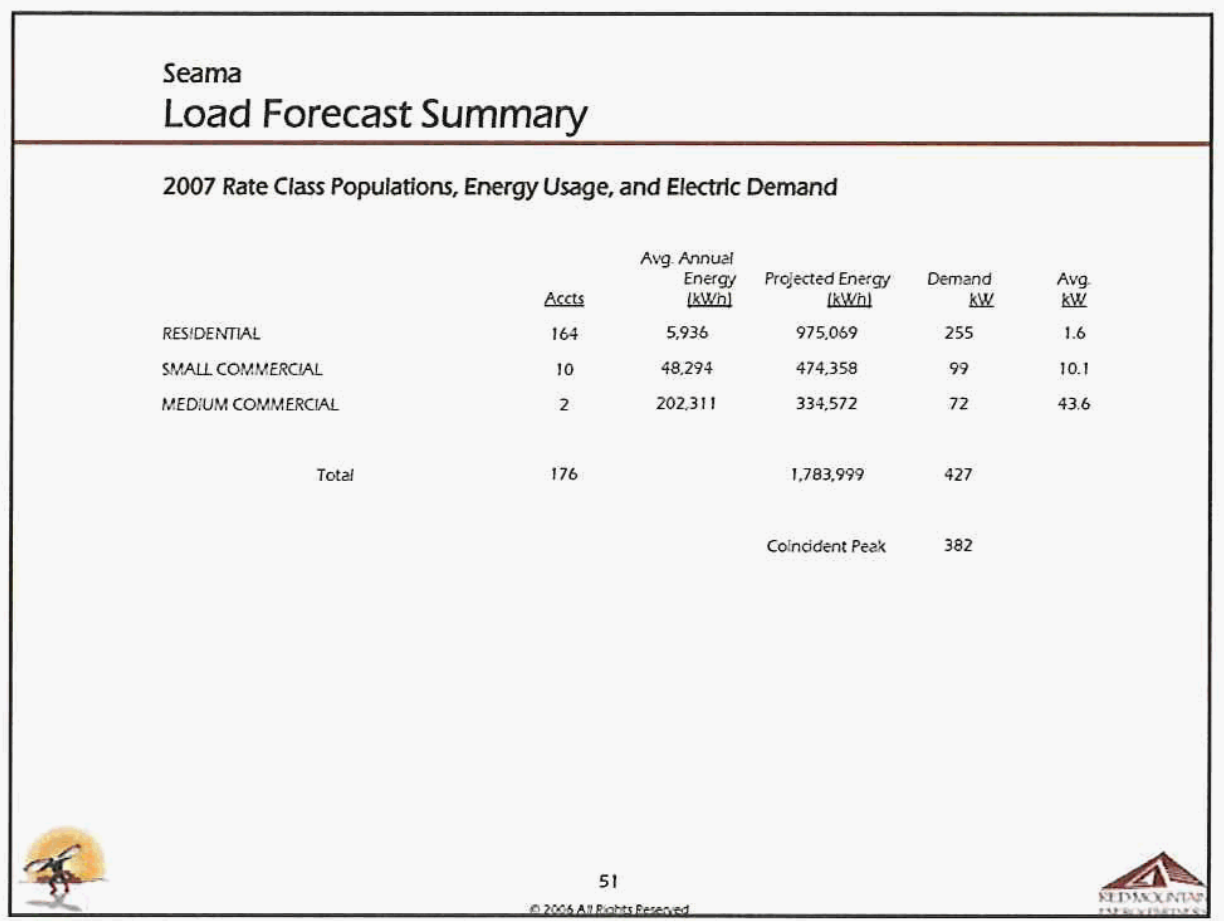



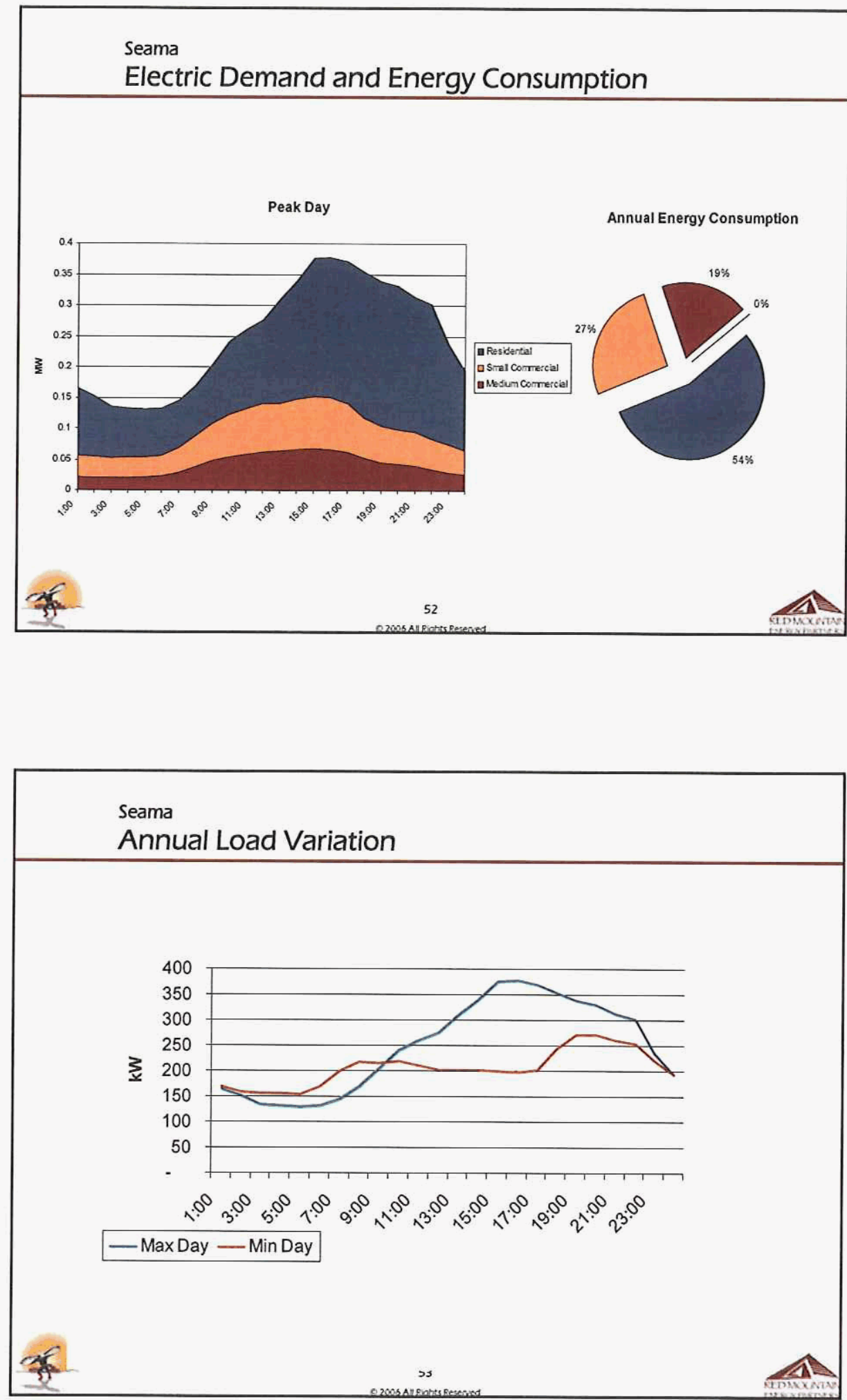

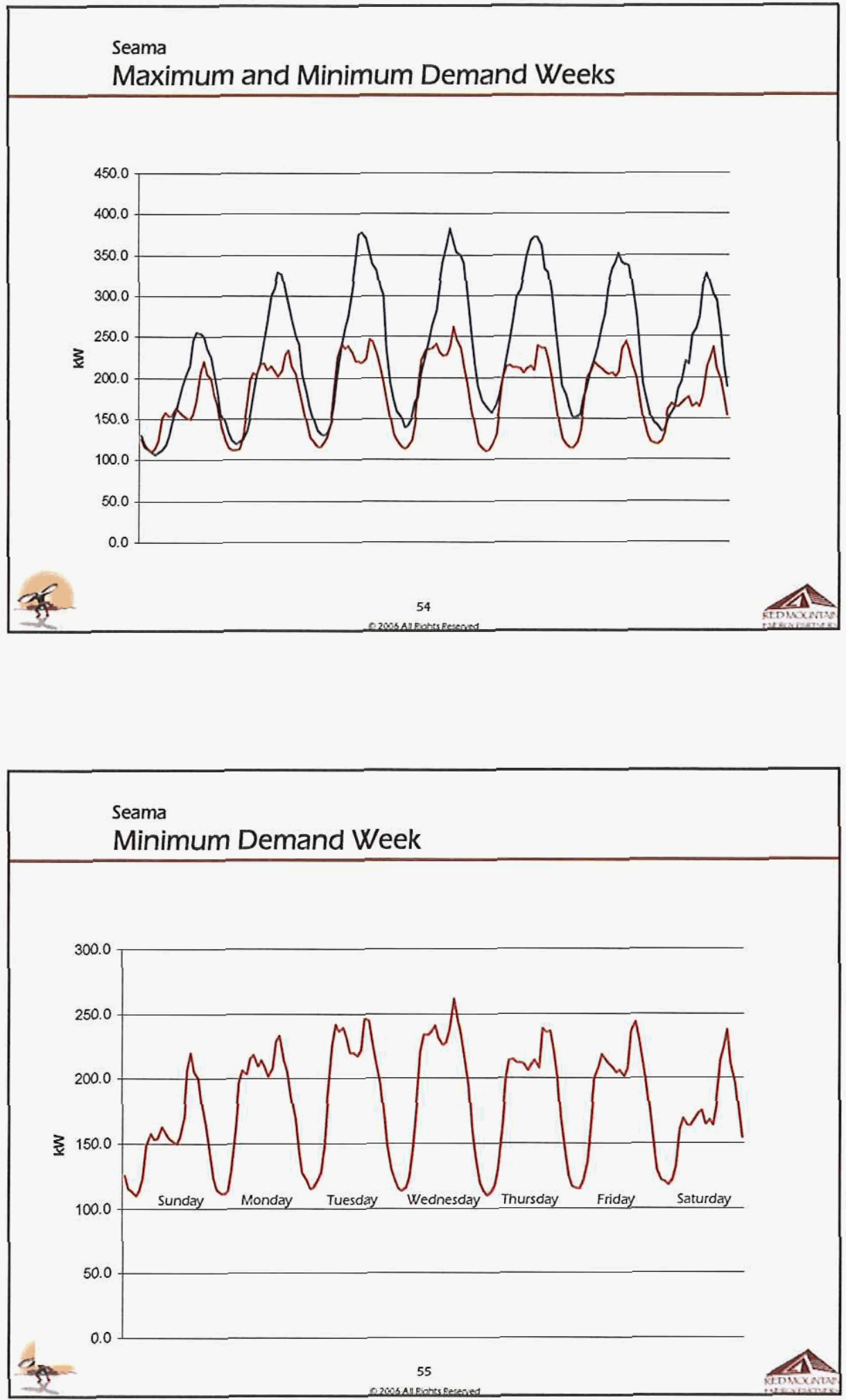


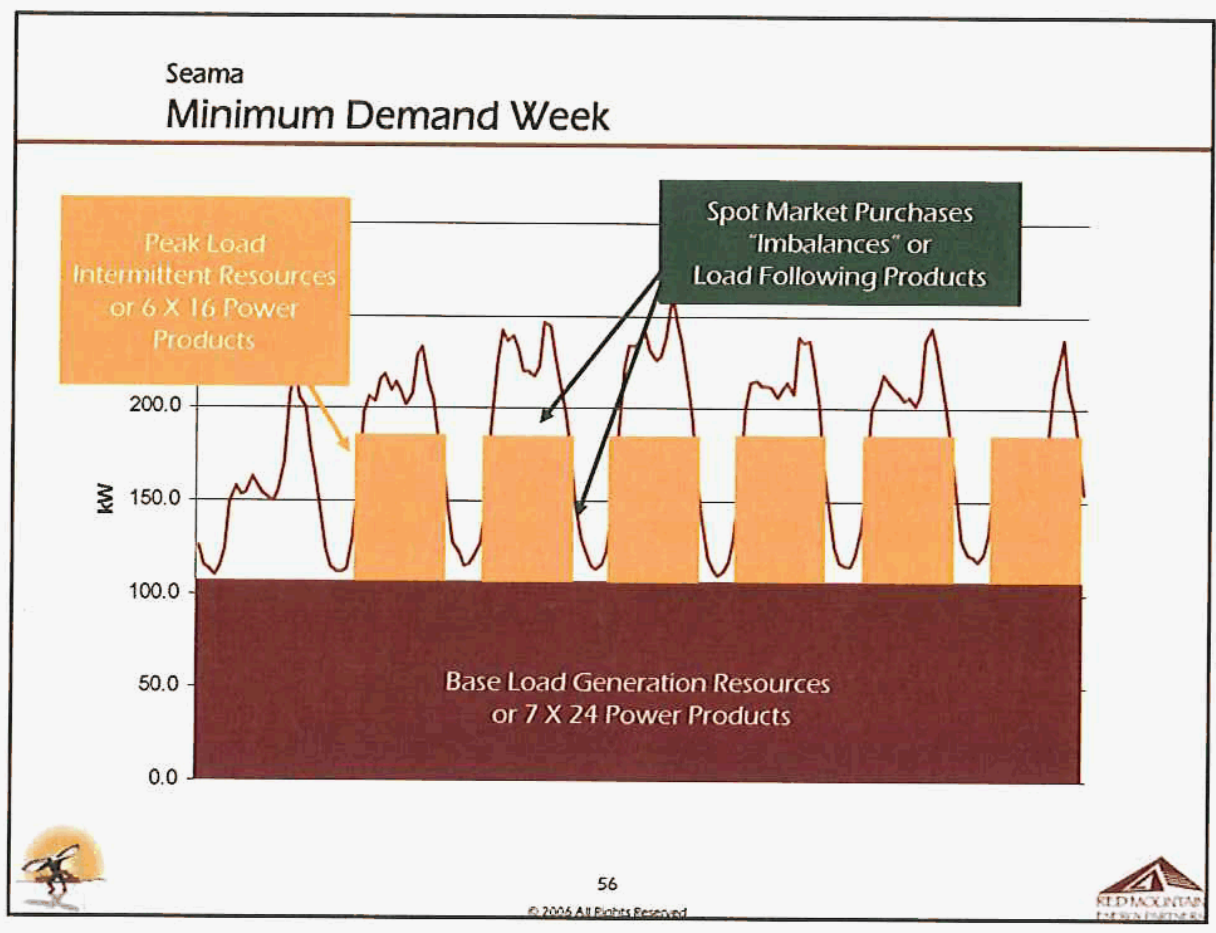




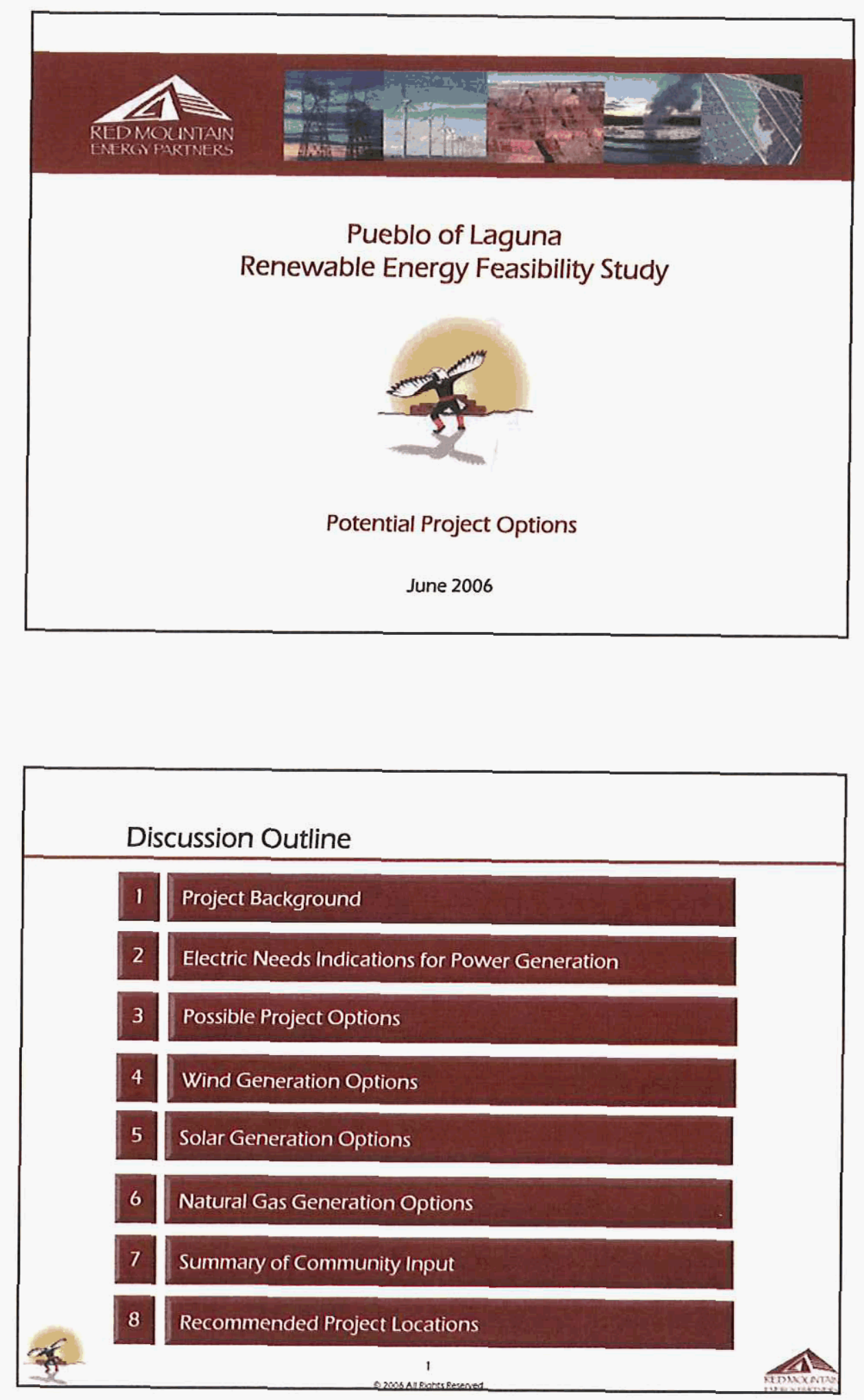

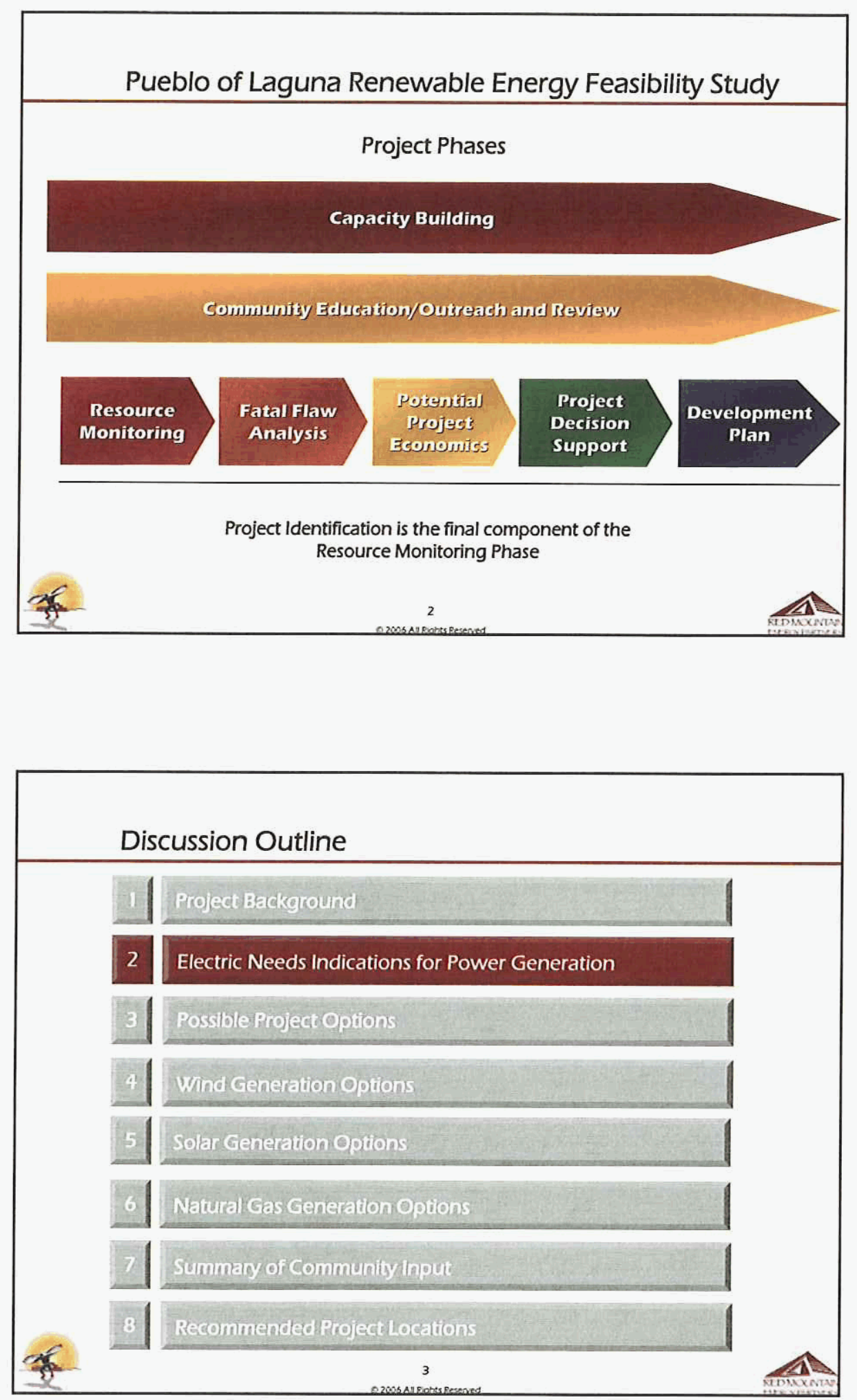


\section{Pueblo of Laguna \\ Load Forecast Summary}

\section{PUEBLO OF LAGUNA}

2007 Rate Class Populations, Energy Usage, and Electric Demand

Residential

Small Commercial

Medium Commercial

\begin{tabular}{|c|c|c|c|c|}
\hline Consumers & $\begin{array}{l}\text { Avg. Annual } \\
\text { Energy (kWh) }\end{array}$ & $\begin{array}{c}\text { Projected } \\
\text { Energy (kWh) }\end{array}$ & $\begin{array}{c}\text { Demand } \\
\underline{\mathrm{kW}}\end{array}$ & $\begin{array}{l}\text { Avg. } \\
\mathrm{kW}\end{array}$ \\
\hline 1,294 & 6,230 & $8,063,459$ & 2,112 & 1.6 \\
\hline 93 & 66,676 & $6,174,853$ & 1,293 & 14.0 \\
\hline 17 & 567,186 & $9,379,839$ & 2,021 & 122.2 \\
\hline 1,403 & & $23,618,151$ & 5,426 & \\
\hline & & Coincident Peak & 4,845 & \\
\hline
\end{tabular}

\section{Electric Loads}

\begin{tabular}{|c|c|c|c|c|c|c|c|}
\hline & Encinal & Laquna & Mesita & Paquate & Parce & Seama & All Villages \\
\hline \multicolumn{8}{|l|}{ Consumers } \\
\hline Residential & 61 & 429 & 247 & 192 & 211 & 155 & 1.294 \\
\hline Smal Commercial & 1 & 48 & 1 & 10 & 22 & 10 & 93 \\
\hline Medum Commeroial & 0 & 8 & 2 & 0 & 5 & 2 & 17 \\
\hline Total Consumers & 62 & 485 & 250 & 202 & 238 & 167 & 1.403 \\
\hline perenteofteral & 4.46 & $345 \%$ & 178 in & 14.4\% & 17.06 & $11.9 \%$ & \\
\hline \multicolumn{8}{|l|}{ Energy Requirements (kWh) } \\
\hline Residential & 380,347 & $2,634,849$ & $1,549,074$ & $1,203.288$ & 1.320 .831 & 975.059 & $8,063,459$ \\
\hline Smal Commercial & 12.676 & 3.712 .765 & 103.942 & 750,585 & $1.120,527$ & 474,358 & $6,174,853$ \\
\hline Medum Commercial & & $2,859,823$ & $2,420,635$ & & $3,764,809$ & 334,572 & $9,379,839$ \\
\hline $\begin{array}{l}\text { Total Energy } \\
\text { percent or tocal }\end{array}$ & $\begin{array}{c}393,023 \\
178\end{array}$ & $\begin{array}{c}9,207,437 \\
39.04\end{array}$ & $\begin{array}{c}4.073 .652 \\
17.266\end{array}$ & $\begin{array}{c}1,953,873 \\
8366\end{array}$ & $\begin{array}{c}6,206,168 \\
26,3 \% 6\end{array}$ & $\begin{array}{c}1.783 .959 \\
7.6 \%\end{array}$ & $23,618,151$ \\
\hline \multicolumn{8}{|l|}{ Demand $(\mathrm{kW})$} \\
\hline Residential & 99.6 & 690.2 & 405.8 & 315.2 & 346.0 & 255.4 & 2,1122 \\
\hline Small Commercial & 2.7 & 777.2 & 21.8 & 157.1 & 234.5 & 99.3 & 1,2925 \\
\hline Medum Commercial & & 616.1 & 521.5 & & 811.1 & 72.1 & 2.020 .9 \\
\hline Peak Demand & 102 & 2,083 & 949 & 472 & 1.392 & 427 & 5.426 \\
\hline Peak Coincident Demand & 101 & 1.842 & 864 & 421 & 1.263 & 382 & 4,845 \\
\hline percent of tocal & $21 \%$ & $38.0 \%$ & $178 \%$ & $27 \%$ & $261 \%$ & 2.96 & \\
\hline & & & 5 & & & & \\
\hline
\end{tabular}



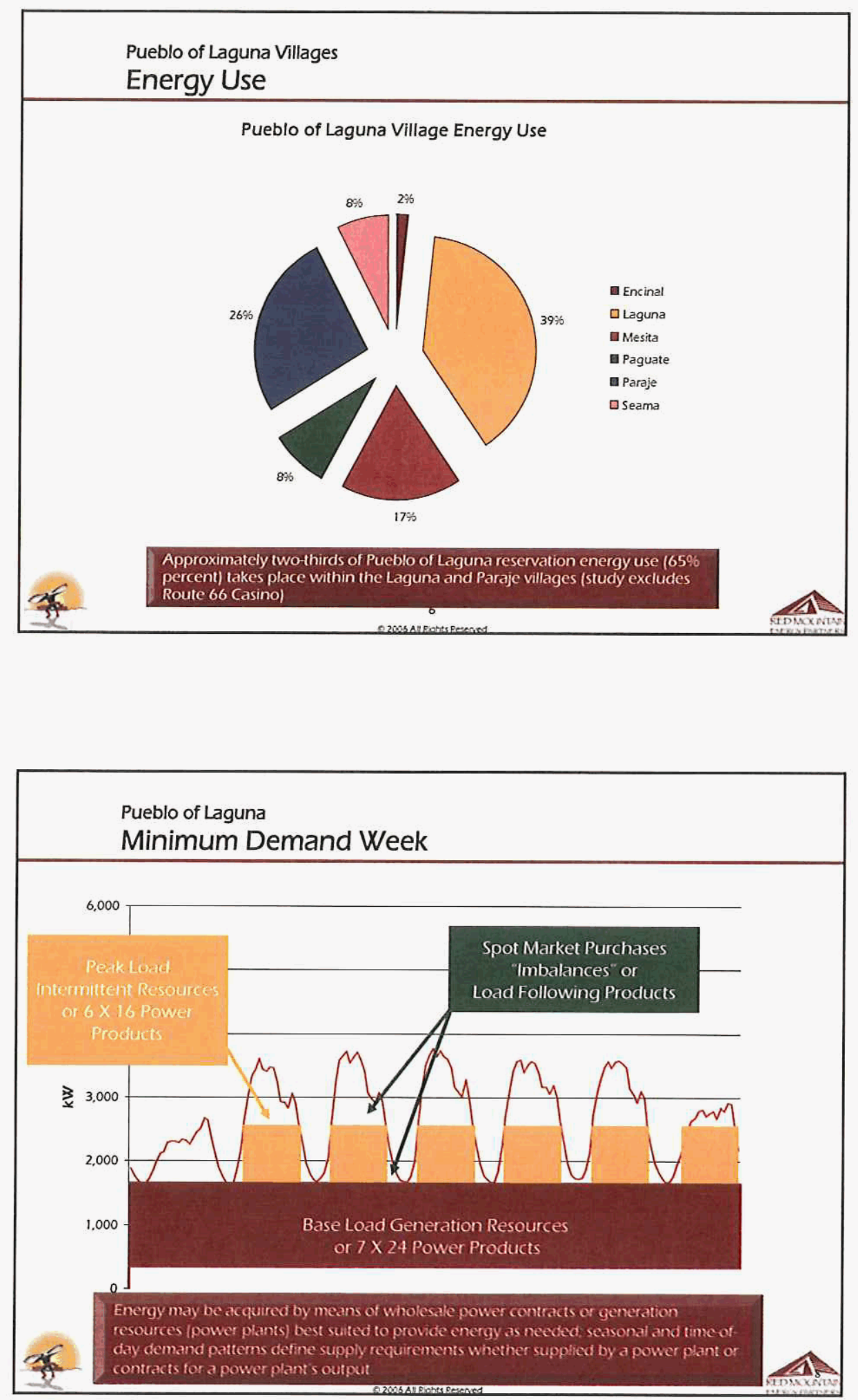

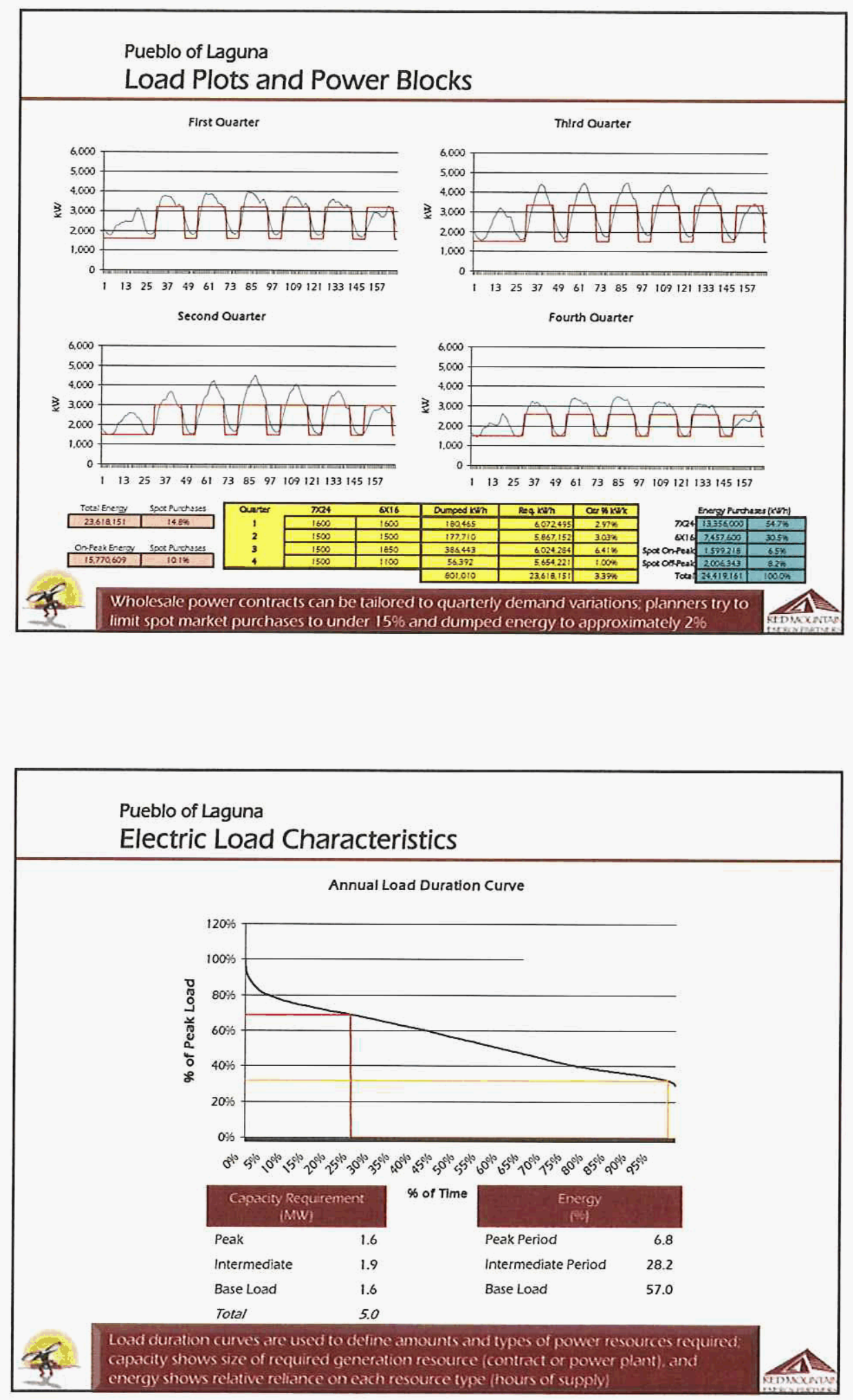


\section{Key Observations}

When addressing Pueblo of Laguna total electric energy requirements:

- The majority of Pueblo of Laguna energy demand is associated with nonresidential activity (66\%)

- Among Pueblo of Laguna villages, the villages of Laguna and Paraje comprise the majority of energy demand $(65 \%)$

- Peak demand is approximately $5 \mathrm{MW}$, however loads can vary as much as 3.2 $M W$ in a single day

2 Resources required to serve the total reservation electric demand:

- 1.6 MW base load supply $(7 \times 24)$

- $1.9 \mathrm{MW}$ intermediate $(6 \times 16)$

- 1.6 MW Load following (spot market purchases)

- $57 \%$ of all energy consumed can be provided by constant duty cycle base load $(7 \times 24)$ generation resources

> $28 \%$ of all energy consumed can be provided by intermediate resources (including solar and wind power generation)

- Remaining energy can be procured on the spot market without exceeding 15\% (appropriately avoiding market uncertainties)

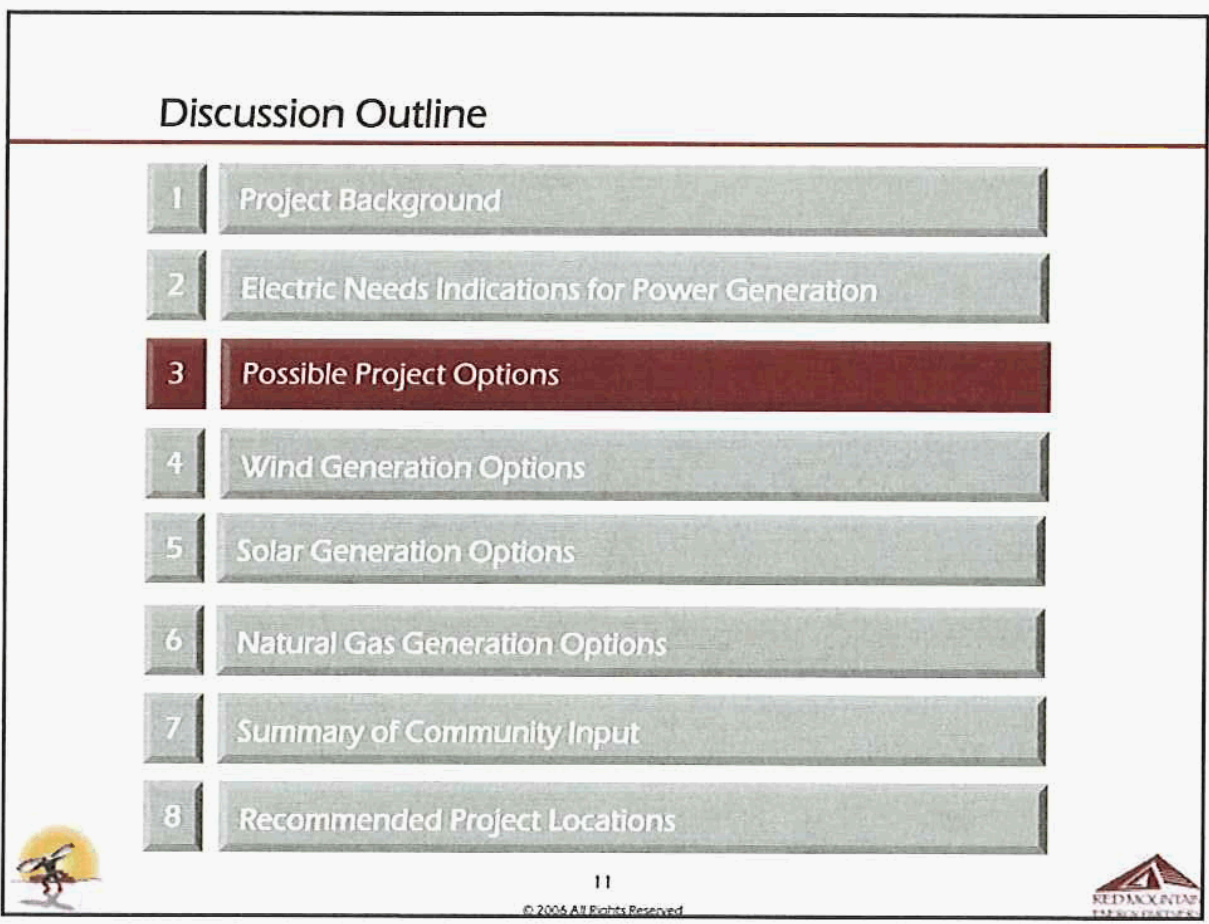




\section{Possible Project Option Considerations}

- Project should generate adequate power to meet a portion or all of specific electric needs
* Reservation
* Village
* Entity

- Customers generally assumed to have continued connection to the grid

- Projects were not intended to meet $100 \%$ of needs

- Laguna renewable projects considered do not generate firm power

- Solar

* Wind

- Purpose of gas-generation would be to firm a renewable resource

Possible Project Summary

\begin{tabular}{|c|c|c|c|c|c|c|c|c|}
\hline $\begin{array}{c}\text { Type of } \\
\text { Generation }\end{array}$ & $\begin{array}{l}\text { Annual } \\
\text { Demand } \\
\mathrm{KW}\end{array}$ & $\begin{array}{l}\text { Annual } \\
\text { Usage } \\
\text { Milon } \\
\text { kWh/ }\end{array}$ & Wind & Solar & Bomass & Geothermal & $\begin{array}{c}\text { Natural } \\
\text { Gas }\end{array}$ & $\begin{array}{c}\text { Hyordd } \\
\text { RE/Natural } \\
\text { Gas }\end{array}$ \\
\hline $\begin{array}{l}\text { Laguna } \\
\text { Reservaton- } \\
\text { scatet } \\
\text { Generation }\end{array}$ & 5.426 & 236 & $\mathrm{x}$ & $x$ & & & $\mathrm{x}$ & $x$ \\
\hline $\begin{array}{l}\text { Laguna } \\
\text { vilizgescate } \\
\text { Generaton } \\
\end{array}$ & 2.093 & 92 & $\bar{x}$ & $x$ & & $x$ & $x$ & $x$ \\
\hline 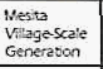 & 949 & 4.1 & $x$ & $x$ & & $x$ & $x$ & $x$ \\
\hline 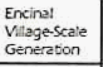 & 102 & .4 & $x$ & $x$ & & $x$ & $x$ & $x$ \\
\hline $\begin{array}{l}\text { Seamz } \\
\text { Vulagescrete } \\
\text { Generariton }\end{array}$ & 427 & 1.8 & $x$ & $x$ & & $x$ & $\mathrm{x}$ & $x$ \\
\hline $\begin{array}{l}\begin{array}{l}\text { Paguate } \\
\text { Villagescate } \\
\text { Generazsion }\end{array} \\
\end{array}$ & 472 & 1.9 & $x$ & $x$ & & $x$ & $x$ & $x$ \\
\hline $\begin{array}{l}\text { Parje } \\
\text { Viligascsale } \\
\text { Gegerston } \\
\end{array}$ & 1.392 & 62 & $x$ & $x$ & & $x$ & $x$ & $x$ \\
\hline $\begin{array}{l}\text { Focily-scate } \\
\text { Generzaton }\end{array}$ & Veries & Veries & $x$ & $x$ & $x$ & $x$ & $x$ & $x$ \\
\hline
\end{tabular}




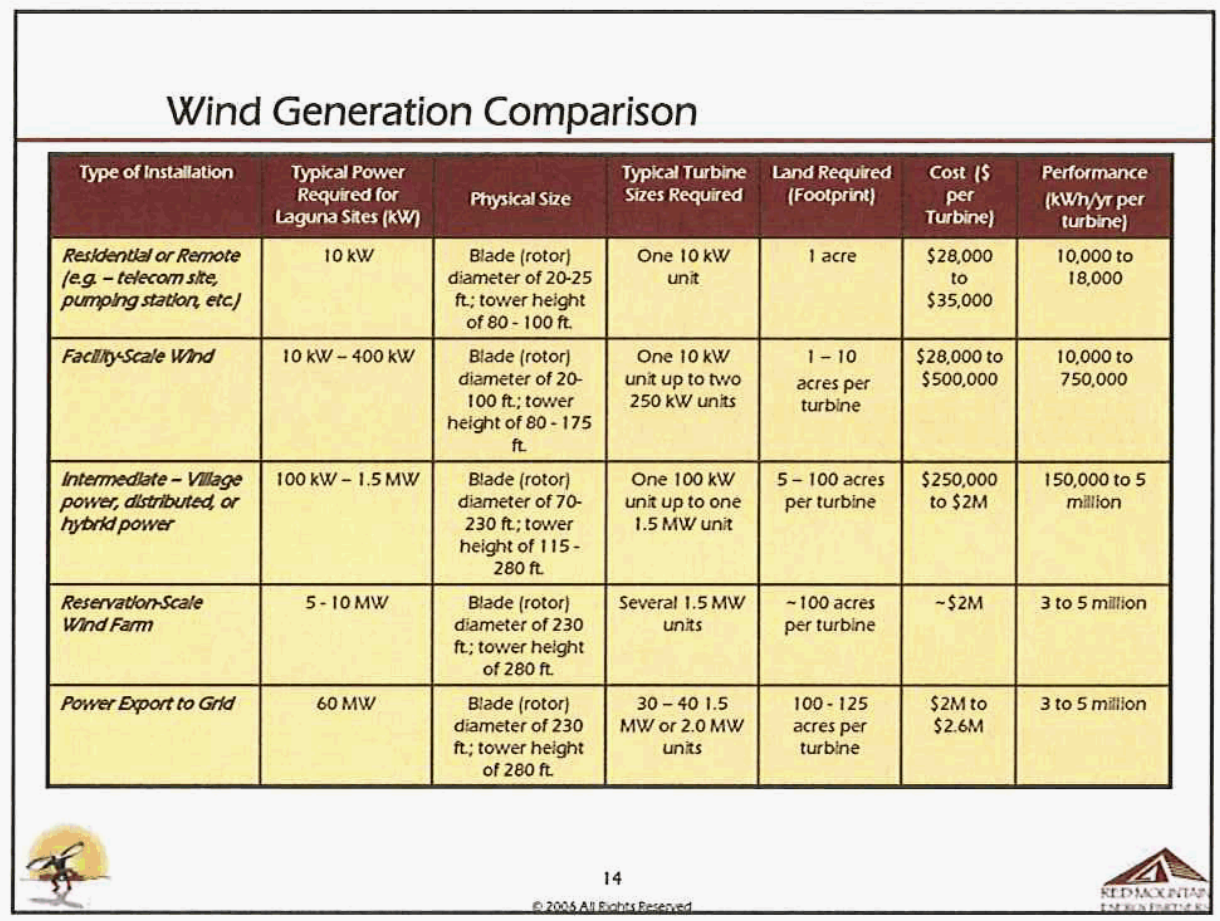

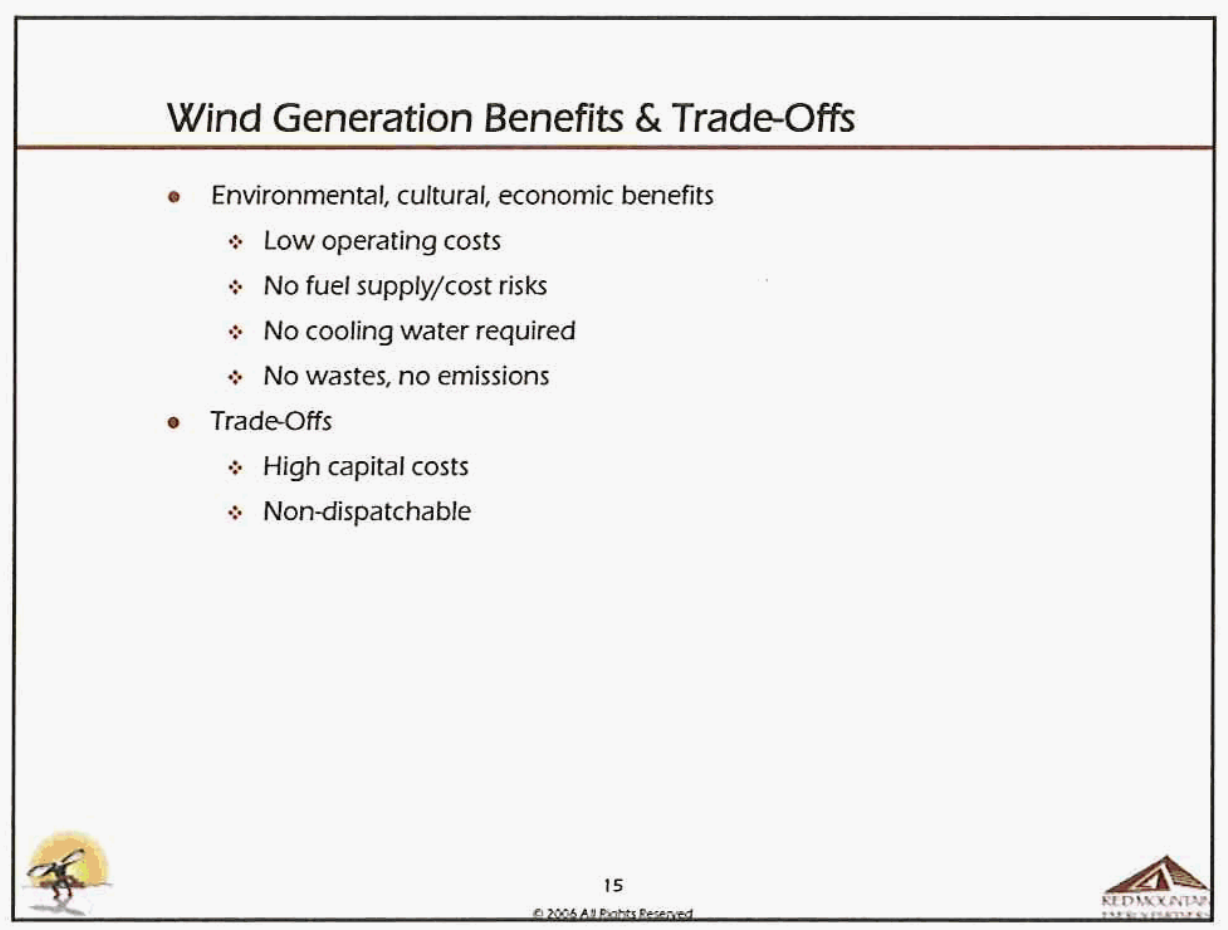




\section{Solar Generation Comparison}

\begin{tabular}{|c|c|c|c|c|c|c|}
\hline Type of Installation & $\begin{array}{l}\text { Typical } \\
\text { Power } \\
\text { Required } \\
\text { for Laguna } \\
\text { Sites }\end{array}$ & Physical Size & $\begin{array}{l}\text { Typical } \\
\text { Technologies } \\
\text { Applicable }\end{array}$ & $\begin{array}{l}\text { Land Required } \\
\text { (Footprint) }\end{array}$ & $\begin{array}{l}\text { Cost w/o } \\
\text { BOS! } \\
\text { (\$ per } \\
\text { kW] }\end{array}$ & $\begin{array}{c}\text { Performance } \\
\text { (kWh/ys per } \\
\text { I kW } \\
\text { installed) }\end{array}$ \\
\hline $\begin{array}{l}\text { Resldentlal or Remore } \\
\text { (e.g - telecom site, } \\
\text { pumpling station, etc) }\end{array}$ & $1.5 \mathrm{~kW}$ & $\begin{array}{c}\text { Arrays ranging from } \\
8 \times 24^{\prime} \text { to } 16^{\circ} \times 20^{\circ} \\
\text { (typical for foxed flat } \\
\text { plat) }\end{array}$ & $\begin{array}{l}\text { Fixed or tïted } \\
\text { roof-mounted } \\
\text { flat plate PV } \\
\text { arrays, ground- } \\
\text { mounted } \\
\text { tracking PV } \\
\text { arrays }\end{array}$ & $\begin{array}{l}\text { Array dimensions } \\
\text { for fixed flat plate; } \\
1.5-2 \text { times } \\
\text { spacing for tited } \\
\text { arrays. Addition } \\
\text { spacing required } \\
\text { for ground- } \\
\text { mounted tracking }\end{array}$ & $\$ 5,000$ to & $\begin{array}{l}1.25 \text { miltion } \\
\text { to } \\
1.5 \text { million }\end{array}$ \\
\hline Facilbyscale solar & $\begin{array}{l}10 \mathrm{~kW}- \\
400 \mathrm{~kW}\end{array}$ & $\begin{array}{l}\text { Arrays ranging from } \\
24^{\circ} \times 60^{\prime} \text { for flat } \\
\text { plate up to } 45^{\circ} \times 55^{\prime} \\
\text { per } 25 \mathrm{~kW} \text { module } \\
\text { for tracking }{ }^{2} \\
\text { concentrator } \\
\text { systems }\end{array}$ & $\begin{array}{l}\text { Fxed or titted } \\
\text { roof-mounted } \\
\text { flat plate PV } \\
\text { arrays, ground- } \\
\text { mounted } \\
\text { tracking PV } \\
\text { arrays, tracking } \\
\text { concentrator } \\
\text { systems }\end{array}$ & $\begin{array}{c}\text { Roughly } 40^{\circ} \times 48^{\prime} \\
\text { for fixed flat plate } \\
\text { up to } 4 \text { acres for } \\
\text { ground-mounted } \\
\text { tracking } \\
\text { concentrator } \\
\text { systems }\end{array}$ & $\begin{array}{l}\$ 5,000 \text { to } \\
\$ 6,000\end{array}$ & $\begin{array}{l}1.25 \text { milition } \\
\text { to } \\
2 \text { miltion }\end{array}$ \\
\hline \multicolumn{7}{|c|}{$\begin{array}{l}\text { "BOS": Balance of System refers to al other required system components in addition to PV modules, such as inverters, } \\
\text { mounting assembles, and electrical wring and connection equipment installation cost is not included. }\end{array}$} \\
\hline 2. & $\begin{array}{l}\text { n a north-south } \\
\text { d to a fixed arra } \\
\text { pared to a fxed }\end{array}$ & $\begin{array}{l}\text { oneaxis configuration } \\
\text { at near latitude tit, and } \\
\text { array (source: APS) }\end{array}$ & $\begin{array}{l}\text { es roughly } 21 \% \text { to } 2 \\
\text { vo-axis tracking impr }\end{array}$ & $\begin{array}{l}\text { more annual energy } \\
\text { es the annual output }\end{array}$ & $\begin{array}{l}\text { tput when } \\
\text { efgy by } 37 \% \text { \% }\end{array}$ & \\
\hline
\end{tabular}

\section{Solar Generation Comparison}

\begin{tabular}{|c|c|c|c|c|c|c|}
\hline Type of Installation & $\begin{array}{l}\text { Typical } \\
\text { Power } \\
\text { Required } \\
\text { for Laguna } \\
\text { Sites }\end{array}$ & Physical Sze & $\begin{array}{l}\text { Typical } \\
\text { Technologies } \\
\text { Applicable }\end{array}$ & $\begin{array}{l}\text { Land Required } \\
\text { (Footprint) }\end{array}$ & $\begin{array}{c}\text { Cost w/o } \\
\text { BOSt } \\
\text { (\$ per } \\
\text { kW] }\end{array}$ & $\begin{array}{c}\text { Performance } \\
\text { (kWhyr per } \\
\text { I kWW } \\
\text { installed) }\end{array}$ \\
\hline $\begin{array}{l}\text { Intermediate - VWlage } \\
\text { power, dlstributed or } \\
\text { mybrid power }\end{array}$ & $\begin{array}{l}100 \mathrm{~kW}- \\
1.5 \mathrm{MW}\end{array}$ & $\begin{array}{l}\text { Arrays ranging from } 24^{\prime} \\
\times 60^{\prime} \text { for flat plate up to } \\
45^{\prime} \times 55^{\prime} \text { per } 25 \mathrm{~kW} \\
\text { modu'e for tracking } \\
\text { concentrator systems }\end{array}$ & $\begin{array}{l}\text { Fixed or tilted } \\
\text { roof-mounted } \\
\text { flat plate PV } \\
\text { arrays, ground- } \\
\text { mounted } \\
\text { tracking PV } \\
\text { arrays, tracking } \\
\text { concentrator } \\
\text { systems }\end{array}$ & $\begin{array}{l}\text { Roughiy } 40^{\circ} \times 48^{\circ} \\
\text { for fixed flat plate } \\
\text { up to } 4 \text { acres for } \\
\text { ground-mounted } \\
\text { tracking } \\
\text { concentrator } \\
\text { systems }\end{array}$ & $\begin{array}{l}\$ 5,000 \text { to } \\
\$ 6,000\end{array}$ & $\begin{array}{l}1.25 \text { million } \\
\text { to } \\
2 \text { million }\end{array}$ \\
\hline $\begin{array}{l}\text { Reservation-Scale } \\
\text { Wind Farm }\end{array}$ & $5.10 \mathrm{MW}$ & $\begin{array}{l}\text { Arrays of } 45^{\prime} \times 55^{\prime} \text { per } \\
25 \mathrm{~kW} \text { module for } \\
\text { tracking concentrators, } \\
\text { up to } 1-1 / 2 \text { to } 2 \text { acres } \\
\text { per MW for trough solar } \\
\text { field }\end{array}$ & $\begin{array}{l}\text { Tracking } \\
\text { concentrator } \\
\text { systems, dish- } \\
\text { engines, } \\
\text { parabolic } \\
\text { trough }\end{array}$ & $\begin{array}{c}\text { Roughiy } 20-30 \\
\text { acres }\end{array}$ & $\begin{array}{c}\$ 4,000 \text { to } \\
\$ 6,000\end{array}$ & $\begin{array}{l}2 \text { miltion } \\
\text { to } \\
2.5 \text { miltion }\end{array}$ \\
\hline Power Export to Gidd & $60 \mathrm{MW}$ & $\begin{array}{l}\text { Arrays of } 45^{\prime} \times 55^{\prime} \text { per } \\
25 \mathrm{KW} \text { module for } \\
\text { tracking concentrators, } \\
\text { up to } 1-1 / 2 \text { to } 2 \text { acres } \\
\text { per } M W \text { for trough solar } \\
\text { field }\end{array}$ & $\begin{array}{l}\text { Tracking } \\
\text { concentrator } \\
\text { systems, dish- } \\
\text { engines, } \\
\text { parabolic } \\
\text { trough }\end{array}$ & $\begin{array}{l}\text { Roughly } 90- \\
120 \text { acres }\end{array}$ & $\begin{array}{l}\$ 4,000 \text { to } \\
\$ 6,000\end{array}$ & $\begin{array}{l}2 \text { million } \\
\text { to } \\
2.5 \text { million }\end{array}$ \\
\hline
\end{tabular}

1. "BOS", Balance of System refers to all other required system components in addition to PV modules, such as inverters. mounting assembles, and electrical wiring and connection equipment instaliation cost is not included 


\section{Solar Generation Benefits \& Trade-Offs}

- Environmental, cultural, economic benefits
- Low operating costs
$\therefore$ No fuel supply/cost risks
$\therefore$ No cooling water required
$\therefore$ No wastes, no emissions
* Good load following characteristics

- Trade-Offs

$\therefore$ High capital costs

$\therefore$ Non-dispatchable

Gas-Fired Generation Comparison

\begin{tabular}{|c|c|c|c|c|c|}
\hline Iype of Installation & $\begin{array}{l}\text { Typical Power } \\
\text { Required for } \\
\text { lagunasites } \\
\text { (kWV) }\end{array}$ & Project Size & Type & $\begin{array}{l}\text { cost } \\
\text { [s per } \\
\mathrm{kW} \text { ) }\end{array}$ & Efinciengy \\
\hline $\begin{array}{l}\text { Residential or } \\
\text { Remote /eg- } \\
\text { telecom ste, } \\
\text { pumping station } \\
\text { exc/ }\end{array}$ & $10 \mathrm{~kW}$ & $5 \mathrm{~kW}-10 \mathrm{MW}$ & $\begin{array}{l}\text { Reciprocating } \\
\text { Engine }\end{array}$ & $\begin{array}{l}\$ 300- \\
900\end{array}$ & $25-4096$ \\
\hline Fachlinyscale & $\begin{array}{c}10 \mathrm{~kW}-400 \\
\mathrm{~kW}\end{array}$ & $\begin{array}{c}25-500 \mathrm{~kW} \\
5 \mathrm{~kW}-10 \mathrm{~mW}\end{array}$ & $\begin{array}{l}\text { Microturbine } \\
\text { Reciprocating } \\
\text { Engine }\end{array}$ & $\begin{array}{l}\$ 700- \\
1100 \\
\\
\$ 300- \\
900\end{array}$ & $\begin{array}{l}\text { 20-30\%; } \\
\text { w/CHP up to } \\
85 \% \\
25-40 \%\end{array}$ \\
\hline $\begin{array}{l}\text { Intermediate - } \\
\text { Village power, } \\
\text { dstributed or } \\
\text { hybrid power }\end{array}$ & $\begin{array}{c}100 \mathrm{~kW}-1.5 \\
M W\end{array}$ & $\begin{array}{c}500 \mathrm{~kW}-25 \\
M W\end{array}$ & $\begin{array}{l}\text { Combustion } \\
\text { Turbine }\end{array}$ & $\begin{array}{l}\$ 300- \\
1000\end{array}$ & $2045 \%$ \\
\hline $\begin{array}{l}\text { Reservation-Scale } \\
\text { Gas Generation }\end{array}$ & 5.10 MW & $\begin{array}{c}500 \mathrm{~kW}-25 \\
\mathrm{MW}\end{array}$ & $\begin{array}{c}\text { Combustion } \\
\text { Turbine }\end{array}$ & $\begin{array}{l}\$ 300- \\
1000\end{array}$ & $2045 \%$ \\
\hline $\begin{array}{l}\text { Power Export to } \\
\text { Gidd }\end{array}$ & $60 \mathrm{MW}$ & $\begin{array}{c}500 \mathrm{~kW}-25 \\
\mathrm{MW}\end{array}$ & $\begin{array}{l}\text { Combustion } \\
\text { Turbine }\end{array}$ & $\begin{array}{l}\$ 300 \\
1000\end{array}$ & $20-4596$ \\
\hline
\end{tabular}




\section{Gas-Fired Generation Benefits \& Trade-Offs}

Microturbines

- Environmental, cultural, economic benefits

$\therefore$ Small number of moving parts

$\therefore$ Low emissions

$\therefore$ Long maintenance intervals

$\therefore$ Compact

* Dispatchable

* Can utilize waste fuels

* Good efficiencies w/cogeneration

- Trade-offs

$\therefore$ Low fuel to electric efficiencies

* Loss of power output/efficiency with higher ambient temperatures
Reciprocating Engines

- Environmental, cultural, economic benefits

$\therefore$ Low capital costs

* Good efficiencies

* Quick startup

* Fuel flexibility

* High reliability

* Low natural gas pressure required

- Trade-offs

$\therefore$ Emissions

$\therefore$ Noisy

* Frequent maintenance intervals

\section{Gas-Fired Generation Benefits \& Trade-Offs}

Combustion Turbines

- Environmental, cultural, economic benefits

* High efficiency, low cost

* Wide range of power outputs

* Can produce high temperature steam

* Well-established channels

* High power to weight ratio

* Proven reliability/availability

- Trade-offs

* Reduced efficiencies at part load

$\therefore$ Sensitive to ambient conditions

$*$ Cost/efficiency not as good in small systems 

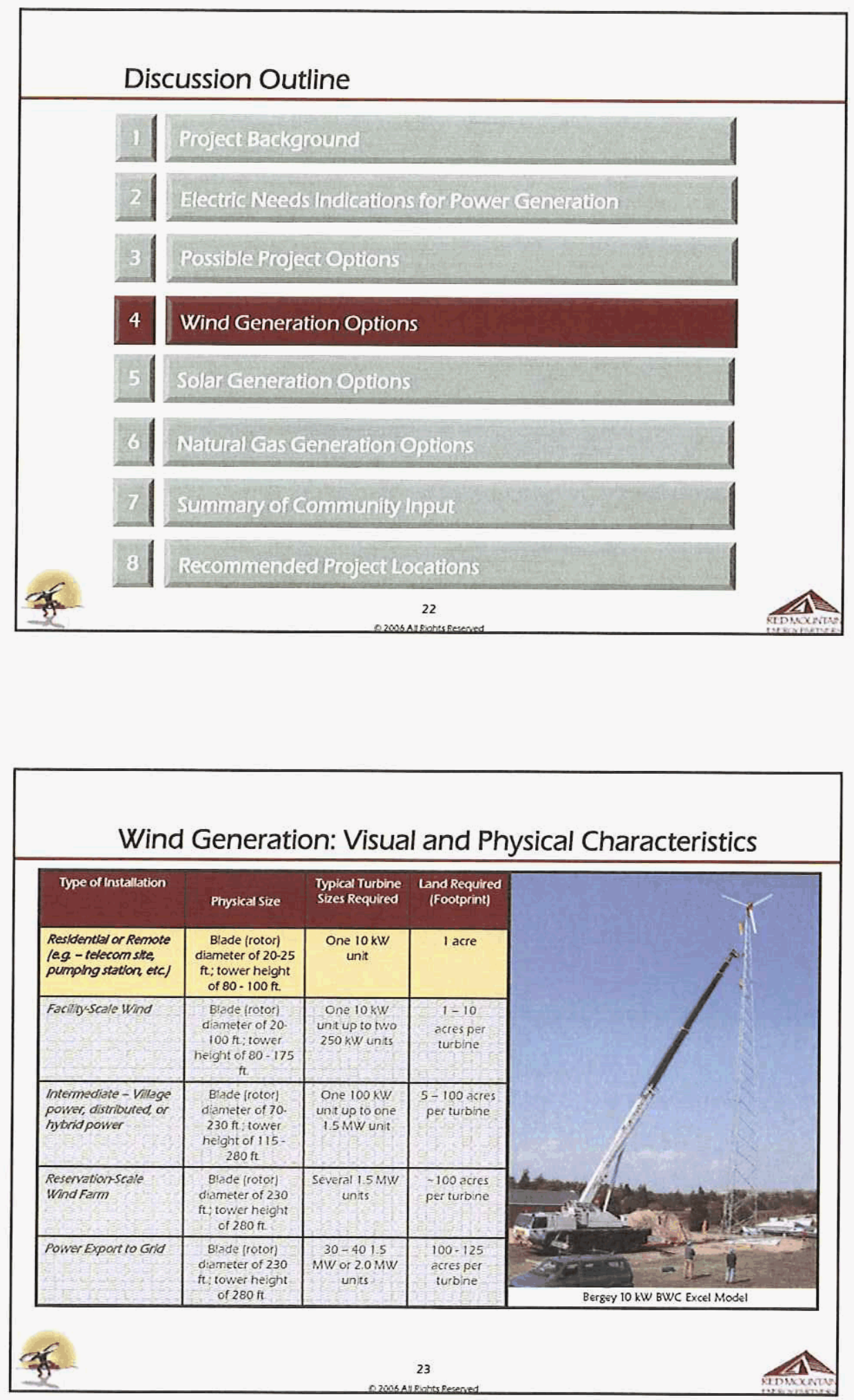

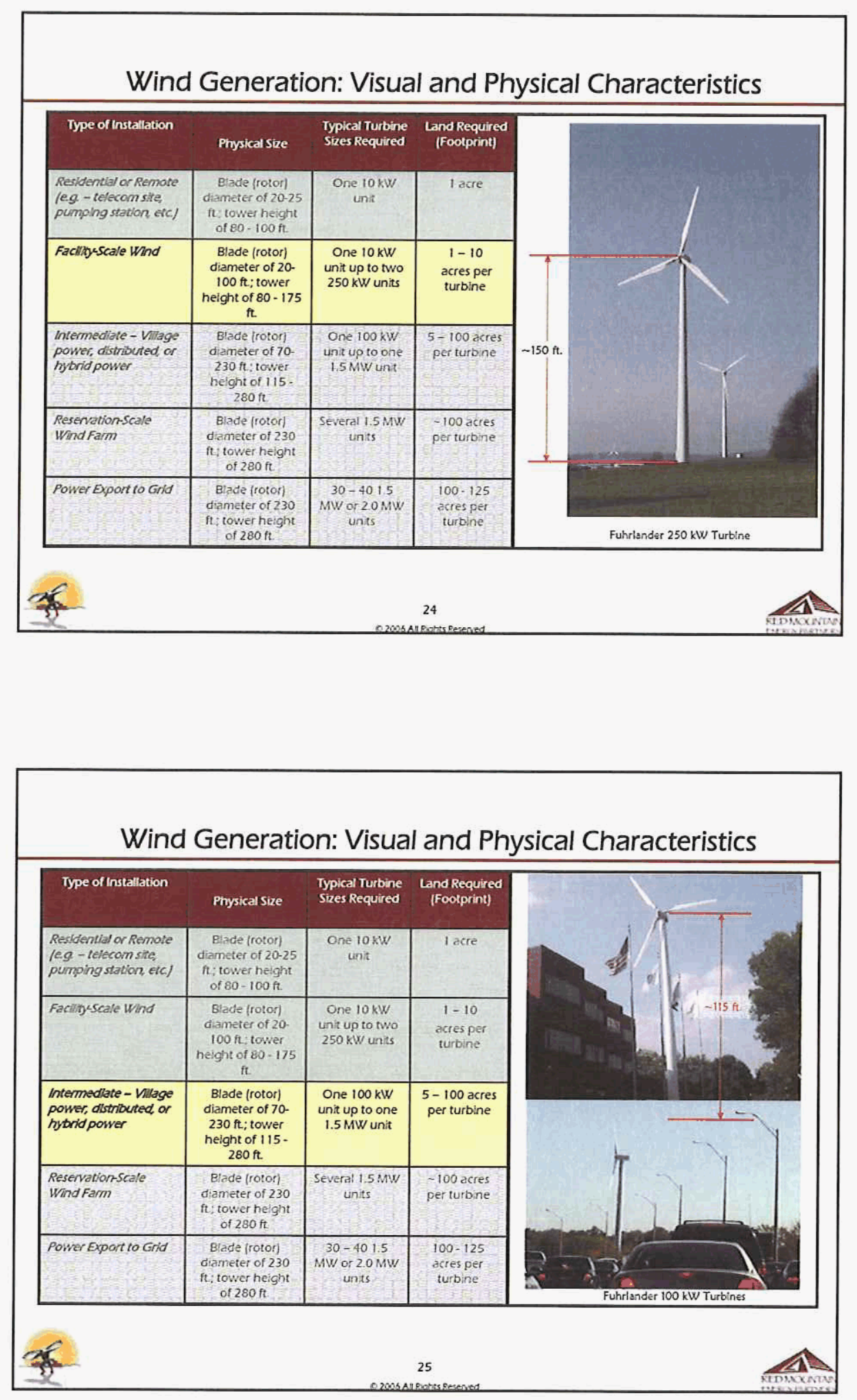

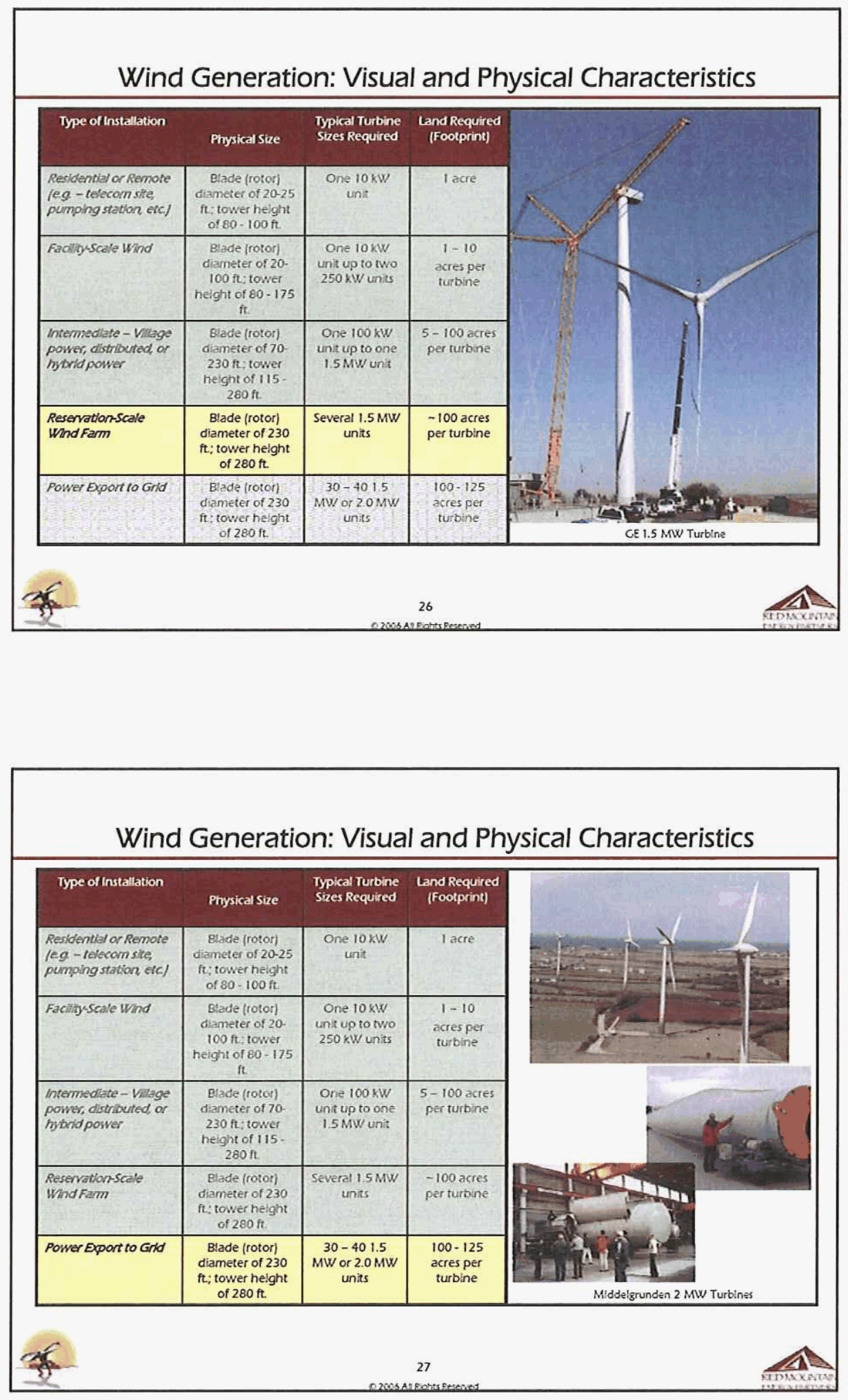


\section{Sizing Considerations for Wind Turbines}

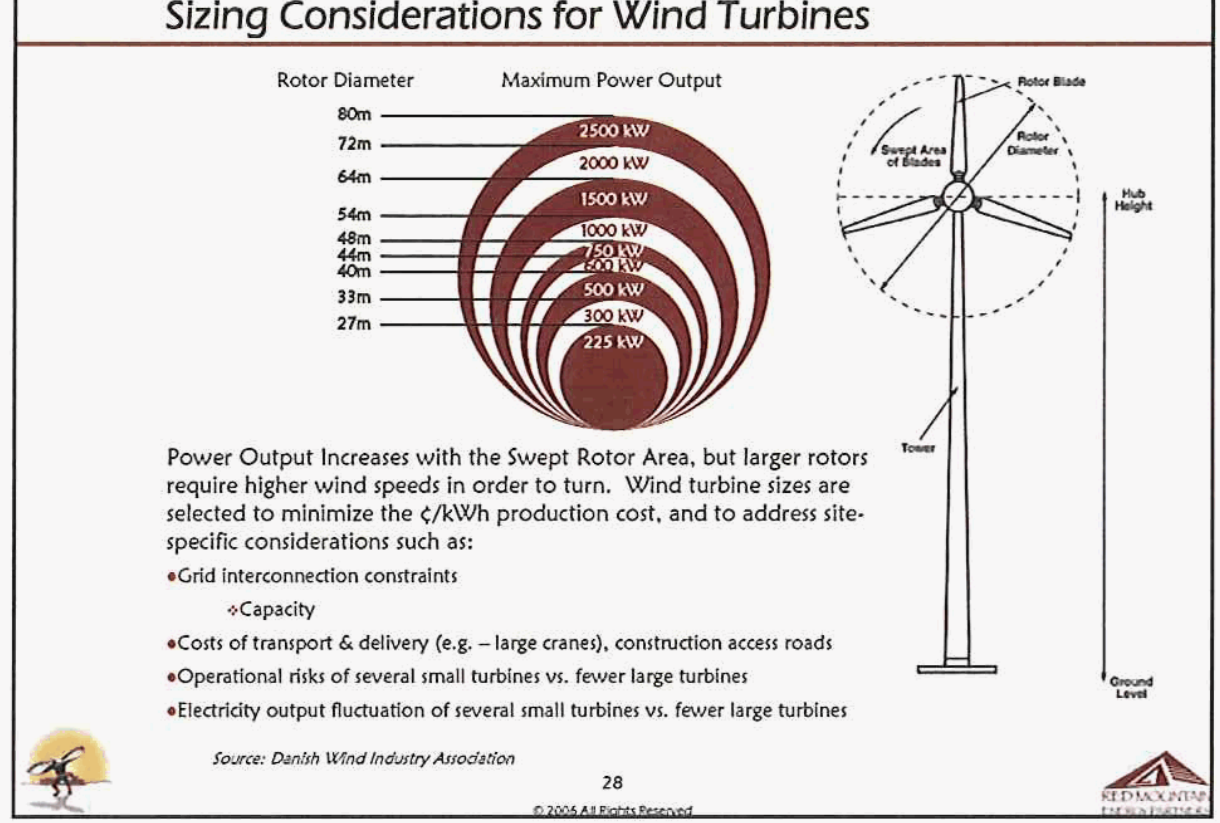

\section{Energy Production Cost Ranges for a $50 \mathrm{MW}$ Wind Farm}

- Private ownership, project financing: 4.95 cents/kWh including PTC, 6.56 cents $/ \mathrm{kWh}$ without PTC.

- IOU ownership, corporate financing: 3.53 cents/kWh including PTC, 5.9 cents $/ \mathrm{kWh}$ without.

- Public utility ownership, internal financing: 2.88 cents $/ \mathrm{kW} / \mathrm{h}$ including REPI, 4.35 cents $/ \mathrm{kWh}$ without.

- Public utility ownership, project financing: 3.43 cents $/ \mathrm{kWh}$ including REPI, 4.89 cents $/ \mathrm{kWh}$ without.

Sensitivity Parameters:

* Utility vs. developer ownership: utilities have access to $7.5 \%$ debt compared to $9.5 \%$ for a developer.

* Utilities enjoy longer debt payment periods (20 years compared to 12 for a developer)

* Developers are subject to a "debt service coverage ratio" (DSCR) requirement, which obligates a wind project to generate enough cash each year to exceed loan payments. Typically, this results in a smaller loan than would be most advantageous for the developer.

* Investors have higher return on equity requirements for wind projects in general (typically $18 \%$ ) than those for gas projects (typically $12 \%$ ).

- Above LCOEs assume a 50-MW wind farm with an installed cost of $\$ 1.000 / \mathrm{kW}$, a 30\% capacity factor, and operations and maintenance (OSiM) expenses of 0.65 cents/kWh

- Source: Ryan Wiser and Edward Kahn of Lawrence Berkeley Laboratory's Energy and Environment Division 


\section{Laguna Wind Sizing \& Configuration Considerations}

Expected Turblne Performance Based on Laguna Wind Data

\begin{tabular}{|c|c|c|c|c|c|c|c|c|c|c|c|}
\hline \multicolumn{2}{|c|}{ Bergey Excel $10 \mathrm{~kW}$} & \multicolumn{2}{|c|}{ Fuhrlander $30 \mathrm{~kW}$} & \multicolumn{2}{|c|}{ Fuhrlander $100 \mathrm{~kW}$} & \multicolumn{2}{|c|}{ Fuhriander $250 \mathrm{~kW}$} & \multicolumn{2}{|c|}{ Vestas $660 \mathrm{~kW}$} & \multicolumn{2}{|c|}{ GE $1.5 \mathrm{MW}$} \\
\hline $\begin{array}{c}\text { Capacity } \\
\text { Factor }\end{array}$ & $\begin{array}{l}\text { Annual } \\
\text { kWh } \\
\text { Output }\end{array}$ & $\begin{array}{c}\text { Capacity } \\
\text { Factor }\end{array}$ & $\begin{array}{l}\text { Annual } \\
\text { kWh } \\
\text { Output }\end{array}$ & $\begin{array}{l}\text { Capacity } \\
\text { Factor }\end{array}$ & $\begin{array}{l}\text { Annual } \\
\mathrm{kWh} \\
\text { Output }\end{array}$ & $\begin{array}{l}\text { Capacity } \\
\text { Factor }\end{array}$ & $\begin{array}{l}\text { Annual } \\
\mathrm{kWh} \\
\text { Output }\end{array}$ & $\begin{array}{l}\text { Capacity } \\
\text { Factor }\end{array}$ & $\begin{array}{l}\text { Annual } \\
k W h \\
\text { Output }\end{array}$ & $\begin{array}{l}\text { Capacity } \\
\text { Factor }\end{array}$ & $\begin{array}{l}\text { Annual } \\
k W h \\
\text { Output }\end{array}$ \\
\hline $6.86 \%$ & 6,388 & $6.06 \%$ & 56.400 & $24.20 \%$ & 225,223 & $19.90 \%$ & 462,921 & $16.61 \%$ & $1.087,382$ & $20.56 \%$ & $3,059,491$ \\
\hline
\end{tabular}

\section{Possible Wind Project Configurations for Laguna}

\begin{tabular}{|c|c|c|c|c|c|c|c|c|}
\hline $\begin{array}{l}\text { Village or } \\
\text { Reservation } \\
\text { Application }\end{array}$ & $\begin{array}{l}\text { Peak } \\
\text { Demand } \\
\text { (KW) }\end{array}$ & $\begin{array}{l}\text { Annual } \\
\text { Usage } \\
(\mathrm{kWW})\end{array}$ & $\begin{array}{l}\text { Load } \\
\text { Factor }\end{array}$ & $\begin{array}{l}\text { Possible Project } \\
\text { Configurations (AI } \\
\text { Grid- } \\
\text { Supplemented) }\end{array}$ & $\begin{array}{l}\text { Peak } \\
\text { Power } \\
\text { (kW) }\end{array}$ & $\begin{array}{l}\text { Projected } \\
\text { Capaciy } \\
\text { Factor }\end{array}$ & $\begin{array}{l}\text { Projected } \\
\text { Annual kWh }\end{array}$ & $\begin{array}{l}\text { Initial } \\
\text { Capital } \\
\text { Cost }\end{array}$ \\
\hline $\begin{array}{l}\text { Laguna industries } \\
\text { Generation }\end{array}$ & 544 & $1.620,000$ & $34.0 \%$ & $\begin{array}{l}\text { Two Fuhriander } \\
250 \mathrm{~kW} \text { Turbines }\end{array}$ & 500 & $21.14 \%$ & 871,507 & $\begin{array}{l}\$ 1.25 \mathrm{M}- \\
\text { S1.75M }\end{array}$ \\
\hline $\begin{array}{l}\text { Encinal Village } \\
\text { Scale Generation }\end{array}$ & 102 & 393,023 & $44.0 \%$ & $\begin{array}{l}\text { One Fuhriander } \\
100 \mathrm{~kW} \text { Turbine }\end{array}$ & 100 & 24.246 & 225.223 & $\begin{array}{l}\$ 250 \mathrm{k}- \\
\$ 300 \mathrm{k}\end{array}$ \\
\hline $\begin{array}{l}\text { Laguna Viliage- } \\
\text { Scale Generation }\end{array}$ & 2,083 & $9,207,437$ & $50.5 \%$ & $\begin{array}{l}\text { One GE } 1.5 \mathrm{MW} \\
\text { Turbine }\end{array}$ & 1,500 & $20.6 \%$ & $3.059,491$ & $\begin{array}{l}\$ 2.0 M- \\
\$ 225 \mathrm{M}\end{array}$ \\
\hline $\begin{array}{c}\text { Mesita Vilizge } \\
\text { Scale Generation }\end{array}$ & 949 & $4,073,652$ & 49.096 & $\begin{array}{l}\text { One Fuhriander } \\
600 \mathrm{~kW} \text { Turbine }\end{array}$ & 600 & & $1,000,000$ & $\begin{array}{l}5950 \mathrm{k}- \\
51 \mathrm{M}\end{array}$ \\
\hline $\begin{array}{l}\text { Paguate Village- } \\
\text { Sca'e Generation }\end{array}$ & 472 & $1,953,873$ & $47.3 \%$ & $\begin{array}{l}\text { Two Fuhriander } \\
250 \mathrm{~kW} \text { Turbines }\end{array}$ & 500 & $21.14 \%$ & 871,507 & $\begin{array}{l}\$ 1.25 \mathrm{M}- \\
\$ 1.75 \mathrm{M}\end{array}$ \\
\hline $\begin{array}{c}\text { Paraje Village- } \\
\text { Scale Generation }\end{array}$ & 1,392 & $6,206,168$ & $50.9 \%$ & $\begin{array}{l}\text { One GE } 1.5 \mathrm{MW} \\
\text { Turbine }\end{array}$ & 1.500 & $20.6 \%$ & $3,059,491$ & $\begin{array}{l}\$ 2.0 M- \\
\$ 2.25 \mathrm{M}\end{array}$ \\
\hline $\begin{array}{c}\text { Seama Viliage } \\
\text { Scale Generation }\end{array}$ & 427 & $1,783,999$ & $47.7 \%$ & $\begin{array}{l}\text { Two Fuhriander } \\
250 \mathrm{~kW} \text { Turbines }{ }^{2}\end{array}$ & 500 & $21.14 \%$ & 871,507 & $\begin{array}{l}\text { S1.25M- } \\
\text { S1.75M }\end{array}$ \\
\hline $\begin{array}{c}\text { Laguna } \\
\text { Reservation-Scale } \\
\text { Generation }\end{array}$ & 5.426 & $23,618,151$ & $49.7 \%$ & $\begin{array}{l}\text { Four GE } 1.5 \mathrm{MW} \\
\text { Turbines }\end{array}$ & 6.000 & 20.695 & $12,237,964$ & $\begin{array}{l}58 M- \\
59 M\end{array}$ \\
\hline $\begin{array}{c}\text { Power Export to } \\
\text { Grid }\end{array}$ & $6 M W$ & $N / A$ & N/A & $\begin{array}{c}\text { Forty GE } 1.5 \mathrm{MW} \\
\text { Turbines }\end{array}$ & $6 \mathrm{MW}$ & $120.6 \%$ & $122,379,640$ & $\begin{array}{l}575 \mathrm{M} \text { - } \\
585 \mathrm{M}\end{array}$ \\
\hline
\end{tabular}

1. Initial capital costs are rough estimates only and do not inciude "allin" development costs. Actual costs wil vary

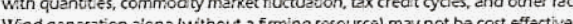

ceffective at this Village sca'e, but may prove viable upon further analysis and/or consideretion of hybrid configurations.

31

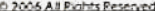



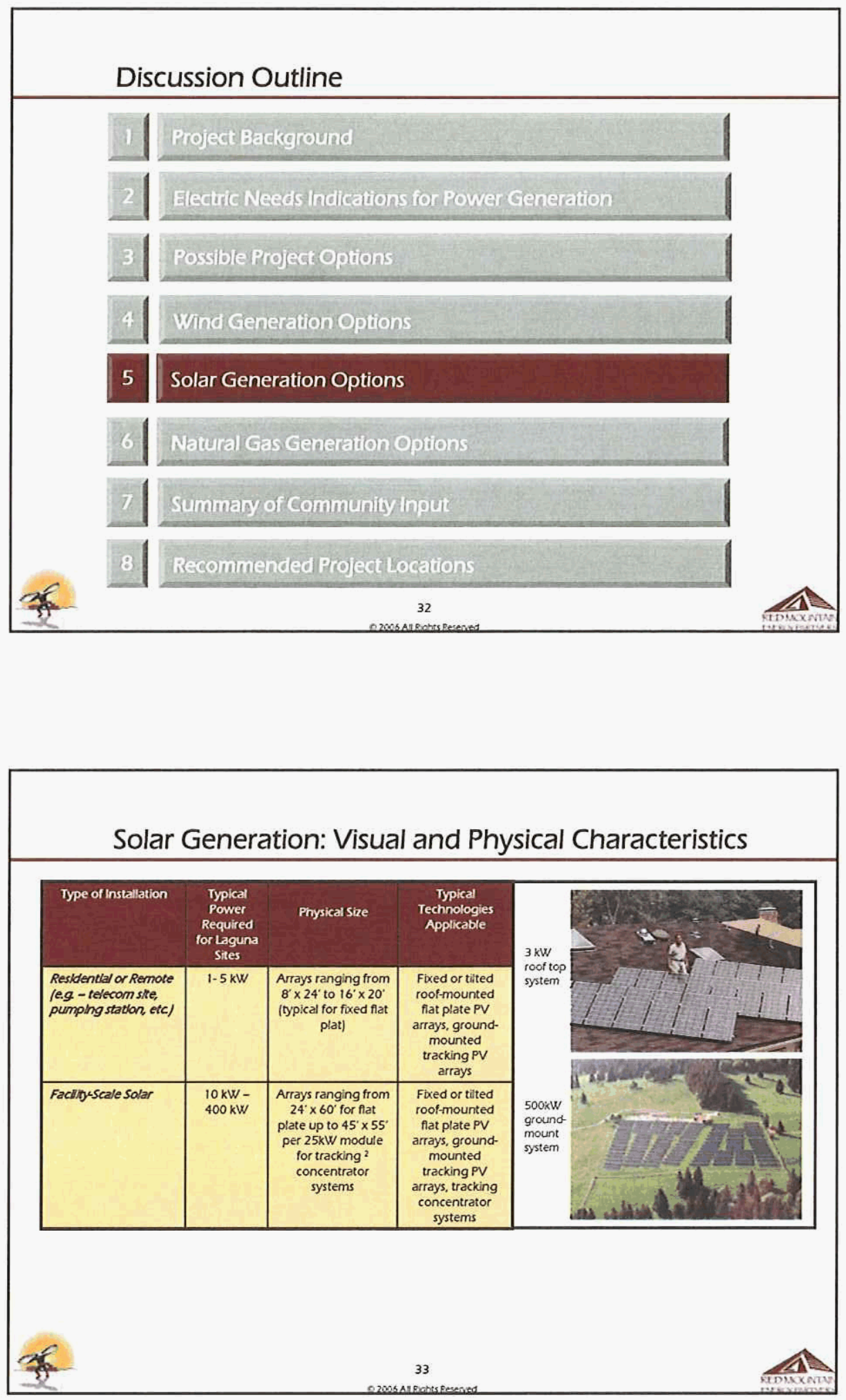

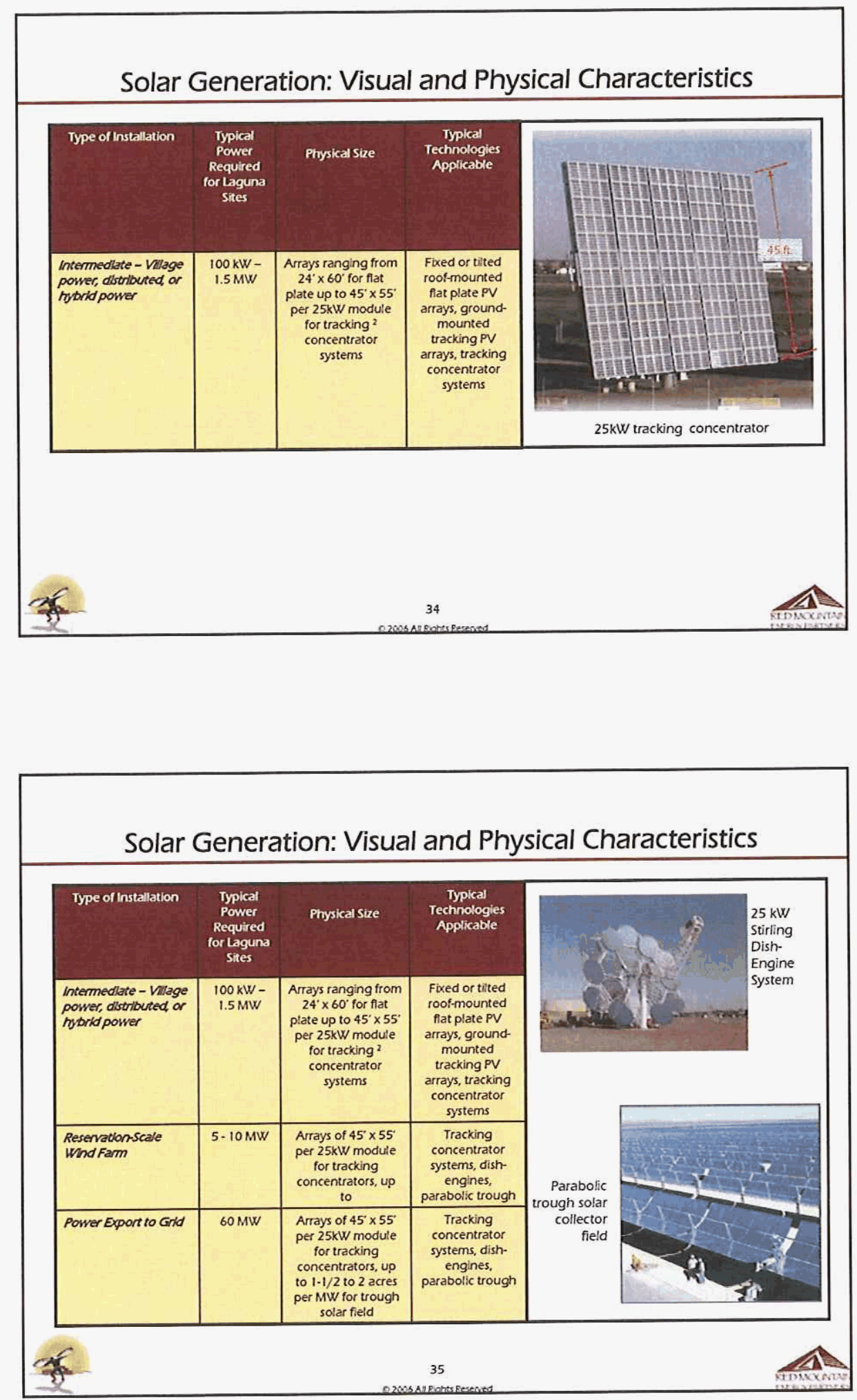


\section{Solar Generation: Economies of Scale Impacts}

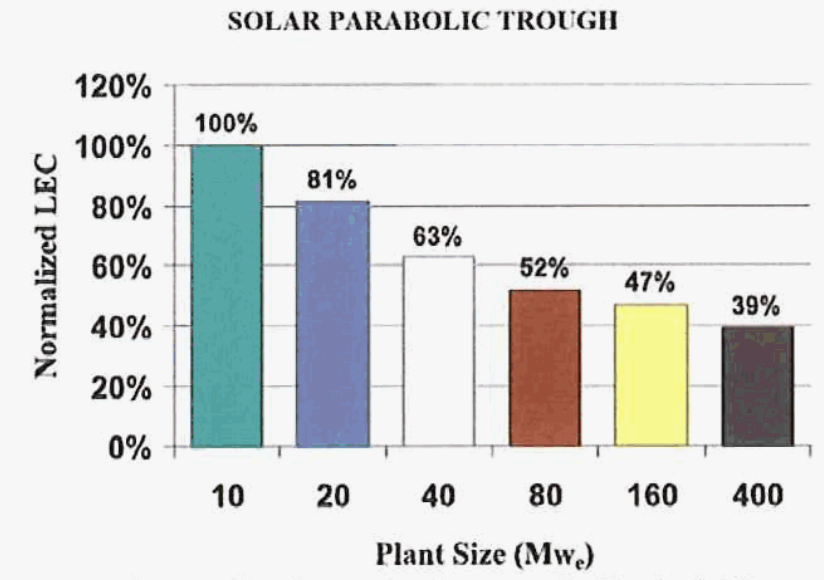

Figure 5. Effect of power plant size on nomalized levelized $\mathrm{COE}$.

\begin{tabular}{|c|c|c|c|c|c|c|c|c|}
\hline \multicolumn{9}{|c|}{ Possible Solar Project Configurations for Laguna } \\
\hline $\begin{array}{l}\text { Village or } \\
\text { Reservation } \\
\text { Appilication }\end{array}$ & $\begin{array}{l}\text { Peak } \\
\text { Demand } \\
{[\mathrm{kW}]}\end{array}$ & $\begin{array}{l}\text { Annual } \\
\text { Usage } \\
(\mathrm{kW} / \mathrm{h})\end{array}$ & $\begin{array}{l}\text { Load } \\
\text { facior }\end{array}$ & $\begin{array}{l}\text { Possible Projed } \\
\text { Configurations (it Gia } \\
\text { Suppiementeor) }\end{array}$ & $\begin{array}{l}\text { Peak } \\
\text { Power } \\
(\mathrm{kW})\end{array}$ & $\begin{array}{l}\text { Expected } \\
\text { Copacity } \\
\text { Factor }\end{array}$ & $\begin{array}{l}\text { Profected } \\
\text { Annual } \\
\mathrm{kWh}\end{array}$ & $\begin{array}{l}\text { Intival } \\
\text { Capital } \\
\text { cost' }\end{array}$ \\
\hline $\begin{array}{l}\text { Laguna Industries } \\
\text { Generation }\end{array}$ & 544 & $1,620,000$ & 34.096 & $\begin{array}{l}\text { Twenty } 25 \mathrm{~kW} \text { Dish- } \\
\text { Engine Modules }\end{array}$ & $500 \mathrm{~kW}$ & $25 \%$ & $1.1 \mathrm{GWh}$ & $525 \mathrm{M}$ \\
\hline $\begin{array}{l}\text { Enoinal Village } \\
\text { Scale Generation }\end{array}$ & 102 & 393.023 & $44.0 \% 6$ & $\begin{array}{l}\text { Four } 25 \mathrm{~kW} \text { Amonix } \\
\mathrm{HCPV}^{2} \text { arrays }\end{array}$ & $100 \mathrm{~kW}$ & $23 \%$ & 203.000 & $5600 \mathrm{k}$ \\
\hline $\begin{array}{l}\text { Laguna Viliage } \\
\text { Scale Generation }\end{array}$ & 2.083 & 9.207 .437 & $50.5 \%$ & Parabolic Trough & $2 M W$ & $25 \%$ & $4.4 \mathrm{GWh}$ & $58 \mathrm{M}$ \\
\hline $\begin{array}{l}\text { Mesita Village Scate } \\
\text { Generation }\end{array}$ & 949 & $4,073,652$ & $49.0 \% 5$ & Parebolic Trough & IMW & $25 \%$ & $2.2 \mathrm{GWh}$ & $54 \mathrm{M}$ \\
\hline $\begin{array}{l}\text { Paguate Vilizge } \\
\text { Scle Generation }\end{array}$ & 472 & 1.953 .873 & $47.3 \%$ & $\begin{array}{l}\text { Twenty } 25 \mathrm{~kW} \text { Dish- } \\
\text { Engine Modules }\end{array}$ & $500 \mathrm{~kW}$ & $25 \%$ & $1.1 \mathrm{GWh}$ & $\$ 2.5 \mathrm{M}$ \\
\hline $\begin{array}{l}\text { Paraje Villagescale } \\
\text { Generation }\end{array}$ & 1.392 & $6,205,168$ & $50.9 \%$ & Parabolic Trough & $1 M W$ & $25 \%$ & $22 \mathrm{GWh}$ & $54 \mathrm{M}$ \\
\hline $\begin{array}{l}\text { Seama Vilizage } \\
\text { Scale Generation }\end{array}$ & 427 & $1,783.999$ & $47.7 \%$ & $\begin{array}{l}\text { Soxteen } 25 \mathrm{~kW} / \text { Amonix } \\
\mathrm{HCPV}^{2} \text { arrays }\end{array}$ & $400 \mathrm{~kW}$ & $23 \%$ & 812,000 & $\$ 2.4 \mathrm{M}$ \\
\hline $\begin{array}{l}\text { Laguna } \\
\text { Reservation-Scale } \\
\text { Generation }\end{array}$ & 5.426 & 23.618 .151 & $49.7 \%$ & Parabolic Trough & $5 M W$ & $25 \%$ & $10.9 \mathrm{GWh}$ & $520 \mathrm{M}$ \\
\hline $\begin{array}{l}\text { Power Export to } \\
\text { Grid }\end{array}$ & $60 \mathrm{Mw}$ & $\mathrm{N} / \mathrm{A}$ & N/A & Parabolic Trough & $\begin{array}{l}\text { Sized to } \\
\text { project } \\
\text { need }\end{array}$ & $25 \%$ & $132 \mathrm{GWh}$ & $\$ 240 \mathrm{M}$ \\
\hline \multicolumn{9}{|c|}{ 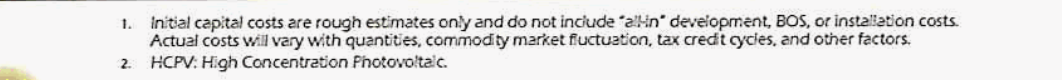 } \\
\hline & & & & 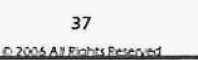 & & & & \\
\hline
\end{tabular}



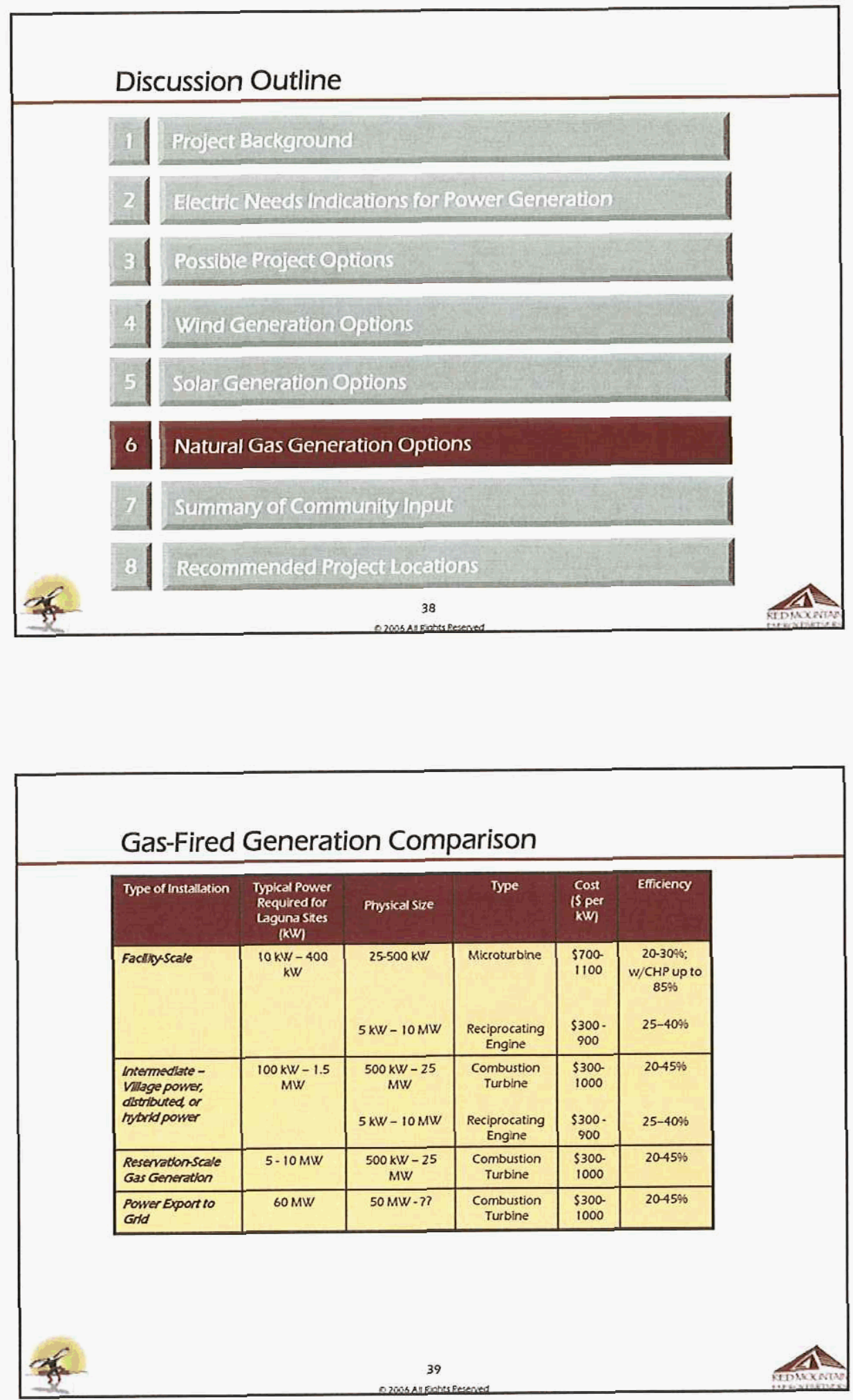

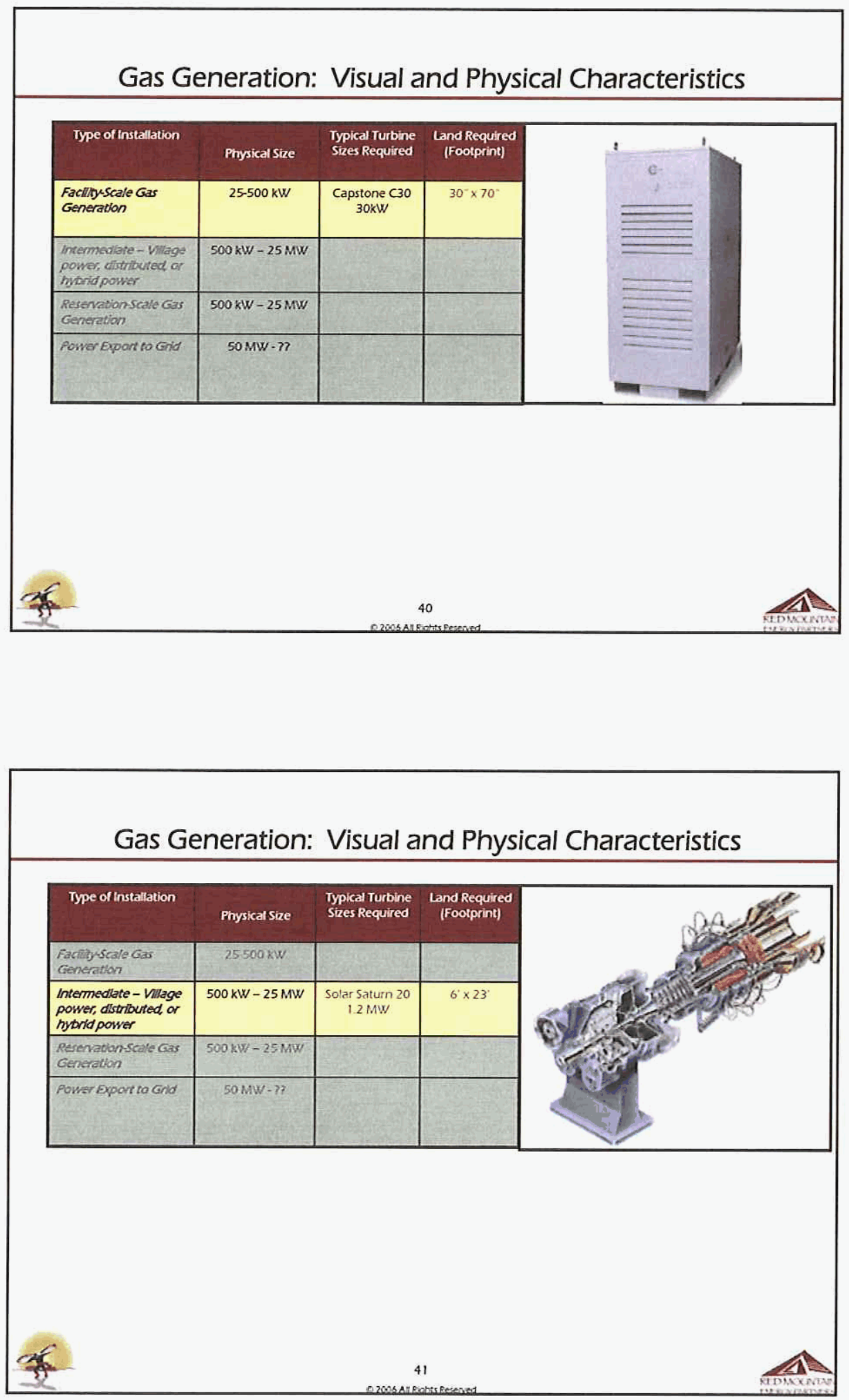


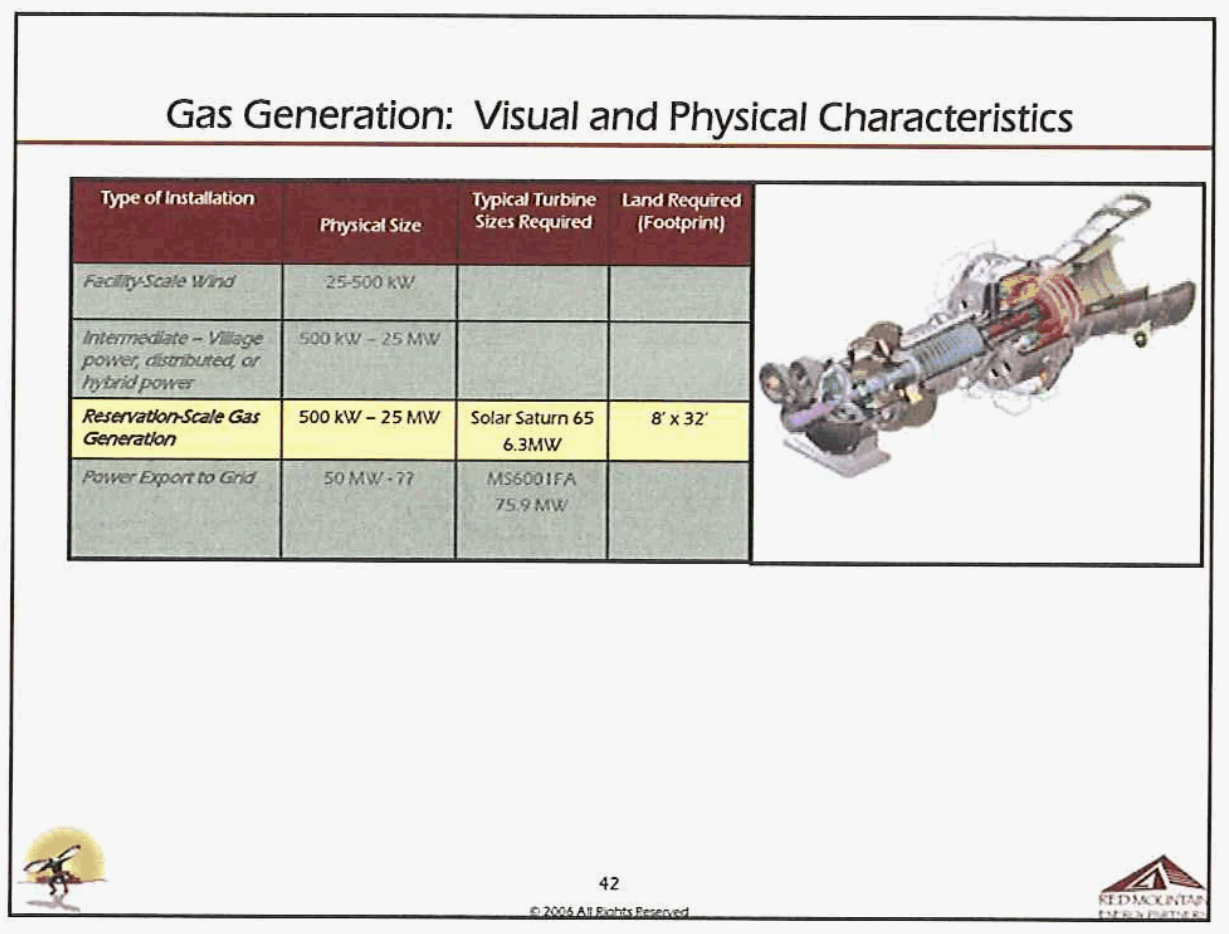

Gas Generation: Visual and Physical Characteristics

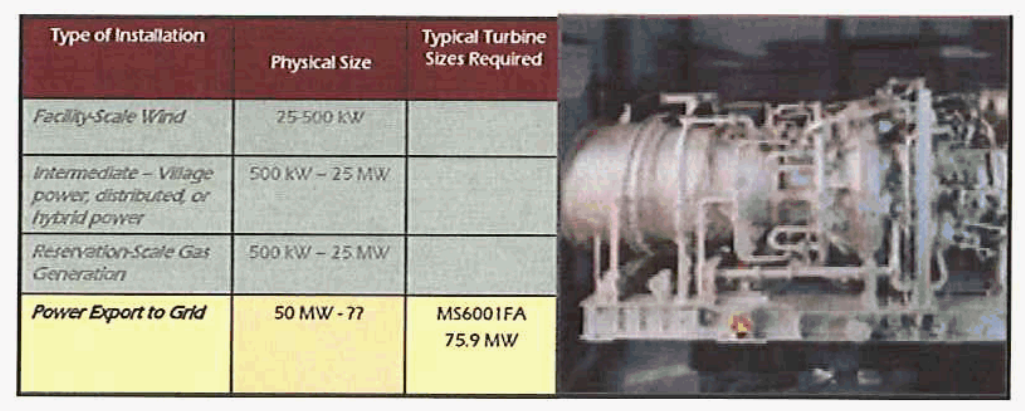




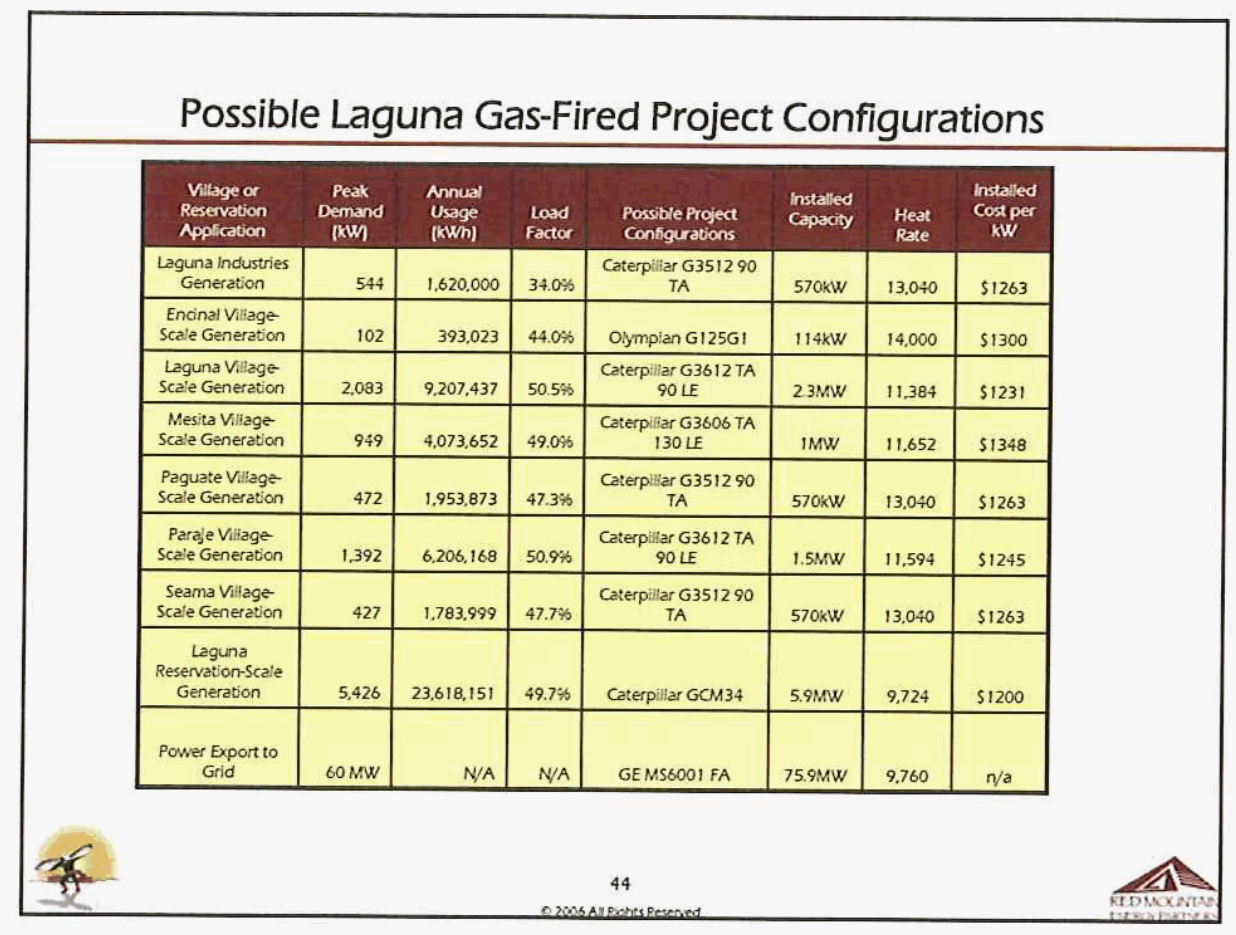

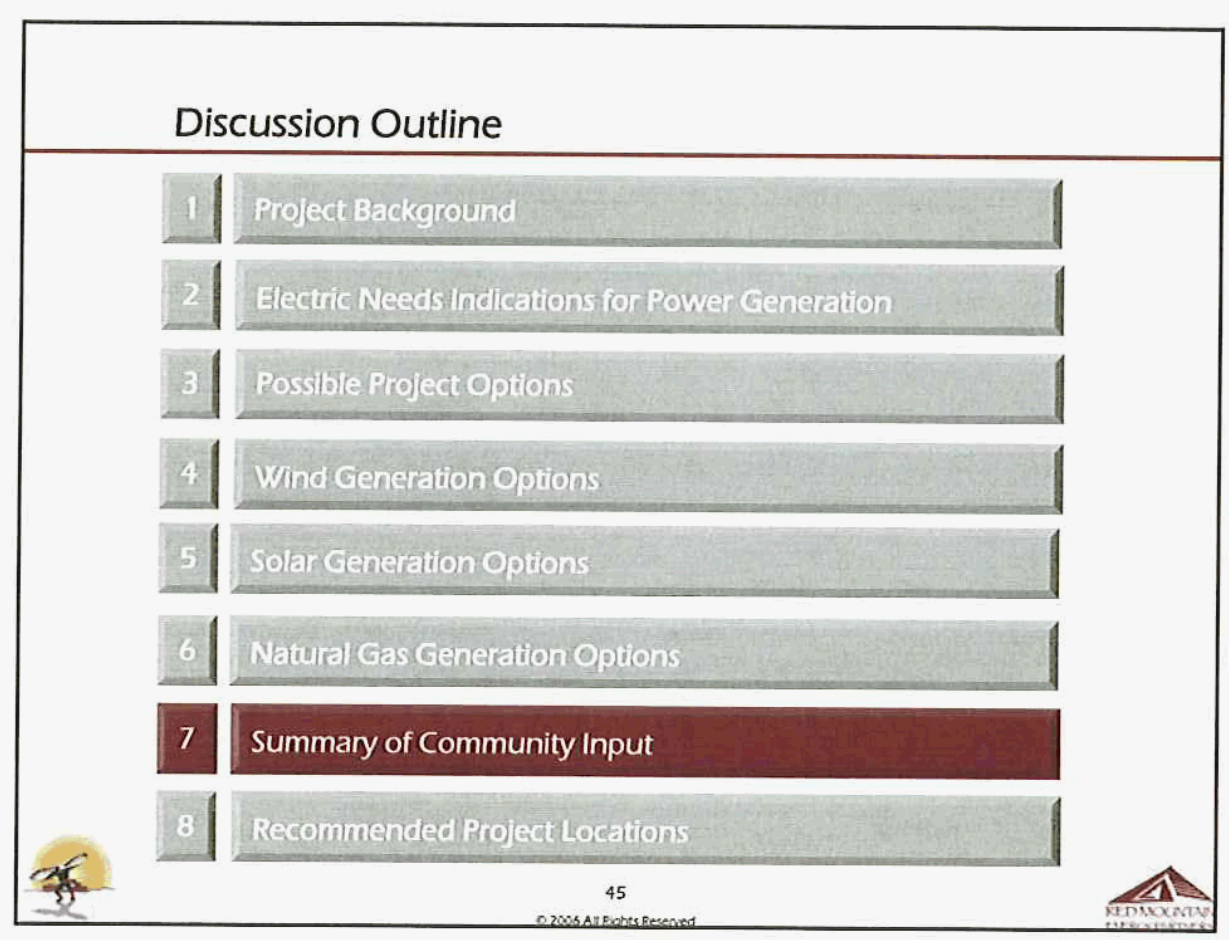




\section{Summary of Community Inputs to Date}

- Visual impacts of renewable technologies may not be acceptable for villagespecific projects

- Anticipate better support for facility or reservation-wide projects in areas with limited visibility

- Improved likelihood of success if projects were located in existing industrial or commercial areas

- Potential interest in Casa Blanca area, near Rainbow and LA JR/SR High School

- Potential interest for LDC facilities/partnering opportunity with PNM

- Best wind resources, and minimal visual impacts will be off mesas currently being monitored

- Further input needed from community

- Suggest multiple venues (Laguna newspaper, UA newsletter, additional village/reservation-wide meetings) to provide education, seek input

\section{Discussion Outline}

Project Background

Electric Needs indications for Power Generation

Possible project Options

Wind Generation Options

Solar Generation Options

Natural Gas Generation Options

Sulminany of Community (n)put

8 Recommended Project Locations 


\section{Recommended Project Locations}

- Casa Blanca

* Optimally sized wind/gas or solar/gas southeast of Rainbow Center

- Mesita

* Optimally sized solar/gas southeast of village

- Laguna

* Optimally sized solar/gas north of transfer station

- Rio Puerco

* Optimally sized solar/gas near eastern edge of reservation

- Paraje

* Optimally sized solar/gas in general vicinity of old high school

- Key sizing considerations

$\therefore$ Economics

$\therefore$ Project size dependent on Utility Authority involvement with electric operations

- Reservation-sized if operating electric utility system

- Facility/village-sized if not operating electric utility system

\section{Further Information}

Contact:
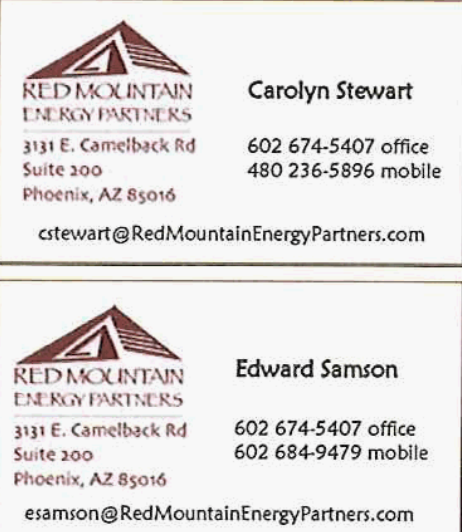
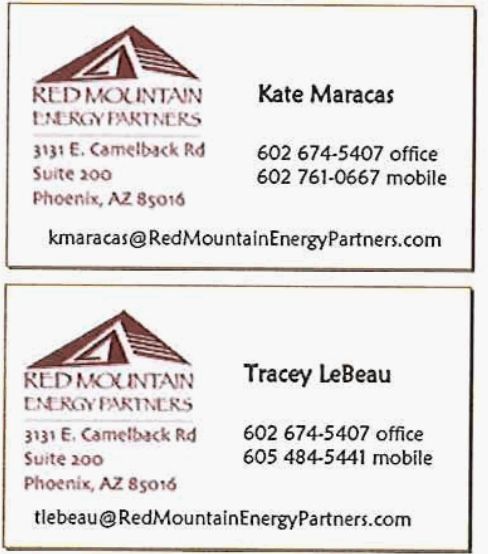

49 


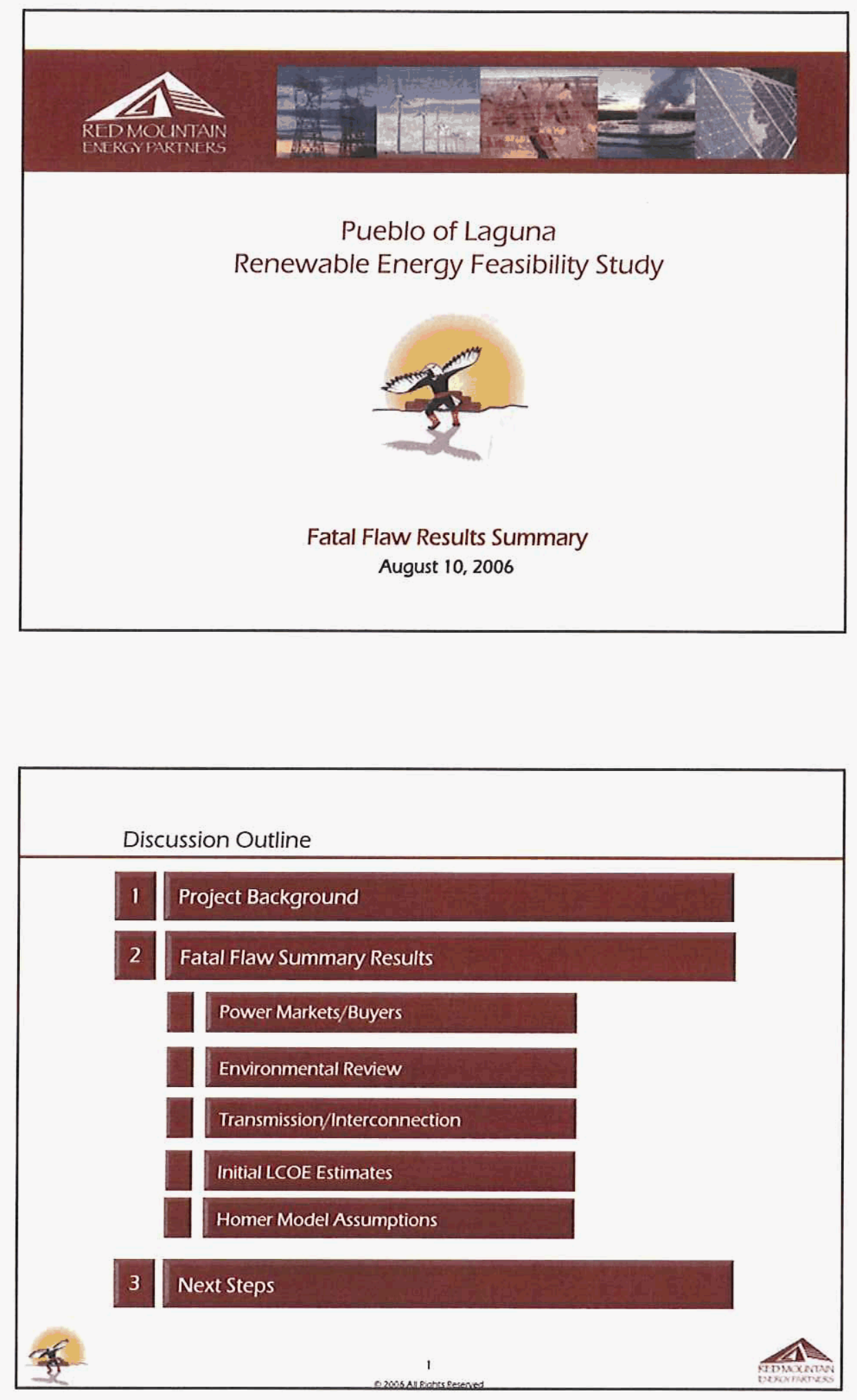


\section{Renewable Energy Feasibility Study}

Department of Energy Funded Study Project Objectives:

- Increase energy knowledge and capacity

- Improve quality and reliability of electric service

- Contribute to development of environmentally clean energy

- Provide data/analysis to support Laguna involvement in renewable

energy projects as an owner or participant

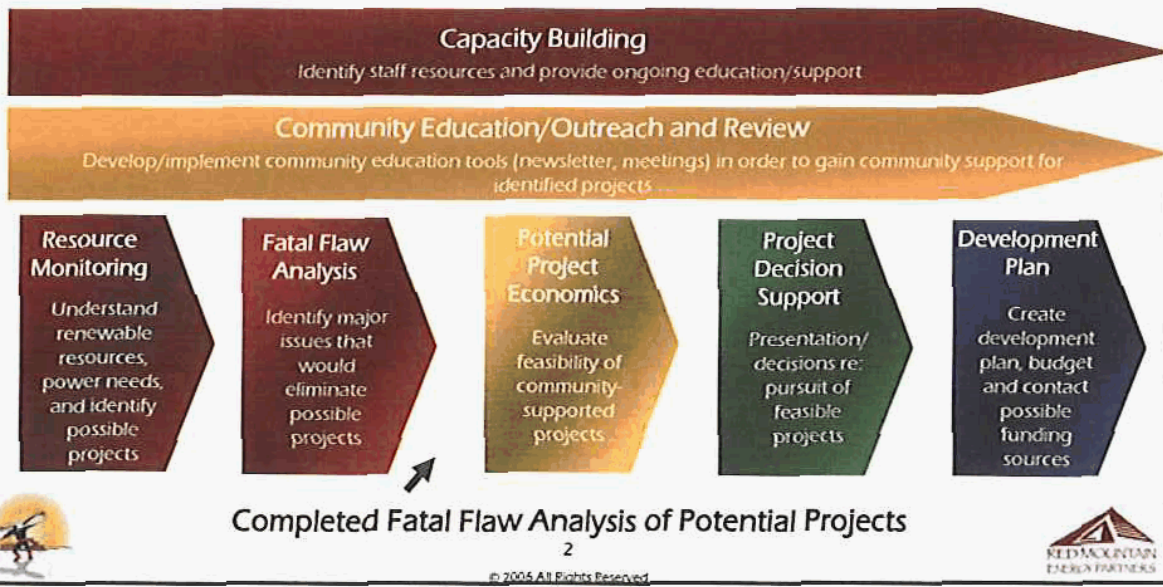

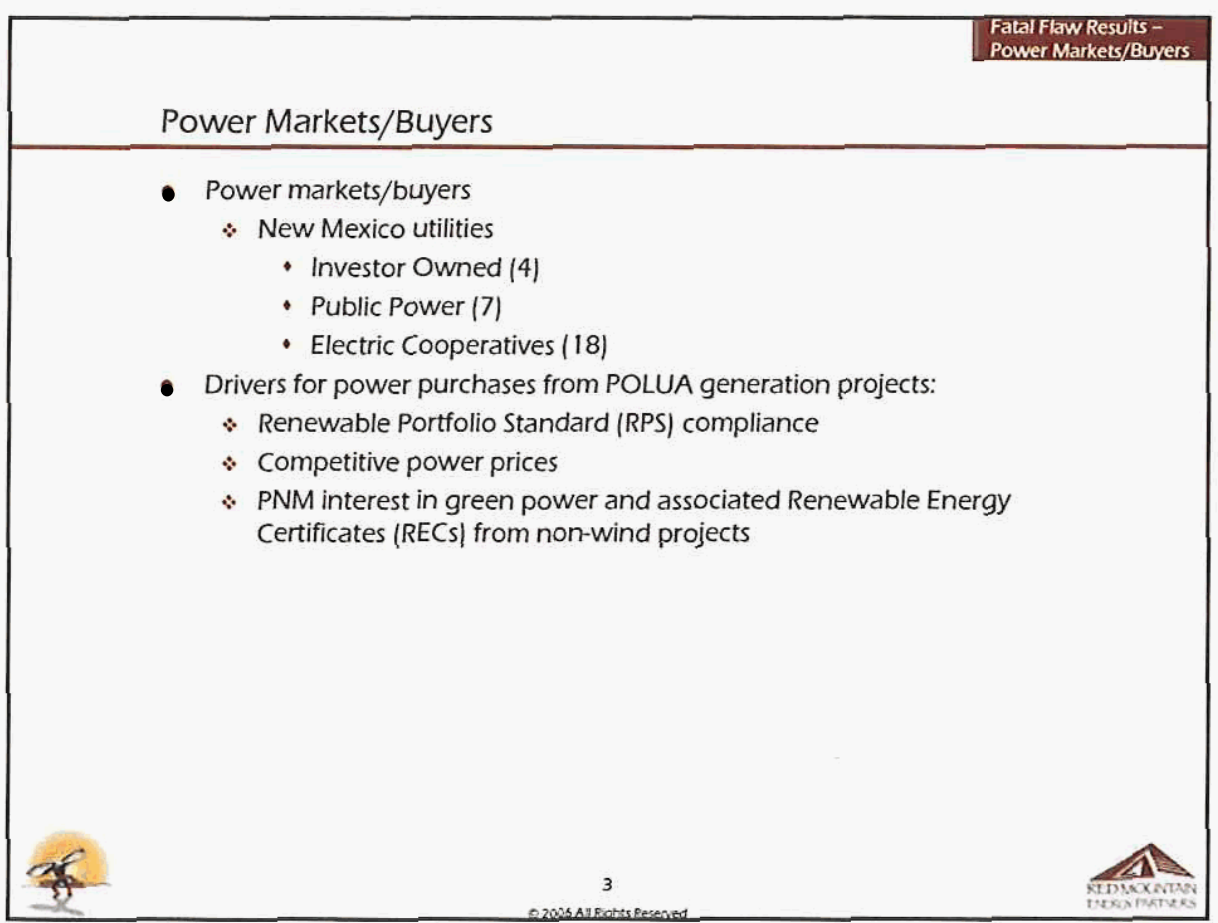



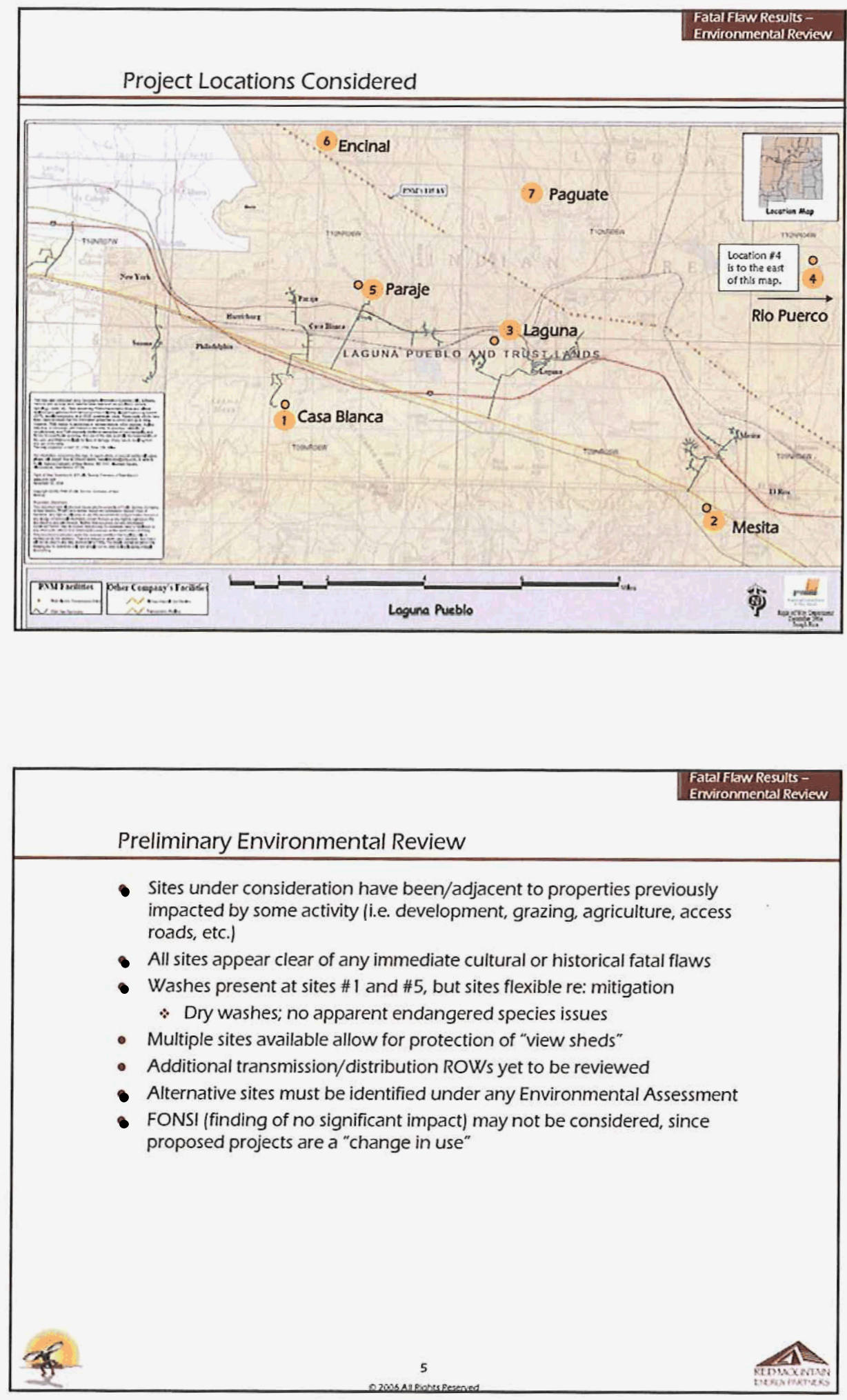


\begin{tabular}{|c|c|c|c|c|c|c|c|}
\hline En & vironmen & al Revien & S Summar & & & \multicolumn{2}{|c|}{$\begin{array}{l}\text { Fatal Flaw Results - } \\
\text { Environmental Review }\end{array}$} \\
\hline Issue & $\begin{array}{l}\text { Casa } \\
\text { Blanca } \\
\end{array}$ & Mesita & Laguna & Rio Puerco & Paraje & Encinal & Paguate \\
\hline $\begin{array}{l}\text { General Site } \\
\text { Description }\end{array}$ & $\begin{array}{l}\text { SE of Laguna } \\
\text { Acoma } \\
\text { Highschool }\end{array}$ & $\begin{array}{l}\text { East of Viliage, } \\
\text { South of } 1-40\end{array}$ & $\begin{array}{l}\text { North of } \\
\text { Transfer Station }\end{array}$ & $\begin{array}{l}\text { Near Eastern } \\
\text { Edge of } \\
\text { Reservation }\end{array}$ & $\begin{array}{l}\text { NE of Otd High } \\
\text { School }\end{array}$ & West of Viliage & East of Village \\
\hline $\begin{array}{l}\text { Geological/ } \\
\text { Water Use }\end{array}$ & $\begin{array}{l}\text { None } \\
\text { Observed }\end{array}$ & $\begin{array}{l}\text { None } \\
\text { Observed }\end{array}$ & $\begin{array}{l}\text { None } \\
\text { Observed }\end{array}$ & $\begin{array}{l}\text { None } \\
\text { Observed }\end{array}$ & $\begin{array}{l}\text { None } \\
\text { Observed }\end{array}$ & $\begin{array}{l}\text { None } \\
\text { Observed }\end{array}$ & \begin{tabular}{|l} 
None \\
Observed
\end{tabular} \\
\hline $\begin{array}{l}\text { Geological } \\
\text { Hazard/ } \\
\text { Soil Erosion }\end{array}$ & Yes & $\begin{array}{l}\text { None } \\
\text { Observed }\end{array}$ & $\begin{array}{l}\text { None } \\
\text { Observed }\end{array}$ & $\begin{array}{l}\text { None } \\
\text { Observed }\end{array}$ & Yes & \begin{tabular}{|l} 
None \\
Observed
\end{tabular} & $\begin{array}{l}\text { None } \\
\text { Observed }\end{array}$ \\
\hline Water Quality & $\begin{array}{l}\text { Specific to Gas } \\
\text { Firming } \\
\text { Technology }\end{array}$ & \begin{tabular}{|l|} 
Specific to Gas \\
Firming \\
Technology
\end{tabular} & \begin{tabular}{|l|} 
Specific to Gas \\
Firming \\
Technology
\end{tabular} & $\begin{array}{l}\text { Specific to Gas } \\
\text { Firming } \\
\text { Technology }\end{array}$ & $\begin{array}{l}\text { Specific to Gas } \\
\text { Firming } \\
\text { Technology }\end{array}$ & $\begin{array}{l}\text { Specific to Gas } \\
\text { Firming } \\
\text { Tecinnology }\end{array}$ & $\begin{array}{l}\text { Specific to Gas } \\
\text { Firming } \\
\text { Technology }\end{array}$ \\
\hline $\begin{array}{l}\text { Airborne } \\
\text { Dust }\end{array}$ & $\begin{array}{l}\text { During } \\
\text { Construction } \\
\text { Only }\end{array}$ & $\begin{array}{l}\text { During } \\
\text { Construction } \\
\text { Only }\end{array}$ & $\begin{array}{l}\text { During } \\
\text { Construction } \\
\text { Only }\end{array}$ & $\begin{array}{l}\text { During } \\
\text { Construction } \\
\text { Only }\end{array}$ & \begin{tabular}{|l|} 
During \\
Construction \\
Only
\end{tabular} & $\begin{array}{l}\text { During } \\
\text { Construction } \\
\text { Only }\end{array}$ & $\begin{array}{l}\text { During } \\
\text { Construction } \\
\text { Only }\end{array}$ \\
\hline Noise & Nominal & Nominal & Norninal & Nominal & Nominat & Nominal & Nominal \\
\hline 36. & $\begin{array}{l}\text { tain sunveys } \\
\text { tee regarded }\end{array}$ & 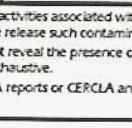 & 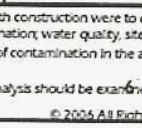 & 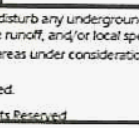 & 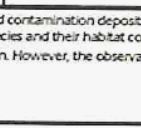 & $\begin{array}{l}\text { Which in turn could af } \\
\text { dote effected } \\
\text { ons ate rightovel and }\end{array}$ & 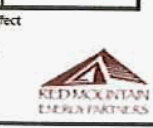 \\
\hline
\end{tabular}

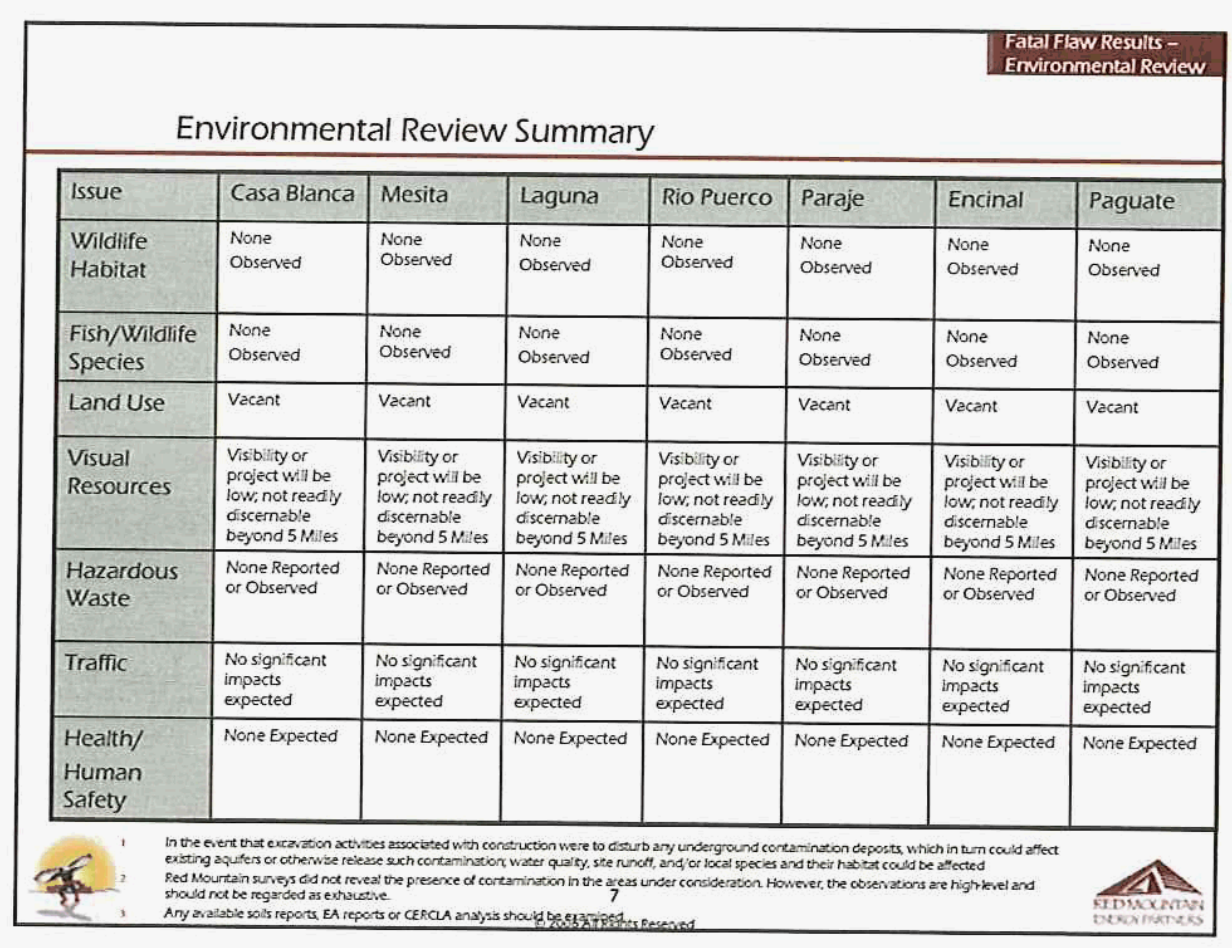




\begin{tabular}{|c|c|c|c|c|c|c|c|}
\hline \multicolumn{8}{|c|}{ Other Considerations Summary } \\
\hline Issue & $\begin{array}{l}\text { Casa } \\
\text { Blanca }\end{array}$ & Mesita & Laguna & Rio Puerco & Paraje & Encinal & Paguate \\
\hline $\begin{array}{l}\text { Cuitural } \\
\text { Resources (I) }\end{array}$ & $\begin{array}{l}\text { None } \\
\text { Documented }\end{array}$ & $\begin{array}{l}\text { None } \\
\text { Documented }\end{array}$ & $\begin{array}{l}\text { None } \\
\text { Documented }\end{array}$ & $\begin{array}{l}\text { None } \\
\text { Documented }\end{array}$ & $\begin{array}{l}\text { None } \\
\text { Documented }\end{array}$ & $\begin{array}{l}\text { None } \\
\text { Documented }\end{array}$ & $\begin{array}{l}\text { None } \\
\text { Documented }\end{array}$ \\
\hline $\begin{array}{l}\text { Community } \\
\text { Concerns }\end{array}$ & TBA & TBA & TBA & TRA & TBA & TBA & TBA \\
\hline $\begin{array}{l}\text { Construction } \\
\text { Access }\end{array}$ & Excellent & Excellent & Good & Moderate & Excellent & Moderate & Moderate \\
\hline $\begin{array}{l}\text { Topography } \\
\text { Observations }\end{array}$ & $\begin{array}{l}\text { Generally } \\
\text { Level and } \\
\text { sloping } \\
\text { toward wash }\end{array}$ & $\begin{array}{l}\text { Generally } \\
\text { Level }\end{array}$ & Generally Level & Generally Level & \begin{tabular}{|l|} 
Generally level \\
with existing \\
buildings on site
\end{tabular} & General'y Level & Generally Level \\
\hline $\begin{array}{l}\text { Interconnection } \\
\text { issues }\end{array}$ & $\begin{array}{l}\text { See Gircuit } \\
51000 \text { and } \\
\text { Gas } \\
\text { Distribution } \\
\text { Map }\end{array}$ & $\begin{array}{l}\text { See Gircuit } \\
51000 \text { and } \\
\text { Gas } \\
\text { Distribution } \\
\text { Map }\end{array}$ & $\begin{array}{l}\text { See Gircuit } \\
51000 \text { and Gas } \\
\text { Distribution } \\
\text { Map }\end{array}$ & $\begin{array}{l}\text { See Grcuit } \\
51000 \text { and Gas } \\
\text { Distribution } \\
\text { Map }\end{array}$ & $\begin{array}{l}\text { See Grcuit } \\
51000 \text { and Gas } \\
\text { Distribution } \\
\text { Map }\end{array}$ & $\begin{array}{l}\text { See Gircuit } \\
51000 \text { and Gas } \\
\text { Distribution } \\
\text { Map }\end{array}$ & $\begin{array}{l}\text { See Grcuit } \\
51000 \text { and Gas } \\
\text { Distribution } \\
\text { Map }\end{array}$ \\
\hline Other & Reserved & Reserved & Reserved & Reserved & Reserved & Reserved & Reserved \\
\hline 32. & 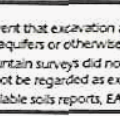 & $\begin{array}{l}\text { reves the presence } \\
\text { reptre. }\end{array}$ & 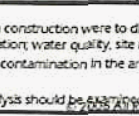 & 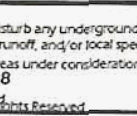 & amination depost & 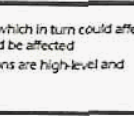 & $\underset{x \rightarrow \infty}{x \rightarrow \infty}$ \\
\hline
\end{tabular}

\begin{tabular}{|l}
\hline Major Environmental Assumptions \\
\hline $\begin{array}{l}\text { Siting will be done in accordance with Pueblo laws, ordinances, and } \\
\text { Environmental Review }\end{array}$ \\
customary processes \\
- Transmission interconnection and distribution will be overnead on tribal \\
lands, or in public rights-of-way \\
- Underground transmission may be necessary onsite depending on the scale \\
of the specific project \\
Construction will be done using generally accepted construction methods for \\
runoff and dust mitigation \\
- As siting is preliminary, community concerns will still need to be addressed \\
and mitigated \\
Construction can be completed in 6-18 months depending on the scale of \\
the project \\
Additional environmental assessments may be necessary as specific projects \\
are identified and proceed to more in-depth studies \\
of
\end{tabular}




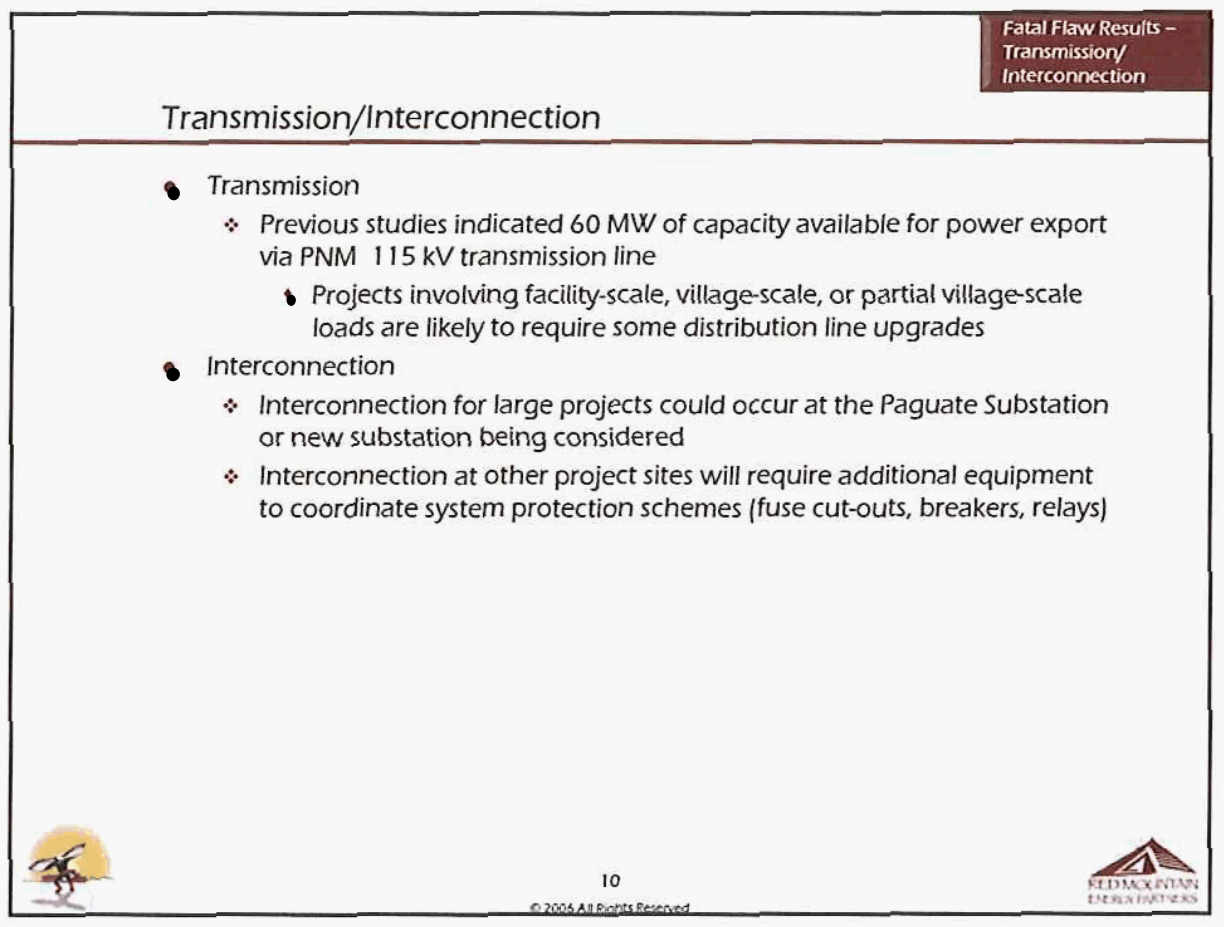

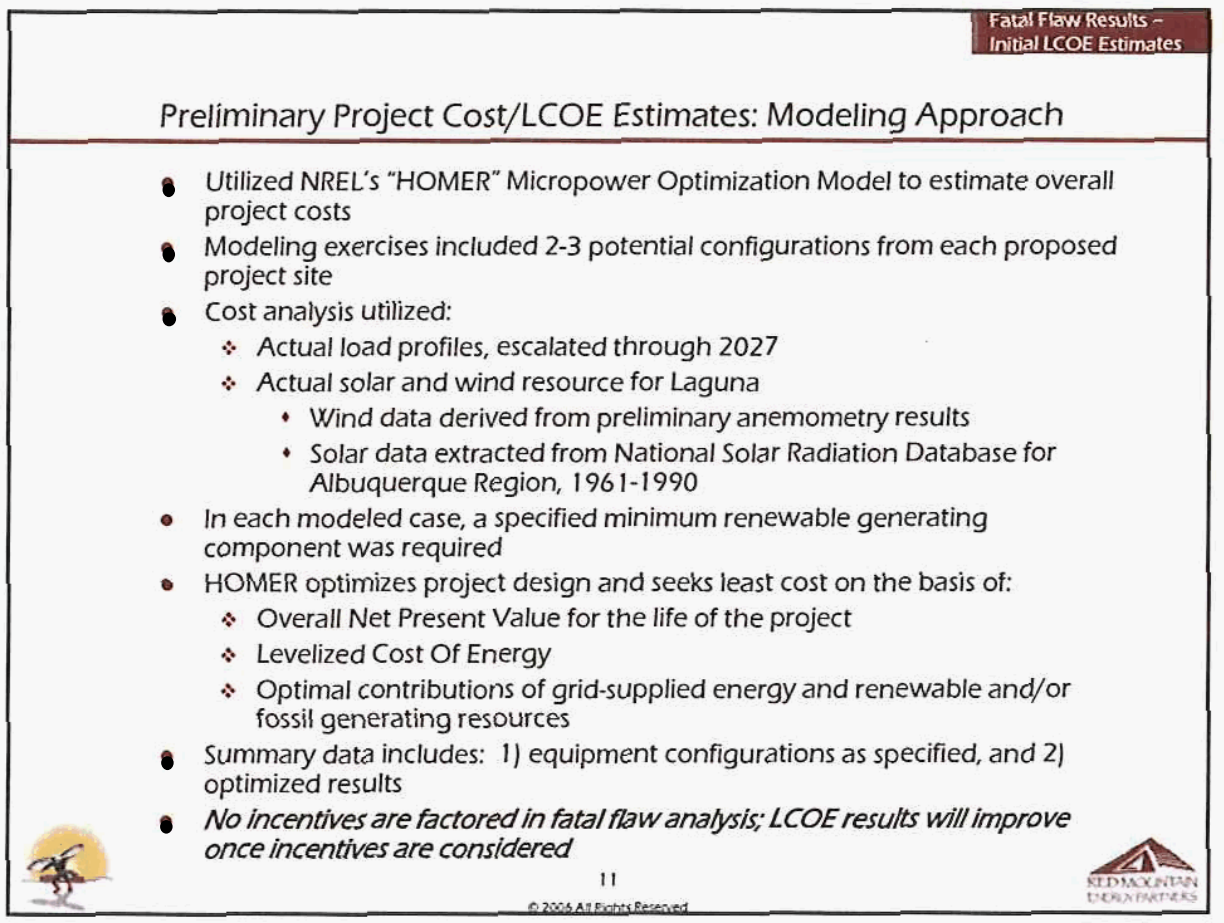




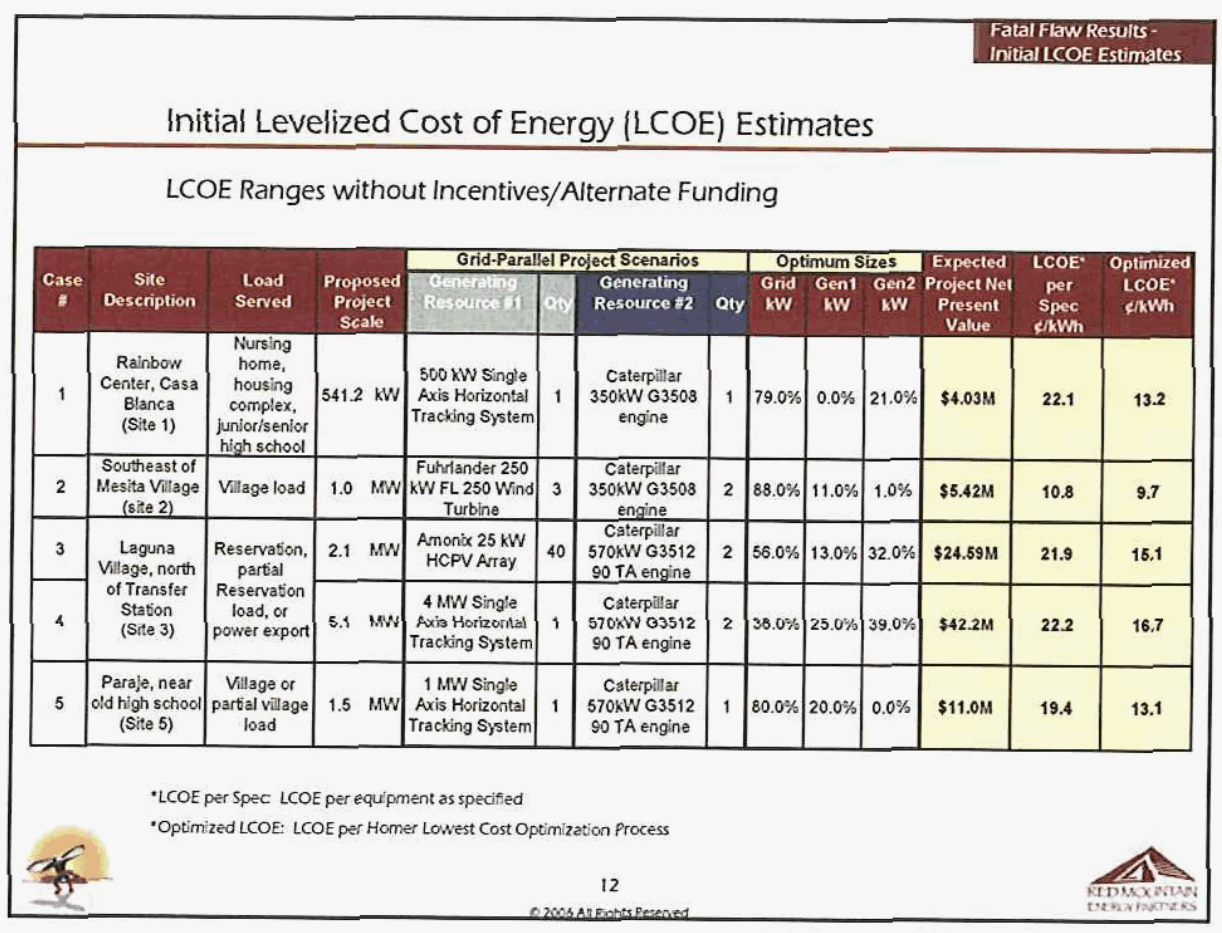

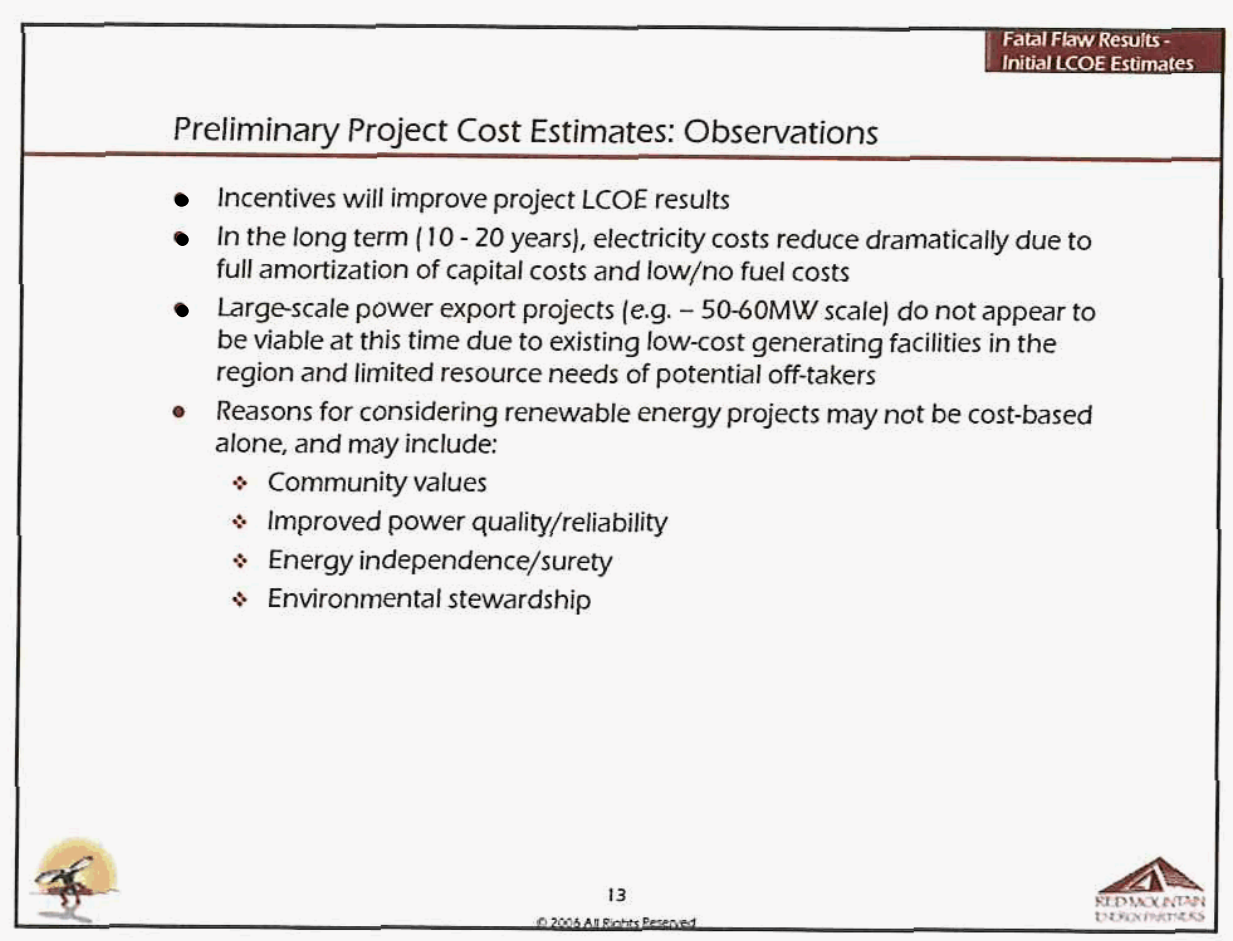




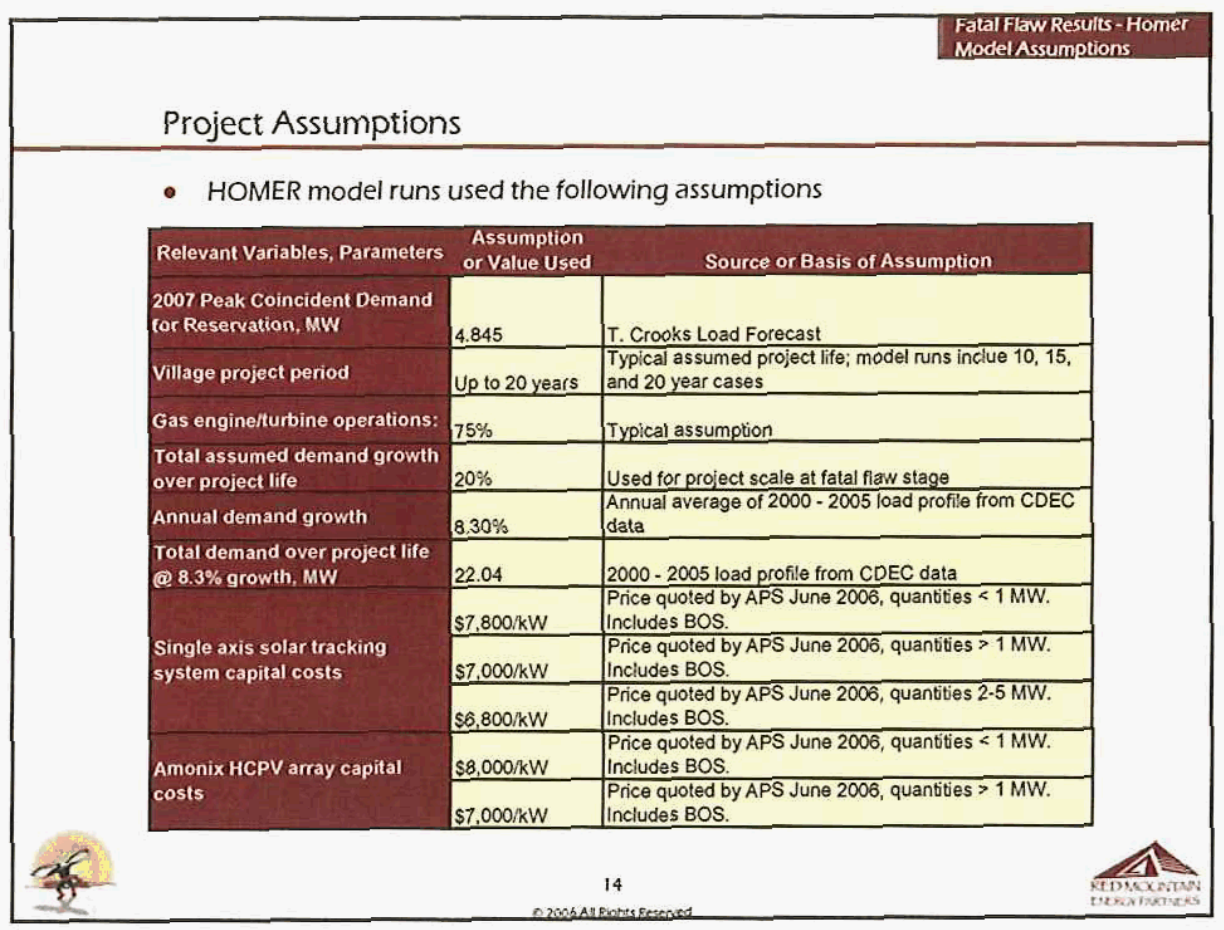

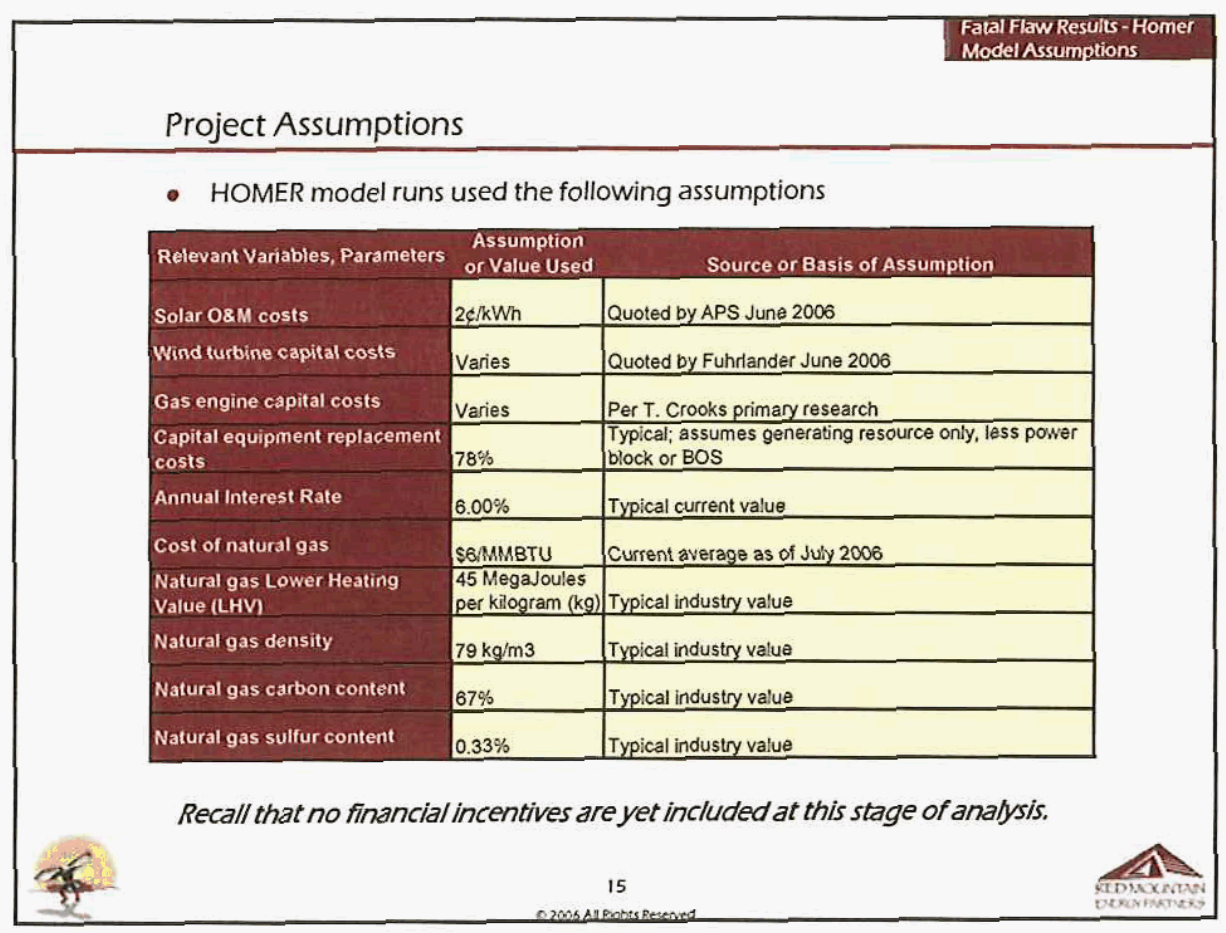


Next Steps

- Continue community education activities

- Gather community feedback on preliminary projects identified

- Further define selected projects and determine financial architecture, partnership structures, etc.

- Begin further wind site testing, as appropriate

- Complete detailed economic analyses of accepted projects

- Prepare development plan

Further Information

Contact:
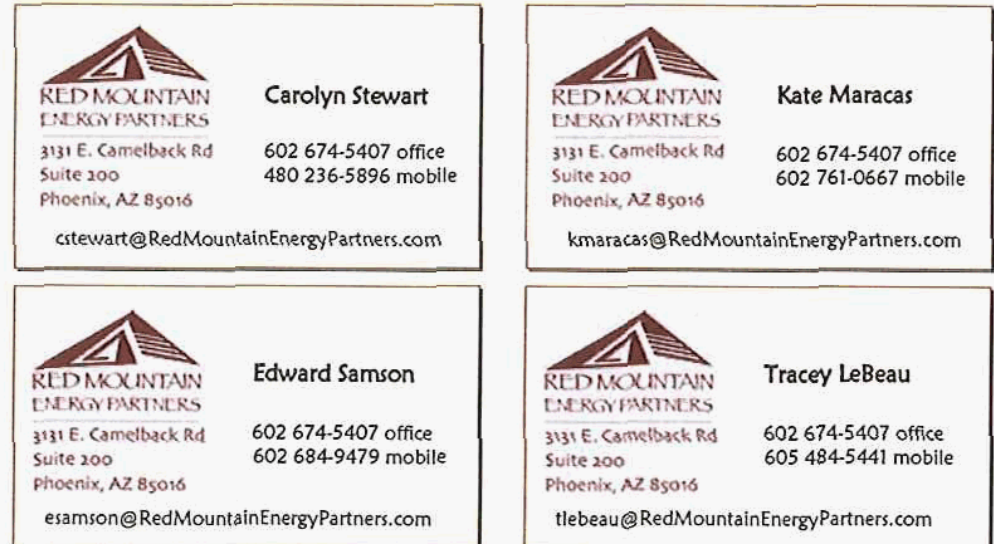



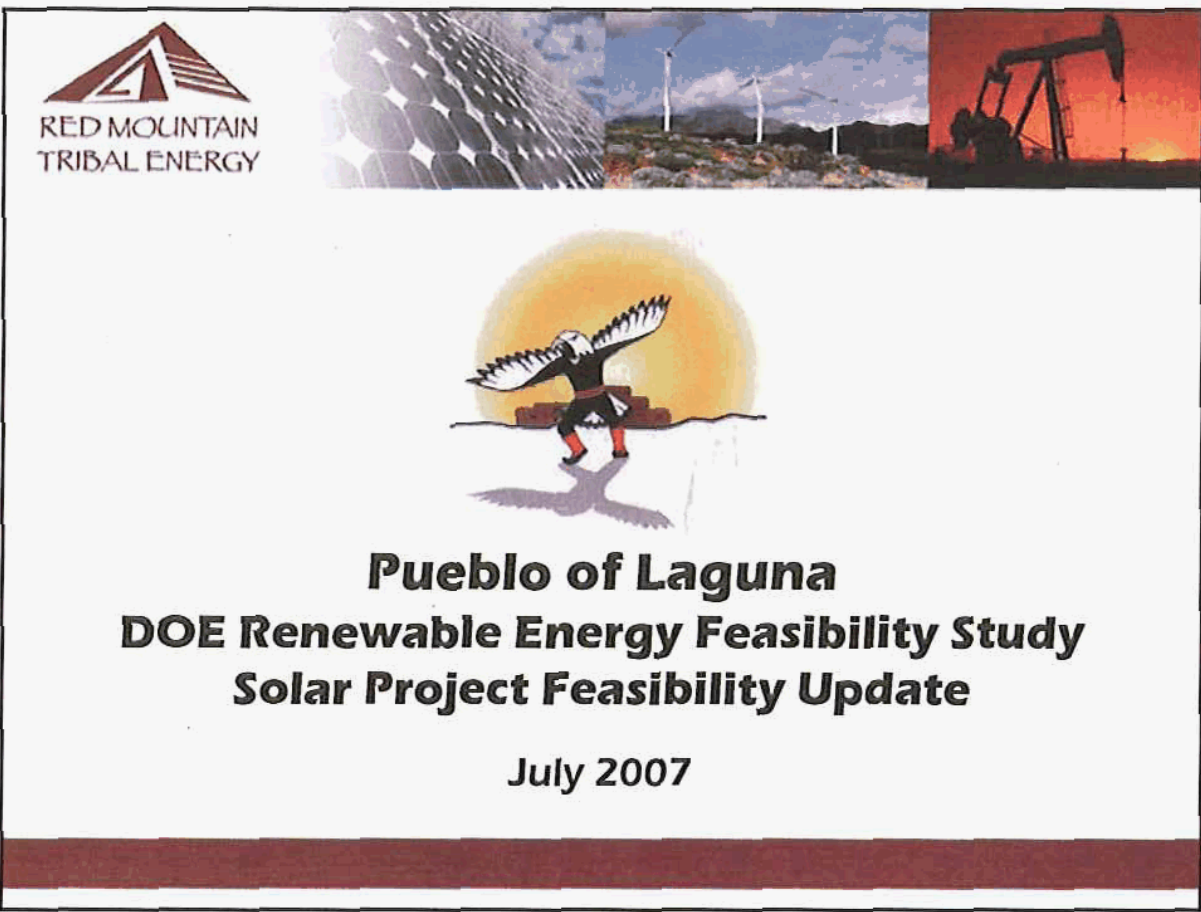

\section{Discussion Outline}

- Project Scope/Progress to Date

- Initial Solar Project Feasibility Review

- Laguna Solar Resource

$\checkmark P V$

$\checkmark$ Concentrating Solar

- Proposed Project Location

- Solar Technology Options

- Solar Project Concepts/Capital Cost Estimates

- Project Development/Ownership Structure Options

- Project Economics/PPA Indications

- Next Steps

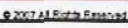




\section{Renewable Energy Feasibility Project Overview}

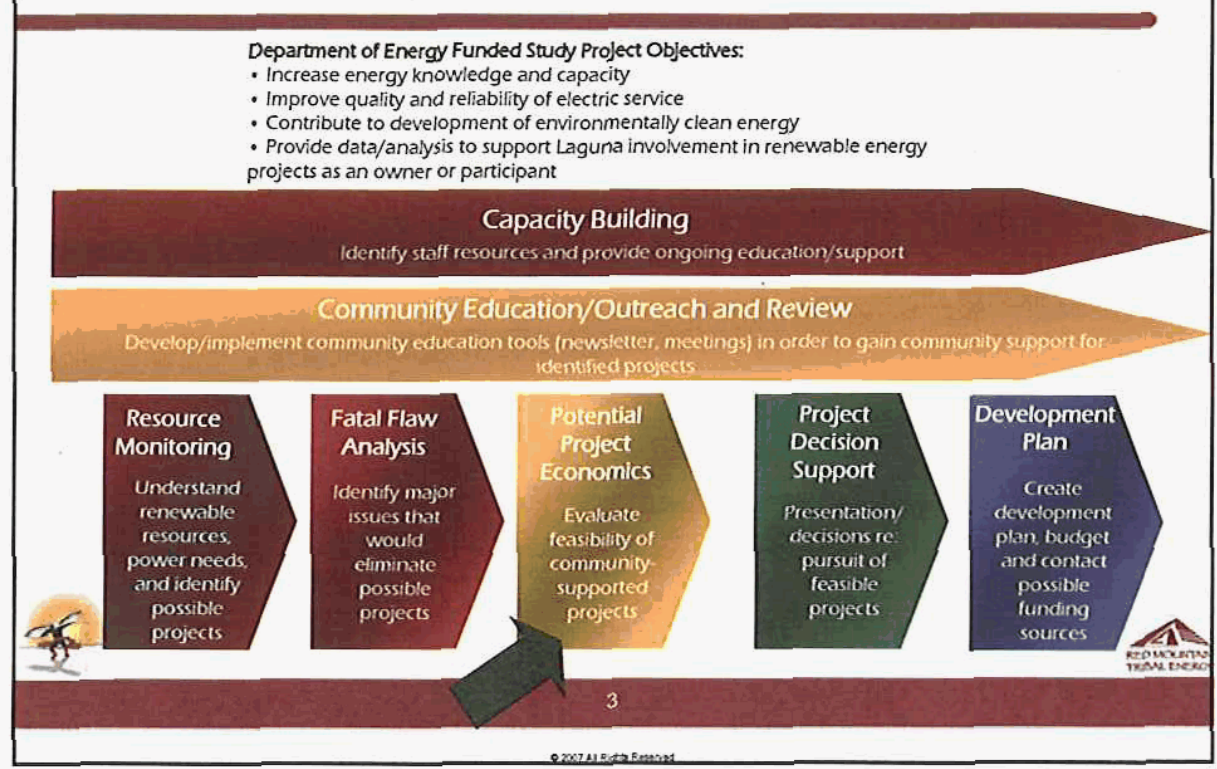

\section{Site Concept/Site Sellection Process}

- Various community and large-scale project concepts and locations were identified throughout the study period and evaluated for fatal flaws

- Questionnaires developed for community input

- Combination of electric system analysis and renewable feasibility study contributed to identification of potential Mesita site

- Feasibility of solar project concepts being analyzed

" PV vs. Concentrating Solar

- Community Scale vs. Large Scale 


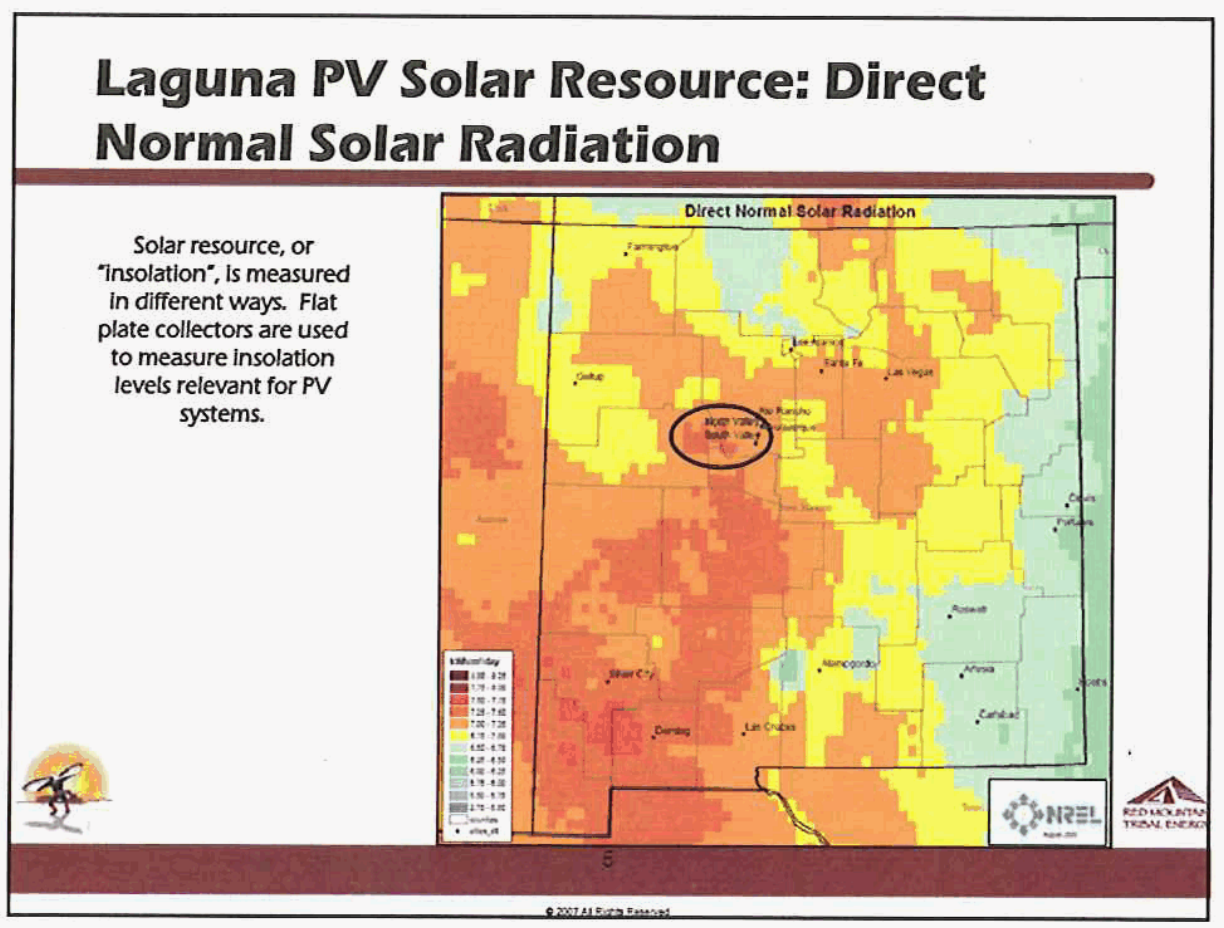

\section{Laguna Solar Resource: Insolation for Flat- Plate Collector Facing South, Fixed Tilt}

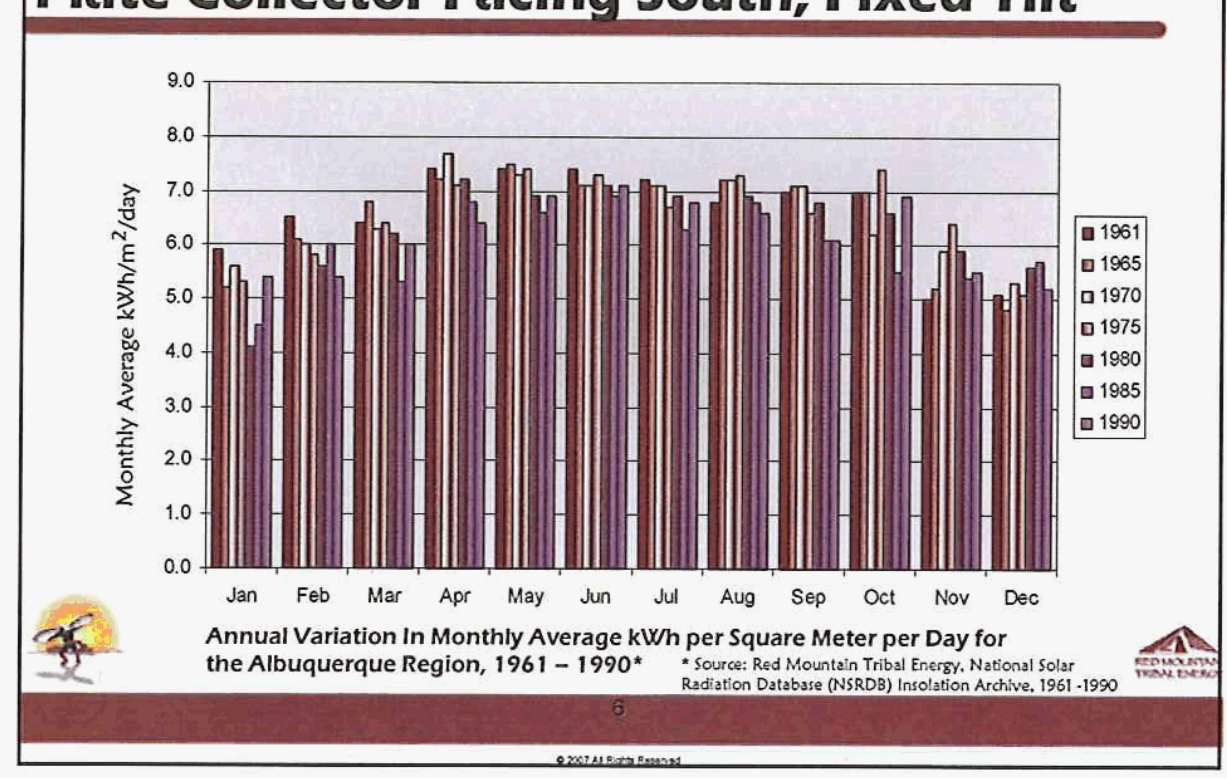




\section{Laguna Solar Resource: Insolation for Flat- Plate Collector Facing South, Fixed Tilt}

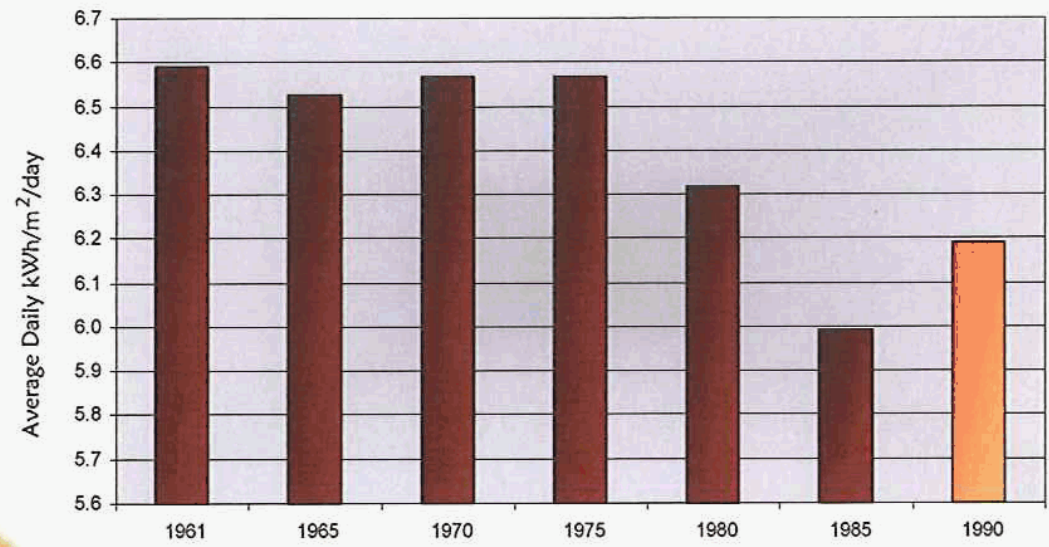

Annual Average kWh per Square Meter per Day, by Year for the Albuquerque Region, $1961-1990^{*}$

\section{Laguna Solar Resource: Concentrating Solar Power}

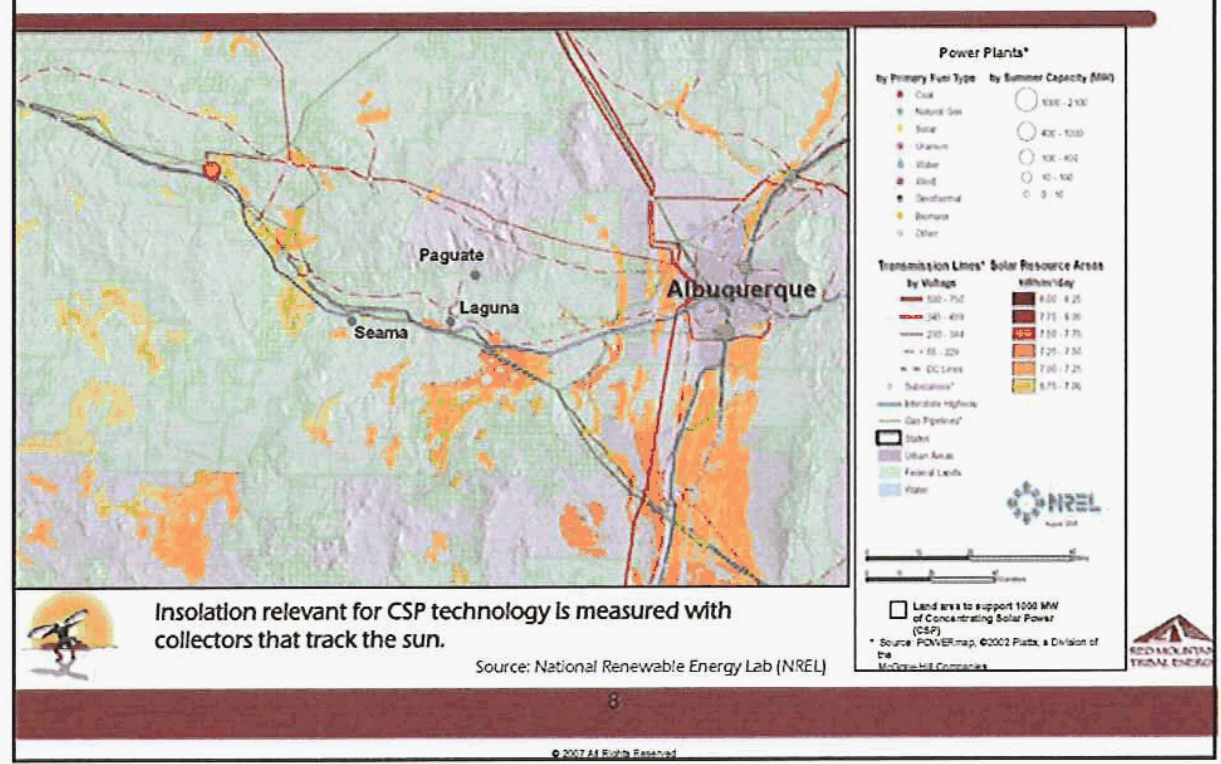




\section{Laguna Sollar Resource : Insolation for Two- Axis Tracking Concentrating Collector}

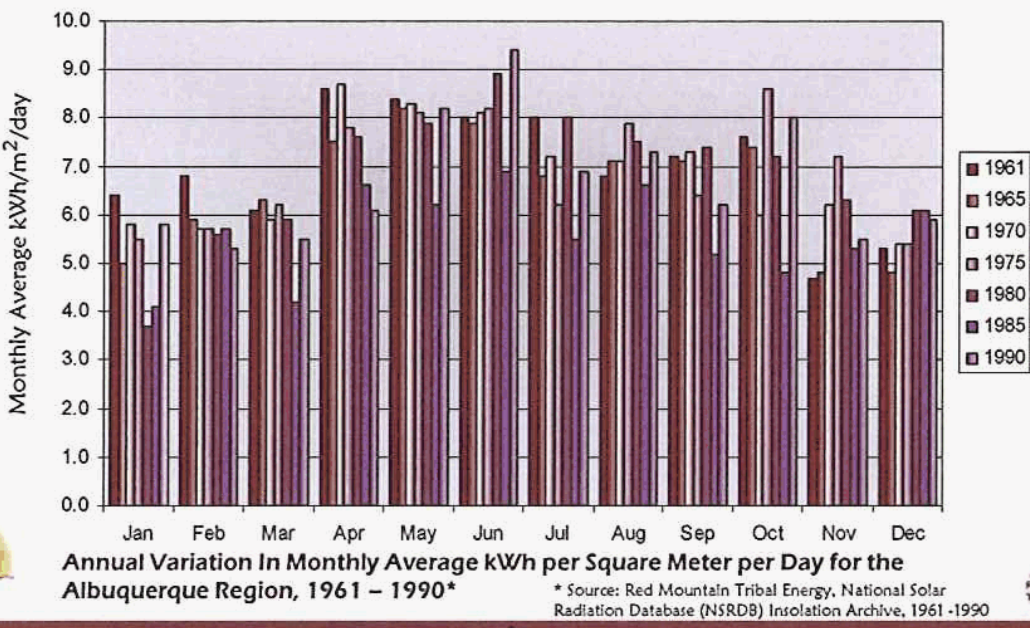

\section{Laguna Solar Resource: Insolation for Two- Axis Tracking Concentrating Collector}

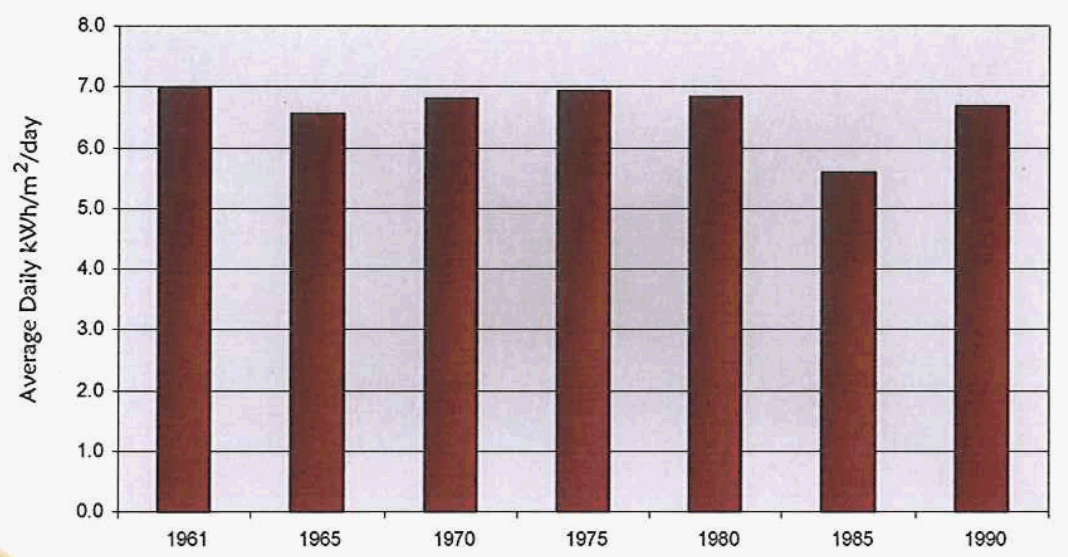

Annual Average $\mathrm{kW} / \mathrm{h}$ per Square Meter per Day, by Year for the Albuquerque Region, $1961-1990^{*}$ 


\section{Possible Project Site}

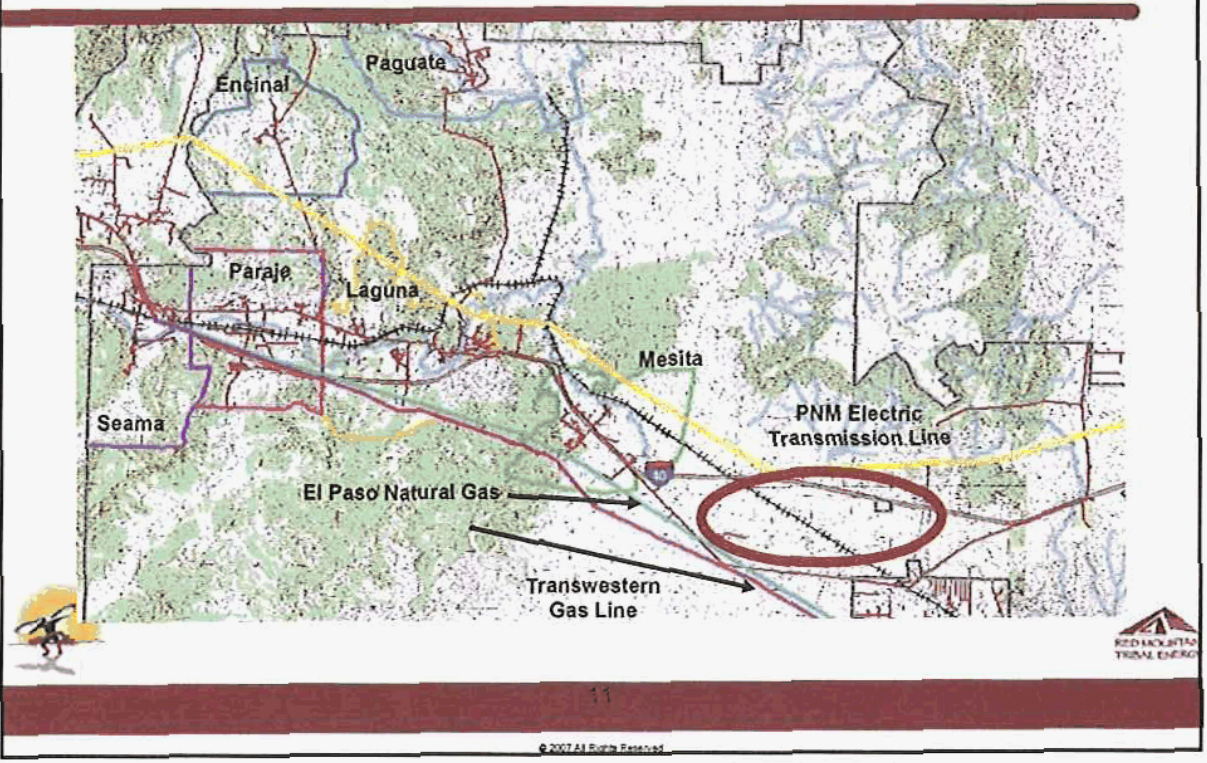

\section{Resource Indications at Possible Project Site}

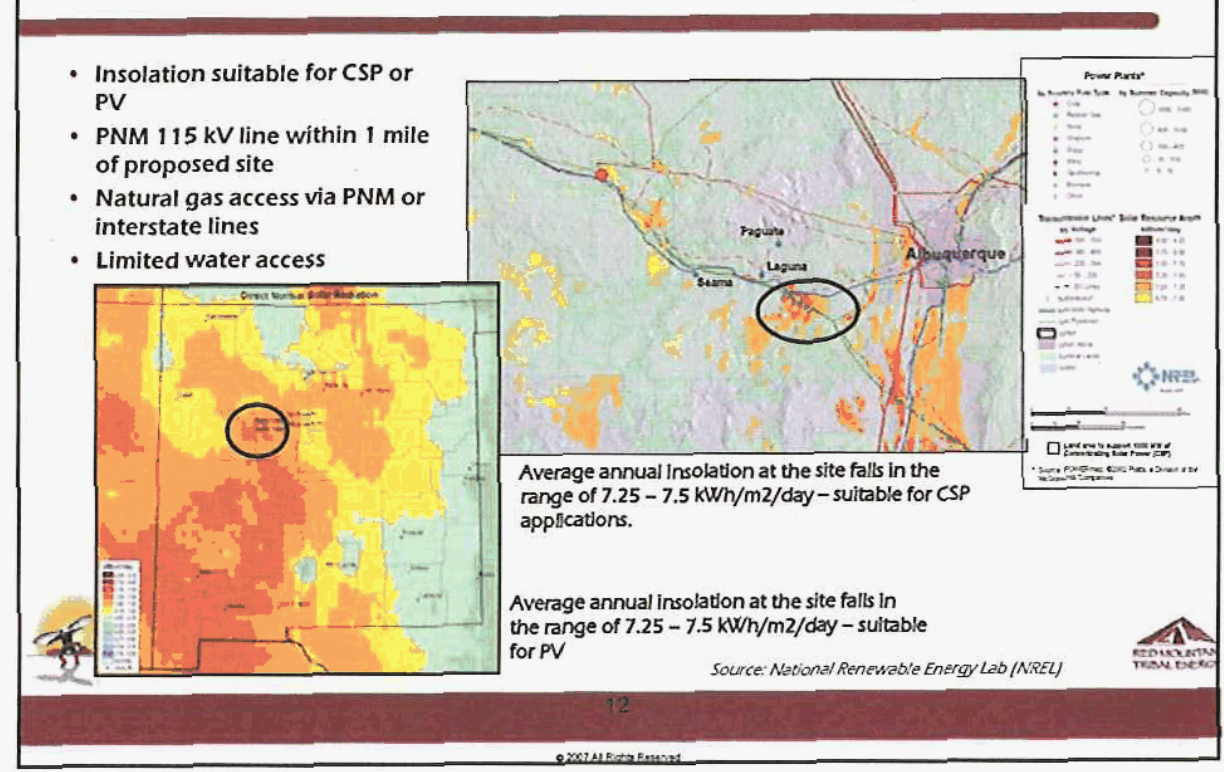




\section{Preliminary Site Assessment}

- Site evaluated in late 2006

- No fatal flaws evident

- Anticipated community support for renewable projects - both small and large

- Some community interest in projects east of Mesita

\section{Solar Technollogy Options}

- PV

- Concentrated Solar 


\section{PV Technology Options}

- Configurations

- Fixed, flat plate

- Single axis tracking

- Dual axis tracking

- Many commercial applications, but usually on a smaller scale

- Few projects over 1 MW

\section{Concentrating Solar Technology Options}

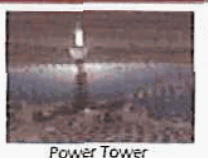

Power Tower

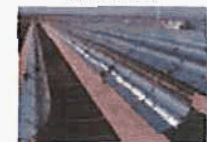

Parabolictrough

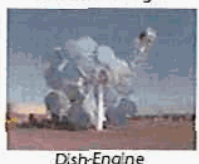

Dishrengine

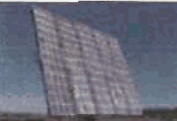

HCPV

- Power Tower: Uses a circular field array of individually-tracking mirrors to focus sunlight onto a central receiver mounted on top of a tower, which produces steam to power a conventional turbine generator to produce electricity

- Parabolic Trough: Uses trough-shaped mirrors to focus sunlight on thermally efficient receiver tubes that contain a heat transfer fluid; fluid is heated and pumped through a series of heat exchangers to produce superheated steam, which powers a conventional turbine generator to produce electricity

Dish-Engine: Uses an array of dish-shaped mirrors to focus solar energy onto a receiver located at the focal point of the dish; fluid in the receiver is heated an used to generate electricity in a small engine or turbine attached to the receiver

- High Concentration Photovoltaics (HCPV): Uses high efficiency PV cells with concentrating (Fresnel) lenses that multiply the sun's intensity, requiring hundreds of times less photovoltaic material to achieve the same energy output as PV cells without concentration 


\section{Concentrating Solar Technology Options}

- Power Tower

- Tested in CA for many years

- No US commercial applications to date

- Anticipated cost/efficiency improvements

- Parabolic Trough

- 350 MW in commercial operation since 1990s

- Water requirements pose a challenge

- Dish-Engine

- No commercially operated systems to date

- Cost improvements promised; not yet delivered

- HCPV

- Field tested in $A Z$ and NV for $15+$ years

- Small scale NV and AZ commercial operations

- Anticipated cost/efficiency improvements

\section{HCPV Technology}

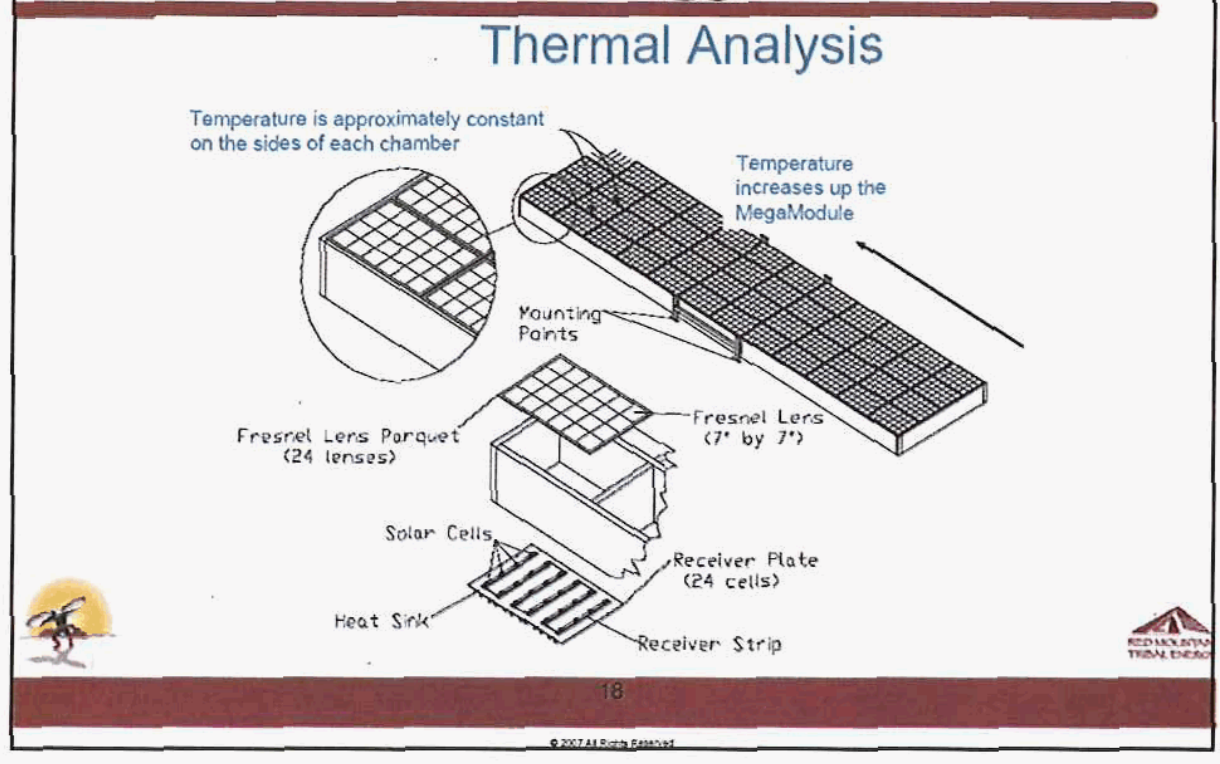




\section{PV vs. Concentrated Sollar Comparison}

\begin{tabular}{|c|c|}
\hline Installation & $\begin{array}{c}\text { Cost } \\
\text { (\$) N/att) }\end{array}$ \\
\hline Single Axis Tracking PV & 8.50 \\
\hline HCPV (Amonix) & $4.90-5.20$ \\
\hline HCPV - Future & 3.00 \\
\hline $\begin{array}{l}\text { Parabolic Trough (for } \\
\text { comparison only) }\end{array}$ & 4.00 \\
\hline
\end{tabular}

\section{Possible Sollar Project Concepts}

- Laguna Solar Project concepts under study

- 3 MWV community scale PV

$\checkmark$ Single axis tracking

- 50 MWV large scale PV or HCPV

$\checkmark$ Single axis tracking vs. high concentration PV

- 100 MWV large scale PV or HCPV

$\checkmark$ Single axis tracking vs. high concentration PV 


\section{MW Single Axis Tracking PV}

- 330 sections, consisting of 52 Sharp NT-180U1-175/180 - watt

photovoltalc modules and 2 satcon

Powergate, ae-500 kW Inverters

- Approximately 30 acres required
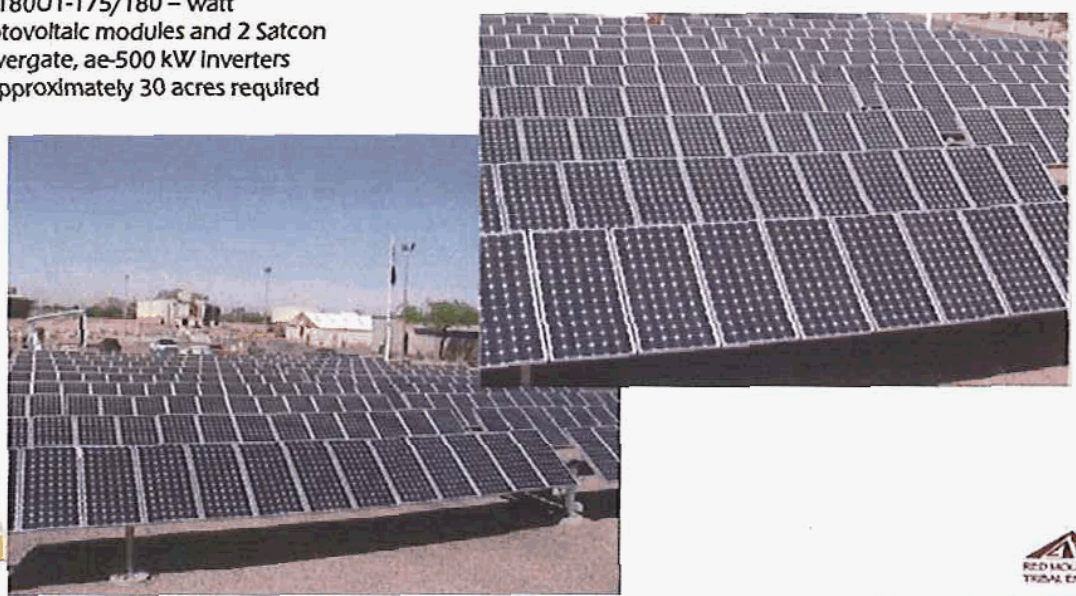

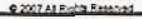

\section{MW Large Scalle PV}

- 5500 sections, each consisting of 52 Sharp NT-180U1-175/180 - watt photovoltaic modules and 2 Satcon

Powergate, ae-500 kW inverters

- Approximately 500 acres required
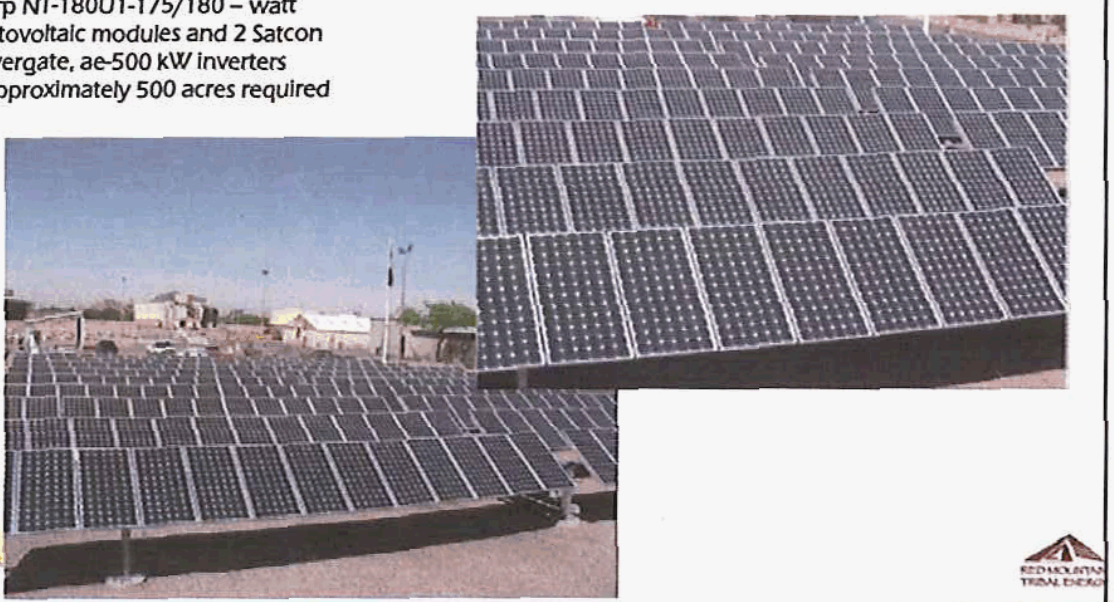


\section{MW Large Scalle HCPV}

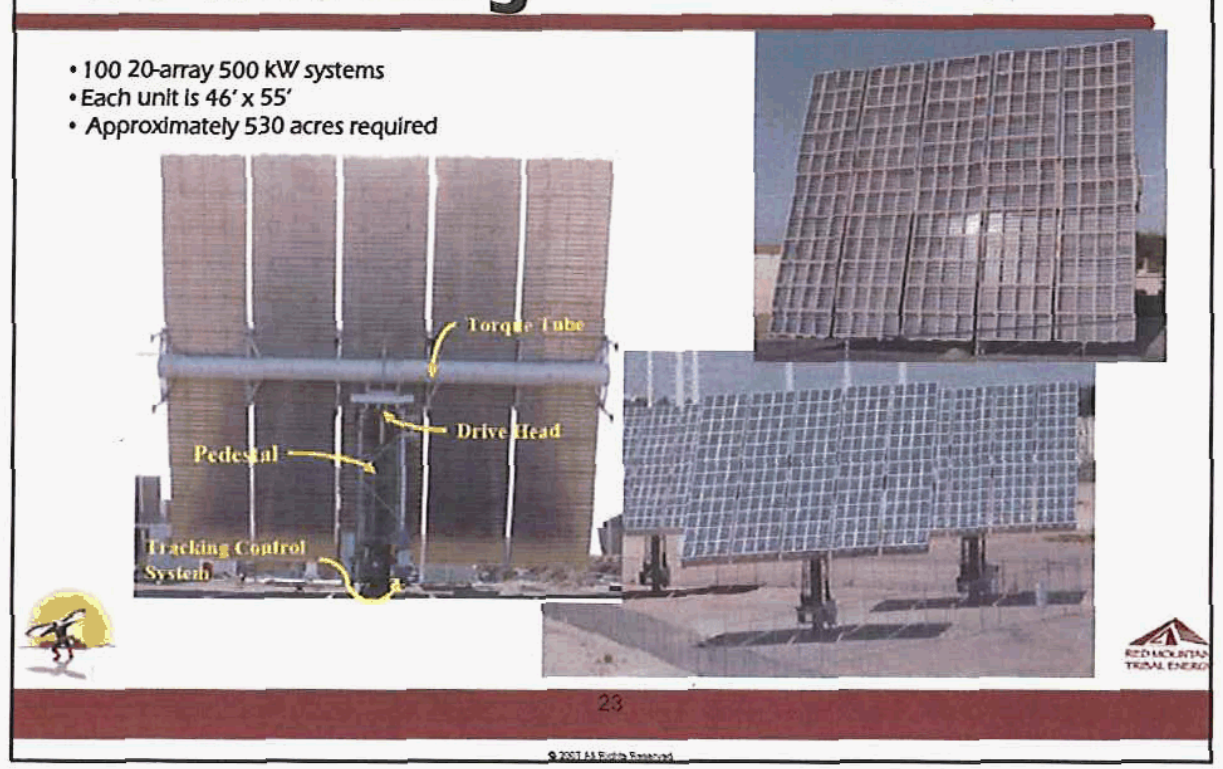

\section{MW Large Scalle PV}

- 11000 sections, each consisting of 52 Sharp NT-180U1-175/180 - watt

photovoltalc modules and 2 Satcon

Powergate, ae-500 kW Inverters

- Approximately 1000 acres required

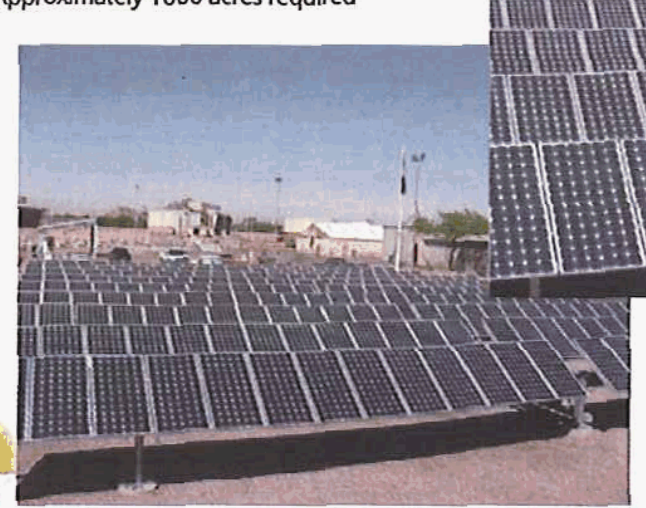




\section{MW Large Scalle HCPV}

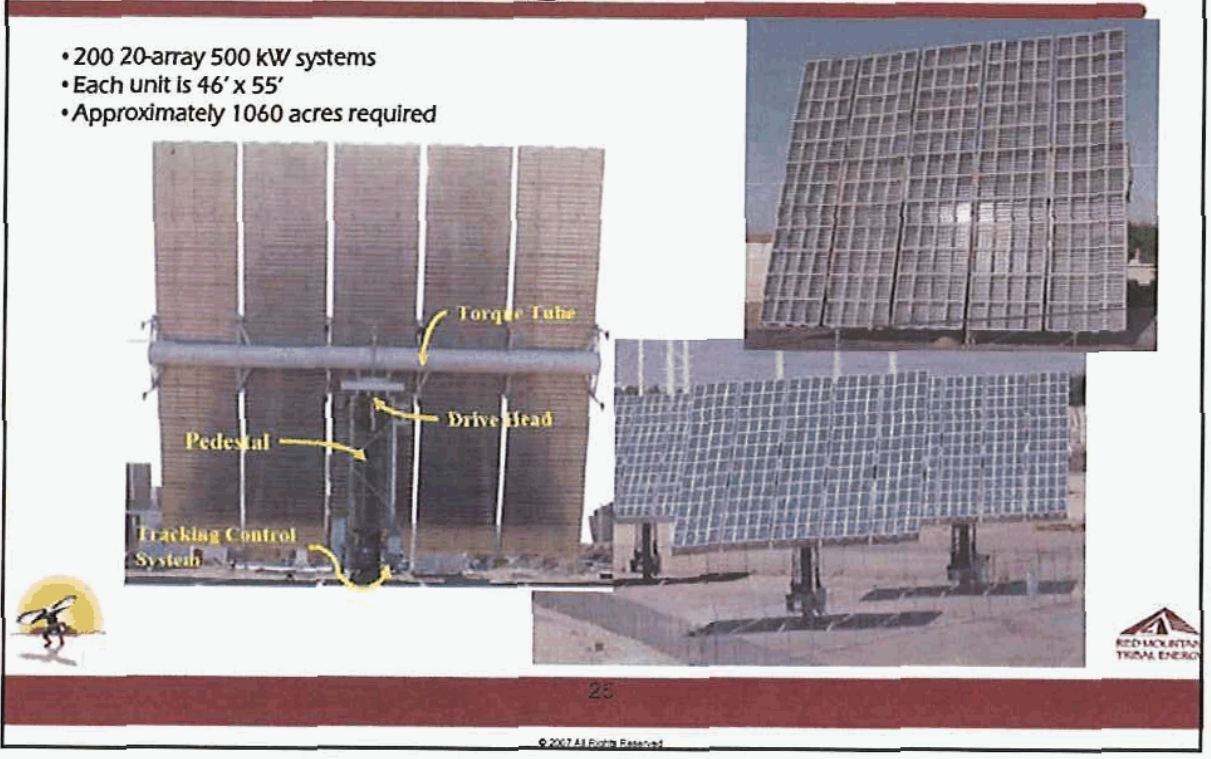

\section{Laguna Solar Project Comparison}

\begin{tabular}{|l|c|c|c|c|}
\hline & $\begin{array}{c}\text { Capital Cost } \\
\text { (millions) }\end{array}$ & $\begin{array}{c}\text { Acreage } \\
\text { Required }\end{array}$ & $\begin{array}{c}\text { Energy } \\
\text { Production } \\
\text { (million kW/hs) }\end{array}$ & $\begin{array}{c}\text { Levelized Cost } \\
\text { of Energy (\$.00 } \\
\text { per kW/h) }\end{array}$ \\
\hline $\begin{array}{l}3 \text { MW/ Single } \\
\text { axis tracking PV }\end{array}$ & $\$ 26$ & 30 & & \\
\hline $\begin{array}{l}50 \text { MWV Single } \\
\text { axis tracking PV }\end{array}$ & 425 & 500 & & \\
\hline 50 MW HCPV & 260 & 530 & & \\
\hline $\begin{array}{l}100 \text { MW/ Single } \\
\text { axls tracking PV }\end{array}$ & 850 & 1000 & & \\
\hline 100 MW/ HCPV & 480 & 1060 & & \\
\hline
\end{tabular}




\section{Project Development/Ownership Options}

- Community Scale

- Developer owned/operated

- Laguna owned/operated w/grant funding

- Large Scale

- Developer owned/operated

- Tribal/Tax Partner Joint Venture

\section{Project Economics/PPA Indications}

- Community Scale

- Laguna owned/operated w/grant funding likely to provide best economics

$\checkmark$ Grants reduce capital outlay

$\checkmark$ Net metered power

$\checkmark$ Sell RECs separately

- Large Scale

- Tribal/Tax Partner Joint Venture likely to provide best economics

$\checkmark$ Tax partner takes advantage of tax incentives (ITC)

$\checkmark$ Sell power/RECs bundled

$\checkmark$ Sell power/RECs separately

$\checkmark$ Utilities vs. financial institutions 


\section{Renewable Energy Project Plan - May 2007}

- Indicated Next Steps - May 2007

"Distribute and analyze results from renewable energy questionnaires

-Monitor wind at other sites

-Develop feasibility study for a large-scale solar project east of Mesita

- Next Steps

- Complete preliminary solar feasibility analysis

-Decision support (presentation materials) -Development plan (steps, partners, funding options)
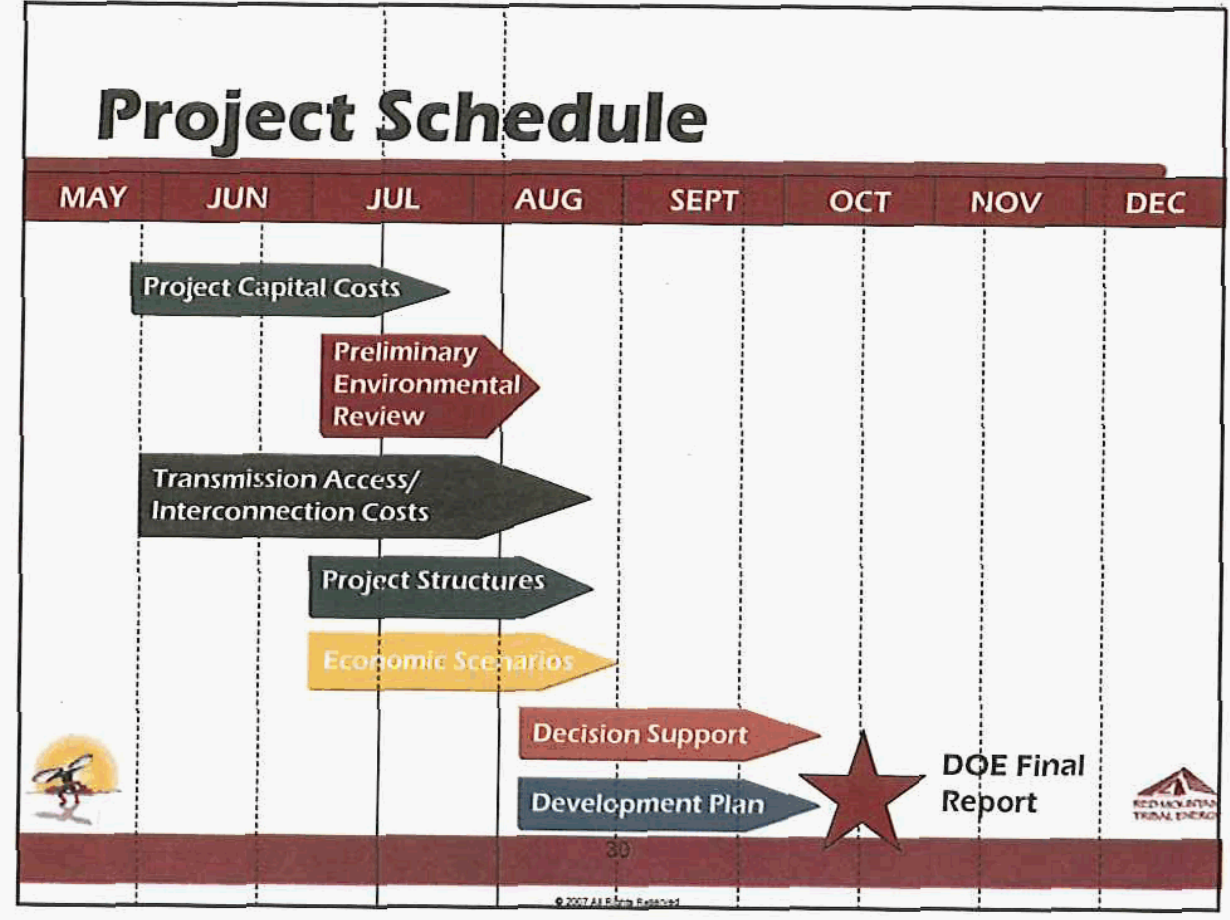


\section{Contact Information}
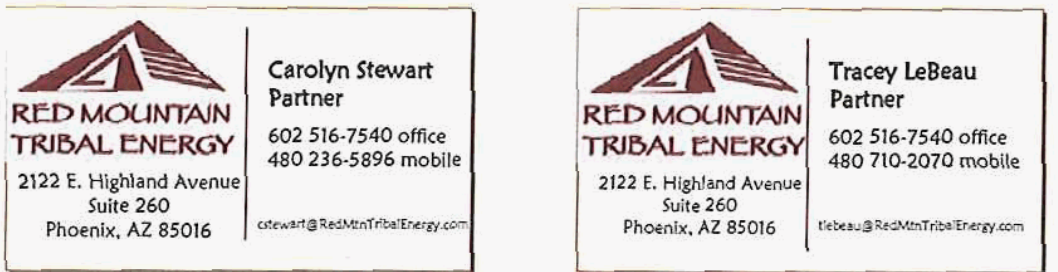

www.RedMtnTribalEnergy.com 



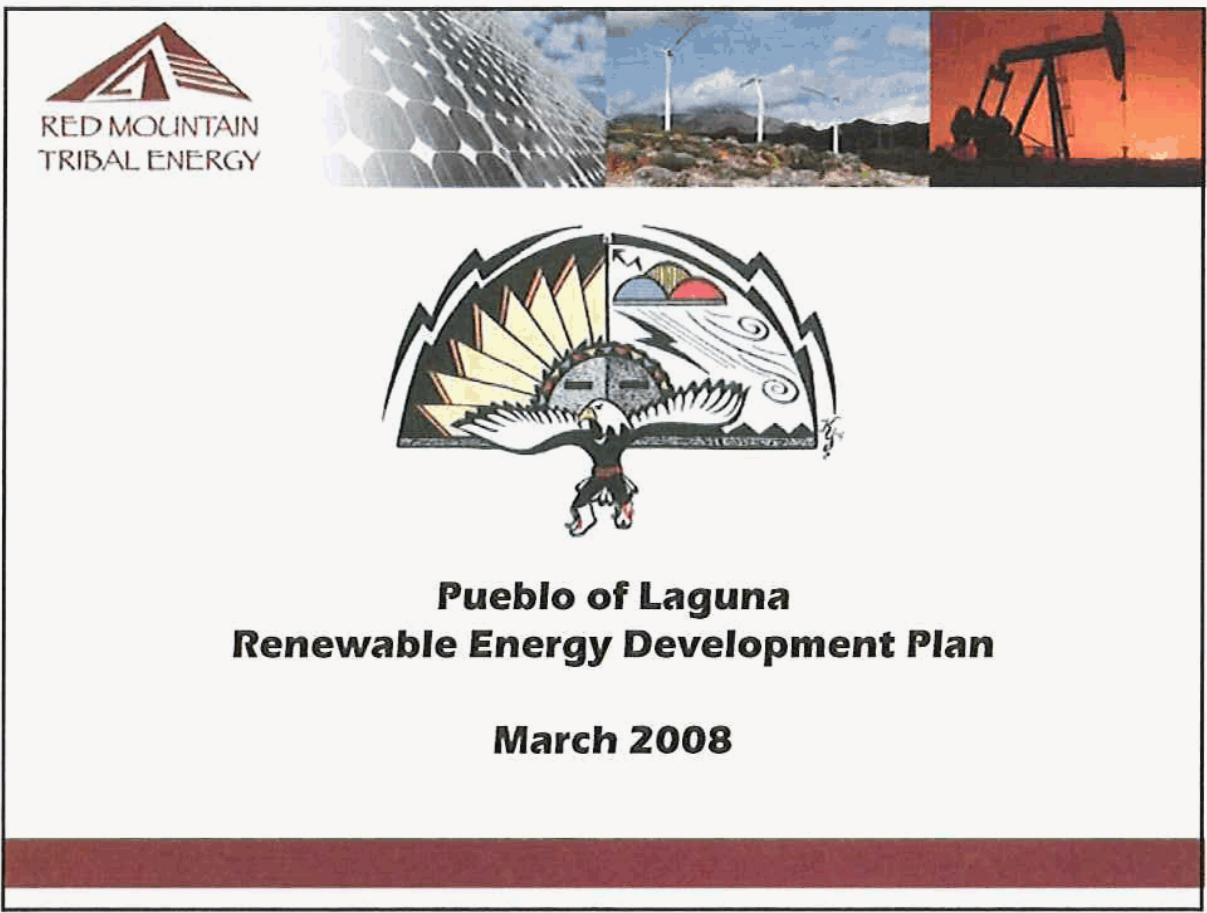

\section{Plan Outline}

- Plan Components and Rationale

- Laguna Community Description Summary

- Community Member Services Plan

$\checkmark$ Community Member Solar Program

$\checkmark$ Community Center Solar Pilot Project

$\checkmark$ LIHEAP/Weatherization Coordination

$\checkmark$ Gross Receipts Tax Exemption Support

- Reservation-Scale Solar Plan

- Large-Scale Solar Plan 


\section{Laguna Renewable Energy Devellopment Plan Purpose}

- Provides a mechanism to economically empower the Laguna community

- Serve as a benchmark for progress in becoming energy selfsufficient

- Moves Laguna away from reliance on fossil fuels, which provides no community benefits

- Enhances livability for community members

- Promotes awareness of the need for community sustainability

- Allows Laguna the opportunity to take environmentally progressive action, and identifies Laguna as being more responsible and conscious about its impact on the earth

\section{Laguna Target Community Summary}

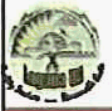




\title{
Laguna Target Community Demographics
}

\author{
Heating Assistance Customer Fuel \\ Use
}

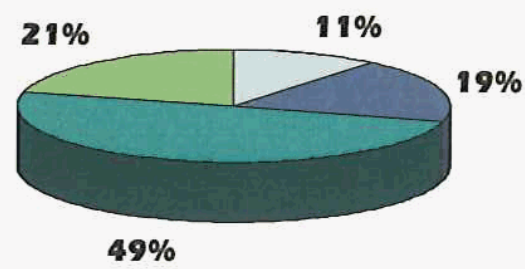

$\square$ Wood $\square$ Propane $\square$ Na\&ural Gas $\square$ Electric

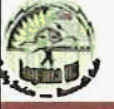

\section{Laguna Target Community Housing Summary}

\section{Heating Assistance Customer}

Home Types

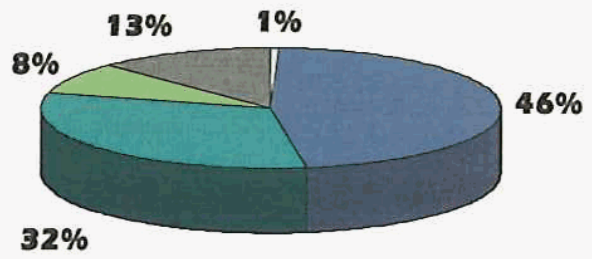




\section{Laguna Target Community Household Annual Energy Use}

-Average Laguna residential kW/h/yr consumption for 2006 is approximately $6,230 \mathrm{~kW} / \mathrm{h} / \mathrm{yr}$

- Space heating typically $30 \%$ of total use

- Water heating typically $20 \%$ of total use

- Laguna has 1,294 residential household customers

\begin{tabular}{|cc|cc|cc|}
\hline \multicolumn{2}{|c|}{ Total Consumption } & \multicolumn{2}{|c|}{ Water Heating Use } & \multicolumn{2}{c|}{ Space Heating Use } \\
$\mathrm{kWh} / \mathrm{yr}$ & $\mathrm{Mbtu} / \mathrm{yr}$ & $\mathrm{kWh} / \mathrm{yr}$ & $\mathrm{Mbtu} / \mathrm{yr}$ & $\mathrm{kWh} / \mathrm{yr}$ & $\mathrm{Mbtu} / \mathrm{yr}$ \\
\hline $6,000.0$ & 20.5 & $1,200.0$ & 4.1 & $1,800.0$ & 6.1 \\
$12,000.0$ & 40.9 & $2,400.0$ & 8.2 & $3,600.0$ & 12.3 \\
$18,000.0$ & 61.4 & $3,600.0$ & 12.3 & $5,400.0$ & 18.4 \\
\hline
\end{tabular}

Eल:

\section{Community Member Services Plan: Community Member Solar Program}




\section{Community Member Solar Program Purpose}

- Reduce Laguna member energy cost burden

- Nearly 200 members eligible in 2006-7 heating season

- Improve reliability of energy services

- Promote use of renewable energy resources

- Community input indicates support for use of renewables, and consistency with community values and traditions

\section{Community Member Solar Program concept}

- Laguna UA provides solar power generation, solar hot water heating and solar heating equipment to community members with the greatest need, where conditions indicate

- Heating assistance

- Appropriate location for solar

- Community member requests (and can take the tax credit)

- Laguna UA provides equipment at significantly reduced cost , assuming grant funding is available

- Purchase wholesale and act as distributor

- Support member tax credit analysis

- Laguna UA bundles any applicable credits for community, for use in funding the program

- White tags (energy conservation)

- Carbon credit offsets

- Renewable Energy Credits

- Laguna UA can provide education and training for community members or ongoing operations and maintenance support, as requested 


\section{Community Member Solar Program: Solar PV Modulle Specified}

\section{- Kyocera KC175 GT}

- High efficiency multi-crystal

- 175 watt, max de power

- 23.6V, max voltage

- 7.42A, max current

- $35.3 \mathrm{lbs}$ module weight

" 50.8" x 39.0" dimensions

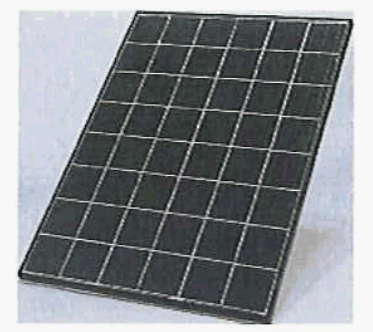

End

(t)

\section{Community Member Solar Program: Residentiall Solar PV Array}

1.05 kW/ PV Expandable System

- KACO 1501i 1500 watt inverter

- 120 " rail kit mounting

- 600V dc disconnect

- Meter socket

- Permit pulling fee

- General parts and labor

Cost estimate: $\$ 10,333.52$

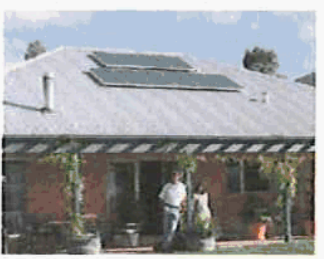

*Sacred Power/LUZ Energy Corp cost estimate, including installation; Laguna UA distributor purchase/installation likely to be lower cost 


\section{Community Member Solar Program: PV Array System Characteristics}

- Assumptions made for 1.05 kW array

- Temperature loss coefficient: .05\% per degree C

- $3 \%$ loss due to component mismatch

- $4 \%$ loss due to dirt

- $10 \%$ loss due to inverter efficiency

- NOCT $47 \mathrm{C}$

- Estimated capacity factor

- $25 \%$ (avg)

- Estimated dc power rating

. $.90 \mathrm{~kW}$ (avg)

- Estimated ac power rating

. $.75 \mathrm{~kW}$ (avg)

- Estimated energy production

- $1939 \mathrm{~kW} / \mathrm{h} / \mathrm{yr}$ (assume avg insolation of $6.4 \mathrm{hr} / \mathrm{d}$ peak sun)

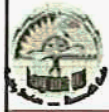

\section{Community Member Solar Program: PV Array Illustration}

- 1 kW PV Array = (6) $175 \mathrm{~W}$ Panels

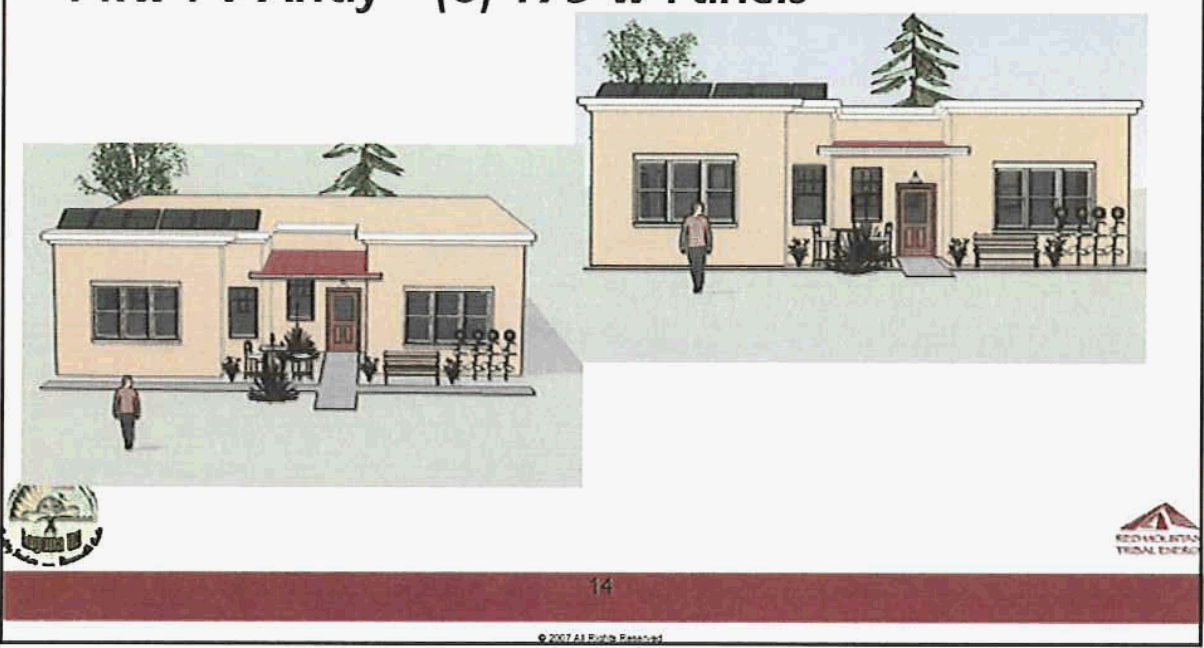




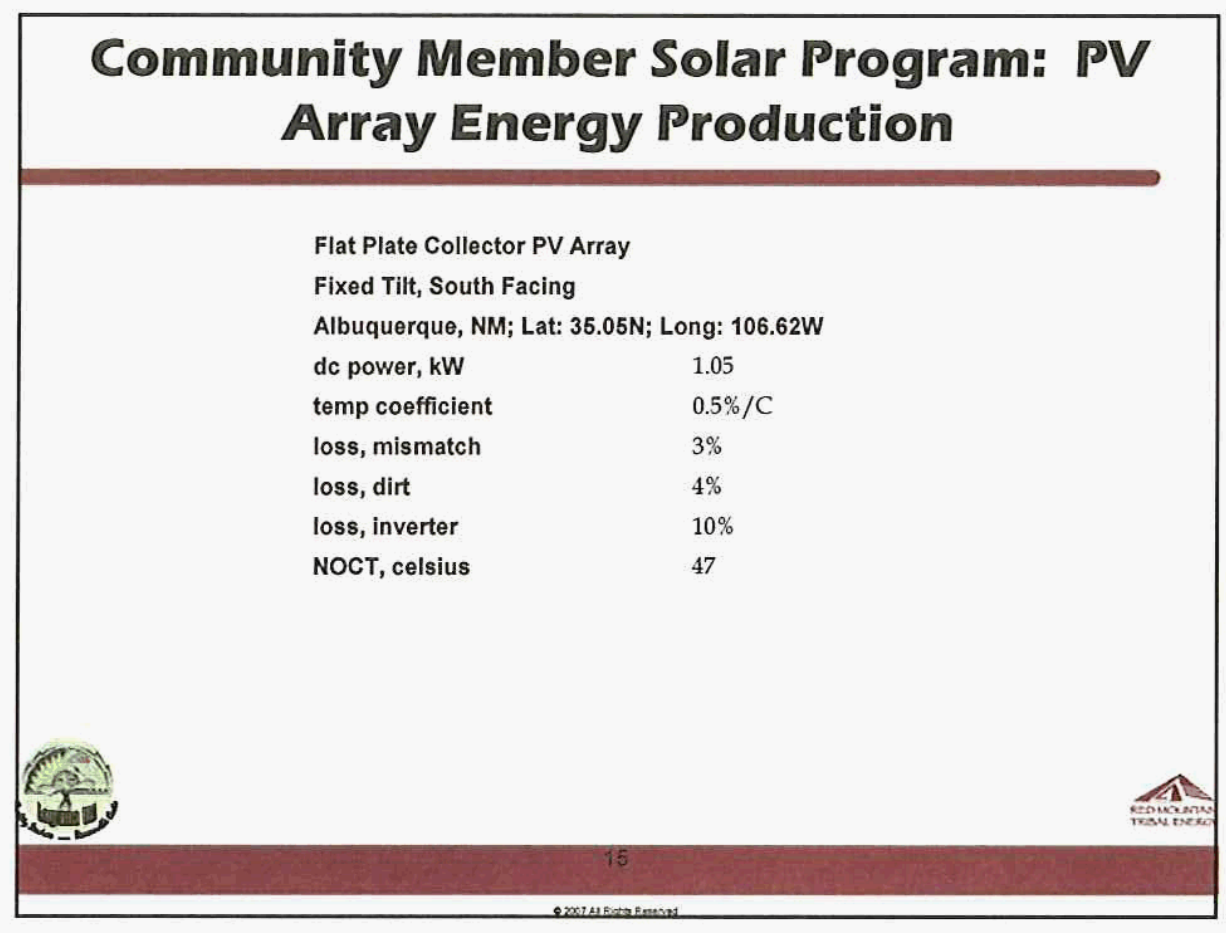

Community Member Solar Program: PV Array Energy Production at Latitude +15 Degree Tilt

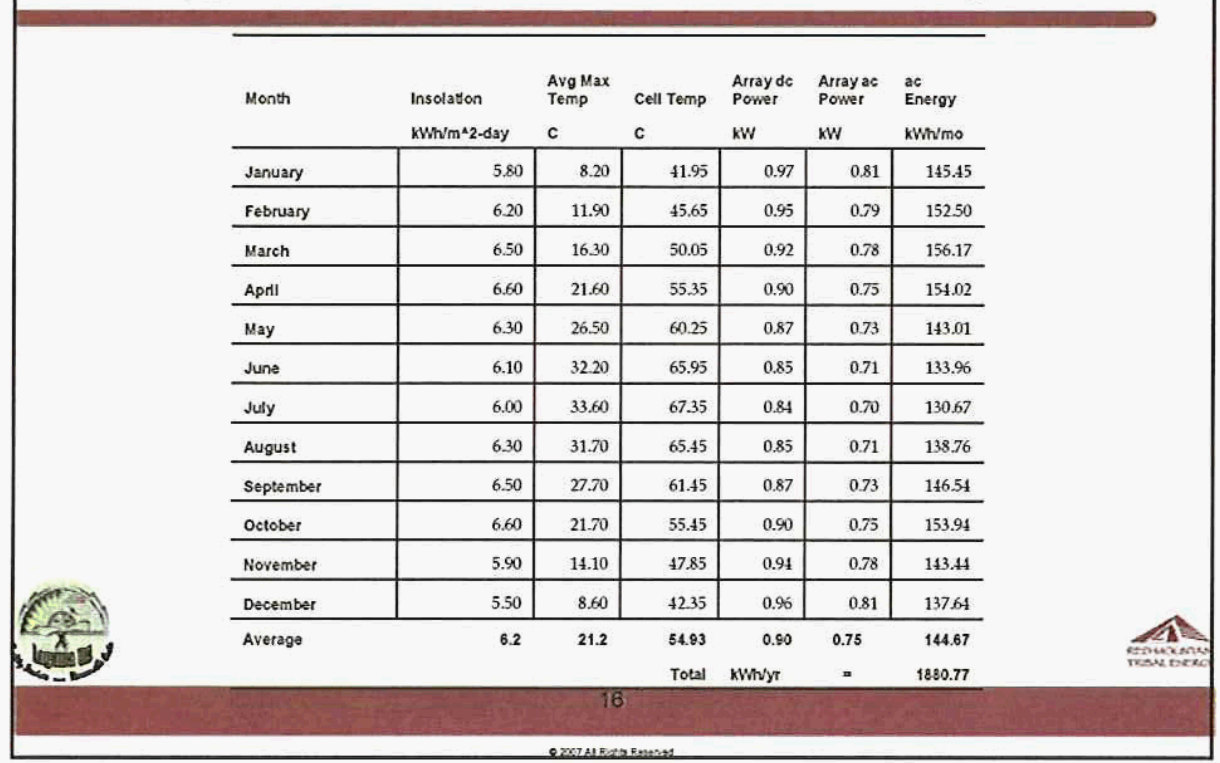




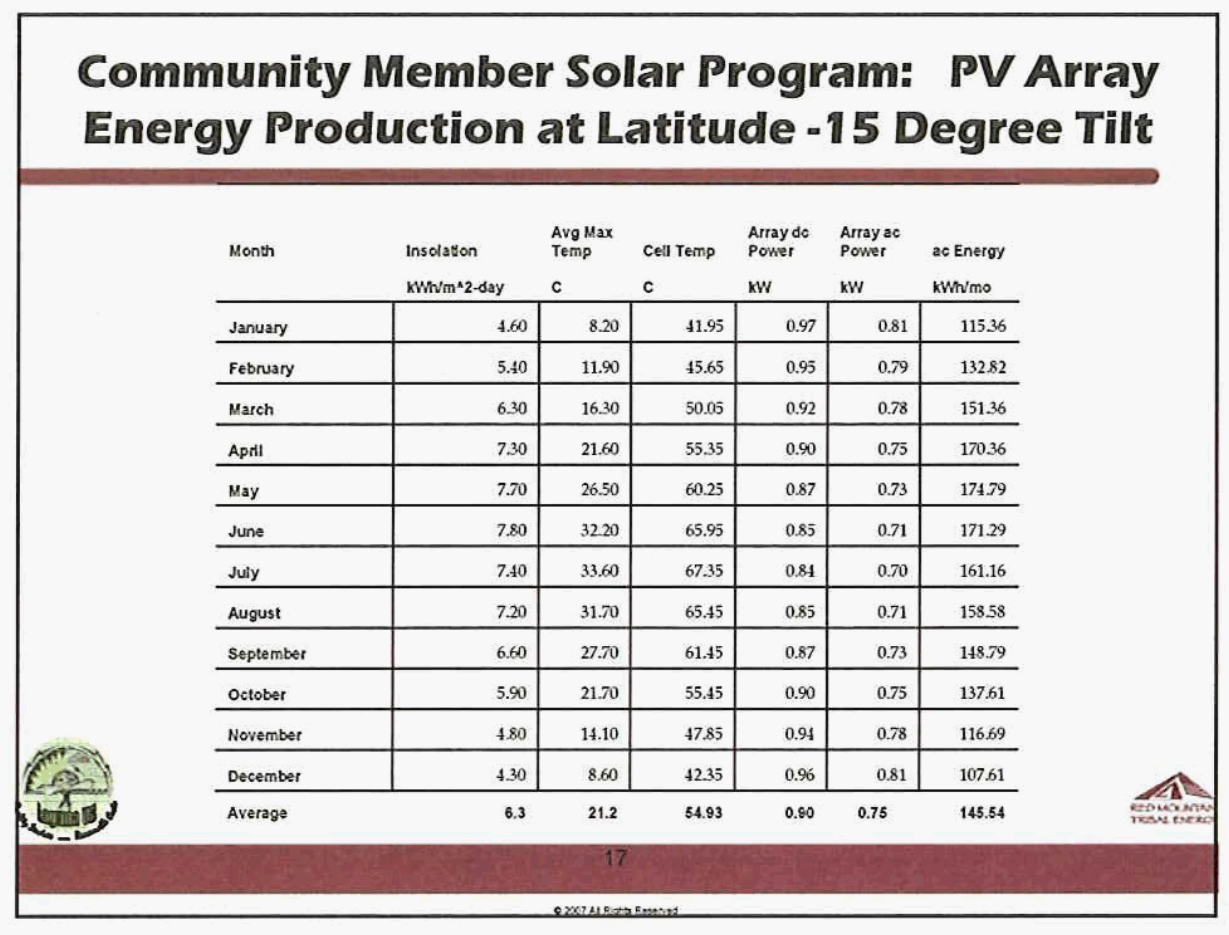

\section{Community Member Solar Program: PV Array} Energy Production at Latitude Tilt

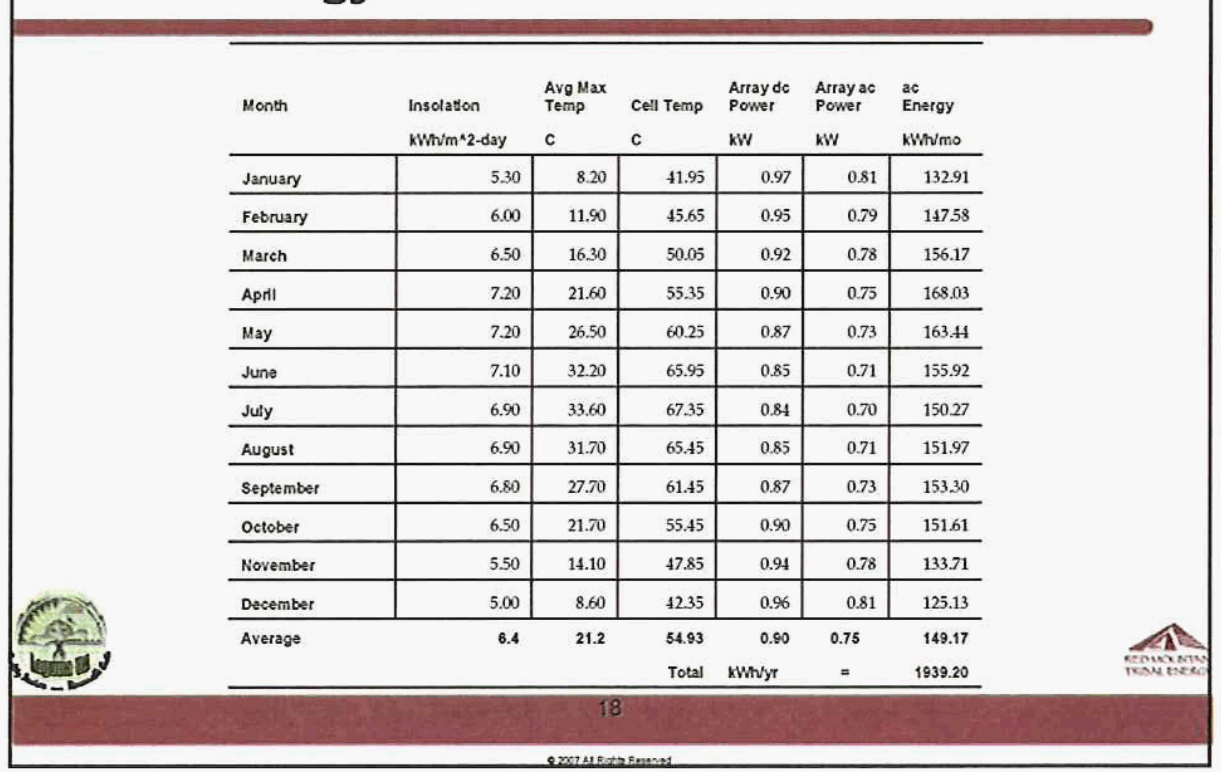




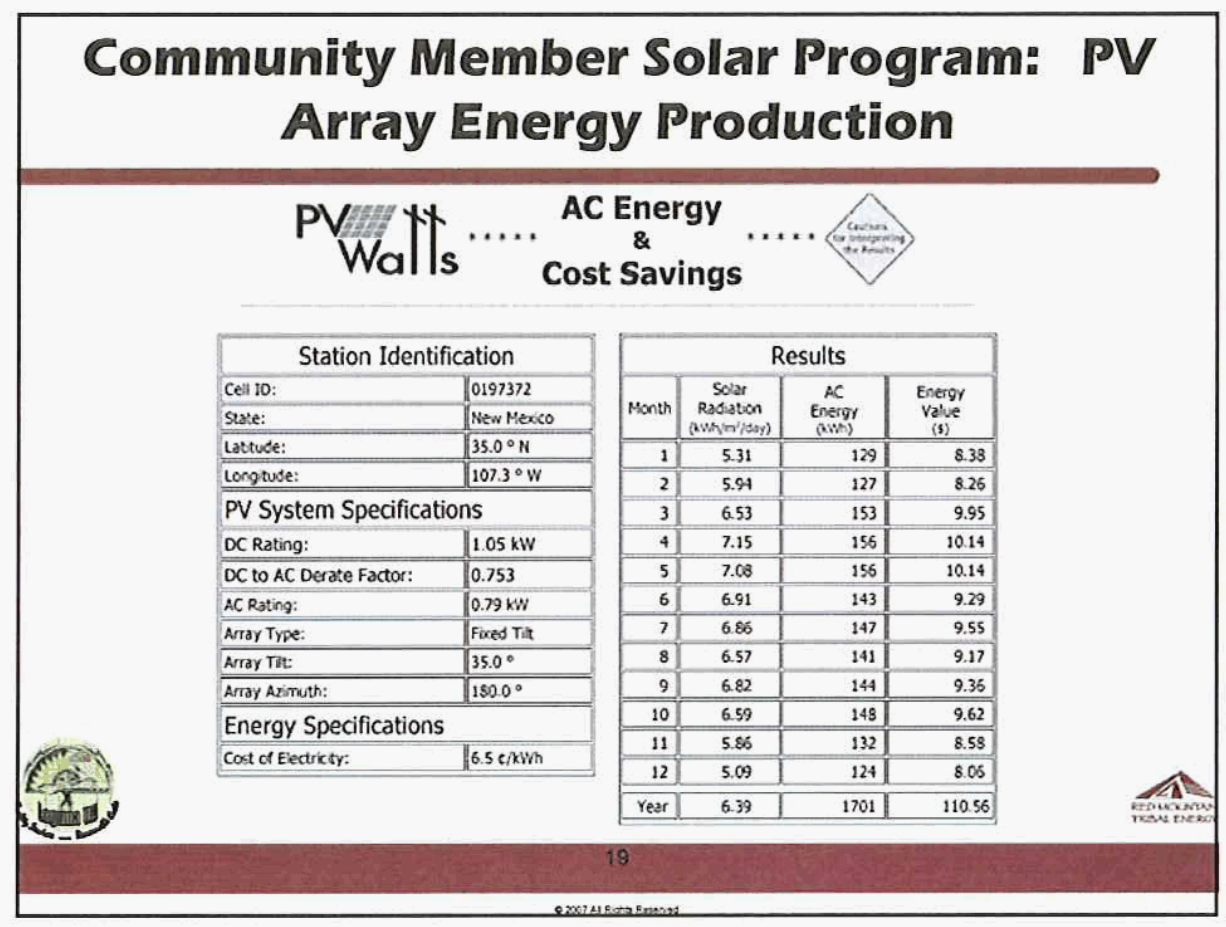

\section{Community Member Solar Program: Solar Hot Water System}

Heliodyne Helio-Pak HX PVC Domestic Hot Water System Components

- 3366 Gobi Collector, 26.8 sq ft

- Rack kit for 1 collector

- PV pump station

- Helix SS heat exchanger

- 40 Gas hot water heater

- Parts, labor, permit pulling fee

Cost estimate: $\$ \mathbf{5}, \mathbf{4 3 5 . 7 5}$

*Sacred Power/LUZ Energy Corp cost estimate; Laguna UA distributor purchase/installation likely to be lower cost

\section{Gobi Solar Colleotor}

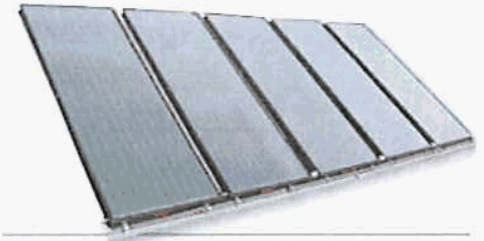




\section{Community Member Solar Program: Solar Hot Water System Characteristics}

\section{- Assumptions made for Gobi 3366}

- Clear sky with $6.4 \mathrm{~h} / \mathrm{d}$ peak sun insolation

- 40 gallon storage (sized for 2-person household)

- Propane cost: $\$ 2.82 /$ gallon (estimate from UA LIHEAP data)

- Natural gas cost: \$12.64/1000 CF (EIA 2007 New Mexico state avg)

- Electricity cost: $\$ 0.0916 / \mathrm{kWh}$ (current CDEC residential rate)

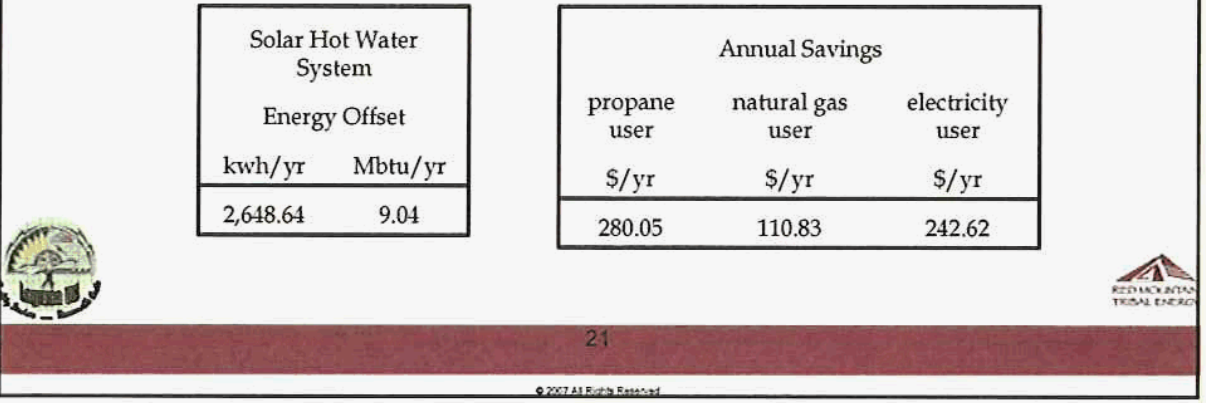

\section{Community Member Solar Program: Solarsheat Space Heating}

1500 G SolarSheat

Hot Arr Collector With PV Assembly

- Ventilation duct and blower

- Vents and insulation

- General parts and labor

Cost estimate: $\$ 4,799.05$

*Sacred Power/LUZ Energy Corp

cost estimate; Laguna UA

distributor purchase/installation likely to be lower cost
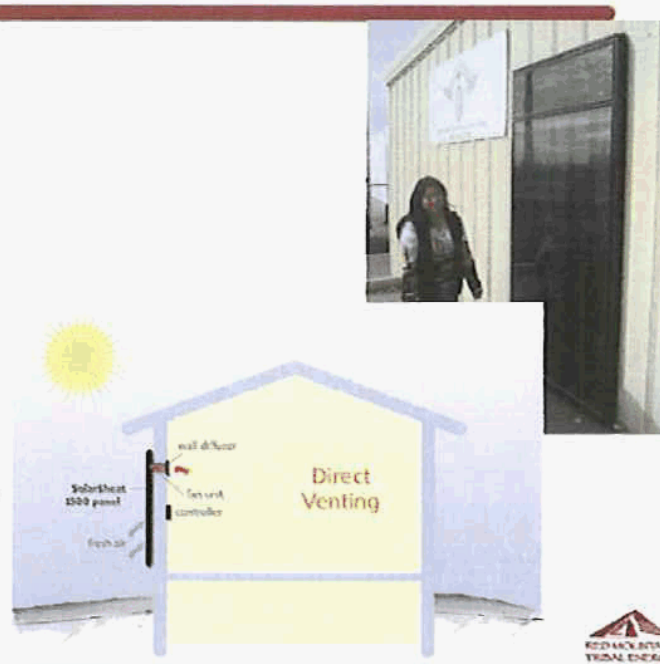


\section{Community Member Solar Program: SolarSheat Space Heating}

\section{- SolarSheat SH1500G}

- Can be wall or roof mounted

- 6 Mj SRCC rating for mildly cloudy day, category C

- 6 kBtu per day output

- Tempered glazed glass

- 15.5 watts PV system

- 71 CFM flowrate

- .35 ton per year carbon dioxide reduction

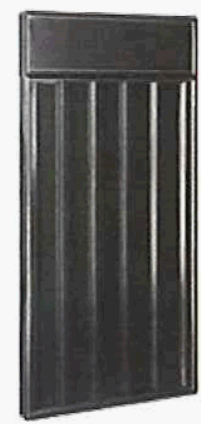

\section{Community Member Solar Program: SolarSheat Characteristics}

- Assumptions:

- 6 Mj SRCC rating for mildly cloudy day, category C

- 6 kBtu output per day

- Propane cost: \$2.82/gallon (estimate from UA LIHEAP data)

- Natural gas cost: $\$ 12.64 / 1000$ CF (EIA 2007 New Mexico state avg)

- Electricity cost: $\$ 0.0916 / \mathrm{kW} / \mathrm{h}$ (current CDEC residential rate)

\begin{tabular}{|c|c|}
\hline \multicolumn{2}{|c|}{ Solar Space Heating } \\
\hline Energ & fffset \\
\hline $\mathrm{kwh} / \mathrm{yr}$ & $\begin{array}{c}\text { Mbtu/ } \\
\text { yr }\end{array}$ \\
\hline 654.16 & 2.23 \\
\hline
\end{tabular}

\begin{tabular}{|ccc|}
\hline \multicolumn{3}{|c|}{ Annual Savings } \\
$\begin{array}{c}\text { propane } \\
\text { user }\end{array}$ & $\begin{array}{c}\text { natural gas } \\
\text { user }\end{array}$ & $\begin{array}{c}\text { electricity } \\
\text { user }\end{array}$ \\
$\$ / \mathrm{yr}$ & $\$ / \mathrm{yr}$ & $\$ / \mathrm{yr}$ \\
\hline 69.17 & 27.34 & 59.92 \\
\hline
\end{tabular}




\section{Community Member Solar Program: Energy Offset Components}

- Solar PV system, solar hot water system, and Solarsheat space heating technologies combine for a total energy reduction of over $5,000 \mathrm{~kW} / \mathrm{h} / \mathrm{yr}$

- Energy offset components (estimated):

- Hot Water Heating: $\quad 2,649 \mathrm{~kW} / \mathrm{h} / \mathrm{yr}$

- Space Heating:

$654 \mathrm{~kW} / \mathrm{h} / \mathrm{yr}$

- PV Array:

$\frac{1,726 \mathrm{~kW} / \mathrm{h} / \mathrm{yr}}{5,029 \mathrm{~kW} / \mathrm{h} / \mathrm{yr}}$

\section{Community Member Solar Program: Energy Offsets By Usage Type}

\begin{tabular}{|c|c|c|c|c|c|c|c|c|c|}
\hline \multicolumn{2}{|c|}{ total consumption } & \multicolumn{2}{|c|}{ water heating $\left({ }^{*}\right)$} & \multicolumn{2}{|c|}{$\begin{array}{l}\text { net water heat } \\
\text { energy need }\end{array}$} & \multicolumn{2}{|c|}{ space heating (**) } & \multicolumn{2}{|c|}{$\begin{array}{l}\text { net space heat } \\
\text { energy need }\end{array}$} \\
\hline KWhyr & Mboustr & kWhyr & Mbeusyr & $\mathrm{kWh} / \mathrm{sr}$ & Mbtustr & $k W / n / g r$ & Mbtustr & $k \mathrm{Wh} / \mathrm{gr}$ & Mbtustr \\
\hline $6,000.0$ & 20.5 & $1,200.0$ & 4.1 & $1,448.6$ & -4.9 & $1,800.0$ & 6.1 & $1,145.8$ & 3.9 \\
\hline $12,000.0$ & 40.9 & $2,400.0$ & 8.2 & -248.6 & -0.9 & $3,600.0$ & 12.3 & $2,945.8$ & 10.1 \\
\hline $18,000.0$ & 61.4 & $3,600.0$ & 12.3 & 951.4 & 3.2 & $5,400.0$ & 18.4 & $4,745.8$ & 16.2 \\
\hline \multicolumn{10}{|c|}{$\begin{array}{l}\left({ }^{\star}\right) \text { water heating assumed to be } 20 \% \text { of total household consumption } \\
\left({ }^{\star *}\right) \text { space heating assumed to be } 30 \% \text { of total household consumption } \\
\text {-Important concepts from this table: }\end{array}$} \\
\hline$\frac{\text { Enil }}{x-1}$ & & & & & & & & & Ant \\
\hline
\end{tabular}




\section{Community Member Solar Program: Energy Cost Offset Comparison}

\begin{tabular}{|c|c|c|c|c|c|c|}
\hline & & & \multicolumn{3}{|c|}{ Annual Cost Offsets } & \\
\hline & & & $\begin{array}{l}\text { Propane } \\
\text { user }\end{array}$ & $\begin{array}{c}\text { Natural Gas } \\
\text { user }\end{array}$ & Electric user & \\
\hline \multicolumn{7}{|l|}{$\begin{array}{l}\text { Solar } \\
\text { Tech }\end{array}$} \\
\hline \multicolumn{7}{|l|}{ Solar Hot } \\
\hline Water & $2,648.64$ & 9.04 & 280.05 & 110.80 & 242.62 & \\
\hline Solar Air & 654.16 & 2.23 & 69.11 & 27.34 & 59.92 & \\
\hline PV Array & $1,701.00$ & 5.80 & 112.19 & 112.19 & 155.81 & \\
\hline TOTAL & $5,003.80$ & 17.07 & 461.35 & 250.33 & 458.35 & \\
\hline \multicolumn{7}{|c|}{ *assume PV array performs according to PVWatts calculations, yielding $1701 \mathrm{kWh} / \mathrm{yr}$ per installed $1.05 \mathrm{~kW}$} \\
\hline \multicolumn{7}{|c|}{$\begin{array}{l}\text { - Propane users achieve largest } \$ \text { savings - estimated to be roughly } \$ 460 \text { per year } \\
\text { - Electric users would save } \$ 458 \text { per year and natural gas users would save } \$ 250 \\
\text { per year of their total household energy bills }\end{array}$} \\
\hline
\end{tabular}

\section{Community Member Solar Program: Energy Offsets and Percent Savings}

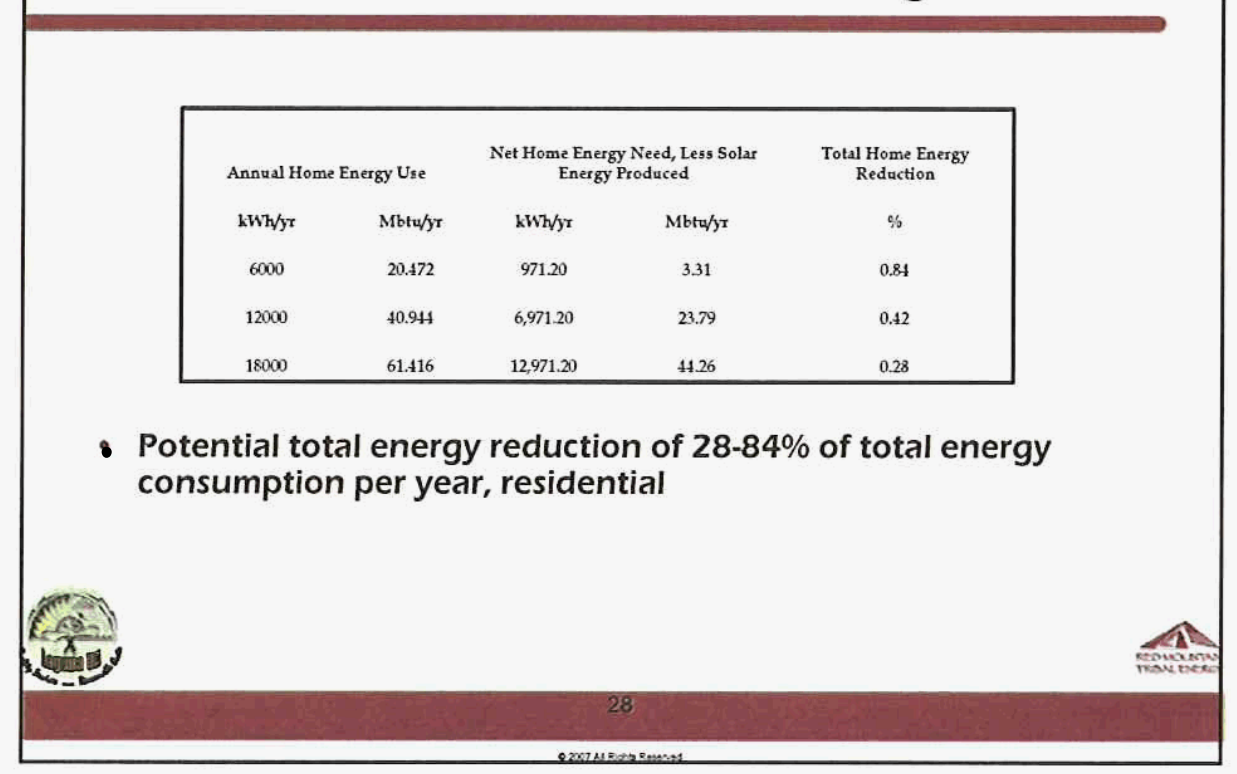




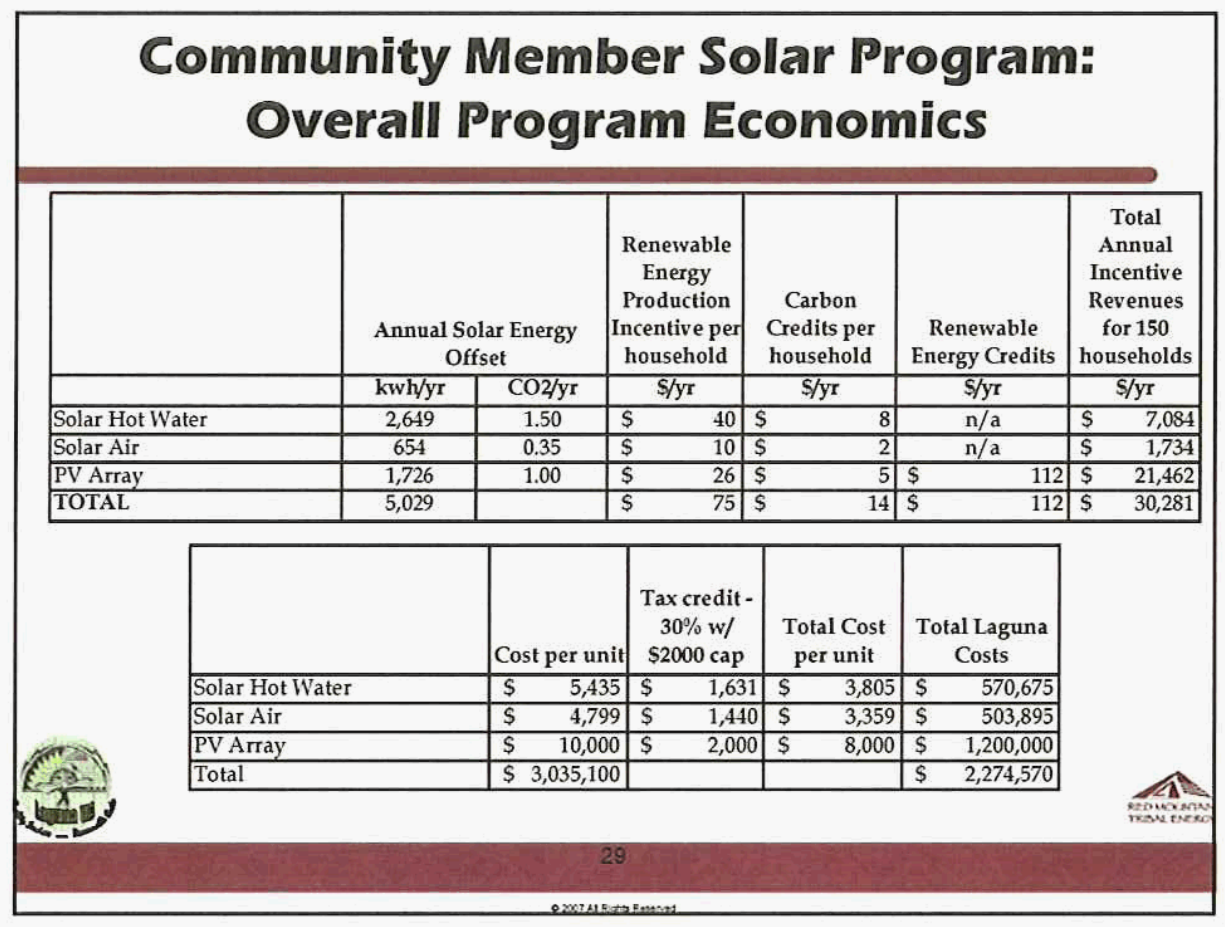

\section{Community Member Solar Program Rationale}

- Laguna recognizes the need to ensure a vibrant and healthy quality of life for community members, including living conditions and housing needs, particularly elders and disadvantaged households

- Electrification provides basic quality of life, and can be addressed by Laguna in an environmentally conscious way

- Use of solar technology is cultural appropriate:

- Aligns with Laguna values of respect and stewardship for the land and environment

- Community member assistance aspects are consistent with Laguna elder care priorities 


\section{Community Member Solar Program Rationale}

- Solar technologies are at various stages of development and feasibility for home and commercial use

- Solar energy exists as both an economy and community defining moment

- Laguna solar development provides recognition of the cleanliness and acceptability of solar technologies and promotes the use of renewable energy in tribal communities

\section{Community Member Solar Program: Funding Plan}

- Applicable Incentives Available

- Personal Tax Credits - Federal/State

- Corporate Tax Credits -Federal/State

- REC sales

- Potential carbon credits

- Renewable production incentives

- Other

- Grant Funding Targets

- DOE Tribal Energy Program

- NM EMNRD

- USDA

- Other 


\section{Community Member Services Plan: Community Center Pilot Solar Project}

\section{Community Center Solar Pillot Project Concept}

- Each of the villages would have the option to have solar equipment (PV panels, solar hot water heating, and solar heating) installed at the their community centers

- Equipment and system costs would be covered by village or available grant funding

- Laguna UA would install/coordinate installation of equipment

- Laguna UA would bundle and sell Renewable Energy Credits and carbon offsets to cover its costs

- Villages would benefit from lower energy costs, due to reduced energy usage resulting from solar equipment installations 


\section{Community Center Solar Pillot Project Purpose}

- Provide villages an opportunity to reduce their energy usage and costs, and free up funding to meet other community needs

- Through information available at the community centers, provide educational programs that help community members understand the benefits of renewable resources

\section{Community Center Solar Pillot Project: Encinal Community Center}

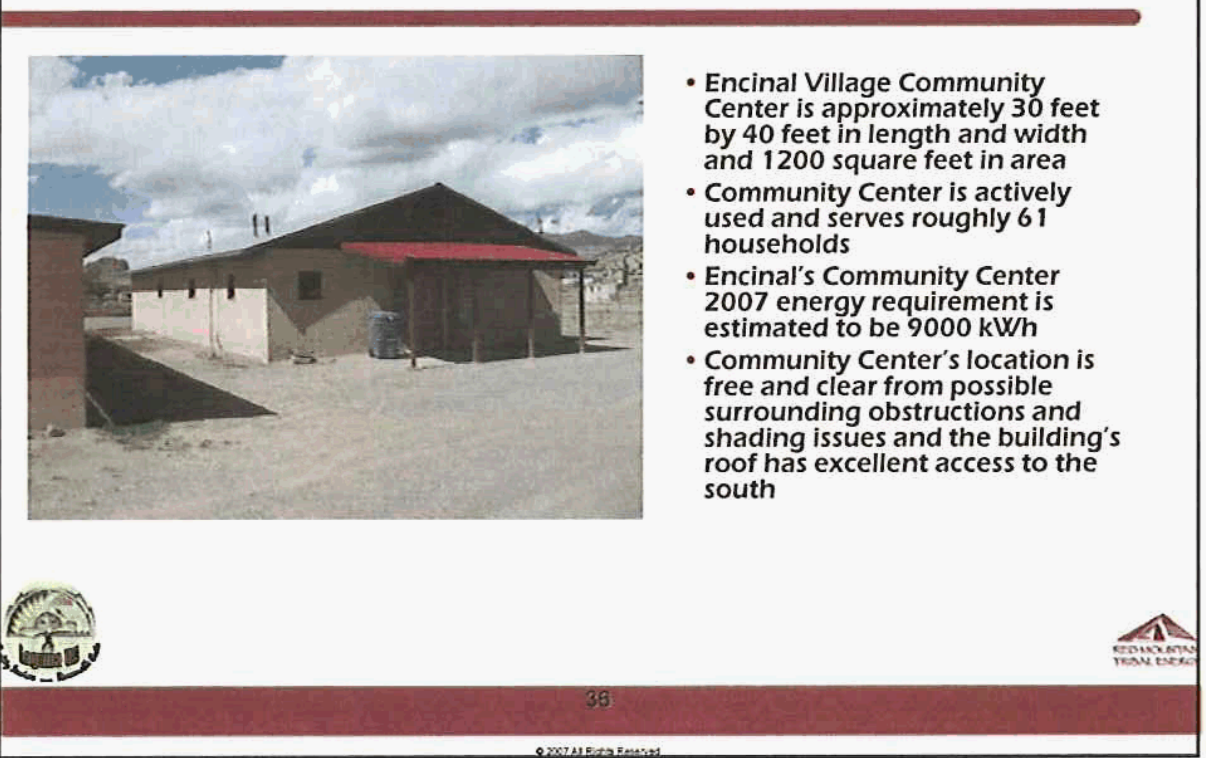




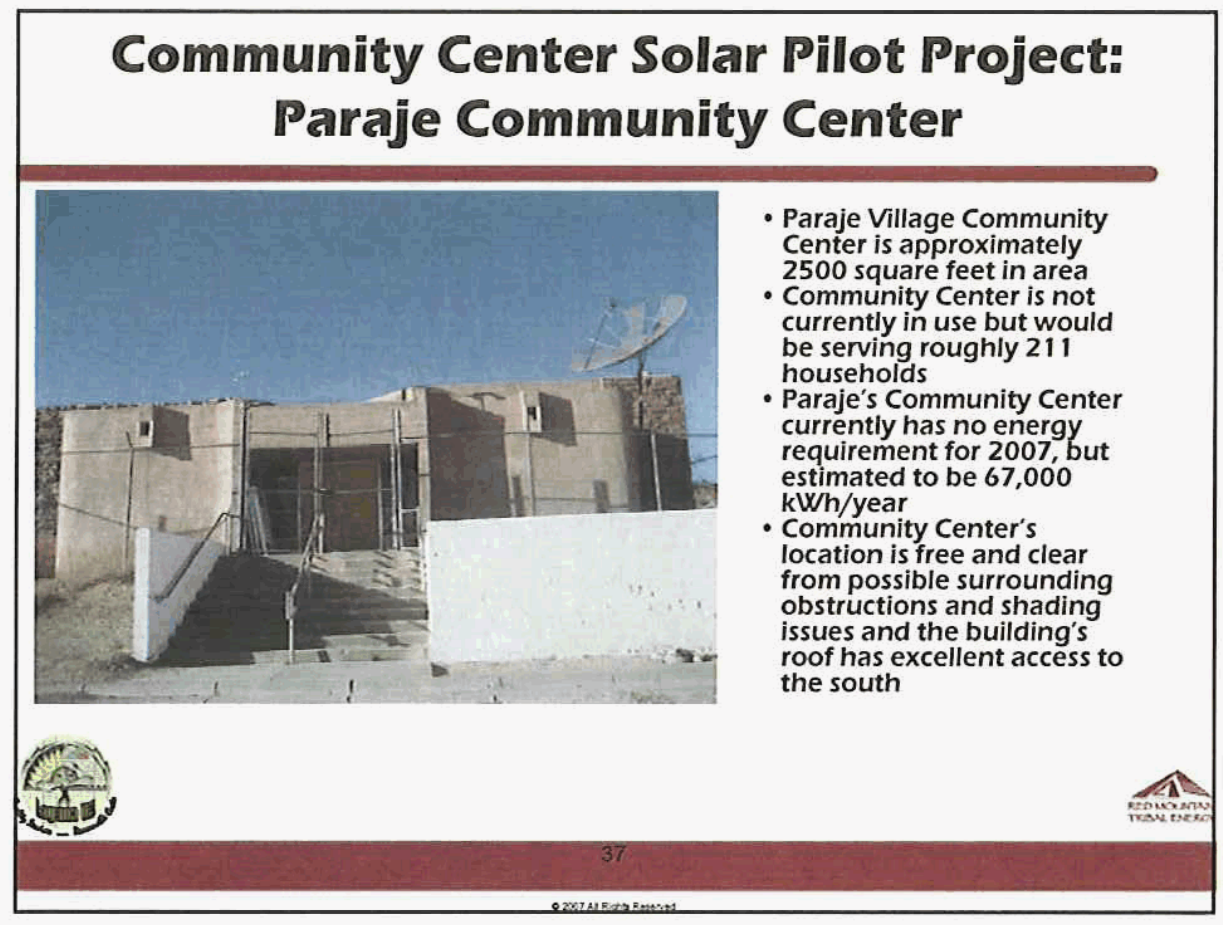

\section{Community Center Solar Pillot Project: Paguate Community Center}

- Paguate Community Center is approximately 65 feet by 30 feet in length and width and 2,000 square feet in area

- Community Center is actively used and serves roughly 192 households

- Paguate Community Center 2007 energy requirement is estimated to be $67,000 \mathrm{~kW} / \mathrm{h} / \mathrm{year}$

- Community Center's location is free and clear from possible surrounding obstructions and shading issues and the building's roof has excellent access to the south
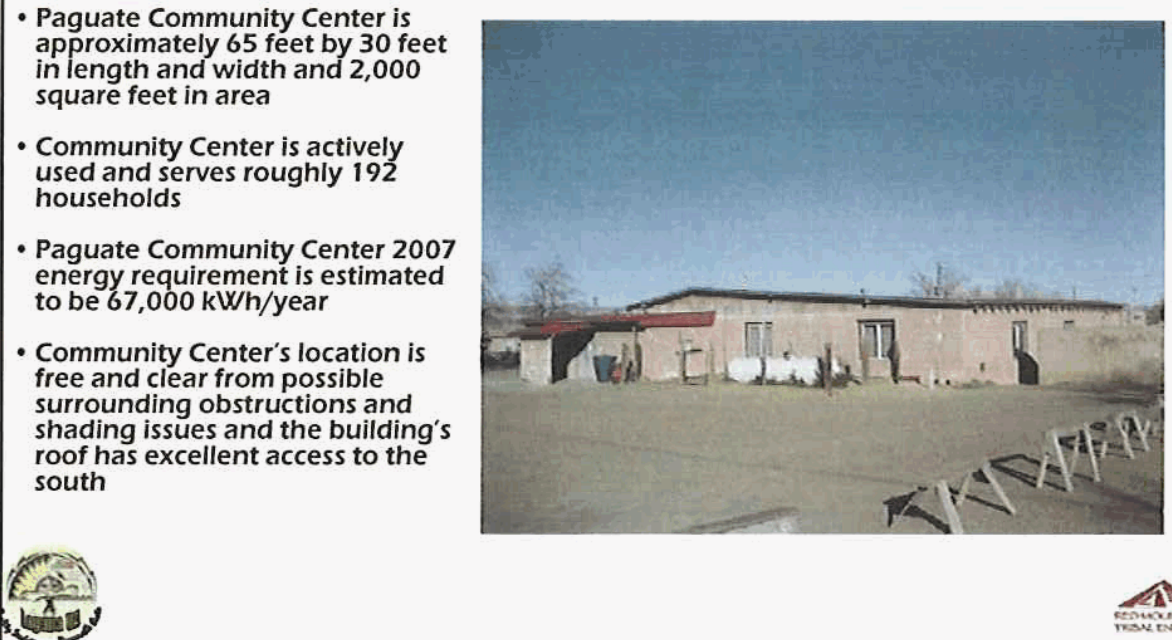


\section{Community Center Solar Pillot Project: Seama Community Center}

- Seama Village Community Center is approximately $\mathbf{5 5}$ feet by 20 feet in length and width and 1100 square feet in area

- Community Center is actively used and serves roughly 155 households

- Seama Community Center 2007 energy requirement is estimated to be $48,300 \mathrm{kWh} /$ year

- Community Center's location gives the building's roof access to the south but there are potential shading issues due to a hill on the building's west side

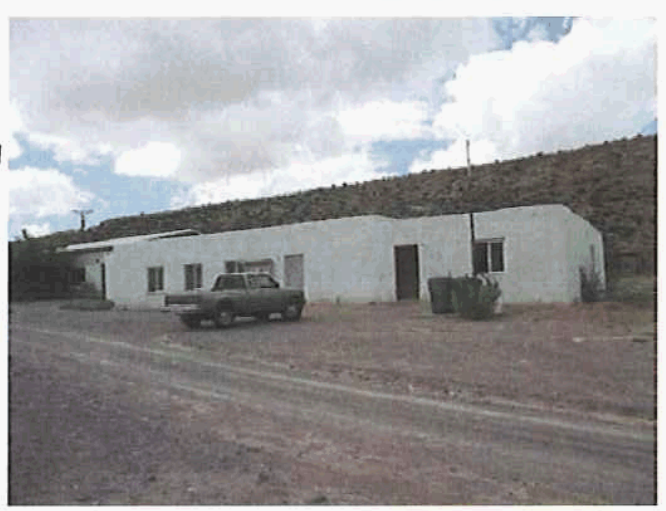

\section{Community Center Solar Pillot Project: Mesita Community Center}

- Village of Mesita has elected not to have solar PV equipment installed at their Community Center

- An alternative site has been designated as available for PV system installation

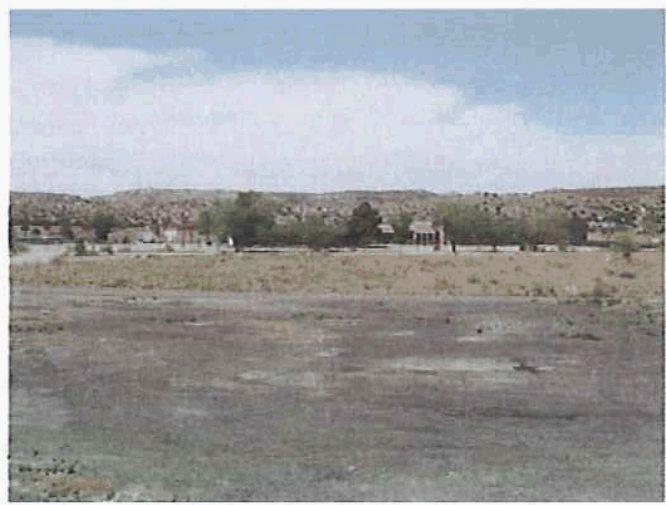




\section{Community Center Solar Pillot Project: Laguna Community/Recreation Center}

- The Laguna Village Community Center is

approximately $\mathbf{2 5 0 0}$ square feet in area

- Community Center is actively used and serves roughly 429 households

- Laguna Community Center 2007 energy requirement is estimated to be 67,000 kW/h/year

- Community Center's Iocation is free and clear from possible surrounding obstructions and shading issues and the

building's roof has excellent access to the south

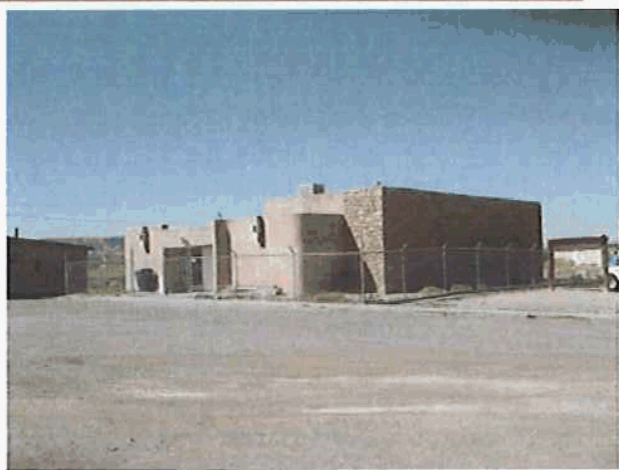

\section{Community Center Solar Pilot Project: Laguna Village Community/Recreation Center}

Laguna Village Community/Recreation Center

- Expansion/renovation

n From 2,500 sq ft to 5,233 sq ft

- Estimated annual consumption

- 6,6676 kW/h/yr

- Assumed to be considered Small Commercial

- Significant roof area for solar technologies
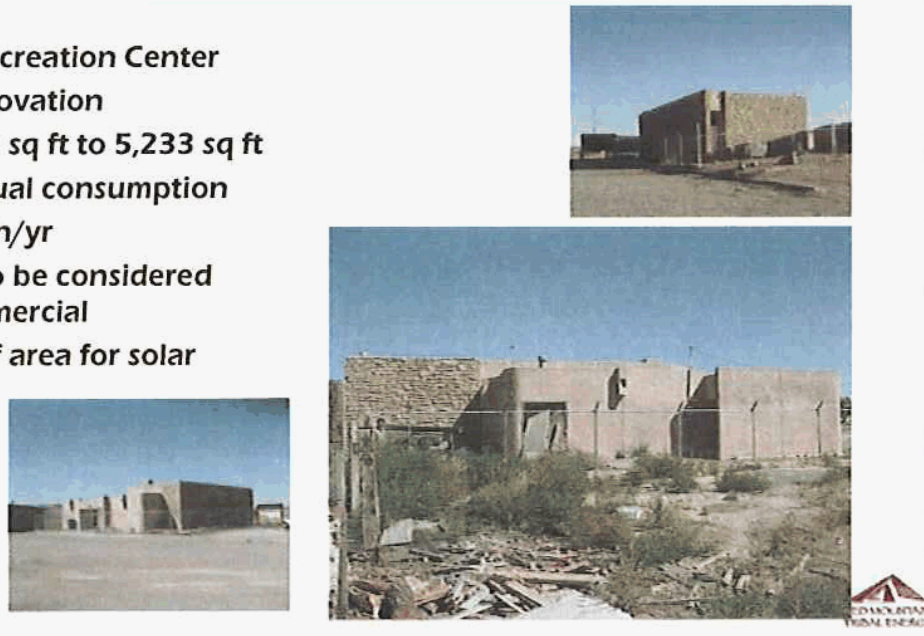


\section{Community Center Solar Pillot Project: Laguna Community/Recreation Center Available Rooftop}

Redesign For Laguna Village Community/Recreation Center

- Rooftop area available for solar PV, Solar Hot Water, SolarSheat heating

- Highlighted area is approximately 2,000 sq ft
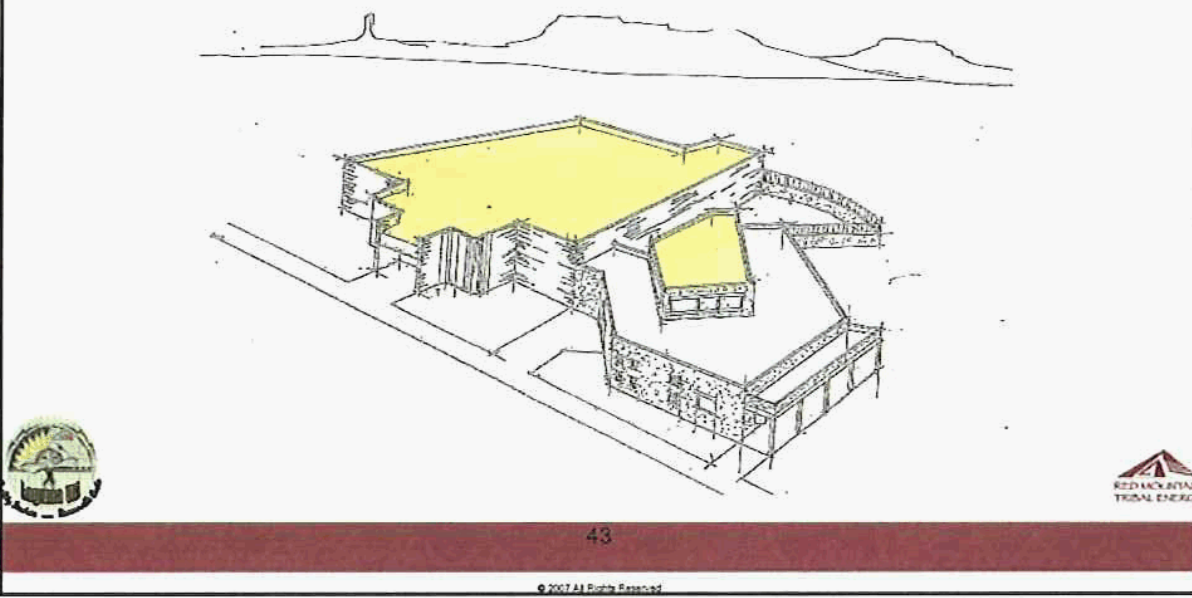

Community Center Solar Pilot Project: Laguna Community/Recreation Center SolarSheat Placement

Redesign Plan View

Possible SolarSheat Placement, highlight area
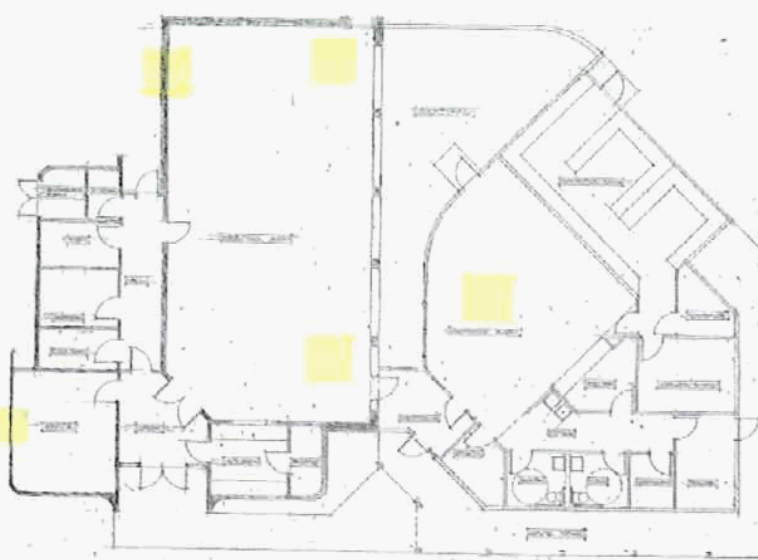

North

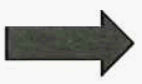

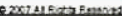




\section{Community Center Solar Pillot Project: Overall Economics}

\begin{tabular}{|c|c|c|c|c|c|c|}
\hline \multirow[b]{3}{*}{ Solar Tech } & \multirow[b]{3}{*}{ Units Installed } & \multirow[b]{3}{*}{$\mathrm{kW} / \mathrm{h} / \mathrm{yr}$} & \multirow[b]{3}{*}{ Motu/yr } & \multicolumn{3}{|c|}{ Annual Cost Offsets } \\
\hline & & & & \multirow{2}{*}{$\begin{array}{l}\text { Propane } \\
\text { User } \\
\$ / y r\end{array}$} & \multirow{2}{*}{$\begin{array}{l}\text { Natural Gas } \\
\text { User } \\
\$ / y r\end{array}$} & \multirow{2}{*}{$\begin{array}{l}\text { Electric } \\
\text { User } \\
\$ / y r\end{array}$} \\
\hline & & & & & & \\
\hline SHWV & 2 & 5,297 & 18 & 560 & 222 & 485 \\
\hline Solar Air & 6 & 3,925 & 13 & 415 & 164 & 360 \\
\hline \multirow[t]{2}{*}{ •PV Array } & $10.15 \mathrm{~kW}, 58$ modules & 16,442 & 56 & 1,739 & 688 & 1,506 \\
\hline & TOTAL & 25,664 & 88 & 2,714 & 1,074 & 2,351 \\
\hline \multicolumn{7}{|c|}{ 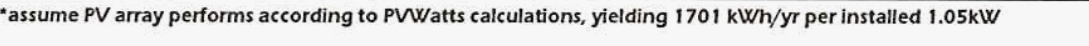 } \\
\hline \multicolumn{7}{|c|}{$\begin{array}{l}\text { Estimated annual energy reduction } 62 \% \text { of } \\
\text { total energy consumption, } \mathrm{kW} / \mathrm{h} / \mathrm{yr}\end{array}$} \\
\hline 13. & & & & & & \\
\hline
\end{tabular}

\section{Community Center Solar Pillot Project: WAPA PV Panel Concept}

- Late in December 2007, WAPA made PV panels available to Tribes, which provided Laguna an opportunity to revise its plans to include these panels, as described below:

- Laguna UA provides WAPA PV panels to each village for community center installation, as requested

- Additional system costs covered by village or available grant funding

- Laguna UA installs/coordinates installation of PV panels

- Laguna UA bundles and sells Renewable Energy Credits and carbon offsets to cover its costs

- Villages benefit from lower electric costs, due to reduced electric usage resulting from solar energy produced by the PV systems 


\section{Community Center Solar Pillot Project: WAPA PV Panel Energy Production/Savings}

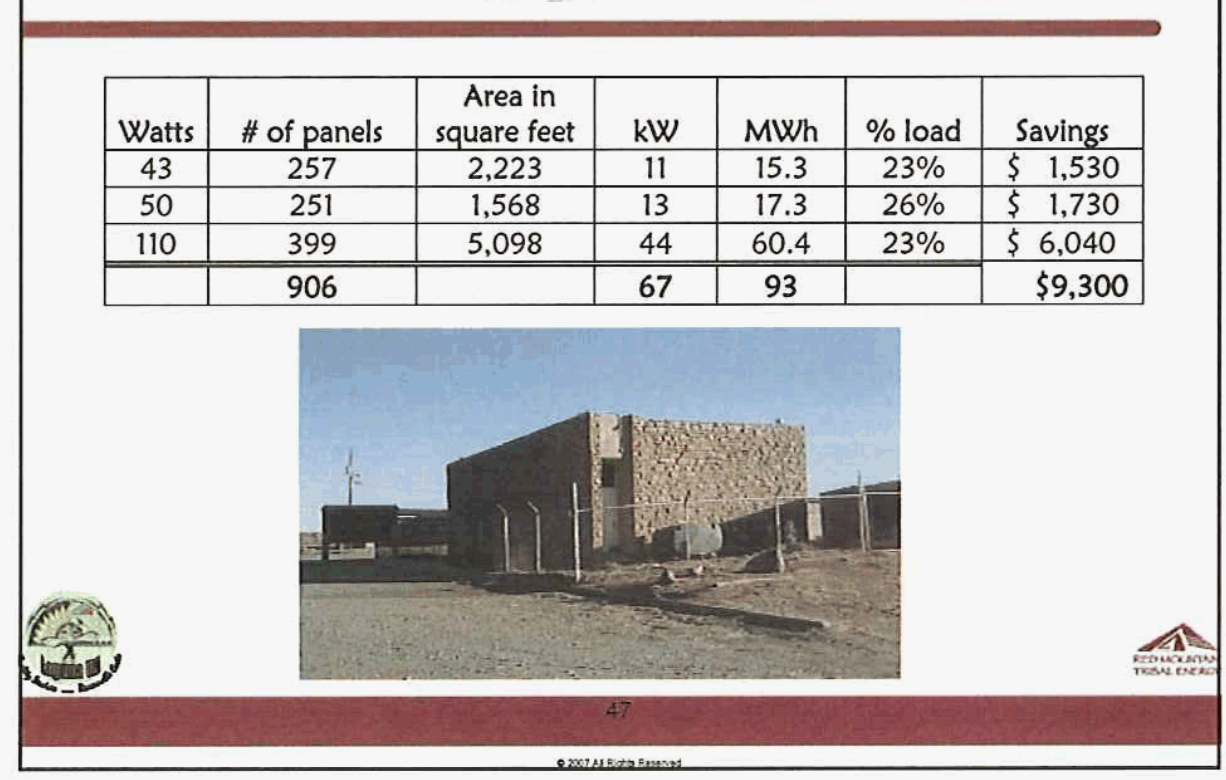

\section{Community Center Solar Pilot Project: Additional System Costs Required for Installation of WAPA PV Panels}

\begin{tabular}{|l|c|c|c|c|}
\hline \multicolumn{5}{|l|}{} \\
\hline System size, $\mathrm{kW}$ & 5.5 & 8 & 10 & 11 \\
\hline Power generated, kWh & 7,227 & 10,500 & 13,100 & 14,500 \\
\hline \% of load provided by solar & $11 \%$ & $16 \%$ & $20 \%$ & $22 \%$ \\
\hline Costs to install & $\$ 35,016$ & $\$ 45,391$ & $\$ 53,451$ & $\$ 58,681$ \\
\hline \# systems & 12 & 8 & 6 & 6 \\
\hline \multicolumn{5}{|l|}{} \\
\\
\end{tabular}




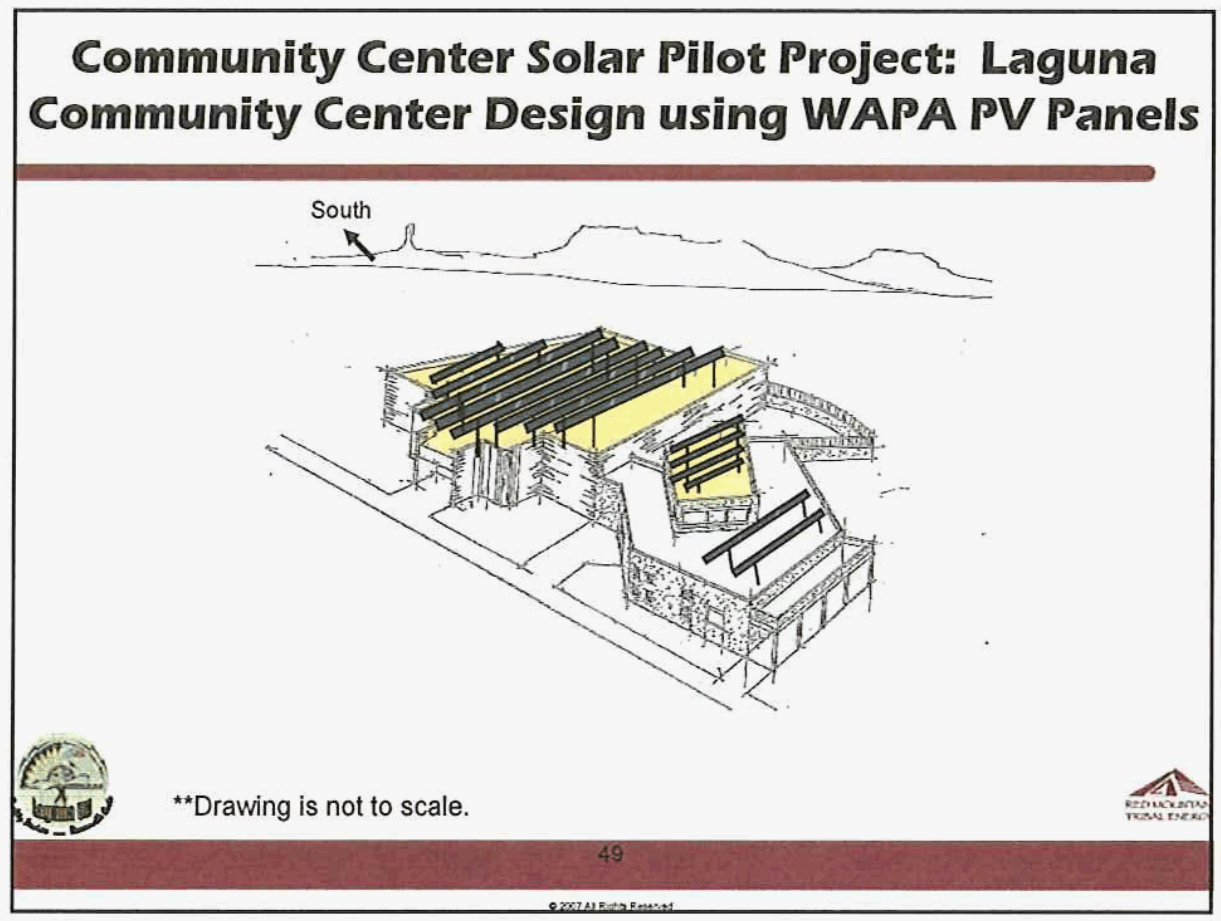

Community Center Solar Pillot Project: PV Panel Installation Training Available

- New Mexico Solar Energy Association www.nmsea.org 5 -day $\$ 500$

- Solar Energy International (SEI) http://www.solarenergy.org/about/index.html 6-day to 10-day workshop - $\$ 950$ PV Lab - \$1,100

- Debby Tewa, PV installer and trainer 


\section{Community Center Sollar Pillot Project Rationale}

- Community centers serve as general meeting places for various cultural, governmental, and social activities

- Community centers also anchor many social programs such as community elder meal programs, child after-school programs, and family celebrations

- Installing PV panels on community centers will create a series of dynamic and multi-dimensional concepts that support and influence Laguna villages at a number of levels:

- Raise energy awareness for Laguna members

- Elevate use of renewable energy for Laguna communities

- Project provides the Laguna UA an opportunity to support community development and member capacity building through energy education and cost-effective use of renewable resources

Project provides the Laguna UA an opportunity to demonstrate (2) its commitment to resourcefully serving community needs

\section{Community Member Services Plan: LIHEAP/Weatherization Coordination}




\section{LIHEAP/Weatherization Coordination}

- LIHEAP - Federal/state/UA funded coordinated effort; initial year completed

- UA implemented the program due to lack of county customer outreach

- UA served almost twice the amount of tribal members compared to the county in 2006

- Weatherization - Similar Laguna-coordinated effort possible to pursue program and funding for residents and tribal entities

- Conduct outreach activities and provide assistance to low income households in meeting their home energy costs, particularly those with the lowest incomes that pay a high proportion of household income for home energy

- Intervene in energy crisis situations

- Provide low-cost residential weatherization and other cost-effective energy-related home repair; and

- Plan, develop, and administer the State's program including leveraging programs 


\section{Prior Laguna LIHEAP Coordination}

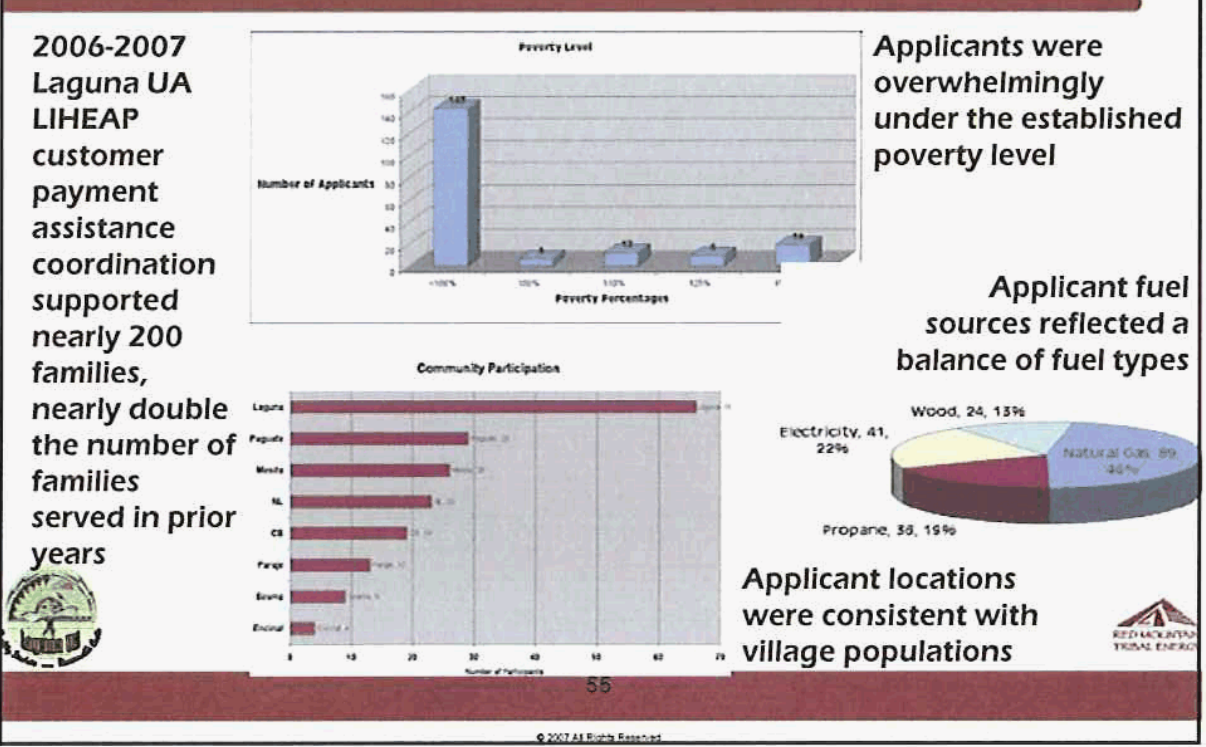

\section{DOE Weatherization Coordination}

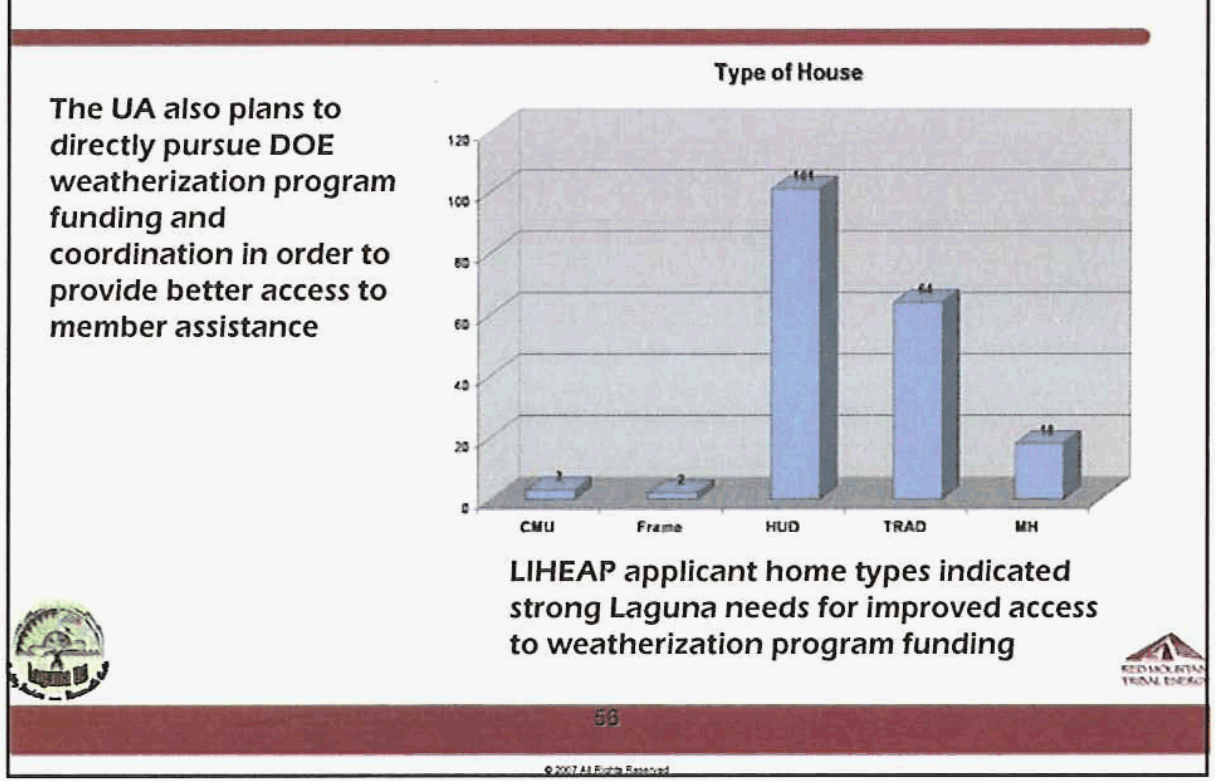




\section{Why Weatherization?}

- Heavy energy burden on low-income

- Typically spend $14 \%$ of annual income on energy, compared with $3.5 \%$ for other households

- Low-income families often choose between heat and other necessities

- 28 million households currently eligible for Weatherization services-many are in Indian Country

\section{Weatherization Benefits}

- Increases energy efficiency of the home

- Reduces energy costs year-round

- Provides long-term relief from expensive energy bills, not just bill paying

- Alleviates arrears, breaks destructive cycle of shut-offs and re-connections

- Breathes new life into marginal housing stock 


\section{How Does Weatherization Work?}

- Client applies for services

- Energy audit conducted; technicians identify energyrelated problems

- List of cost-effective measures developed

- Energy efficiency measures installed

- Measures more technically sophisticated since origins; building science widely practiced

- Range of diagnostic tools used to identify and remediate energy problem

- Test for health and safety hazards (e.g., carbon monoxide, gas leaks, potential for backdrafting)

\section{How Does Weatherization Work?}

- Critical client education component undertaken

- Ensure savings

- Prevent health hazards

- Prolong life of measures/equipment

- Conducted before and after measures are installed

- Post-work inspection accomplished 


\section{Energy Efficiency Measures: Base Load Reduction}

- Electricity consumption can be reduced through lighting, refrigerator, or water heater measures,

- Range from low flow devices and CFL installations to refrigerator and HVAC system replacements

- Utility resources often used to supplement work for such measures as refrigerator and HVAC system replacement

\section{Impacts of Weatherization}

- Energy savings in the range of $30 \%$, depending upon many variables

- New evaluation in the planning stages

- DOE Central Region results range from 25$40 \%$ savings

- On average, cost per weatherized house is $\mathbf{\$ 2 7 4}$

- For every $\$ 1$ invested in Weatherization return of $\$ 1.40$ on average

- For every $\$ 1$ invested (by DOE), another $\$ 1.48$ is "leveraged" from other funding sources such as federal, state, utility and private resources 


\section{Low lncome Energy Funding Sources}

LOW-InCOMe Funding

\section{Low Income Energy Funding History}

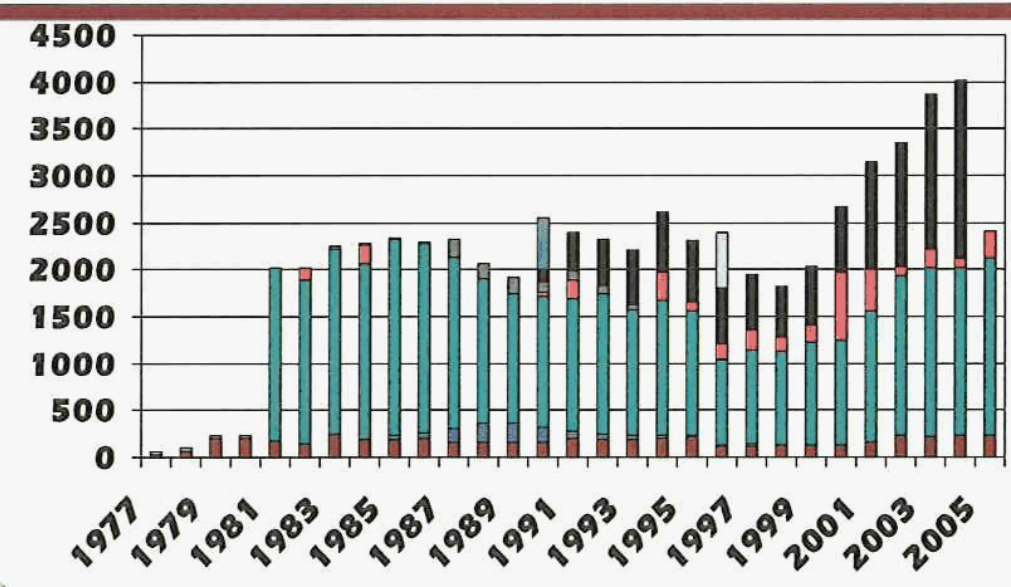




\section{Funding Resource Regions}

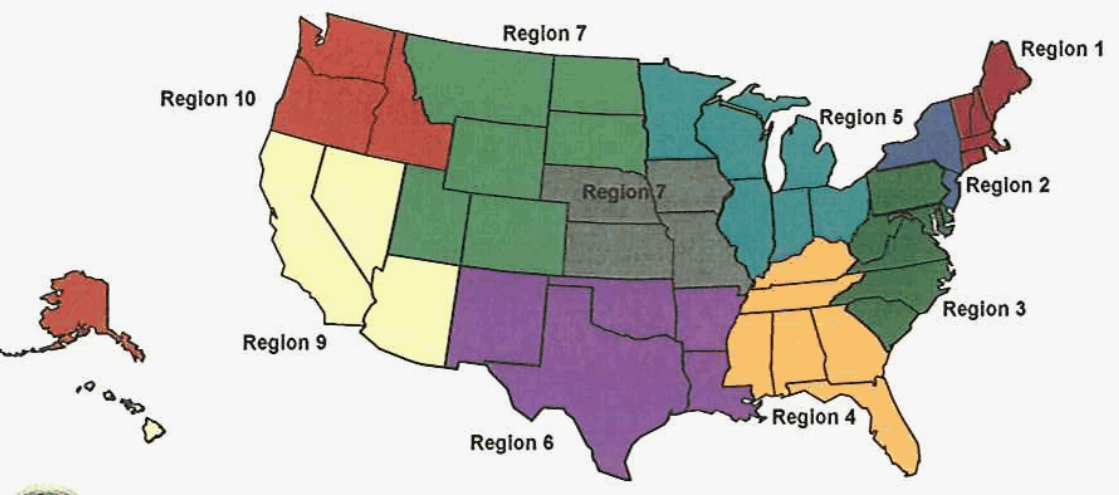

Exin-

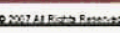

\section{Low-Income Energy Funds - Federal Region 6}

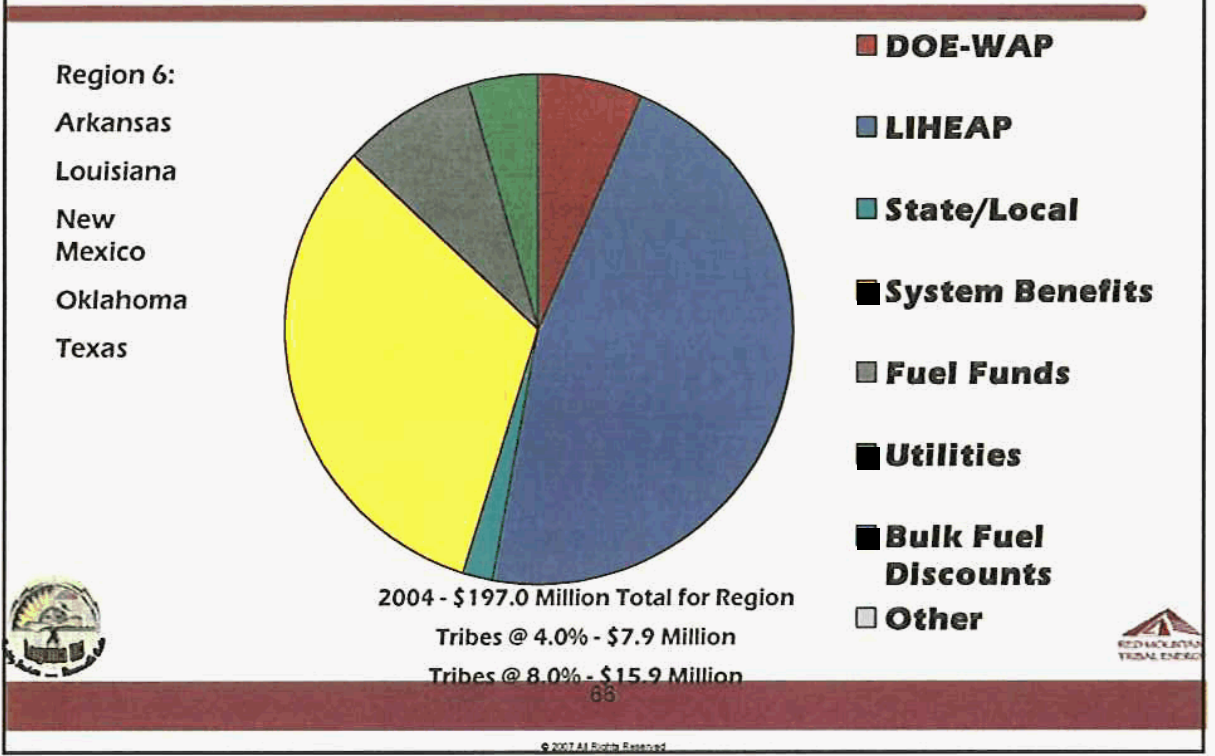




\section{Value of Energy Efficiency}

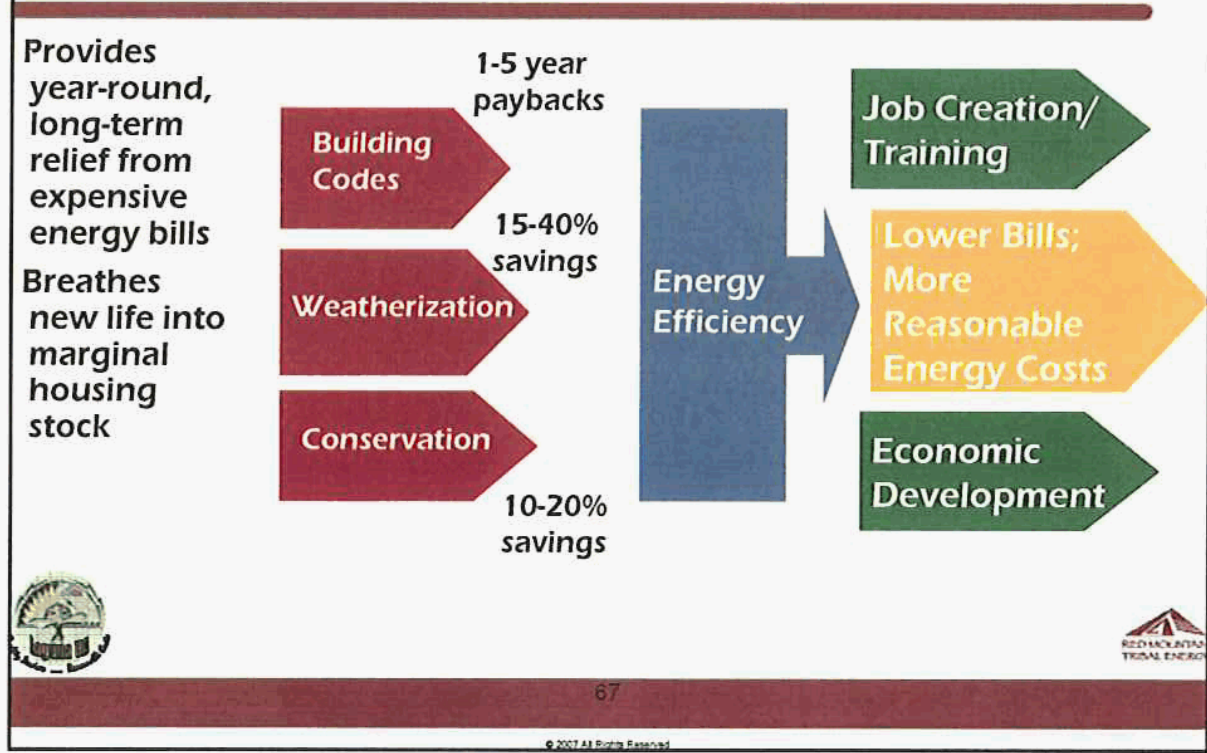

\section{Energy Related Funding Sources}

- DOE - Weatherization Program

- HHS - LIHEAP

- Utilities - Various

- Private organizations -Various

- BIA-HIP

- HUD-NAHASDA

- Others 


\section{DOE Weatherization Program}

- FY2008: \$224MM

- Existed since 1976 (10 CFR Part 440 for DOE)

- Goal to reduce energy costs for low-income families, particularly elderly, disabled, and children, while ensuring health and safety

- Operates in every State, County, District of Columbia, and reservation

- Year-round services free to low-income eligible residents

- Direct Services

$\checkmark$ Advanced Energy Audit; Insulation

$\checkmark$ Blower Doors \& other Diagnostic tools

$\checkmark$ Air Sealing \& Duct Sealing; Windows \& Doors

$\checkmark$ Heating \& Cooling systems

$\checkmark$ Water Heaters

$\checkmark \mathrm{AC}$ and Warm Climate Weatherization Measures

$\checkmark$ Electrical Appliances and Weatherization Base Load Measures Training \& Technical Assistance

\section{DOE - Weatherization Funds Flow}

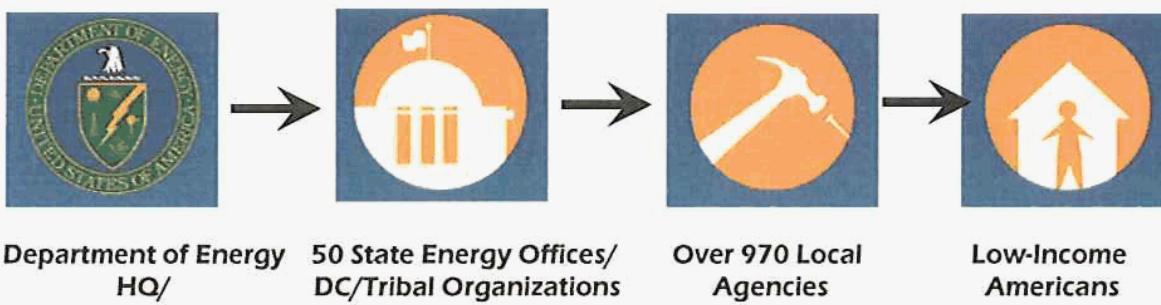

6 Regional Offices 
DOE-WAP Fund Distribution Factors

- F-3 Residential Energy Expenditures by LowIncome Households in each state

- Approximation of financial burden energy use places on low-income households

- Approximation necessary due to lack of statespecific data on residential energy expenditures by low-income households

- F-3 Residential Energy Expenditures by LowIncome Households in each state

- Approximation of financial burden energy use places on low-income households

- Approximation necessary due to lack of statespecific data on residential energy expenditures by low-income households 


\section{Grantees and Sulbgrantees}

- DOE funds "grantees"

- Most are state offices

- Two are tribes

- Grantees fund "subgrantees"

- Community action agencies

- Special purpose agencies

- Units of local government

- Tribes

- Subgrantees have opportunities to participate in State, Regional, and National-level training and technical assistance

2004-5 DOE Weatherization Services Provided

CENTRAL REGION WEATHERIZATION PROGRAM - PROGRAM YEAR 04-05

\begin{tabular}{|c|r|r|r|r|r|r|}
\hline State & $\begin{array}{c}\text { FY 04 DOE } \\
\text { Allocation } \\
\text { (PY 04-05) }\end{array}$ & $\begin{array}{c}\text { State } \\
\text { Recoives } \\
\text { Non- } \\
\text { DOE } \\
\text { Funds? }\end{array}$ & $\begin{array}{c}\text { TOTAL } \\
\text { Number of } \\
\text { Units } \\
\text { Completed }\end{array}$ & $\begin{array}{c}\text { TOTAL } \\
\text { Number of } \\
\text { People } \\
\text { Assisted with } \\
\text { Grant Funds }\end{array}$ & $\begin{array}{c}\text { Number of } \\
\text { Native } \\
\text { Americans } \\
\text { Assisted with } \\
\text { Grant Funds }\end{array}$ & $\begin{array}{c}\% \\
\text { Assisted - } \\
\text { Native } \\
\text { American }\end{array}$ \\
\hline Colorado & $\$ 5,479,996$ & Yes & 4,082 & 10,088 & 143 & $1.42 \%$ \\
\hline Kansas & $\$ 2,530,561$ & Yes & 1,413 & 2,630 & 33 & $1.25 \%$ \\
\hline Louisiana & $\$ 1,731,371$ & No & 1,127 & 1,640 & 2 & $0.12 \%$ \\
\hline Montana & $\$ 2,519,458$ & Yes & 1,354 & 2,447 & 553 & $22.60 \%$ \\
\hline Nebraska & $\$ 2,494,014$ & No & 595 & 1,472 & 74 & $5.03 \%$ \\
\hline New Mexico & $\$ 1,723,006$ & Yes & 869 & 1,703 & 172 & $10.10 \%$ \\
\hline North Dakota & $\$ 2,496,970$ & No & 803 & 1,437 & 217 & $15.10 \%$ \\
\hline Oklahoma & $\$ 2,591,542$ & No & 915 & 2,059 & 187 & $9.08 \%$ \\
\hline South Dakota & $\$ 1,916,788$ & No & 1,049 & 988 & 180 & $18.22 \%$ \\
\hline Texas & $\$ 5,575,530$ & Yes & 1,822 & 4,212 & 6 & $0.14 \%$ \\
\hline Utah & $\$ 2,077,161$ & No & 830 & 2,417 & 143 & $5.92 \%$ \\
\hline Wyoming & $\$ 1,174,532$ & No & 539 & 1,072 & 43 & $4.01 \%$ \\
\hline
\end{tabular}




\section{Find Information/Apply for Services}

- Contact current local provider in your area

- Obtain contact list or Name of State Program Manager

- View Weatherization websites:

- http://www.eere.energy.gov/weatherization/

- http://www.waptac.org/

- Contact DOE's Regional Weatherization Project Manager

- Central Region - Rob DeSoto

\section{Getting Access to Direct Funding}

- W/hat the current federal regulations /10 CFR 440) pertaining to processes for applying for direct grants -

- 10 CFR 440.11 Native Americans

- (1) Low-income members of an Indian Tribe must present to the Support (Regional) Office Director, a case of not receiving benefits equivalent to assistance provided to other low-income persons in a State 


\section{Getting Access to Direct Funding}

- (2) The low-income members of such tribe would be better served by means of a grant made directly to provide such assistance

- The Director reviews, and can make a determination for direct funding, in which case he reserves funding not less than 100 percent, or more than $150 \%$, an amount which bears the same ratio of Native Americans under this determination, to the population of all low-income persons in the State.

\section{Other Options}

- For Providing Weatherization services:

- 10 CFR 440.12 State Application

- (5) A recommendation that a Tribal organization be treated as a local applicant eligible to submit an application pursuant to 440.14 (b) (becoming a "Subgrantee") 


\section{HHS - LIHEAP}

- Tribes can apply for funding/administer programs

- Leveraging awards

- Tribal Reach awards

- To minimize the health and safety risks that result from high energy burdens on low-income Americans, prevent homelessness as a result of inability to pay energy bills, increase the efficiency of energy usage by low income families and target energy assistance to individuals who are most in need

- Implementation and evaluation of energy efficiency education services that meet quality standards established by the Secretary in consultation with the Secretary of Energy, and have the potential for being replicable model designs for other programs

- 148 Tribes and Tribal agencies

- Tribes eligible households as a \% of state eligible households

Section 2604(d)(1) of the LIHEAP statute authorizes the U.S. Department of Health and Human Services to award direct LIHEAP allotments to Indian tribes and tribal organizations

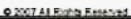

\section{HUD - NAHASDA}

- Native American Housing Assistance and SelfDetermination Program

- Indian Housing Block Grant

- Loan Guarantees

- Tribally Administered

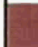

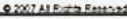




\section{BUA-H\|P}

- Home Improvement Grants

- Available to low-income members

- Awards \$2000

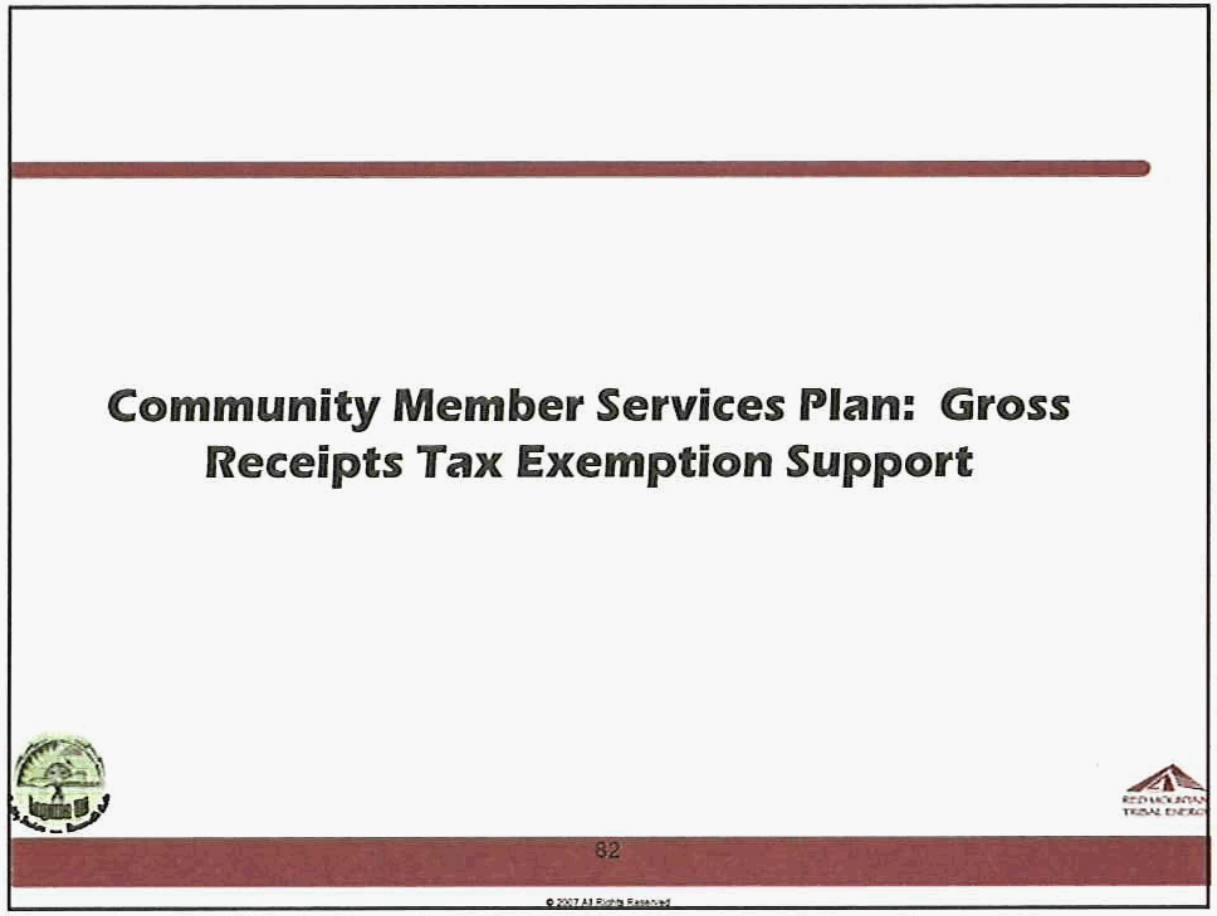




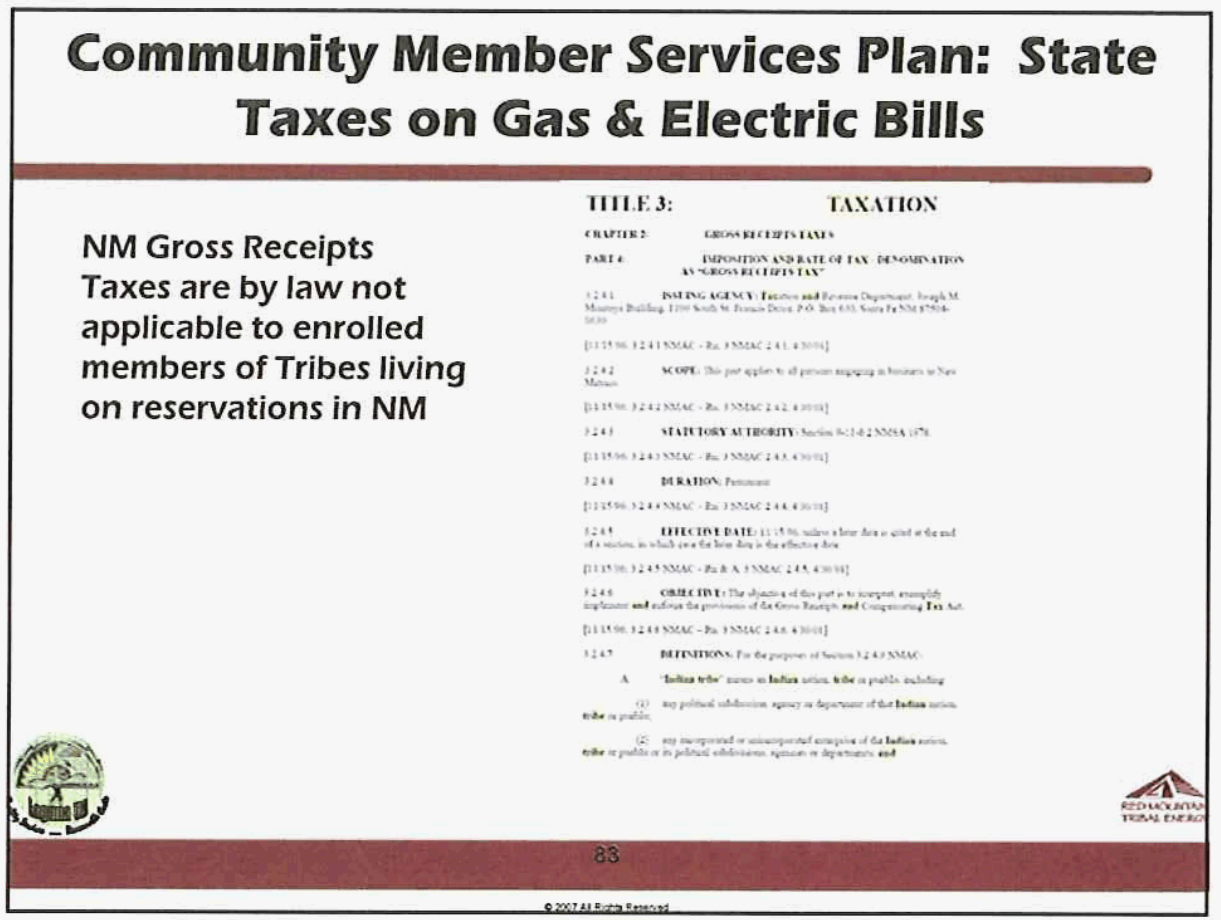

\section{Community Member Services Plan: State Taxes on Gas \& Electric Bills}

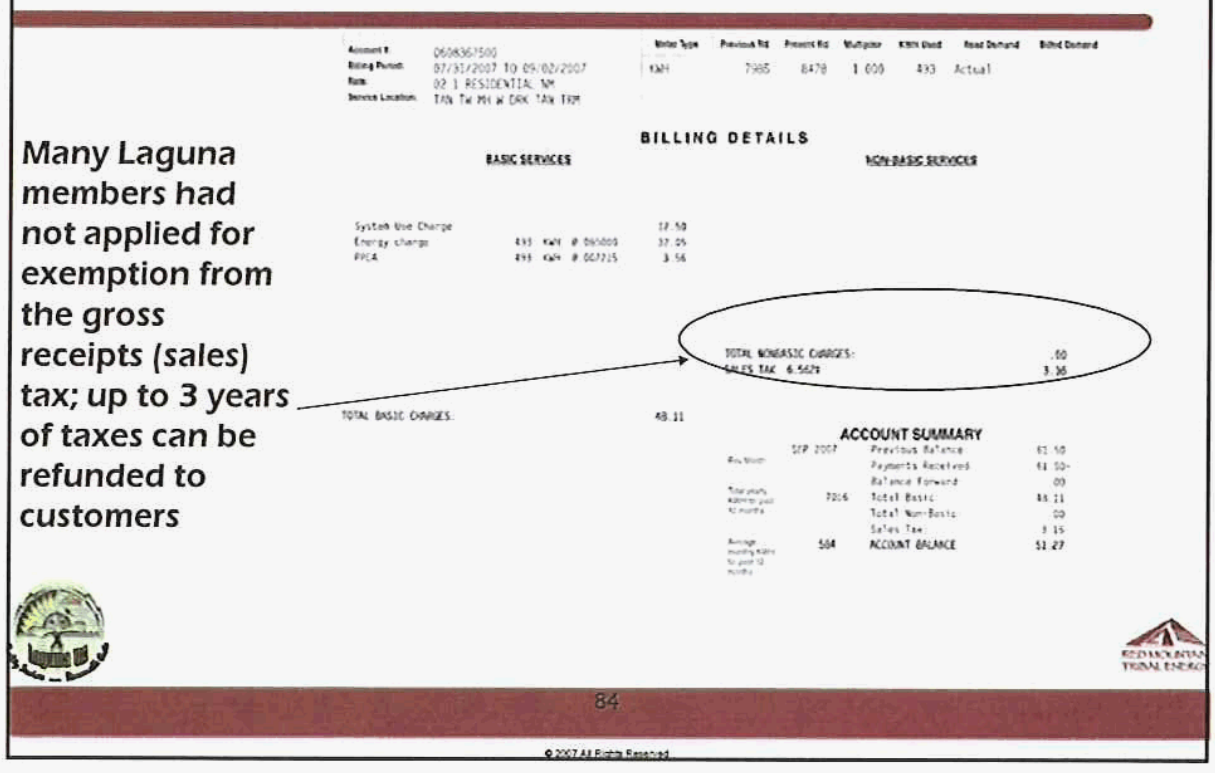




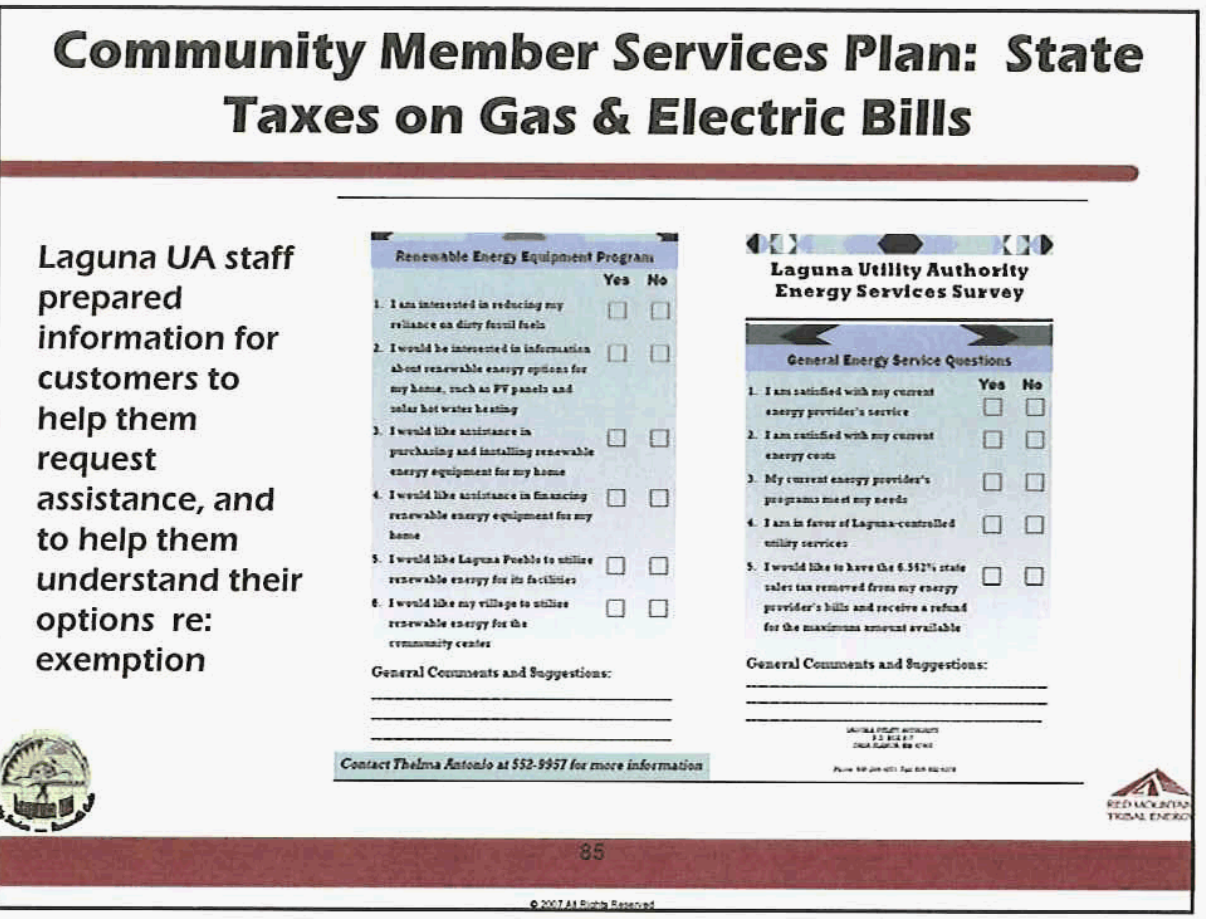

\section{Community Member Services Plan: State Taxes on Gas \& Electric Bills}

Laguna UA staff
prepared
information for
customers to
help them
request
assistance, and
to help them
understand their
options re:
exemption
B.




\section{Reservation-Scalle Solar Project}

\section{Reservation-Scalle Solar Project Concept}

- Laguna could develop and ultimately own (after 5-7 years) a reservation-scale (2 MW) concentrating solar plant, which would help meet peak usage on its system

$\checkmark$ Grant funding would be needed to fund initial development costs

$\checkmark$ Laguna would partner with a financial institution that provided initial equity

$\checkmark$ Economics assume utilization of tax incentives (ITC)

$\checkmark$ Economics assume utilization of New Market Tax Credits available from the New Mexico Finance Authority

$\checkmark$ Power could be net metered or sold to its utility provider

$\checkmark$ Renewable Energy Credits and carbon offsets could sold separately

$\checkmark 13$ acres of flat land needed

$\checkmark$ Minimal water use (washing only) 


\section{Reservation-Scalle Sollar Project Purpose}

- Allow Laguna to leverage its resources (insolation) and provide peak period power to the Laguna electric system, which could lower overall power supply costs

- Generate power at prices acceptable to utilities needing peak power and renewable energy credits, using all available incentives

- Provide Laguna an ownership position and partnership payouts for the 20-year life of the project

- Create several jobs for Laguna members during construction and operation of the project

\section{Reservation-Scalle Solar Project: Applicable Laguna Solar Resources}

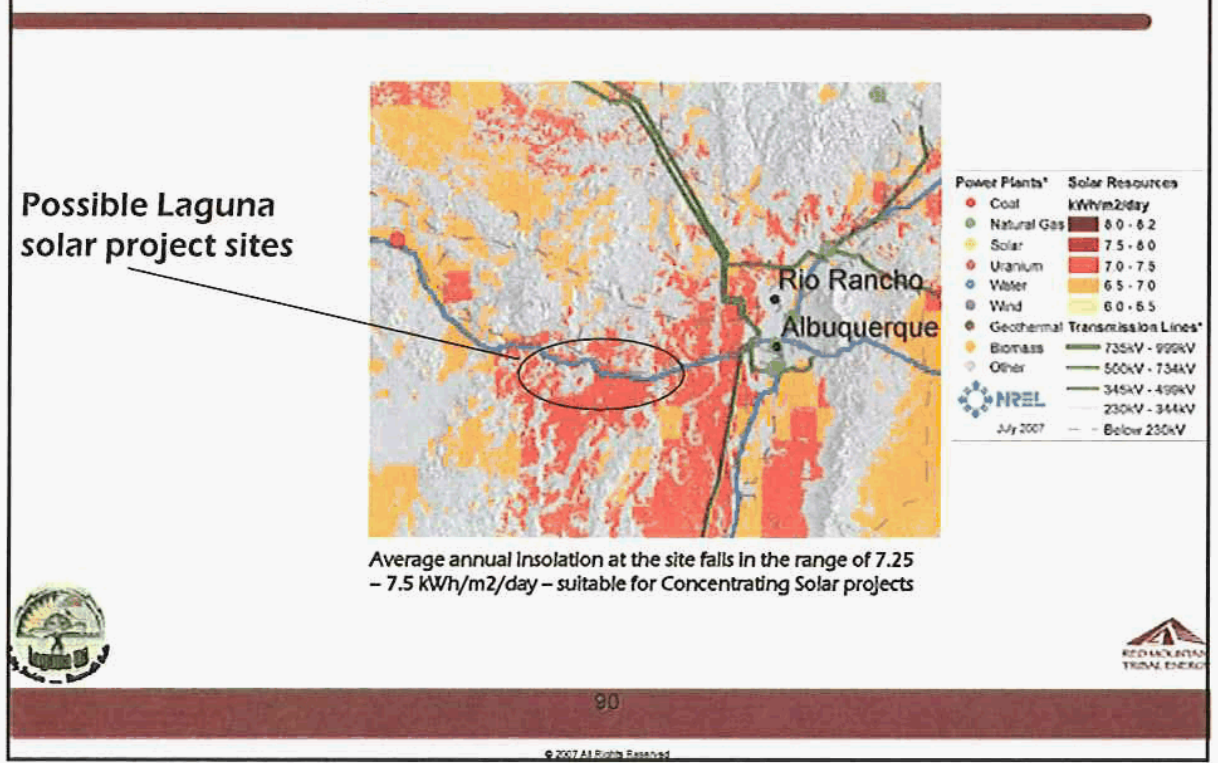




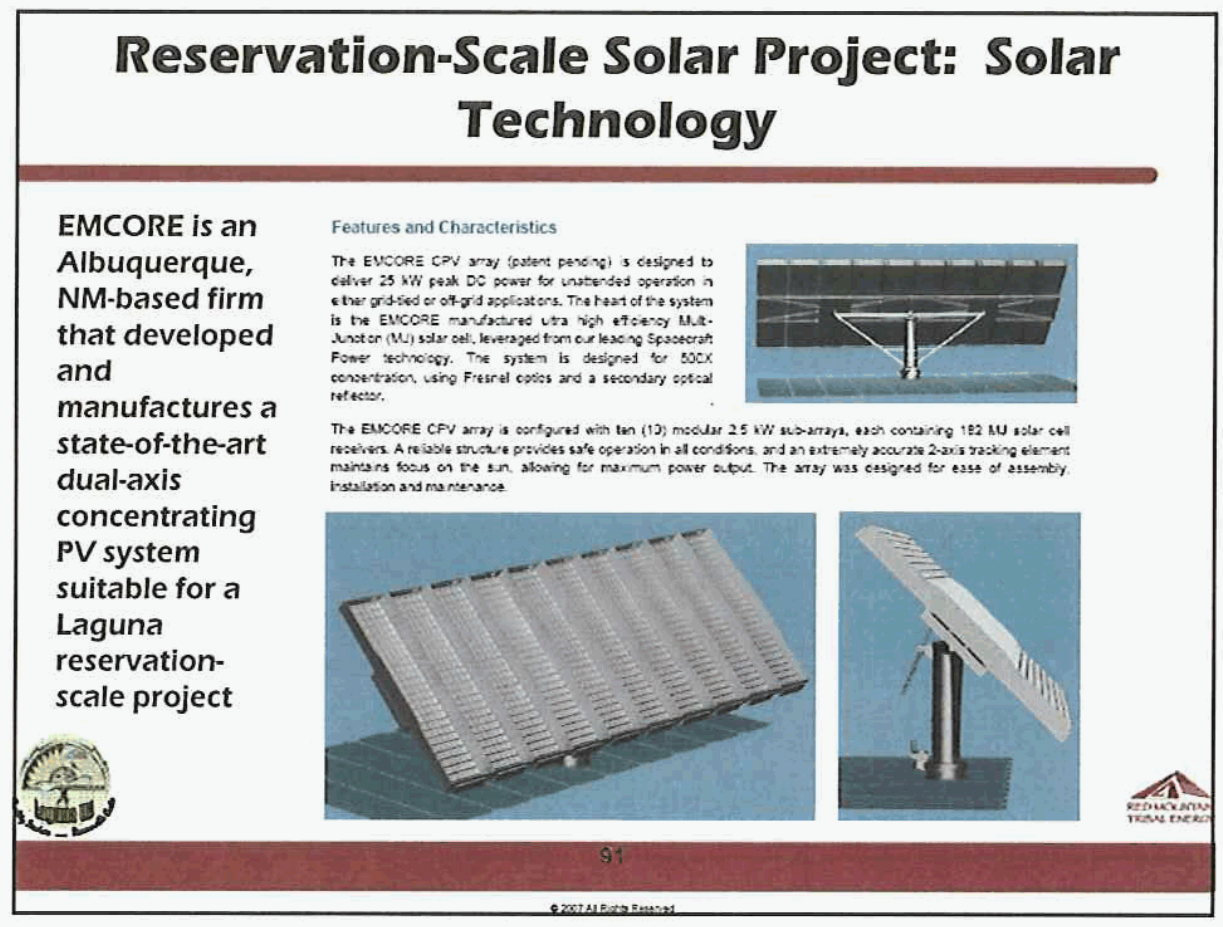

\section{Reservation-Scale Solar Project: Possible Laguna Community Solar Project Locations}

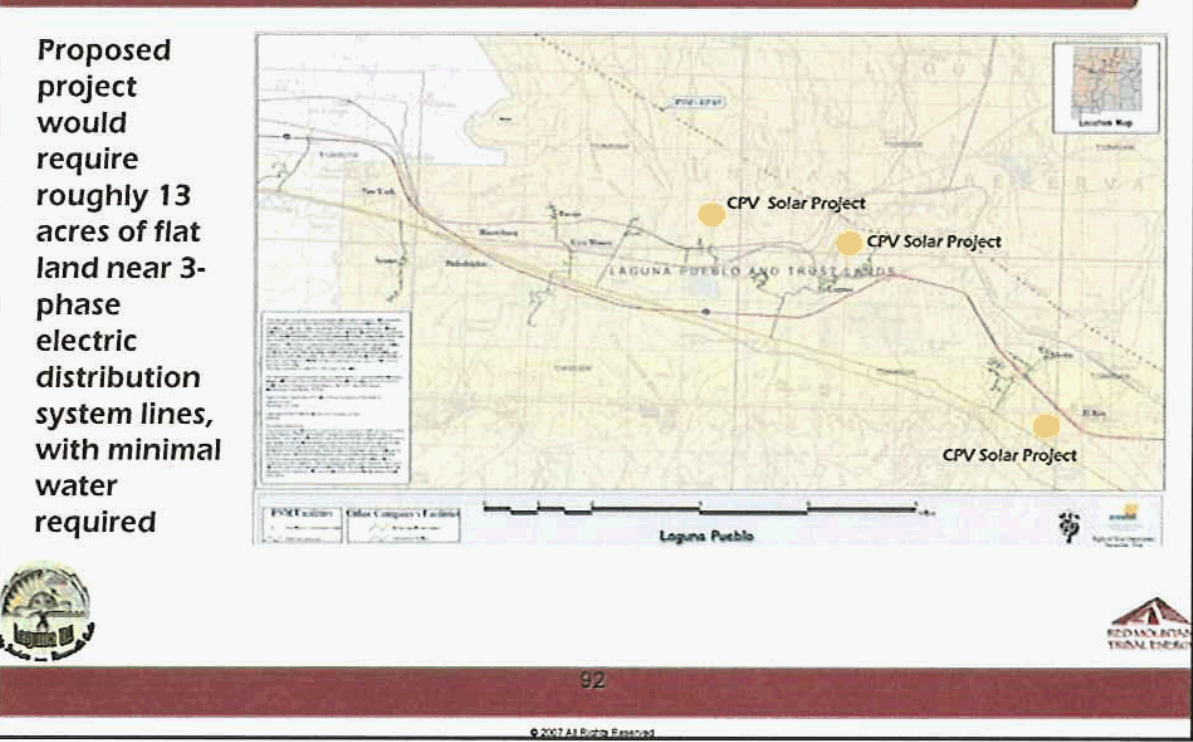




\section{Reservation-Scalle Solar Project: Potential Power Purchasers}

- PNM

- Has sufficient renewable resources to comply with $6 \%$ RPS for 2008-9

- Considering two additional renewable resources

$\checkmark$ Large-scale central station solar facility (100-200 MW)

$\checkmark$ Expansion of customer-owned PV REC program to apply to systems $>10 \mathrm{~kW}$

- Expected to issue an RFP for small scale renewables in early 2008

- Continental Divide Electric Cooperative

- Current RPS requires cooperatives to have $5 \%$ renewable supplied power by 2015

\section{Reservation-Scalle Solar Project: Funding Requirements}

Funding Requirements

- \$14 million in capital costs

- Turnkey equipment and construction for $2 \mathrm{MW}$ of concentrating PV

- System interconnection costs

- $\$ 500,000$ in development costs

- Detailed feasibility analyses

- Environmental reviews

a Project coordination

- Financing studies

- Power purchase negotiations

- Project interconnection studies

Funding Sources

- Development costs - grant funding (DOE, USDA), investor funding 


\section{Reservation-Scalle Solar Project: Possible Project Development Steps and Timing}

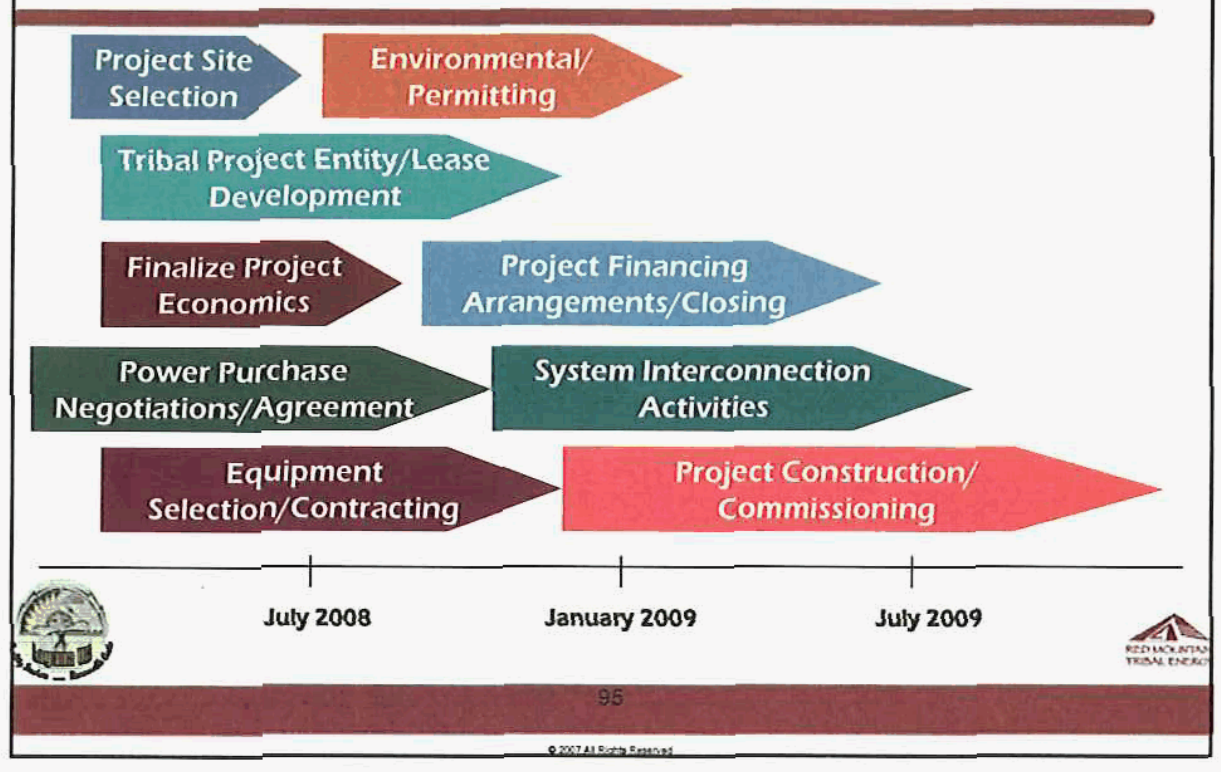

\section{Reservation-Scalle Solar Project: Project Rationalle}

- 2 MW concentrating PV solar project would leverage Laguna solar resources (insolation) and provide peak period power to the Laguna electric system

- Using all available incentives, project power could be generated within a reasonable price range for the peak power and renewable energy credits needed by NM utilities

- Power for the project would not require interconnection through a new substation, but would boost power quality at its interconnection point

- Laguna could develop the project on its own, and benefit from an ownership position and partnership payouts for the life of the project 


\section{Large-Scalle Sollar Project}

\section{Large-Scalle Solar Project Concept}

- Laguna could work with a development partner to develop, and ultimately (after 5-7 years) own and operate a large-scale (100 MW) concentrating solar plant, which would provide power to the grid to help New Mexico utilities meet their renewable energy requirements

- Development partner would fund initial development costs

- Tax partners would provide initial equity

- Economics assume utilization of tax incentives (ITC)

- Economics could assume utilization of New Market Tax Credits available from the New Mexico Finance Authority

- Power would sold to nearby utilities

- Renewable Energy Credits would likely be bundled and sold with project energy

- Carbon offsets could be sold separately

- 650 acres of flat land needed

- Minimal water use (washing only)

- Laguna could benefit from local manufacturing/component integration facilities needed 


\section{Large-Scalle Sollar Project Purpose}

- Allow Laguna to leverage its resources (insolation) and provide peak period power to the NM grid

- Generate power at prices acceptable to utilities needing peak power and renewable energy credits through utilization of all available incentives

- Provide Laguna an opportunity to benefit from an ownership position and significant partnership payouts for the 20-year life of the project

- Create manufacturing positions, as well as construction and operations and maintenance positions for Laguna members

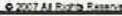

\section{Large-Scalle Solar Project: Applicable Laguna Solar Resources}

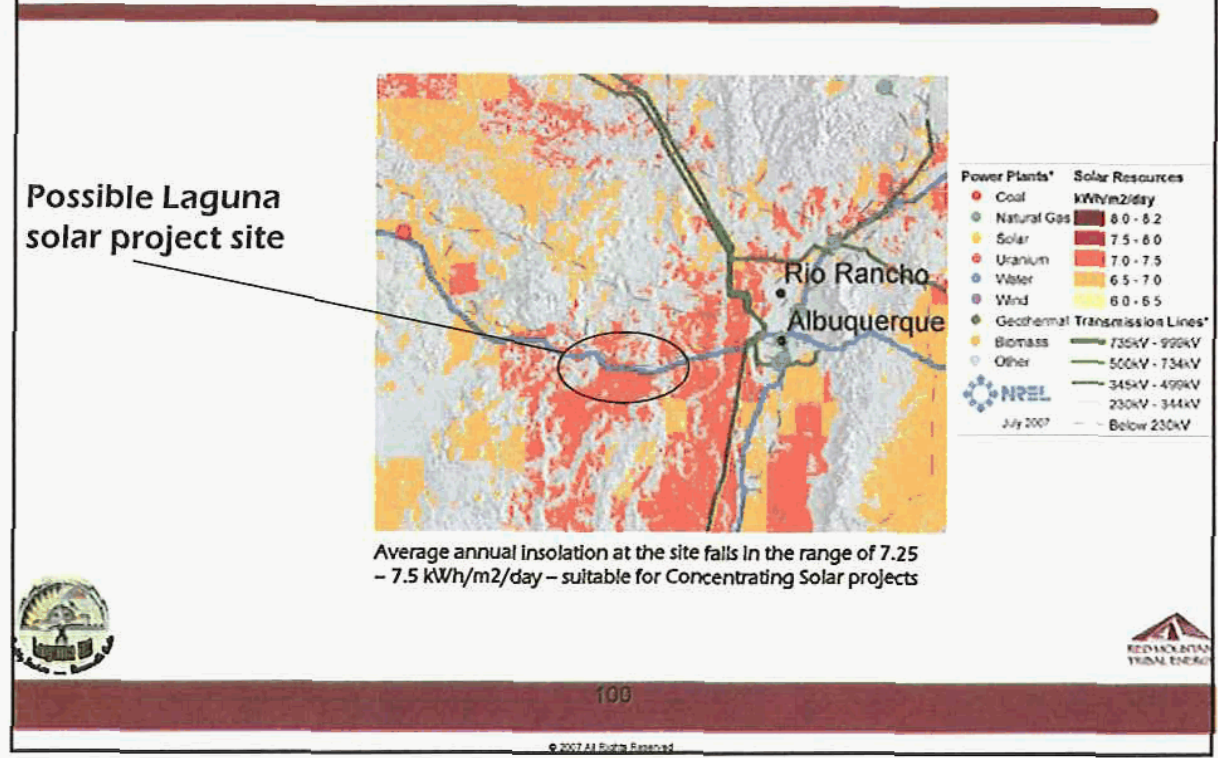




\section{Large-Scalle Solar Project: Solar Technology}

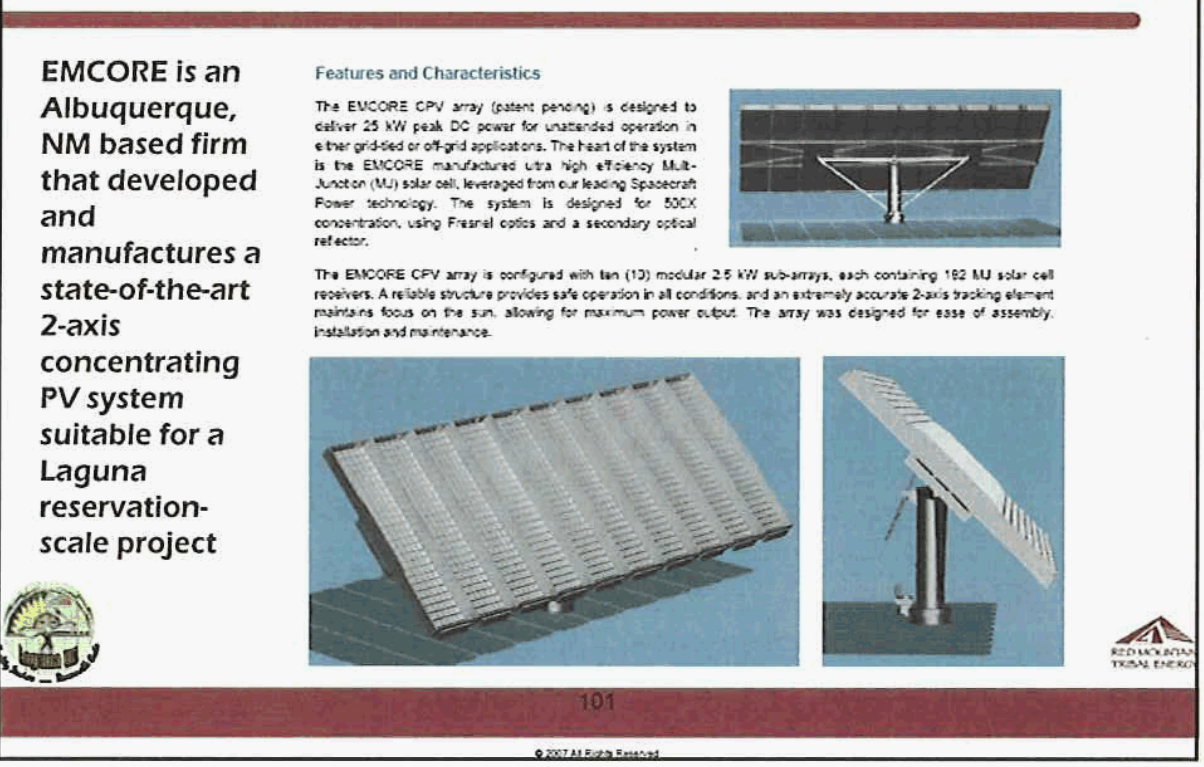

\section{Large - Scalle Solar Project: Proposed Site}

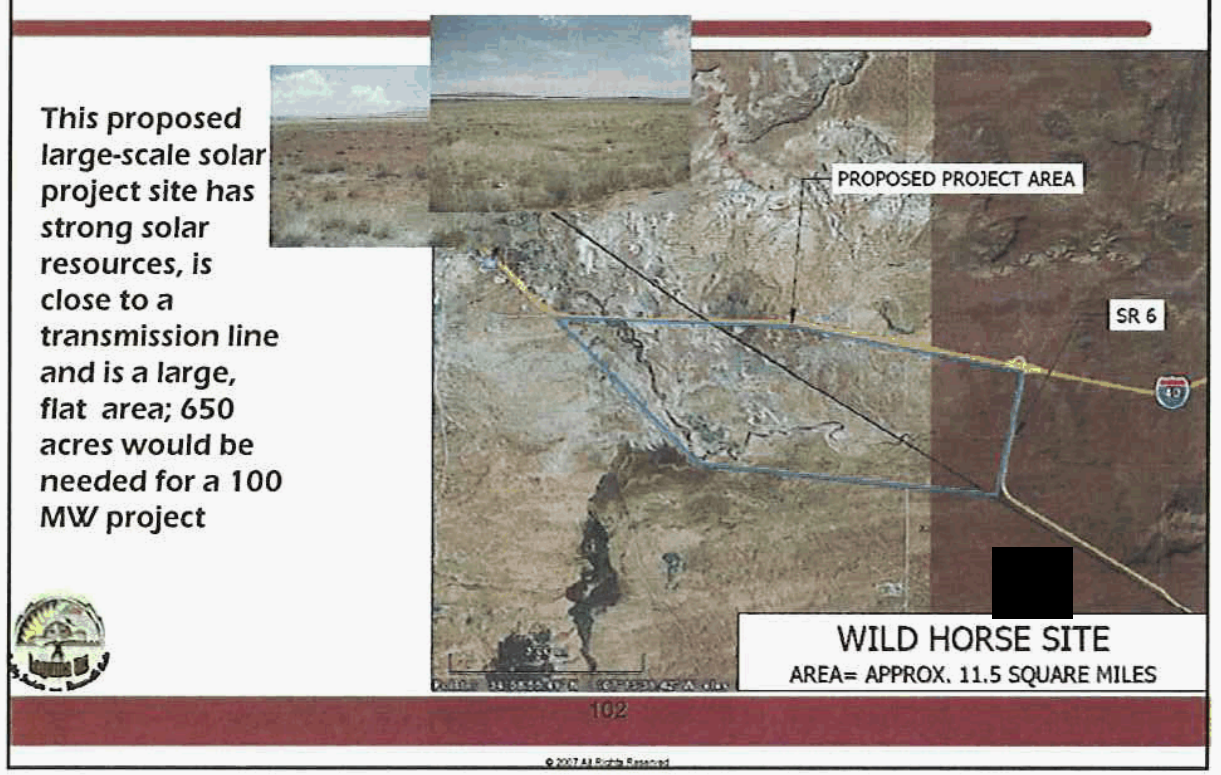




\section{Large-Scalle Solar Project: Potentiall Power Purchasers}

- PNM

- Has sufficient renewable resources to comply with $6 \%$ RPS for 2008-9

- PNM solar solicitation expected in early 2008

$\checkmark$ RPS requirements/solar set aside requires PNM to acquire roughly 200 MW of solar by 2011

- Is considering expansion of customer-owned PV REC program to apply to systems $>10 \mathrm{~kW}$

- Expected to issue an RFP for small scale renewables in early 2008

- Continental Divide Electric Cooperative

- Current RPS requires cooperatives to have $5 \%$ renewable supplied power by $\mathbf{2 0 1 5}$

\section{Large-Scalle Solar Project: Funding Requirements}

Funding Requirements

- \$340-560 million in capital costs

- Turnkey equipment and construction for $100 \mathrm{MW}$ of concentrating PV

- System interconnection costs

- \$14 million in development costs

- Detailed feasibility analysis

- Environmental reviews

- Full-time project coordination

- Financing studies

- Power purchase negotiations

- Project interconnection studies

Funding Sources

- Development costs - grant funding (DOE, USDA), investor funding

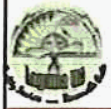




\section{Large-Scalle Sollar Project: Possible Project Development Steps and Timing}

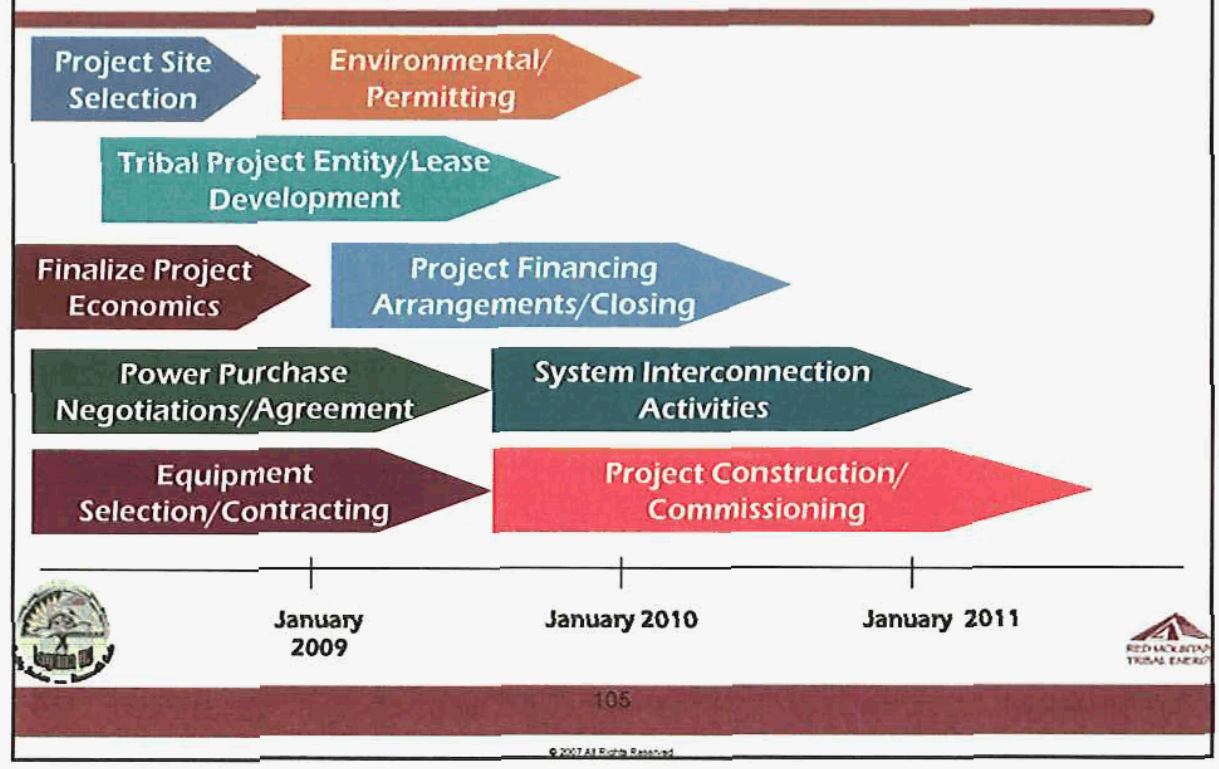

\section{Large-Scalle Solar Project Next Steps}

- Pursue community and Council approval for project development

- Identify site specifics

- Begin environmental, cultural reviews

- Begin discussions with large-scale solar developers

- Several large-scale solar developers have expressed interest in working with Laguna

- Solar technology providers also interested in Laguna potential sites

- Establish contact with potential power purchasers

" Utilities

- Financial entities

- Begin discussions with technology providers

- EMCORE

- Other CPV technology providers 


\section{Large-Scalle Solar Project Rationalle}

- Laguna brings many advantages to a solar project partnership which can help ensure project success

- Location is close to market and transmission lines (115 kV)

- Laguna solar resources and nearby natural gas lines

- Time to market could be short, as permitting process could be accelerated with Tribal leadership support for energy development

- High potential for manufacturing/component integration facilities

- Available incentives and financing considerations can reduce levelized costs of energy

- $30 \%$ Investment Tax Credit for solar equipment

- New Market Tax Credits (39\%) available for similar projects

- Renewable Energy Credits from tribal lands worth 2x for certain power purchasers

- Low-interest loan/loan guarantees/Clean Renewable Energy Bonds

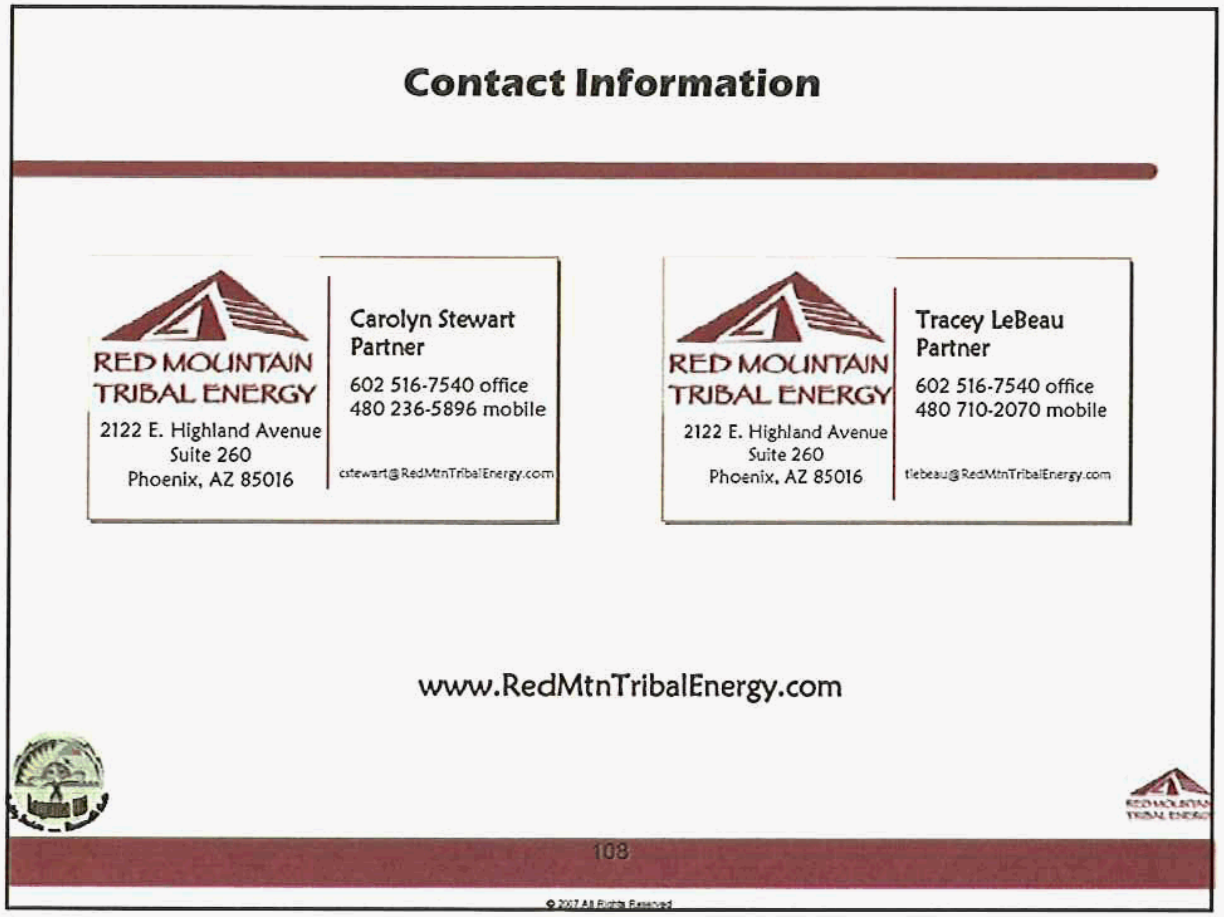



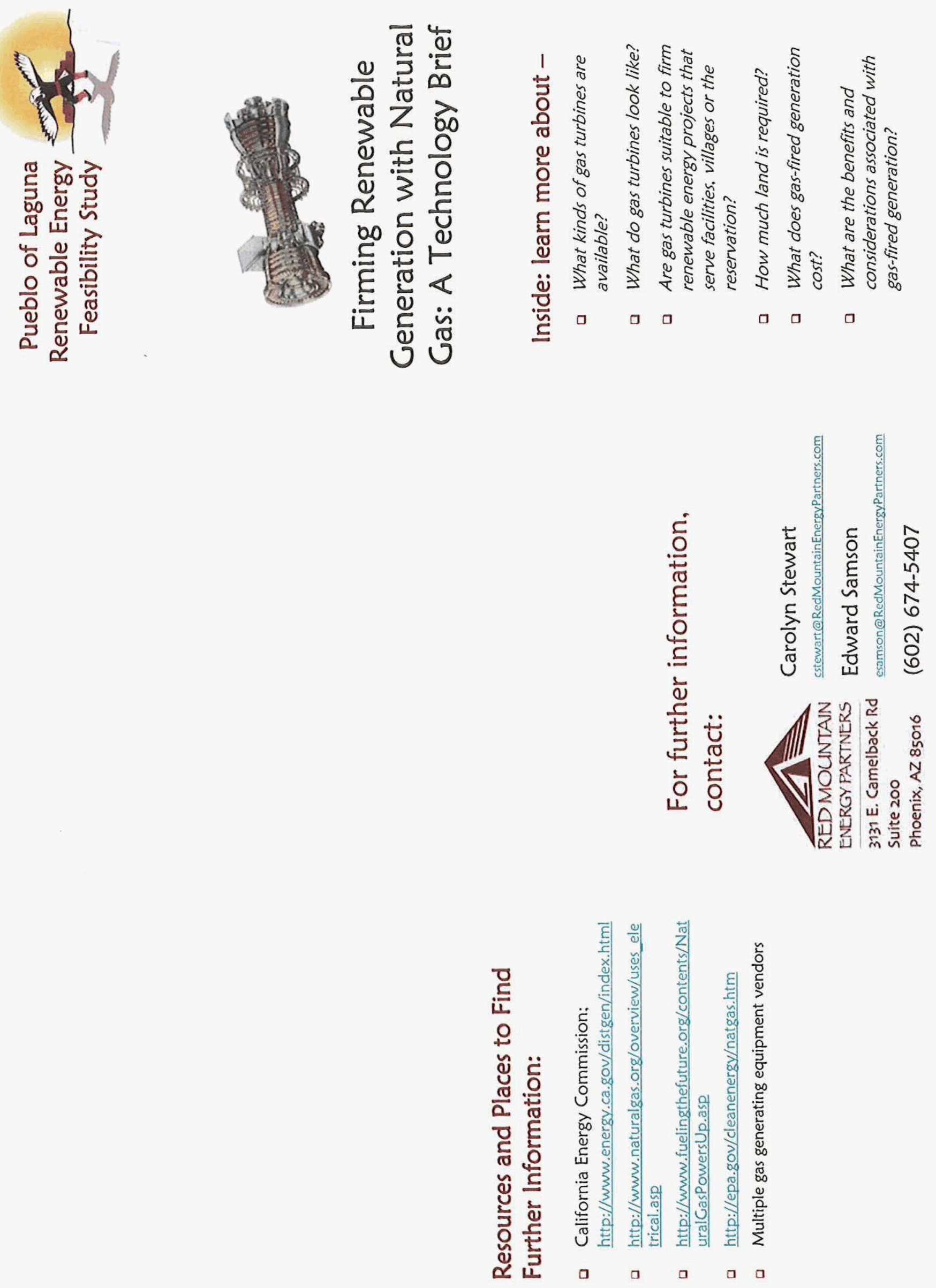


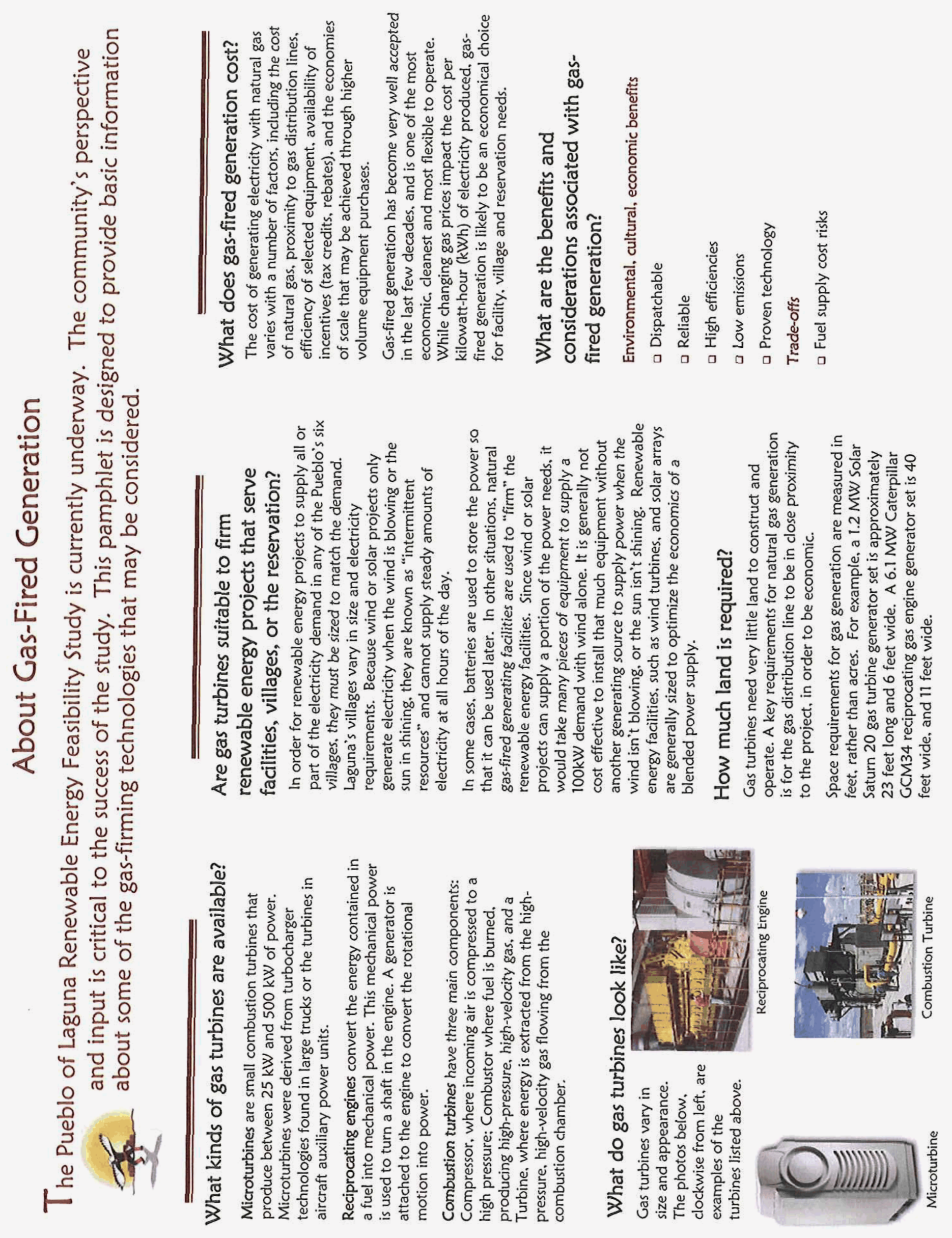





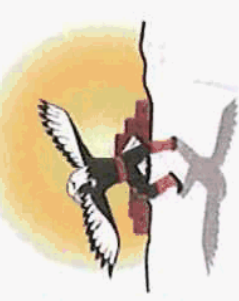

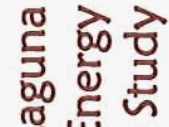
岃岕

을 究

ฉ

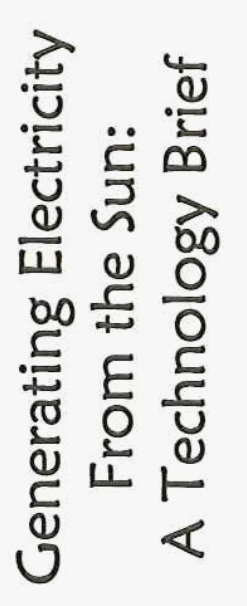

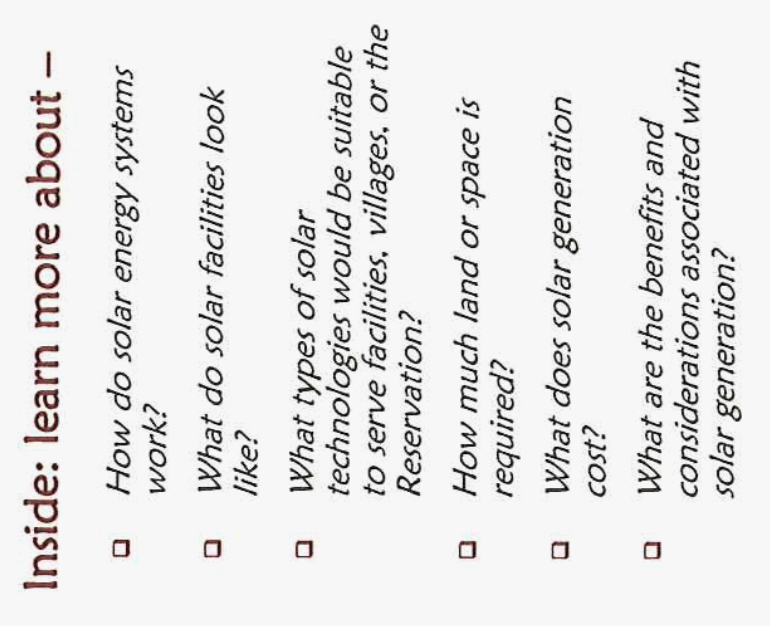

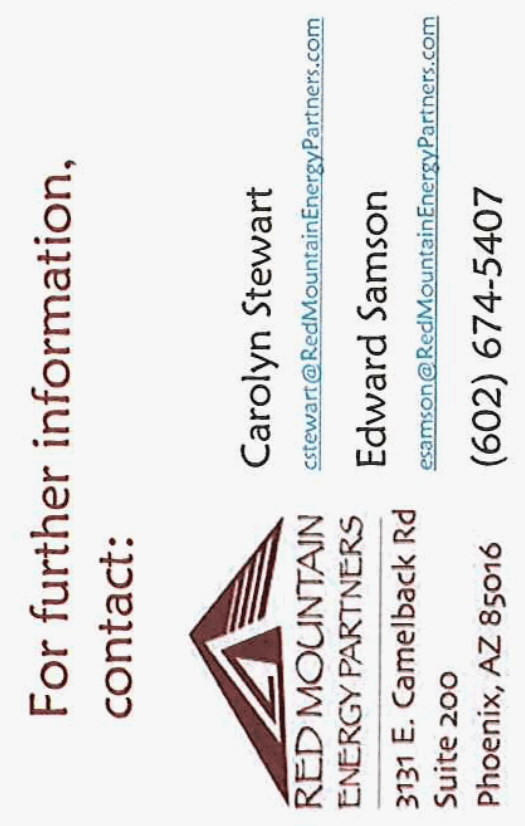




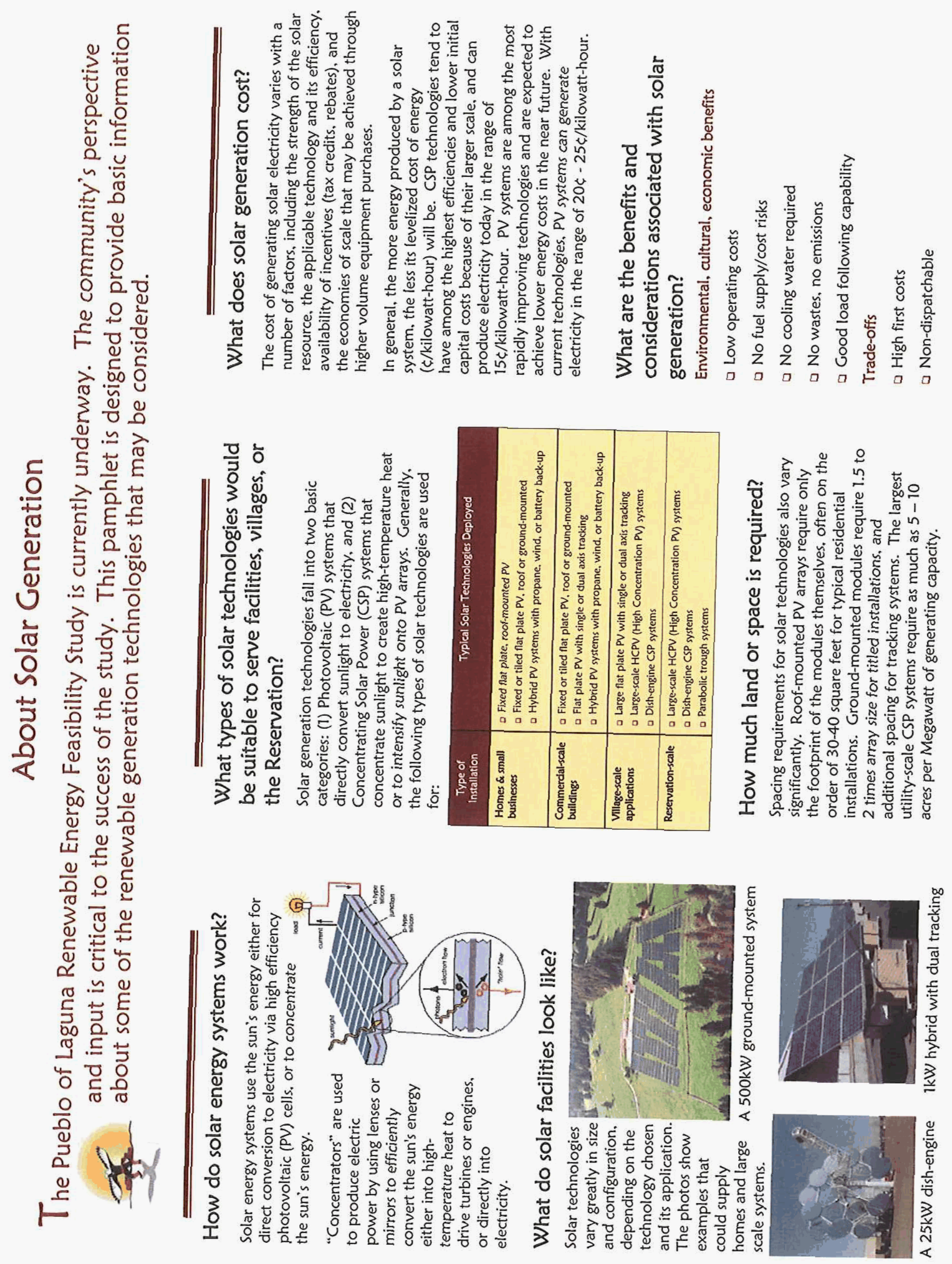





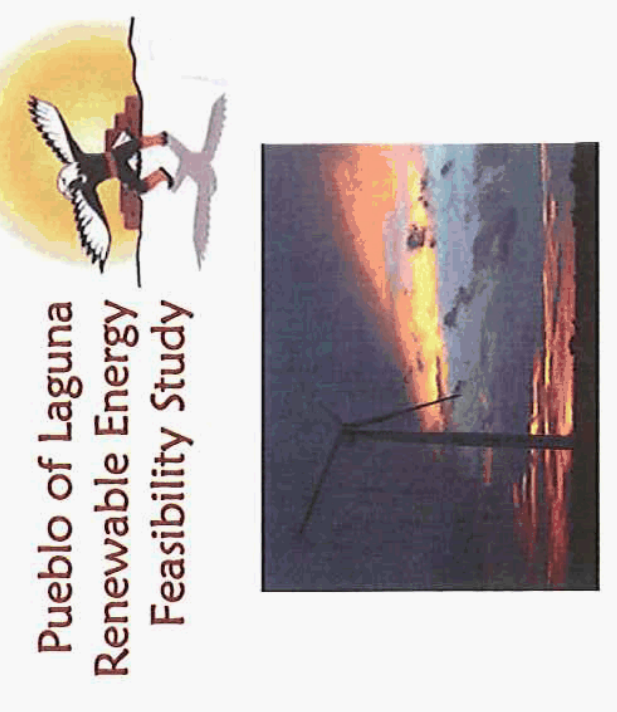

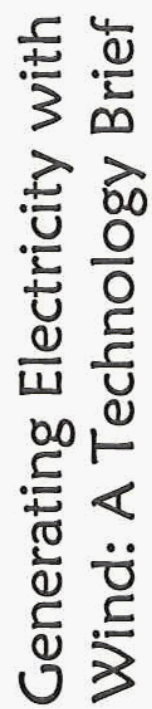
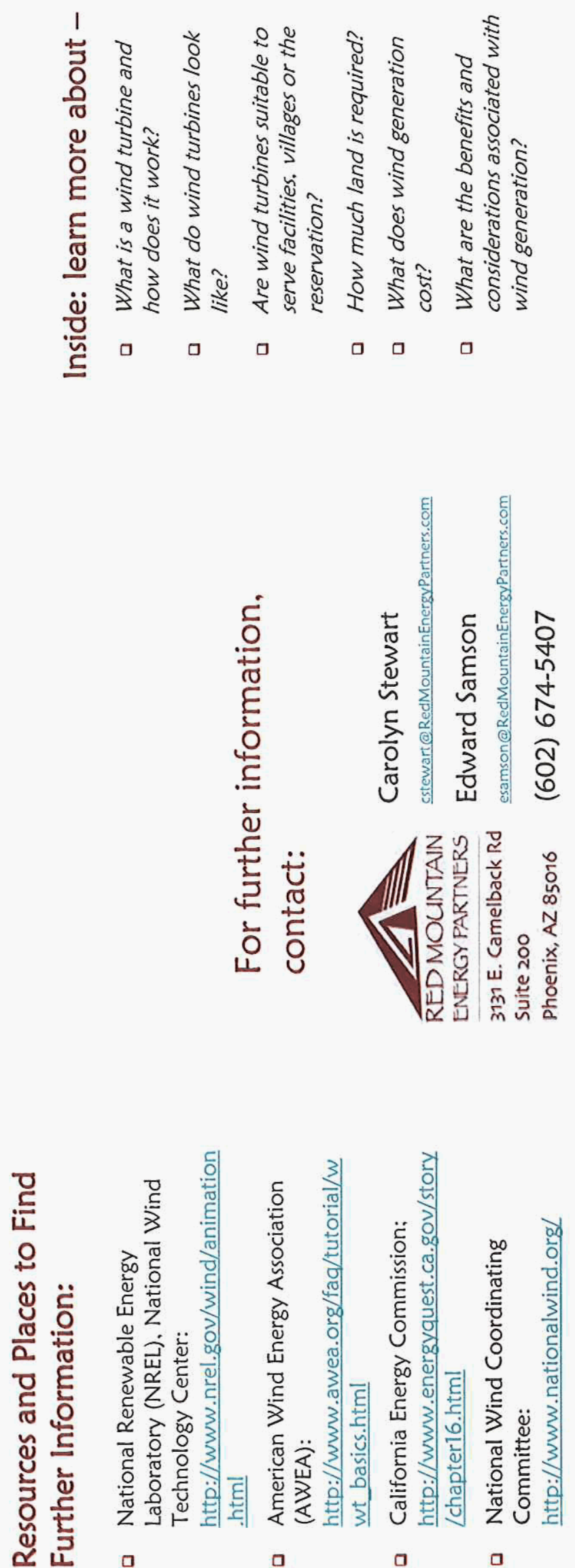


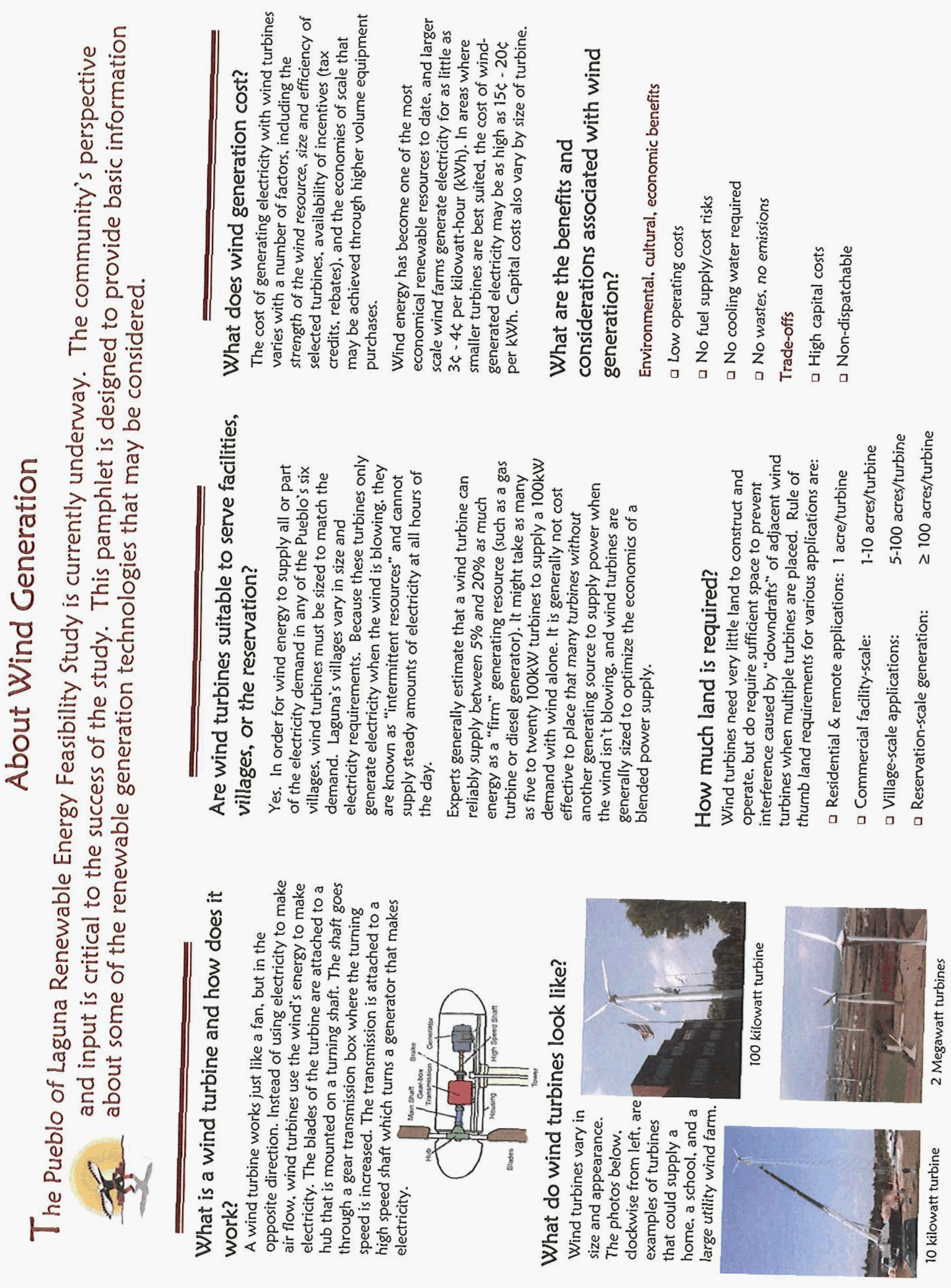




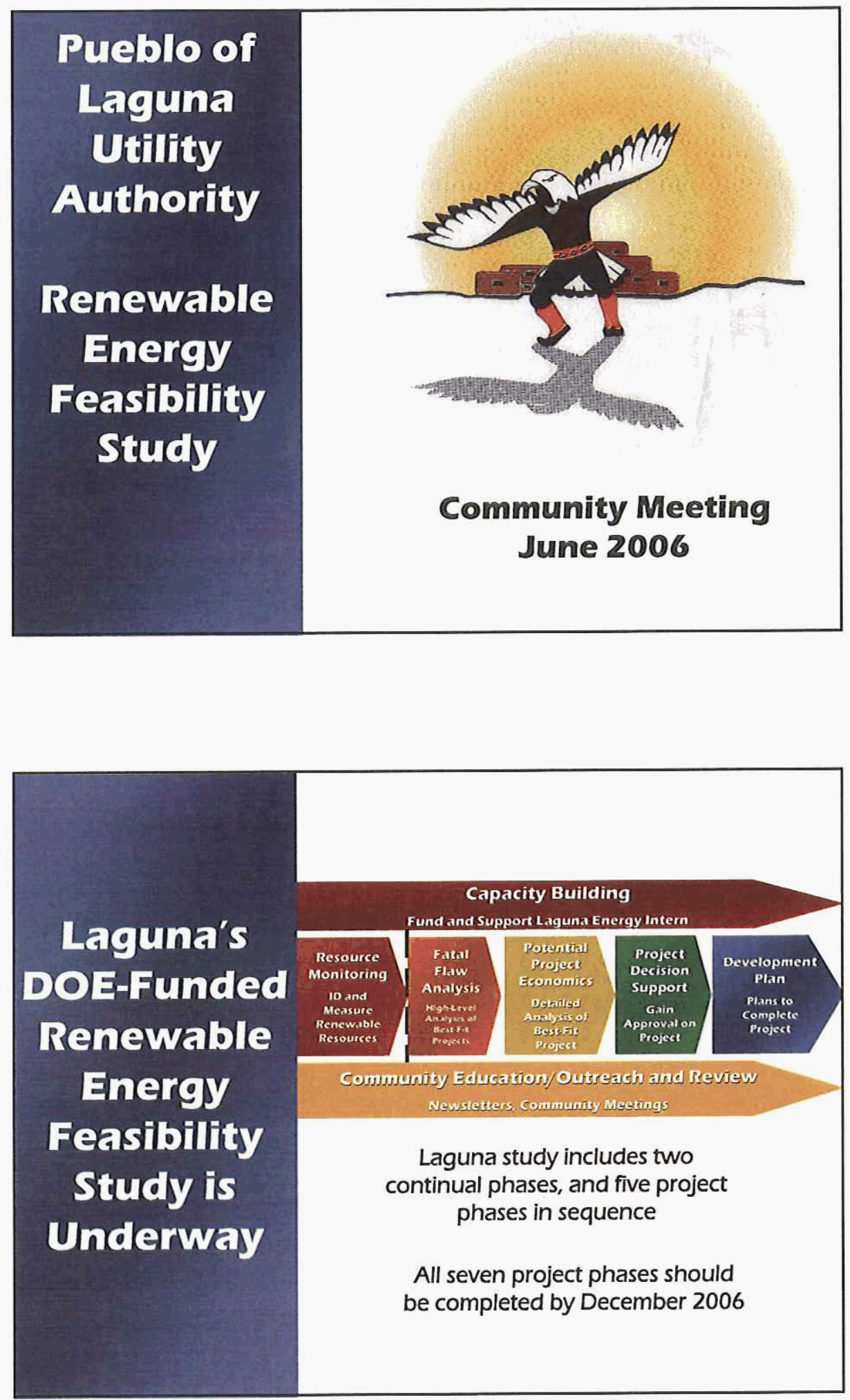

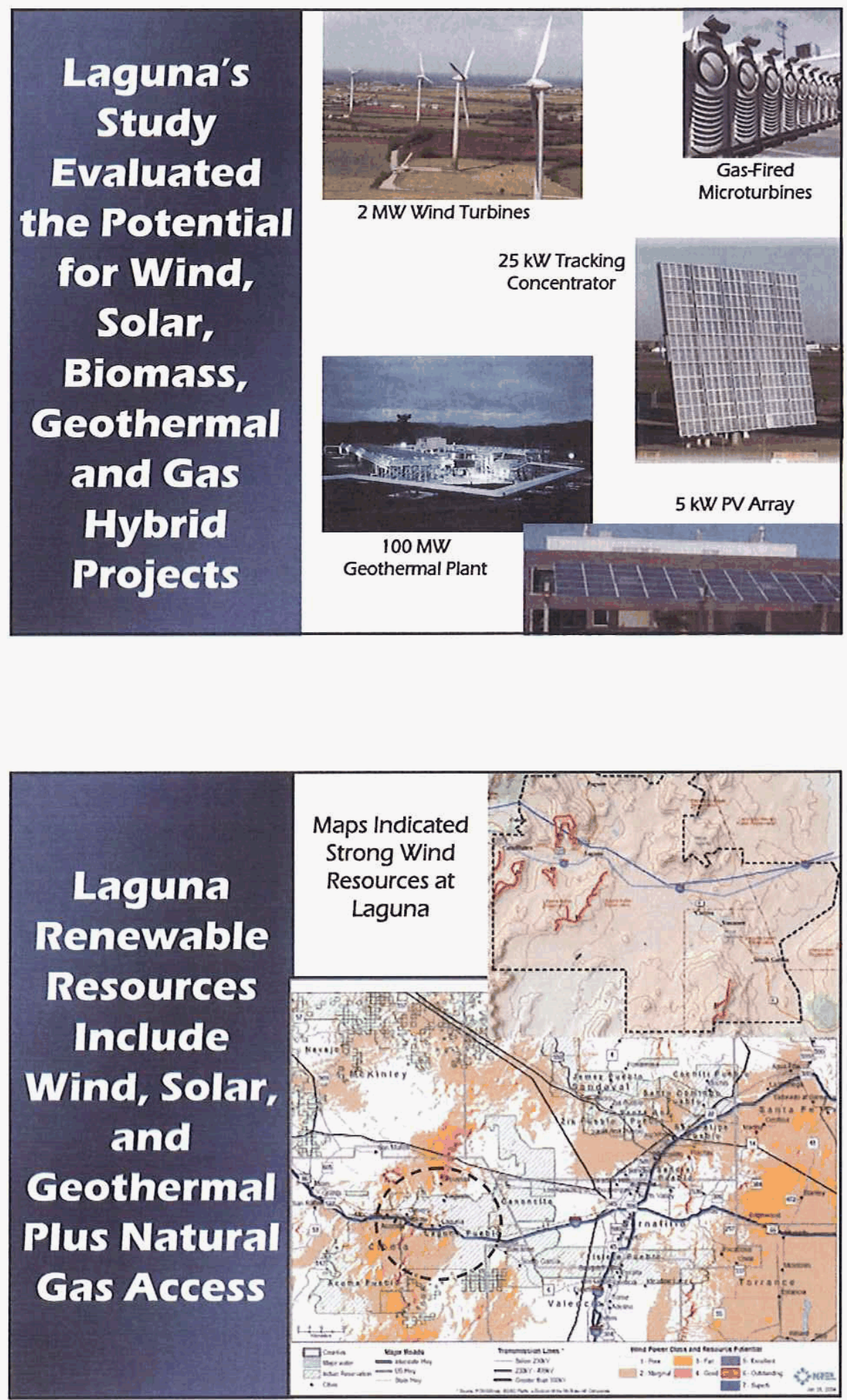

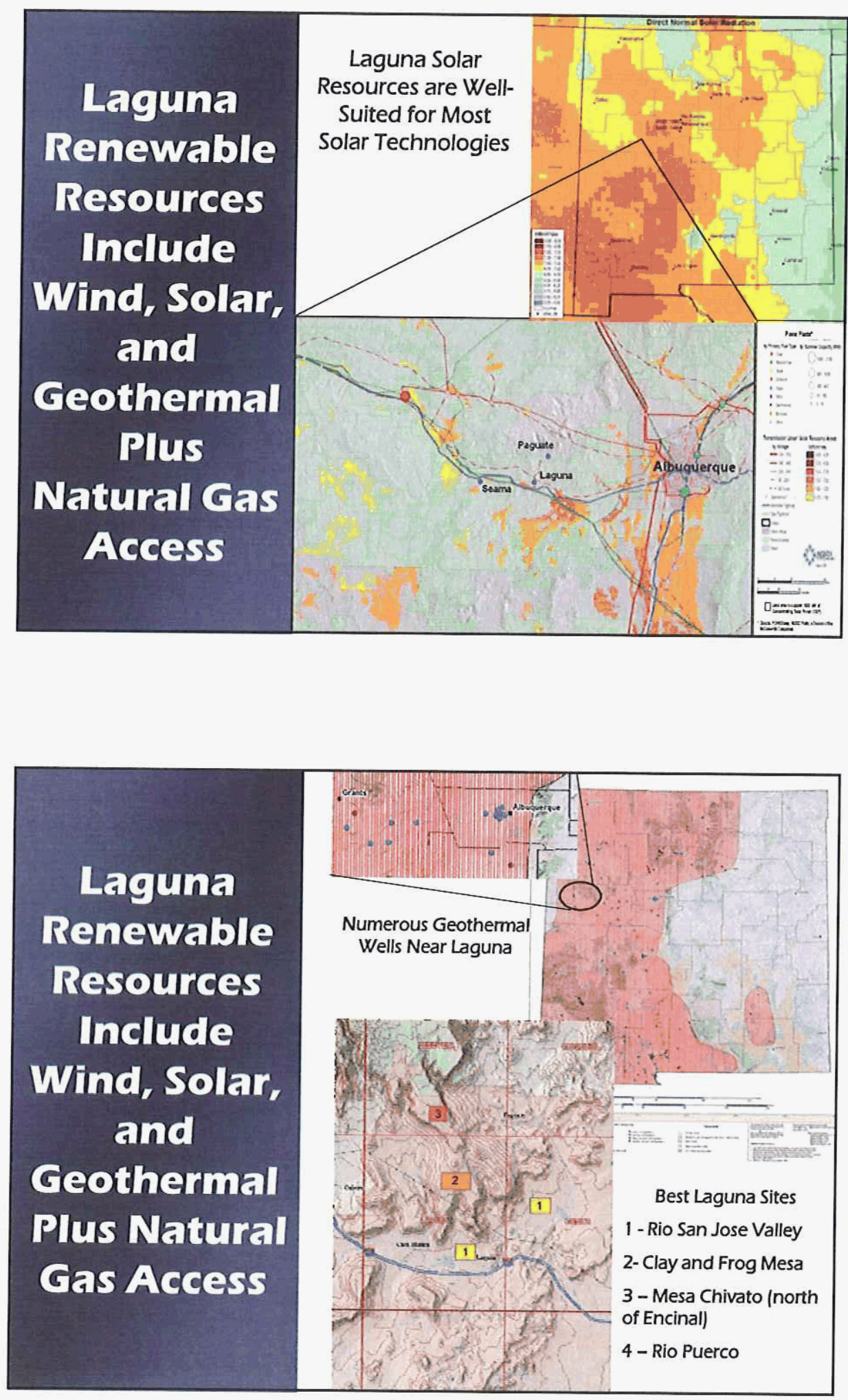

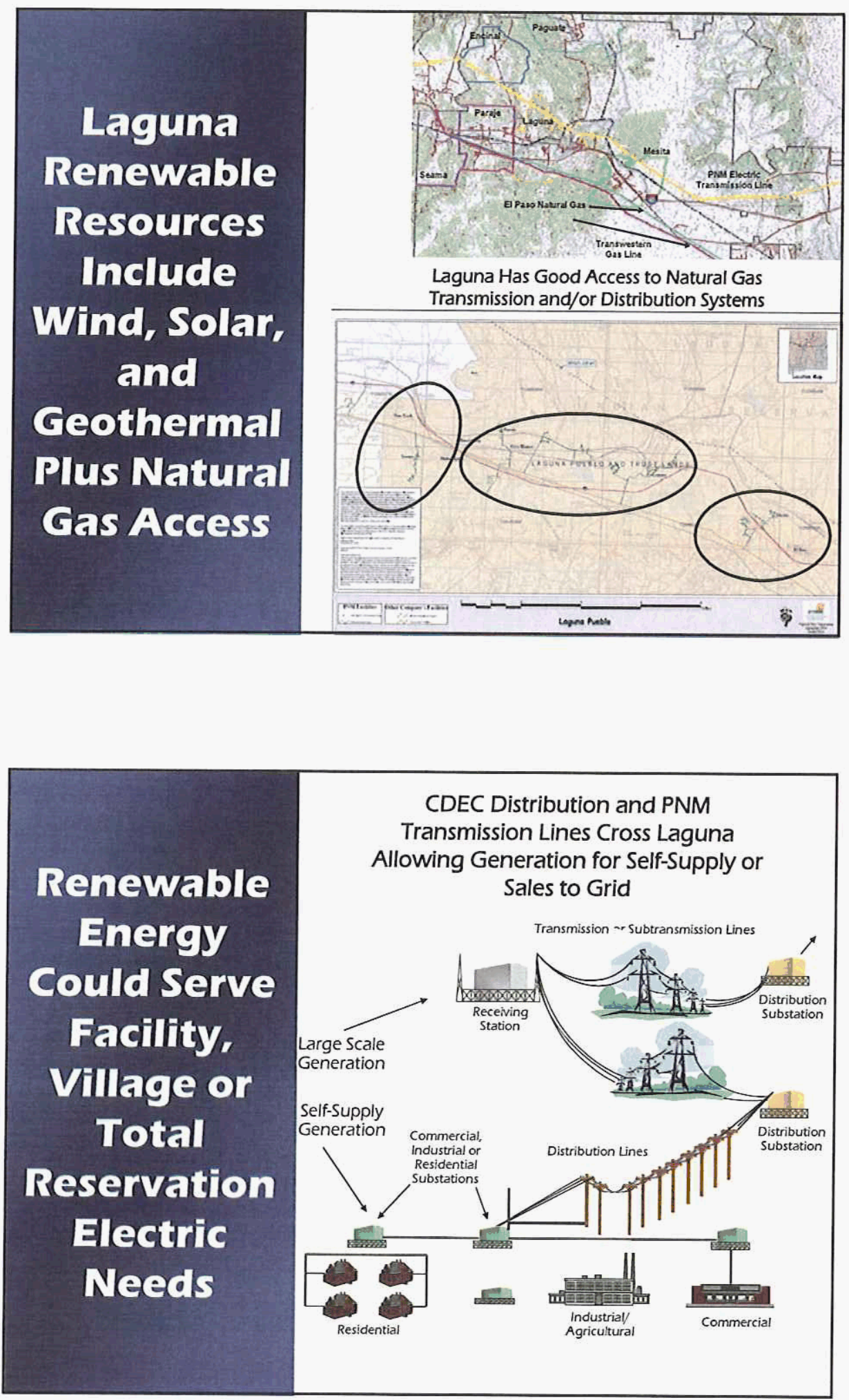

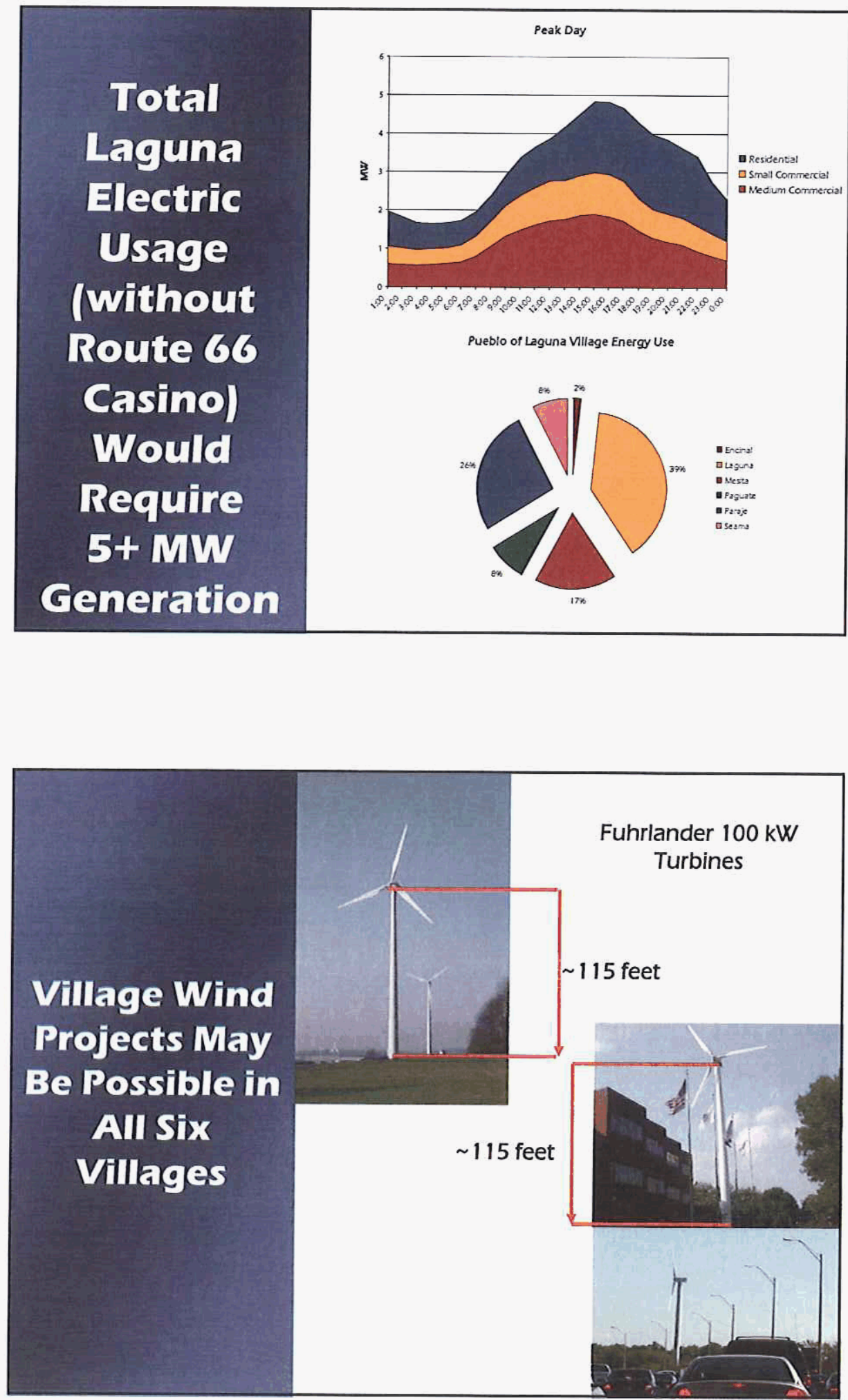

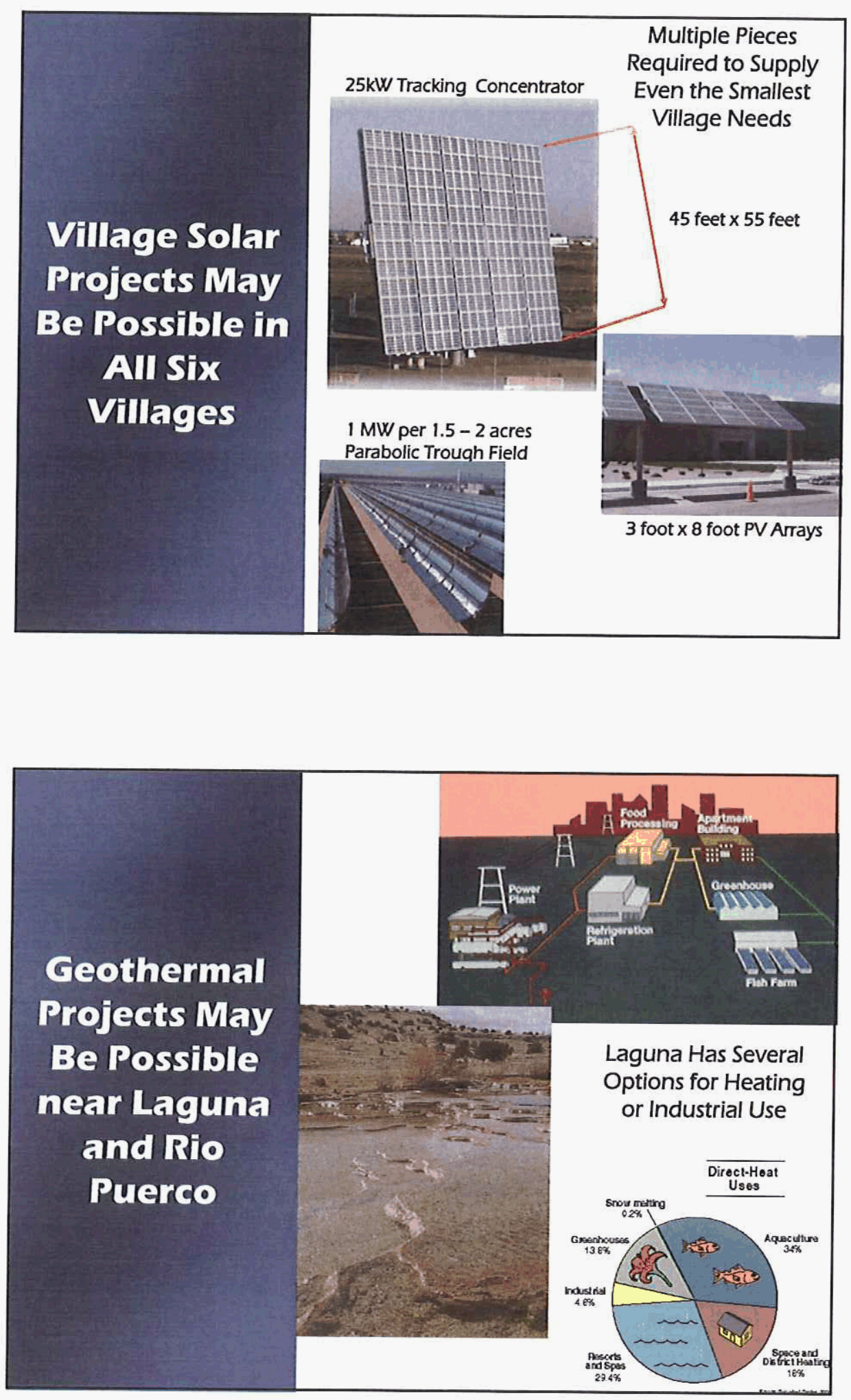

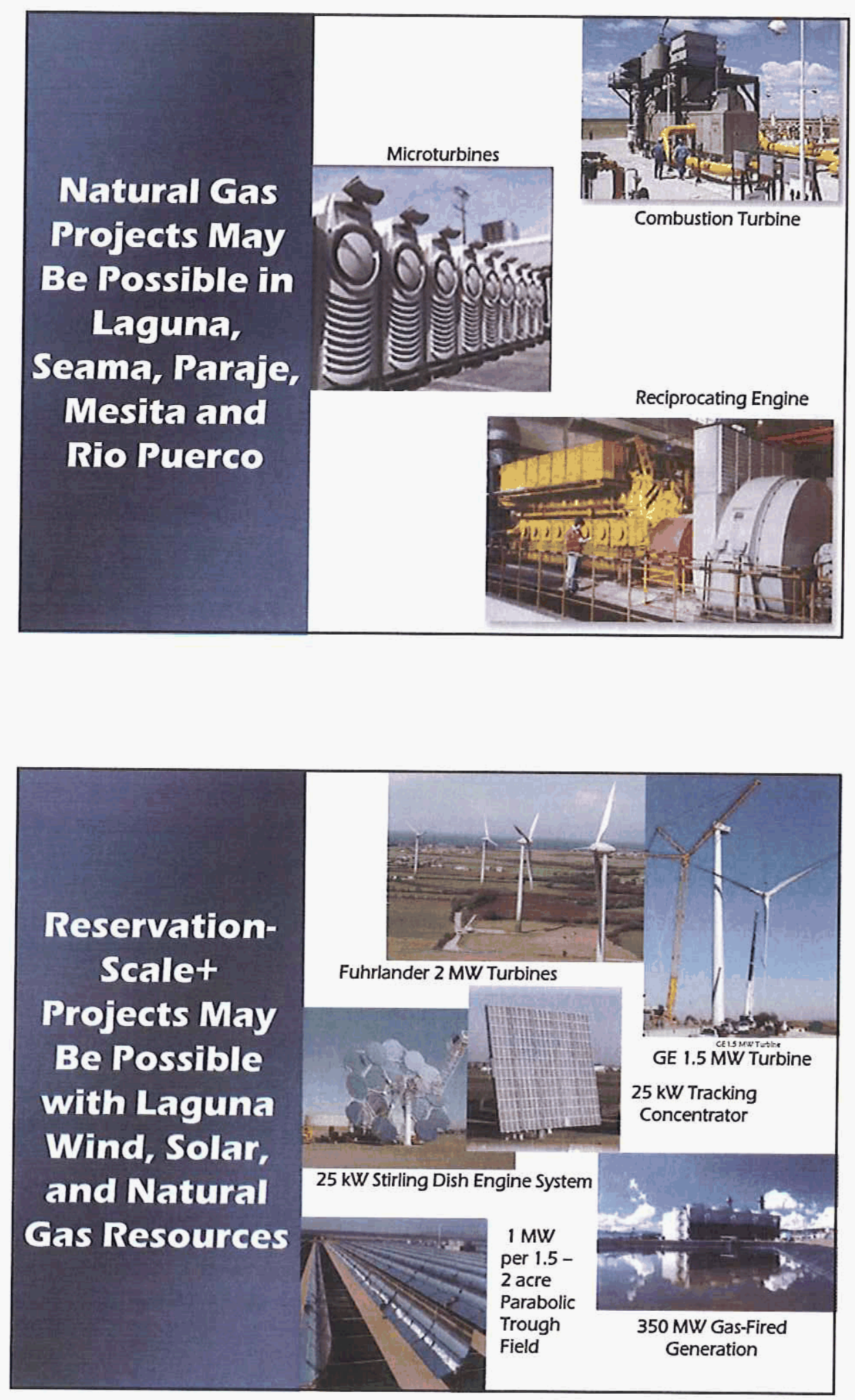

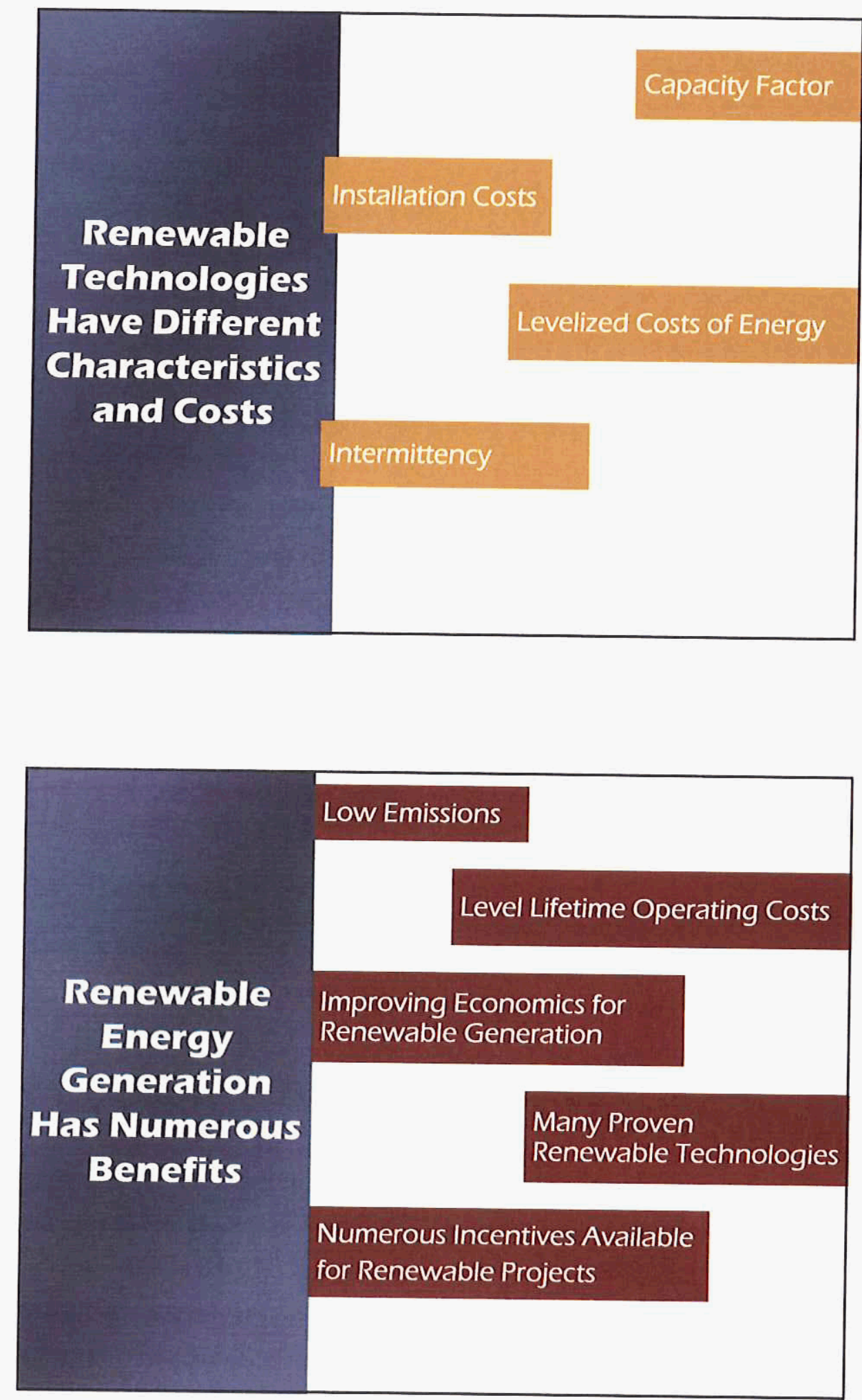


\begin{tabular}{|c|c|c|c|c|c|}
\hline \multirow{7}{*}{$\begin{array}{l}\text { Key Laguna } \\
\text { Considerations } \\
\text { are the Cultural } \\
\text { and } \\
\text { Environmental } \\
\text { Impact of } \\
\text { Projects, } \\
\text { Installation Cost, } \\
\text { and Cost of } \\
\text { Electricity } \\
\text { Produced }\end{array}$} & \multicolumn{5}{|c|}{ Technology Comparisons } \\
\hline & $\begin{array}{l}\text { Type of } \\
\text { Resource }\end{array}$ & $\begin{array}{c}\text { Installed } \\
\text { System } \\
\text { cost } \\
\text { (sikW) } \\
\end{array}$ & $\begin{array}{l}1 \mathrm{MW} \\
\text { Equivalent }\end{array}$ & $\begin{array}{l}\text { Capacity } \\
\text { Factor }\end{array}$ & Key Issues \\
\hline & Wind & 1,200 & $\begin{array}{l}\text { Less than } \\
1 \text { Turbine } \\
\end{array}$ & $\begin{array}{c}\text { Class } 4 \\
\text { wind } \\
30 \% \\
\end{array}$ & Intermittency \\
\hline & $\begin{array}{l}\text { Solar } \\
\text { Thermal }\end{array}$ & $\begin{array}{l}3,000- \\
4,000\end{array}$ & $\begin{array}{l}1 \text { acre e.g. } \\
\text { Walmart } \\
\text { store }\end{array}$ & \begin{tabular}{|c|}
$22-25 \%$ \\
up to \\
$90 \%$ with \\
storage \\
\end{tabular} & $\begin{array}{l}\text { High Capital } \\
\text { Cost }\end{array}$ \\
\hline & \begin{tabular}{|l|}
$\begin{array}{l}\text { Solar PV. } \\
\text { commerclal }\end{array}$ \\
\end{tabular} & 6,500 & 10 acres & $14-20 \%$ & $\begin{array}{l}\text { High Capital } \\
\text { Cost }\end{array}$ \\
\hline & Geothermal & $\begin{array}{l}2,000 \\
2,500\end{array}$ & $15-20 \mathrm{klbs}$ & $90 \%$ & $\begin{array}{l}\text { High Initial } \\
\text { costs; } \\
\text { resource could } \\
\text { deplete }\end{array}$ \\
\hline & Natural Gas & $\begin{array}{l}500 . \\
1,000\end{array}$ & & $75+\%$ & $\begin{array}{l}\text { Permilting; } \\
\text { some } \\
\text { emissions }\end{array}$ \\
\hline
\end{tabular}

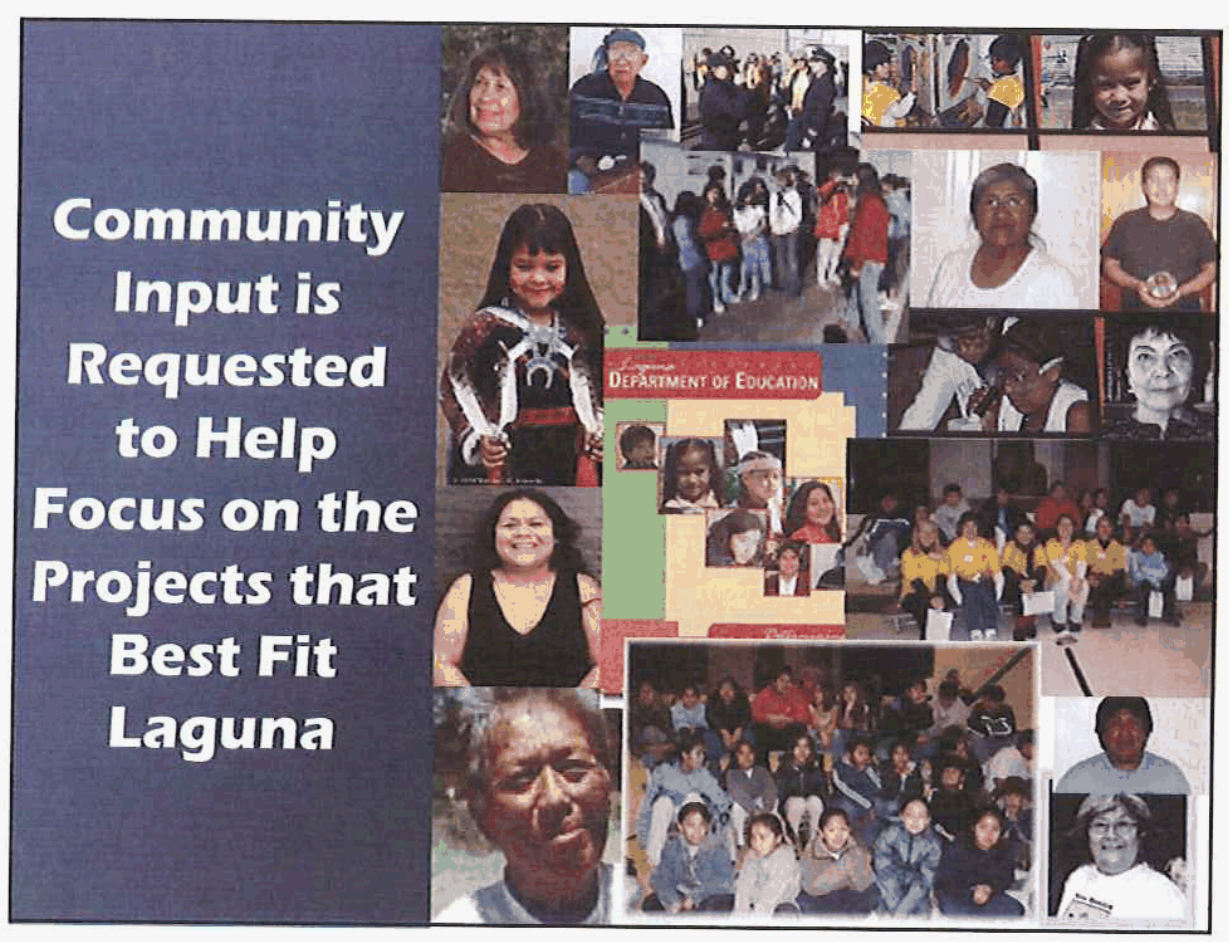





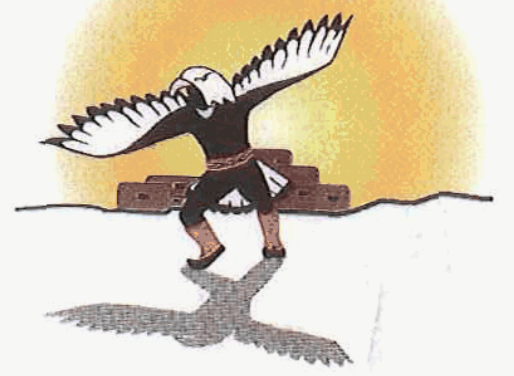

\section{Pueblo of Laguna \\ Renewable Energy Project Options}

Your input is invited.

\section{Background}

Renewable energy resources - those that can be renewed by nature and can provide clean sources of electricity generation, are abundant in the Southwestern U.S. The Pueblo of Laguna's lands are blessed with many such sources, including solar energy and wind, as well as access to natural gas resources and infrastructure. Laguna has considered the potential value of these resources for quite some time, and their potential to offer environmental benefit, improved electric service quality, affordable electricity, energy self-sufficiency, and clean, sustainable growth for future generations.

The Pueblo of Laguna Renewable Energy Feasibility Study has been underway since December 2005. The study, funded by the U.S. Department of Energy ("DOE") and managed by the Pueblo of Laguna Utility Authority, was undertaken to identify, evaluate, and plan the development of renewable energy generation projects that could potentially be built on Tribal lands. The community's perspective and input is critical to the success of the study. This material is designed to provide basic information about some renewable energy project options that have been identified as part of the study, and to gather perspective about your preferences, ideas, concerns, and any other input you would like to offer about the potential projects. Because the projects can only achieve the desired benefit if they succeed in meeting the community's needs and goals, your perspective is vital.

\section{Project Overview}

The map below reflects several Renewable Energy Project Options identified by the project team. We have additional project information on the following pages, and included a sheet requesting your input.

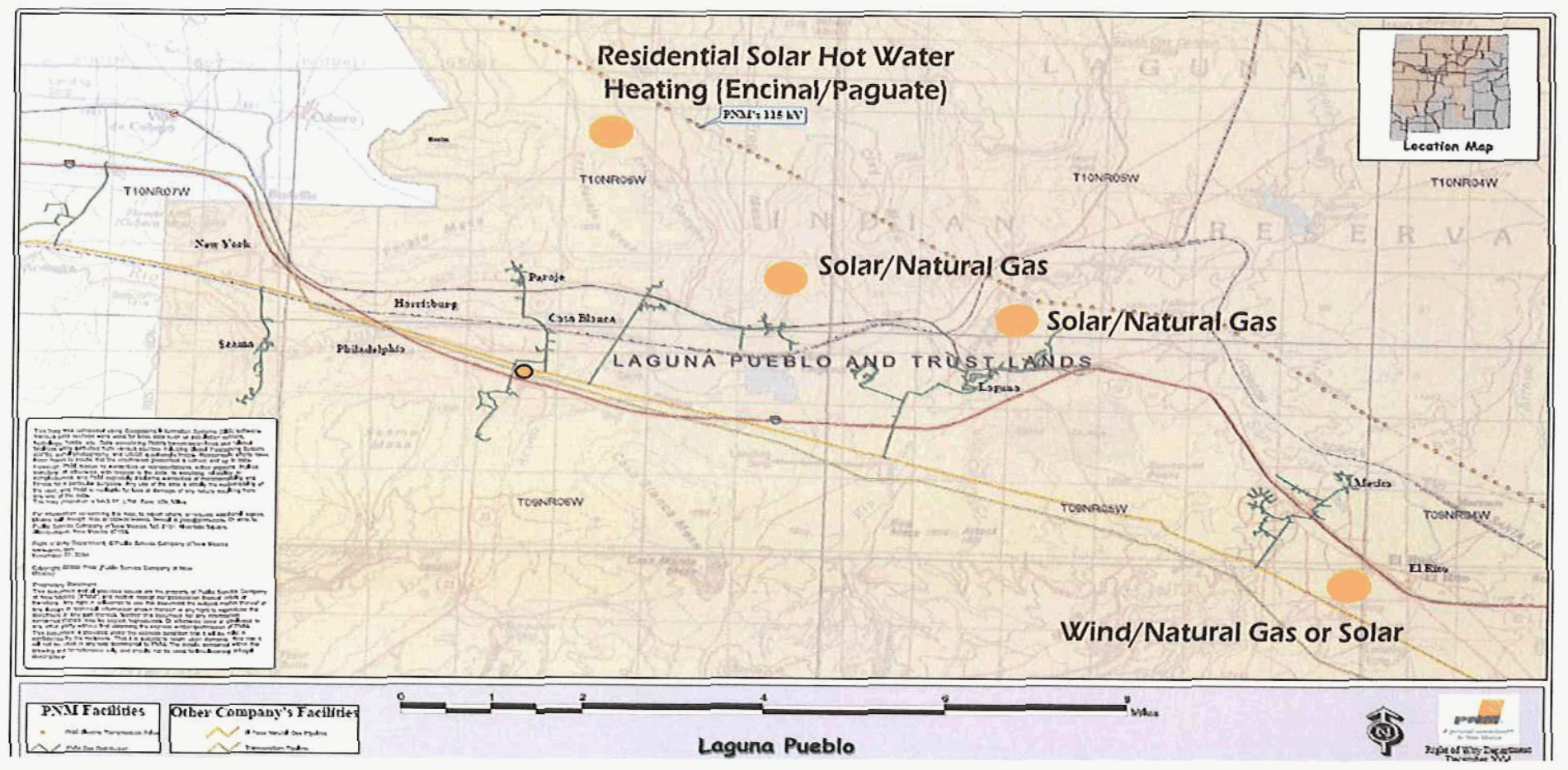




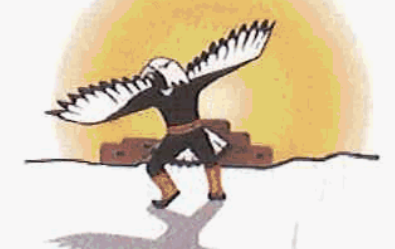

\section{Pueblo of Laguna Renewable Energy Project Options}

Project Descriptions

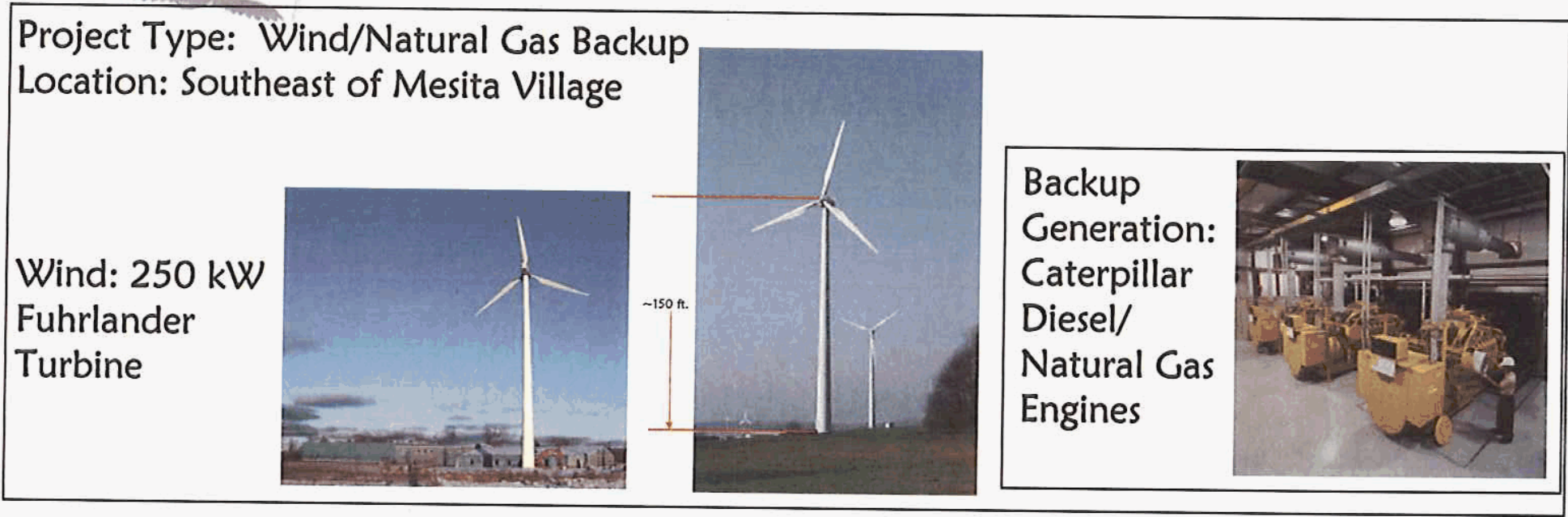

Project Type: Solar or Solar/Natural Gas Backup Location: Laguna Village, north of Transfer Station or Southeast of Mesita Village

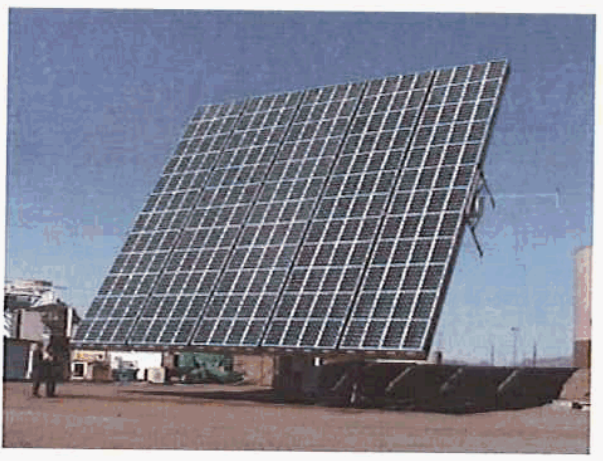

Solar: HCPV (High

Concentration

Photovoltaic)
Solar: Single Axis Tracker PV (Photovoltaic)

\section{Backup Generation:}

Caterpillar Diesel/ Natural Gas Engines

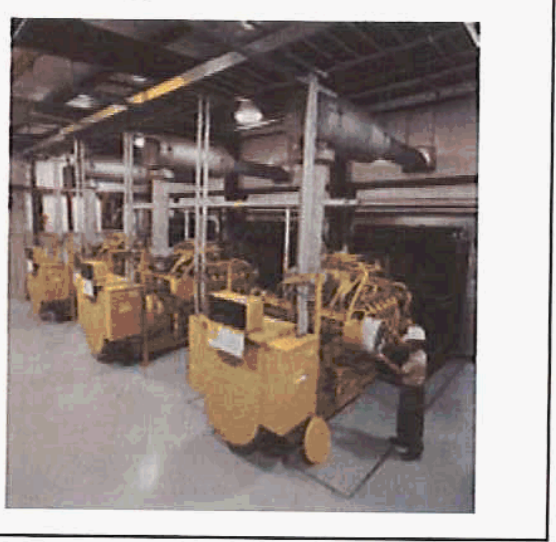

Project Type: Solar Electric or Hot Water Heating Location: Any residence, community center or commercial building
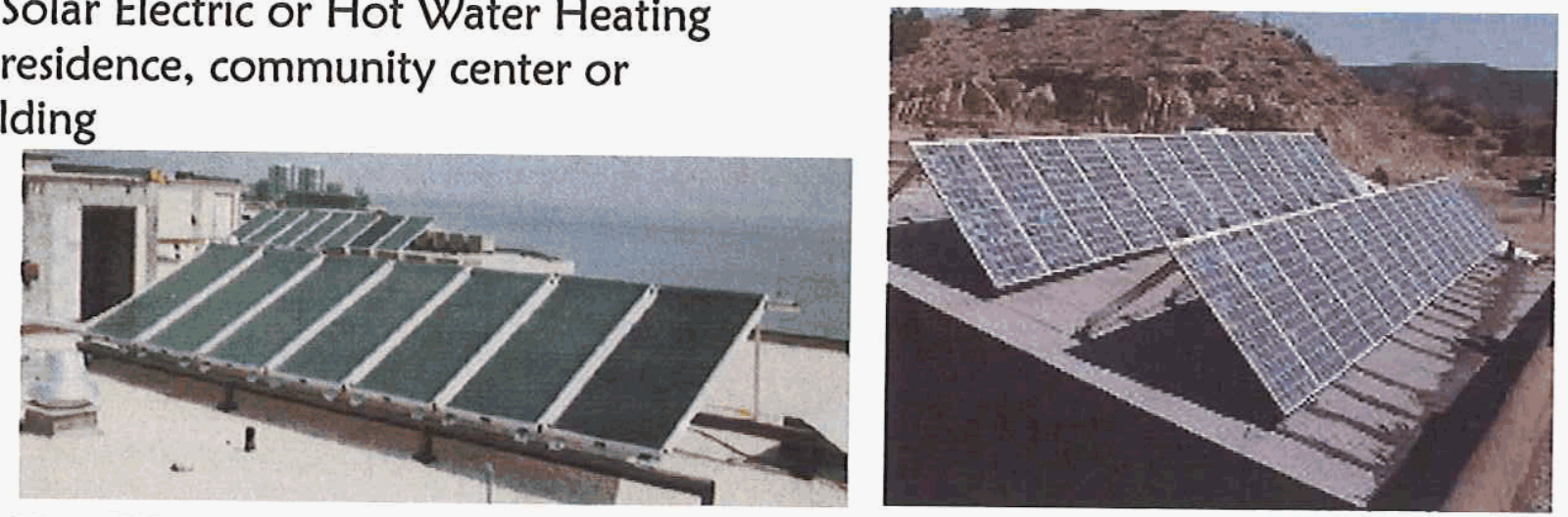


\section{Pueblo of Laguna Renewable Energy Project Community Input Questionnaire}

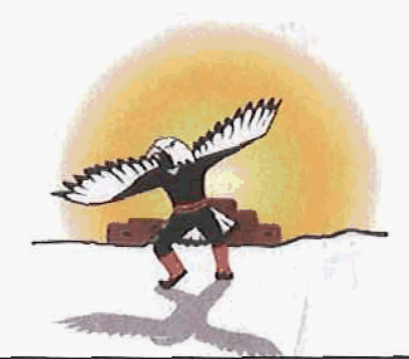

\section{Instructions for Completion}

This material is designed to provide basic information about some renewable energy project options that have been identified as part of Laguna's DOE-funded renewable energy study. Because the projects can only achieve the desired benefit if they succeed in meeting the community's needs and goals, your perspective is vital. Please help us by providing your comments, concerns and questions about the potential renewable energy projects.

\section{Community Input Questionnaire}

What is your feeling about renewable energy?

What is your feeling about solar and wind power generation?

Have you had any experience with renewable energy power generation? Yes No

If yes, please describe:

Do you feel that there is a need for renewable energy power generation within the Pueblo of Laguna? Yes No

Please explain:

Do you feel that the Pueblo of Laguna produce power to sell to others outside of Laguna? Yes No

Please explain: 


\section{Pueblo of Laguna Renewable Energy Project Community Input Questionnaire}

\section{Community Input Questionnaire, Continued}

How do you feel about renewable energy projects at the proposed sites?

Do you have a preference as to which project should be developed?

Would you like additional information on any particular project?

What results would you like to see for Laguna from a renewable energy project?

Do you have any additional questions or comments?

\section{What To Do With Your Completed Questionnaire}

Please return completed surveys to:

Thelma Antonio, Energy Program Coordinator Pueblo of Laguna Utility Authority

PO Box 517

Casa Blanca, NM 87007

$505246-4271$ or $505246-4277$
Please add you contact information here if you would like additional information: 


\section{Project Updates}

$>$ Study Update: Project Identification Meeting

$>$ POLUA Board of Directors: Status Report

$>$ POLUA Board and Entity Update

$>$ DOE Program Review October 2006

$>$ DOE Tribal Energy Program Review October 2007 
Pueblo of Laguna

Renewable Energy Feasibility Study

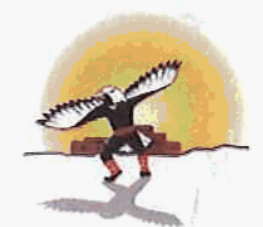

Study Update

Project Identification Meeting

March 2006

Meeting Agenda

- Introductions

- Project Background - Ken Garcia, Dave Melton, Harry Antonio

- Project Overview/Schedule - Carolyn Stewart

- Laguna Renewable Resources - Kate Maracas

- Natural Gas Access/Issues - Tracey LeBeau

- Energy Use Profile - Edward Samson, Kate Maracas

- Laguna Project Considerations - All

- Next Steps - Carolyn Stewart 


\section{Project Background}

- Utility Authority Formation - 1980s-2005

- Utility Formation and Power Development Study - 2004-2005

* Utility Organization, Implementation

$\therefore$ Electric System Issues, Alternatives

$\therefore$ Power Development Potential

- Multiple Grant Applications

$\therefore$ ANA - Utility Implementation (awarded)

* USDA - Electric System Acquisition/Improvements

$\therefore$ DOE - Electric System Negotiations

* DOE - Renewable Energy Feasibility (awarded)

* DOI - Renewable Energy Feasibility (underway)

$\therefore N M$ - Wind Feasibility (awarded)

- Foresight Energy Wind Development Agreement - May 2005

- Utility Authority Operations Begin - August 2005

- DOE Feasibility Study - Now Underway

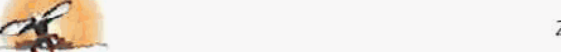

Pueblo of Laguna Utility/Energy Studies

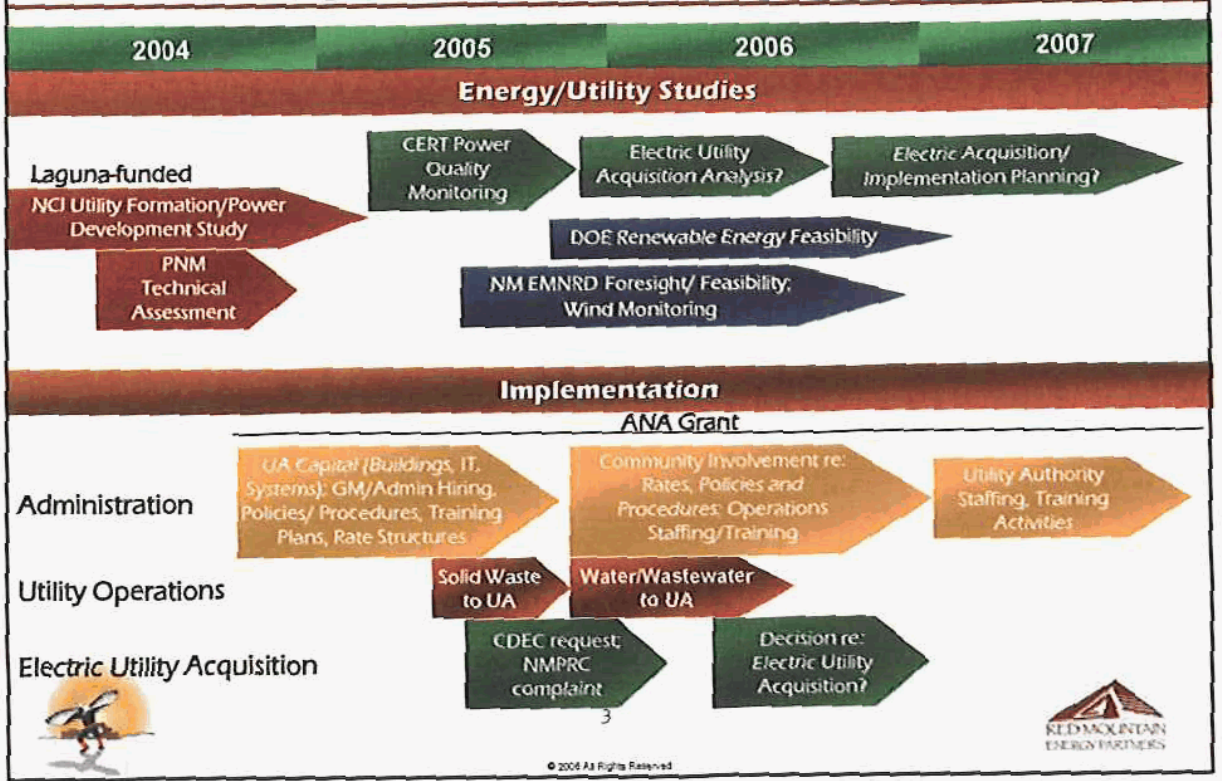




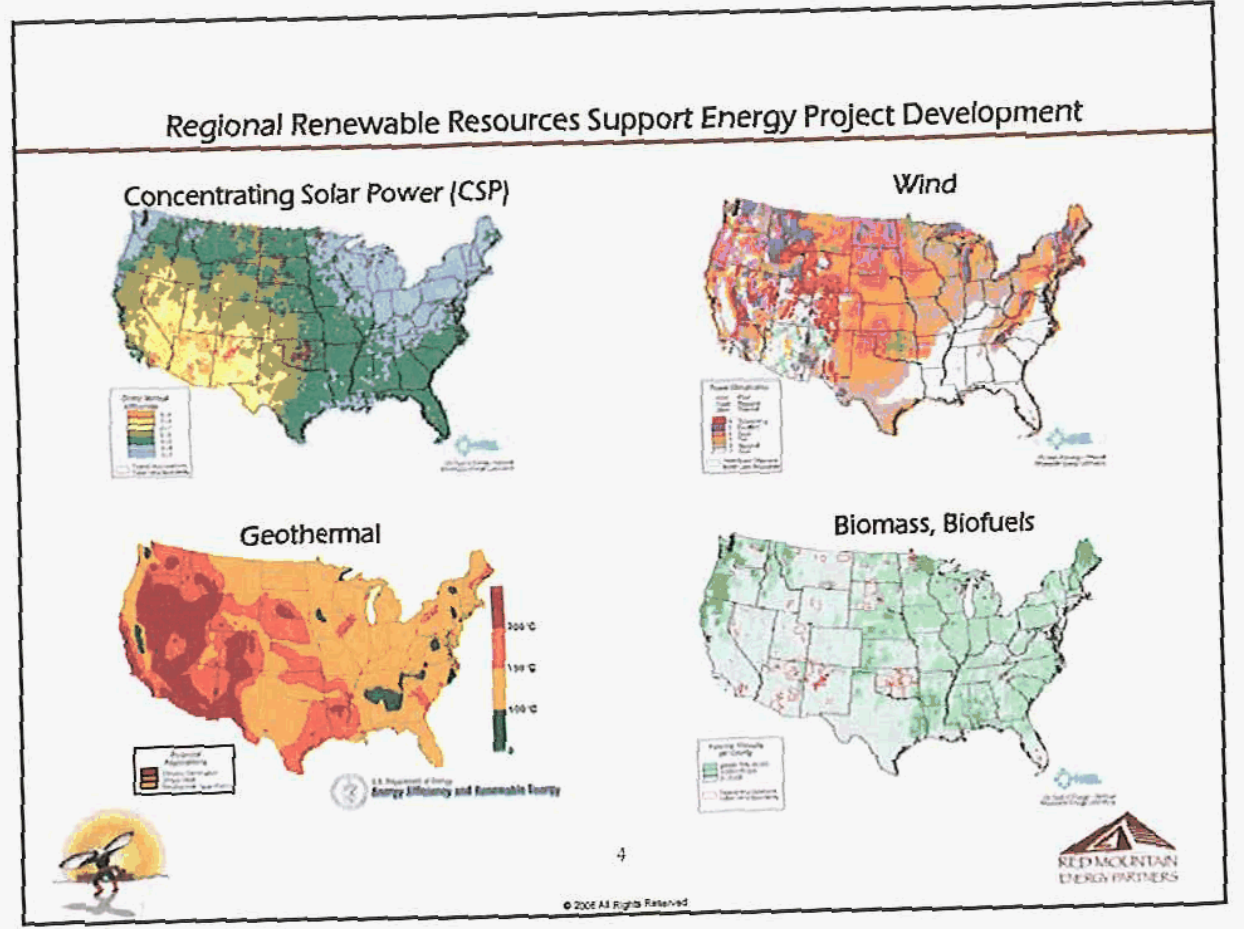

Renewable Energy Feasibility Study Purpose/Objectives

- Increase energy knowledge and capacity

- Improve quality and reliability of electric senvice

- Contribute to development of environmentally clean energy

- Provide data/analysis to support Laguna involvement in renewable energy projects as an owner or participant 
Renewable Energy Feasibility Study Scope

- Capacity Building

* Identify staff resources and provide ongoing education/support

- Community Education/Outreach

* Develop/implement community education tools (newsletter, meetings) in order to gain community support for identified projects

- Resource Monitoring

$\therefore$ Understand renewable resources, needs, and identify possible projects

- Fatal Flaw Analysis

* Identify significant issues for possible projects

- Potential Project Economics

$\therefore$ Evaluate feasibility of community-supported projects

- Project Decision Support

$\because$ Support presentation/decisions re: feasible projects

- Development Plan

$\therefore$ Create development plan, budget and possible funding sources

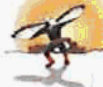

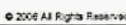
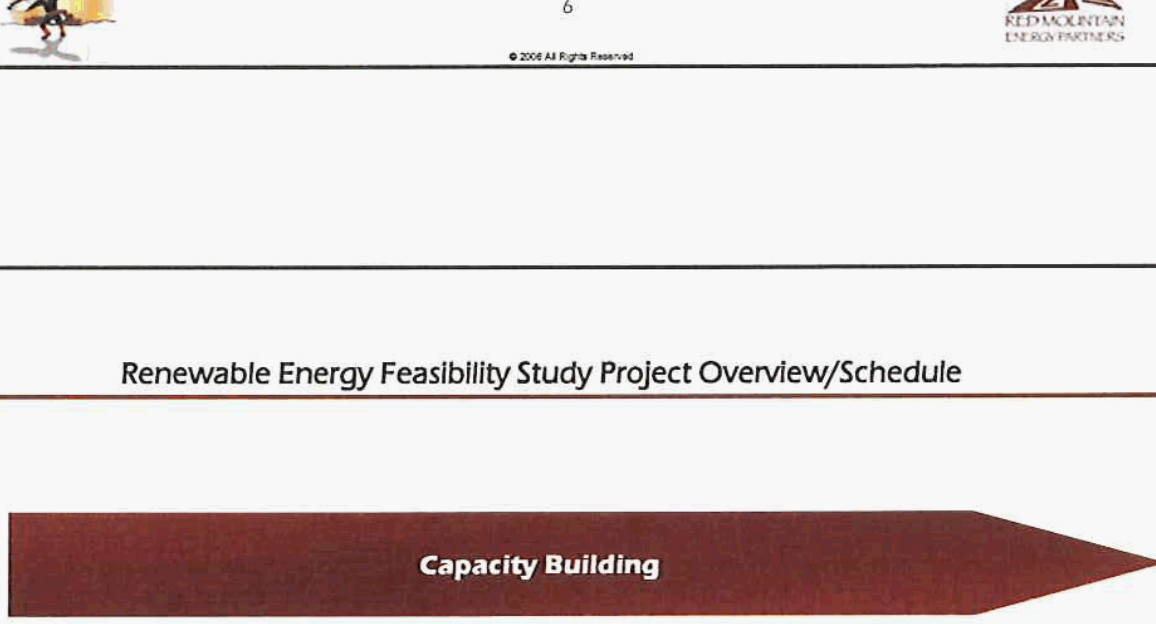

Sonwinity Edration/Outreasts and Bevion

Resource

Monitoring
Fatal Flaw

Analysis
Petental

Project

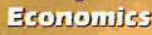

Project

Decision

Support
Development

plan 


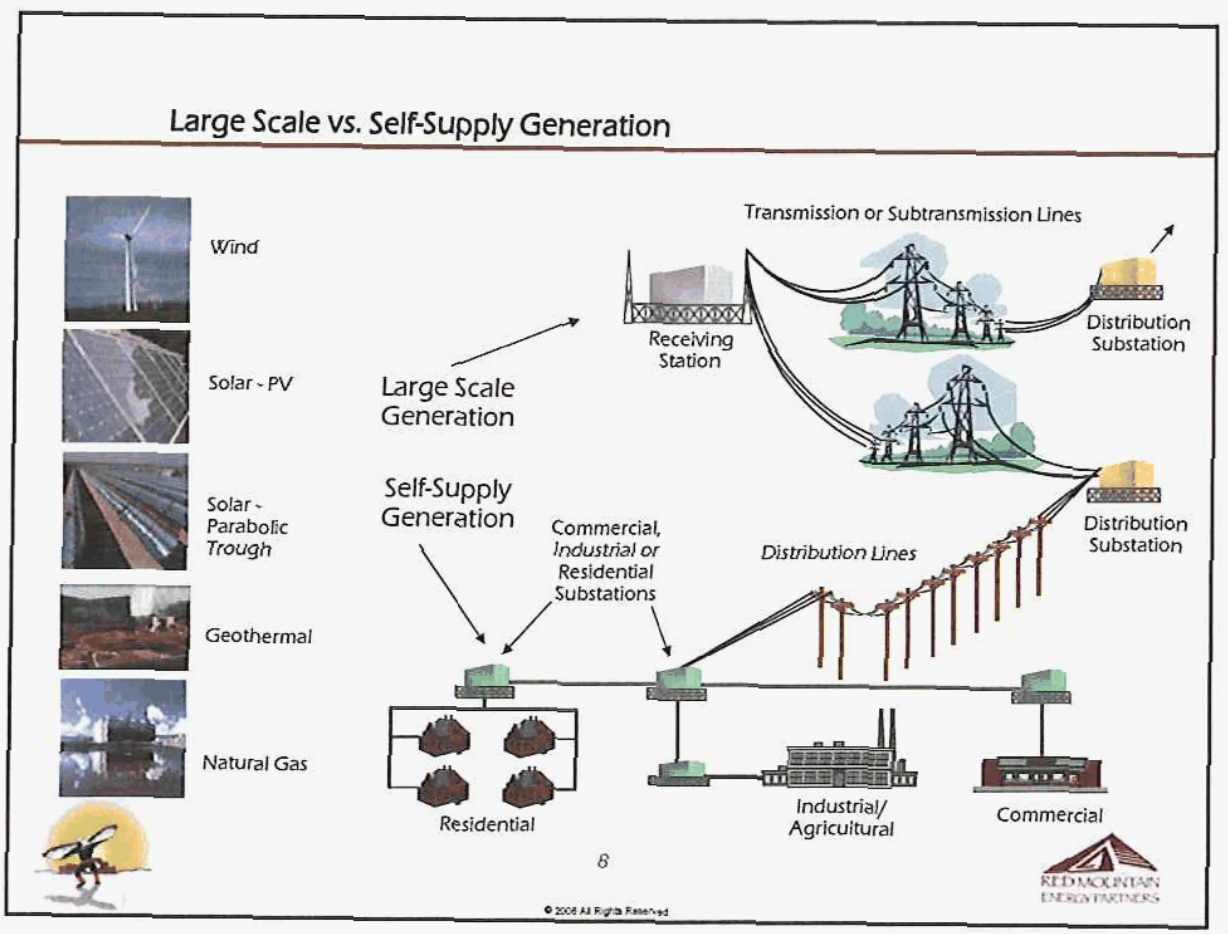

Laguna Renewable Resources Assessed

- Solar

- Wind

- Biomass

- Geothermal 


\section{Laguna Solar Resource - Direct Normal Solar Radiation}

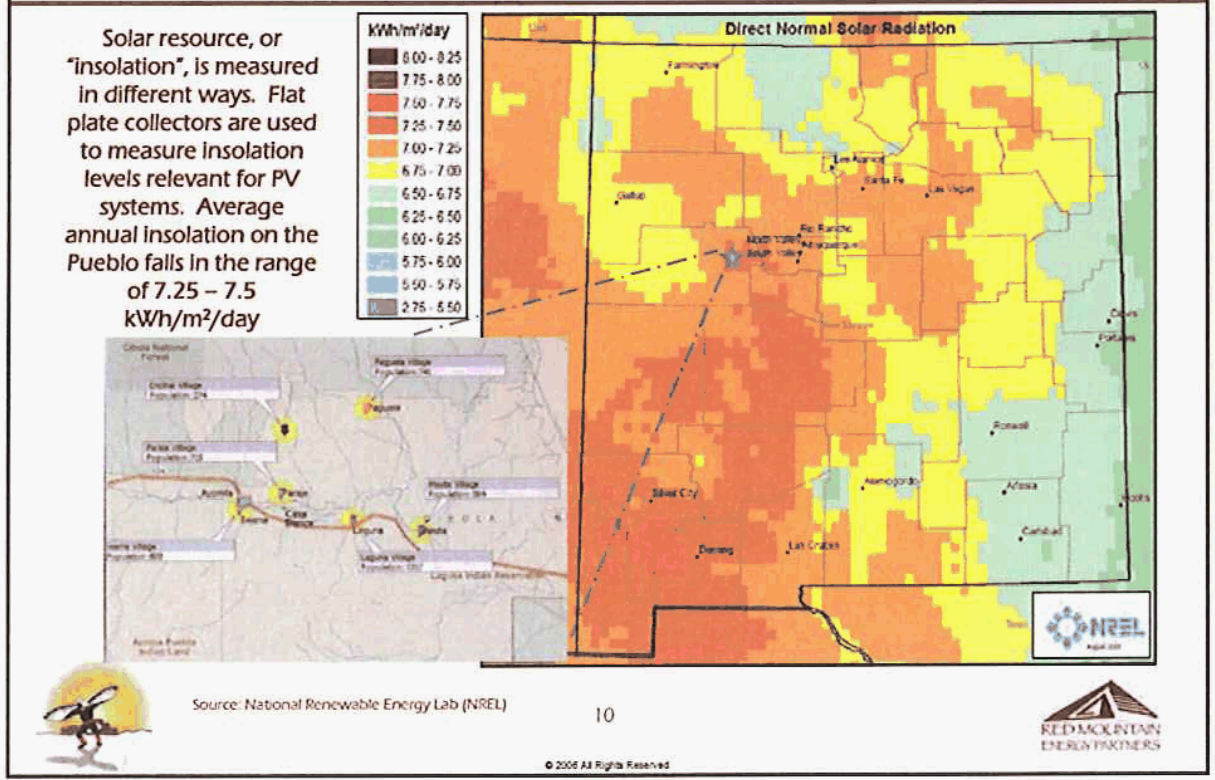

Laguna Solar Resource - Regional Prospects for Concentrating Solar Power

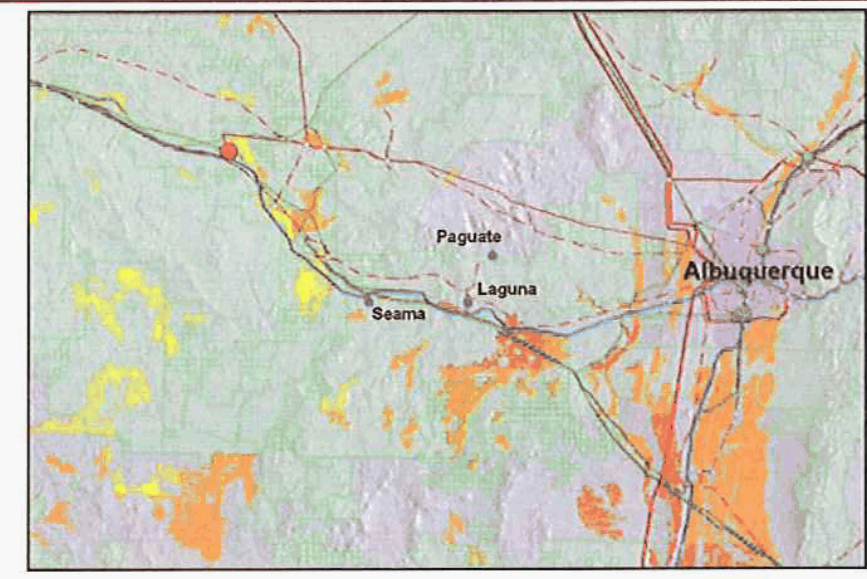

Insolation relevant for CSP technology is measured with collectors that track the sun. Parts of the Pueblo have concentrator insolation levels in the range of $7.25-7.5 \mathrm{kWh} / \mathrm{m}^{2} /$ day, well within the range suitable for large-scale solar projects

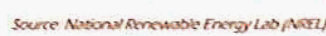

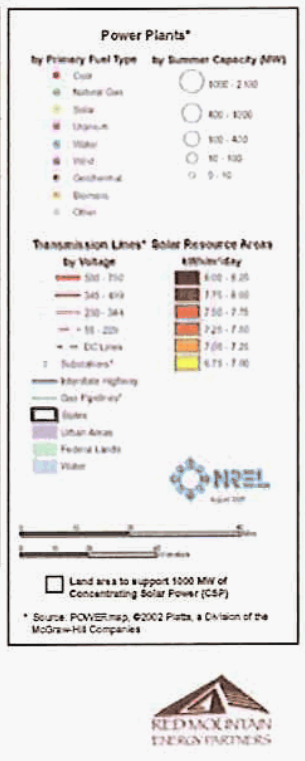



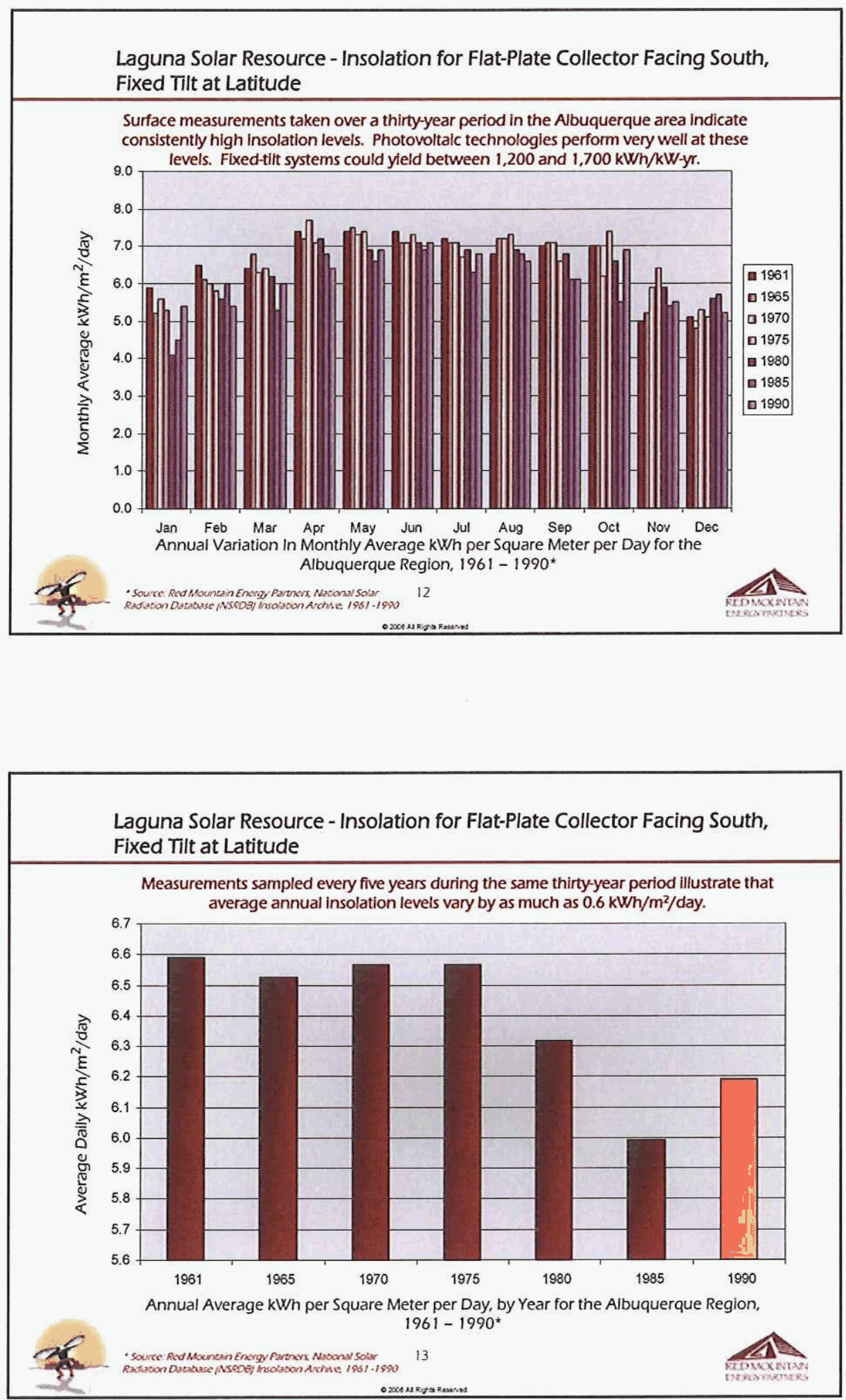

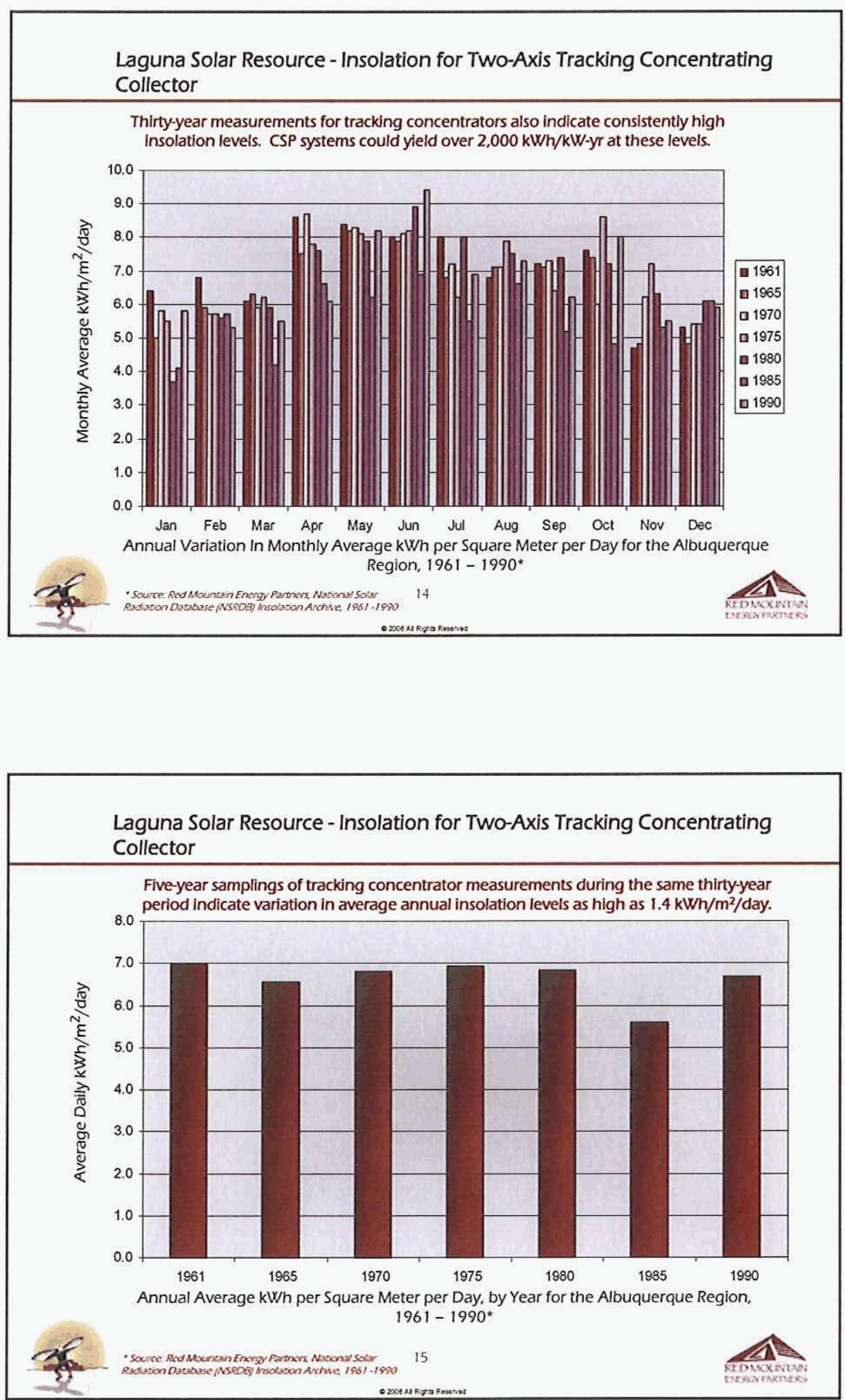
Solar Energy Technology Options: Residential \& Commercial Solar Systems

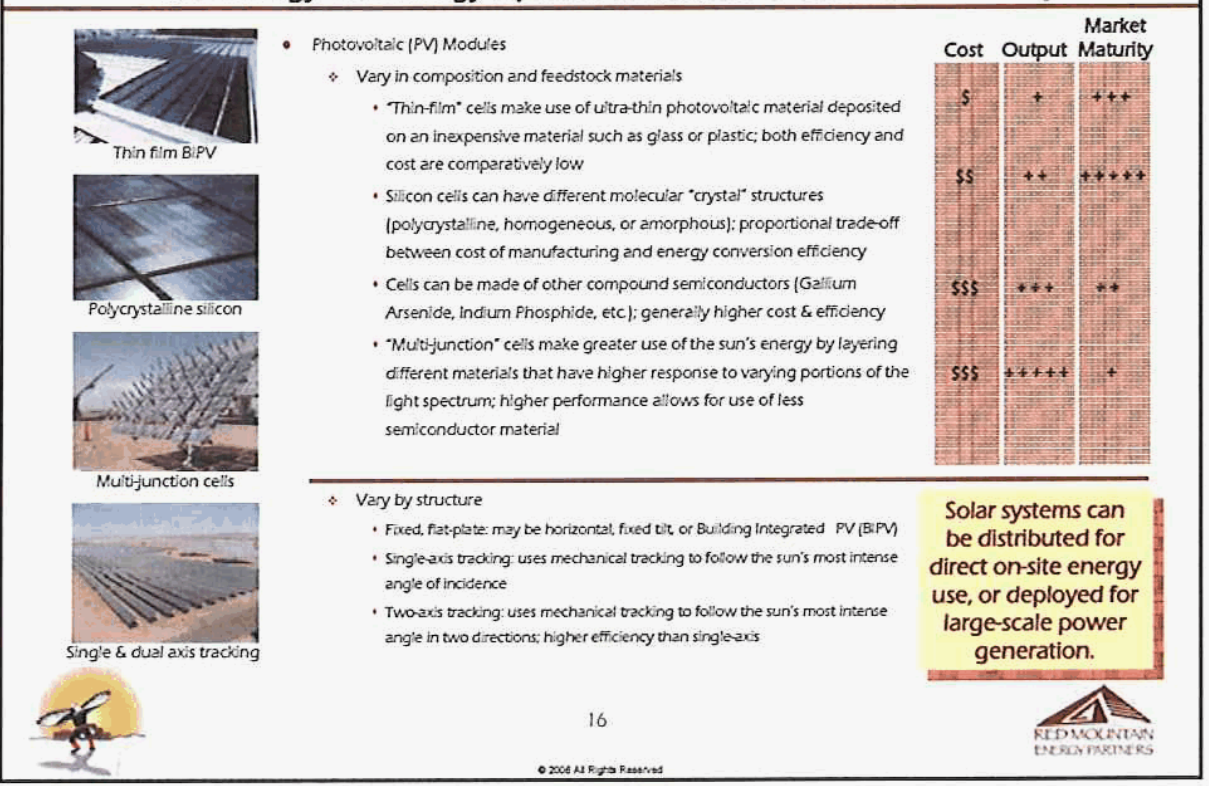

Solar Energy Technology Options: Large-Scale Solar Systems

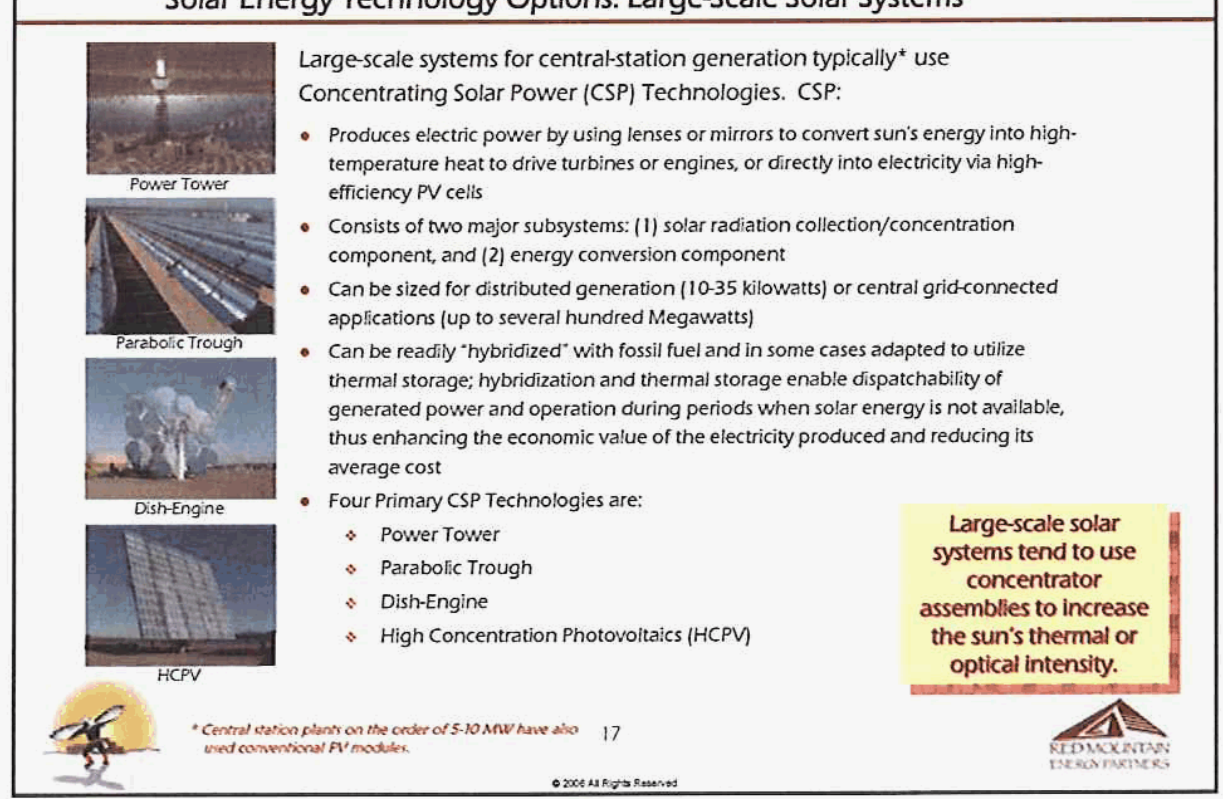




\section{Solar Energy Technology Options: Large-Scale Solar Systems}

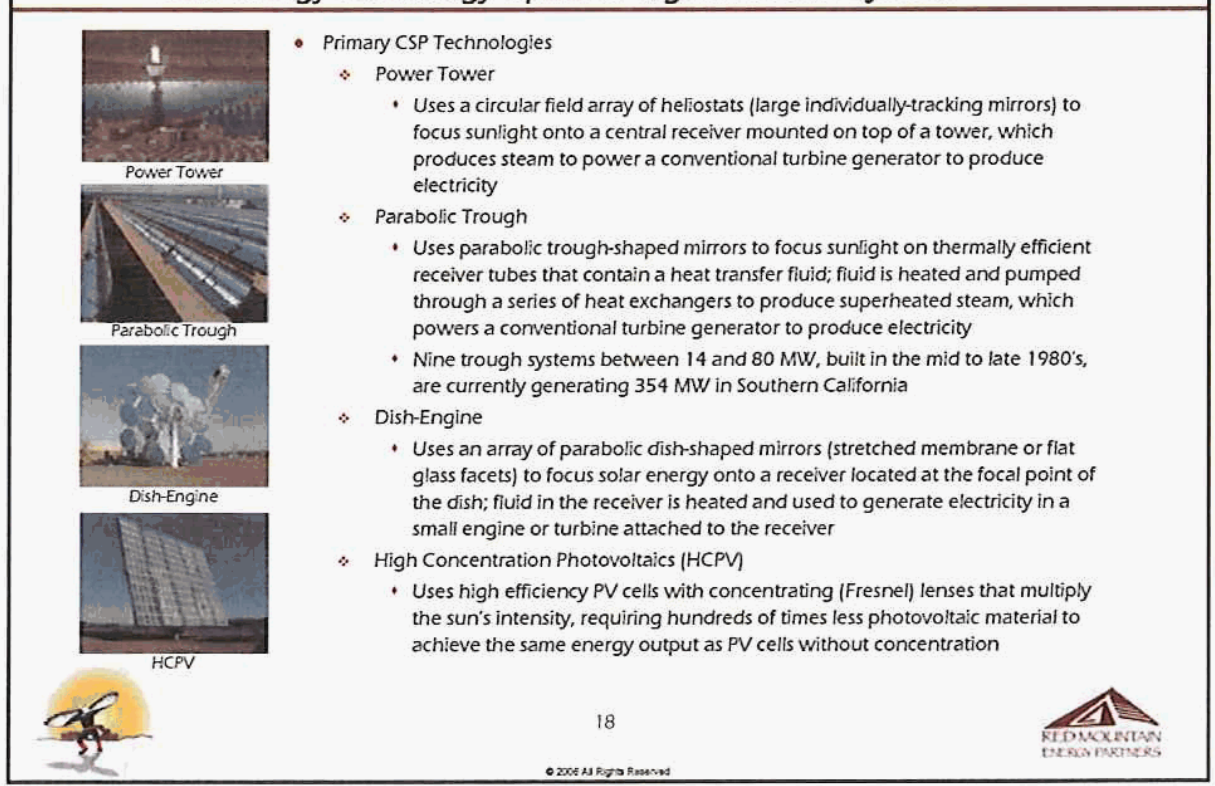

\section{Solar Energy Technology Options: Comparisons of Cost and Performance}

Examination of $\mathrm{kWh}$ delivered per dollar expended is useful in considering technology investment decisions. Some solar technologies may have lower first-costs, but also much lower performance, and thus longer payback periods.

\begin{tabular}{|l|c|c|c|}
\hline Installation & Cost (S/Watt) & $\begin{array}{c}\text { Performance } \\
\text { (kWh/kW/yr) }\end{array}$ & kWh/s \\
\hline Fixed-Horizontal & 5.25 & 1,250 & 4.75 \\
\hline Flxed-Latitude & 5.25 & 1,630 & 6.20 \\
\hline Tracking-Horizontal & 5.50 & 2,350 & 8.55 \\
\hline Tracking-Latitude & 6.50 & 2,450 & 8.25 \\
\hline HCPV & 6.00 & 2,030 & 6.75 \\
\hline Parabolic Trough & 4.00 & 2,000 & 10.00 \\
\hline HCPV-Future & 3.00 & 2,400 & 16.00 \\
\hline Dish/Turblne-Future & 2.50 & 2,400 & 19.20 \\
\hline
\end{tabular}


Technology Fit with Laguna Solar Resource - PV Alternatives

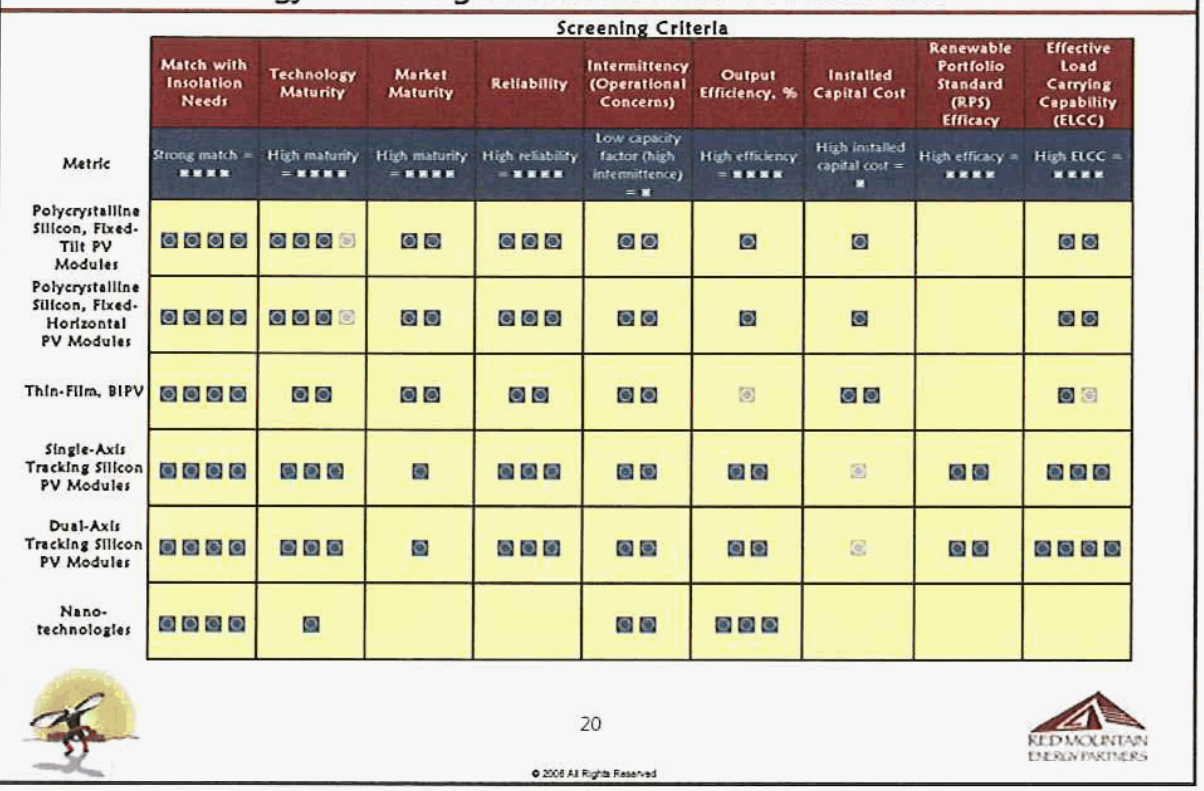

Technology Fit with Laguna Solar Resource - CSP Alternatives

\begin{tabular}{|c|c|c|c|c|c|c|c|c|c|}
\hline & \multicolumn{9}{|c|}{ Screening Criterla } \\
\hline & $\begin{array}{l}\text { March with } \\
\text { insolvilon } \\
\text { Neess }\end{array}$ & $\begin{array}{l}\text { Teeinolony } \\
\text { Maturily }\end{array}$ & $\begin{array}{l}\text { Market } \\
\text { Maturity }\end{array}$ & Rellability & $\begin{array}{l}\text { Intermitirendy } \\
\text { (Opertilonal } \\
\text { Concerms) }\end{array}$ & Oulput & $\begin{array}{l}\text { Instiled } \\
\text { Coplteil Cont }\end{array}$ & 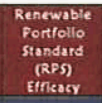 & 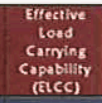 \\
\hline $\begin{array}{l}\text { Matric or } \\
\text { Welghtlng } \\
\text { Costrfleant }\end{array}$ & Mrong match & $\begin{array}{l}\text { High } \\
\text { maturity- } \\
\text { Wer. }\end{array}$ & $\begin{array}{l}\text { Hiles } \\
\text { maturicy:- } \\
\text { Wres: }\end{array}$ & $\begin{array}{l}\text { Miss } \\
\text { melasilizy - }\end{array}$ & 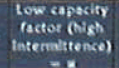 & 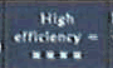 & 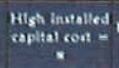 & High encesy & mest nece \\
\hline 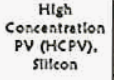 & 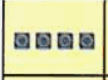 & 임 & 回 & 圆回回 & 回圆 & 图圆 & 圆 & 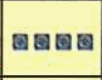 & 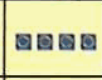 \\
\hline $\begin{array}{l}\text { HCPV, Multt- } \\
\text { Junction PV } \\
\text { Cells }\end{array}$ & 回回回 & 回 & 圆 & 回回 & 国圆 & 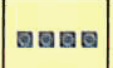 & 回 & 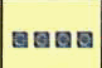 & 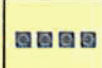 \\
\hline $\begin{array}{c}\text { Dish-Engine } \\
\text { Systems }\end{array}$ & 回园园回 & 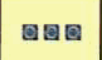 & ㅁ? & 回园 & 빔 & 回回回 & 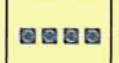 & 미미뭉 & 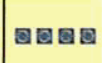 \\
\hline $\begin{array}{l}\text { Dish-Turtine } \\
\text { with Storage }\end{array}$ & 回回回 & & & & 回 & 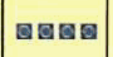 & ๘ & 回国圆圆 & 回回回回 \\
\hline $\begin{array}{l}\text { Parbollte } \\
\text { Trough }\end{array}$ & 回圆回四 & 国圈回 & 圈圆 & 国圆圆圆 & 回回 & 回回圆 & 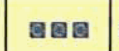 & 娄国圆圆 & 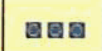 \\
\hline Power Tower & 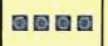 & 圆圆 & E & 娄圆 & 빕 & 圆回回 & 圆 & 回回回圆 & 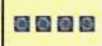 \\
\hline
\end{tabular}


Technology Fit with Laguna Solar Resource

\section{Observations}

- Technology selections for any project should be weighed against considerations specific to the Tribe's needs and objectives

* Insolation levels in and around the Pueblo appear to be well-suited for most solar technologies

* Large scale systems are a better fit if RPS Efficacy, and sales of Renewable Energy Credits (RECS) are a goal

$\because$ For distributed generation applications, ELCC is an important criterion in determining a system's payback period

* Output efficiency and installed capital costs must be examined by pro forma analysis to understand the true costs and revenue potential of produced energy

$\therefore$ Intermittency and operational concerns are relevant to both distributed (on and off-grid) and centralized projects

\section{Observations}

- Insolation levels in and around the Pueblo villages well-suited to support distributed and centralized photovoltaic applications

- Cooler temperatures in the high-desert region yield higher operating efficiency for photovoltaics

- Insolation appears to be sufficient for larger scale CSP technologies, but actual site measurements required to support project investment decisions

* Project economics for installations in even higher insolation areas highly dependent upon financing, project structure, enabling incentives, etc.

* Annual kWh produced and sold will make or break project profitability, and thus accurate resource characterization is critical

* Technology advances and volumetric deployments expected to bring capital costs down in the next several years 

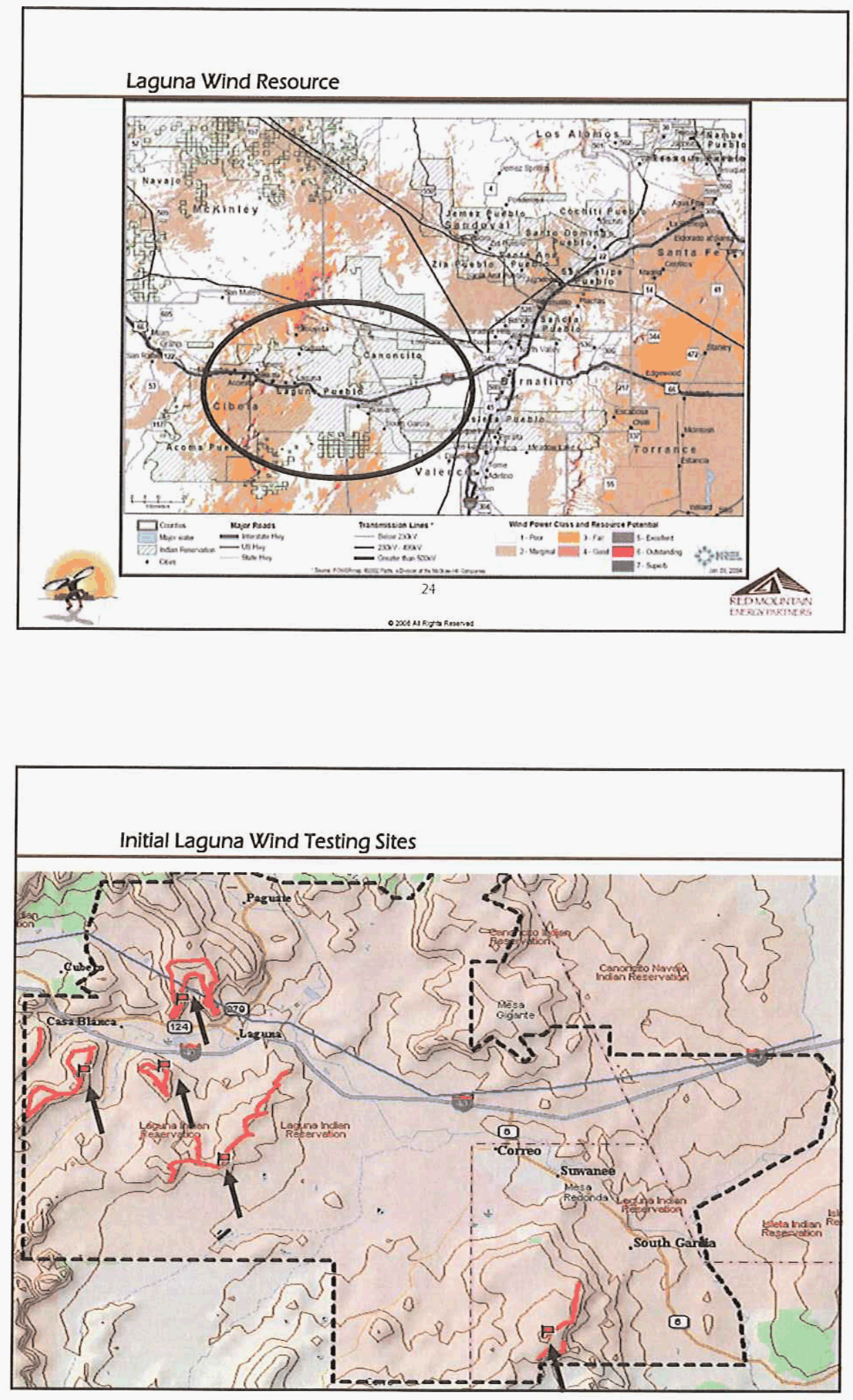

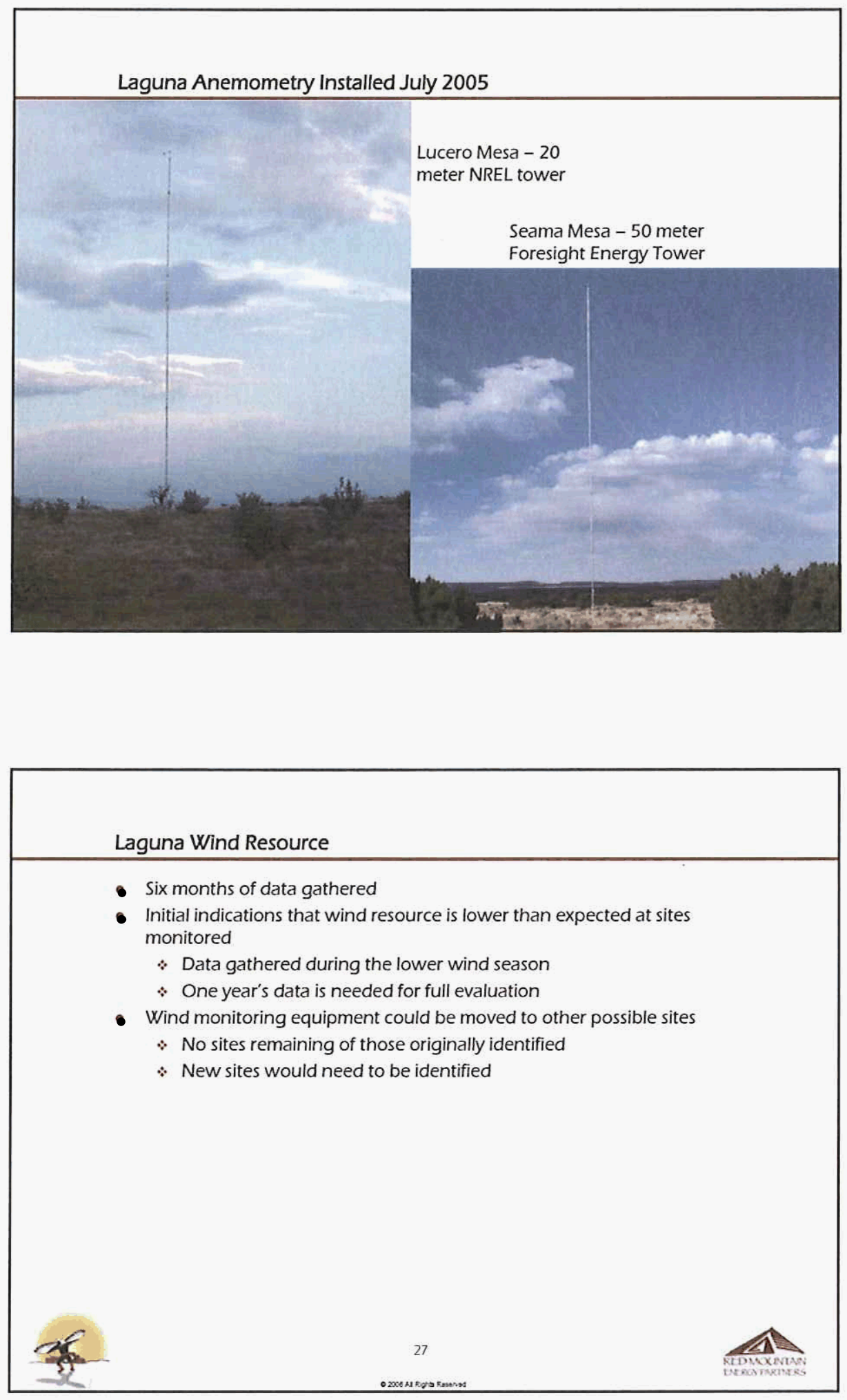
Wind Technology

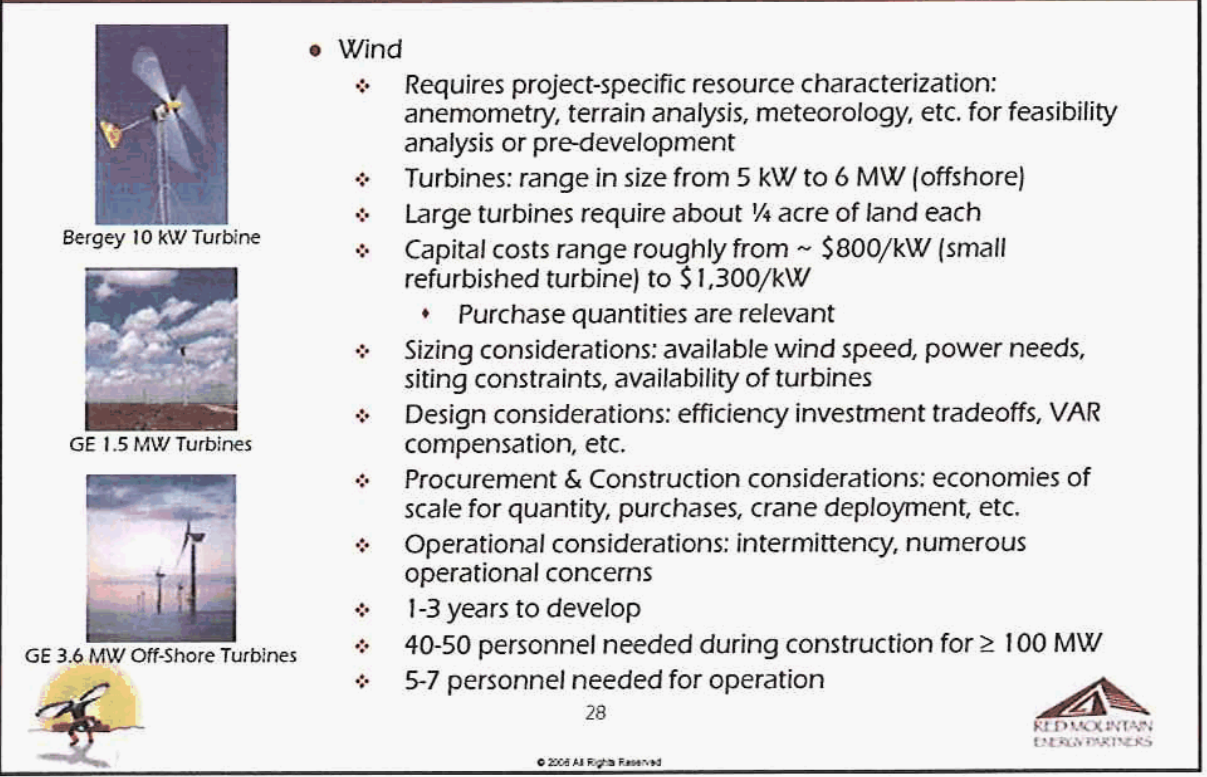

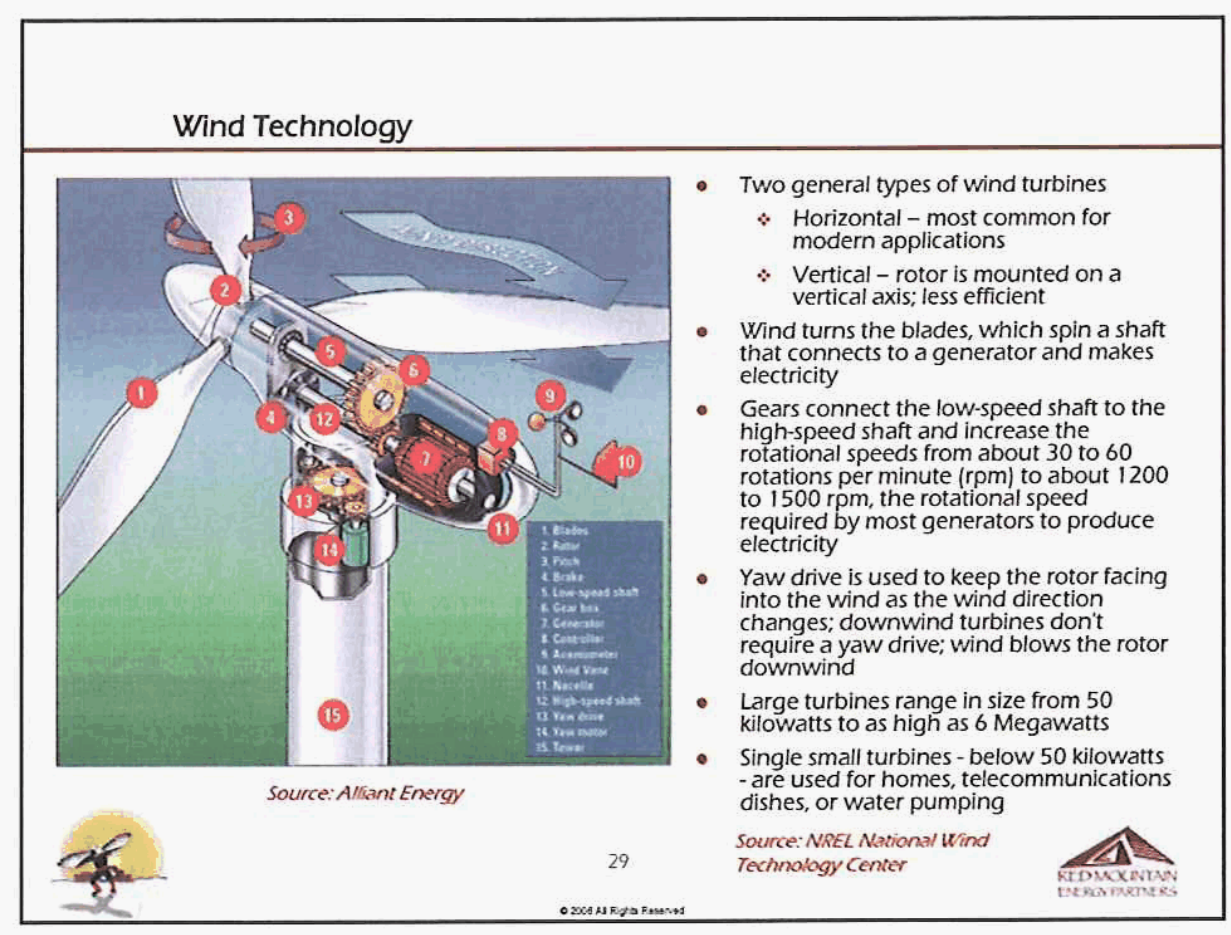




\section{Wind Technology Overview}

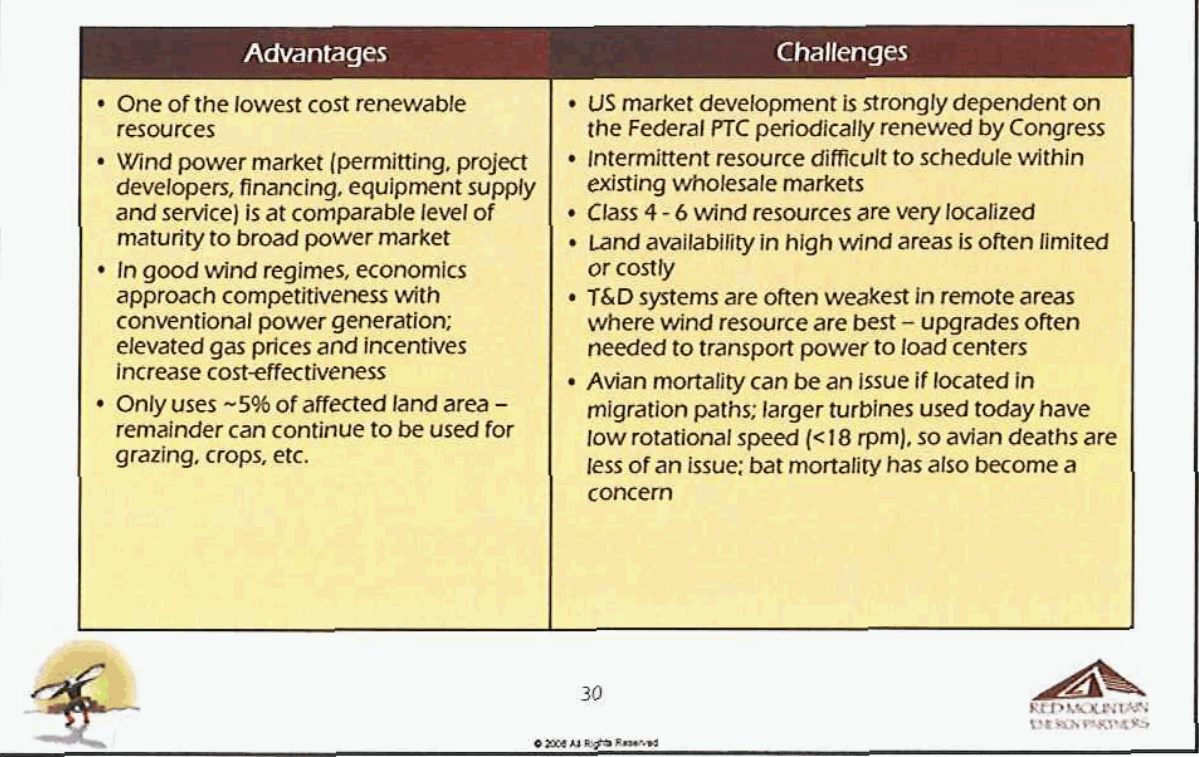

\section{General Wind Project Economics}

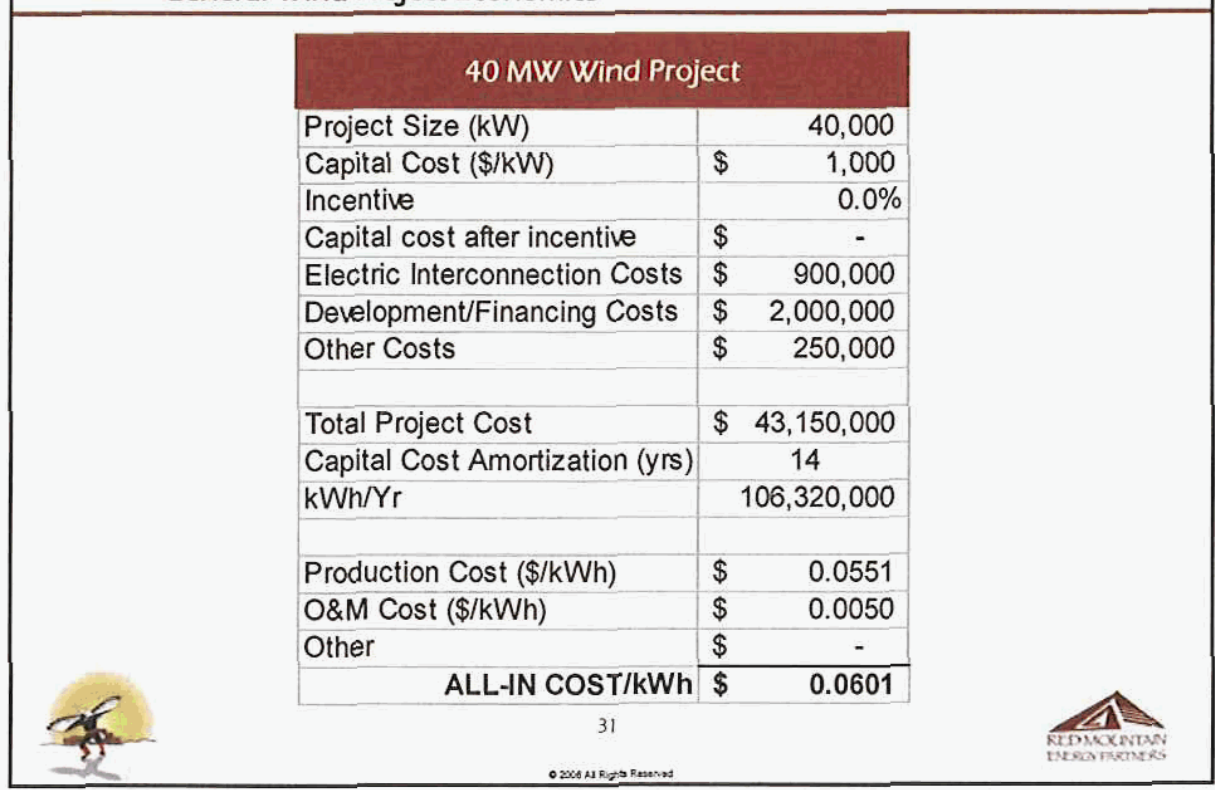


Foresight Development Agreement - Summary of Timeline and Activities Foresight Responsibilities

Phase I

- Identify potential sites, install met testing equipment, collect data $6-12$ mos - Apply for state, federal, and other grants - Prepare technical report describing conditions for each tested site

- Deliver report within 13 months after

installation of met tower

Move towers to alternative sites, if needed

up to 9 months into the term
Phase II

- Physical and cuitural evaluation of proposed site(s)

- Prepare detailed financial projections

- Discuss equity participation agmts

- Execute Power Purchase Agreements

- Prepare Interim Development Report

- Commence land payment option of

$\$ 25,000$ peryear upon execution

an Easement Agreement for site
Phase III

- Continue land option payments

- Finalize PPAs for the Project's output

- Finalize project sale to new owner or

oversee financing and construction of facility

- Finalize financing of Project, utifizing a minimum $50 \%$ project debt, or provide notice that lead equity investor will contribute up to $100 \%$ of capital costs

\section{Mores}

Laguna Responsibilities

- Provide/assist access to potential project sites

for monitoring equipment

- Assist in applying for federal, state, and other grant applications, and administering funds

- If Foresight concludes wind resource is adequate, and provides evidence of ability to commit additional $\$ 250,000$ for

development, Parties extend Agreement.

not, Foresight delivers all data and reports

to Laguna; Agreement will expire

- Seek tribal approval to execute Grant of Easement with B!A

- Allow foresight to undertake legally permissible activities on sites selected

- Administer grant funds and assist

Foresight to develop and market Project

- Parties enter into long-term Easement Agreement for project site.

- Primary Agreement: payments for

greater of 1) $\$ 2,500$ per MW of turbine capacity/yr, escalated by $5 \%$ each five years; or 2) $3 \%$ of Gross Operating Proceeds during Operating Period
Execute Equity Agreement w/option fo up to $20 \%$ equity or provide Laguna annual cash payment of $\$ 50,000$ - Execute Post Operating Period Agreement, owner responsible for removing equipment/restoring land to original condition or transferring $100 \%$ ownership to Laguna. PPA extension extends Easement Agreement and increases Royalty Payments to greater Gross Operating Proceeds

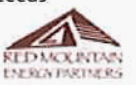

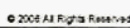

Laguna Biomass Resource

- Organic material available on a renewable or recurring basis:

$\therefore$ Forest-related materials

$*$ Agricultural-related materials

* Animal waste

* Solid woody waste materials

* Crops and trees planted for the purpose of being used to produce energy

* Landfill gas, wastewater treatment gas, and biosolids

* Segregated municipal solid waste ("MSW"), excluding tires, medical and hazardous waste 


\section{Laguna Biomass Resource}

- Forest - Adequate resources, with over 150,000 acres of forest; no existing logging infrastructure/forest management activity

- Agriculture: Extremely limited to non-existent; agriculture limited to small family plots of corn, chile, alfalfa and assorted vegetables. No discernible residue stream

- Animal wastes: Non-existent. No confined animal operations /dairy, feedlot, poultryl

- Landfill gas: Non-existent. Several abandoned landfills. Contents "burned" before backfill, not lined nor monitored. Gas production likely to be low because of small size/capacity and low rainfall in region

- Food residues: Yellow grease from restaurants hauled by septic firms; insufficient amounts to justify biodiesel production

- Wastewater treatment: Mostly uncovered lagoons with no methane recapture; wastewater treatment facility biosolids sent to digester in $A B O$

\section{Laguna Biomass Resource}

- Municipal solid waste: 940 households; handled by Utility Authority

$\therefore$ Delivered to transfer station and then to Albuquerque landfill

* Not likely enough tonnage to warrant consideration of MSW

- Transfer station: Modest amount of woody biomass from local $C \& D$ is ground up for free use by anyone

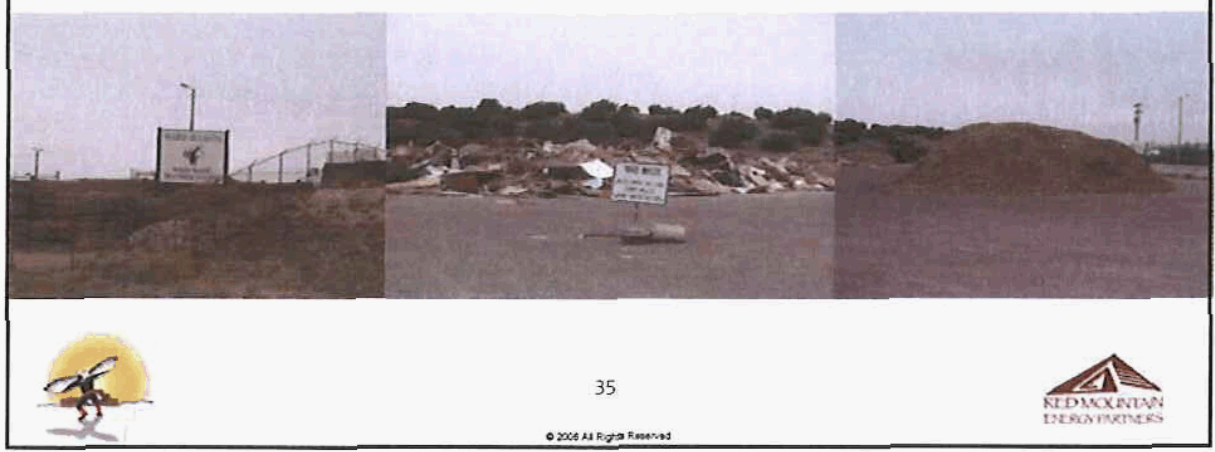




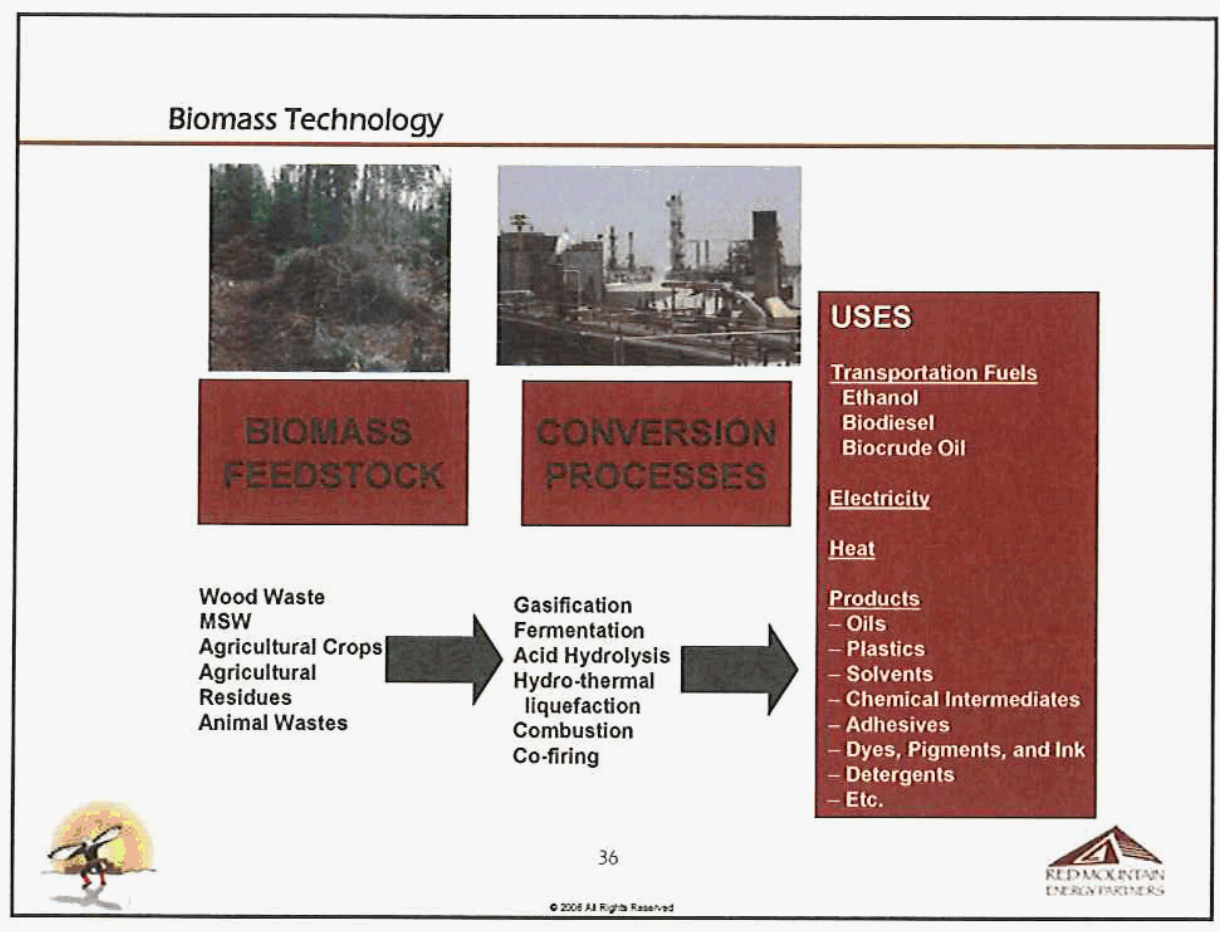

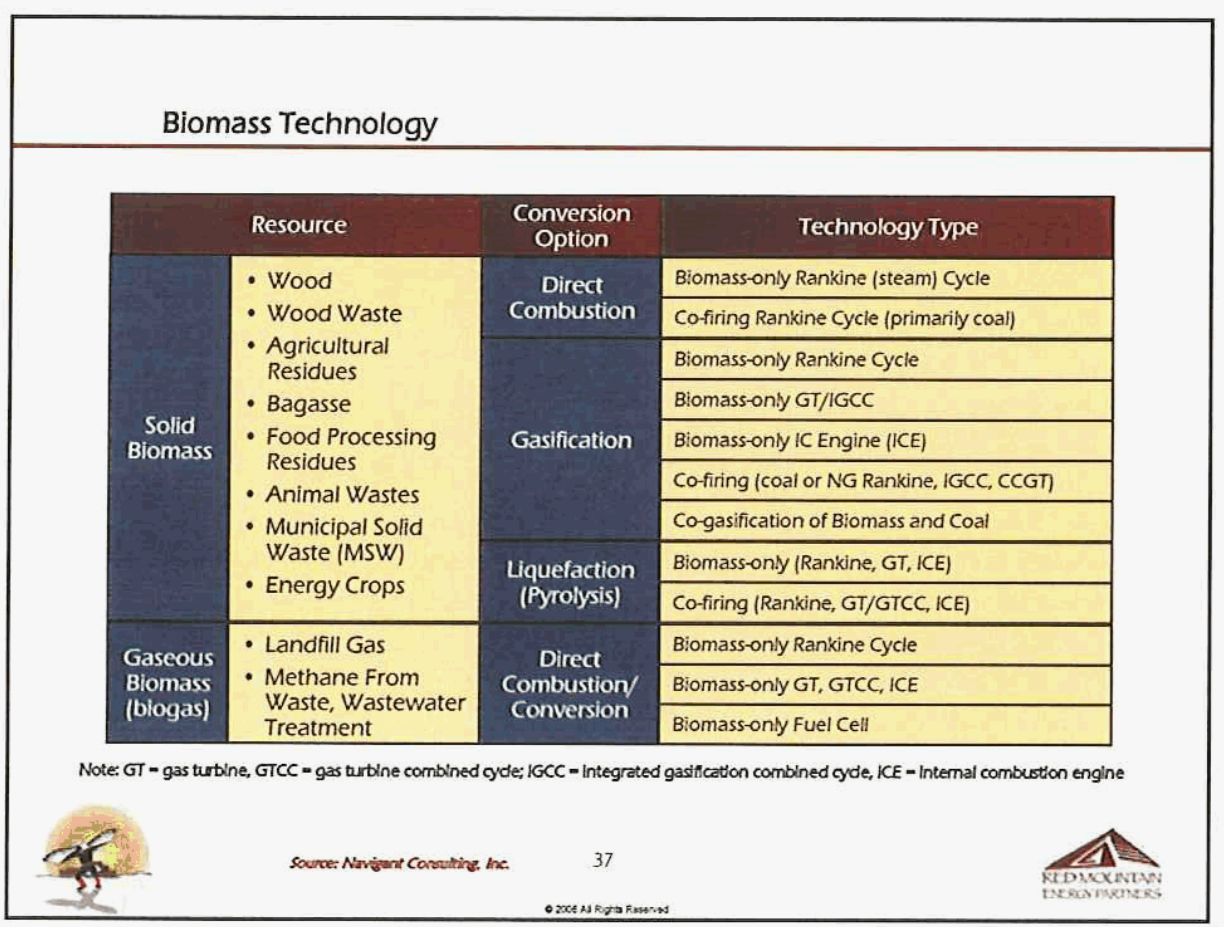




\section{Direct Solid Biomass Combustion and Gasification}

\section{Advantages}

Challenges

- Biomass combustion is proven technology

- Dispatchable, high capacity-factor

- Creates additional economic value for agriculture or biomass-based industries /energy cost savings via cogeneration, or revenue from sale of biomass residues to power plants]

- Adds more capacity to the overall power supply mix (vs. co-firing at existing fossil plants, which only displaces existing capacity)

- Avoids or reduces cost of disposal (waste $=$ fuel)

- Biomass gasification combined cycle promises higher efficiency and lower emissions relative to biomass combustion - should be easier to permit than direct combustion for distributed applications) investment renewable technologies operating a power plant

- High capital costs $(\$ 1,500-2,500 / \mathrm{kW}$, depending on size, higher for distributed applications)

- Combustion has low efficiency (20-25\%) (lower

- Requires long-term biomass fuel supply at reasonable price to avoid risk of stranded

- Limited incentives relative to other major

- Can be difficult to site due to emission concerns

- For distributed systems a lack of business models to address ownership and operation, since site owners may not have the interest in owning and

- Biomass gasification combined cycle is not commercially proven and progress has been slow

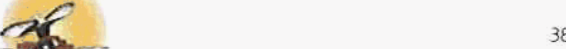

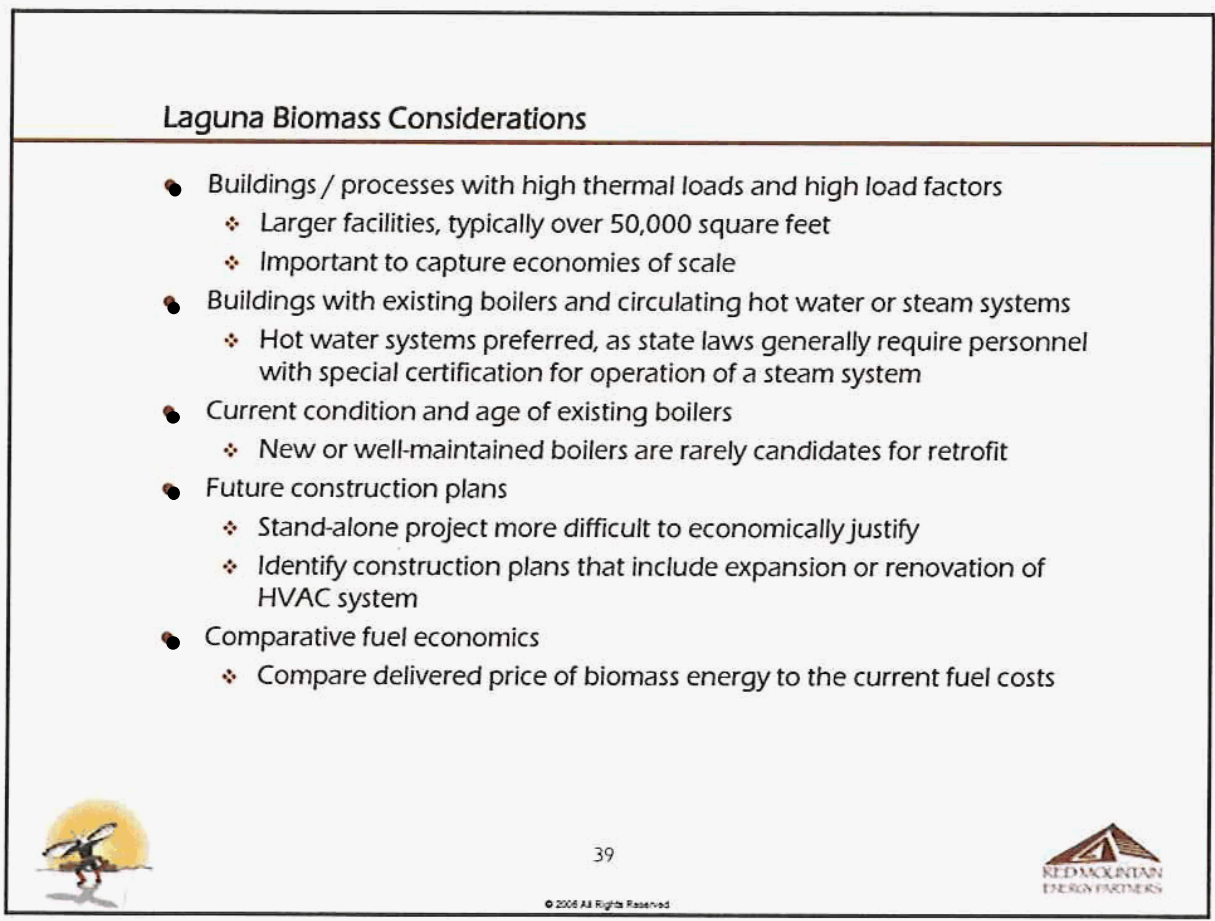




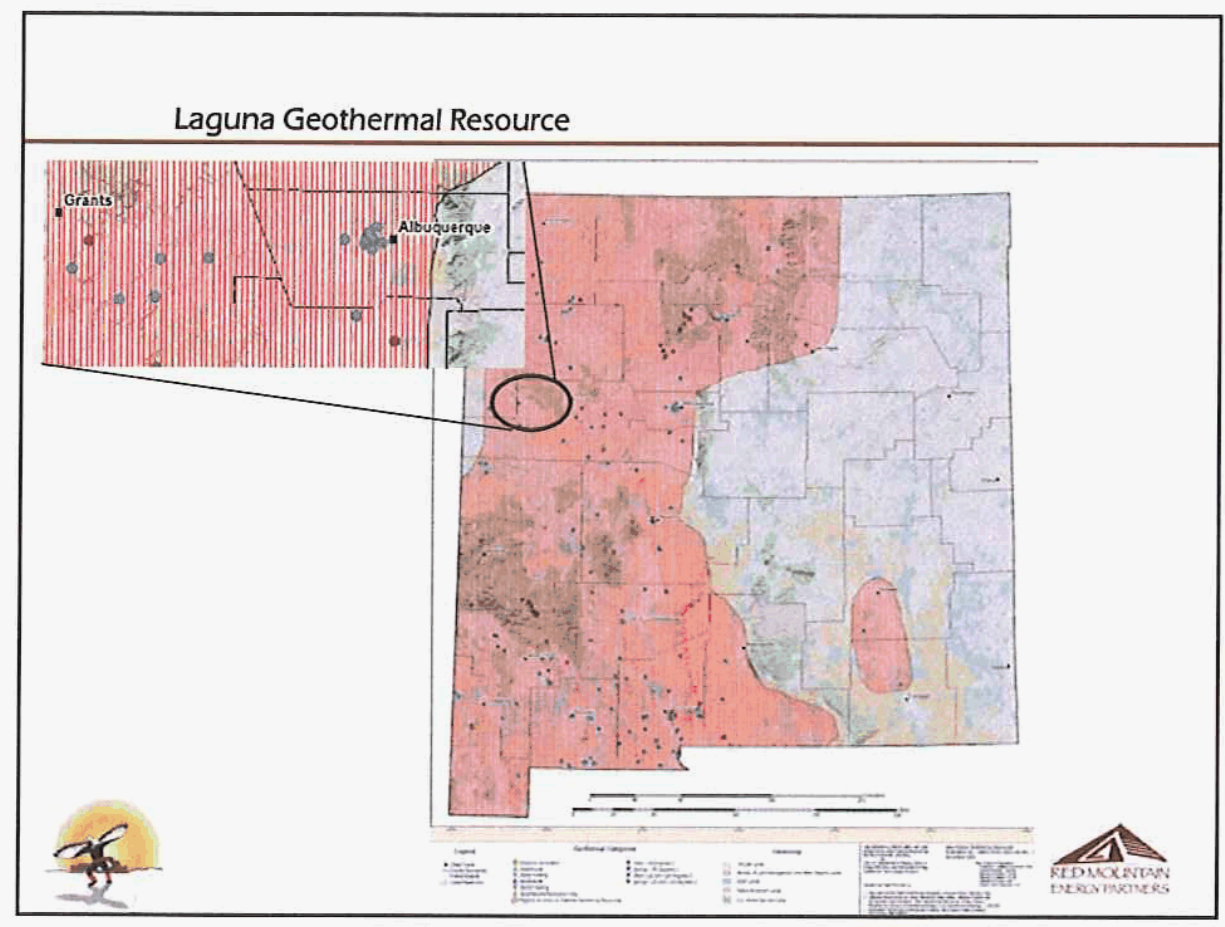

Large-Scale Geothermal Technology

Geothermal power has an established track record, with the US and Philippines accounting for $50 \%$ of the installed capacity.

- Dry steam plants use hydrothermal fluids that are primarily steam. The steam goes directly to a turbine, which drives a generator that produces electricity. These resources are the least common

- Hydrothermal fluids above $360 \mathrm{~F}$ can be used in flash steam plants to make electricity. Fluid is sprayed into a tank held at a much lower pressure than the fluid, causing some of the fluid to rapidly vaporize, or "flash." The vapor then drives a turbine, which drives a generator.

- Binary plants use fluids below 400F. Heat from the geothermal fluid is used to boil a low-boiling point fluid, which then drives the turbines. These resources are the most common.
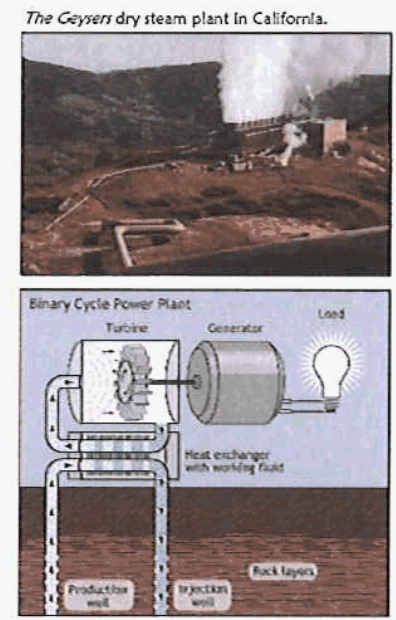

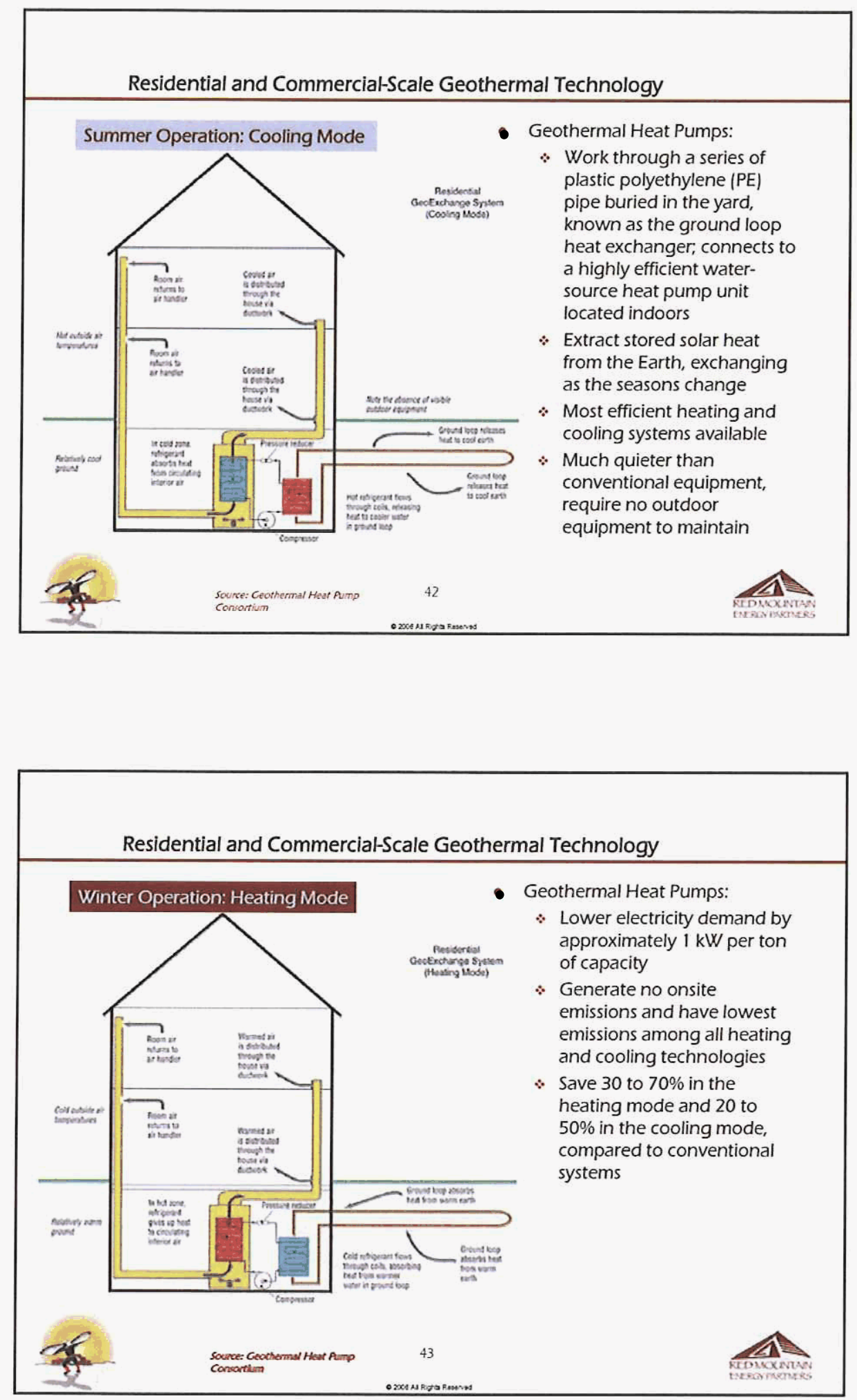

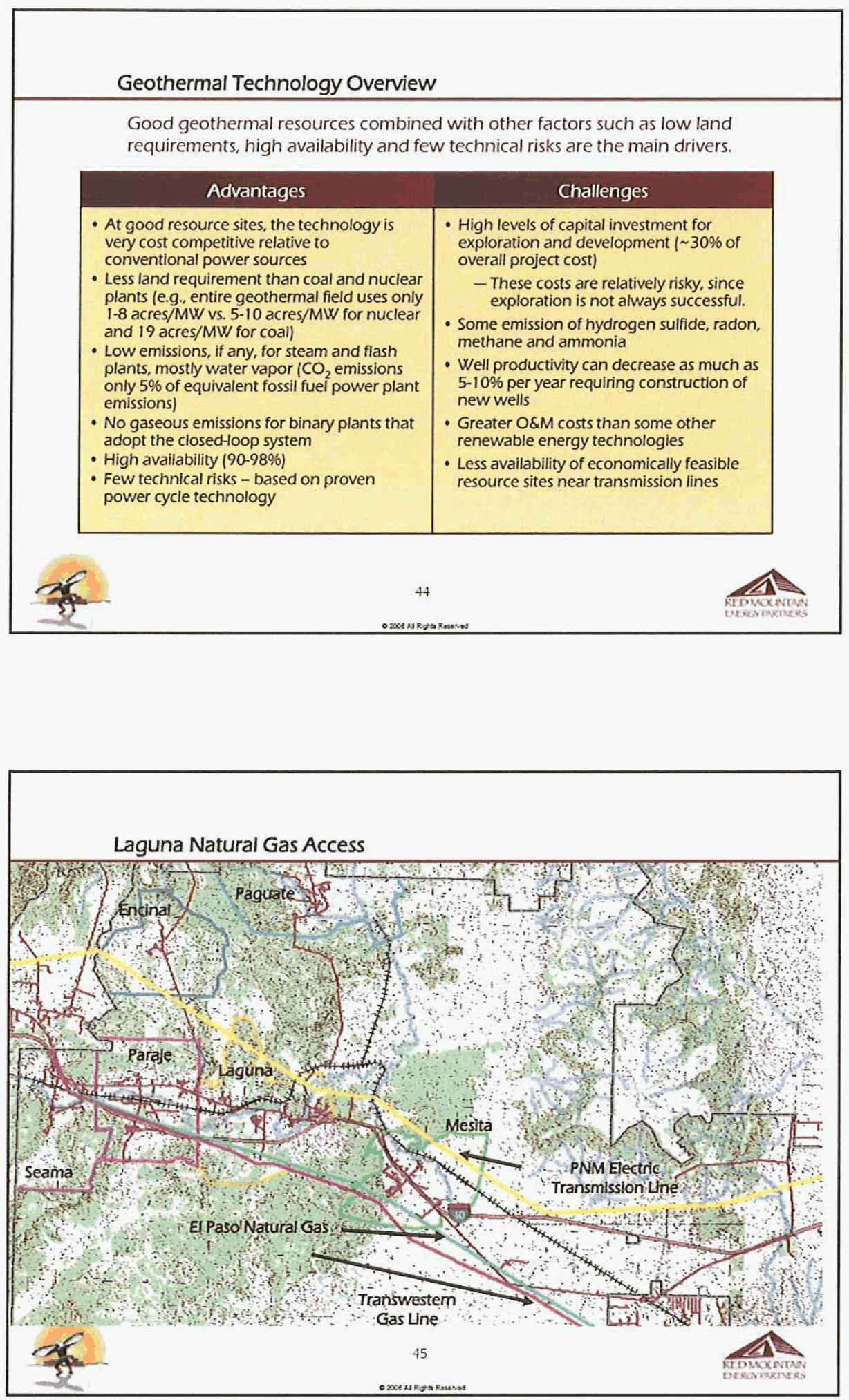


\section{Laguna Natural Gas Access}

- Excellent access to two high-pressure interstate pipelines

- El Paso Natural Gas

* Transwestern

- Access via PNM gas distribution system

* Laguna

$\therefore$ Mesita

* Seama

$\therefore$ Paraje

\section{Laguna Natural Gas Opportunities}

- "Firm" a renewable resource w/natural gas generation

$\therefore$ Large scale

* Small scale

- Operate gas distribution operations

$\therefore$ Provide retail distribution services on reservation

$\therefore$ Poise to provide gas services on reservation to serve current and attract potential commercial, industrial customers

* Potentially provide wholesale gas to gas-fired reservation project 


\section{Laguna Natural Gas Considerations}

- Energy project development

$\therefore$ Project location/cost of project laterals

$*$ Cost and process of interconnection

- Natural gas distribution operations

* Cost of distribution lines/project laterals

- Distance/terrain are a challenge

- Potential to attract new businesses

- Pipeline capacity

- Previously a major issue

- Gas supply

$\therefore$ Gas cost risk

* Tribal supply arrangement

* Hedging options

\section{Natural Gas - Combined Cycle Cogeneration}

- Small Combined Cycle / Cogeneration

- Combined Cycle with steam export for industrial or commercial use

$\therefore \quad$ More fuel efficient and cost effective than separate power and heating/cooling plants

$\therefore$ Capacity from 10 to 100 megawatts

$\therefore \quad$ Requires 5 to 20 acres of land

$\therefore$ Requires 2 to 5 years to develop and construct

* Capital cost between $\$ 50$ million and $\$ 100$ million

* Up to 100 jobs during construction, about 20 jobs during operation

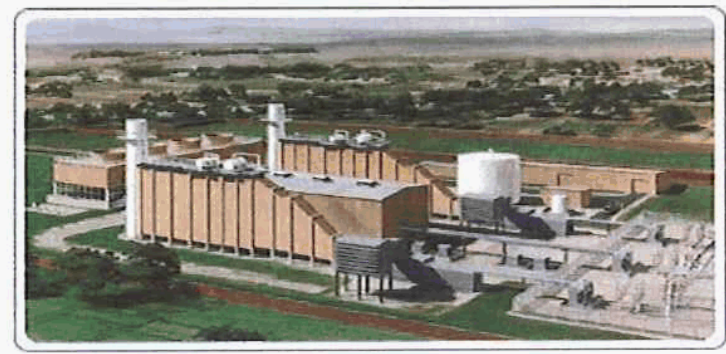

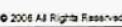


Natural Gas - Simple Cycle

- Combustion Turbine or Reciprocating Engine only

- Lower capital cost, but lower fuel efficiency

- Appropriate for summer peak operation

- Capacity from 20 to 100 megawatts

- Requires 5 to 20 acres of land

- Requires 1 to 3 years to develop and construct

- Capital cost between $\$ 10$ million and $\$ 50$ million

- Up to 50 jobs during construction about 10 during operation

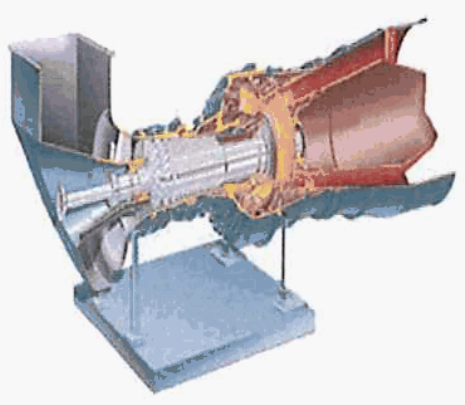

\begin{tabular}{|c|c|c|}
\hline \multicolumn{3}{|l|}{200 MW CCGT } \\
\hline Project Size (MW) & & 200 \\
\hline Capital Cost (\$/MW) & $\$$ & 800,000 \\
\hline Electric Interconnection Costs & $\$$ & $1,000,000$ \\
\hline Ancillary Equipment Costs & $\$$ & $1,000,000$ \\
\hline Fuel Infrastructure Costs & $\$$ & $2,000,000$ \\
\hline Fuel Cost (\$/MMBtu) & $\$$ & 5.00 \\
\hline Heat Rate (Btu/MWh) & & 7.5 \\
\hline Total Project Cost & & $164,000,000$ \\
\hline Capital Cost Amort (yrs) & & 15 \\
\hline Annual Capital & & $24,079,175$ \\
\hline $\mathrm{MWh} / \mathrm{Yr}$ at $80 \% \mathrm{LF}$ & & $1,401,600$ \\
\hline Production Cost (\$/MWh) & $\$$ & 17.1798 \\
\hline O\&M Cost (\$/MWh) & $\$$ & 5.0000 \\
\hline Fuel Cost (\$/MWh) & $\$$ & 37.5000 \\
\hline ALL-IN COST/MWh & $\$$ & 59.6798 \\
\hline
\end{tabular}




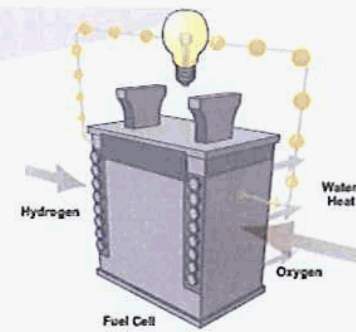

Oxygen enters a fuel cell membrane from the atmosphere, while its hydrogen source may come from natural gas, or renewable energy-based water electrolysis
- Convert the chemical energy of a fuel and an oxidant directly into electrical energy and heat using electrochemical processes

- The simplest type, a Proton Exchange Membrane Fuel Cell (PEMFC), combines hydrogen fuel with oxygen from the air to produce electricity, water, and heat. Consists of:

$\therefore$ an anode (a negative electrode that repels electrons).

* an electrolyte in the center, and

$\therefore$ a cathode (a positive electrode that attracts electrons)

- Hydrogen gives up electrons at the anode, and recombines with the hydrogen ions (the protons) and oxygen at the cathode to form water

- As hydrogen flows into the fuel cell anode, a catalyst, often a platinum coating on the anode, helps to separate the gas into protons (hydrogen ions) and electrons

- Electricity results from free electrons liberated from hydrogen at the anode flowing through an external electrical circuit before recombining with hydrogen ions and oxygen at the cathode to produce water

- Individual fuel cells can be combined in series into a fuel cell "stack" to achieve the desired voltage

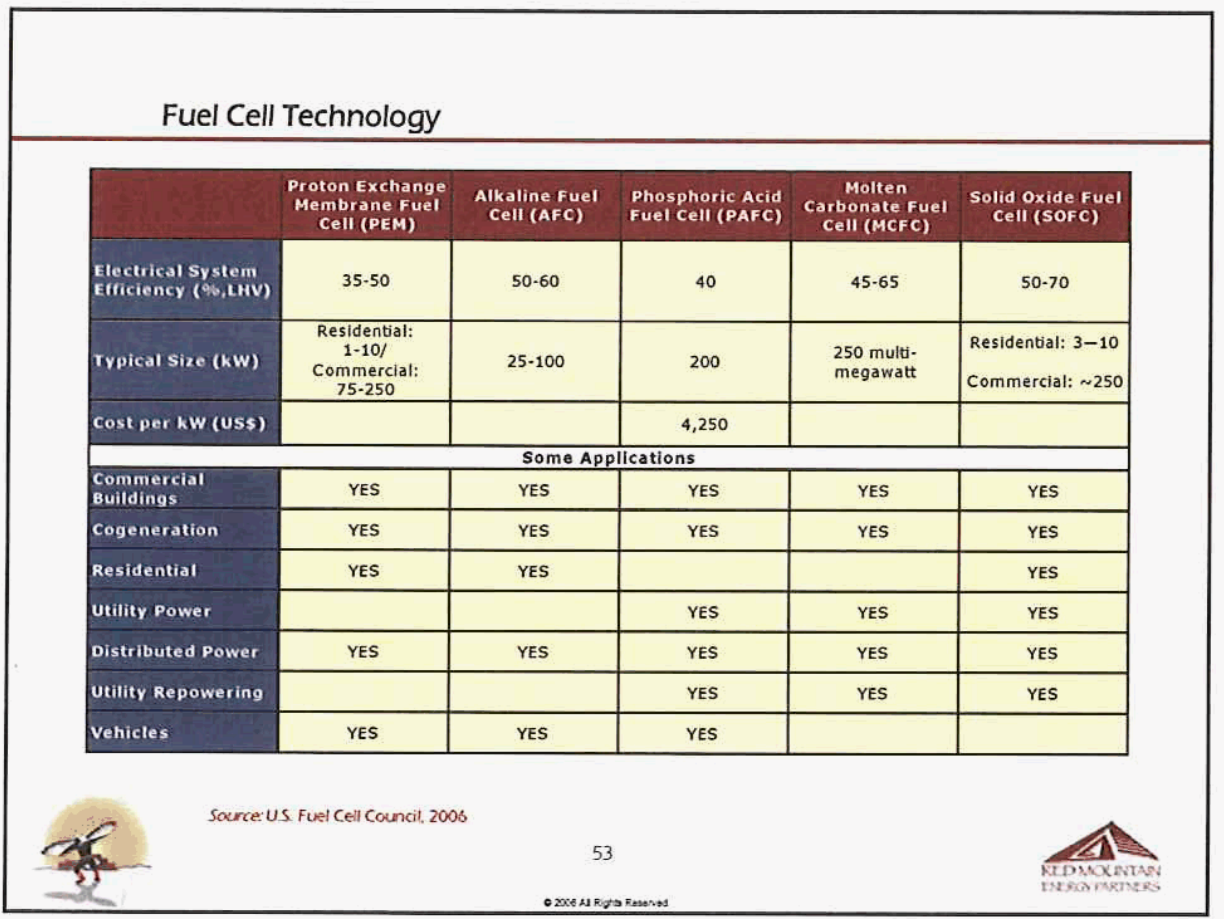




\section{Fuel Cell Technology}

Stationary fuel cell units, used for small power needs and back-up generation are the most mature fuel cell application

\begin{tabular}{|c|c|}
\hline Advantages & Challenges \\
\hline $\begin{array}{l}\text { - High energy conversion efficiency- } \\
\text { may range from } 40 \text { to } 60 \% \text { based on } \\
\text { lower heating value (LHV) of the fuel } \\
\text { - Good part load characteristics; quick } \\
\text { response to load changes } \\
\text { - Modular design and flexibility of size } \\
\text { - Easily sited due to low environmental } \\
\text { signature; no gaseous, solid or noise } \\
\text { emissions } \\
\text { - Suitable for cogeneration - heat } \\
\text { rejected from some fuel cell types such } \\
\text { as the Molten Carbonate and Solid } \\
\text { Oxide fuel cells is at sufficiently high } \\
\text { temperature for process heat } \\
\text { - Can be constructed in a wide range of } \\
\text { electrical output, from less than a kW to } \\
\text { sizes in excess of a MW }\end{array}$ & $\begin{array}{l}\text { - Sensitivity to certain contaminants that } \\
\text { may be present in the fuel such as } \\
\text { sulfur and chlorides } \\
\text { - High capital costs; currently on the } \\
\text { order of } \$ 4,500 / \mathrm{kW} \text { with research } \\
\text { targeted toward a } \$ 400 / \mathrm{kW} \text { price point } \\
\text { - Lack of the field data on } \\
\text { endurance/reliability } \\
\text { - Fuel entering the cell must be gaseous } \\
\text { and in some cases, must be hydrogen } \\
\text { which leads to the requirement of a } \\
\text { fuel pre-processor, or "reformer" - in } \\
\text { some types fuel is processed outside } \\
\text { (externally reformed) or inside } \\
\text { (internally reformed) }\end{array}$ \\
\hline & a fosonst \\
\hline
\end{tabular}

\section{Fuel Cell Applications}

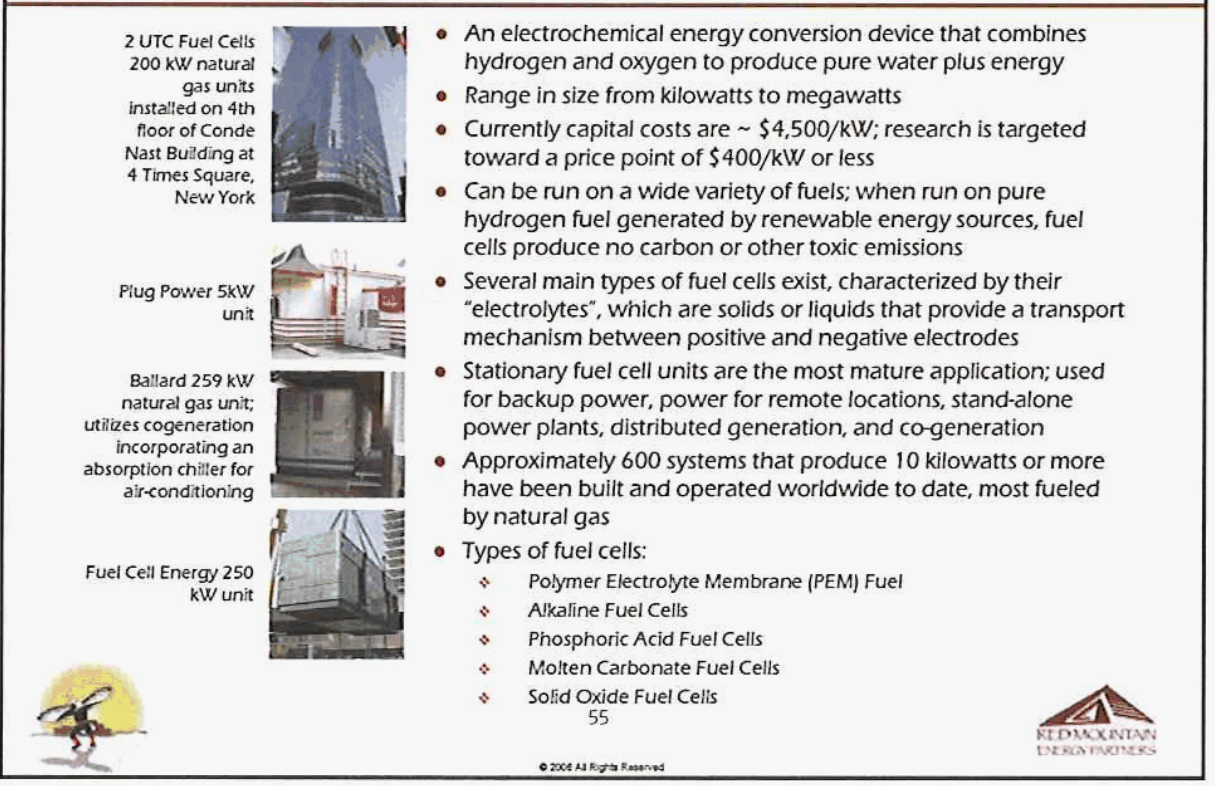




\section{Purpose of Energy Profiling}

- Identification of fit between energy demand and potential generation options

* Best-fit generation options have high correlation between daily energy use profile and the generating resource's daily energy production profile

- Identification of energy management opportunities

* Energy efficiency, conservation, demand management

* Implications for operational flexibility:

- Time-of-use patterns

- "Coincident" usage patterns to avoid demand charge threshold

- Real-time consumption information

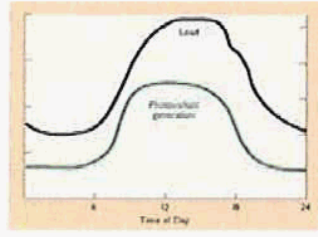

Example of a photovoltaic production profile and a typical demand profile

Laguna Energy Profile

Energy profile depicts a very typical pattern of daily energy use

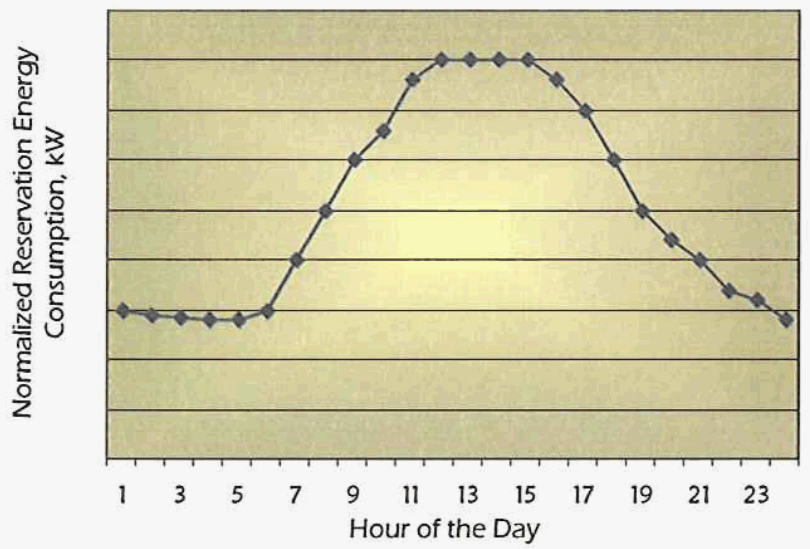

Average Daily Energy Consumption, Reservation-wide, Based Upon Existing Generalized Analysis 


\section{Building an Energy Use Profile}

- Update existing Energy Profile

- Preliminary, generalized annual load profile

* Updates to existing profile required

- Some information incomplete

- New construction to date will be added via housing stocks database

- Validate existing profile through meter data and utility billings

* Data gathering underway, additional requests to utilities needed

* Validation will reflect better representation of actual Reservation electricity load and forecast

Efforts Underway

- Data requests for 2004/2005 utility billings and/or energy ledgers

- Requests to utility providers for detailed data

* Meter types by facility

* Demand or "interval" data, where available

- Applicable electricity tariffs (rate schedules)

* Requests must be made or approved by Tribal customers

- Update of facility and dwelling inventory via analysis of housing stock database

- Discussions with facility/energy managers

- Reconstruction of energy profile with actual data 


\section{System Electric Load vs. Generation}

- The tariff, or "rate schedule", sets price charged for electricity consumed, based upon customer information and energy demand

* Total system load, driven by customer energy demand, varies hourly and seasonally

* Generating capacity must be maintained to serve peak demand

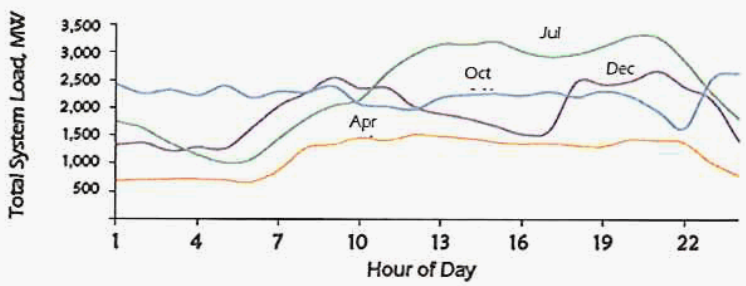

-lllustrative-

Typical

pattern for a

southwestem

U.S. utility

with summer

peak load

Typical daily electrical utility system load varies by season.

OXXsergagranes

System Electric Load vs. Generation

* Cost of generating electricity varies hourly and seasonally, due to need to dispatch more expensive plants during peak demand periods

*Utilities recover their capital costs required to maintain sufficient peak generating capacity through "facility charges", also known as capacity charge, or demand charge

$\therefore$ Utilities also recover the production costs of more expensive electricity by using "time-of-use" rates

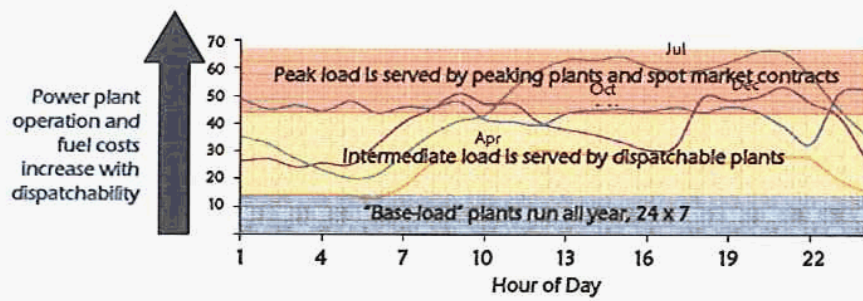

- Illustrative-

Typical

pattern for a

southwestem

U.S. utility

with summer

peak load

Energy costs also vary both hourly and by season. 


\section{Laguna Energy Project Considerations}

- Renewable Resources

$\because$ Wind

* Solar

* Biomass

* Geothermal

- Technology cost effectiveness

- Natural gas complement

- Laguna needs vs. available resource

- Large scale vs. self-supply

- Siting/permitting

- Joint tribal project

Renewable Resource "Benchmark" Traits

\begin{tabular}{|c|c|c|c|}
\hline Type of Resource & Measurement & Typical Values & I MWV Equivalent \\
\hline Wind & $\begin{array}{l}\text { Wind Speed } \\
\text { (Density) }\end{array}$ & $\begin{array}{c}\text { Class } 4.7 \\
15.7+\mathrm{mph}\end{array}$ & Less than 1 Turbine \\
\hline Solar PV & Solar Insolation & $\begin{array}{l}\text { Capacity Factor } \\
\text { Watts } / \mathrm{m} 2\end{array}$ & $\begin{array}{l}\text { I acre e.g. Walmart } \\
\text { store }\end{array}$ \\
\hline Solar Thermal & Solar Insolation & $\begin{array}{l}\text { Capacity Factor } \\
\text { Watts/m2 }\end{array}$ & 10 acres \\
\hline Solid Blomass & Heat/Water Content & $5000-7000 \mathrm{Btu} / \mathrm{lb}$ & I Dry Ton $/ \mathrm{Hr}$ \\
\hline Blogas & Methane Content & $\begin{array}{c}\text { LFG: } 50 \% \text { Methane } \\
\text { Dairy: } 60 \% \\
\text { Methane }\end{array}$ & $\begin{array}{l}1 \text { MM tons WIP; } \\
1000 \text { cattle }\end{array}$ \\
\hline $\begin{array}{l}\text { Geothermal } \\
\text { (Flash/Direct Steam) }\end{array}$ & $\begin{array}{c}\text { Steam Temperature, } \\
\text { Pressure and } \\
\text { Quantity }\end{array}$ & $\begin{array}{l}>350 \mathrm{~F} \\
100 \mathrm{psi}\end{array}$ & $15-20 \mathrm{kibs}$ \\
\hline Small Hydro & $\begin{array}{c}\text { Head }(\mathrm{ft}) \\
\text { Flow(cu.ft/sec) }\end{array}$ & Wide range & $33 \mathrm{ft} @ 500 \mathrm{cu} . \mathrm{ft} / \mathrm{sec}$ \\
\hline
\end{tabular}




\begin{tabular}{|c|c|c|c|c|c|}
\hline \multicolumn{6}{|c|}{ Renewable Resource "Benchmark" Traits } \\
\hline Type of Resource & System Size & $\begin{array}{l}\text { Installed } \\
\text { System Cost } \\
\text { (S/kW) }\end{array}$ & $\begin{array}{l}\text { O\&M Costs } \\
\text { (c/kWh) }\end{array}$ & $\begin{array}{l}\text { Capacity } \\
\text { Factor }\end{array}$ & Key Issues \\
\hline Wind & $\begin{array}{l}\text { Each Turbine: } \\
650-2500 \mathrm{~kW} \\
\end{array}$ & 1,200 & 0.5 & $\begin{array}{l}\text { Class } 4 \text { wind } \\
30 \%\end{array}$ & Intermittency \\
\hline Solar Thermal & $\begin{array}{l}25 \mathrm{~kW}- \\
300 \mathrm{MW}\end{array}$ & $3,000-4,000$ & $0.03-0.04$ & $\begin{array}{l}22-25 \% \text {, up } \\
\text { to } 90 \% \text { with } \\
\text { storage }\end{array}$ & High Capital Cost \\
\hline $\begin{array}{l}\text { Solar PV- } \\
\text { Commerclal }\end{array}$ & $250-1000 \mathrm{~kW}$ & 6,500 & 0.8 & $14-20 \%$ & High Capital Cost \\
\hline Solld Blomass & $\begin{array}{c}15 \mathrm{~kW}-100 \\
\mathrm{MW}\end{array}$ & $1,500-2,000$ & $\begin{array}{c}1.5 \\
(+ \text { fuel) }\end{array}$ & $85 \%$ & Emissions \\
\hline Blogas & $5 \mathrm{MW}$ & $1,200-1,500$ & $\begin{array}{l}1.5 \cdot 2.0 \\
(+ \text { fuel })\end{array}$ & $85 \%$ & $\begin{array}{l}\text { Air quality; gas collection } \\
\text { system }\end{array}$ \\
\hline Geothermal & $50 \mathrm{MW}+$ & $2,000-2,500$ & $1.0-1.5$ & $90 \%$ & $\begin{array}{l}\text { Upfront costs such as drilling } \\
\text { an exploration; resource } \\
\text { could deplete } \\
\end{array}$ \\
\hline Small Hydro & $1.5 \mathrm{MW}$ & $3,500-4,500$ & 0.5 & $45-65 \%$ & Permitting: High Capital Cost \\
\hline & & & 64 & & \\
\hline
\end{tabular}

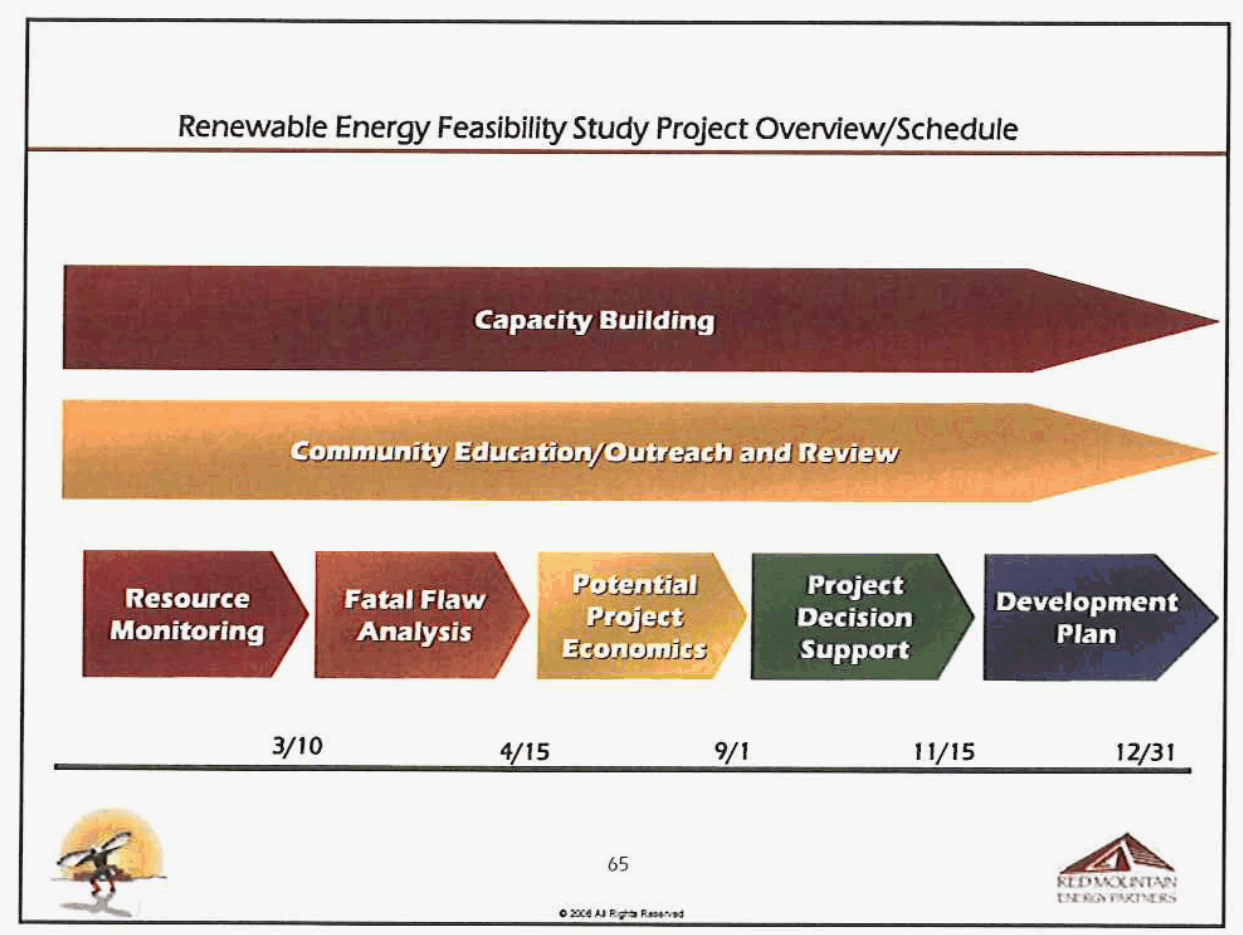




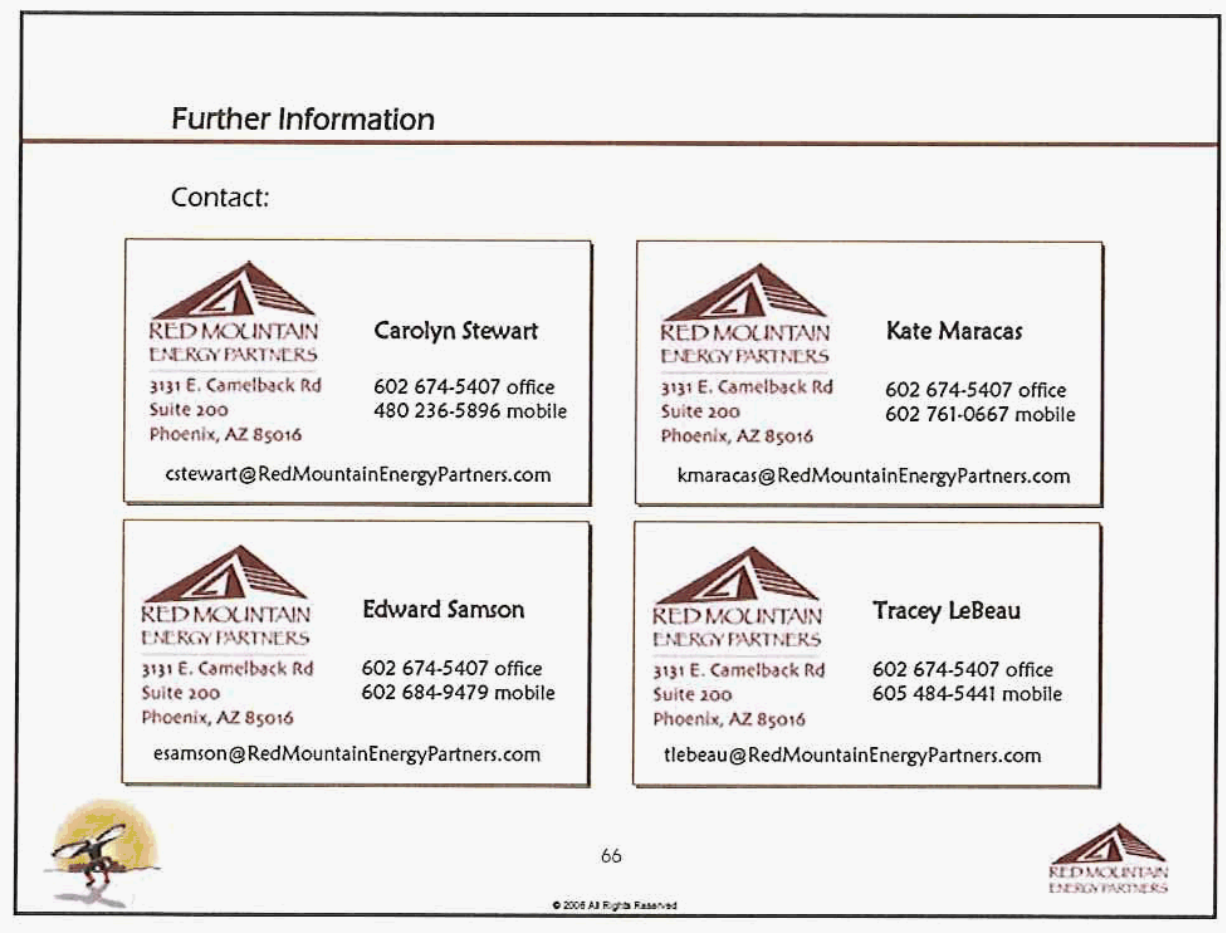



Pueblo of Laguna

Utility Authority Board of Directors Status Report

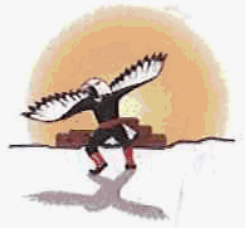

May 2006

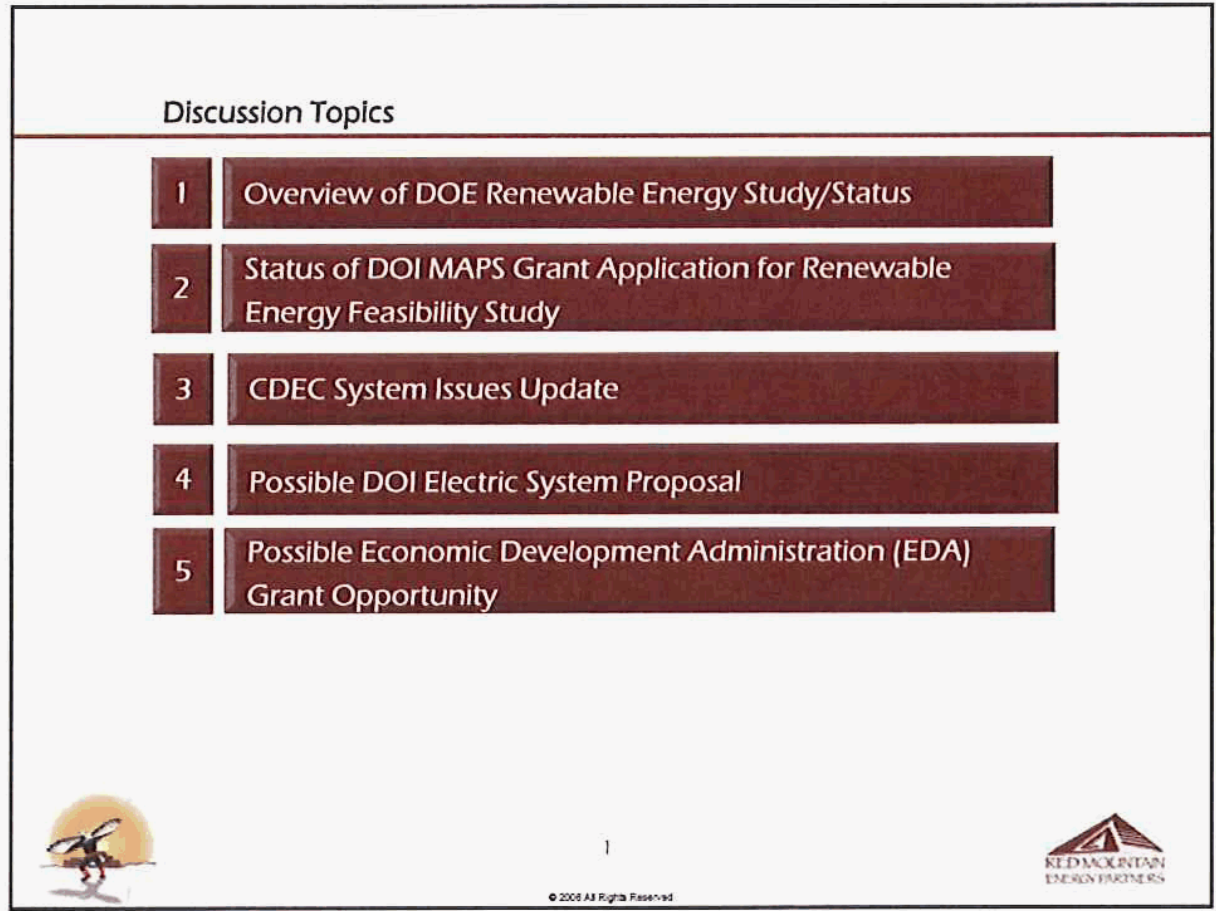




\section{Background Information}

- Utility Authority Formation - 1980s-2005

- Utility Formation and Power Development Study - 2004-2005

* Utility Organization, Implementation

$\therefore$ Electric System Issues, Alternatives

* Power Development Potential

- Multiple Grant Applications

* ANA - Utility Implementation (awarded)

* USDA - Electric System Acquisition/Improvements

* DOE - Electric System Negotiations

$\therefore$ DOE - Renewable Energy Feasibility (awarded)

* DOI-Renewable Energy Feasibility

* NM - Wind Feasibility (awarded)

- Foresight Energy Wind Development Agreement - May 2005

- Utility Authority Operations Begin - August 2005

- DOE Feasibility Study - Now Underway

Pueblo of Laguna Utility/Energy Studies

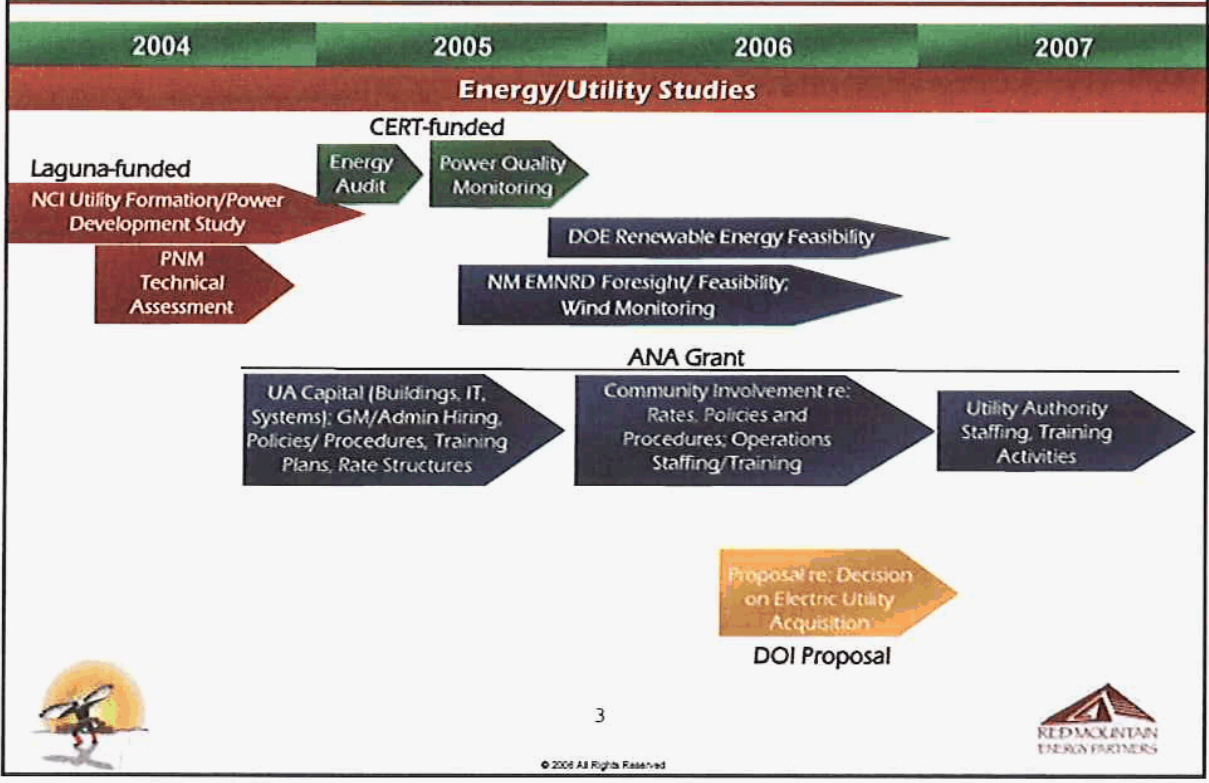



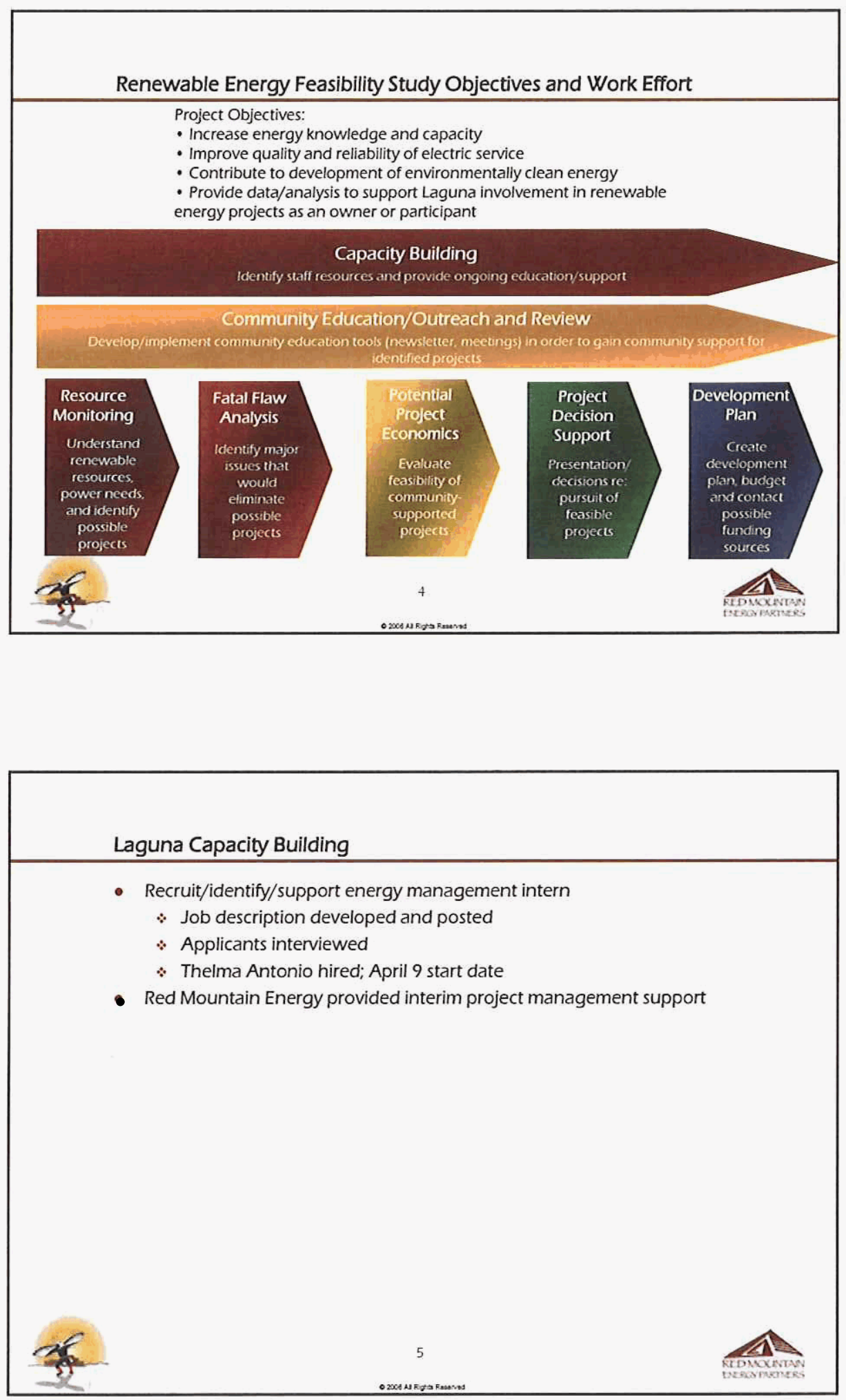


\section{Laguna Community Outreach}

- Newsletter concept developed

- Newsletter article scheme developed

$\therefore$ Lead topic for each edition

$\therefore$ Regular articles on utility related topics

$\therefore$ Schedule developed

* First edition completed and distributed

* Second edition underway

- Community meeting concepts developed

$\therefore$ Series of village and combined meetings

- Initial meeting now scheduled May 17

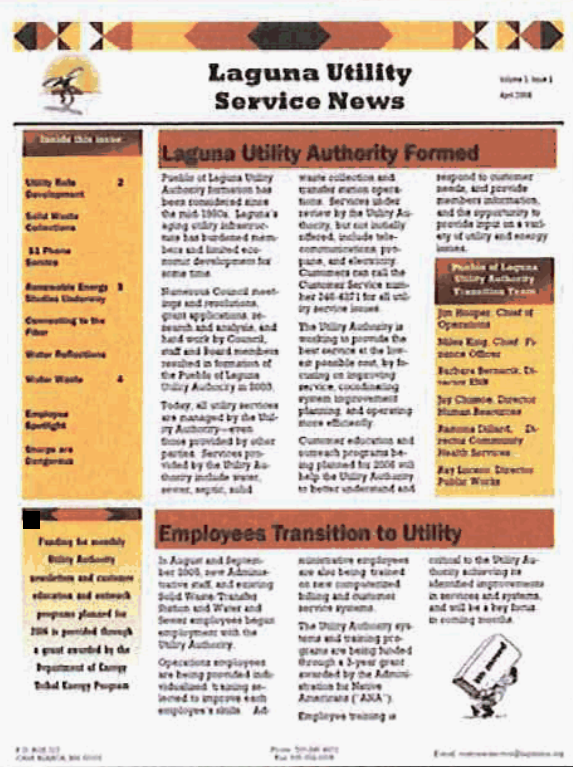

\section{Laguna Solar Resource}

Concentrating Solar Power

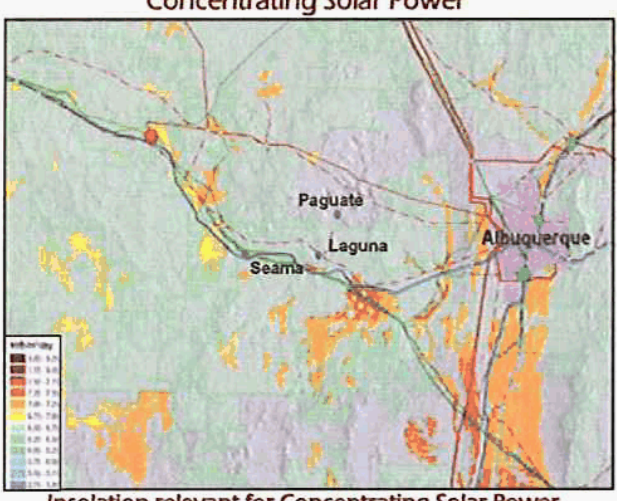

Insolation relevant for Concentrating Solar Power

technology is measured with collectors that track the sun. Parts of the Pueblo have concentrator insolation levels in the range of $7.25-7.5 \mathrm{kWh} / \mathrm{m}^{2} /$ day, well within the range suitable for large-scale solar projects

Direct Solar Radiation (PV)

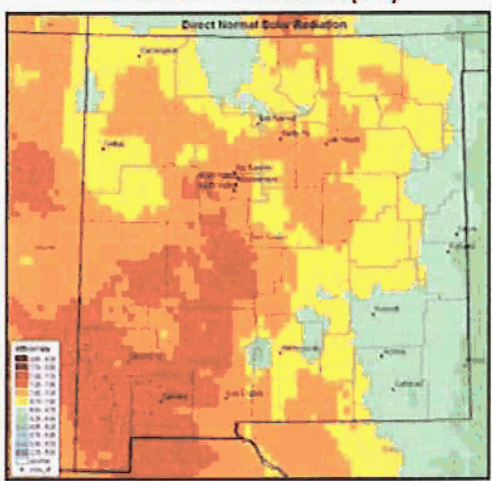

Flat plate collectors are used to measure insolation levels relevant for PV systems. Average annual insolation on the Pueblo falls in the range of $7.25-7.5 \mathrm{kWh} / \mathrm{m} 2 /$ day 

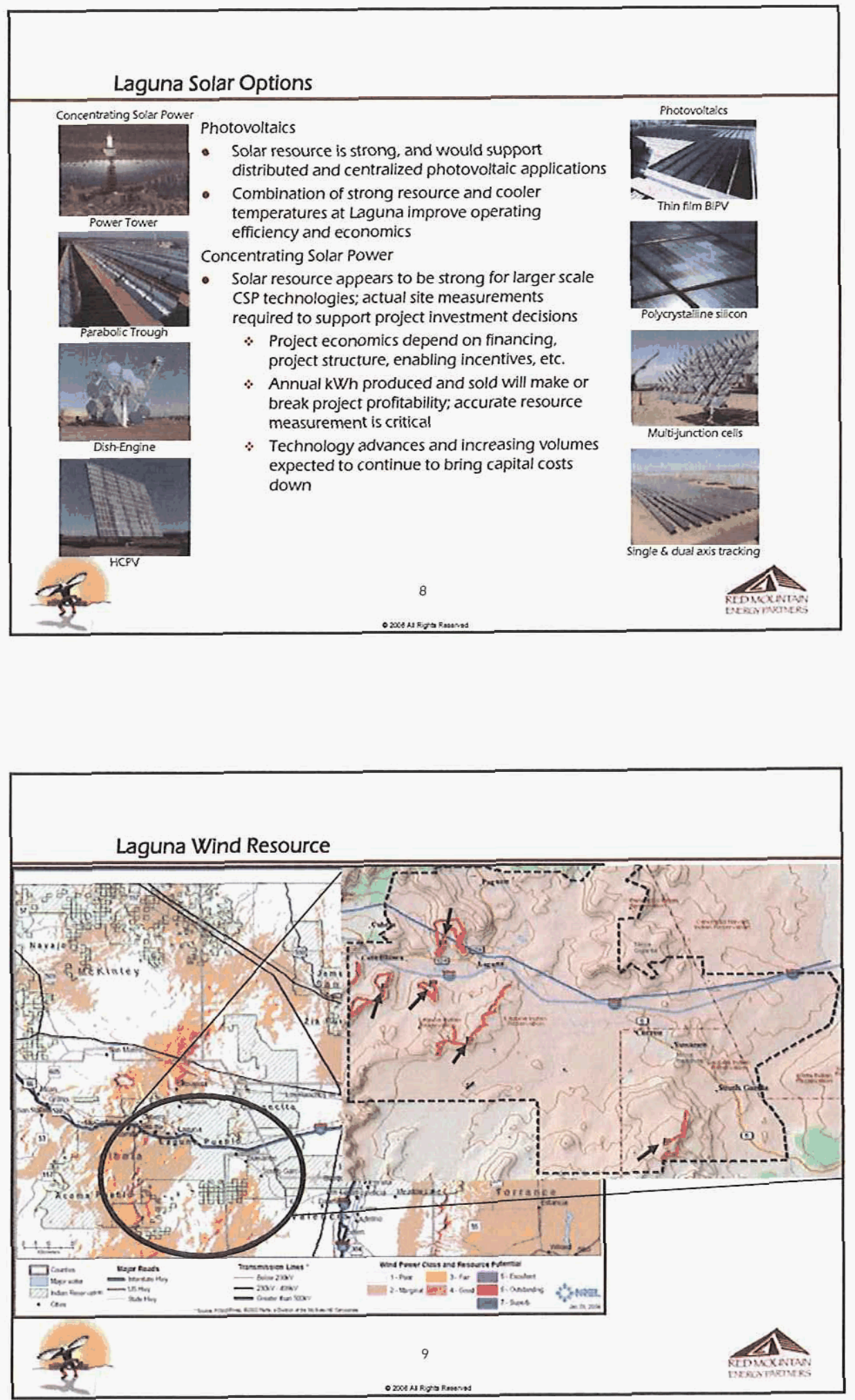


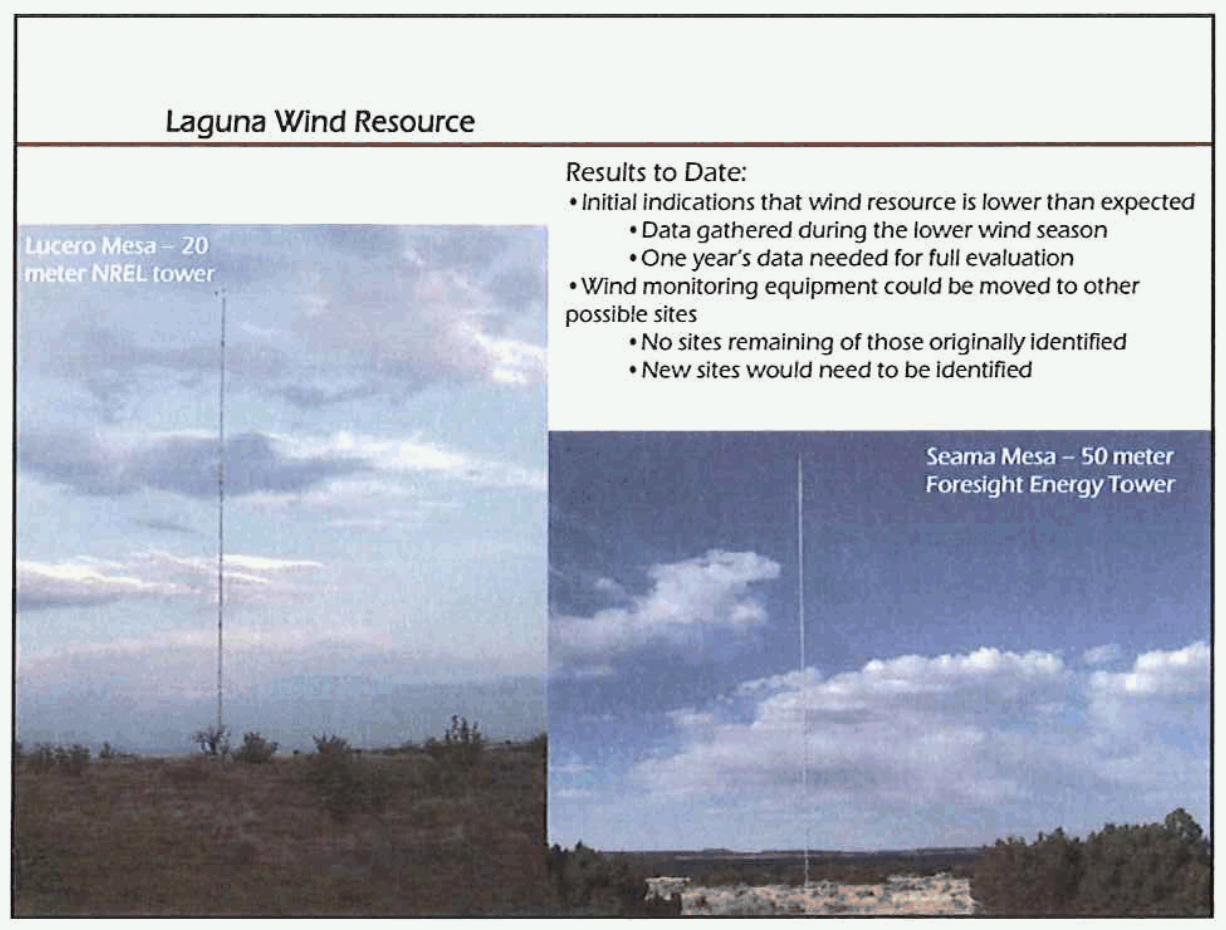

Foresight Development Agreement - Summary of Timeline and Activities Foresight Responsibilities

Phase I

- Identify potential sites, install met testing equipment, collect data $6-12$ mos - Apply for state, federal, and other grants

- Prepare technical report describing

conditions for each tested site

- Deliver report within 13 months after

installation of met tower

- Move towers to alternative sites, if needed,

up to 9 months into the term
Phase II

- Physical and cultural evaluation of proposed site(s)

- Prepare detailed financial projections - Discuss equity participation agmts

- Execute Power Purchase Agreements

- Prepare Interim Development Report

- Commence land payment option of

$\$ 25,000$ per year upon execution of

an Easement Agreement for site

\section{Phase III}

- Continue land option payments - Finalize PPAs for the Project's output - Finalize project sale to new owner or oversee financing and construction of facility

- Finalize financing of Project, utilizing a minimum $50 \%$ project debt, or provid notice that lead equity investor will contribute up to $100 \%$ of capital costs

\section{i}

Laguna Responsibilities

- Provide/assist access to potential project sites for monitoring equipment

- Assist in applying for federal, state, and other grant applications, and administering funds

- If Foresight concludes wind resource is adequate, and provides evidence of ability to commit additional $\$ 250,000$ for

development, Parties extend Agreement. If not, Foresight delivers all data and reports to Laguna; Agreement will expire

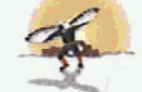

- Seek tribal approval to execute Grant of Easement with $B ! A$

- Allow Foresight to undertake legally permissible activities on sites selected - Administer grant funds and assist

Foresight to develop and market Project

- Parties enter into long-term Easement Agreement for project site.

- Primary Agreement: payments for greater of 1) $\$ 2,500$ per MW of turbine capacity/yr, escalated by $5 \%$ each five years; or 2) 3\% of Gross Operating Proceeds during Operating Period
- Execute Equity Agreement w/option fo up to $20 \%$ equity or provide Laguna annual cash payment of $\$ 50,000$ - Execute Post Operating Period Agreement, owner responsible for removing equipment/restoring land to original condition or transfering to original condition or transferring 1009 ownership to Laguna. PPA extensio extends Easement Agreement and increases Royalty Payments to greater o $\$ 5000$ per MW/year, or $5 \%$ of Project's Gross Operating Proceeds

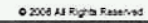

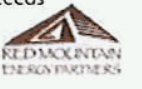




\section{Laguna Wind Options}

- Wait for Foresight Energy report due early August

- Potential for Foresight to end agreement, based on interim results

- Per Foresight Development Agreement, Laguna would be free to pursue other wind development opportunities (small scale or large scale)

$\therefore$ - If community supported concept of wind generation, consider largescale development at other sites

- Further testing to determine if other sites could be viable

- If Foresight continues agreement, Laguna could still pursue village-specific projects

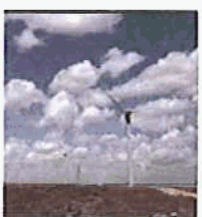

GE 1.5 MW Turbines (large scale)

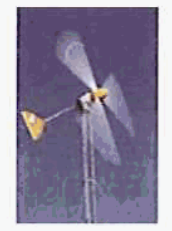

Bergey $10 \mathrm{~kW}$ Turbine (small scale)

- Forest - Unclear if adequate resources; no existing logging infrastructure/forest management activity

- Agriculture: Extremely limited; agriculture limited to small family plots of corn, chile, alfalfa and assorted vegetables; no discernible residue stream

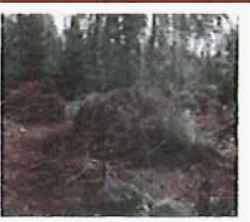

- Animal wastes: None

- Landfill gas: None; several abandoned landfills; contents "burned" before backfill, not lined nor monitored; gas production likely to be low

- Food residues: Yellow grease from restaurants hauled by septic firms; insufficient amounts to justify biodiesel production

- Wastewater treatment: Mostly uncovered lagoons with no methane recapture; wastewater treatment facility biosolids sent to digester in $A B O$

- Municipal solid waste: 940 households

* Delivered to transfer station and to $A B Q$; not likely enough tonnage to warrant consideration

- Transfer station: Modest amount of woødy biomass from local $C \& D$ is ground up for free use by anyone
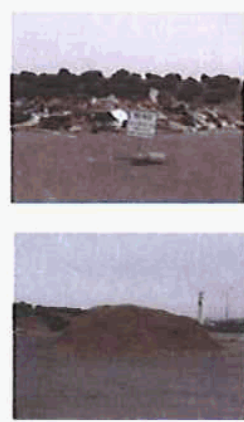

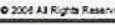




\section{Laguna Biomass Options}

- No obvious opportunities, given limited resources

- High interest in use of salt cedar

- Best options:

$\therefore$ Focus on resources for buildings / processes with high thermal loads and high load factors

- Larger facilities, typically over 50,000 square feet

- Buildings with older existing boilers and circulating hot water or steam systems

* Consider for future construction that includes expansion or renovation of HVAC system

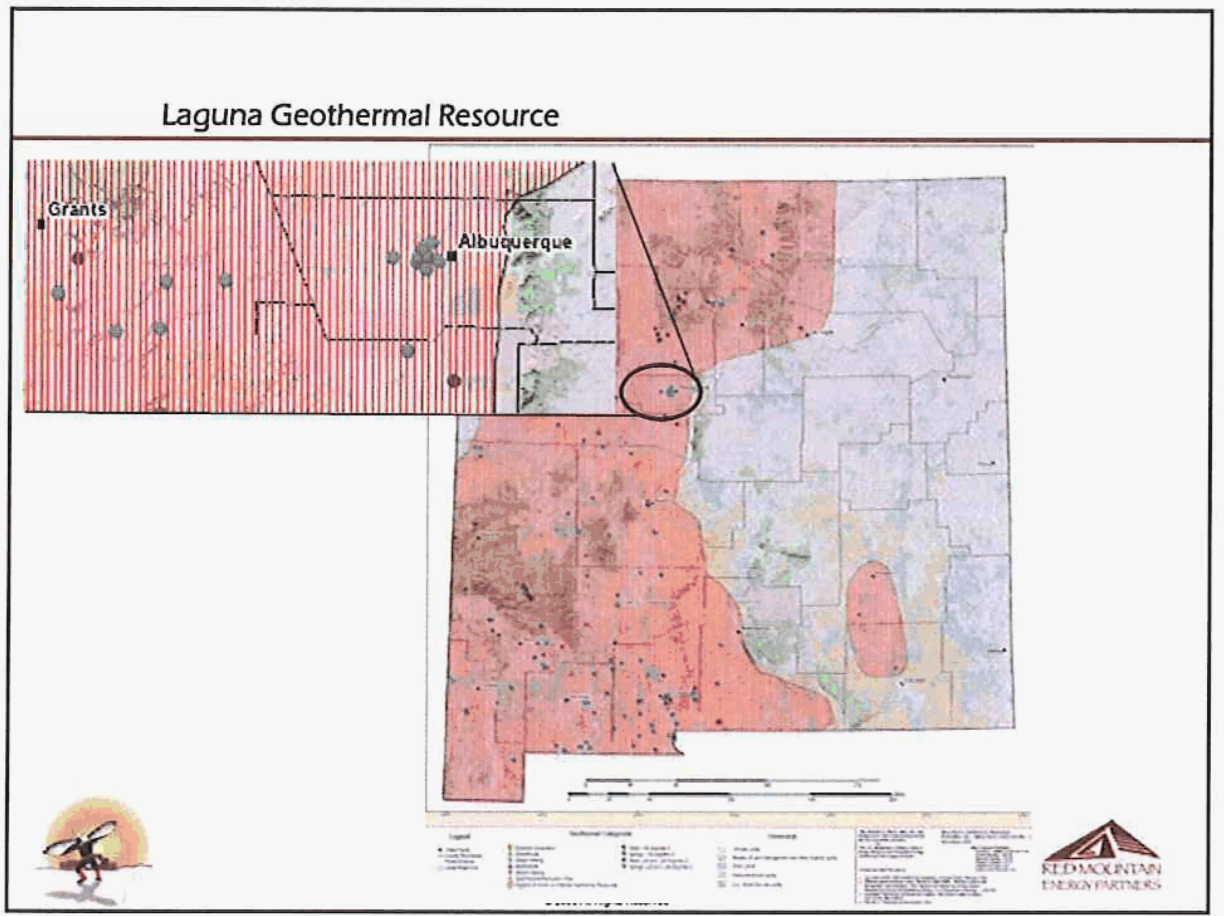


Laguna Geothermal Resource

- Scenario 1 (yellow boxes)

* Rio San Jose Valley

* Flat surface with shallow fresh water

* Elevation 5,700 to 5,900 ft

- Scenario 2 (orange box)

* Clay and Frog Mesa south of Wheat Mountain

$\therefore$ Relatively flat surface

* Elevation 6,600 to $6,700 \mathrm{ft}$

- Scenario 3 (red box)

$*$ Mesa Chivato north of Encinal

$\therefore$ Relatively flat surface

$\therefore$ Elevation 7,200 to $7,400 \mathrm{ft}$
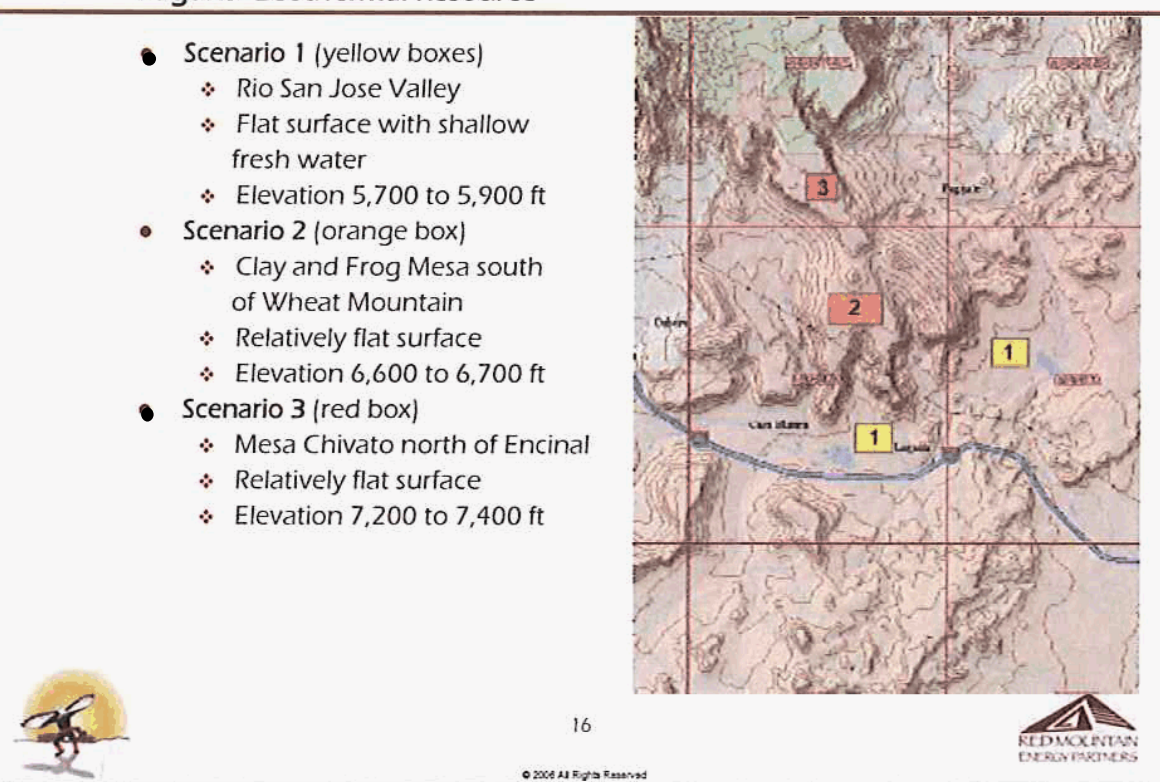

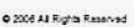

\section{Laguna Geothermal Options}

- Potential Direct-Use Geothermal

* Curing and drying

* District space heating

- Greenhouses

* Spa/resort

* Aquaculture (fish farm)

* Any process of interest to Pueblo of Laguna that requires large amounts of low-grade heat

- Geothermal Heat Pumps (ground heat exchange)

* Heating and cooling

* Large buildings

* Homes 


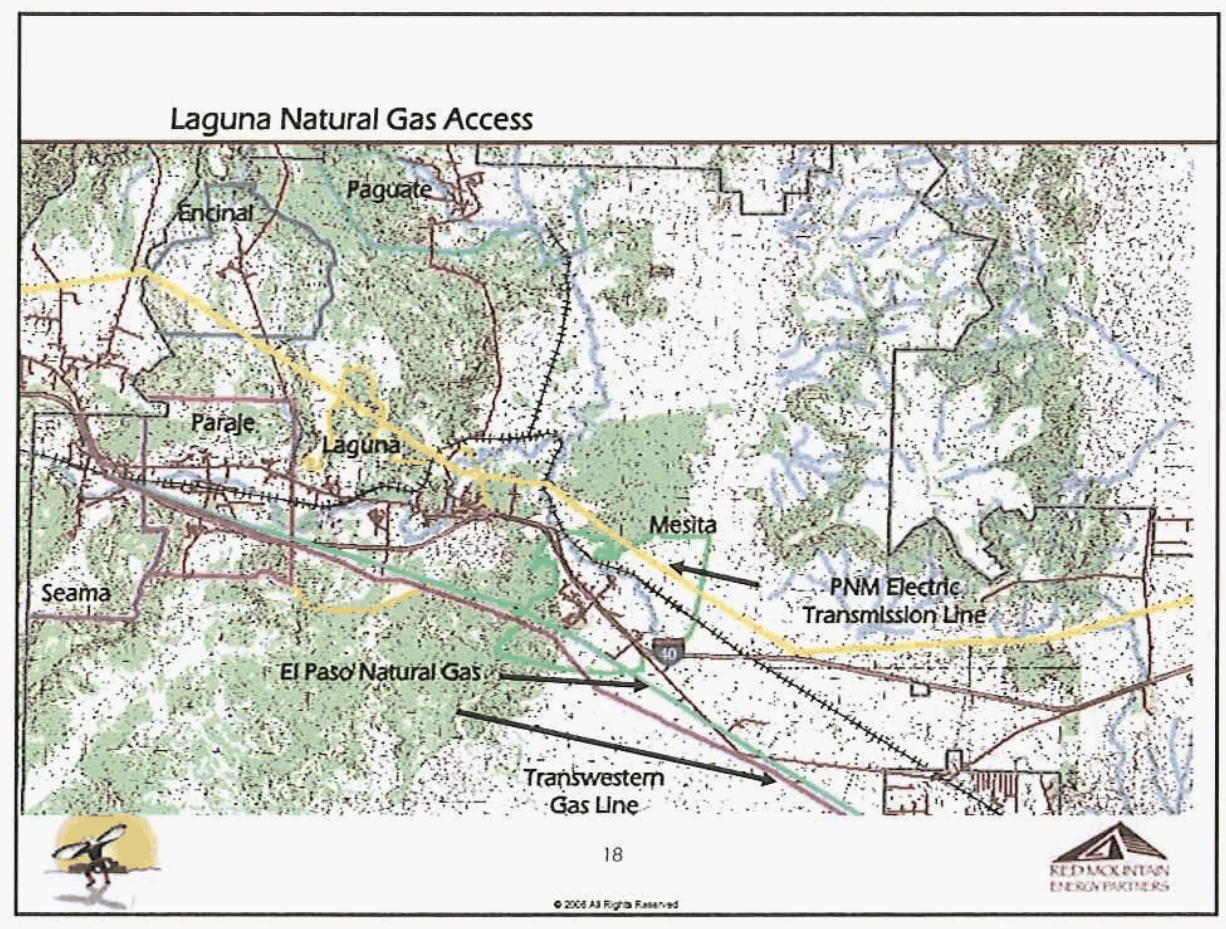

\section{Pueblo of Laguna Natural Gas Line Access}

- Interstate pipelines on Laguna provide interconnection potential for very high-volume natural gas use

- TransWestern Pipeline

* El Paso Natural Gas Pipeline

- PNM transmission/distribution system service on portions of Laguna provides access for industrial. commercial, government and residential access
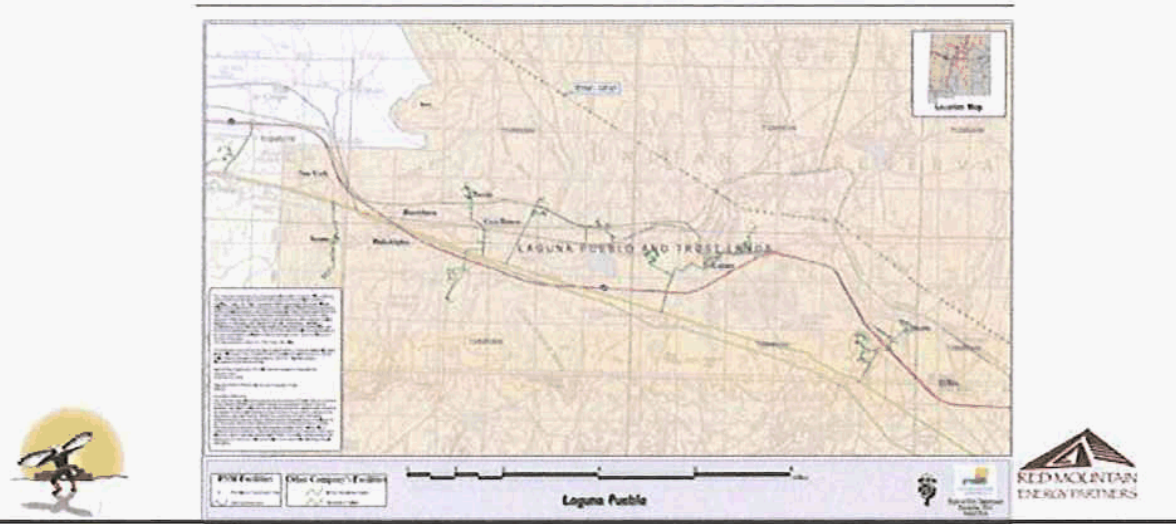


\section{Laguna Natural Gas Options}

- Large scale gas-fired generation

$\therefore$ Meet reservation load, plus sales to grid

* Consideration of economies of scale in relation to possible interconnection and pipeline lateral costs

- "Firm" a renewable resource w/natural gas generation

* Large scale

* Small scale

- Village-scale microturbine

- Facility location

$\therefore$ Turbine or CHP

- Gas distribution operations

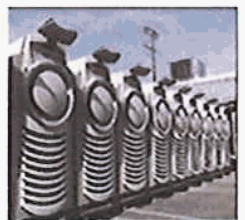

Microturbine

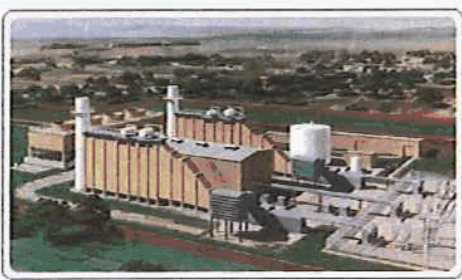

Large scale combined cycle/combined heat/power plant

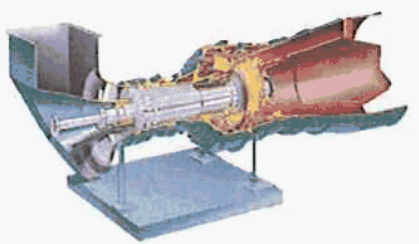

Combustion turbine or recip engine

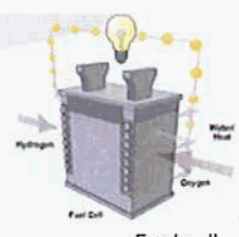

20

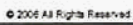

Laguna Electric Load Assessment

- Usage

* Reservation

- Villages

$\therefore$ Major entities

- Profile

* Time of use

* Level of use

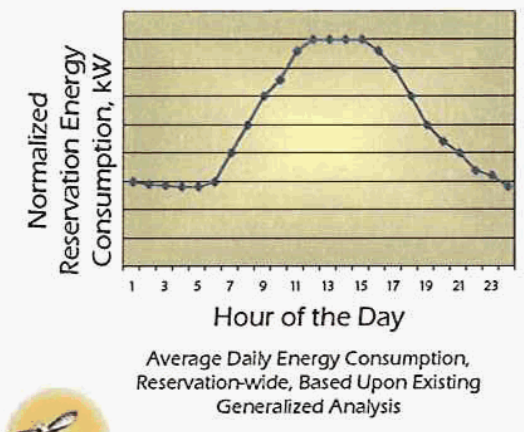

- Purposes/uses of electric load assessment:

* Identify "fit" between energy demand and potential generation options

- High correlation between daily energy use profile and energy production profile of generating resource

* Identification of energy management opportunities

- Energy efficiency, conservation, demand management

* Identify impacts on operational flexibility

- Time-of-use patterns (certain hours)

- "Coincident" usage (all at once) patterns to avoid demand charge

- Real-time consumption information 


\section{Laguna Potential Project Identification}

Match between:

- Resource available $\therefore$ Equipment types

- Electric usage/type of use

* Provide a portion or all of power supply

- High-level project economics

- Community considerations

$\therefore$ Cultural fit

$\therefore$ Environmental impact

$\therefore$ Footprint

$\therefore$ Views

$\therefore$ Power cost impact

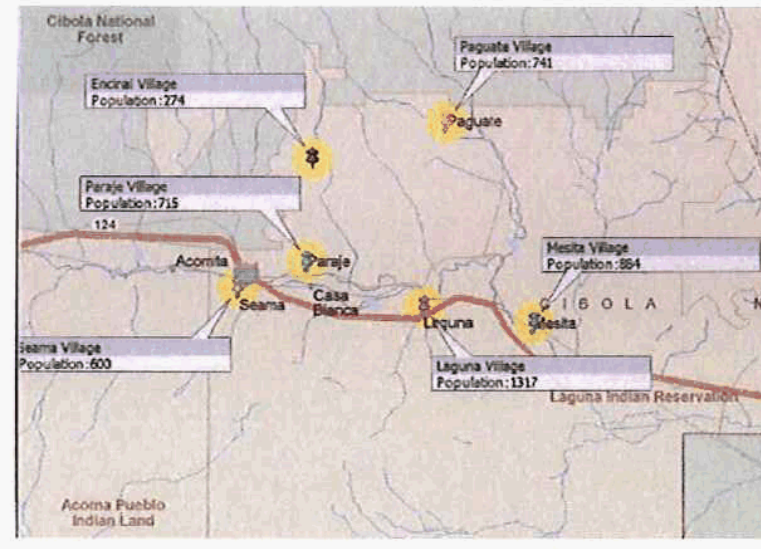

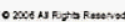

Project Identification - Renewable Resource Comparisons

\begin{tabular}{|c|c|c|c|c|c|}
\hline $\begin{array}{l}\text { Type of } \\
\text { Resource }\end{array}$ & System Size & Measurement & $\begin{array}{l}\text { Capacity } \\
\text { Factor }\end{array}$ & $\begin{array}{l}\text { Installed System } \\
\text { Cost (\$/kW) }\end{array}$ & 1 MW Equivalent \\
\hline Wind & $\begin{array}{l}\text { Each Turbine: } \\
650-2500 \mathrm{~kW}\end{array}$ & $\begin{array}{l}\text { Wind Speed } \\
\text { (Density) }\end{array}$ & $\begin{array}{c}\text { Class } 4 \text { wind } \\
30 \%\end{array}$ & 1.200 & Less than 1 Turbine \\
\hline Solar Thermal & $\begin{array}{l}25 \mathrm{~kW}- \\
300 \mathrm{MW}\end{array}$ & Solar Insolation & $\begin{array}{l}22-25 \% \text {, up to } \\
90 \% \text { with } \\
\text { storage }\end{array}$ & $3,000-4,000$ & $\begin{array}{l}10 \text { acres acre e.g. } \\
\text { Walmart store }\end{array}$ \\
\hline $\begin{array}{l}\text { Solar PV - } \\
\text { Commerclal }\end{array}$ & $250-1000 \mathrm{~kW}$ & Solar Insolation & $14-20 \%$ & 6,500 & 1 acre (Walmart) \\
\hline Solid Biomass & $\begin{array}{c}15 \mathrm{~kW}-100 \\
\mathrm{MW}\end{array}$ & $\begin{array}{l}\text { Heat/Water } \\
\text { Content }\end{array}$ & $85 \%$ & $1,500-2,000$ & I Dry Ton $/ \mathrm{Hr}$ \\
\hline Blogas & $5 M W$ & $\begin{array}{l}\text { Methane } \\
\text { Content }\end{array}$ & $85 \%$ & $1,200-1,500$ & $\begin{array}{l}1 \text { MM tons WWIP; } \\
1000 \text { cattle }\end{array}$ \\
\hline Geothermal & $50 \mathrm{MW}+$ & $\begin{array}{c}\text { Steam } \\
\text { Temperature, } \\
\text { Pressure and } \\
\text { Quantity }\end{array}$ & $90 \%$ & $2,000-2,500$ & $15-20 \mathrm{klbs}$ \\
\hline Small Hydro & $1.5 \mathrm{MW}$ & $\begin{array}{c}\text { Head }(\mathrm{ft}) \\
\text { Flow(cu.ft/sec) }\end{array}$ & $45-65 \%$ & $3,500-4,500$ & $33 \mathrm{ft} @ 500 \mathrm{cu} \cdot \mathrm{ft} / \mathrm{sec}$ \\
\hline
\end{tabular}



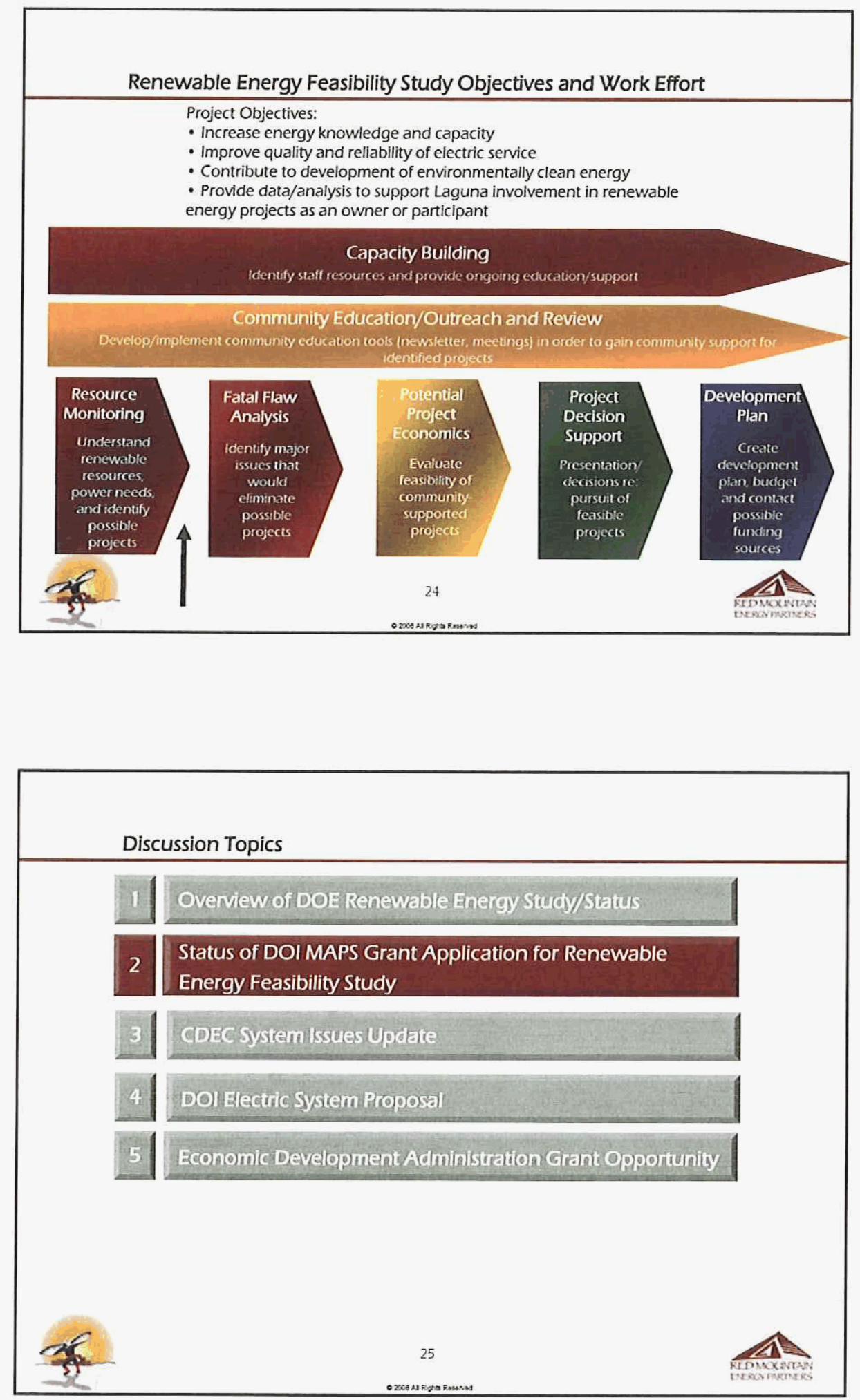
(2) 

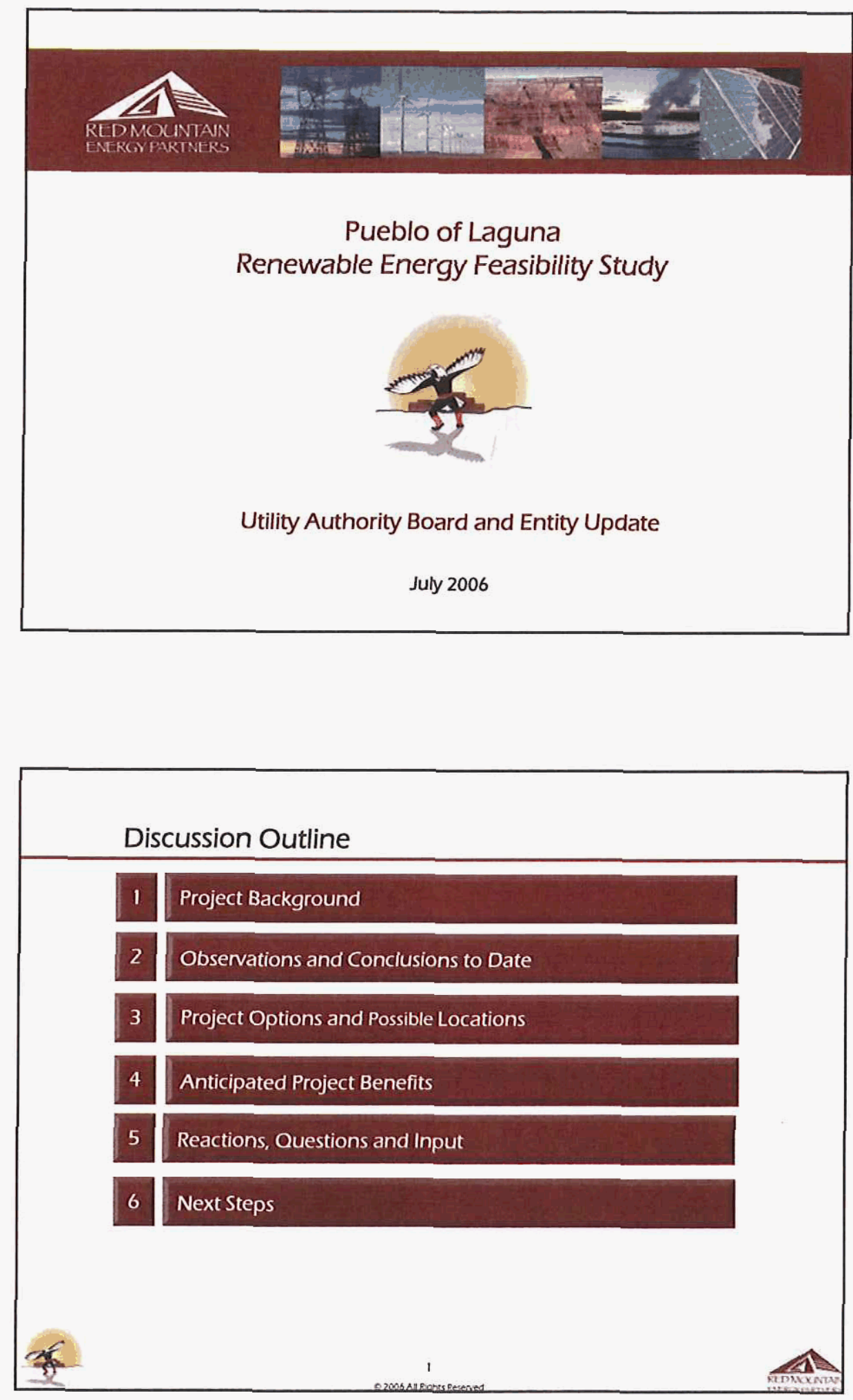


\section{Renewable Energy Feasibility Study}

\begin{tabular}{|l} 
Department of Energy Funded Study Project Objectives: \\
- Increase energy knowledge and capacity \\
- Improve quality and reliability of electric service \\
- Contribute to development of environmentally clean energy \\
- Provide data/analysis to support Laguna involvement in renewable \\
energy projects as an owner or participant
\end{tabular}

\section{Renewable Resources/Natural Gas}

- Wind

* Waiting for final anemometry results

* Preliminary results indicate small project potential, but likely not adequate for large wind project (Class 4 or better)

- Solar

* Strong solar resource; appears adequate for photovoltaic or concentrating solar applications

- Geothermal

* Power generation not likely; other business uses indicated

- Biomass

* Resource still unknown; waiting to review forest resource assessment

- Natural gas

* Good access to gas transmission and distribution in most locations

* Available for firming or backup 


\section{Electric Usage}

- Majority of energy demand is nonresidential; $66 \%$ in Laguna and Paraje

* Peak demand is approximately $5 \mathrm{MW}$; varies up to $3.2 \mathrm{MW}$ in a day

* Resources required to serve the total reservation electric demand:

- 1.6 MW base load supply $(7 \times 24)(57 \%)$

- $1.9 \mathrm{MW}$ intermediate $(6 \times 16)(28 \%$ includes solar/wind)

- 1.6 MW load following (spot market purchases)

Pueblo of Laguna Village Energy Use
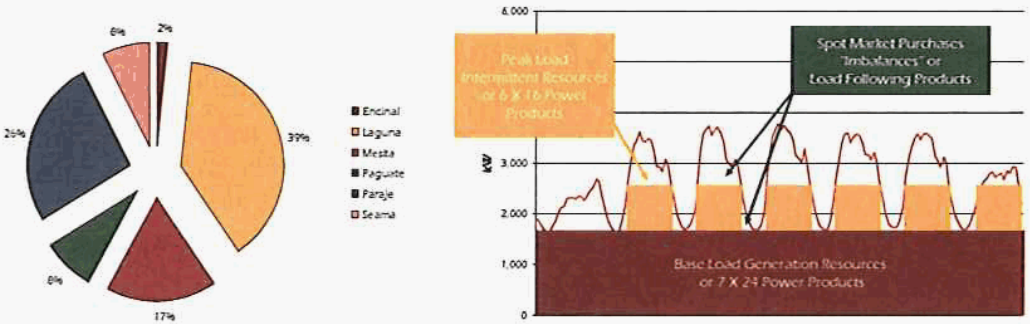

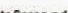

\section{Project Considerations}

- Laguna renewable projects considered generate intermittent power

$$
\therefore \text { Solar }
$$$$
\text { * Wind }
$$

- Customers assumed to have continued connection to electric grid
- Projects would not meet $100 \%$ of needs

- Purpose of gas-fired generation would be to firm a renewable resource

- Project could generate adequate power to meet a portion or all of specific electric needs

* Reservation+

* Village

* Entity/facility 


\section{Community Input}

- Visual impacts may not be acceptable for village-specific projects

- Anticipate better support for facility or reservation-wide projects in areas with limited visibility

- Improved likelihood of success if projects were located in existing industrial or commercial areas

- Best wind resources, and minimal visual impacts will be off mesas currently being monitored

- Further input needed from community

* Multiple methods (Laguna newspaper, UA newsletter, additional village/reservation-wide meetings) to provide education, seek input

\section{Project Options}

\begin{tabular}{|c|c|c|c|c|c|c|}
\hline ste & $\begin{array}{l}\text { Ste } \\
\text { Description }\end{array}$ & $\begin{array}{l}\text { Losd } \\
\text { Served }\end{array}$ & $\begin{array}{l}\text { Extimated } \\
2027 \text { Locat } \\
\text { Demand }\end{array}$ & $\begin{array}{l}\text { Possible } \\
\text { Project } \\
\text { Configurations }\end{array}$ & $\begin{array}{l}\text { Tolat } \\
\text { Project Peak } \\
\text { Copscity }\end{array}$ & $\begin{array}{l}\text { Approdimate } \\
\text { Project Size } \\
\text { (Land Requirement }\end{array}$ \\
\hline \multirow{3}{*}{1} & \multirow{3}{*}{$\begin{array}{l}\text { Raintow Center, } \\
\text { Casa Blance }\end{array}$} & \multirow{3}{*}{$\begin{array}{l}\text { Nursing home, } \\
\text { housing complex } \\
\text { junior/senior high } \\
\text { school }\end{array}$} & \multirow{3}{*}{$541 \mathrm{~kW}$} & $\begin{array}{l}\text { Lage-scale Coneentrathy Solar Arrays, firmed } \\
\text { with Naturat Gas Combustion Engine }\end{array}$ & $783 \mathrm{~kW}$ & 5 acres \\
\hline & & & & $\begin{array}{l}\text { Large-Scale Treding Solar Arrays, frmed with } \\
\text { Natural Gas Combustion Engine }\end{array}$ & $490-783 \mathrm{~kW}$ & 45 acres \\
\hline & & & & $\begin{array}{l}\text { Two } 250 \mathrm{~kW} \text { Wind Turbines, firmed with Natural } \\
\text { Gas Combustion Engine }\end{array}$ & $788 \mathrm{~kW}$ & Up to 10 acres \\
\hline \multirow{3}{*}{2} & \multirow{3}{*}{$\begin{array}{l}\text { Southeast of } \\
\text { Mesita Vilage }\end{array}$} & \multirow{3}{*}{ Village losd } & \multirow{3}{*}{$1.0 \mathrm{MW}$} & $\begin{array}{l}\text { Large-scale Concentrating Solar Anfays, firmed } \\
\text { with Natural Gas Combuston Engine }\end{array}$ & $157 \mathrm{MN}$ & 810 acres \\
\hline & & & & 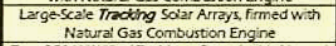 & $1.05 .128 \mathrm{MW}$ & $5-8$ acres \\
\hline & & & & $\begin{array}{l}\text { Two } 250 \mathrm{~kW} \text { Wind Turbines, fimed with Netural } \\
\text { Gas Combustion Engine }\end{array}$ & $1.33 \mathrm{MW}$ & Up to 10 acres \\
\hline \multirow{4}{*}{3} & \multirow{4}{*}{$\begin{array}{c}\text { Laguna Vilage. } \\
\text { north of Transfer } \\
\text { Station }\end{array}$} & \multirow{4}{*}{$\begin{array}{l}\text { Reservation, partial } \\
\text { Reservation load. or } \\
\text { power export }\end{array}$} & \multirow{4}{*}{$5.8 \mathrm{MW}$} & $\begin{array}{c}\text { Largescale Concentrating Solar Arrays, firmed } \\
\text { with Natura' Gas Combustion Eng ine }\end{array}$ & $214 \mathrm{MW}$ & 10 acres \\
\hline & & & & $\begin{array}{c}\text { Large-Scale Tradihg Solar Arrays, firmed with } \\
\text { Natural Gas Combustion Engine }\end{array}$ & $0.8-5.14 \mathrm{MW}$ & 5.25 acres \\
\hline & & & & $\begin{array}{l}\text { Large-Scale Trading Solar Arrajs, firmed with } \\
\text { Natural Gas Turbhe }\end{array}$ & $6.6 \mathrm{MW}$ & 35 aces \\
\hline & & & & Power Grid-Sca'e Natural Gas Combustion Turbine & $75.9 \mathrm{MW}$ & $?$ \\
\hline \multirow{4}{*}{4} & \multirow{4}{*}{$\begin{array}{l}\text { Rio Puerco, near } \\
\text { Route 6s Casino }\end{array}$} & \multirow{4}{*}{$\begin{array}{l}\text { Reservation partiat } \\
\text { Reservation hasd, or } \\
\text { power export }\end{array}$} & \multirow{4}{*}{$5.8 \mathrm{MN}$} & 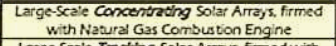 & $2.14 \mathrm{MN}$ & 10 acres \\
\hline & & & & $\begin{array}{l}\text { Largescrate Trodking Solar Arrays, firmed with } \\
\text { Natural Gas Combustion Engine }\end{array}$ & $0.85 .14 \mathrm{MW}$ & 5.25 acres \\
\hline & & & & $\begin{array}{l}\text { Large Scale Trocking Solar Arrays fimed with } \\
\text { Natural Gas nurbhe }\end{array}$ & $6.6 \mathrm{MN}$ & 35 acres \\
\hline & & & & Power Grid-Sate Natural Gas Combustion Turbine & $75.9 \mathrm{MW}$ & $?$ \\
\hline \multirow[t]{2}{*}{5} & \multirow{2}{*}{$\begin{array}{c}\text { Parje, near oid } \\
\text { high school }\end{array}$} & \multirow{2}{*}{$\begin{array}{l}\text { Village or partial } \\
\text { village load }\end{array}$} & \multirow{2}{*}{$1.5 \mathrm{MN}$} & $\begin{array}{l}\text { Large-Scale Concentrating Solar Arrays, frmed } \\
\text { with Natural Gas Combustion Engine }\end{array}$ & $1.57 \mathrm{MW}$ & 8-10xres \\
\hline & & & & $\begin{array}{l}\text { Large-Scale Tracking Solar Arrays, firmed With } \\
\text { Natural Gas Combustion Engine }\end{array}$ & $0.8-1.57 \mathrm{MW}$ & $5-10$ actes \\
\hline
\end{tabular}

Note: All projects could be configured as natural gas or renewable only, rather than a hybrid arrangement; renewable-only projects would provide intermittent power 

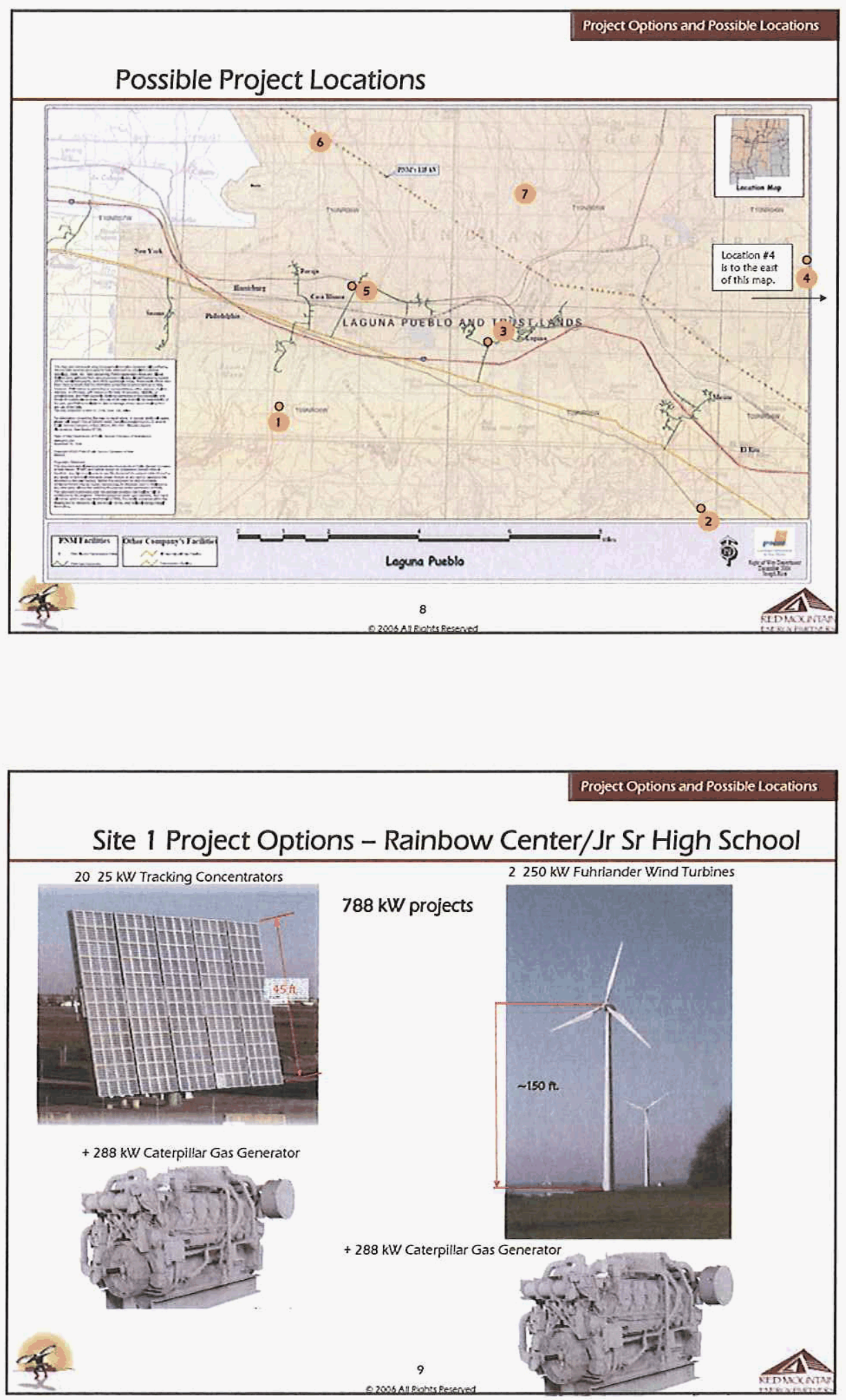


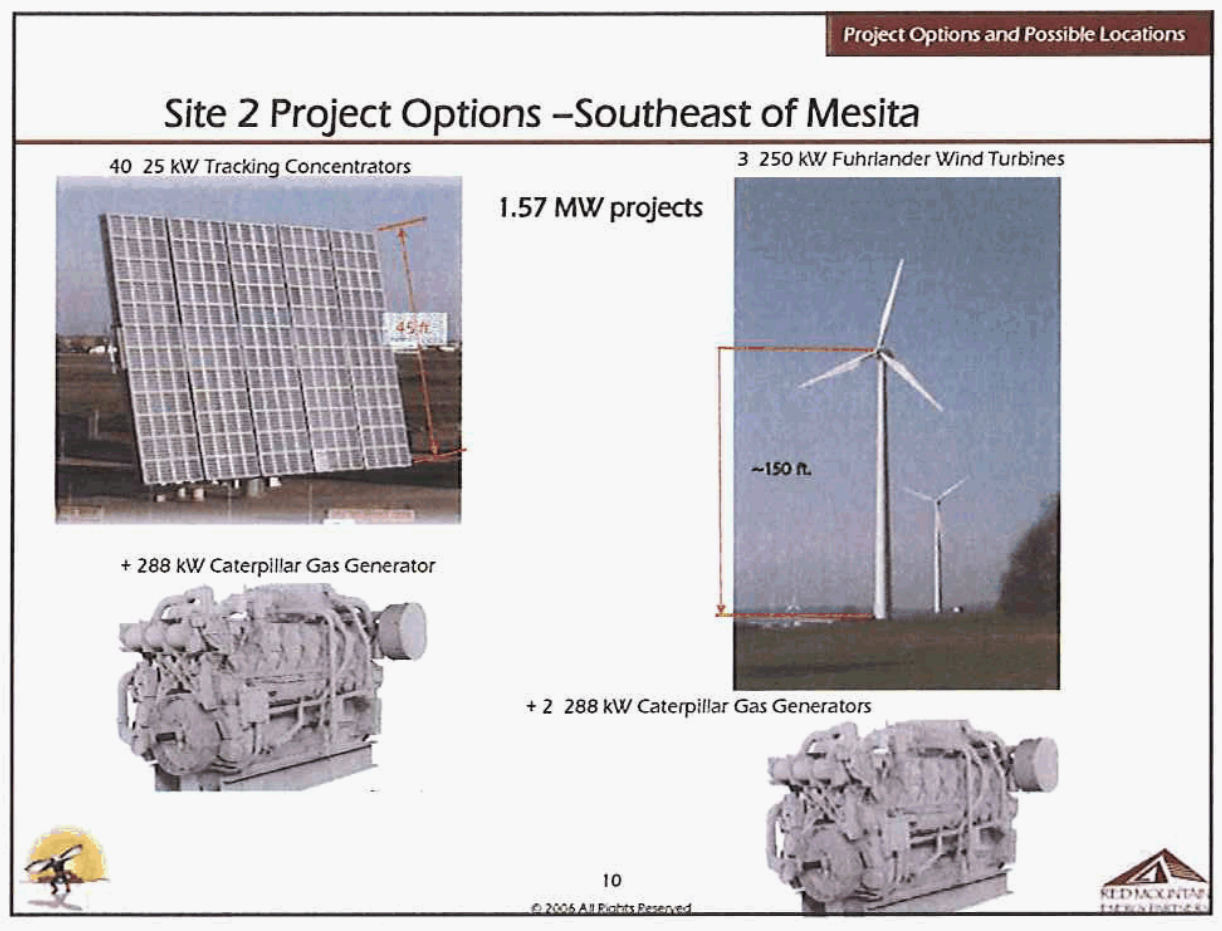

Project Options and Possible Locations

Site 3 Project Options - Laguna, north of Transfer Station

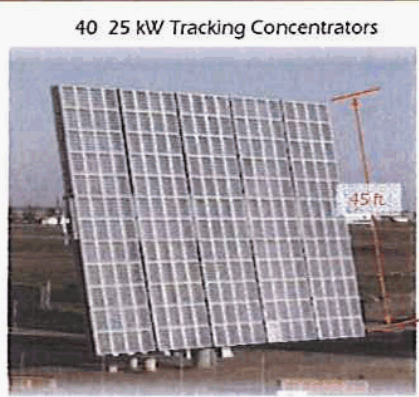

$2.14 \mathrm{MW}$ project

+2570 kW Caterpillar Gas Generator
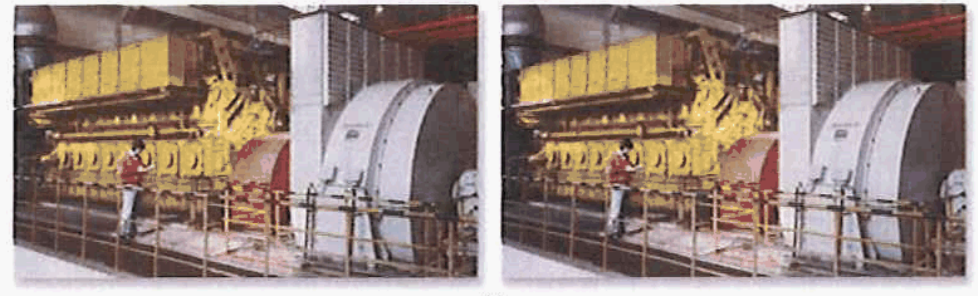

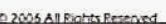



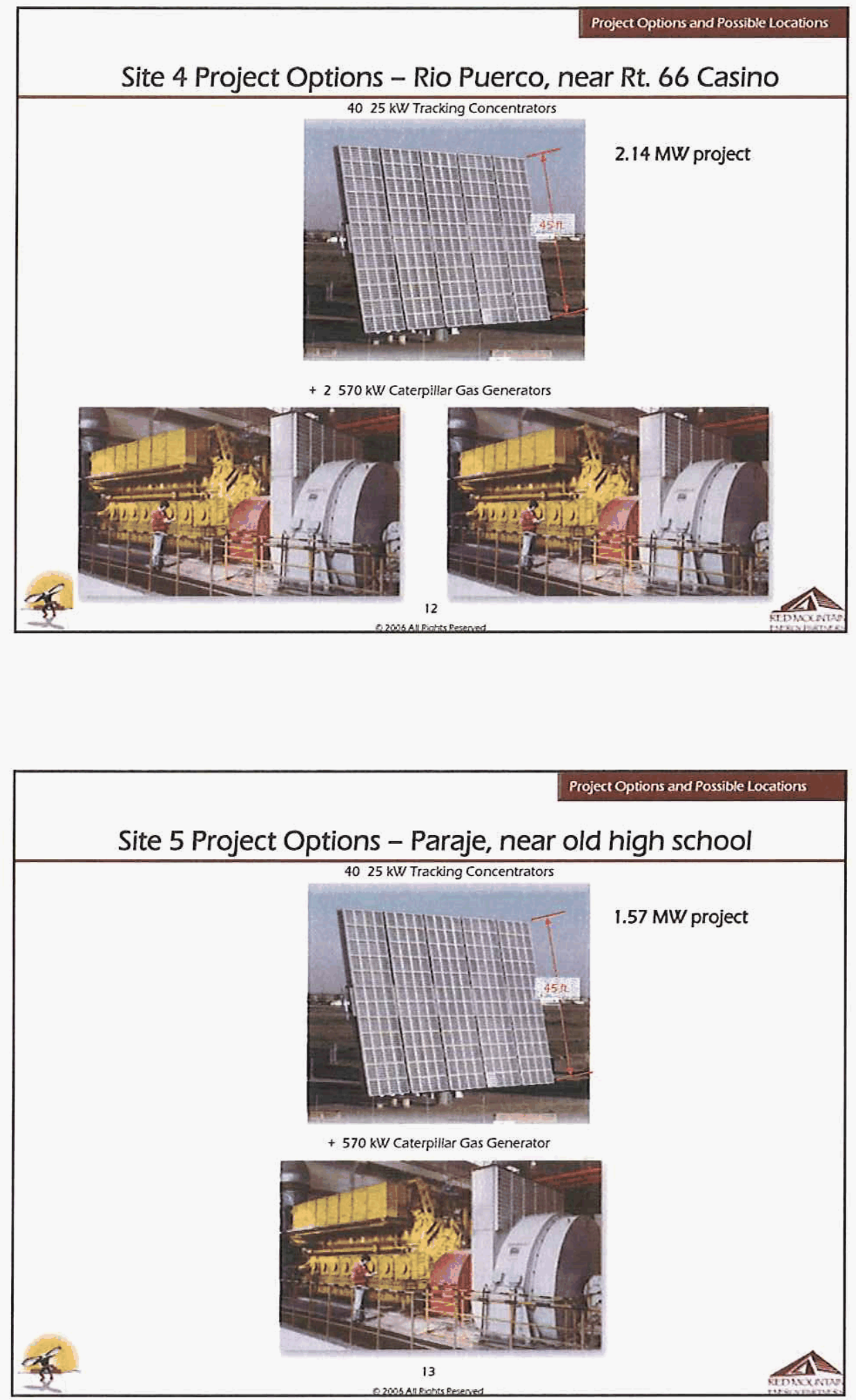

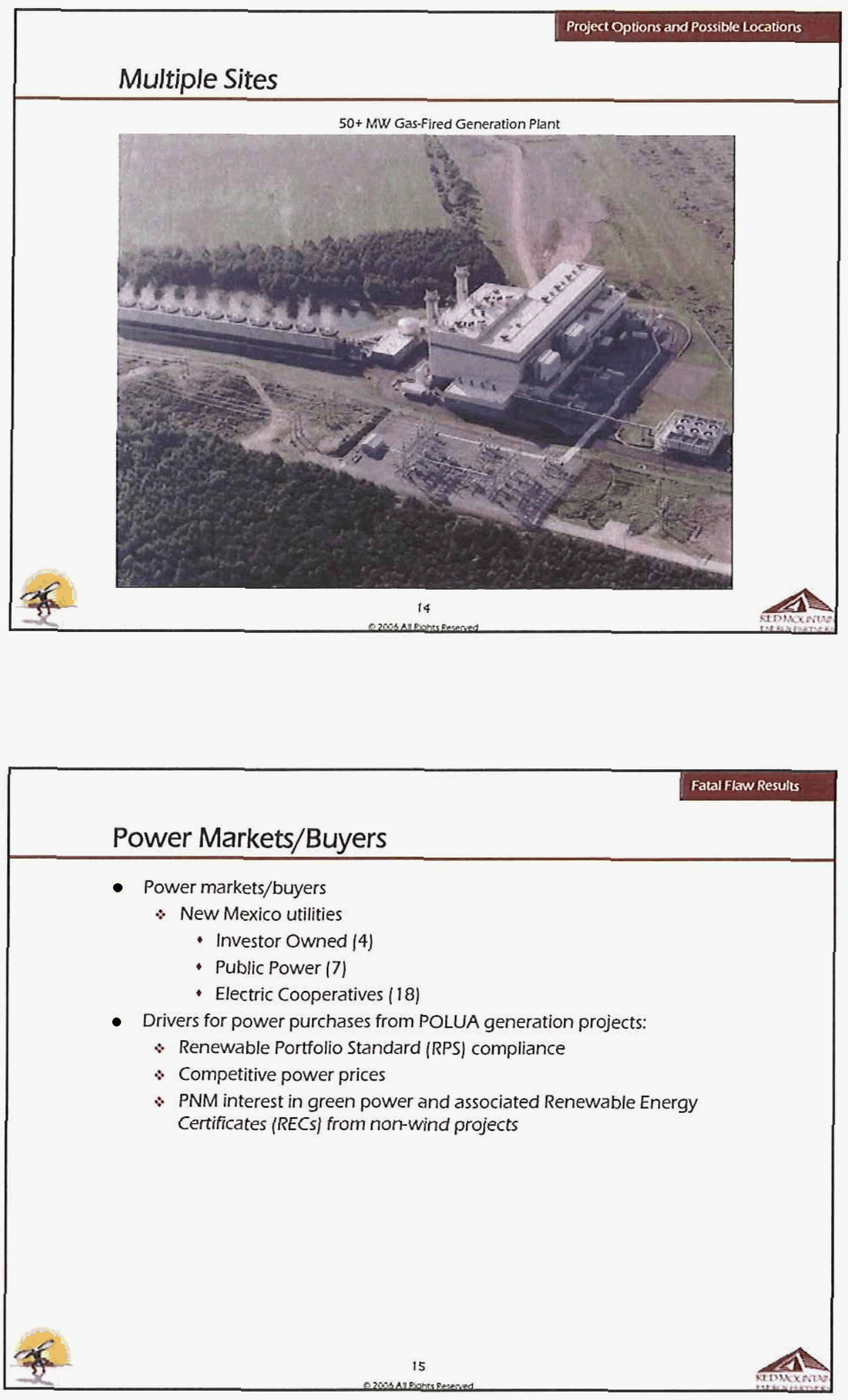


\section{Preliminary Environmental Review}

- Sites under consideration have been/adjacent to properties previously impacted by some activity (i.e. development, grazing, agriculture, access roads, etc.)

- All sites appear clear of any immediate cultural or historical fatal flaws

- Washes present at sites \#1 and \#5, but sites flexible re: mitigation

*Dry washes; no apparent endangered species issues

- Multiple sites available allow for protection of "view sheds"

- Additional transmission/distribution ROWs yet to be reviewed

- Alternative sites must be identified under any Environmental Assessment

- FONSI (finding of no significant impact) may not be considered, since proposed projects are a "change in use"

\section{Transmission/Interconnection}

- Transmission

* Previous studies indicate $60 \mathrm{MW}$ of capacity available for power export via PNM $115 \mathrm{kV}$ transmission line

- Projects involving facility-scale, village-scale, or partial village-scale loads are likely to require some distribution line upgrades

- Interconnection

* Interconnection for large projects could occur at the Paguate Substation or new substation being considered

* Interconnection at other project sites will require additional equipment to coordinate system protection schemes (fuse cut-outs, breakers, relays) 


\section{Renewable Generation}

Wind Solar

- Environmental, cultural, economic benefits

$\therefore$ Low operating costs

$\therefore \quad$ No fuel supply/cost risks

$\therefore \quad$ No cooling water required

* No wastes, no emissions

- Trade-Offs

- High capital costs

* Non-dispatchable
- Environmental, cultural, economic benefits

* Low operating costs

- No fuel supply/cost risks

$\therefore$ No cooling water required

$\therefore$ No wastes, no emissions

* Good load following characteristics

- Trade-Offs

* High capital costs

$\therefore$ Non-dispatchable

\section{Gas-Fired Generation}

Microturbines

- Environmental, cuitural, economic benefits

* Small number of moving parts

* Low emissions

* Long maintenance intervals

* Compact

* Dispatchable

* Canutilize waste fuels

* Good efficiencies w/cogeneration

- Trade-offs

- Low fuel to electric efficiencies

s Loss of power output/efficiency with higher ambient temperatures
Reciprocating Engines

- Environmental, cuitural, economic benefits

* Low capital costs

- Good efficiencies

- Quick startup

- Fuel flexibility

- High reliability

- Low natural gas pressure required

- Trade-offs

* Emissions

s Noisy

- Frequent maintenance intervals

Combustion Turbines

- Environmental, cultural, economic benefits

* High efficiency, low cost

- Wide range of power outputs

- Can produce high temperature steam

* Wellestablished channels

* High power to weight ratio

* Proven reliability/availability

- Trade-offs

* Reduced efficiencies at part load

s Sensitive to ambient conditions

- Cost/efficiency not as good in small systems 

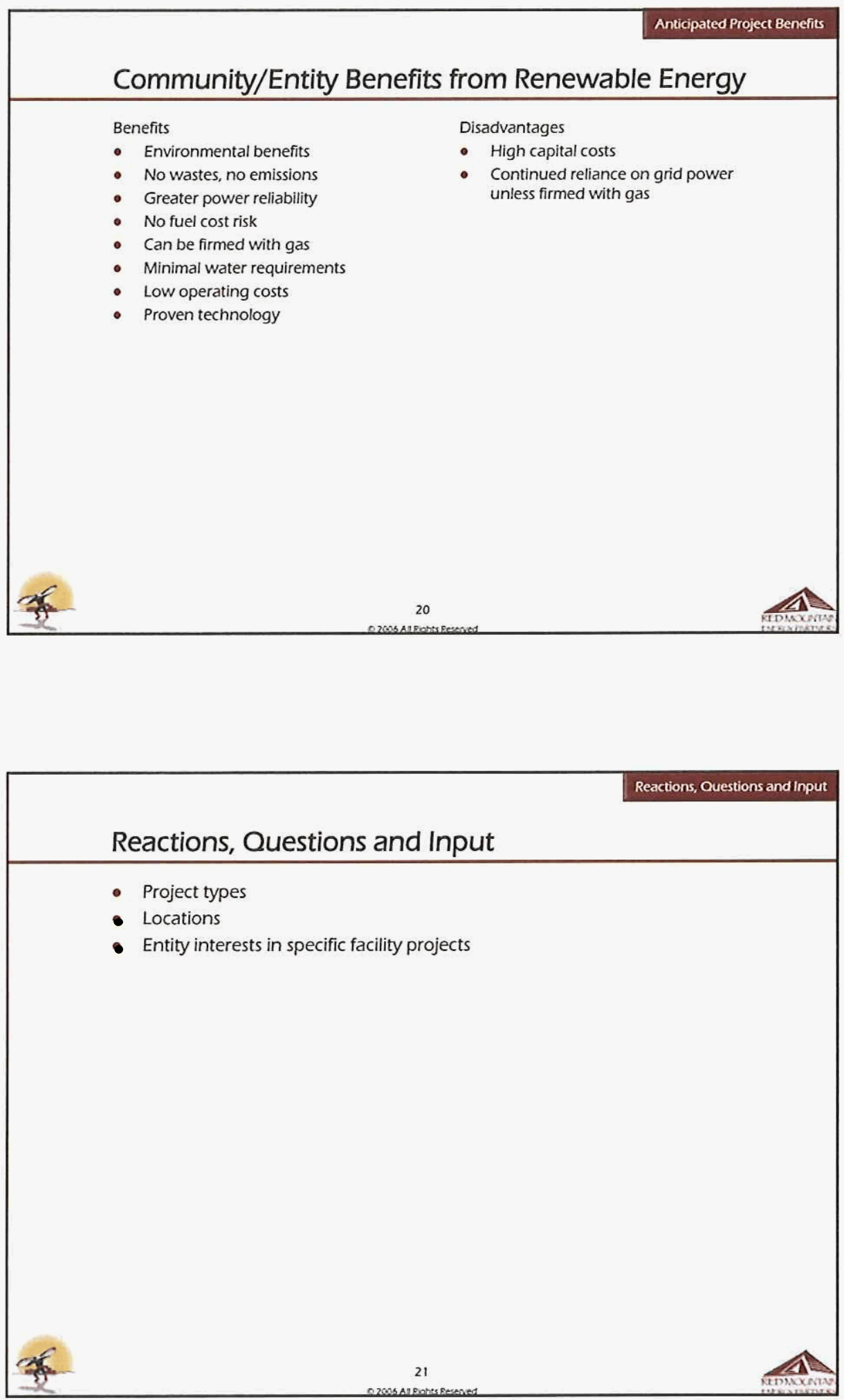


\section{Next Steps}

- Continue community education activities

- Continue to analyze most probable project(s) and locations

- Complete technical and financial analyses of probable projects

- Prepare development plan

\section{Further Information}

Contact:
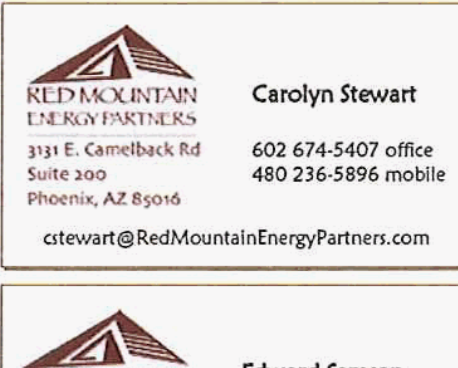

REDMOXINTAN

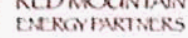

3131 E. Camelback Rd

Suite 200

Phoenix, AZ 85016

esamson@RedMountainEnergyPartners.com

Edward Samson

$602674-5407$ office 6026849479 mobile

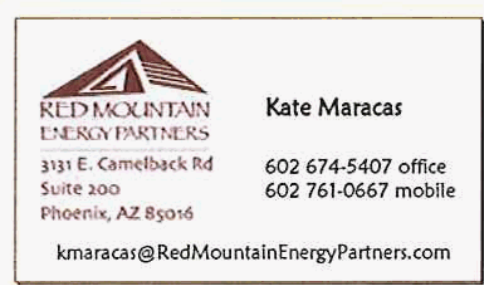

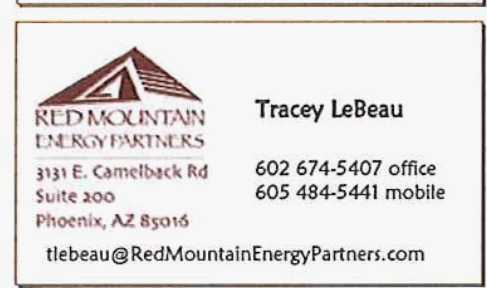

23 


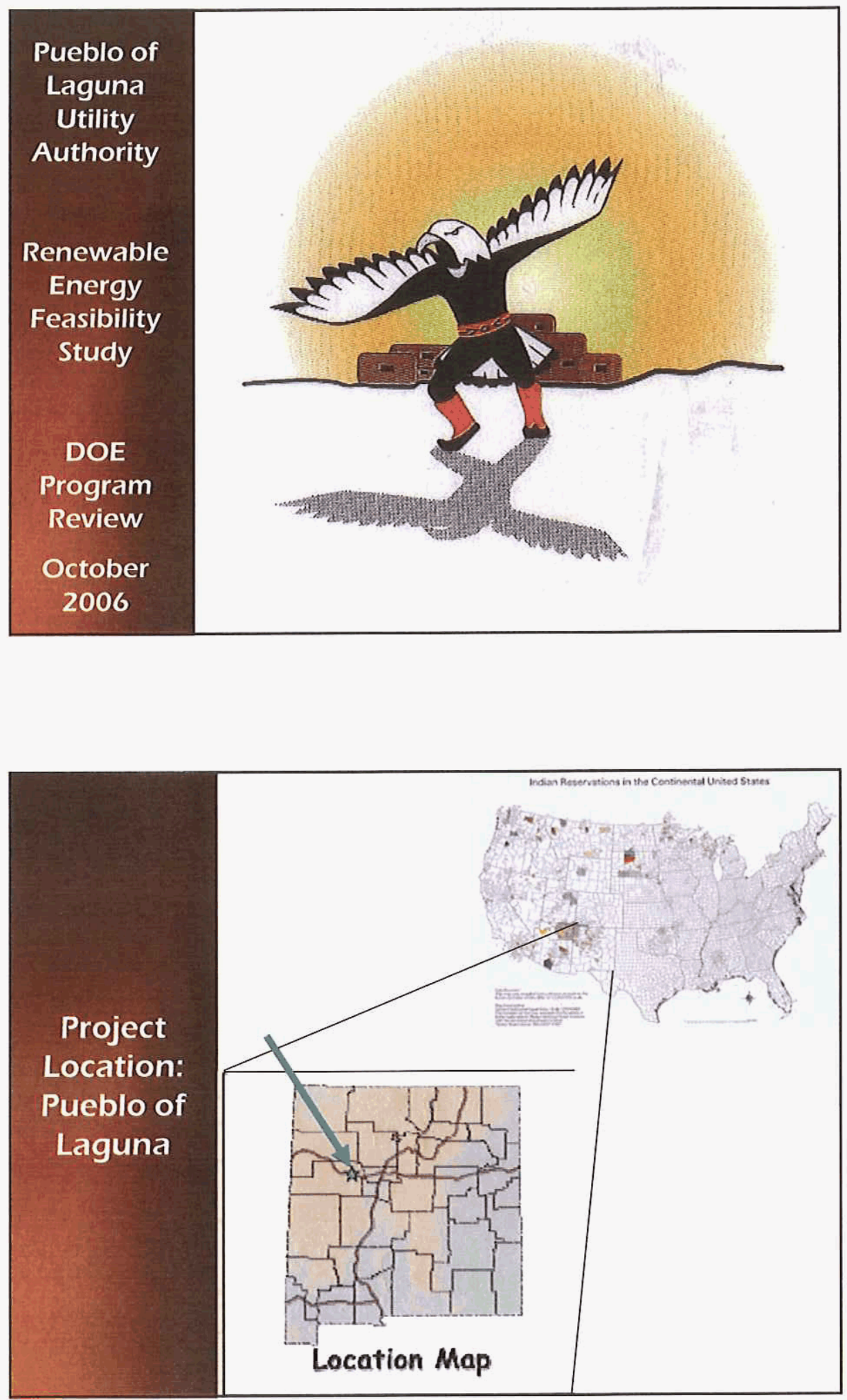

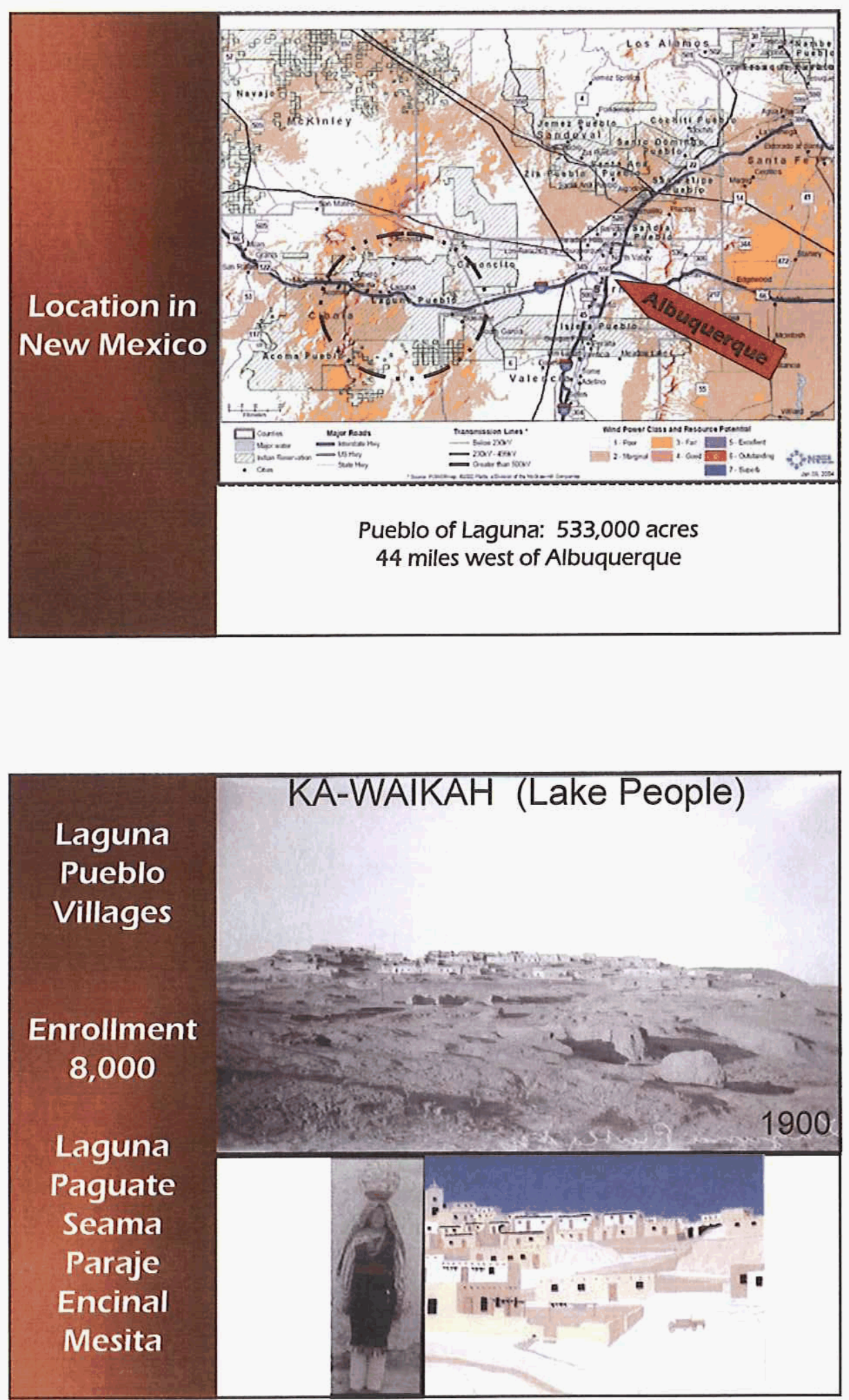

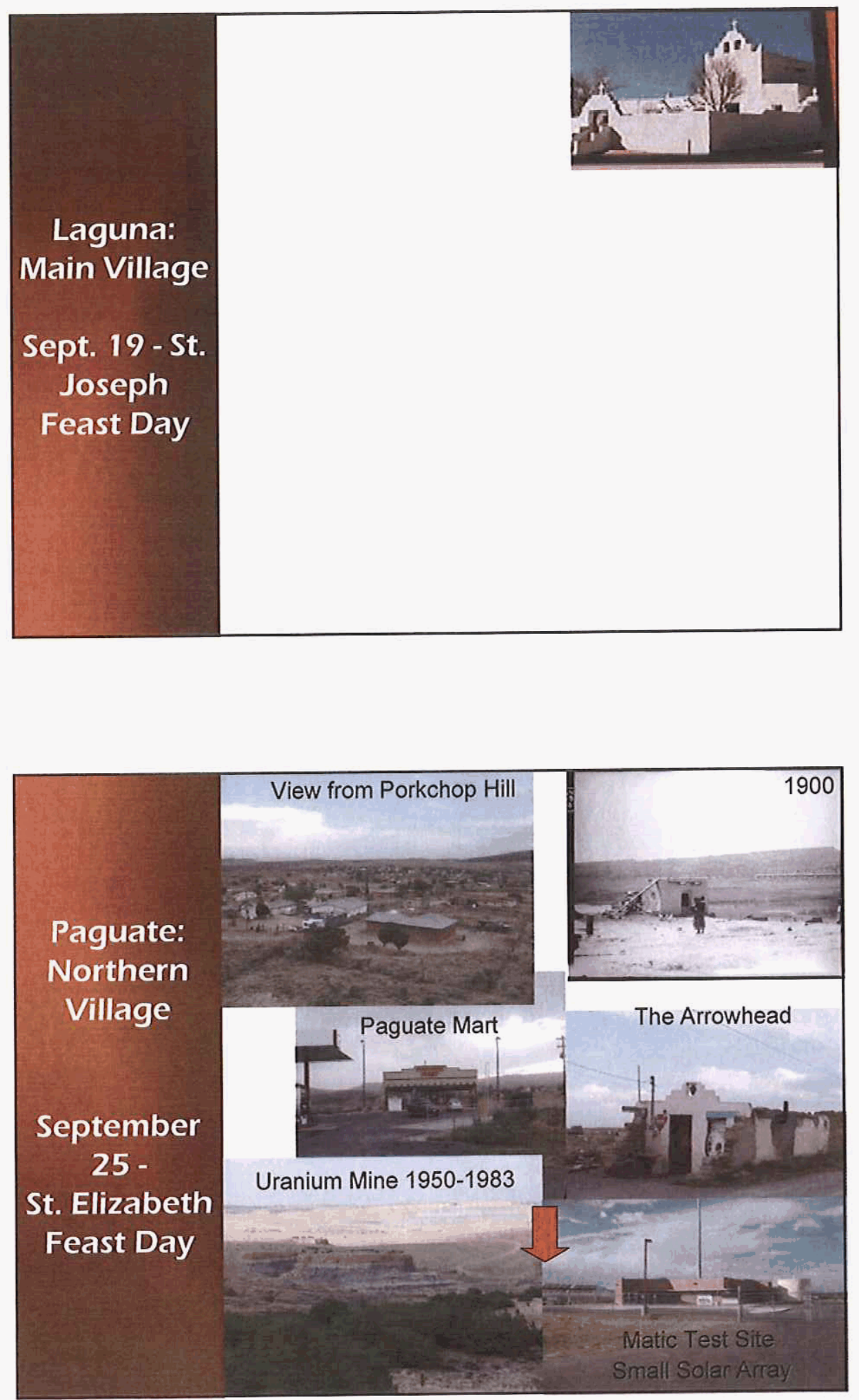

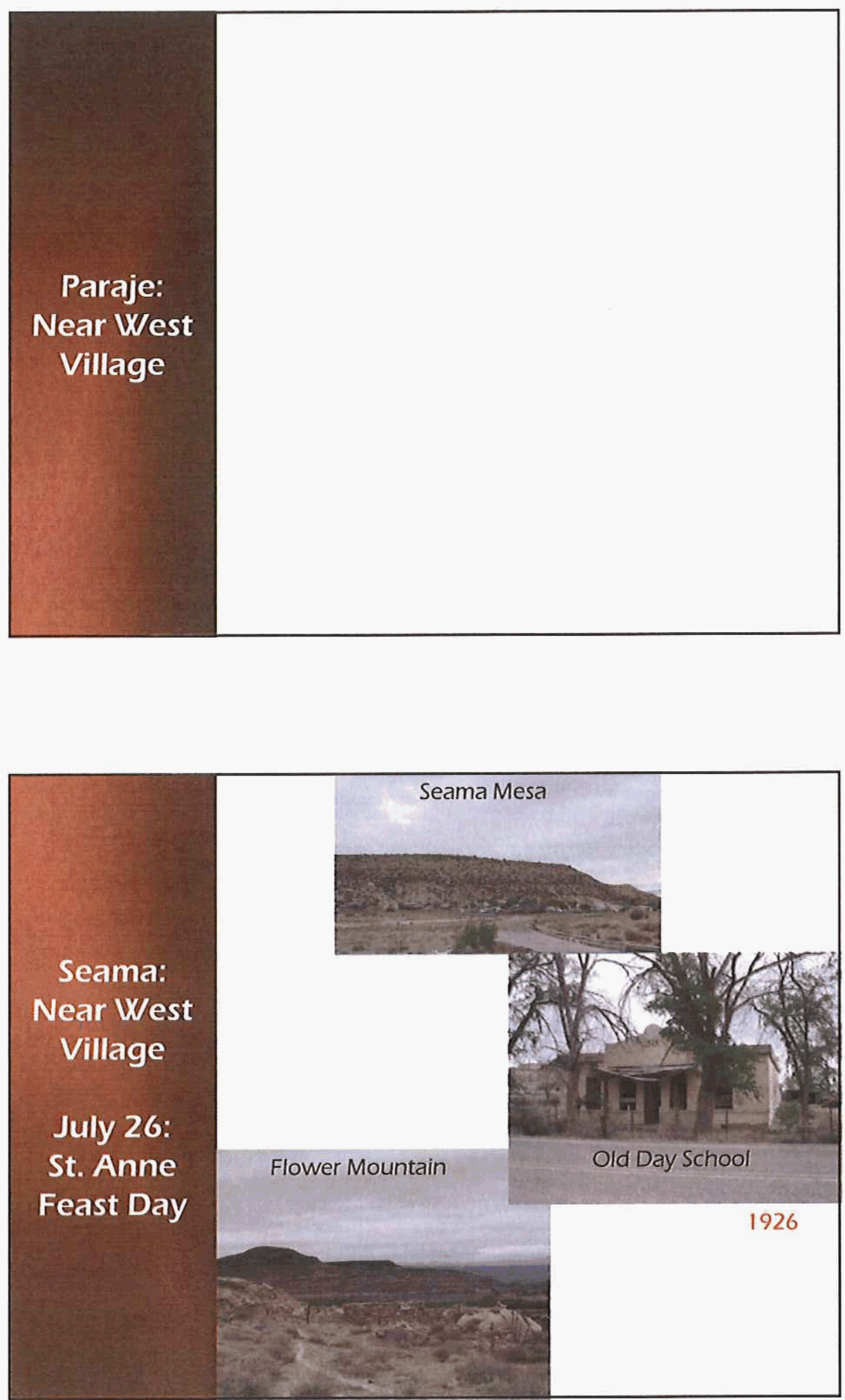

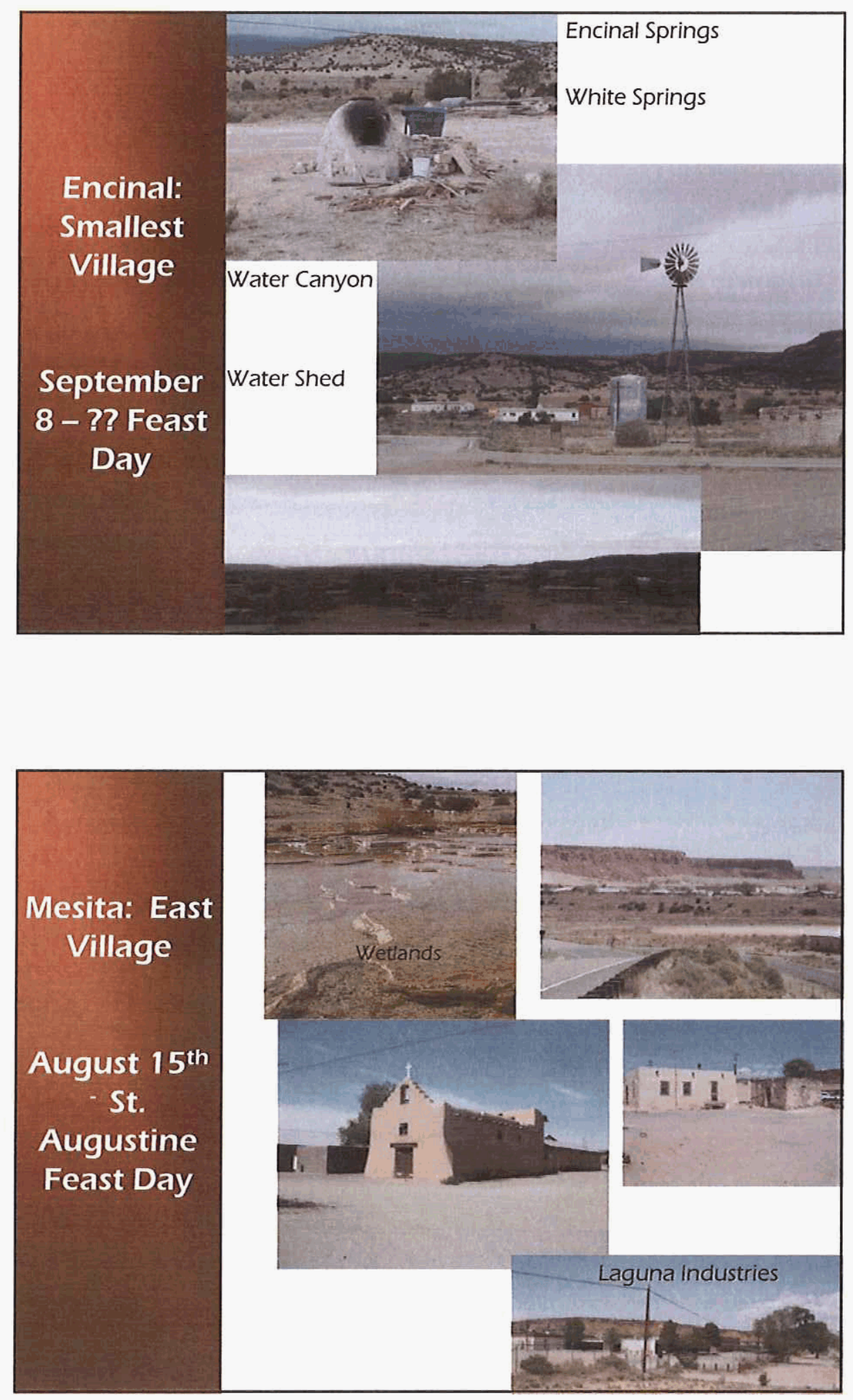


\section{Project Objectives}

- Assess feasibility of renewable energy generation projects

\section{Project}

focuses on

community

involvement

in addition

to technical

studies

- Build on Pueblo interest in renewable energy focused on wind, solar and biomass resources

- Prior projects included solar facilities at Majors Ranch and Laguna Industries

- Consider options to leverage nearby gas pipelines to develop a "hybrid" project, providing "firm" power

- Encourage community involvement and participation through education

- Expand on previously completed work

- Utility formation and development

- Energy self-sufficiency options

- Integration of energy management with economic development

- Support energy capacity building

\begin{tabular}{|c|c|}
\hline & Project Participants \\
& Tribal and Staff Participants: \\
& - Pueblo of Laguna Utility Authority and \\
& Board of Directors \\
Project is & - Pueblo of Laguna Tribal Council and Staff \\
being led by & Officers \\
Utility & - Pueblo of Laguna Villages \\
Authority & - Pueblo of Laguna Entities/Facility Managers \\
personnel & Project Consultant: \\
& - Red Mountain Energy Partners \\
& \\
& \\
& \\
\hline
\end{tabular}



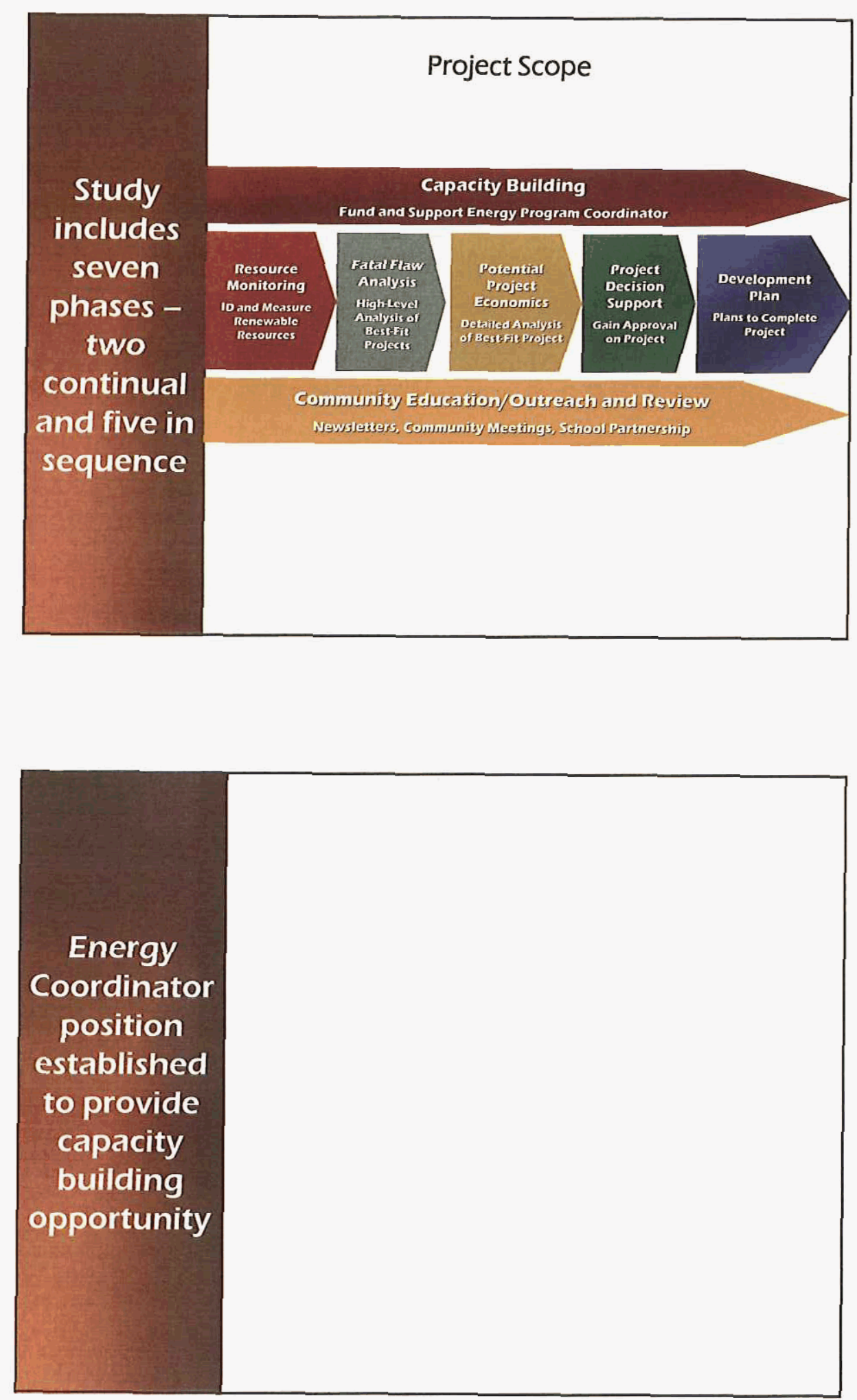

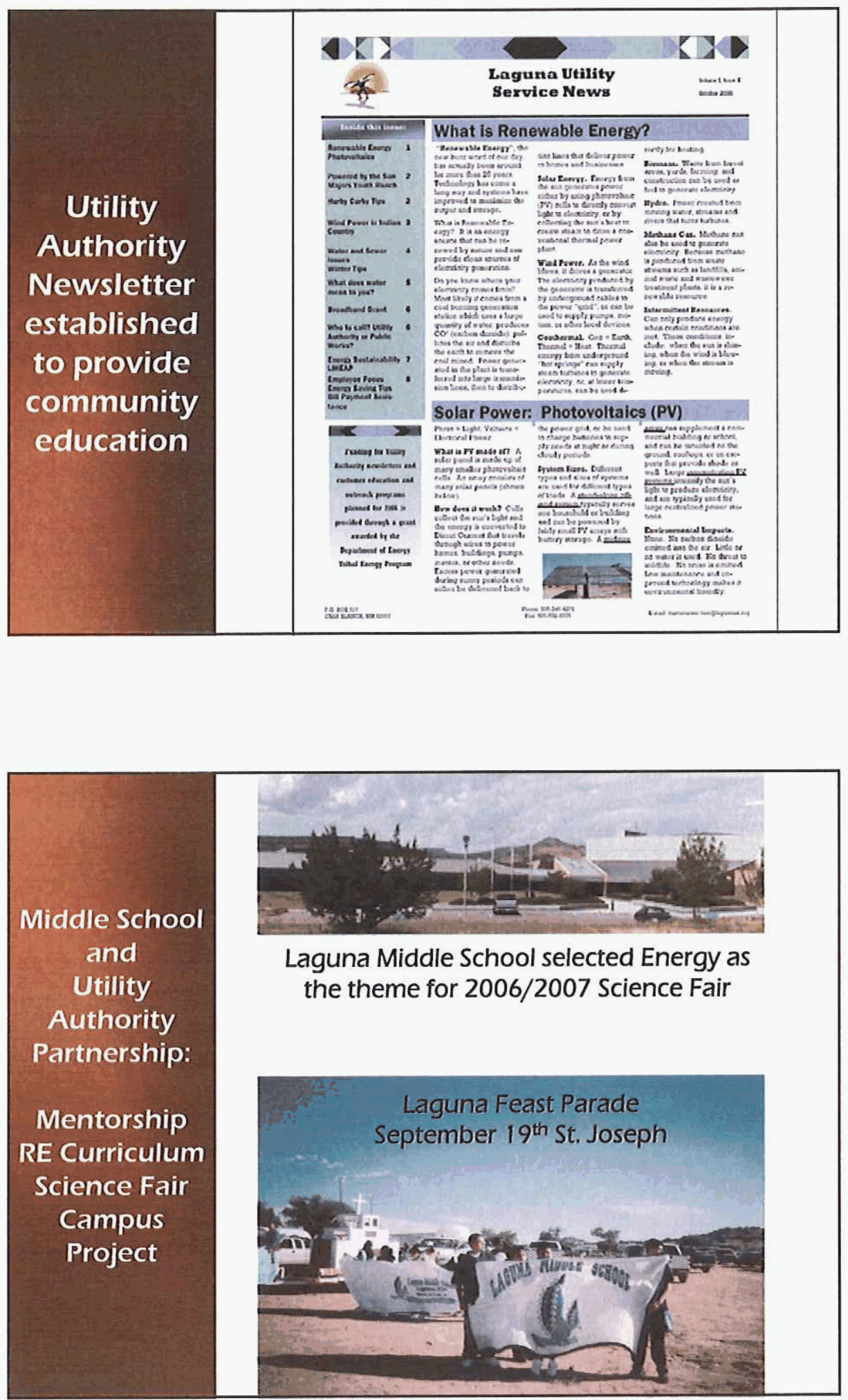

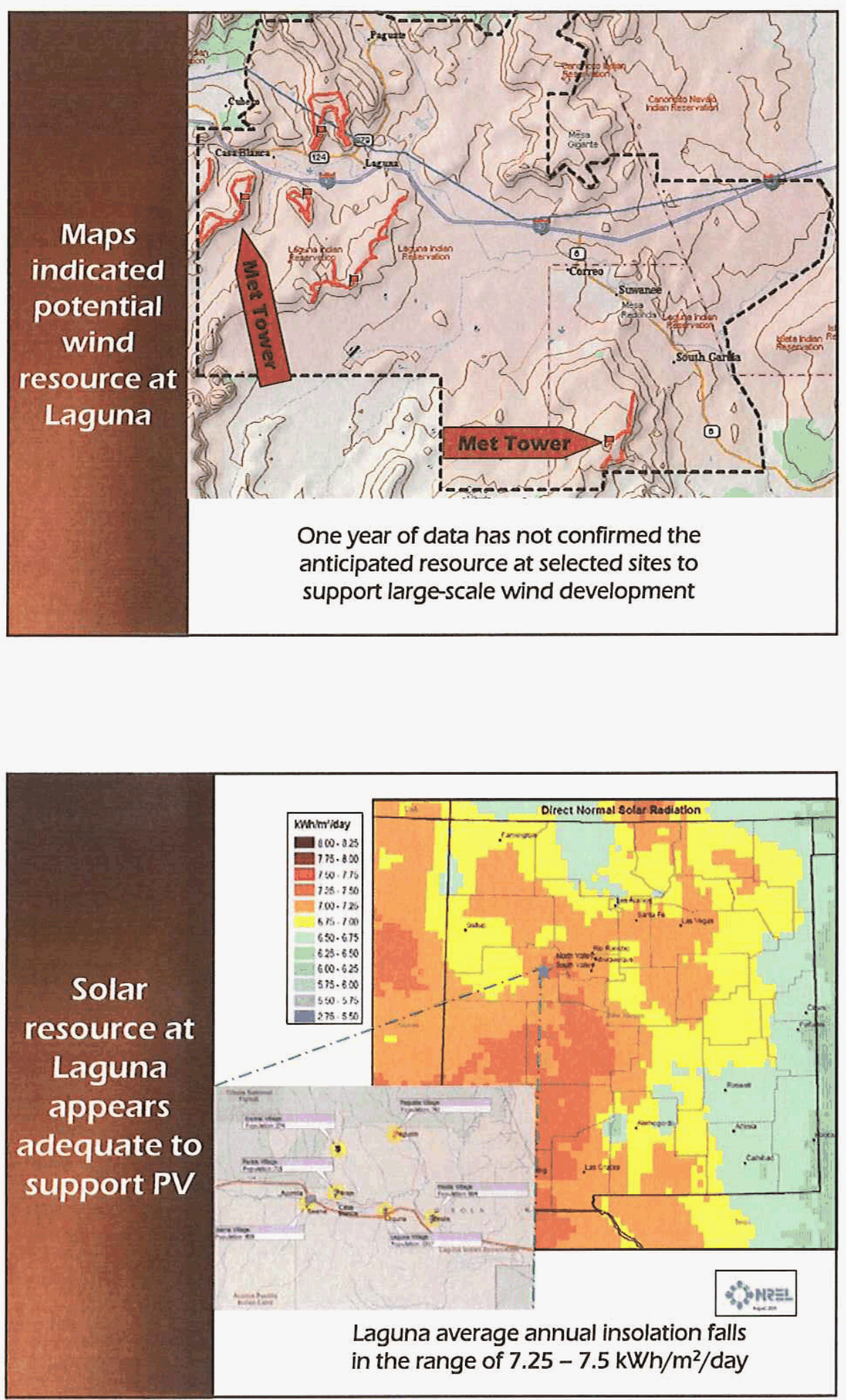

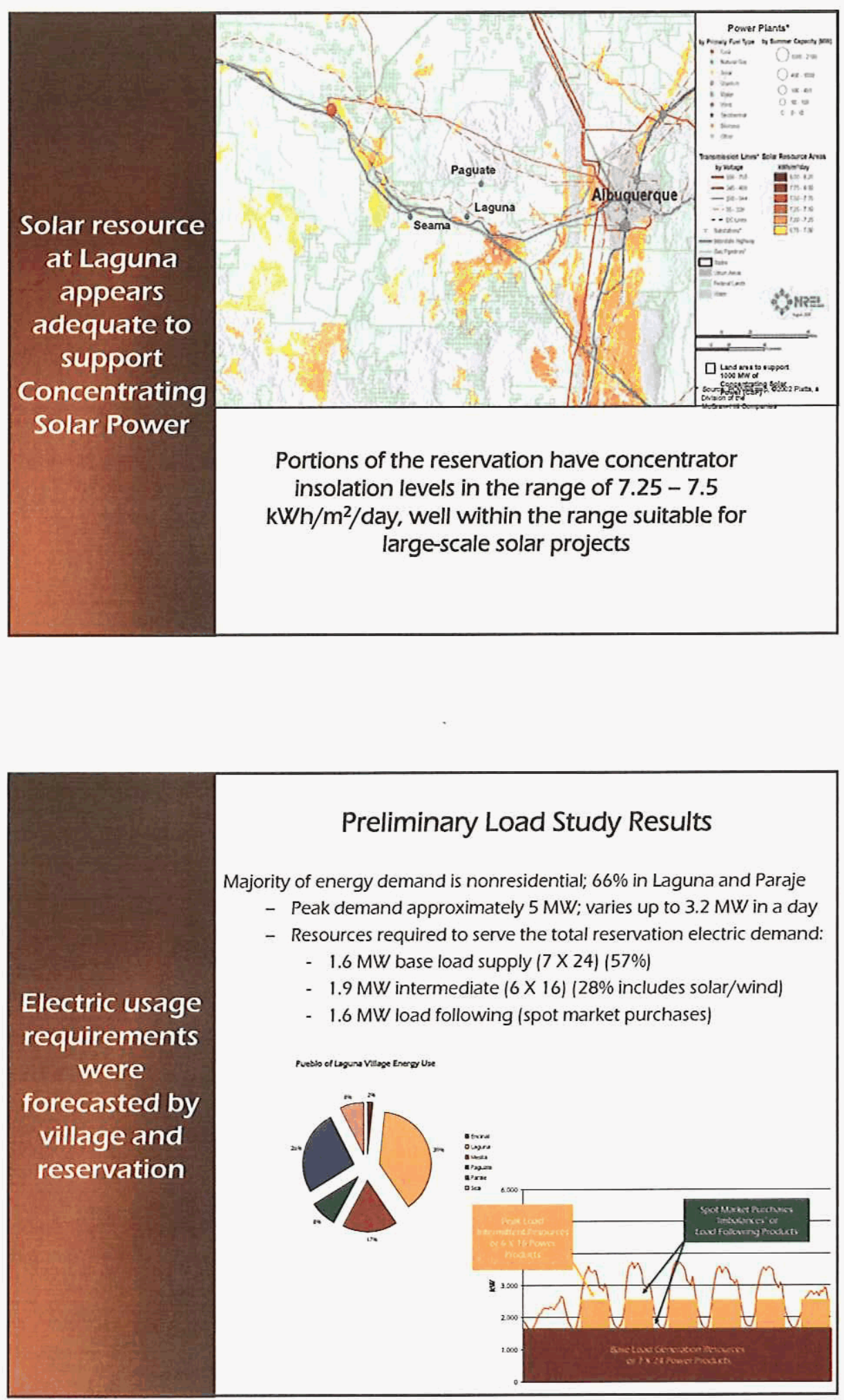


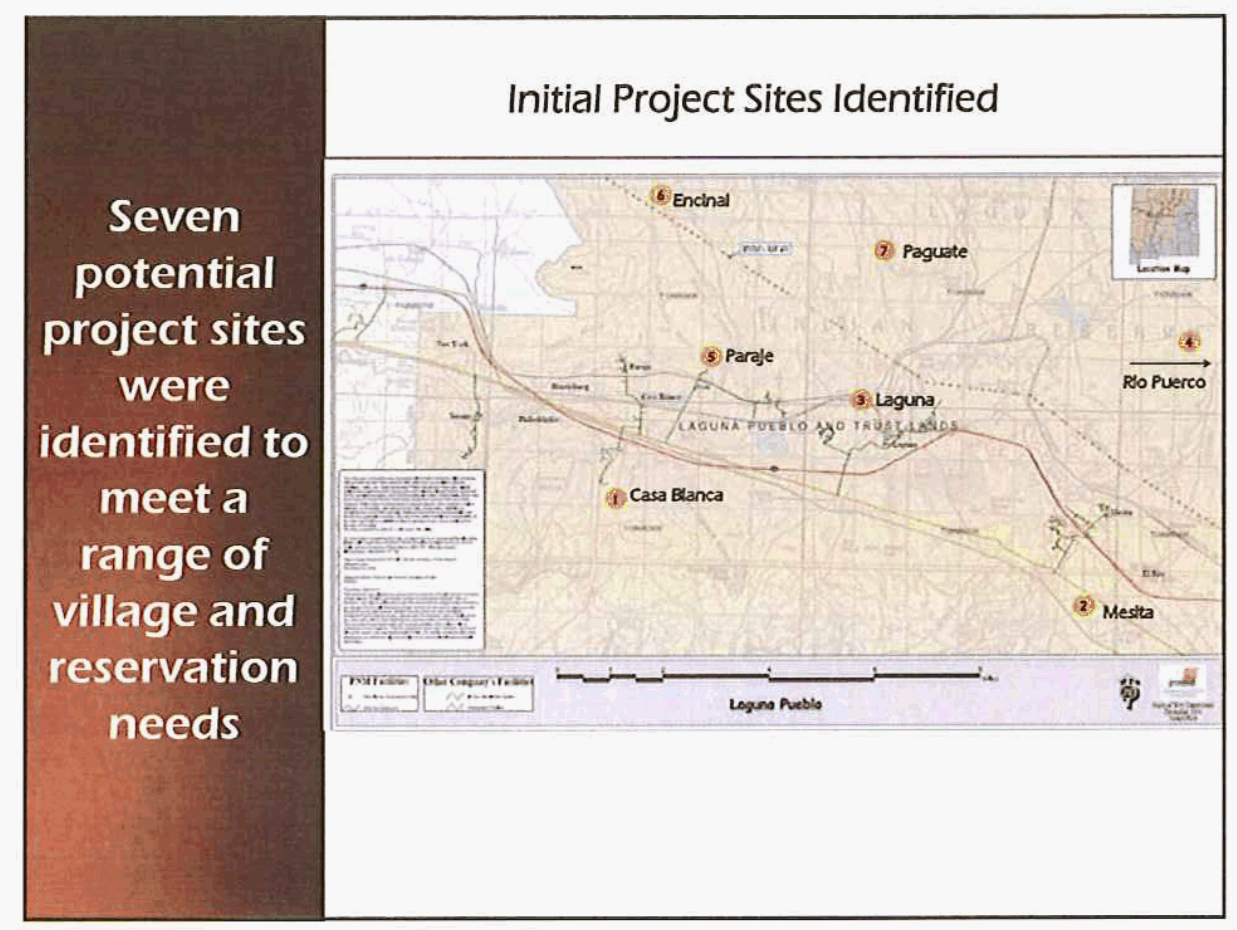

\begin{tabular}{|c|c|c|c|c|c|c|c|c|c|}
\hline \multirow[b]{3}{*}{ Multiple } & \multicolumn{9}{|c|}{ Initial Wind Project Configurations } \\
\hline & 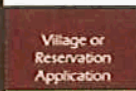 & $\begin{array}{c}\text { Peak } \\
\text { Demand } \\
\text { awl }\end{array}$ & $\begin{array}{l}\text { Anual } \\
\text { Uase } \\
\text { akin }\end{array}$ & $\begin{array}{l}\text { Lowd } \\
\text { fatiox }\end{array}$ & 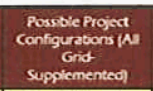 & $\begin{array}{l}\text { Peat } \\
\text { Pencer } \\
\text { paty }\end{array}$ & $\begin{array}{l}\text { Propected } \\
\text { Coacty } \\
\text { Fatar }\end{array}$ & $\begin{array}{l}\text { Prokeded } \\
\text { Annuakikn }\end{array}$ & $\begin{array}{l}\text { Intivit } \\
\text { capet } \\
\text { cost' }\end{array}$ \\
\hline & $\begin{array}{c}\text { Laguna industries } \\
\text { Generation }\end{array}$ & 544 & 1.620 .000 & $340 \%$ & \begin{tabular}{|c|}
$\begin{array}{c}\text { Two Funtrander } \\
250 \mathrm{KW} \text { Turbines }\end{array}$ \\
\end{tabular} & 500 & $21.14 \%$ & 871.507 & $\begin{array}{l}\$ 1.2 \mathrm{MM} \\
\text { S1.55M } \\
\end{array}$ \\
\hline wind & $\begin{array}{l}\text { Encinal Vilage } \\
\text { Scaze Generzion } \\
\end{array}$ & 102 & 393,023 & $44.0 \%$ & $\begin{array}{l}\begin{array}{l}\text { One Fuhrlander } \\
100 \text { kW Turbine }\end{array} \\
\end{array}$ & 100 & $242 \%$ & 225.223 & $\begin{array}{ll}\$ 250 \%- \\
\$ 300 \%\end{array}$ \\
\hline oroject & $\begin{array}{l}\text { Laguna Village } \\
\text { Scele Generzion }\end{array}$ & 2,083 & $9.207,437$ & $50.5 \%$ & $\begin{array}{c}\text { One GE } 1.5 \mathrm{MW} \\
\text { Turbine }\end{array}$ & 1.500 & $20.6 \%$ & $3.059,491$ & $\begin{array}{ll}\$ 20 \mathrm{M}- \\
\$ 22 \mathrm{sM}\end{array}$ \\
\hline & $\begin{array}{l}\text { Mesta Vilage } \\
\text { Scé'e Generation }\end{array}$ & $9 \div 9$ & $4,073.652$ & $49.0 \%$ & $\begin{array}{l}\text { Once Furrtander } \\
600 \mathrm{KW} \text { Tubine } 2\end{array}$ & 600 & & $1,000,000$ & $\begin{array}{l}5950 \%- \\
\text { SiM- }\end{array}$ \\
\hline options & \begin{tabular}{|l} 
Paguate Viliage \\
Scaie Generation
\end{tabular} & 472 & 1.953 .873 & $47.3 \%$ & \begin{tabular}{|c|} 
Two Funtlander \\
$250 \mathrm{~kW}$ Turones ${ }^{2}$ \\
\end{tabular} & 500 & $21.14 \%$ & 871.507 & \begin{tabular}{|l}
$1.25 \mathrm{M}-$ \\
S1.75M \\
\end{tabular} \\
\hline vere & $\begin{array}{l}\begin{array}{c}\text { Peraje Vilibge } \\
\text { Scale Generdion }\end{array} \\
\end{array}$ & 1.392 & $6,205,168$ & $50.9 \%$ & $\begin{array}{l}\begin{array}{c}\text { One GE } 1.5 \mathrm{MN} \\
\text { Turbine }\end{array} \\
\end{array}$ & 1.500 & $20.6 \%$ & $3,059,491$ & \begin{tabular}{|l|l|}
$520 \mathrm{M}-$ \\
$\$ 2259 \mathrm{M}$ \\
\end{tabular} \\
\hline considered & $\begin{array}{l}\text { Seama Viltage } \\
\text { Scale Generzaton }\end{array}$ & 427 & $1.783,999$ & $47.7 \%$ & \begin{tabular}{|l|} 
Two Funhtander \\
$250 \mathrm{KW}$ Tutbines \\
\end{tabular} & 500 & $21.14 \%$ & 871.507 & \begin{tabular}{|l}
$5125 \mathrm{M}-$ \\
S1.75M \\
\end{tabular} \\
\hline at identified & $\begin{array}{c}\text { Laguna } \\
\text { Seservationscale } \\
\text { Generation }\end{array}$ & 5,426 & \begin{tabular}{|l|}
23.618 .151 \\
\end{tabular} & $49.7 \%$ & $\begin{array}{c}\text { Four GE } 1.5 \mathrm{MN} \\
\text { Turbines }\end{array}$ & 6.000 & $20.6 \%$ & 12237,964 & $\begin{array}{c}\text { SEM- } \\
\text { S9M } \\
\end{array}$ \\
\hline sites & $\begin{array}{c}\text { Power Export to } \\
\text { Grid }\end{array}$ & 6мN & $N / A$ & $\mathrm{~N} / \mathrm{A}$ & $\begin{array}{c}\text { Forty GE } 15 \mathrm{MN} \\
\text { Turbines } \\
\end{array}$ & $6 \mathrm{MN}$ & $120.6 \%$ & 122.379 .640 & $\begin{array}{l}\text { S75M. } \\
585 \mathrm{M} \\
\end{array}$ \\
\hline & \multicolumn{9}{|c|}{$\begin{array}{l}\text { Initial wind project capital costs identified } \\
\text { ranged from } \$ 250,000 \text { to } \$ 85 \text { million }\end{array}$} \\
\hline
\end{tabular}




\begin{tabular}{|c|c|c|c|c|c|c|c|c|c|}
\hline & \multicolumn{9}{|c|}{ Initial Solar Project Configurations } \\
\hline \multirow{3}{*}{ Multiple } & 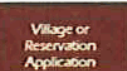 & 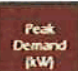 & 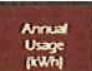 & $\begin{array}{l}\operatorname{lol} x \\
\text { fatox }\end{array}$ & 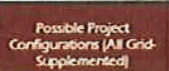 & $\begin{array}{l}\text { Peat } \\
\text { pensed } \\
\text { pand }\end{array}$ & 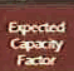 & 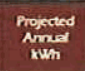 & $\cos _{\cos 2}$ \\
\hline & 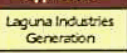 & 54 & 1.620 .000 & $340 \%$ & $\begin{array}{l}\text { Twerny } 25 \text { KW Dish } \\
\text { Engremodies }\end{array}$ & $5000 \mathrm{~N}$ & $25: 5$ & $1.1 \mathrm{Gm}$ & $525 \mathrm{M}$ \\
\hline & 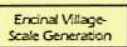 & 102 & 33,023 & 44006 & 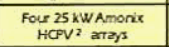 & $1000 \mathrm{~N}$ & 236 & 203000 & s600k \\
\hline \multirow{3}{*}{$\begin{array}{c}\text { solar project } \\
\text { options }\end{array}$} & 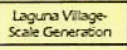 & 2.033 & $9,207,437$ & $505 \%$ & Parasosctrough & $2 \mathrm{MN}$ & $25 \%$ & $4.40 \mathrm{~mm}$ & $\operatorname{sen}$ \\
\hline & 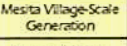 & $9 \div 9$ & 4.073.652 & 49060 & Prasabecic Trough & $\mathrm{imw}$ & $25 \%$ & $220 \mathrm{wn}$ & $\operatorname{sen}$ \\
\hline & 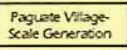 & 472 & 1.9538 .83 & $473 \%$ & 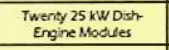 & $5000 \mathrm{~N}$ & $25 \%$ & $1.1 \mathrm{Gm}$ & $525 \mathrm{M}$ \\
\hline \multirow{4}{*}{$\begin{array}{c}\text { were } \\
\text { considered } \\
\text { at identified } \\
\text { sites }\end{array}$} & 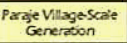 & 1,392 & $6.206,168$ & 50.5s & Perabotic Trough & $\operatorname{Ims}$ & $25 \%$ & $22 \mathrm{Gm}$ & $\sin$ \\
\hline & 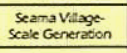 & 427 & $1,733.998$ & $47.7 \%$ & 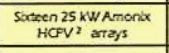 & $4000 \mathrm{~W}$ & $23 \%$ & 812000 & $522 \mathrm{M}$ \\
\hline & 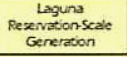 & 5.426 & 23.618 .151 & 49.756 & Perabolic Trough & $\sin \omega$ & $25 \%$ & $1090 \mathrm{~mm}$ & s2om \\
\hline & $\begin{array}{l}\text { Power Epront to } \\
\text { Grid }\end{array}$ & GoMn & $N / A$ & N/A & Prababoc Trough & 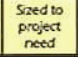 & $25 \%$ & $132 \mathrm{own}$ & s2tan \\
\hline & \multicolumn{9}{|c|}{$\begin{array}{l}\text { Initial solar project capital costs identified } \\
\text { ranged from } \$ 600,000 \text { to } \$ 240 \text { million }\end{array}$} \\
\hline
\end{tabular}

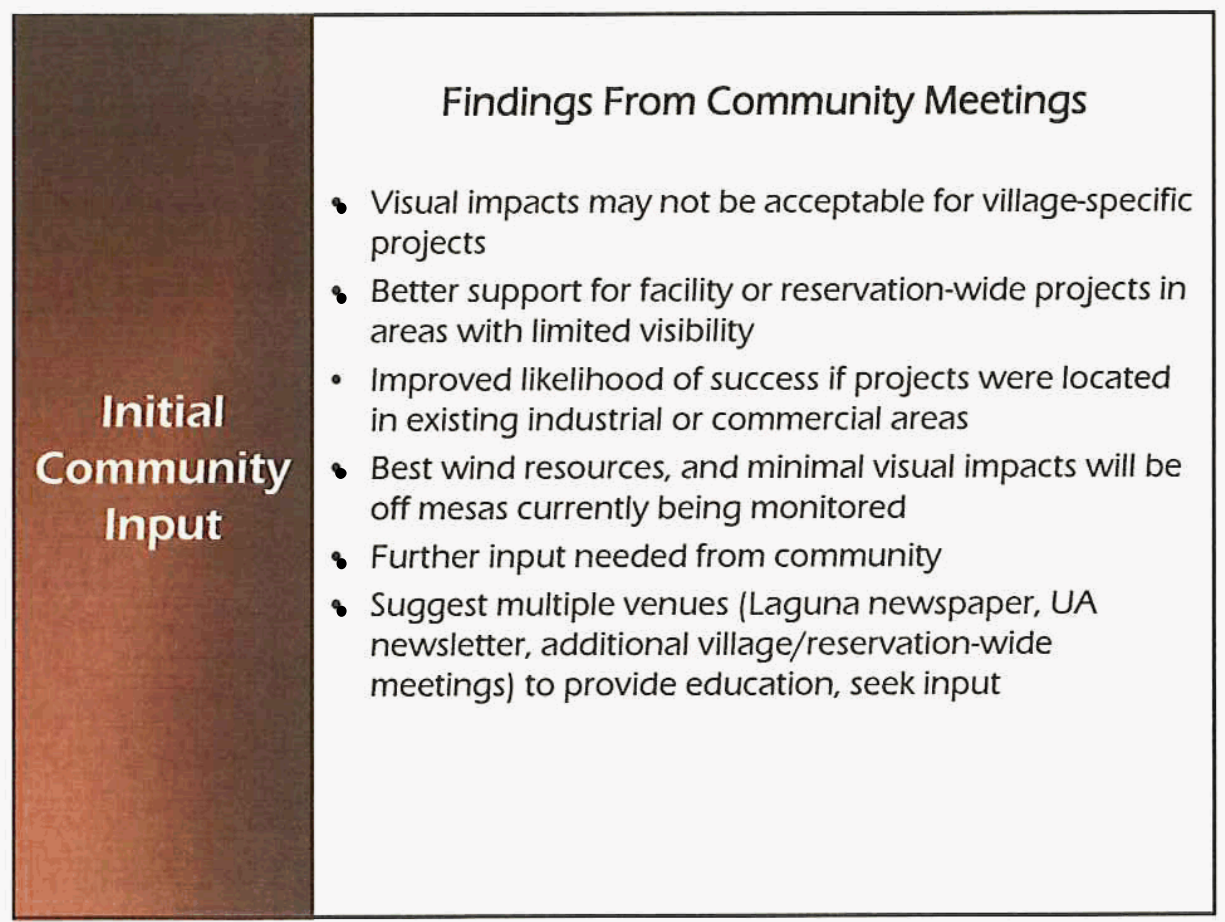




\section{Preliminary Findings}

- Sites under consideration adjacent to properties previously impacted by some activity (i.e. development, grazing, agriculture, access roads, etc.)

- All sites appear clear of any immediate cultural or historical fatal flaws

Preliminary environmental findings did not elliminate any sites
- Washes present at sites \# 1 and \#5, but sites flexible re: mitigation

- Dry washes; no apparent endangered species issues

- Multiple sites available allow for protection of "view sheds"

- Additional transmission/distribution ROWs yet to be reviewed

- Alternative sites must be identified under any Environmental Assessment

- FONSI (finding of no significant impact) may not be considered, since proposed projects are a "change in use"

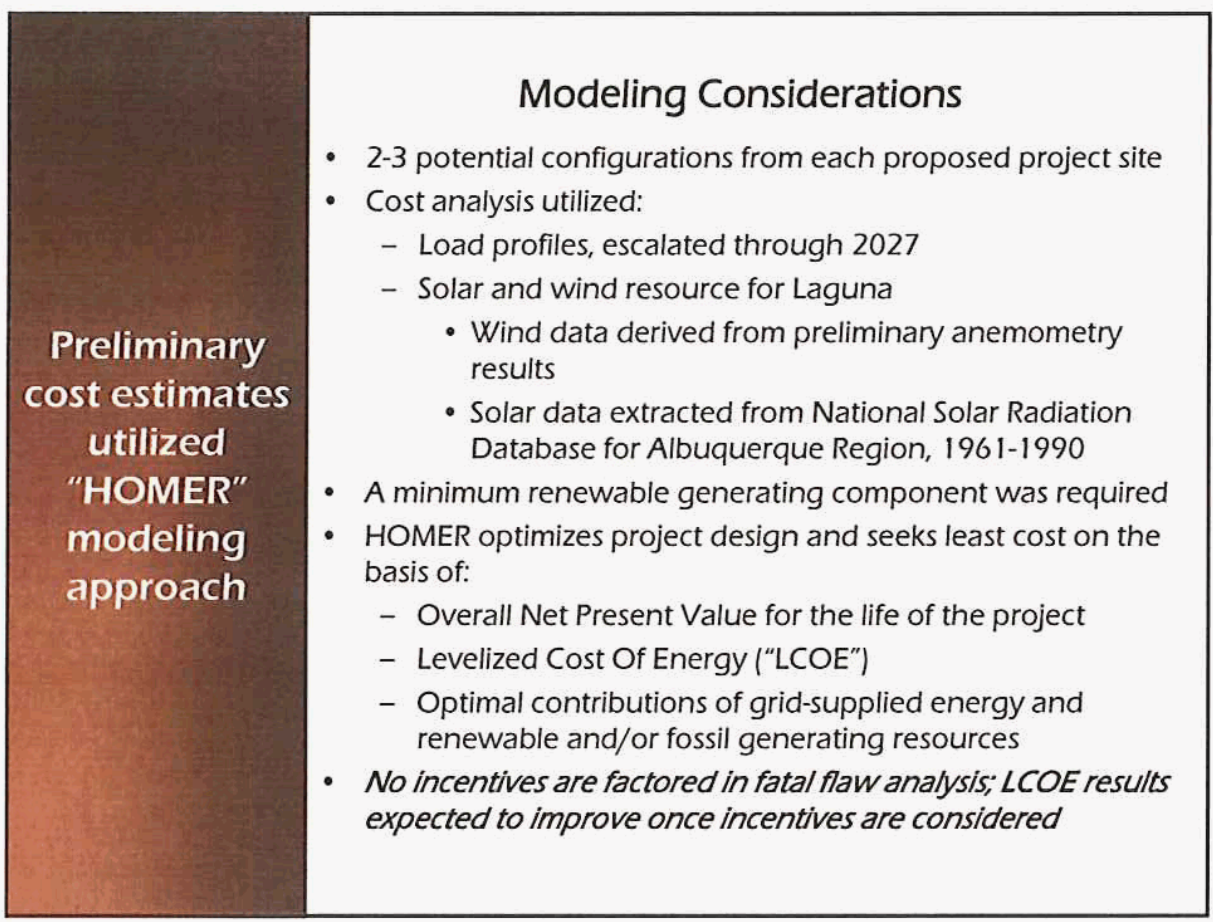




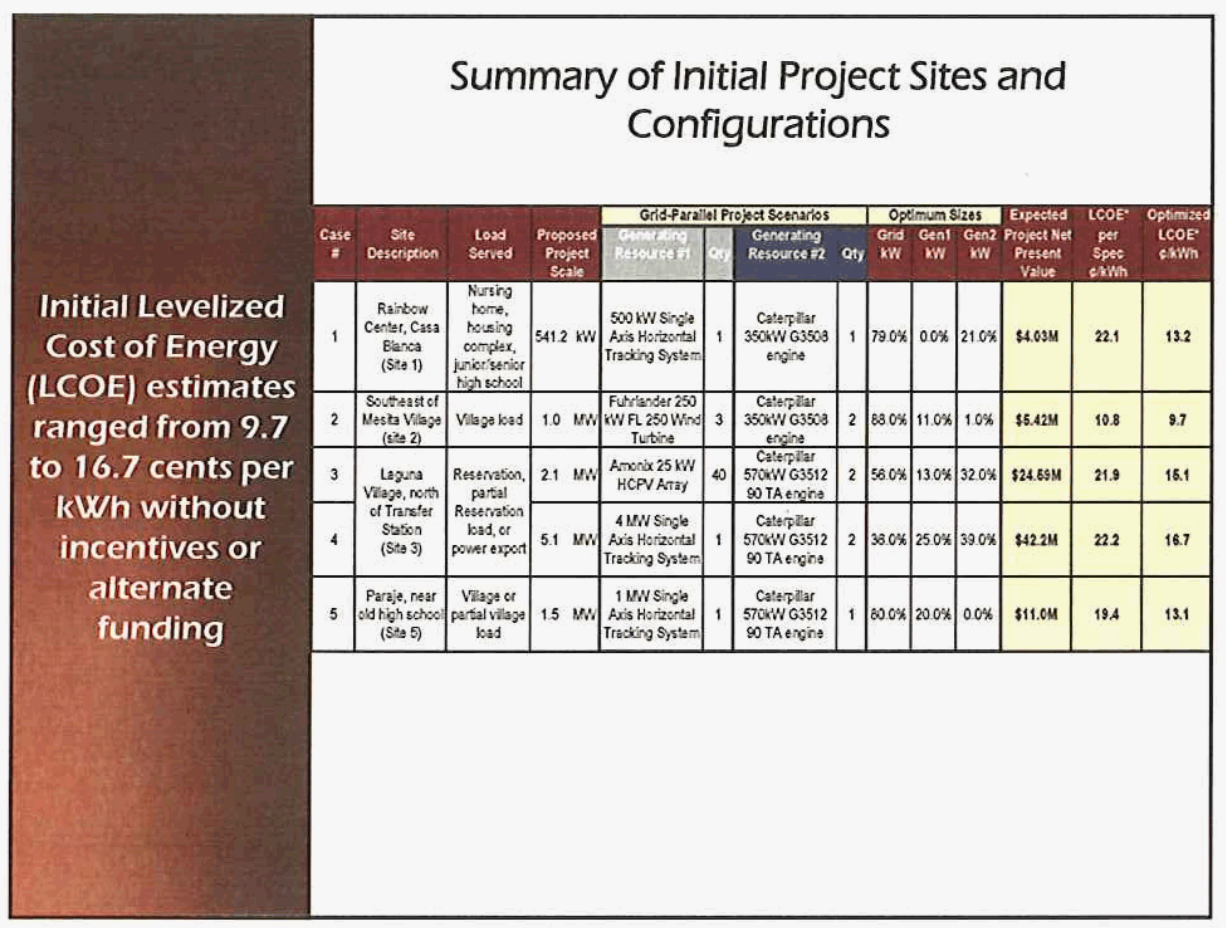

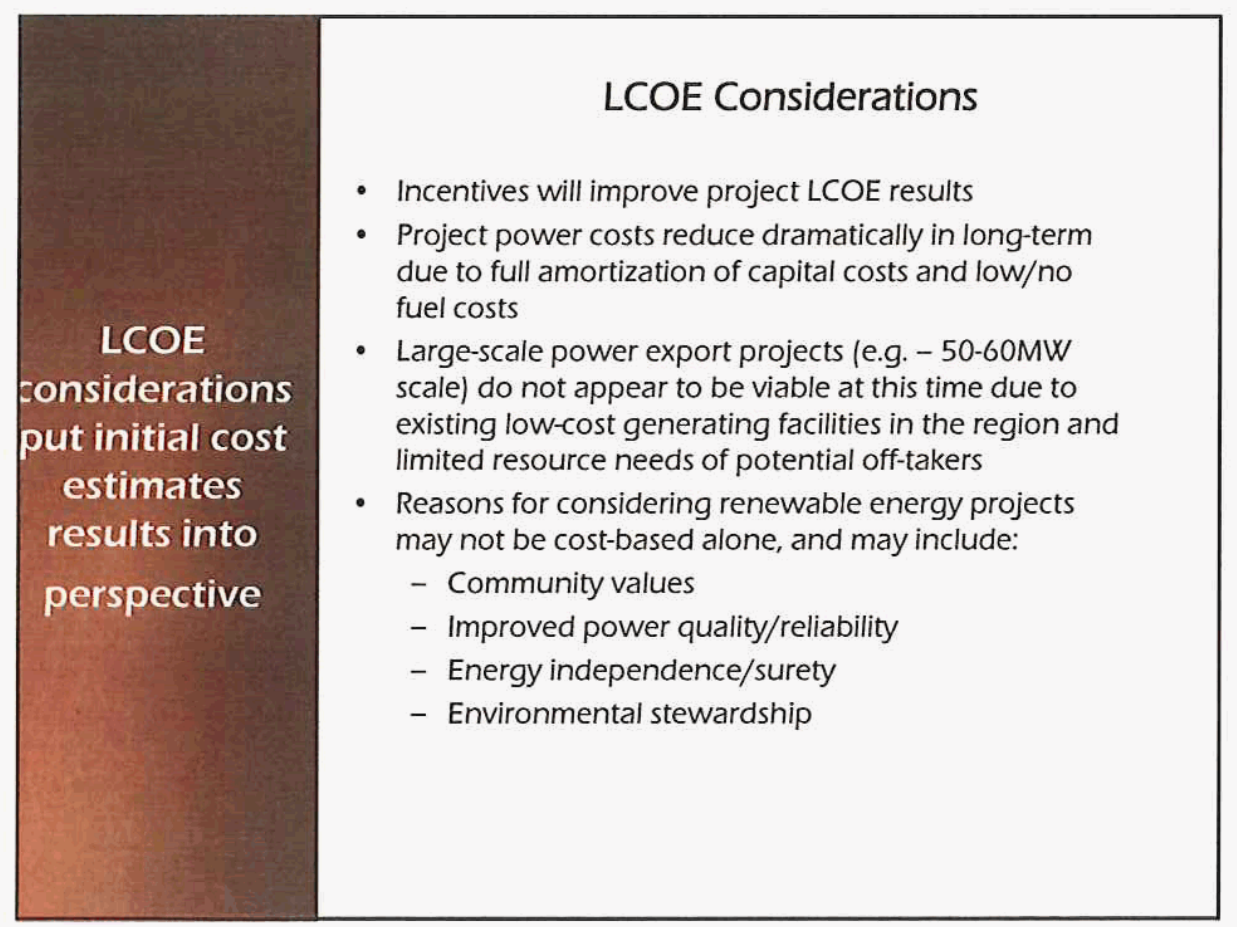




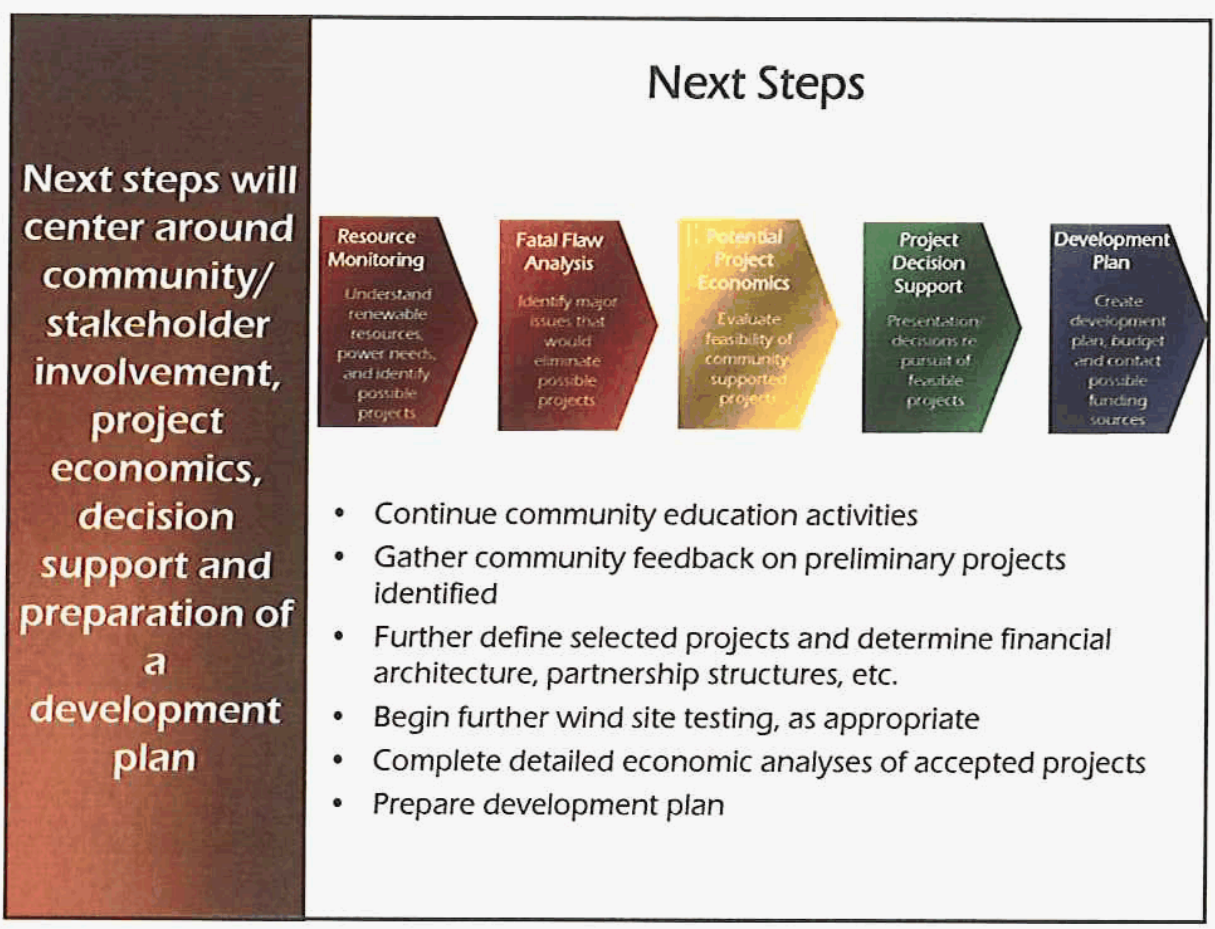




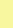



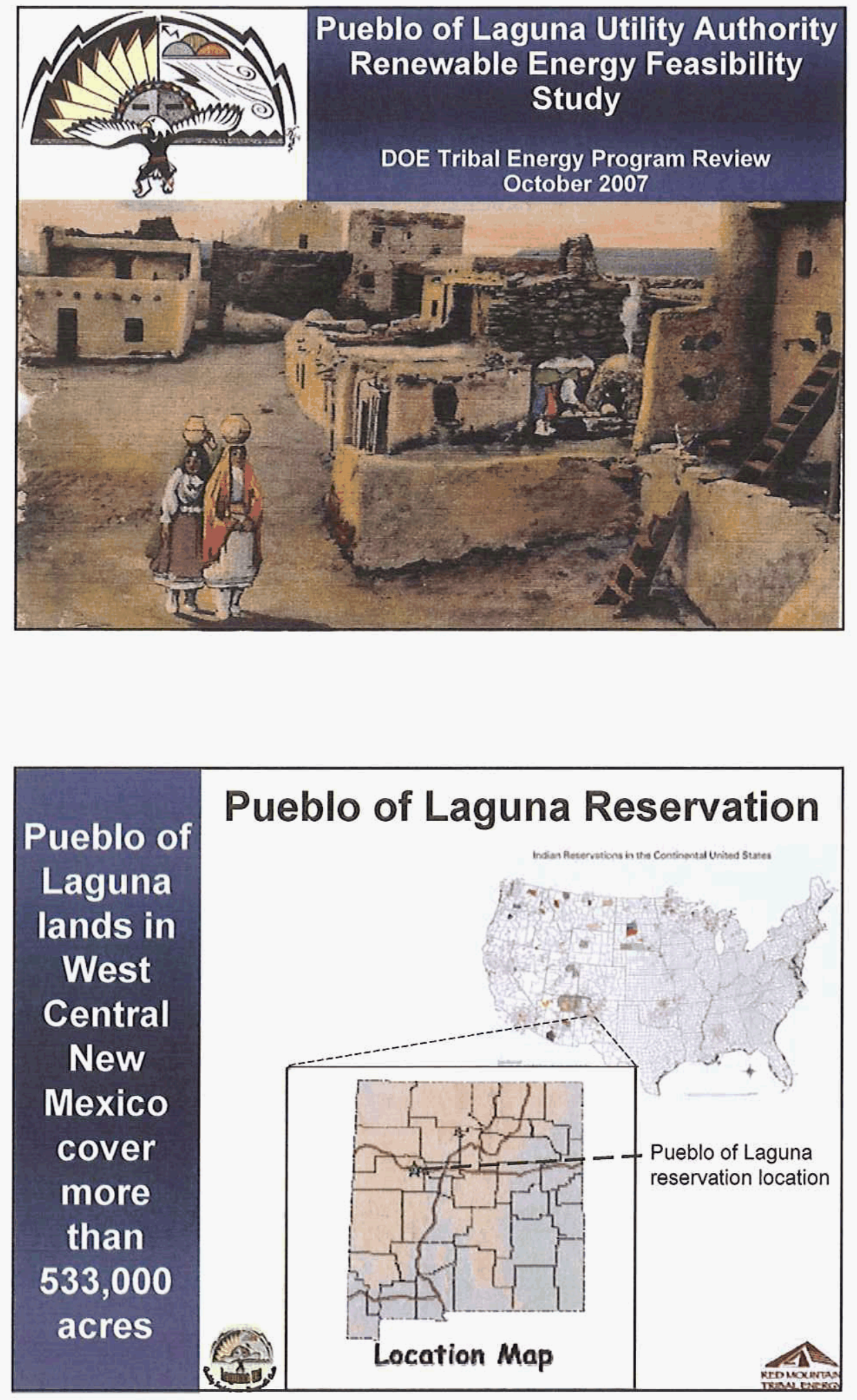

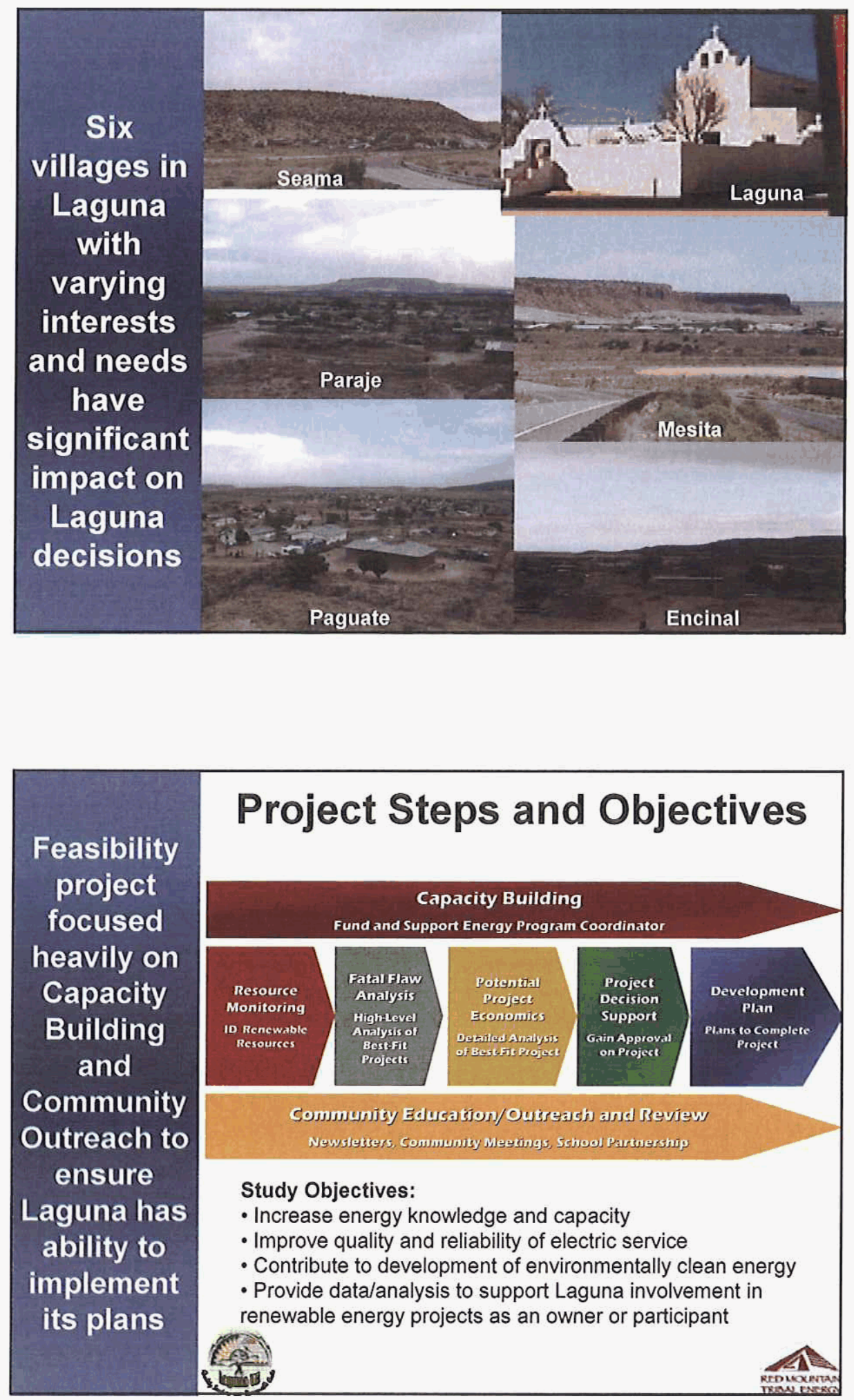


\begin{tabular}{|c|c|}
\hline \multirow{3}{*}{$\begin{array}{l}\text { Project is } \\
\text { being led }\end{array}$} & \multirow{3}{*}{ Project Participants } \\
\hline & \\
\hline & \\
\hline by Laguna & - Tribal and Staff Participants: \\
\hline UA & - Pueblo of Laguna Utility Authority and \\
\hline personnel, & Board of Directors \\
\hline with & - Pueblo of Laguna Tribal Council and \\
\hline technical & - Pueblo of Laquna Villages \\
\hline support & - Pueblo of Laguna Entities/Facility \\
\hline $\begin{array}{l}\text { provided } \\
\text { bv Red }\end{array}$ & Managers \\
\hline $\begin{array}{l}\text { D)y Red } \\
\text { Mountain }\end{array}$ & - Project Consultant: \\
\hline $\begin{array}{l}\text { Mountain } \\
\text { Tribal }\end{array}$ & - Red Mountain Tribal Energy \\
\hline Energy & \\
\hline & \\
\hline
\end{tabular}

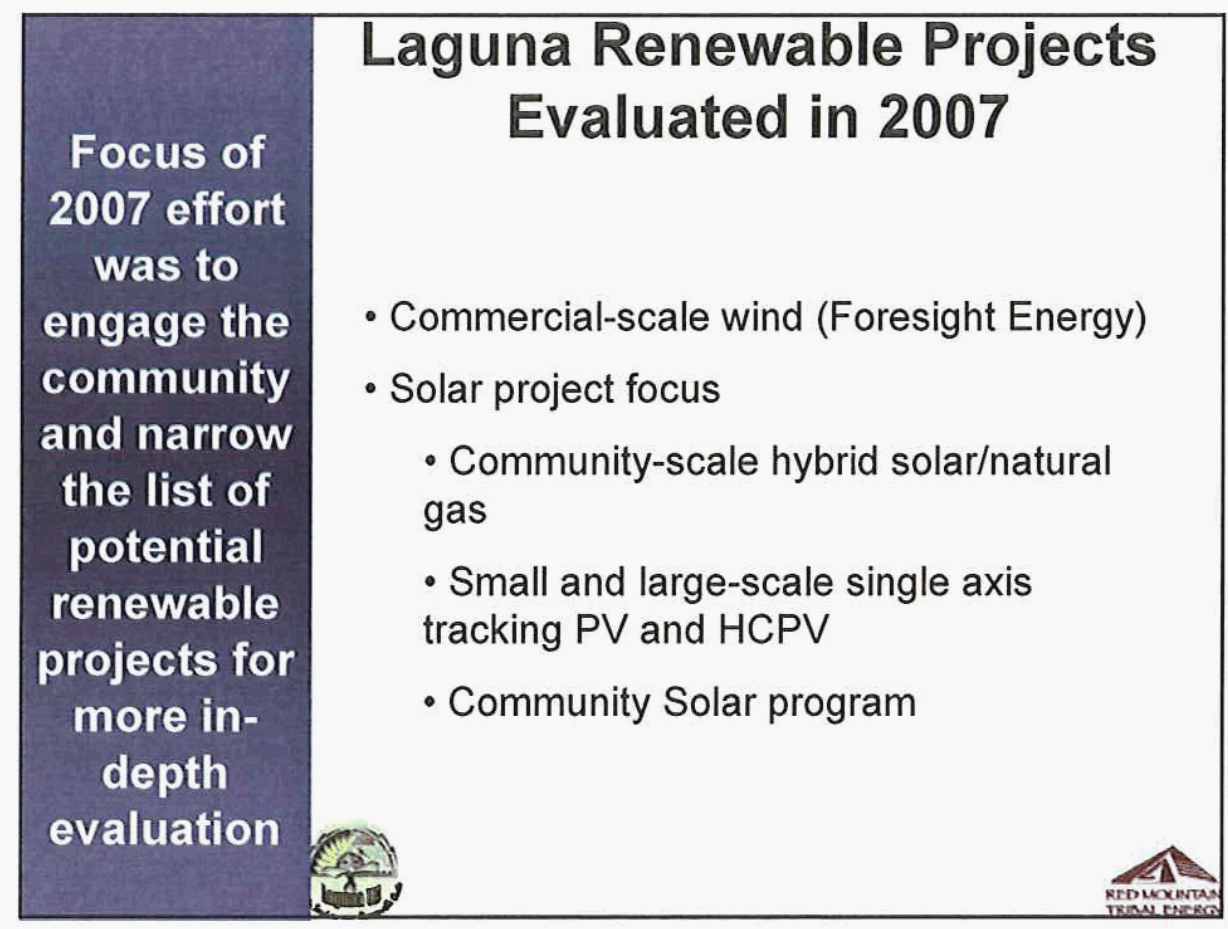



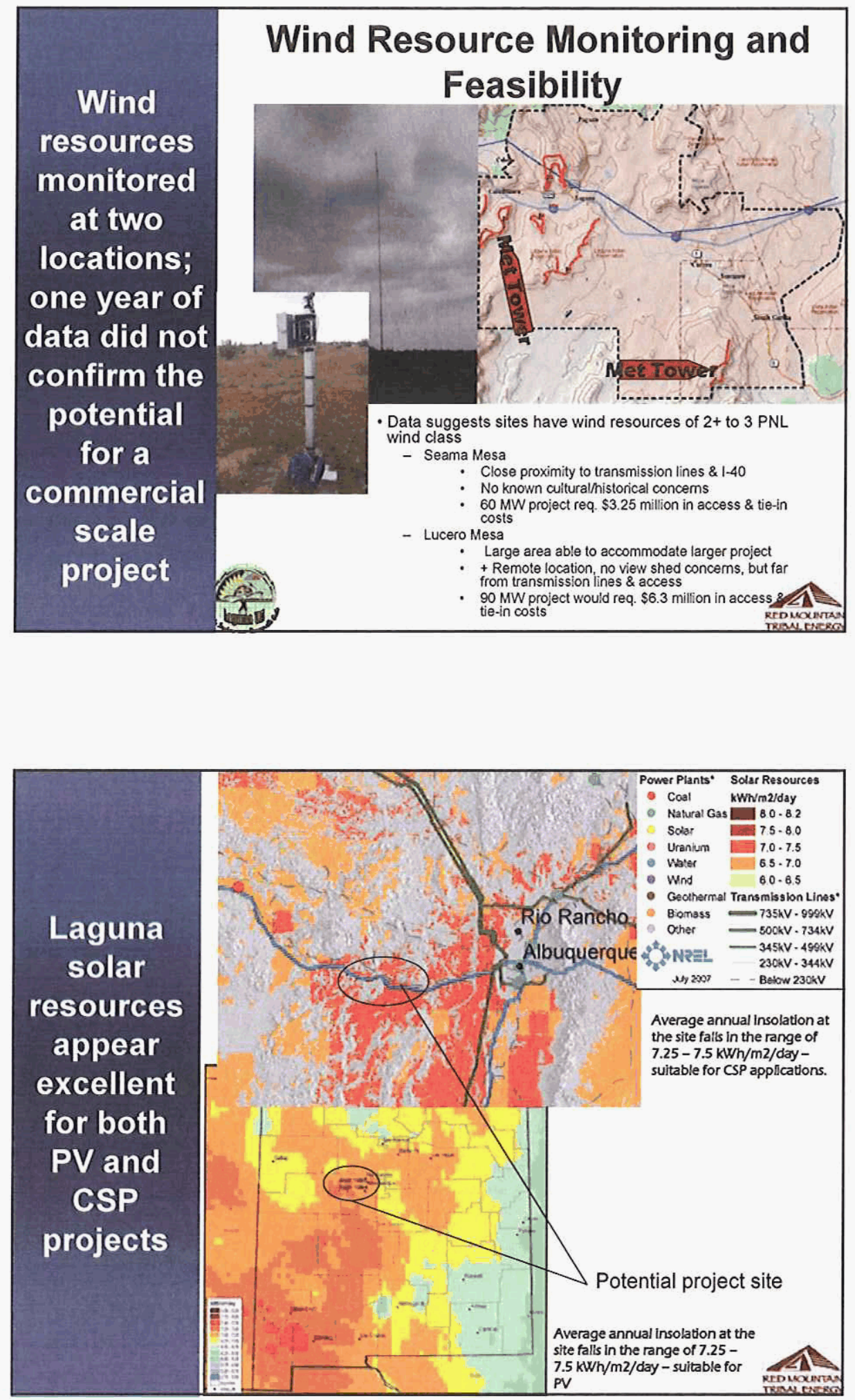


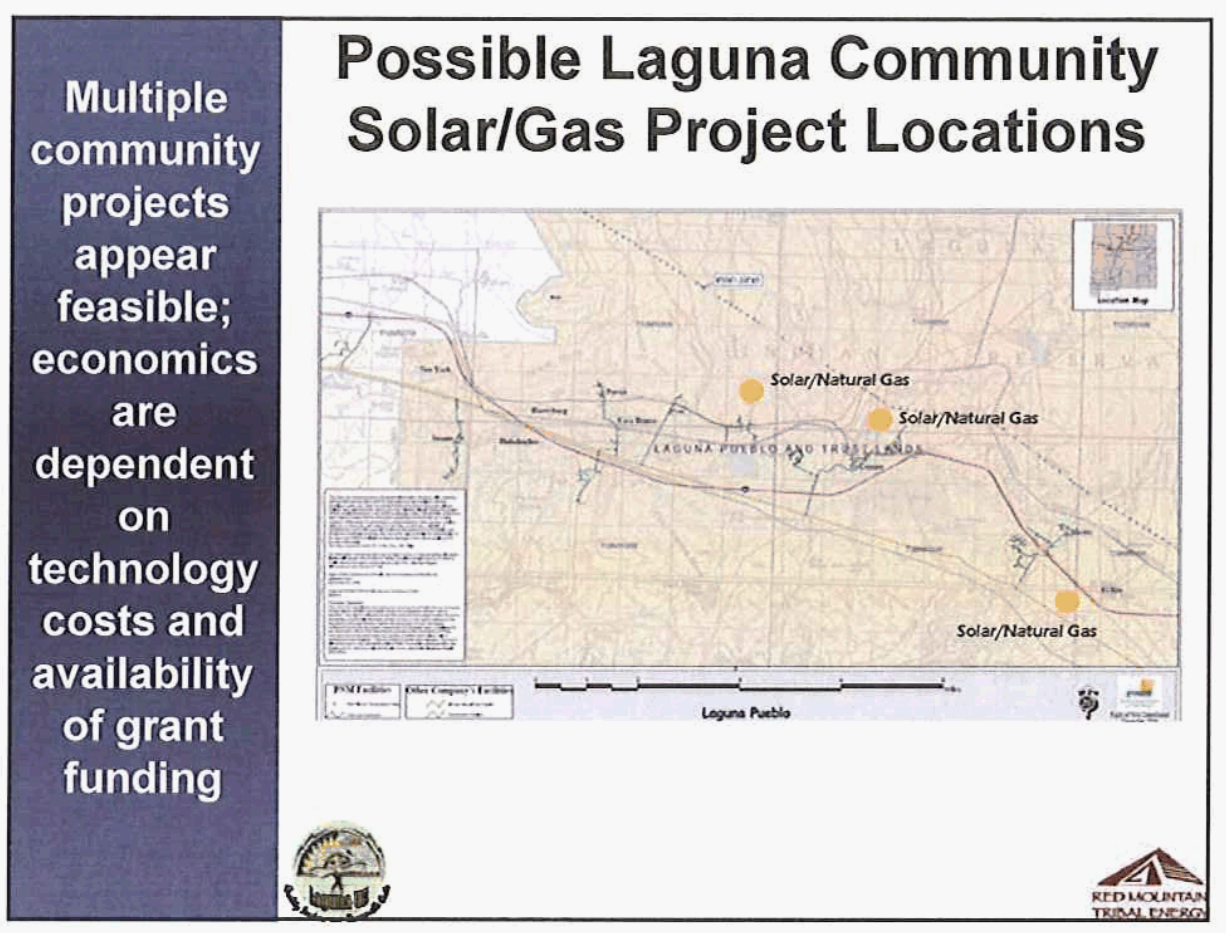

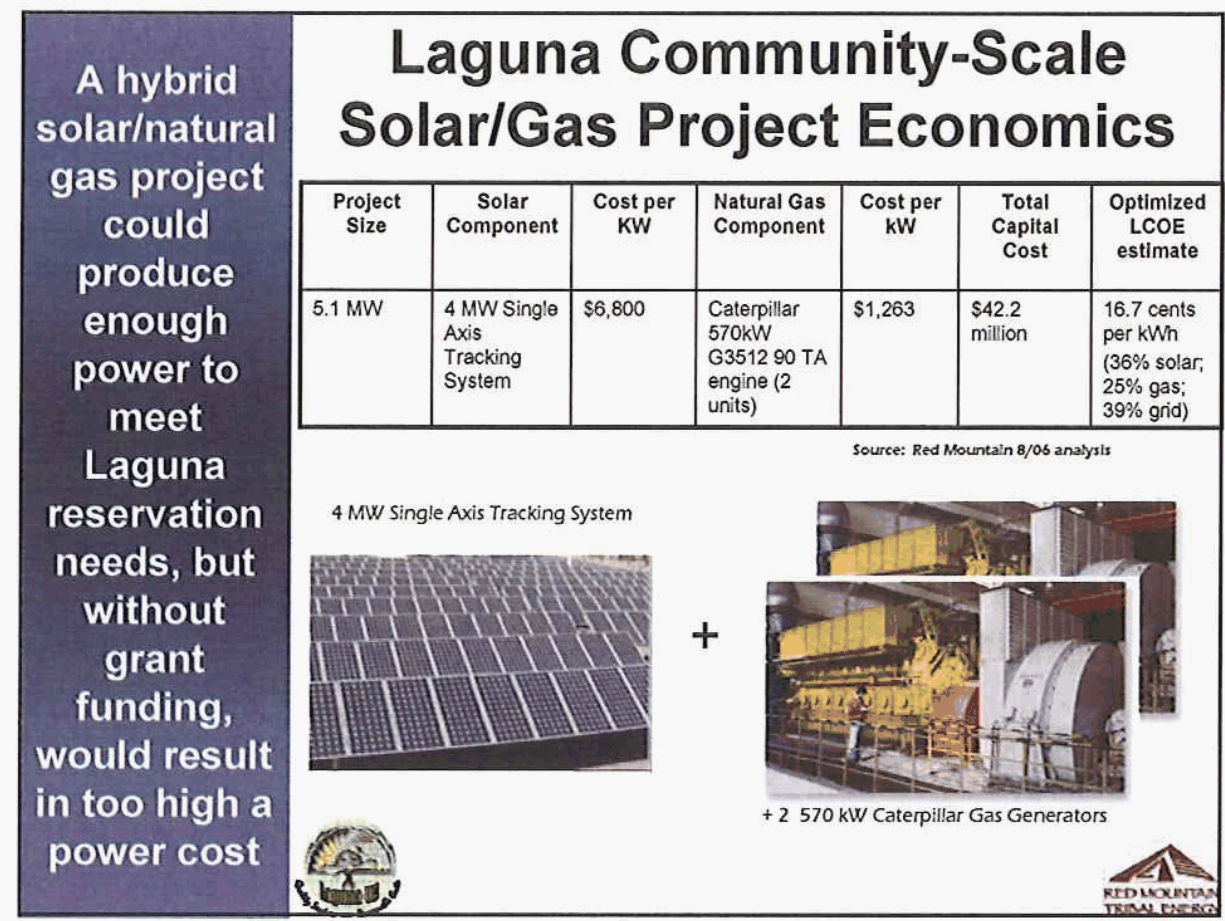



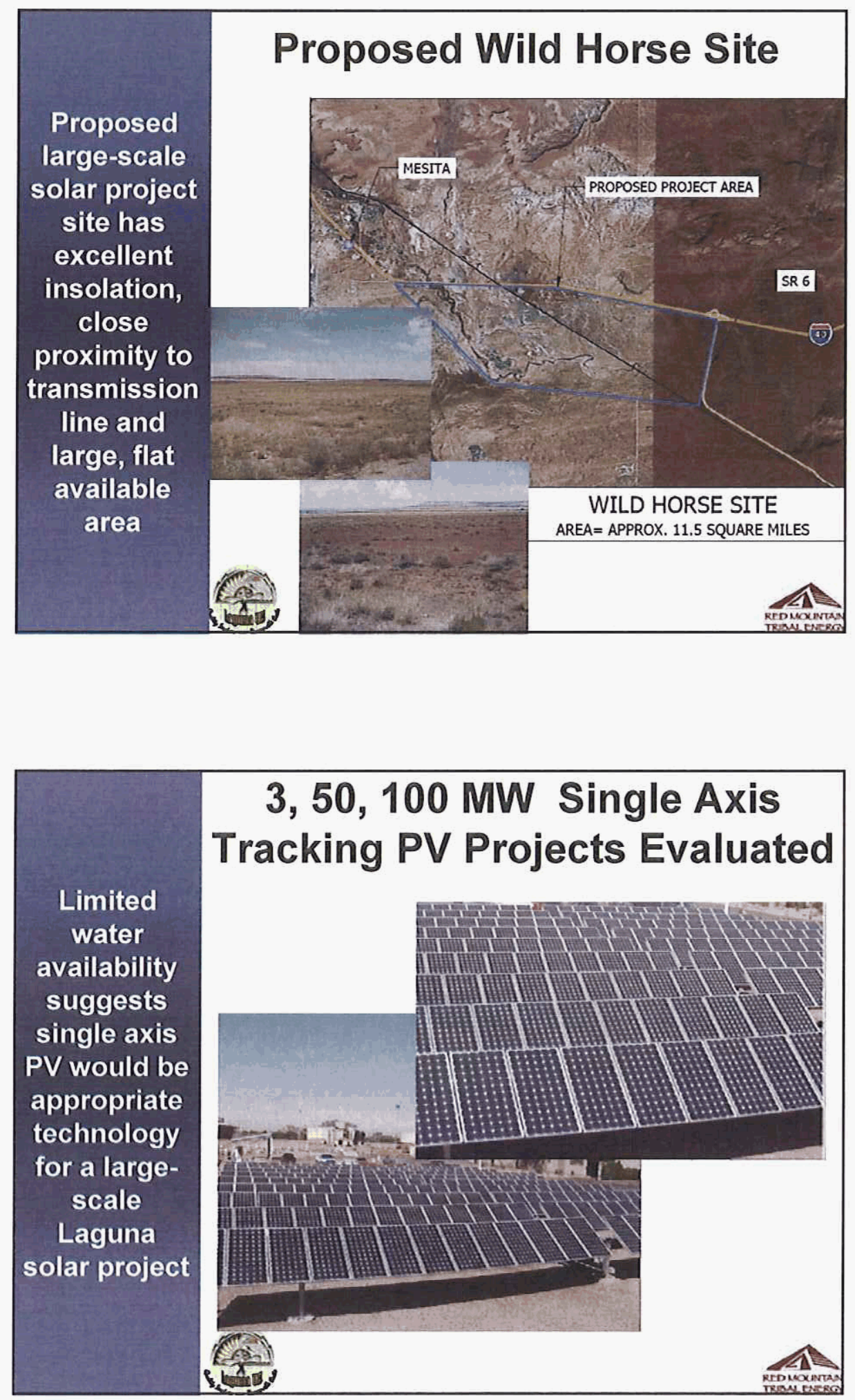


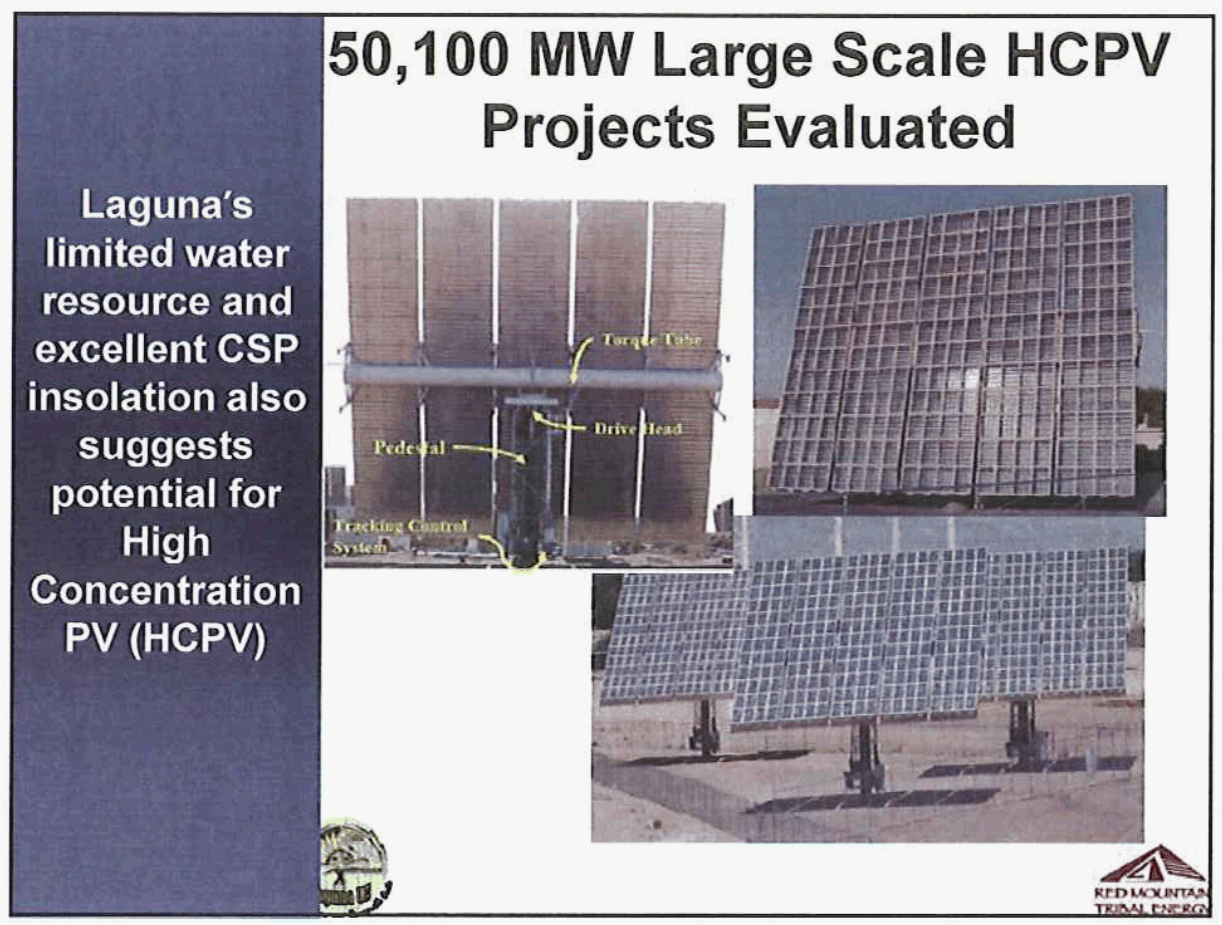

\begin{tabular}{|c|c|}
\hline & $\begin{array}{c}\text { Laguna Solar Project Economic } \\
\text { Considerations }\end{array}$ \\
\hline $\begin{array}{l}\text { Scale of } \\
\text { Laguna } \\
\text { project and } \\
\text { available } \\
\text { technology/ } \\
\text { costs } \\
\text { suggest } \\
\text { different } \\
\text { ownership } \\
\text { approaches }\end{array}$ & $\begin{array}{l}\text { - Laguna Community Scale Solar/Gas Energy Project } \\
\text { - Laguna owned/operated w/grant funding likely } \\
\text { to provide best economics } \\
\text { - Grant funding could reduce capital outlay } \\
\text { - Net metered power/use as self-generation } \\
\text { - RECs sold separately } \\
\text { - Laguna Large Scale Solar Energy Project } \\
\text { - Developer/Tax Partner Joint Venture likely to } \\
\text { provide best economics } \\
\text { - Tax partner can take advantage of tax } \\
\text { incentives (ITC) } \\
\text { - Project could sell power/RECs bundled or } \\
\text { separately } \\
\text { - Utility or developer could partner with Laguna }\end{array}$ \\
\hline & 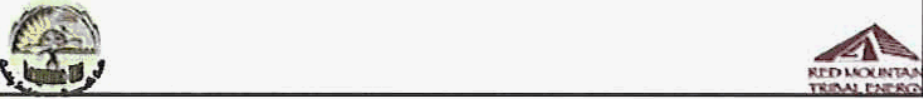 \\
\hline
\end{tabular}




\begin{tabular}{|c|c|c|c|c|c|}
\hline \multirow{3}{*}{$\begin{array}{l}\text { Laguna } \\
\text { renewable } \\
\text { resources } \\
\text { allowed for } \\
\text { multiple } \\
\text { projects to } \\
\text { be }\end{array}$} & \multicolumn{5}{|c|}{$\begin{array}{r}\text { Laguna Solar Pro } \\
\text { Comparison }\end{array}$} \\
\hline & & $\begin{array}{l}\text { Project Capital } \\
\text { Cost (includes } \\
\text { development, } \\
\text { transmission } \\
\text { interconnection } \\
\text { costs) }\end{array}$ & $\begin{array}{l}\text { Acreage } \\
\text { Required }\end{array}$ & $\begin{array}{l}\text { Levelized } \\
\text { Cost of } \\
\text { Energy * } \\
\text { (Cents per } \\
\text { kWh) }\end{array}$ & $\begin{array}{c}\text { Capital Cost } \\
\text { Considerations }\end{array}$ \\
\hline & \begin{tabular}{|l|}
3 MW \\
Single axis \\
tracking PV
\end{tabular} & $\$ 28.3$ million & 30 & & $\begin{array}{l}\text { Assumes lower- } \\
\text { cost ClGS } \\
\text { modules }\end{array}$ \\
\hline $\begin{array}{l}\text { evaluated; } \\
\text { with current }\end{array}$ & $\begin{array}{l}50 \mathrm{MW} \\
\text { Single axis } \\
\text { tracking PV }\end{array}$ & $\$ 410$ million & 500 & & $\begin{array}{l}\text { Assumes lower- } \\
\text { cost CIGS } \\
\text { modules; } 20 \% \\
\text { economies of scale }\end{array}$ \\
\hline $\begin{array}{c}\text { technology } \\
\text { cost }\end{array}$ & $\begin{array}{l}50 \text { MW } \\
\text { HCPV }\end{array}$ & $\$ 188$ million & 530 & & $\begin{array}{l}\text { Assumes projected } \\
\text { CPV cost } \\
\text { reductions }\end{array}$ \\
\hline performance & $\begin{array}{l}100 \mathrm{MW} \\
\text { Single axis } \\
\text { tracking PV }\end{array}$ & $\$ 604$ million & 1000 & & $\begin{array}{l}\text { Assumes lower- } \\
\text { cost CIGS } \\
\text { modules; } 30 \% \\
\text { economies of scale }\end{array}$ \\
\hline $\begin{array}{l}\text { 10OONAV } \\
\text { HCPV }\end{array}$ & $\begin{array}{l}100 \mathrm{MW} \\
\text { HCPV }\end{array}$ & $\$ 340$ million & 1060 & & $\begin{array}{l}\text { Assumes projected } \\
\text { CPV cost } \\
\text { reductions }\end{array}$ \\
\hline $\begin{array}{c}\text { appears } \\
\text { most viable }\end{array}$ & $\frac{25}{x+1}$ & $\begin{array}{l}\text { mes Tribal/ta } \\
\text { unding for ca }\end{array}$ & $\begin{array}{l}\text { eer JV; } \\
\text { ttlays }\end{array}$ & & lue or \\
\hline
\end{tabular}

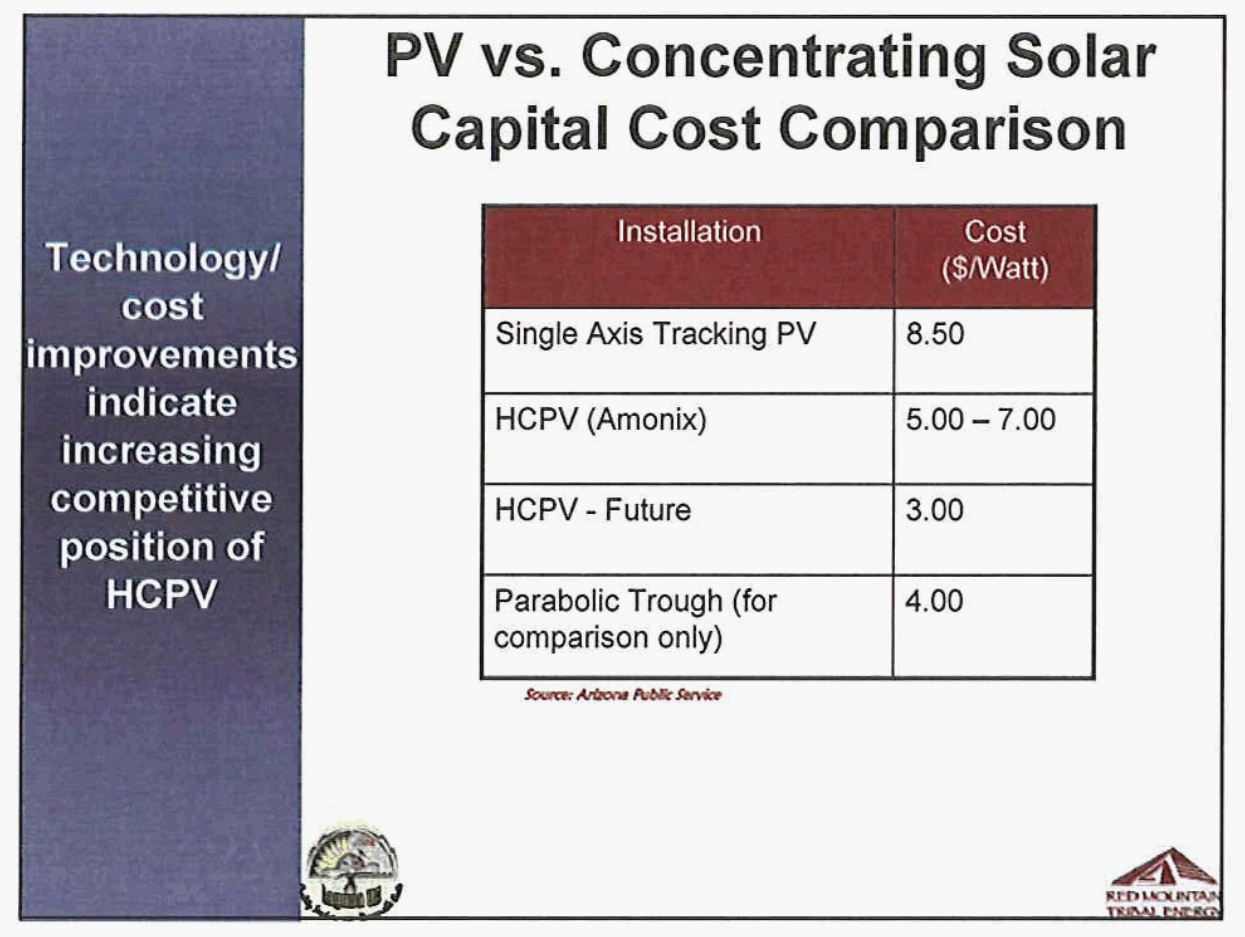




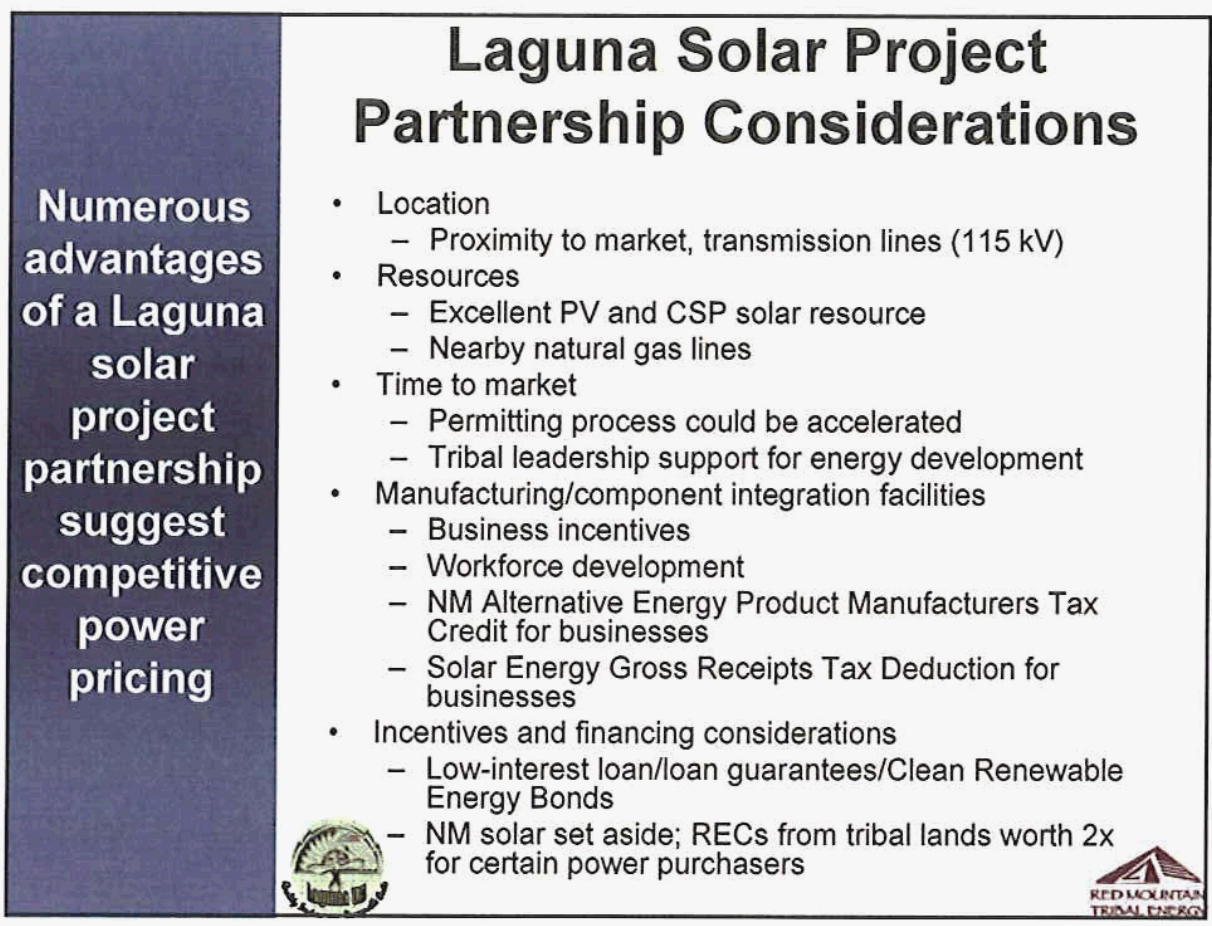

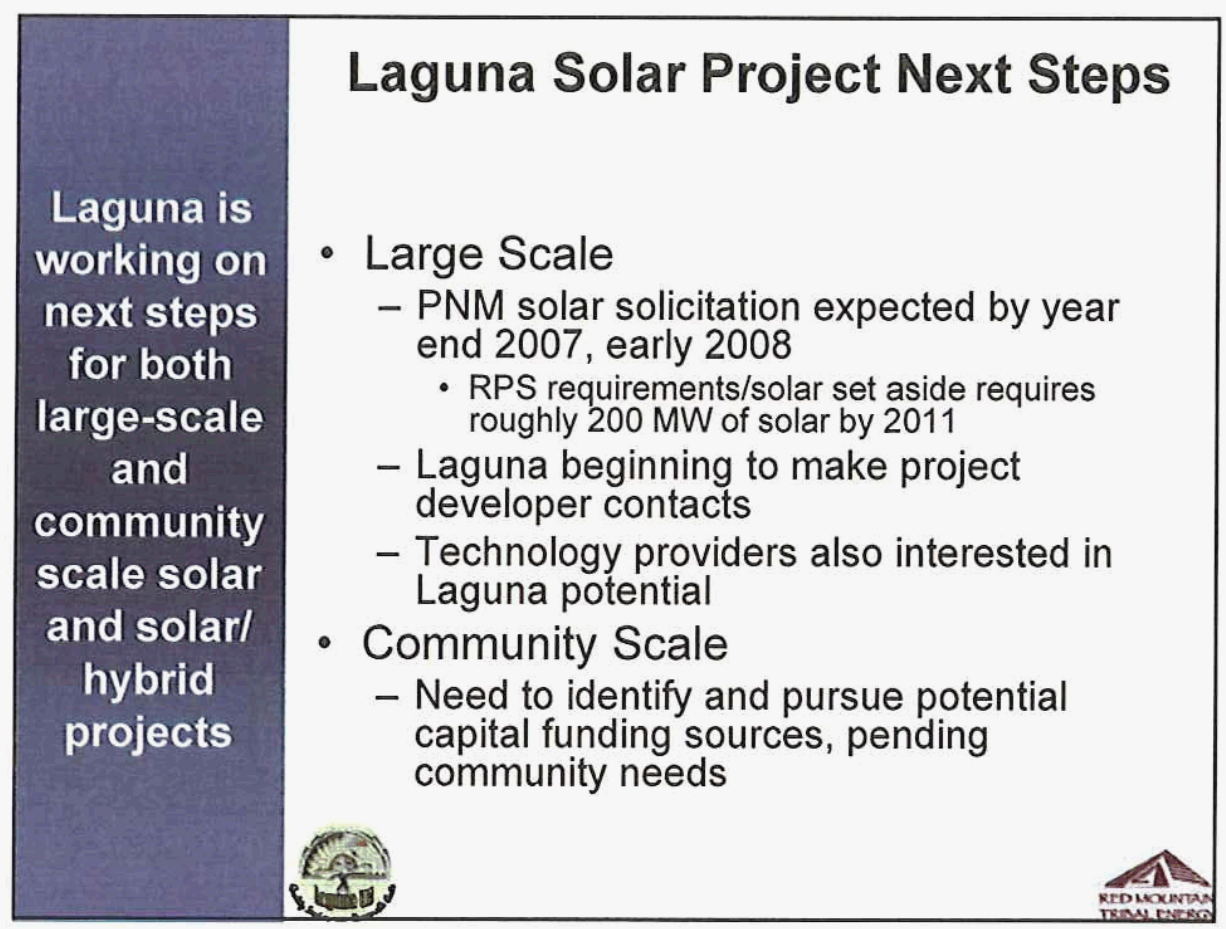




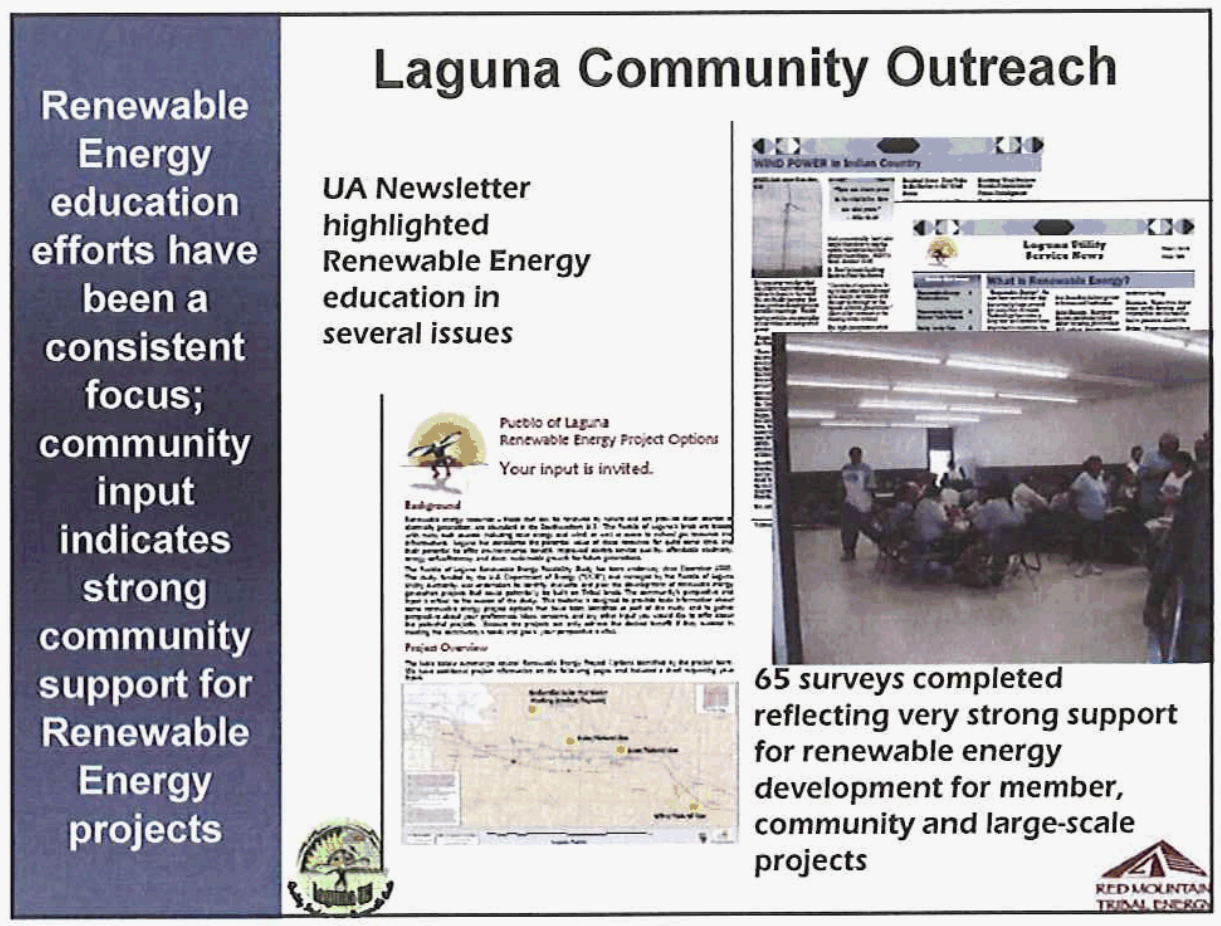

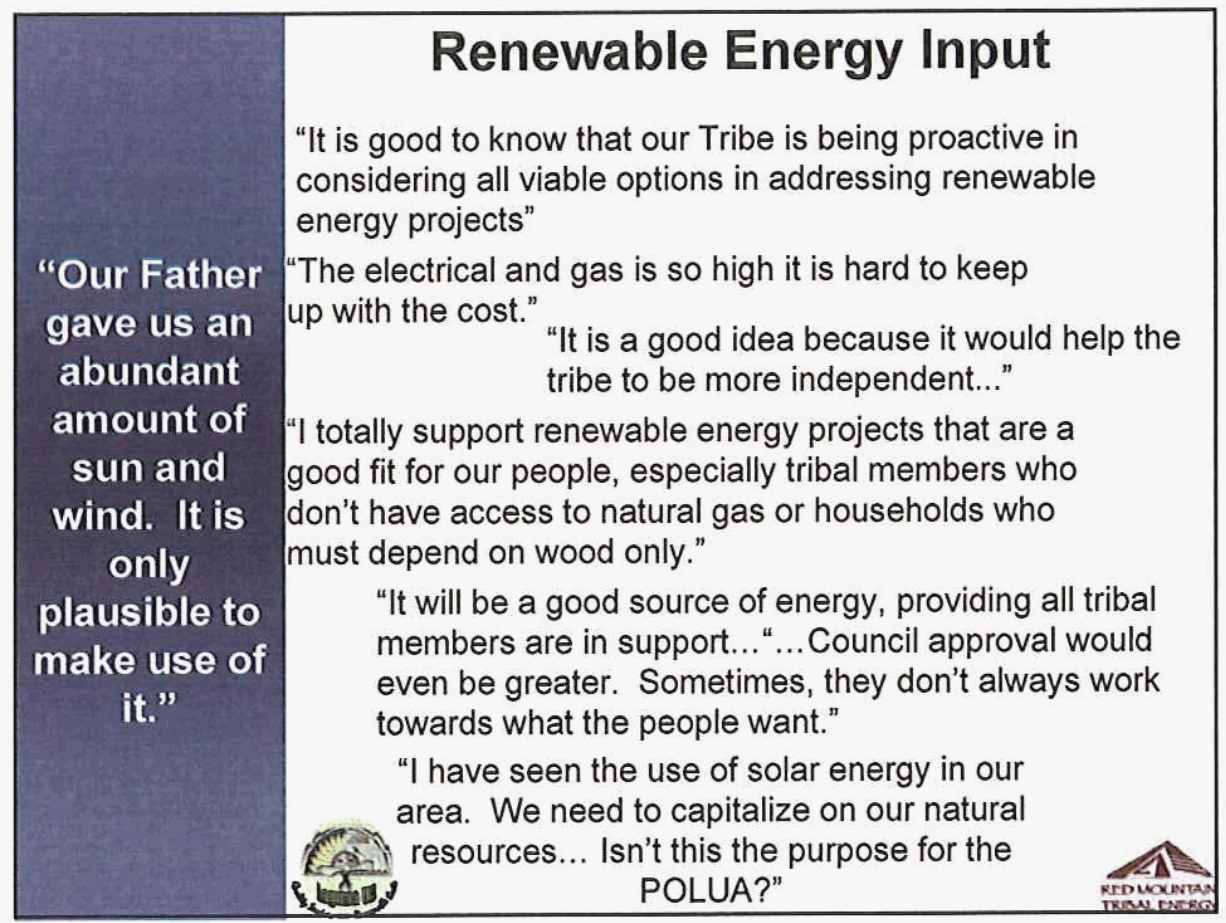




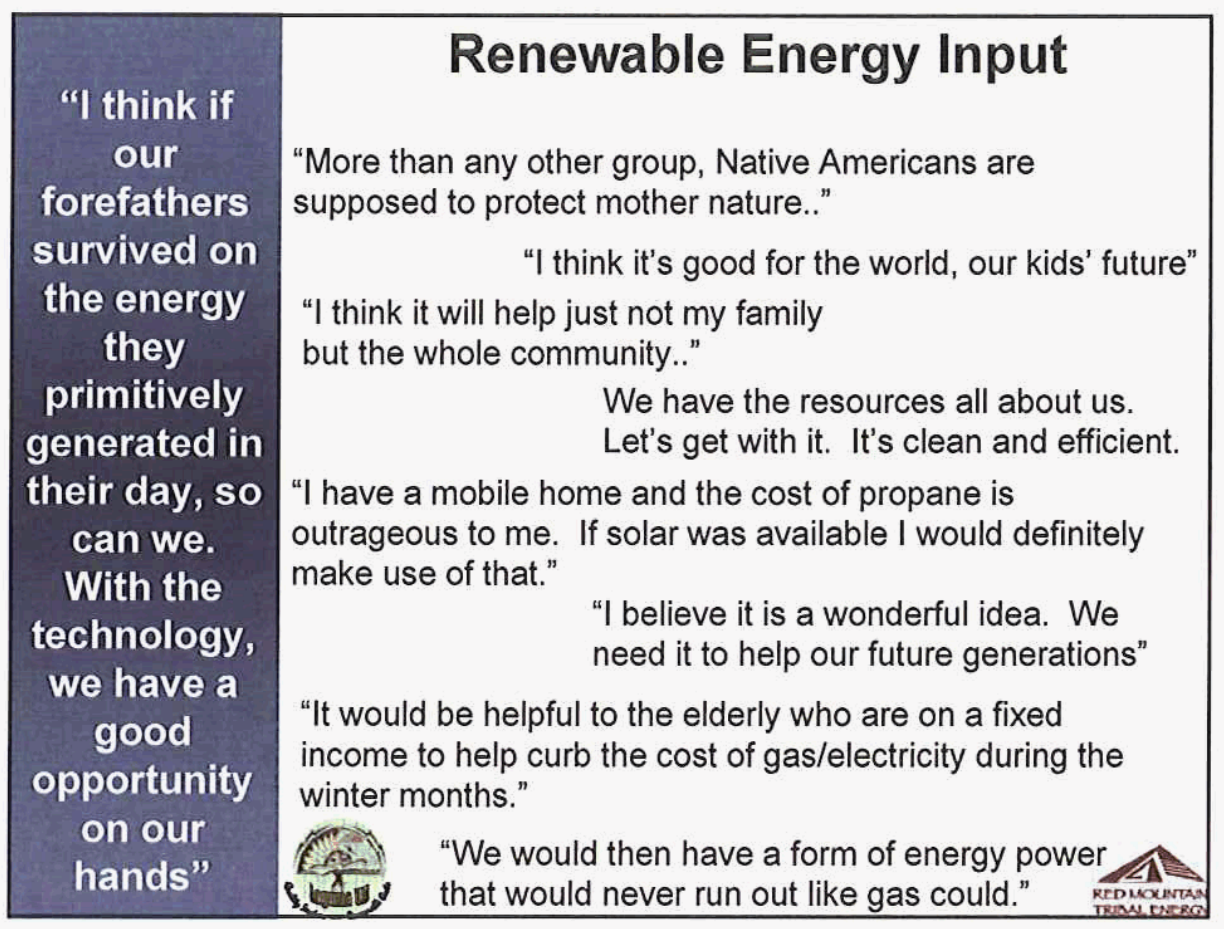

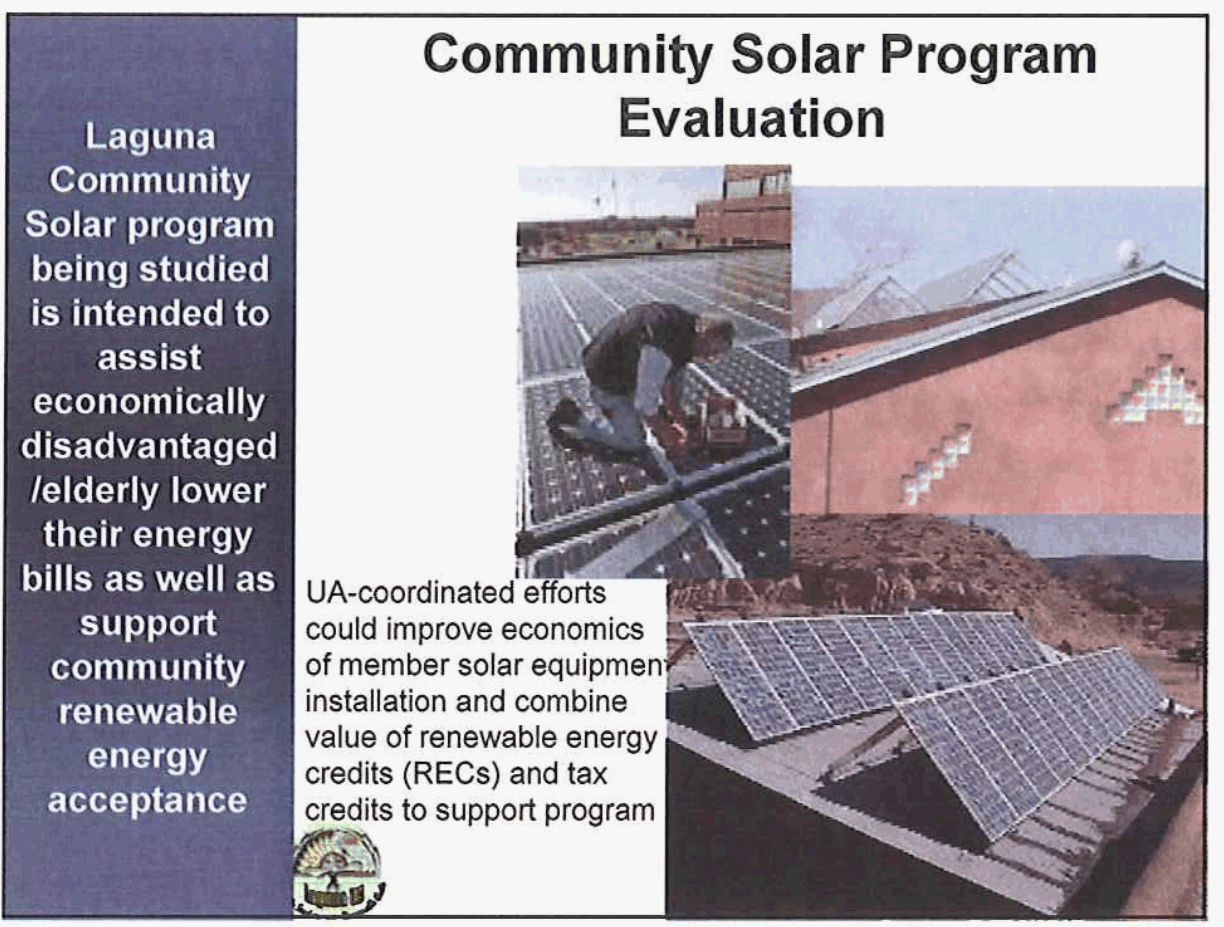




\begin{tabular}{|c|c|}
\hline $\begin{array}{c}\text { Initial } \\
\text { efforts are } \\
\text { focused on } \\
\text { installing } \\
\text { equipment } \\
\text { at six } \\
\text { community } \\
\text { centers and } \\
\text { several } \\
\text { homes to } \\
\text { serve as a } \\
\text { pilot for a } \\
\text { broader } \\
\text { Community } \\
\text { Solar } \\
\text { program }\end{array}$ & $\begin{array}{l}\text { Laguna Community Solar Program } \\
\text { - Purpose to aid elderly/disadvantaged } \\
\text { members } \\
\text { - UA program concept } \\
\text { - Purchase solar/PV equipment locally } \\
\text { - Fund purchases via bundled REC sales + tax } \\
\text { credits available } \\
\text { - Leverage any other grants/incentives available } \\
\text { to UA } \\
\text { - Make available to low-income households } \\
\text { - } 161 \text { customers already approved for payment } \\
\text { assistance } \\
\text { - UA arranges net metering with utility } \\
\text { - Meter runs backwards when sun is shining } \\
\text { - Customer bill credit would need to be } \\
\text { negotiated with CDEC }\end{array}$ \\
\hline
\end{tabular}

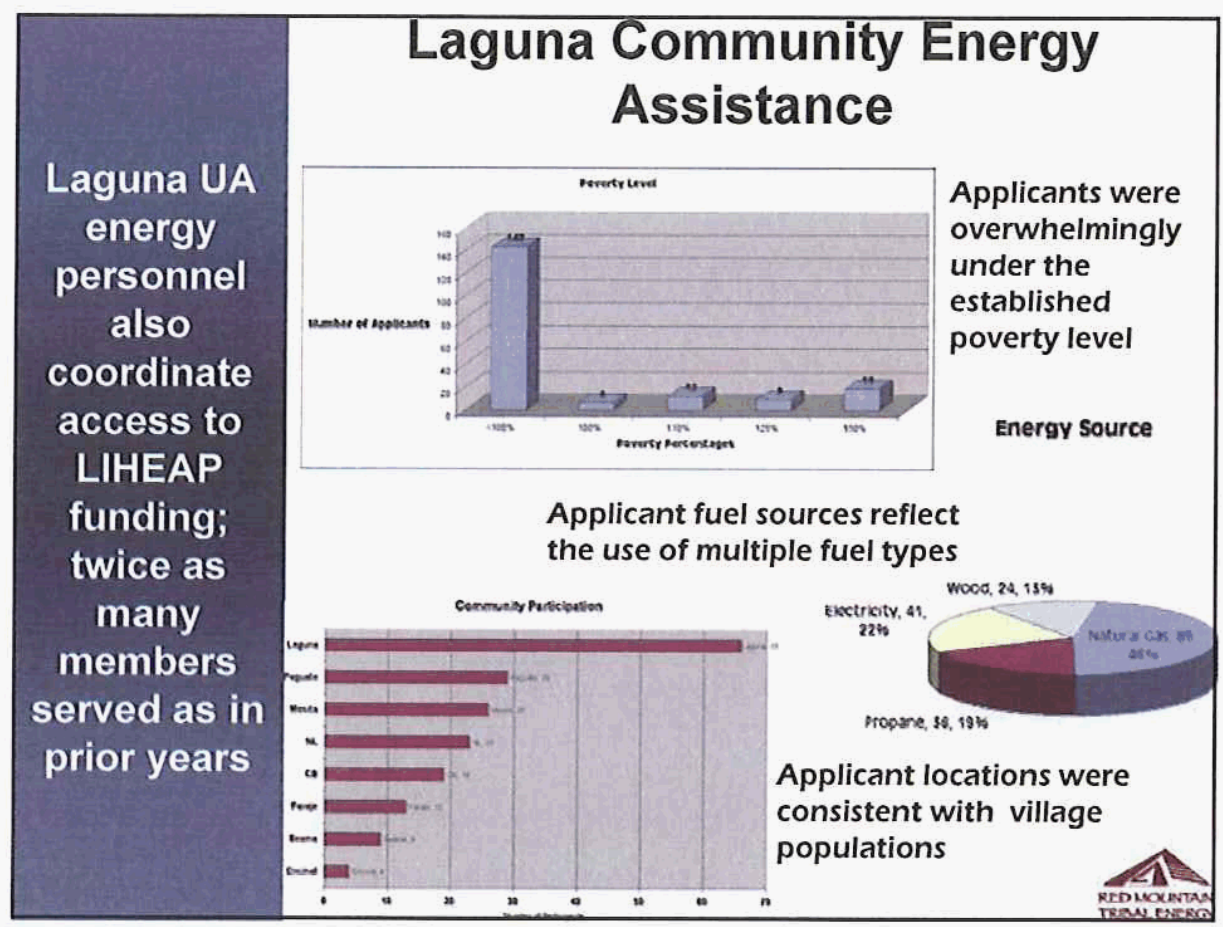




\begin{tabular}{|c|}
\hline Contact Information \\
Pueblo of Laguna Utility Authority: \\
Thelma Antonio, Energy Coordinator \\
$505246-4277$ \\
thelma.antonio@lagunaua.org \\
Ken Garcia, General Manager \\
505 246-4272 \\
ken.garcia@lagunaua.org \\
\\
\\
\\
Red Mountain Tribal Energy: \\
Carolyn Stewart, Partner/Principal \\
$602516-7540$ \\
cstewart@RedMtnTribalEnergy.com \\
\end{tabular}

